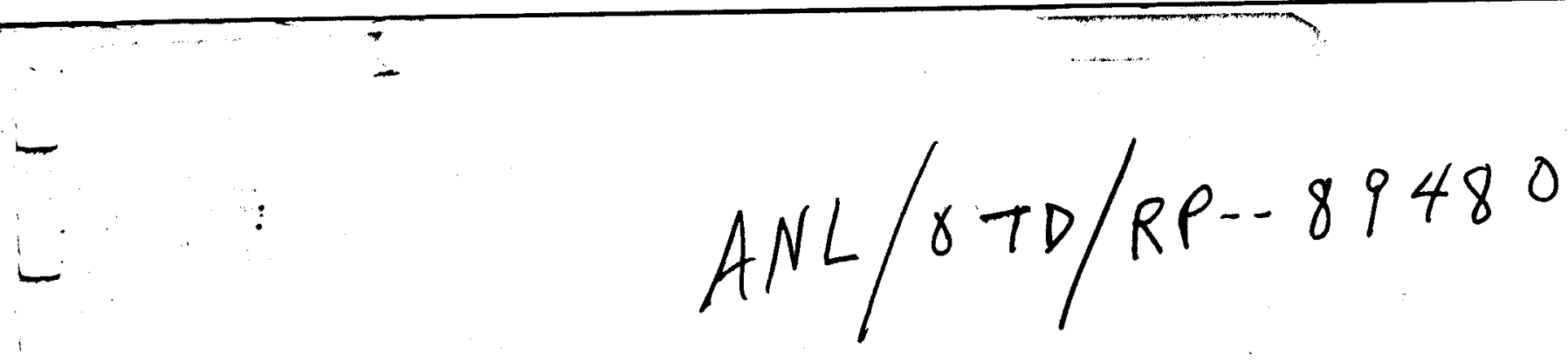

\title{
ARGONNE NATIONAL LABORATORY ANNUAL REPORT OF LABORATORY DIRECTED RESEARCH AND DEVELOPMENT PROGRAM ACTIVITIES FOR FY 1995
}

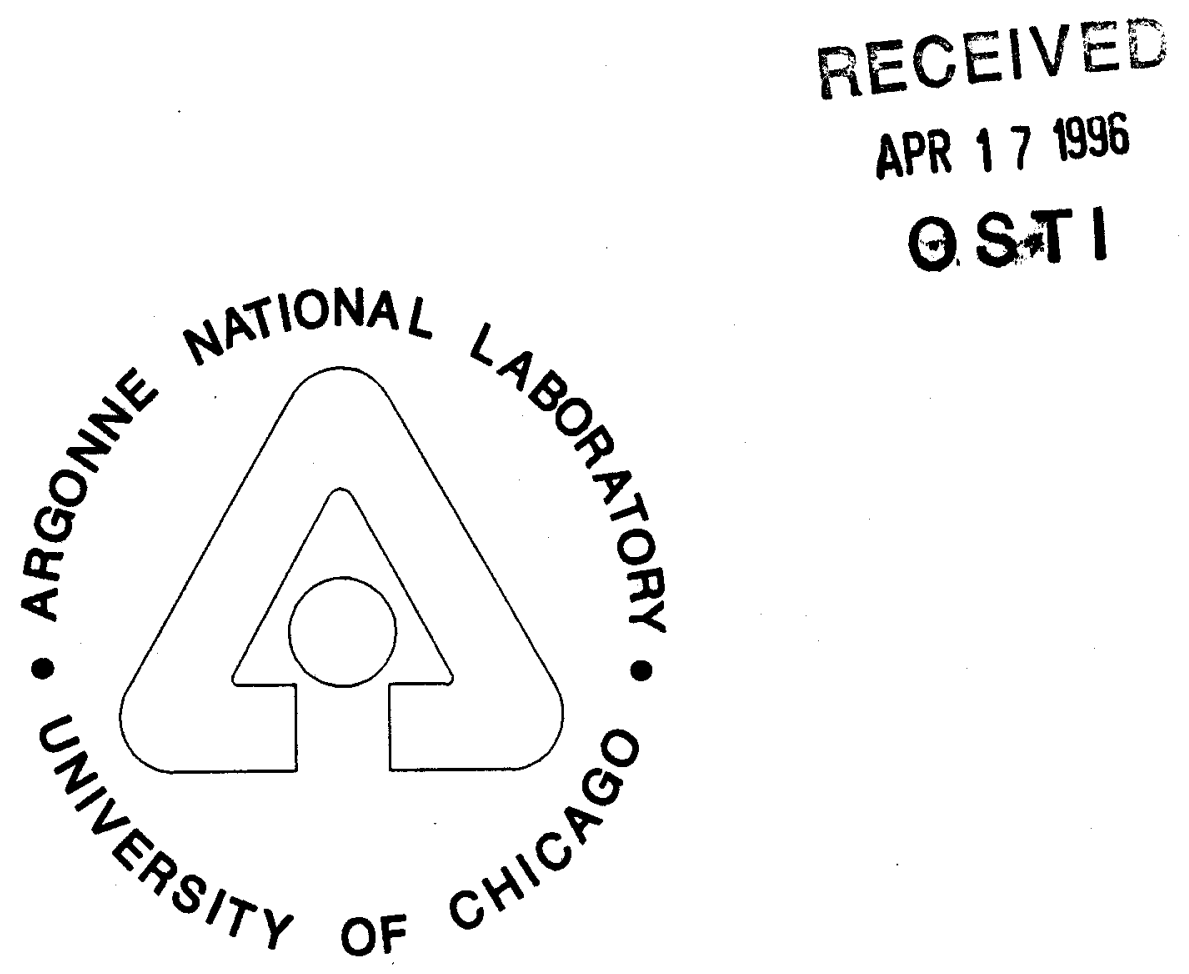

Strategic Planning Group

Office of The Director

February 15, 1996 


\section{DISCLAIMER}

This report was prepared as an account of work sponsored by an agency of the United States Government. Neither the United States Government nor any agency Thereof, nor any of their employees, makes any warranty, express or implied, or assumes any legal liability or responsibility for the accuracy, completeness, or usefulness of any information, apparatus, product, or process disclosed, or represents that its use would not infringe privately owned rights. Reference herein to any specific commercial product, process, or service by trade name, trademark, manufacturer, or otherwise does not necessarily constitute or imply its endorsement, recommendation, or favoring by the United States Government or any agency thereof. The views and opinions of authors expressed herein do not necessarily state or reflect those of the United States Government or any agency thereof. 


\section{DISCLAIMER}

Portions of this document may be illegible in electronic image products. Images are produced from the best available original document. 


\title{
DISCLAIMER
}

This report was prepared as an account of work sponsored by an agency of the United States Government. Neither the United States Government nor any agency thereof, nor any of their employees, makes any warranty, express or implied, or assumes any legal liability or responsibility for the accuracy, completeness, or usefulness of any information, apparatus, product, or process disclosed, or represents that its use would not infringe privately owned rights. Reference herein to any specific commercial product, process, or service by trade name, trademark, manufacturer, or otherwise does not necessarily constitute or imply its endorsement, recommendation, or favoring by the United States Government or any agency thereof. The views and opinions of authors expressed herein do not necessarily state or reflect those of the United States Government or any agency thereof.

\section{ARGONNE NATIONAL LABORATORY ANNUAL REPORT OF LABORATORY DIRECTED RESEARCH AND DEVELOPMENT PROGRAM ACTIVITIES FOR FY 1995}

\begin{abstract}
PATENT HOLD
This document copy, sinceits transmitted in advance of patent clearancer by Aroenne National Laboratory, is made avaitable in coplidence solely for use in performanee of work under contracts with the U.S. Department of Energy. This decument is pot to be published nor its contento otbenvise disseminated or used for purposes othor than epecifiod above before patent approval for suet release or use has been secured, upon requestrom the Argonne Patent Dept. Argonne National Laboratory, 9700 South Cass Avenue, Argonne, IL 60439.
\end{abstract}

\author{
Strategic Planning Group \\ Office of the Director \\ February 15, 1996
}


The submitted manuscript has been authored

by a contractor of the U.S. Government

under contract No. W-31-109ENG-38.

Accordingly, the U. S. Government retains a

nonexclusive, royalty-free license to publish

or reproduce the published form of this

or reproduce the published form of this
contribution, or allow others to do so. for

U. S. Government purposes.

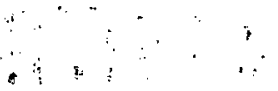




\section{Argonne National Laboratory \\ Annual Report of Laboratory Directed Research and \\ Development Program Activities \\ for FY 1995}

CONTENTS

\section{INTRODUCTION}

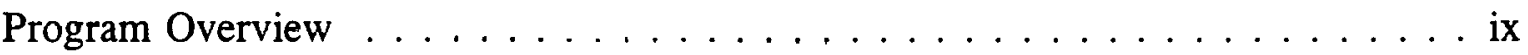
1994 Areas of Technical Impact $\ldots \ldots \ldots \ldots \ldots \ldots \ldots$. . . . . . . . . . . . LDRD Management Process . . . . . . . . . . . . . . . . . xv

Figure 1 - Argonne's LDRD Administrative Process:

Centralized Program . . . . . . . . . . . . . . . xvii

Figurc 2 - Argonne's LDRD Administrative Process:

Figure 3 - Argonne's LDRD Administrative Process:

Decentralized Program . . . . . . . . . . . . . . xvix

Figure 4 - Argonne's LDRD Administrative Process:

Individual Investigator Program $\ldots \ldots \ldots \ldots \ldots \ldots$

Exhibit A - Call for Proposals to Technical Staff . . . . . . . . . . . . xxi

Exhibit B - Call for Proposals to Lab Management . . . . . . . . . . . . . xxiii

Exhibit C - Call for Science and Technology LDRD Preproposals . . . . . . xxv

Exhibit D - Call for Individual Investigator Proposals . . . . . . . . . . xxvii

Exhibit E - LDRD Guidelines and Procedures . . . . . . . . . . . xxix

Exhibit F - LDRD Program Proposal Form . . . . . . . . . . . xxxi

Exhibit G - LDRD Program Proposal Evaluation Form . . . . . . . . . xxxiii

Exhibit H - Director's Individual Investigator LDRD Program

Proposal Submission Guidelines . . . . . . . . . . . xxxv

Exhibit I - Charge to the Coordination Council for Science and Technology xxxix

Exhibit J - Charge to the LDRD Director's Review Committee . . . . . . . xli

Exhibit K - LDRD Data Collection Form for FY $1995 \ldots \ldots \ldots$. . . . . . . xlv

PROJECT FUNDING TABLE $\ldots \ldots \ldots \ldots \ldots \ldots \ldots \ldots \ldots \ldots$ xlvii

PROJECT SUMMARIES BY TECHNICAL AREA

Advanced Accelerator and Detector Technology

95-001N Rapidly Cycling Synchrotron Performance Optimization . . . . . . . 1

94-057R1 Development of Basic Concepts and Components for the

Acceleration of Radioactive Beams . . . . . . . . . . . . . . . 5

92-182R3 Target Station Studies for the Intense Pulsed Neutron Source

Upgrade . . . . . . . . . . . . . . . . . . . . . . 9

93-151R2 Millimeter Wave Linac and Undulator Development Using

Microfabrication Techniques . . . . . . . . . . . . 11 
94-144R1 Short Wavelength Pulsed Magnetic Undulator Development Using Microfabrication Techniques . . . . . . . . . . . . . 17

94-191R1 In-Situ Surface Monitoring System for Synchrotron Optics in High Heat Load Environment . . . . . . . . . . . . . . . . 21

94-001R1 Amorphous Silicon Sensors: A New Detector Technology . . . . . 29 95-271N Booster Target Studies for the Intense Pulsed Neutron Source . . . 33

X-Ray Techniques for Research in the Biological and Physical Sciences

94-193R1 Development of X-Ray Scattering Techniques with $\mu \mathrm{eV}-\mathrm{meV}$

Resolution . . . . . . . . . . . . . . . . . . 37

95-012N New Synchrotron Anomalous Scattering Techniques . . . . . . . 49

93-152R2 Development of Interferometer-Based Fourier Transform X-Ray

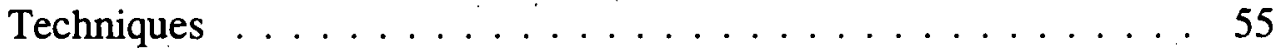

94-142R1 Inelastic X-Ray Scattering Studies Using High and Ultra High Energy Resolution . . . . . . . . . . . . . . . . . . 59

95-007N Development of a Monochromator System for the BESSRC X-Ray Beamlines at the APS . . . . . . . . . . . . . . . 63

95-171N Development of a Fast Spectrometer for Studying X-ray Induced Processes . . . . . . . . . . . . . . . . . . . . . . . . 69

93-041R2 Development of Synchrotron Radiation Analytical Capabilities at the Center for Advanced Microstructures and Devices . . . . . . 71 95-013N Optical Luminescence X-Ray Absorption Fine Structure: A New Method to Probe the Coordination and Valence of f-Elements in Amorphous Materials . . . . . . . . . . . . . . . . . . 75

93-150R2 Novel Applications of Undulator Radiation: Holography, Intensity Interferometry, and Speckle . . . . . . . . . . . . . . 79

95-003N High Power X-ray Induced Modification of Materials . . . . . . 85

95-006N Development of Cryogenically Cooled Monochromator Crystals for an APS Undulator-A Beamline . . . . . . . . . . . . . . . 89

93-007R2 Development and Characterization of Transition Metal Cluster Beams for X-Ray Absorption Studies . . . . . . . . . . . . 93

93-004R2 Atomic, Molecular, and Optical Physics with Synchrotron Radiation . . . . . . . . . . . . . . . . . . . 95

Nuclear Technology

94-046R1 Sodium Imaging System (SIS), Phase II _. . . . . . . . . . . . 99

95-040N Optimization of Fuel Handling and Processing at ANL-West . . . 103

95-027N Use of Spent Experimental EBR-II Fuels to Address Potential

Materials Problems under Dry Storage Conditions . . . . . . . . . 107

95-054N Removal of Lithium Oxide and Lithium from Lithium

Chloride-Based Process Salts . . . . . . . . . . . . . . 111

93-043R2 Implement Reliability Centered Maintenance and Condition

Monitoring at EBR-II . . . . . . . . . . . . . . . . . . . . . 115

95-037N Investigation of Fast Neutron Radiography . . . . . . . . . . . 119

94-187R1 Nuclear Decontamination and Decommissioning Cutting

Technology Development . . . . . . . . . . . . . . . . . 123

95-221N Alkali Metal Laser Doppler Velocimetry . . . . . . . . . . . . . 127 
94-015R1 Development of Real Time X-Ray Examination for Hot Cell Applications . . . . . . . . . . . . . . . . . . . . . . 129

93-040R2 Laser Based Hot Cell Elemental and Isotopic Analysis System . . 131

95-041N Utilization of Waste Hardware . . . . . . . . . . . . . . 135

95-030N Development of Analytical Methods to Perform Waste

Characterization of Activated Metals . . . . . . . . . . . . 139

95-049N Hydroclones for Removal of Suspended Material from

Fused Salts . . . . . . . . . . . . . . . . . . . . . 145

94-114R1 Weapons Plutonium Disposition - An Evaluation of

Fuel Cycle and Technology Options . . . . . . . . . . . . 153

95-061N Preconceptual Design of a Facility for Conditioning Spent

Nuclear Fuel Using Pyroprocess . . . . . . . . . . . . . . . . . 157

95-280N Engineering Evaluation of Utilization of ANL-W Facilities

to Support Boron Neutron Capture Therapy . . . . . . . . . 161

Materials Science and Technology

94-032R1 Interpreting Crack Propagation in Critical Components of

Mechanized Systems . . . . . . . . . . . . . . . . . . 163

94-055R1 New Materials Synthesis Strategies for High Temperature

Superconductors Composed of $\mathrm{Cu}-\mathrm{O}$ Planes Separated by

Silicate-Based Layers . . . . . . . . . . . . . . . . . . . . . . 167

95-063N Modeling and Applications of Composite Structures . . . . . . 171

95-160N Plasma Synthesis of Novel Catalysts . . . . . . . . . . . . 177

95-062N Development and Testing of a Simulation System to Assess

and Predict the Structural Properties of Welds . . . . . . . . . . 179

94-169R1 Plasma Modification and Characterization to Improve

Micromanufacturing and Etching Technology . . . . . . . . 185

95-178N High Critical Currents and Patterning by Splayed and Columnar

Defects in Superconductors . . . . . . . . . . . . . . . . . . . 189

95-182N Synthesis and Characterization of Nanocrystalline

Barium Titanate . . . . . . . . . . . . . . . . . . . . 195

95-184N Exploratory Development of Sulfide Fiber Glass Mat . . . . . 199

95-163N Use of Self-Assembled Monolayers as Templates for

Growth of Novel Microporous Molecular Sieve Films . . . . . . . 203

95-196N Feasibility Studies in Application of Phosphate Based

Composites for Light Weight Construction Materials . . . . . . . . 209

94-058R1 X-Ray Synchrotron Radiation Studies of Semiconductor/

Semiconductor Heterophase Epitaxy _. . . . . . . . . . . . 213

94-060R1 Investigations of Time-Resolved and Anomalous SAXS Studies

for Critical Problems in Advanced Materials Research . . . . . . . 217

94-103R1 Development of a Code for Simulating Welding Processes . . . . 223

95-020N Development of Laser Technology for the Decontamination

of Surfaces . . . . . . . . . . . . . . . . . . . . . . 229

94-182R1 Removal of Contaminant $\mathrm{Cu}$ from Remelted Steel Scrap . . . . . 235

95-162N Ion-Specific Sensors Based on Conductive Organic Polymers . . . 239

94-165R1 Development and Applications of Novel Biomimetic $\mathrm{TiO}_{2}$

Photocatalysts for Selective Heavy Metals Removal . . . . . . . . . 243 
94-179R1 Application of Advanced Materials and Methods to Cermet Gas Sensor Development . . . . . . . . . . . . . . . . . . . 249

95-253N Applications of Nanoparticle Technology to the Development of a New Class of Energy-Efficient Industrial Heat Transfer Fluids . . 261

95-260N Development of Smooth Diamond Films for Cost-Effective Machining and Wear Applications . . . . . . . . . . . . 267

95-305N Theory of Macroscopic Quantum Phenomena and Disordered Materials . . . . . . . . . . . . . . . . . . . . . . . . 279

Computational Science and Technology

94-054R1 Large-Scale Wavelet-Based Signal Processing . . . . . . . . . 283

95-010N CAVE Virtual Environment Technology Development . . . . . . 285

95-070N A Human-Computer Expert System for Automated

Highway System . . . . . . . . . . . . . . . . . . . . . . 287

94-190R1 Integrating MultiMedia with High Performance Computing and Communications . . . . . . . . . . . . . . . . . . . 289

95-072N Recurrent Artificial Neural Networks for Modeling of Nonlinear Systems . . . . . . . . . . . . . . . . . . . . 293

95-247N Artificial Intelligence Applied to Adaptive Mesh Refinement for Nonlinear Finite Element Analysis . . . . . . . . . . . . . . . . 297

94-047R1 2D-3D/Holographic Images and Virtual Reality Projections for Control Room/Simulator Designs . . . . . . . . . . . . . . . 301

95-269N Computer Aided Drug Design Using Virtual Reality . . . . . . . 305

95-167N Optimization Methods for Macromolecular Conformation on High-Performance Architectures . . . . . . . . . . . . . . . . . 309

95-241N Improved Fuel Injector Spray Modeling for Internal Combustion

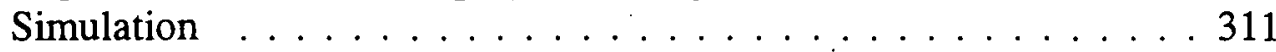

95-279N Evaluation of the ANL ICRKFLO Code for Simulation of a Commercial-Scale Fluid Catalytic Cracker System . . . . . . . . 315

Biological Sciences

92-180R3 Molecular Biology and Biotechnology of Hyperthermophilic Organisms: The Chaperone Release Factor . . . . . . . . . . 321

92-108R2 Structural Biology and the Human Genome: Crystal Structures of PNA . . . . . . . . . . . . . . . . . . . . . 323

95-176N Solution Structures of GroEL and GroES Complexes with Proteins . . . . . . . . . . . . . . . . . . . . . . . . . . 329

95-164N Reconstructing Metabolic Function from Genome Sequence Data . . . . . . . . . . . . . . . . . . . . . . . . 333

95-183N Structure-Function Characterizations of Protein Regulators of Programmed Cell Death . . . . . . . . . . . . . . . . . 337

95-011N Time Domain Molecular Structure Determination for Metastable Reaction Intermediates in Photochemical Processes Using X-Ray Absorption . . . . . . . . . . . . . . . . . . . . 341

93-090R2 Computational Structural Biology: Computer Simulation of Enzyme Reactions . . . . . . . . . . . . . . . . . . . 345 
95-270N Lipogenin, A Potential Human "Hormone" that Acts as a General Inducer of Lipid Formation . . . . . . . . . . . . . . . 351

Environmental Science

91-034R2 Integrated Land Use Management Using Remote Sensing and Ecological Models . . . . . . . . . . . . . . . . . . . 355

95-272N Three-Dimensional Real-Time Imaging of the Planetary Boundary Layer on Watershed Scales . . . . . . . . . . . . 359

Environmental Control and Waste Management Technology

95-050N Development of Performance Assessment Capability for

Waste Disposal . . . . . . . . . . . . . . . . . . 363

95-095N Beneficial Uses for Nuclear Waste . . . . . . . . . . . . . . 371

93-094R2 $\mathrm{No}_{x}$ Reduction in Diesel Engine Exhaust Using a Nitrogen

Plasma . . . . . . . . . . . . . . . . . . . . . . . . . . . 375

94-167R1 Waste Management of Chlorofluorocarbons . . . . . . . . . 379

95-075N New Technology for Removal of Heavy Noble Gases from

Argon and from Air . . . . . . . . . . . . . . . 385

95-071N Risk Evaluation of Nuclear Waste - Uncertainty and Sensitivity

Analyses . . . . . . . . . . . . . . . . . . . . . . 389

95-051N Evaluation of TRU-Bearing Disposal Alloys . . . . . . . . . . 393

95-229N Neural Network NOx Emission Controller for Fossil Plants . . . . 399

Novel Concepts in Other Areas

95-217N Feasibility Study for the Development of a Miniature Rapid-Scan

Passive Remote Fourier Transform Infrared (FTIR) Sensor . . . . . 403

95-170N Nucleon Amplitudes in QCD . . . . . . . . . . . . . . . 407

95-191N Near-Field Scanning Optical Microscopy . . . . . . . . . . 411

94-003R1 Intelligent Robotics Glove . . . . . . . . . . . . . . . . . 415

95-159N Ultrapurification of Tungsten Hexafluoride for Semiconductor

Use . . . . . . . . . . . . . . . . . . . . . . 419

94-168R1 A Computer Simulation of an Intelligent Vehicle Highway

System for the Chicago Metropolitan Area . . . . . . . . . . . 423

95-265N High-Sensitivity Infrared Chemical Sensor Development . . . . . . 425

APPENDIX A - 1995 LDRD Project Summary . . . . . . . . . . . . . . A-1

*APPENDIX B - 1995 Data Collection Results . . . . . . . . . . . . . B-1

*Issued separately to limited distribution. 
blank page 


\section{INTRODUCTION}

\section{Program Overview}

The purposes of Argonne's Laboratory Directed Research and Development (LDRD) Program are to encourage the development of novel concepts, enhance the Laboratory's R\&D capabilities, and further the development of its strategic initiatives. Projects are selected from proposals for creative and innovative R\&D studies which are not yet eligible for timely support through normal programmatic channels. Among the aims of the projects supported by the Program are establishment of engineering proof-of-principle; assessment of design feasibility for prospective facilities; development of an instrumental prototype, method, or system; or discovery in fundamental science or exploratory development

Several of these projects are closely associated with major strategic thrusts of the Laboratory as described in Argonne's Five Year Institutional Plan, although the scientific implications of the achieved results extend well beyond Laboratory plans and objectives. The projects supported by the Program are distributed across the major programmatic areas at Argonne as indicated in the Laboratory's LDRD Plan for FY 1995. The following table displays the area of principal emphasis (indicated by an "X") as well as additional area(s) (indicated by an "O") to which a contribution was made by each FY 1995 project.

A brief description of Argonne's LDRD management process and a table of funding profiles for each project active in FY 1995 then follow. The FY 1995 DOE approved funding cap was $\$ 16.9$ million or about $3.5 \%$ of Argonne's estimated FY 1995 operating budget. Actual expenditures amounted to $\$ 13.6$ million. Individual reports summarizing the purpose, approach, results and accomplishments of projects funded under Argonne's LDRD Program for FY 1995 comprise the bulk of this report. Appendix A summarizes funding and topics of new projects begun to date in FY 1996.

The LDRD program has been identified as one of the Laboratory's functional operations areas under the new performance-based contract for Argonne between DOE and the University of Chicago. This annual report, in addition to descriptions of scientific and technological matters, includes data relevant to applicable LDRD performance measurcs. In general these are found in the "Specific Accomplishments" paragraphs of the separate project reports. Those data that are quantifiable have been collected and integrated with separately collected data (using a data update form illustrated in exhibit $\mathrm{K}$ ). The resulting summary of performance is found in Appendix B (distributed as a separate document to appropriate offices of Argonne National Laboratory, the University of Chicago, and the Department of Energy). 
blank page 


\begin{tabular}{|c|c|c|c|c|c|c|c|c|c|}
\hline Proposal Number & $\begin{array}{c}\text { Advanced } \\
\text { Accelerator } \\
\text { and Detector } \\
\text { Technology }\end{array}$ & $\begin{array}{c}\text { X-Ray } \\
\text { Techniques } \\
\text { for Research } \\
\text { in the } \\
\text { Biological and } \\
\text { Physical } \\
\text { Sciences }\end{array}$ & $\begin{array}{c}\text { Nuclear } \\
\text { Technology }\end{array}$ & $\begin{array}{l}\text { Materials } \\
\text { Science and } \\
\text { Technology }\end{array}$ & $\begin{array}{c}\text { Compu- } \\
\text { tational } \\
\text { Science and } \\
\text { Technology }\end{array}$ & $\begin{array}{l}\text { Biological } \\
\text { Sciences }\end{array}$ & $\begin{array}{l}\text { Environ- } \\
\text { mental } \\
\text { Science }\end{array}$ & $\begin{array}{c}\text { Environ- } \\
\text { mental } \\
\text { Control and } \\
\text { Waste } \\
\text { Manage-ment } \\
\text { Technology }\end{array}$ & $\begin{array}{c}\text { Novel } \\
\text { Concepts in } \\
\text { Other Areas }\end{array}$ \\
\hline $95-001 \mathrm{~N}$ & $x$ & & & & & & & & \\
\hline 94-057R1 & $\mathrm{x}$ & & & & & & & & \\
\hline 92-182R3 & $x$ & & & 0 & & & & & \\
\hline 93-151R2 & $x$ & & & 0 & & & & & \\
\hline 94-144R1 & $x$ & & & 0 & & & & & \\
\hline 94-191R1 & $x$ & & & 0 & & & & & \\
\hline 94-001R1 & $x$ & & & 0 & & & & & \\
\hline $95-271 N$ & $x$ & & & 0 & & & & & \\
\hline 94-193R1 & & $x$ & & & & & & & \\
\hline $95-012 N$ & & $x$ & & & & & & & \\
\hline 93-152R2 & & $x$ & & & & & & & \\
\hline 94-142R1 & & $x$ & & & & & & & \\
\hline $95-007 N$ & & $x$ & & & & & & & \\
\hline $95-171 N$ & 0 & $x$ & & & & & & & \\
\hline 93-041R2 & & $x$ & 0 & & & & & & \\
\hline $95-013 N$ & & $x$ & & 0 & & & & & \\
\hline 93-150R2 & & $x$ & & 0 & & & & & \\
\hline $95-003 \mathrm{~N}$ & & $x$ & & 0 & & & & & \\
\hline $95-006 \mathrm{~N}$ & & $\mathrm{x}$ & & $\mathrm{O}$ & & & & & \\
\hline 93-007R2 & & $\mathrm{X}$ & & 0 & & & & & \\
\hline 93-004R2 & & $x$ & & & & & & & 0 \\
\hline 94-046R1 & & & $x$ & & & & & & \\
\hline $95-040 \mathrm{~N}$ & & & $\mathrm{x}$ & & & & & & \\
\hline $95-027 N$ & & & $x$ & & & & & & \\
\hline $95-054 \mathrm{~N}$ & & & $\bar{x}$ & & & & & & \\
\hline $93-043 R 2$ & & & $x$ & & & & & & \\
\hline $95-037 N$ & 0 & & $x$ & & & 0 & & & \\
\hline 94-187R1 & & & $x$ & 0 & & & & & \\
\hline $95-221 \mathrm{~N}$ & & & $x$ & 0 & & & & & \\
\hline
\end{tabular}




\begin{tabular}{|c|c|c|c|c|c|c|c|c|c|}
\hline Proposai Number & $\begin{array}{c}\text { Advanced } \\
\text { Accelerator } \\
\text { and Detector } \\
\text { Technology }\end{array}$ & $\begin{array}{c}\text { X-Ray } \\
\text { Techniques } \\
\text { for Research } \\
\text { in the } \\
\text { Biological and } \\
\text { Physical } \\
\text { Sciences }\end{array}$ & $\begin{array}{l}\text { Nuclear } \\
\text { Technology }\end{array}$ & $\begin{array}{l}\text { Materials } \\
\text { Science and } \\
\text { Technology }\end{array}$ & $\begin{array}{c}\text { Compu- } \\
\text { tational } \\
\text { Science and } \\
\text { Technology }\end{array}$ & $\begin{array}{l}\text { Biological } \\
\text { Sciences }\end{array}$ & $\begin{array}{l}\text { Environ- } \\
\text { mental } \\
\text { Science }\end{array}$ & $\begin{array}{c}\text { Environ- } \\
\text { mental } \\
\text { Control and } \\
\text { Waste } \\
\text { Manage-ment } \\
\text { Technology }\end{array}$ & $\begin{array}{c}\text { Novel } \\
\text { Concepts in } \\
\text { Other Areas }\end{array}$ \\
\hline 94-015R1 & & & $x$ & 0 & 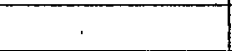 & & & . & 0 \\
\hline 93-040R2 & & & $x$ & 0 & & & & & 0 \\
\hline $95-041 \mathrm{~N}$ & & & $\mathrm{X}$ & & & & & 0 & \\
\hline $95-030 \mathrm{~N}$ & & & $x$ & & . & & & 0 & \\
\hline $95-049 \mathrm{~N}$ & & & $x$ & & . & & & 0 & \\
\hline 94-114R1 & & & $\mathrm{x}$ & & & & & 0 & \\
\hline $95-061 \mathrm{~N}$ & & & $\mathrm{x}$ & & & & & 0 & \\
\hline $95-280 \mathrm{~N}$ & & & $\mathrm{X}$ & & & 0 & & & \\
\hline 94-032R1 & & & & $x$ & & & & & \\
\hline 94-055R1 & & & & $x$ & & & & & \\
\hline $95-063 \mathrm{~N}$ & & & & $x$ & & & & & \\
\hline $95-160 \mathrm{~N}$ & & 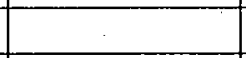 & & $\mathrm{x}$ & & & & & \\
\hline $95-062 \mathrm{~N}$ & & & & $x$ & & & & & \\
\hline 94-169R1 & & & & $x$ & & & & & \\
\hline $95-178 \mathrm{~N}$ & & & & $x$ & & & & & \\
\hline $95-182 \mathrm{~N}$ & & & & $x$ & & & & & \\
\hline $95-184 \mathrm{~N}$ & & & . & $x$ & & & & & \\
\hline $95-163 N$ & & . & & $\mathrm{x}$ & & & . & & \\
\hline $95-196 \mathrm{~N}$ & & & & $x$ & & & & & \\
\hline 94-058R1 & & 0 & & $x$ & & & & & \\
\hline 94-060R1 & & 0 & & $x$ & & & & & \\
\hline 94-103R1 & & & & $x$ & 0 & & & & \\
\hline $95-020 \mathrm{~N}$ & & & & $x$ & & & & 0 & \\
\hline $94-182 R 1$ & & & & $x$ & & & & 0 & \\
\hline $95-162 N$ & & & & $x$ & & & & 0 & \\
\hline 94-165R1 & & & & $x$ & & & & 0 & \\
\hline 94-179R1 & & & & $x$ & & & & 0 & \\
\hline $95-253 N$ & & & & $\mathrm{x}$ & & & & & \\
\hline $95-260 N$ & & & & $x$ & . & & & & \\
\hline
\end{tabular}




\begin{tabular}{|c|c|c|c|c|c|c|c|c|c|}
\hline Proposal Number & $\begin{array}{c}\text { Advanced } \\
\text { Accelerator } \\
\text { and Detector } \\
\text { Technology }\end{array}$ & \begin{tabular}{|c|} 
X-Ray \\
Techniques \\
for Research \\
in the \\
Biological and \\
Physical \\
Sciences
\end{tabular} & $\begin{array}{c}\text { Nuclear } \\
\text { Technology }\end{array}$ & $\begin{array}{l}\text { Materials } \\
\text { Science and } \\
\text { Technology }\end{array}$ & $\begin{array}{c}\text { Compu- } \\
\text { tational } \\
\text { Science and } \\
\text { Technology }\end{array}$ & $\begin{array}{l}\text { Biological } \\
\text { Sciences }\end{array}$ & $\begin{array}{l}\text { Environ- } \\
\text { mental } \\
\text { Science }\end{array}$ & $\begin{array}{c}\text { Environ- } \\
\text { mental } \\
\text { Control and } \\
\text { Waste } \\
\text { Manage-ment } \\
\text { Technology }\end{array}$ & $\begin{array}{c}\text { Novel } \\
\text { Concepts in } \\
\text { Other Areas }\end{array}$ \\
\hline $95-305 \mathrm{~N}$ & & & & $\mathrm{x}$ & & & & & \\
\hline 94-054R1 & & & & & $x$ & & & & \\
\hline $95-010 \mathrm{~N}$ & & & & & $x$ & & & & \\
\hline $95-070 \mathrm{~N}$ & & & & & $x$ & & & & \\
\hline 94-190R1 & & & & & $x$ & & & & \\
\hline $95-072 \mathrm{~N}$ & & & & & $x$ & & & & \\
\hline $95-247 N$ & & & & & $\mathrm{x}$ & & & & \\
\hline 94-047R1 & & & 0 & & $x$ & & & & \\
\hline $95-269 N$ & & & & & $x$ & 0 & & & \\
\hline $95-167 N$ & & & & & $\mathrm{x}$ & 0 & & & \\
\hline $95-241 \mathrm{~N}$ & & & & & $x$ & & & 0 & \\
\hline $95-279 N$ & & & & & $x$ & & & & \\
\hline 92-180R3 & & & & & & $\mathrm{X}$ & & & \\
\hline 92-108R2 & & & & & & $\mathrm{x}$ & & & \\
\hline $95-176 \mathrm{~N}$ & & & & & & $\mathrm{x}$ & & & \\
\hline $95-164 N$ & & & & & & $x$ & & & \\
\hline $95-183 \mathrm{~N}$ & & & & & & $x$ & & & \\
\hline $95-011 \mathrm{~N}$ & & 0 & & & & $x$ & & & \\
\hline 93-090R2 & & & & & $\mathrm{O}$ & $x$ & & & \\
\hline $95-270 N$ & & & & & & $x$ & & & \\
\hline $91-034 R 2$ & & & & & 0 & & $x$ & & \\
\hline $95-272 \mathrm{~N}$ & & & & & & & $x$ & & \\
\hline $95-050 \mathrm{~N}$ & & & & & & & & $x$ & \\
\hline $95-095 \mathrm{~N}$ & & & & & & & & $x$ & \\
\hline 93-094R2 & & & & & & & & $x$ & \\
\hline 94-167R1 & & . & & & & & & $x$ & \\
\hline $95-075 \mathrm{~N}$ & & & 0 & & & & & $x$ & \\
\hline $95-071 \mathrm{~N}$ & & & 0 & & & & & $\mathrm{x}$ & \\
\hline $95-051 \mathrm{~N}$ & & & 0 & & & & & $x$ & \\
\hline
\end{tabular}




\section{AREAS OF TECHNICAL IMPACT}

\begin{tabular}{|c|c|c|c|c|c|c|c|c|c|}
\hline . & $\begin{array}{l}\text { Advanced } \\
\text { Accelerator } \\
\text { and Detector } \\
\text { Technology }\end{array}$ & \begin{tabular}{|} 
X-Ray \\
Techniques \\
for Research \\
in the \\
Biological and \\
Physical \\
Sciences
\end{tabular} & $\begin{array}{c}\text { Nuclear } \\
\text { Technology }\end{array}$ & $\begin{array}{l}\text { Materials } \\
\text { Science and } \\
\text { Technology }\end{array}$ & $\begin{array}{c}\text { Compu- } \\
\text { tational } \\
\text { Science and } \\
\text { Technology }\end{array}$ & $\begin{array}{l}\text { Biological } \\
\text { Sciences }\end{array}$ & $\begin{array}{l}\text { Environ- } \\
\text { mental } \\
\text { Science }\end{array}$ & $\begin{array}{c}\text { Environ- } \\
\text { mental } \\
\text { Control and } \\
\text { Waste } \\
\text { Manage-ment } \\
\text { Technology }\end{array}$ & $\begin{array}{l}\text { Novel } \\
\text { Concepts in } \\
\text { Other Areas }\end{array}$ \\
\hline $95-229 N$ & & & & & 0 & & & $x$ & \\
\hline $95-217 N$ & & & & & . & & & & $\bar{x}$ \\
\hline $95-170 \mathrm{~N}$ & & & & & & & & & $x$ \\
\hline $95-191 \mathrm{~N}$ & & & & & & & & & $x$ \\
\hline 94-003R1 & & & 0 & & & & & & $x$ \\
\hline $95-159 N$ & & & & 0 & & & & & $\mathrm{X}$ \\
\hline 94:168R1 & & & & & 0 & & & & $x$ \\
\hline $95-265 \mathrm{~N}$ & & & & & & & & 0 & $x$ \\
\hline
\end{tabular}




\section{$\underline{\text { LDRD Management Process }}$}

Research and development activities at Argonne are organized under four associate laboratory directors who oversee the research conducted in 23 programmatic divisions. At the top of the organization is the laboratory director. He is assisted by the chief operations officer (who manages the Laboratory's physical plant and support functions), the chief financial officer, and the deputy to the laboratory director (who heads the Strategic Planning Office). This group of eight sit as the Strategic Planning Board and make final recommendations concerning laboratory directed R\&D.

Responsibility for all final decisions concerning Argonne's LDRD program resides with the laboratory director. Certain responsibilities regarding funding, oversight, proposal evaluation, and project direction are delegated. The laboratory director also delegates selection of a fraction of LDRD projects to individual associate laboratory directors and retains the prerogative to make his own selections as well. Beginning in FY 1995, and as described in the FY 1995 LDRD Plan, another fraction is reserved for support of the Director's Individual Investigator LDRD Program, which uses a peer review committee to evaluate relatively small, short-term, single investigator proposals. The remainder of projects is selected by the Strategic Planning Board, which is also charged with recommending the proportion to be allocated to other LDRD components. In FY 1995 the Board retained selection prerogative for projects totaling $72 \%$ of the LDRD budget. In FY 1996 the corresponding figure is about 76\%. The laboratory director coordinates plans for the various components of the program. In FY 1994, the laboratory director authorized a special emphasis on proposals that integrate basic and applied research and instituted a screening panel, the Coordination Council for Science and Technology (CCST), to solicit and present proposals to the Strategic Planning Board. An addendum to Argonne's FY 1994 LDRD Plan describes this fully. This component continues in FY 1995 and FY 1996. Proposals of all types are reviewed by the Strategic Planning Office for adherence to DOE guidelines and laboratory administrative procedures. They are then reported to the laboratory director, who must approve them in terms of content and aggregate budget. At the end of the fiscal year, accomplishments of all LDRD projects are reported to the laboratory director and DOE. As examples, calls for proposals and proposal guidance issued in 1995 are attached as exhibits A throngh H. The charge to the CCST is attached as exhibit I. The charge to the Director's Review Committee for the Individual Investigator Program is attached as exhibil J.

Coordination, oversight, and administration of the Laboratory's LDRD program is the responsibility of the deputy to the laboratory director. He is assisted by the associate director of the Strategic Planning Group, who has direct responsibility for the day-to-day administration of the LDRD program, oversight of the individual investigator component peer review process, assessment of the scientific and technological character of proposals in the context of DOE's LDRD program guidance and DOE Order 5000.4A, and for coordination of LDRD reports and plans. Responsibility for the actual conduct of all laboratory directed R\&D resides individually with the associatc laboratory directors and their line managers. Flow charts attached here as Figures 1 through 4 schematically describe the administrative processes for the various components of the Program.

The LDRD program is funded lab-wide through the Laboratory's indirect budget which derives from a uniform levy against all program operating budgets. The bulk of the initially planned 
LDRD budget is committed near the beginning of the fiscal year after most proposals have been evaluated (during September through early November). The laboratory director and the Strategic Planning Board maintain the option to fund new starts during the year either by increasing the total LDRD budget plan (within the DOE approved cap) or by redirection of previously authorized funds. Although many projects propose two or three year durations, funding levels and project selection are determined annually, based on technical progress and the Laboratory's strategic goals and resources.

Under the new performance based contract between DOE and the University of Chicago governing the operation of Argonne National Laboratory, LDRD has been identified as a functional area for which a specific set of performance measures apply. Data pertaining to these measures and upon which the LDRD program will, at least in part, be judged have been assembled in a separate document. Those copies of this annual report distributed to specific offices at Argonne, the University of Chicago, and DOE include the performance measures summary document as Appendix B. The management processes employed to collect these data include surveys of current and former investigators, analysis of project annual reports, and validation against records of the Industrial Technology Development Center (ITD) and the Information and Publishing Division (IPD), as well as the Work for Others (WFO) office. The data collection form currently in use is included here as exhibit $\mathrm{K}$. 
Figure 1

Argonne's LDRD Administrative Process:

Centrallzed Program

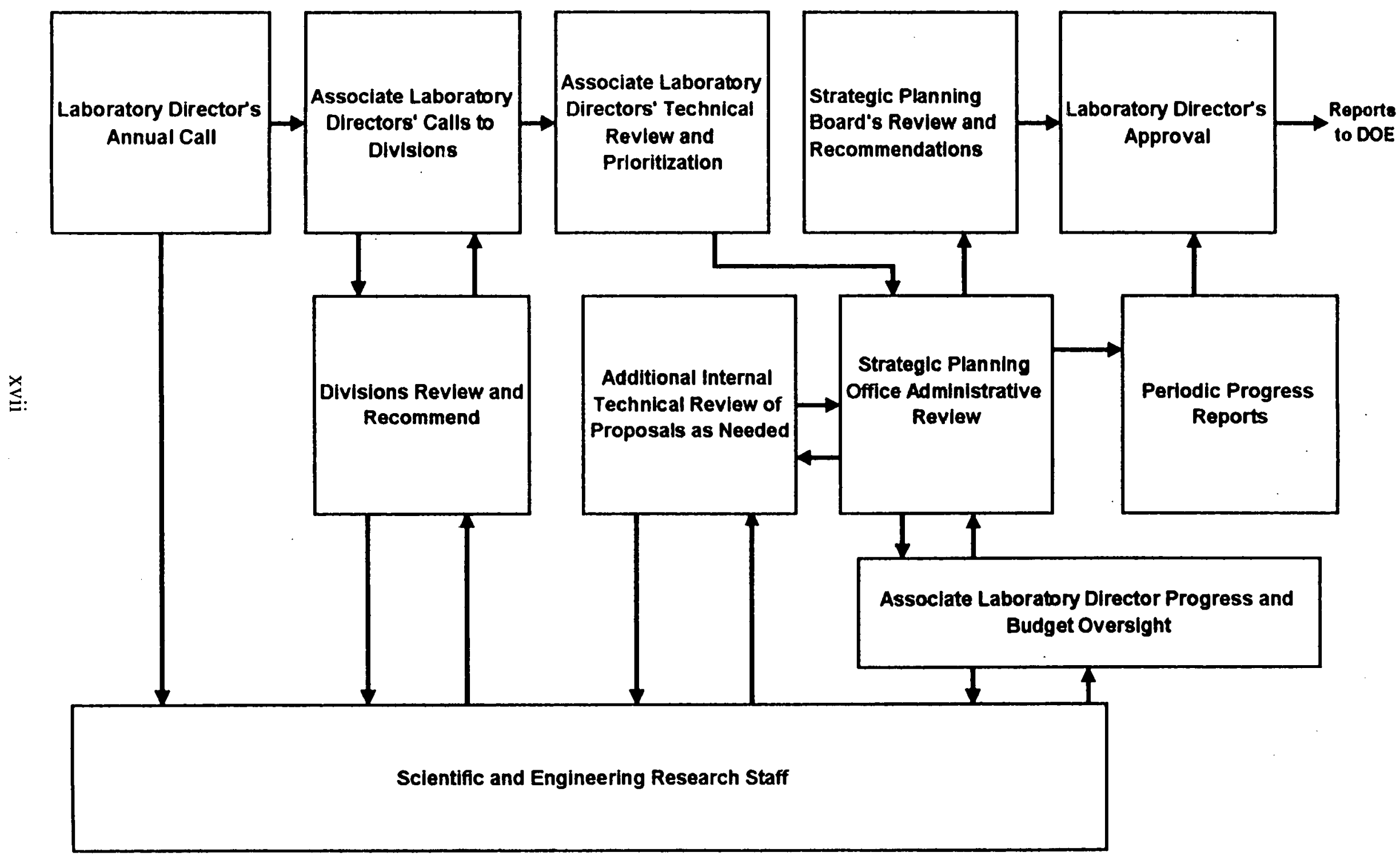




\section{Figure 2}

Argonne's LDRD Administrative Process:

Coordinating Council for Sclence and Technology (CCST)

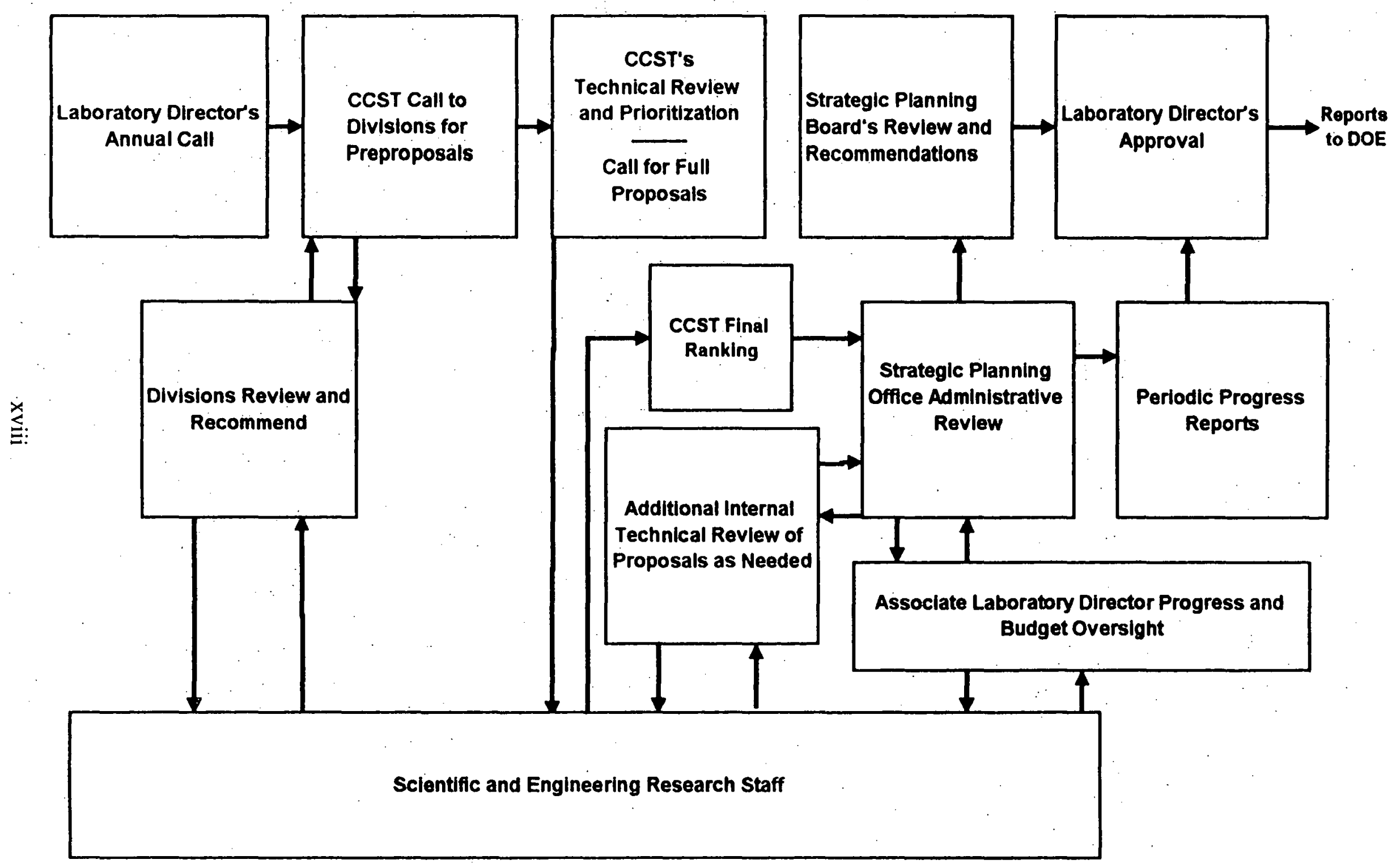


Figure 3

Argonne's LDRD Administrative Process:

Decentrailzed Program

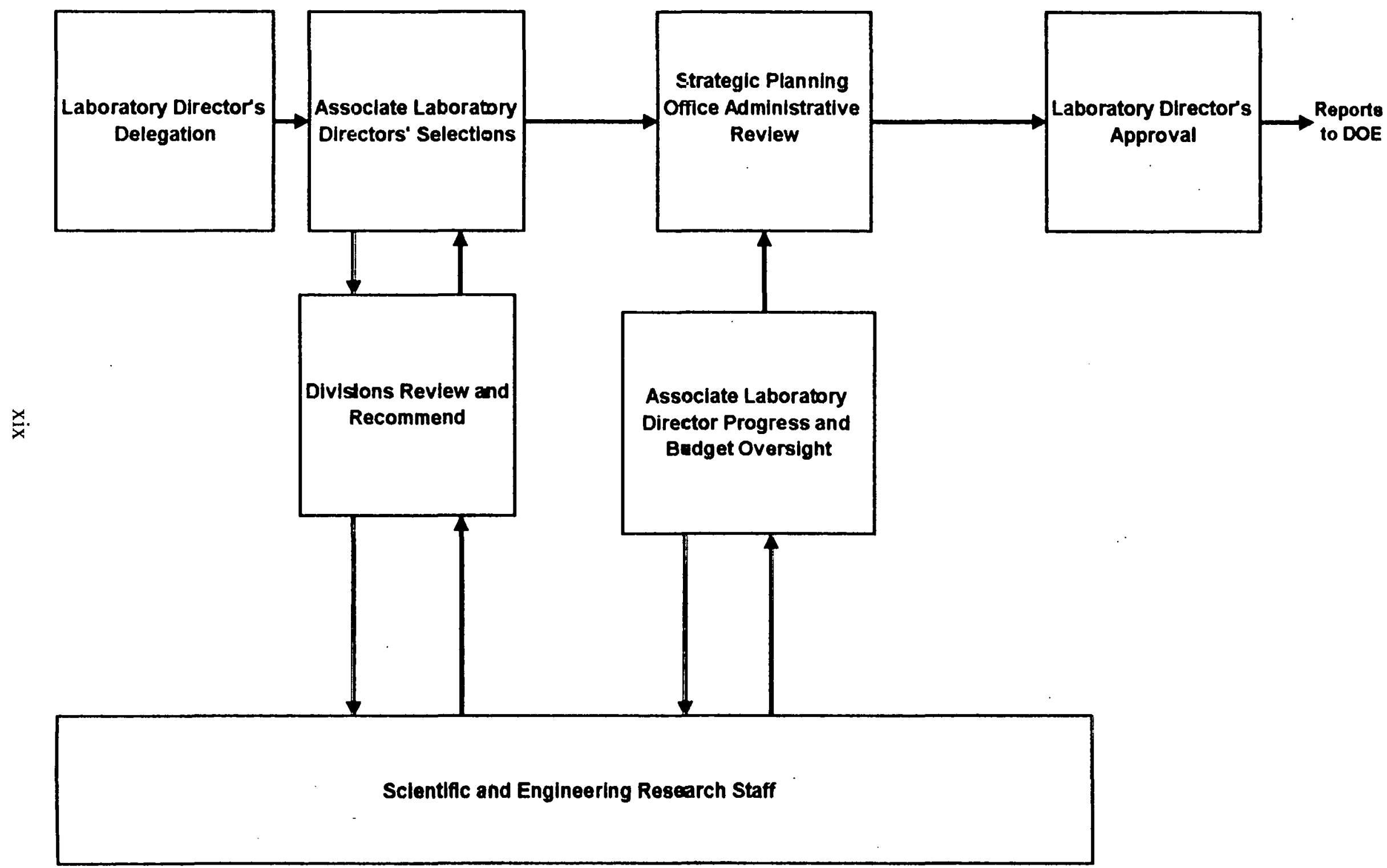


Figure 4

Argonne's LDRD Administrative Process:

Indivldual Investlgator Program

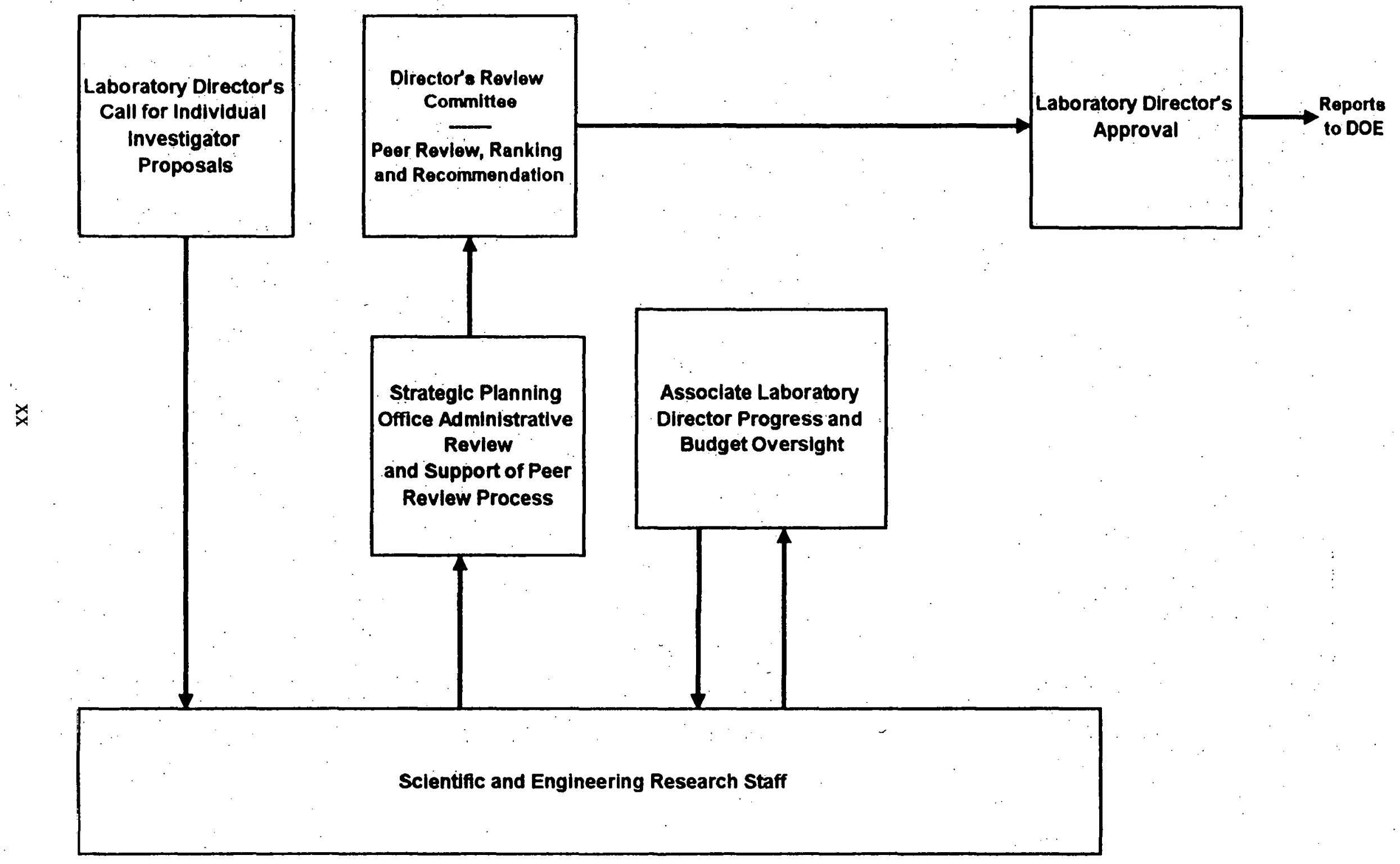




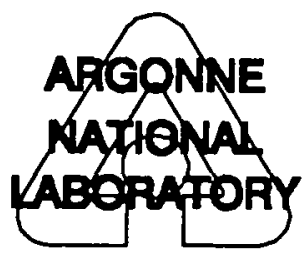

$\iota$

DATE: $\quad$ May 1, 1995

FO: Argonne Technical Staff

SUBJECT: Call for FY 1996 LDRD Proposals

It is again time to consider offering proposals to Argonne's Laboratory Directed Research and Development (LDRD) Program. Proposals of novel and innovative ideas are being solicited for fiscal year 1996. The LDRD program is relatively small in comparison to other Laboratory programs, however I expect several projects you propose for next year will be building blocks for Argonne programs of the future just as past LDRD projects were the basis for some of our large programs today. LDRD is an important mechanism to explore new avenues for the Laboratory, and I encourage you to participate.

Current guidelines for LDRD proposals are being distributed to Associate Laboratory Directors and will be available from division offices. The basic format and general criteria applicable to all LDRD proposals can be found there. As in fiscal year 1995, three avenues are available for proposal submission. General proposals should be submitted through division offices. Proposals specifically linking basic and applied studies will be solicited by Argonne's Coordinating Council for Science and Technology which will issue a supplementary call and submission guidance specifically for such proposals. Small, short-term, single investigator projects may be proposed directly to my office in accordance with the guidelines for the LDRD Individual Investigator Program announced in a separate memorandum. Once again, I anticipate an impressive set of innovative proposals for FY 1996.

\section{AS/ENK:jr}

c: J. Asbury

H. Drucker

F. Fradin

E. Kaufmann

D. Moncton

J. O'Kelley

R. Teunis

C. Till 
blank page 


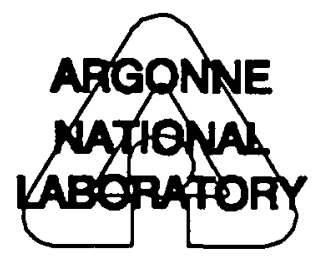

DATE: $\quad$ May 1, 1995

TO: $\quad$ H. Drucker

F. Fradin

D. Moncton

R. Teunis

C. Till

FROM:

A. Schriesheinf

SUBJECT: Call for FY 1996 Laboratory Directed Research and Development Proposals

Guidelines for the submission of general proposals for consideration of support under the Laboratory Directed Research and Development Program are attached. Please transmit this call for proposals to staff in your area. With your divisions and staff, you should assure that each proposal received is technically reviewed and is screened for conformance with $\mathrm{DOE}$ and Laboratory guidelines and acceptance criteria. Proposals which do not meet LDRD criteria will be returned. Proposals to either the Coordinating Council for Science and Technology (CCST) or the Director's Individual Investigator Program (IIP) should follow submission guidance specific to those LDRD components.

DOE 5000.4(a), the relevant Order defining LDRD procedures and content, is attached for your reference. Section 9 of the Order is most pertinent for responding to this call.

A sample proposal format is also attached and must be followed to facilitate record keeping and reporting requirements. In addition, a one-page evaluation form must be completed for each proposal.

We estimate that the FY 1996 funding level for the LDRD Program will not exceed this year's, unless future budget developments suggest a change. All proposals submitted by your staff, a memo stating your recommended priorities, and a list of technical staff who aided in the technical review process should be submitted to Elton Kaufmann, Strategic Planning Office, no later than July 3, 1995. I plan to complete the review and allocation process with the Strategic Planning Board during September. Once our FY 1996 Laboratory budget is better defined, I will notify you of the amounts, if any, set aside for our decentralized (ALD discretionary) LDRD program. 
Subsidiary calls for proposals and corresponding guidance are forthcoming for the Director's Individual Investigator Program and the Coordinating Council for Science and Technology that add special criteria to the general ones in the attached. Do not submit duplicate proposals either through the IIP, CCST, or directly to the Strategic Planning Office.
AS/ENK:jr
Attachments
c: J. Asbury
J. Day
M. Hennebry
R. Huebner
E. Kaufmann
L. LeSage
S. Morss
J. O'Kelley 
TO: $\quad$ Division Directors

Department Heads

Program Managers

FROM: $\quad$ H. Drucker $\div$.

F. Fradin FF/mo

ALD, EEST

ALD, PR

SUBJECT: Call for Science and Technology LDRD Preproposals

We assume that the Coordination Council for Science and Technology (CCST) will be involved again in reviewing LDRD requests for projects bringing together basic and applied scientists in pursuit of technologies that might be of interest to DOE and, eventually to DOE's new industrial partners. As was the case last year, we will be using the criteria developed by CCST and, of course, the standard LDRD criteria. As was the case last year, we ask for preproposals one page in length per the format attached. SINCE WE RECEIVE MANY PROPOSALS, LET US. WARN YOU THAT PREPROPOSALS LONGER THAN ONE PAGE SELDOM GET READ. This point, we trust, is clear.

Please provide proposals to Dr. Drucker by June 30 for review by CCST. CCST will help, when appropriate, in identifying potential collaborators. Preliminary decisions on proposals will be made in early July, and full proposals will be due by July 31 .

Given the excellence of last year's proposals, we believe that this program has the strong support of all concerned. Please give this appropriate distribution such that we can repeat last year's success.

$\mathrm{HD} / \mathrm{FF}: \mathrm{njv}$

Attachments: 3

NOTE: For FY96 the CCST committee determined that no new starts would be requested. 
blank page 


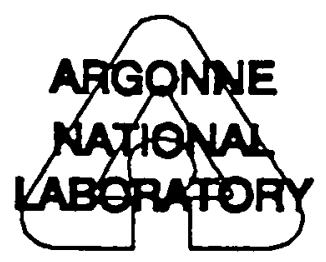

DATE: $\quad$ May 1, 1995

TO: Argonne Technical Stafe

FROM: Alan Schriesheim, Laboratory Director

SUBJECT: LDRD Call for Proposals for FY 1996: Director's Individual Investigator Awards

Guidelines for the submission of proposals to the Laboratory Directed Research and Development Individual Investigator Program are attached. Projects selected in this category will be funded for a single year at a total cost not to exceed $\$ 75,000$. Successful proposals will be selected through a Laboratory-wide competition. Criteria against which proposals will be judged are also provided in the attachment. A Director's Review Committee will evaluate and rank submissions and report its recommendations directly to me. The Individual Investigator component of our LDRD Program adds significantly to Argonne's ability to build on your innovative talent in fulfillment of our current and future missions.

Our traditional LDRD Program will continue according to the guidelines and schedule announced in a separate memo.

AS:jr

Attachment

c: Strategic Planning Board

E. Kaufmann 
blank page 


\section{LABORATORY DIRECTED RESEARCH AND DEVELOPMENT \\ GUIDELINES AND PROCEDURES}

\section{PURPOSE}

The ANL Laboratory Directed Research and Development Fund (LDRD) is established to support and encourage new ideas and novel approaches to scientific and technical problems. It is expected that efforts supported by the LDRD program will lead to new funded programs and new directions for the Laboratory, while taking maximum advantage of the talents and creativity of Argonne staff.

\section{PROCEDURES}

1. LDRD projects to be funded will be selected on a competitive basis from all proposals submitted by Argonne staff.

2. Each proposal will identify the principal investigator(s), state the purpose of the project, define the project's scope, and provide a brief summary of the planned project. The proposal should specify the amount of funding required and, if the project is expected to extend over more than one year, the funding needed until completion. Projects may not be extended beyond three years unless exceptional circumstances obtain.

3. A new proposal must explicitly state that no prior LDRD funding was devoted to any aspect of the work. If part or all of the project received prior-year LDRD funding, proposals must specify amounts by fiscal year and trace any changes in project title which have occurred. Any initiation or termination of subtasks within a project during or between fiscal years must be reported and explained.

4. The Strategic Planning Board will review the proposals submitted, obtain any additional information considered necessary, and submit its recommendations for funding to the laboratory director.

5. Budgets will be established for each of the approved projects, and appropriate accounts will be opened to collect charges.

6. Proposals may be submitted to the Strategic Planning Board at any time. However, a majority of the funds will be committed at the beginning of the fiscal year. A limited amount (up to $20 \%$ ) may be reserved to allow the Laboratory to support new projects or to respond to opportunities. In addition, funding allocations may be changed during the year by the Strategic Planning Board in response to new needs or opportunities. 
7. The resources to be made available for the LDRD program will be established by the laboratory director, within the DOE approved maximum, prior to the beginning of the fiscal year.

8. A written report will be prepared by the principal investigator(s). for each year of each funded project. The report should summarize the technical results obtained by the study in sufficient detail and at a level of presentation so that a non-specialist can appreciate the content and accomplishments. The report should also identify opportunities for new funding resulting from the study. The report should be submitted within one month after the completion of the project or within one month after the end of the fiscal year, whichever comes first. Explicit guidelines on content and format of the report will be distributed before fiscal-year end.

\section{PROGRAM SELECTION GUIDELINES}

1. The LDRD program will be administered according to the guidelines established by DOE for the use of LDRD Funds (DOE Order 5000.4A, 4/9/92).

2. LDRD projects should emphasize scientific and technical excellence and be at the forefront of science and technology.

3. LDRD projects should offer the promise to enhance the Laboratory's capabilities and be relevant to the long-term strategic view of the Laboratory (see Section V.D of the FY 1996 FY 2001 Institutional Plan).

4. LDRD projects should involve adequate effort and resources to insure proposed ideas are thoroughly tested. However, LDRD projects will generally not exceed 1.5 FTE of effort plus M\&S (total $\$ 200 \mathrm{~K}$ in any one year and, if a multi-year project, should not exceed $\$ 500 \mathrm{~K}$ in total). Separately identifiable tasks that can stand alone as LDRD projects should not be combined into larger, single PI, projects.

5. LDRD projects may not be used to substitute for or supplement funds from DOE or other sponsors. LDRD projects may not be used to prepare conceptual design reports, or to fund construction line-item projects, facility maintenance, or capital expenditures of a general purpose nature. 


\section{LDRD PROGRAM PROPOSAL FORMAT}

(On cover page to be attached to each proposal submitted to Strategic Planning Office) PROJECT TITLE:

PRINCIPAL INVESTIGATOR(S):

ANL DIVISION(S):

ALD OFFICE:

PRIOR LDRD FUNDING:

CURRENT REQUEST:

FUTURE LDRD FUNDING:

PURPOSE: (One paragraph statement of the nature and purpose of the project.)

(Body of proposal must incorporate all, but no more than, the following elements:)

PROJECT TITLE: (Repeated from cover page)

PURPOSE:

(One paragraph statement of the nature and purpose of the project).

(Repeated from cover page)

WORK SCOPE: (Delineation by reference only of the principal types of activities to be carried out. Details should be deferred to the BRIEF PROJECT SUMMARY paragraph below. Taken together with the PURPOSE paragraph, this should give enough detail so that a non-specialist will fully understand intent and scope.)

BACKGROUND: (Description of technical/scientific opportunity or need in the context of the state of the field and prior work, if any, at ANL and elsewhere.)

BRIEF PROJECT SUMMARY:

(More complete description of scientific/technical activities referenced in the WORK SCOPE including anticipated approach, tools, milestones, collaborations, etc. Preparation of reports, proposals, presentations and organizational aspects of collaborations may NOT be principal activities.)

JUSTIFICATION: (Estimation of likely scientific/technical benefits and potential for follow-on research support.)

BUDGET AND EFFORT:

(Budget \& effort plan broken down as effort (in \$ and FTE), M\&S, low value procurement, and subcontracts. No expenditure for capital equipment is provided for under LDRD).

ESTIMATE OF COMPARATIVE CONTRIBUTION TO LDRD PROGRAM OBJECTIVES:

Development of a novel R\&D idea

Development of a new lab capability

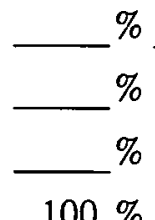

TOTAL: 
blank page

xxxii 


\section{Exhibit G}

\section{LDRD PROGRAM}

\section{PROPOSAL EVALUATION FORM}

Project Title:

P.I./Division:

Yes No Unknown

(1) Will this funding be used to initiate a new project which is beyond the exploratory phase?

(2) Will this funding be used to substitute for or increase funding for tasks normally funded by DOE or other users of the Laboratory?

(3) Will this allocation create an implicit commitment of multiyear funding by initiating projects which will require significant funding in future years to reach a useful stage of completion?

(4) Will this allocation be used to fund construction design (conceptual design, Title I, or more advanced design): or

(5) Fund construction line-item projects, in whole or in part; or

(6) Fund facility maintenance; or

(7) Fund capital expenditures of a general purpose nature?

Reviewer's Evaluation/Judgment of proposal: (Circle) (Must be completed or proposal will be returned)

Scientific/Technical Merit:

Relatedness to Lab Strategy:

Innovative Appeal/Exploratory

Nature:

Expected Contribution to

Science/Engineering Advance:

Prospects for Future Support:
Outstanding Good

Outstanding Good

Outstanding Good Adequate Marginal Poor

Outstanding Good Adequate Marginal Poor

Outstanding Good Adequate Marginal Poor

Other Comments: 
blank page 


\title{
DIRECTOR'S INDIVIDUAL INVESTIGATOR LDRD PROGRAM
}

\author{
Proposal Submission Guidelines
}

\section{A. Selection Criteria:}

Proposals will be ranked according to five principal criteria.

1. Quality: Inherent scientific and technical merit. Is the proposed project technically sound and at the forefront of its technical area?

2. Innovative appeal and/or exploratory nature: The Director's Review Committee will be seeking indications that the proposed work constitutes a new direction in research and development activity at Argonne.

3. Likelihood of success: Are the goals of the proposed project reasonable and commensurate with the resources and time scale of the program?

4. Expected contribution to science or expected advance in engineering: What technical advances will be achieved and why will they be valuable?

5. Leverage for new avenues of R\&D: If successful, what are the prospects that the project would lead to an expanded effort funded from other sources (including Argonne's traditional LDRD program)?

While no specific relative weights are applied to the five criteria, weakness in any area is likely to disqualify a proposal owing to the intense nature of the competition for funds.

\section{B. Content:}

1. The proposal must be self contained and provide sufficient information to address explicitly the five selection criteria used by the Director's Review Committee.

2. The introductory "purpose" paragraph should be written in a way accessible to a technical nonspecialist.

3. Most small projects must rely on use of existing Laboratory facilities and other resources in order to reach a useful stage of completion. The proposal must specify what and where these resources are.

C. Format:

1. Proposal format must adhere strictly to the same format required of proposals to the traditional LDRD Program (obtainable from division and associate laboratory director offices), but be no longer than ten pages including the cover page. The top line of the 
cover page should indicate "FOR SUBMISSION TO THE DIRECTOR'S INDIVIDUAL INVESTIGATOR LDRD PROGRAM." Signature lines at bottom of the cover page for all cognizant division directors and associate laboratory directors should be added.

2. The lead investigator should append a curriculum vitae no longer than two pages to the proposal. Similar material for collaborators may also be appended.

D. Requirements and Restrictions:

1. Proposals not consistent with requirements of DOE Order 5000.4A (copies available from ALD offices or directly from the LDRD manager in the Office of the Director) will be returned without review.

2. The laboratory director wishes to fund as many proposals in the individual investigator category as possible. Projects will normally be funded for a duration of one year and a total cost not exceeding $\$ 75,000$. Under extenuating circumstances and after consultation with the LDRD office, individual investigator LDRD projects funded in the previous fiscal year may be proposed for an extension of at most one additional year and would compete with new proposals.

3. Proposed projects may not be supplements to or continuations of current or previously funded LDRD projects. The emphasis of the Individual Investigator Program is on new directions and new research. The appearance that a proposal is an extension of on-going research and development work is viewed negatively.

4. Only one lead principal investigator may submit each proposal. Collaborating investigators may be named. Involvement of post-doctoral researchers is encouraged. Substantial portions of the budget should not be directed to outside consultants or subcontracts. The lead investigator and principal collaborators may not be current members of the Director's Review Committee.

5. The division director(s) and associate laboratory director(s) in whose area(s) the work will be performed must sign the cover page attesting to the availability of the lead investigator and collaborators as well as the facilities needed by the project, if it is funded.

E. Submission:

An original and four copies of the completed proposal (with necessary signatures on the cover page) should be submitted to the LDRD manager in the Office of the Director no later than close of business July 3, 1995.

F. Reviews and Reports:

1. Proposals will be reviewed by the Director's Review Committee (DRC) composed of Laboratory scientists and engineers appointed by the laboratory director and drawn 
from the broad spectrum of Argonne divisions. In addition to evaluation of the written proposal, Committee review procedures may include, in exceptional circumstances:

- Interviews of the principal investigators by selected members of the DRC;

- Reviews by experts not on the DRC or by reviewers external to Argonne, at the discretion of the DRC chairperson.

2. The chairman of the DRC will forward the full list of proposals, as ranked by the DRC, to the Office of the Director. The laboratory director will make awards based on this list and available funds.

3. All review discussions and documentation will be held in confidence by the DRC and the LDRD manager's office (except as noted in F.1 above).

4. The list of projects approved by the laboratory director will be published.

5. The annual report requirement for the Individual Investigator LDRD Program is the same as that for the traditional LDRD Program. Reports from both components will be collected into a single program report for transmission to DOE. The DRC may request brief midyear progress reports or presentations at its discretion.

6. Any proposed change in scope or change in availability of resources must be reported to the LDRD manager immediately. 
blank page 
Coordination Council for Science and Technology

\section{CHARGE}

To identify and develop opportunities for integrating the Laboratory's basic research capabilities in support of the technology missions and programs of the Laboratory and the Department of Energy; to promote the establishment of joint basic and technology development programs in support of this goal; to monitor the progress of such programs; and to advise Laboratory management concerning policies for the effective integration of basic research and technology development programs.

The Council will be co-chaired by the Associate Laboratory Directors for Physical Research and for Energy, Environmental and Biological Research. Other standing members will include the following directors of programmatic divisions: Materials Science, Chemistry, Mathematics and Computing Sciences, Environmental Research, Energy Systems, Environmental Assessment and Information Systems, (APS) Experimental Facilities, Chemical Technology, Engineering Physics, and Materials and Components Technology. A rotating membership of senior scientists and engineers will also be appointed by the Laboratory Director.

To support the goals and activities of the Council, the Laboratory Director will set aside, each fiscal year, a portion of funds for Laboratory Directed R\&D (LDRD) Program for allocation to joint basic-applied projects. The Council shall recommend annually projects for consideration by the Strategic Planning Board and the Laboratory Director for LDRD funding. 
blank page 


\title{
LDRD DIRECTOR'S REVIEW COMMITTEE
}

(Established by the Laboratory Director 6/94)

Purpose: LDRD DIRECTOR'S REVIEW COMMITTEE conducts a peer review of proposals submitted to the Laboratory Director for LDRD funding under the Director's Individual Investigator LDRD Program. It applies criteria as described in submission guidelines for the Program and ranks submissions against them. The mechanics of the peer review process are determined each year by the Committee to conform to the number and distribution of proposals and the distribution of technical expertise on the Committee. The Committee's goal is to provide a rank ordered list of proposals to the Laboratory Director for final approval and determination of the Program's funding level each year. The Committee members adhere to guidelines governing confidentiality of deliberations and avoidance of conflict of interest. The Chairperson of the Committee may authorize consulting with nonmembers where special technical expertise is needed for the peer review. The Committee also assumes responsibility for monitoring the progress of projects it has recommended in order to identify changes in scope of work or changes in the availability of resources required by the projects.

$\begin{array}{ll}\text { Members: } & \text { John P. Ackerman, Chairman } \\ & \text { Edmond L. Berger } \\ & \text { Richard R. Cirillo } \\ & \text { Joseph L. Dehmer } \\ & \text { Joanne K. Fink } \\ & \text { Julie D. Jastrow } \\ & \text { Tuncer M. Kuzay } \\ & \text { John Noonan } \\ & \text { Douglas L. Porter } \\ & \text { John P. Schiffer } \\ & \text { Lee C. Teng } \\ & \text { Randall E. Winans }\end{array}$

\author{
Samuel D. Bader \\ John M. Carpenter \\ Dennis W. Dees \\ John D. Ditmars \\ David J. Hill \\ Elton N. Kaufmann, Executive Director \\ Ewing L. Lusk \\ Basil Picologlou \\ William W. Schertz, Vice Chairman \\ Jeffrey Schwartz \\ Richard A. Valentin
}

The Committee initially comprises twenty members. All members are appointed by the Laboratory Director after consultation with line managers and current and former Committee members. The total number of members may be adjusted in response to the number of proposals received. No member will hold a management position of division director level or above. Each technical directorate of the Laboratory shall be represented by no less than four members or one-fifth of the total number of members, which ever is greater. To the extent possible, while adhering to the previous criterion, no division shall have more than one member at any time. Normally members' terms are three years except for the case of inaugural members whose terms are distributed from one to three years to allow staggering of the appointments and replacement of about one-third of the members annually. The Laboratory Director appoints a chair and vice chair in the inaugural year and thereafter a vice chair annually. The vice chair becomes the chair in the following year. The Laboratory's LDRD Manager serves as executive director for the Committee and the Manager's office supports the Committee's administrative needs.

Meetings: As required. 
blank page 


\title{
LABORATORY DIRECTED RESEARCH AND DEVELOPMENT
}

\section{DIRECTOR'S REVIEW COMMITTEE}

\author{
Guidelines and Responsibilities
}

\section{Proposal Receipt, Peer Review, and Ranking:}

Proposals to the Individual Investigator component of the LDRD Program will be received in the Office of the Director by the LDRD manager where they will be logged in and screened for compliance with DOE and Laboratory requirements and for adherence to the attached Proposal Submission Guidelines. Screened proposals will be forwarded to the Chairperson of the Director's Review Committee who will, with the advice of the Committee, oversee a distribution and review procedure taking into account proposal topics and Committee-member areas of expertise.

The process will insure that all Committee members become sufficiently familiar with all proposals to knowledgeably rank them according to the criteria specified in the Proposal Submission Guidelines. Review procedures should include an opportunity for lead investigators to present and explain their proposal to, at a minimum, a subgroup of the Committee. As many meetings of the full Committee as are necessary to adequately discuss all submissions should be convened. Committee meetings will normally be closed, but may be open when investigators are delivering oral presentations at the Committee's request. Proposals that do not present a sufficiently clear and/or detailed picture of the proposed work may either be rejected without further consideration or returned for augmentation, depending on the Committee's judgment of its potential.

The products of the review procedure will be a rank ordered list of proposals provided to the laboratory director by September 30 and a short paragraph critiquing each proposal which will be returned to the lead investigator of each submitted proposal (whether funded or not). The laboratory director, with advice from the Strategic Planning Board, determines the size of the Individual Investigator component of the Program. Feedback to investigators of rejected (low ranked) proposals should be specific and helpful so chances for future awards are enhanced.

It is the purpose of the Individual Investigator component of LDRD to provide an avenue for funding of modest projects that are highly deserving but would likely not have been funded through the traditional LDRD mechanism. The same proposal may therefore not be submitted to both components of the LDRD Program in the same year. However, if the Committee determines that the character of the proposal is such that it more properly should have been considered in the traditional component of the Program, it may be so redirected.

In exceptional cases, e.g., when the natural pace of a project demands it, the Committee may recommend spreading an award over two years, subject to adequate progress.

The Committee may determine that some projects will require monitoring after funds are awarded. Particularly high risk projects may have crucial midyear milestones, or a project's prosecution may depend on the availability of special equipment which could be delayed or withdrawn. Also, the LDRD manager may have advised the Committee of an investigator initiated change in work scope. The Committee may request midyear progress reports and/or interviews and may recommend early termination of a project as warranted. Unspent funds would, at the discretion of the laboratory director, be available for midyear starts of other Individual Investigator projects. 
The final reports of all funded projects will be made available to the Committee for comments. Any such comments will be made a part of the project's record.

Committee Operations and Member Roles and Responsibilities:

The laboratory director will appoint a chairperson and vice chairperson who will chair meetings of the Committee and make Committee assignments to accomplish the review process. The chairperson may enlist nonmembers of the Committee (inside or external to Argonne) when particular expertise is not available among the members. The chairperson's term as chair will be one year and he/she will be succeeded by the vice chairperson. Initial member appointments will be for terms of one, two, or three years and subsequent appointments for three years in order to stagger terms.

The LDRD manager will function as executive director for the Committee, provide information on DOE and Laboratory guidelines, serve as liaison to the Office of the Director, and arrange for clerical and secretarial support as required.

All scientific and engineering staff below division director grade and not excluded by conflict-of-interest considerations (see below) are eligible for appointment to the Committee by the laboratory director: It is anticipated that the majority of members will have substantial experience in their technical fields and be familiar with the peer review process. Membership of the Committee will be chosen to reflect the diversity of, and to represent proportionately, all major research organizations and interests of the Laboratory. The laboratory director will accept nominations for Committee membership from expiring members and from associate laboratory directors. The Committee shall initially comprise approximately twenty (20) members. The number may be adjusted based on numbers of proposals received and experience with the time requirements of the review process.

\section{Conflict of Interest:}

Neither lead investigators nor principal collaborators on proposals to this component of the LDRD Program may accept appointment to the Committee. However, members may be investigators on projects funded through the traditional LDRD process. Members in line or program management positions must abstain from ranking of proposals originating in their areas. Any other special relationship of a member to a particular proposal should be disclosed to the Committee chairperson.

\section{Confidentiality:}

Committee members must agree to maintain strict confidentiality concerning the status of any proposal during review and concerning the written or verbal content of Committee deliberations, during or after the review process, whether these occur during official Committee activities or during informal interactions among Committee members or with proposing investigators. Committee meeting minutes will not document discussions of the merits of proposals, only preserving a record of decisions taken by the whole Committee. Any nonmember reviewers who help the Committee must agree to the same confidentiality requirements to which members are bound. 


\section{LDRD DATA COLLECTION FORM FOR FY 1995 \\ (return to Judy Reedy, OTD/201, by $1 /$ )}

ALD:

PROPOSAL NUMBER:

WORK PROJECT NUMBER:

TITLE:

PRINCIPAL INVESTIGATOR(S):

1. Subject Category (from OSTI's International Energy Subject Categories and Scope):

Primary _ Secondary _ Tertiary

2. (a) Did or will all or part of the research performed under this LDRD project continue (with or without interruption) under other auspices (such as redirected or new program funds, work-forothers, CRADAs, etc.)?

Yes $\square \quad$ No $\square$

(b) If yes, list the sources (principal source first) of follow-on funding:

(c) Number of proposals submitted:

3. Number of refereed publications based solely on the work supported by LDRD funds and done during the active period of this project:

4. Number of students and postdocs (combined total) directly supportcd by this LDRD project while this project was active (do not include any supported by follow-on funds).

5. Number of new, permanent, full-time, technical staff hired as a direct result of and as direct participants in this LDRD Project:

6. Number of copyrights derived both directly from this LDRD project and from any follow-on efforts to date:

7. Number of invention disclosures submitted to the Laboratory's Industrial Technology Development Center that derived both from this LDRD project directly and from any follow-on efforts to date:

8. Number of patent applications granted and pending derived from both this LDRD project directly and from any follow-on efforts to date.

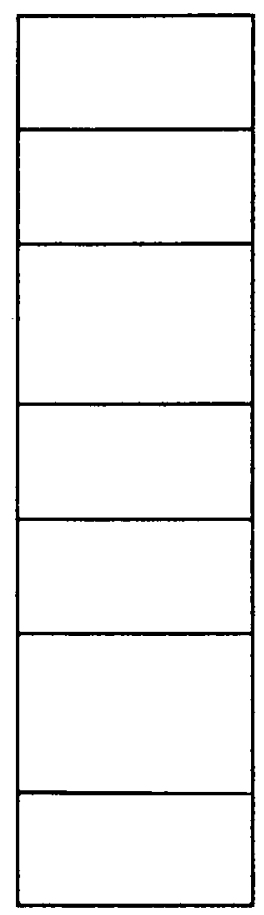

9. Remarks (add extra sheets if more space is needed): 
blank page 


\section{LDRD FIVE YEAR FUNDING SUMMARY \\ IN \$K}

(for projects active in FY 1995)

\begin{tabular}{|c|c|c|c|c|c|c|}
\hline Proposal No & 1993 & 1994 & 1995 & 1996 & $1997^{2}$ & TOTAL \\
\hline $95-001 \mathrm{~N}$ & -0 & $-0-$ & 619.1 & 600.0 & 600.0 & $1,819.1$ \\
\hline $94-057 \mathrm{R} 1$ & -0 & 187.2 & 296.5 & 300.0 & -0 & 783.7 \\
\hline $92-182 \mathrm{R} 3$ & 3 & 213.9 & 202.6 & -0 & -0 & 416.5 \\
\hline $93-151 R 2$ & 187.3 & 207.3 & 216.1 & -0 & -0 & 610.7 \\
\hline 94-144R1 & -0 & 69.4 & 114.2 & 155.0 & -0 & 338.6 \\
\hline 94-191R1 & -0 & 84.0 & 122.8 & 130.0 & -0 & 336.8 \\
\hline $94-001 \mathrm{R} 1$ & -0 & 141.2 & 189.3 & -0 & -0 & 330.5 \\
\hline $95-271 \mathrm{~N}$ & -0 & -0 & 203.1 & -0 & -0 & 203.1 \\
\hline 94-193R1 & -0 & 84.5 & 136.6 & 180.0 & -0 & 401.1 \\
\hline $95-012 \mathrm{~N}$ & -0 & -0 & 130.4 & 100.0 & 200.0 & 430.4 \\
\hline $93-152 \mathrm{R} 2$ & 183.1 & 150.7 & 148.6 & -0 & $-0-$ & 482.4 \\
\hline 94-142R1 & -0 & 198.8 & 150.4 & 150.0 & -0 & 499.2 \\
\hline $95-007 \mathrm{~N}$ & -0 & -0 & 200.9 & 150.0 & 200.0 & 550.9 \\
\hline $95-171 N$ & -0 & -0 & 56.3 & -0 & -0 & 56.3 \\
\hline $93-041 R 2$ & $137.9^{4}$ & $223.6^{5}$ & $343.1^{5}$ & $150.0^{5}$ & -0 & 854.6 \\
\hline $95-013 \mathrm{~N}$ & $-0-$ & -0 & 81.5 & 50.0 & -0 & 131.5 \\
\hline $93-150 \mathrm{R} 2$ & 145.3 & 81.5 & 130.3 & $-0-$ & -0 & 357.1 \\
\hline $95-003 \mathrm{~N}$ & $-0-$ & $-0-$ & 43.3 & 119.0 & 130.0 & 292.3 \\
\hline $95-006 \mathrm{~N}$ & -0 & -0 & 220.9 & 200.0 & -0 & 420.9 \\
\hline 93-007R2 & 58.3 & 98.8 & 92.5 & -0 & -0 & 249.6 \\
\hline $93-004 R 2$ & 312.0 & 353.1 & 257.9 & -0 & -0 & 923.0 \\
\hline $94-046 \mathrm{R} 1$ & -0 & 89.7 & 152.3 & -0 & -0 & 242.0 \\
\hline $95-040 \mathrm{~N}$ & -0 & -0 & 158.2 & -0 & -0 & 158.2 \\
\hline $95-027 \mathrm{~N}$ & -0 & $-0-$ & 215.2 & -0 & $-0-$ & 215.2 \\
\hline $95-054 \mathrm{~N}$ & -0 & -0 & 294.4 & -0 & -0 & 294.4 \\
\hline $93-043 R 2$ & 248.2 & 225.2 & 223.9 & -0 & -0 & 697.3 \\
\hline
\end{tabular}




\begin{tabular}{|c|c|c|c|c|c|c|}
\hline Proposal $\mathrm{No}$ & 1993 & 1994 & $1995 \%$ & $1996 \%$ & $1997^{2}$ & TOTAL \\
\hline $95-037 \mathrm{~N}$ & $-0-$ & $-0-$ & 35.7 & $-0-$ & $-0-$ & 35.7 \\
\hline 94-187R1 & $-0-$ & 226.0 & 201.7 & 200.0 & -0 & 627.7 \\
\hline $95-221 \mathrm{~N}$ & -0 & -0 & 57.1 & $-0-$ & -0 & 57.1 \\
\hline $94-015 \mathrm{R} 1$ & $-0-$ & 111.3 & 99.9 & -0 & $-0-$ & 211.2 \\
\hline $93-040 R 2$ & 216.2 & 124.4 & 132.5 & -0 & -0 & 473.1 \\
\hline $95-041 \mathrm{~N}$ & $-0-$ & -0 & 239.9 & 240.0 & 240.0 & 719.9 \\
\hline $95-030 \mathrm{~N}$ & -0 & $-0-$ & 198.9 & 150.0 & 150.0 & 498.9 \\
\hline $95-049 \mathrm{~N}$ & $-0-$ & $-0-$ & 298.6 & $-0-$ & -0 & 298.6 \\
\hline $94-114 \mathrm{R} 1$ & -0 & 314.7 & 148.8 & $-0-$ & -0 & 463.5 \\
\hline $95-061 \mathrm{~N}$ & $-0-$ & $-0-$ & 297.2 & -0 & -0 & 297.2 \\
\hline $95-280 \mathrm{~N}$ & -0 & $-0-$ & 25.0 & 200.0 & 275.0 & 500.0 \\
\hline $94-032 R 1$ & -0 & 160.8 & 137.5 & 141.0 & $-0-$ & 439.3 \\
\hline $94-055 \mathrm{R} 1$ & $-0-$ & 155.1 & 25.2 & $-0-$ & -0 & 180.3 \\
\hline $95-063 \mathrm{~N}$ & -0 & $-0-$ & 188.0 & 200.0 & -0 & 388.0 \\
\hline $95-160 \mathrm{~N}$ & $-0-$ & $-0-$ & 75.2 & -0 & -0 & 75.2 \\
\hline $95-062 \mathrm{~N}$ & $-0-$ & -0 & 172.6 & 200.0 & 200.0 & 572.6 \\
\hline 94-169R1 & $-0-$ & 162.4 & 141.4 & 70.0 & -0 & 373.8 \\
\hline $95-178 \mathrm{~N}$ & $-0-$ & -0 & 77.9 & -0 & $-0-$ & 77.9 \\
\hline $95-182 \mathrm{~N}$ & $-0-$ & -0 & 75.4 & $-0-$ & $-0-$ & 75.4 \\
\hline $95-184 \mathrm{~N}$ & $-0-$ & $-0-$ & 74.3 & $-0-$ & -0 & 74.3 \\
\hline $95-163 \mathrm{~N}$ & $-0-$ & -0 & 73.7 & -0 & $-0-$ & 73.7 \\
\hline $95-196 \mathrm{~N}$ & -0 & -0 & 72.2 & $-0-$ & $-0-$ & 72.2 \\
\hline 94-058R1 & -0 & 209.5 & 142.5 & 100.0 & $-0-$ & 452.0 \\
\hline 94-060R1 & $-0-$ & 56.3 & 57.9 & $-0-$ & -0 & 114.2 \\
\hline 94-103R1 & -0 & 218.4 & 131.0 & 100.0 & $-0-$ & 449.4 \\
\hline $95-020 \mathrm{~N}$ & $-0-$ & $-0-$ & 207.1 & $-0-$ & -0 & 207.1 \\
\hline 94-182R1 & $-0-$ & 144.4 & 148.2 & $-0-$ & $-0-$ & 292.6 \\
\hline $95-162 \mathrm{~N}$ & $-0-$ & $-0-$ & 79.9 & 60.0 & -0 & 139.9 \\
\hline 94-165R1 & $-0-$ & 176.9 & 149.5 & 140.0 & -0 & 466.4 \\
\hline 94-179R1 & -0 & 140.2 & 136.4 & 140.0 & $-0-$ & 416.6 \\
\hline
\end{tabular}

xlviii 


\begin{tabular}{|c|c|c|c|c|c|c|}
\hline Proposal No. & 1993 & 1994 & 1995 & $1996^{\prime}$ & $1997^{2}$ & TOTAL \\
\hline $95-253 N$ & -0 & $-0-$ & 100.5 & 140.0 & 150.0 & 390.5 \\
\hline $95-260 \mathrm{~N}$ & $-0-$ & $-0-$ & 100.0 & 140.0 & $-0-$ & 240.0 \\
\hline $95-305 \mathrm{~N}$ & $-0-$ & $-0-$ & 53.3 & 60.0 & 80.0 & 193.3 \\
\hline 94-054R1 & $-0-$ & 160.6 & 168.0 & $-0-$ & $-0-$ & 328.6 \\
\hline $95-010 \mathrm{~N}$ & -0 & -0 & 176.3 & 200.0 & -0 & 376.3 \\
\hline $95-070 \mathrm{~N}$ & $-0-$ & $-0-$ & 198.0 & 200.0 & 200.0 & 598.0 \\
\hline 94-190R1 & $-0-$ & 158.9 & 140.8 & -0 & $-0-$ & 299.7 \\
\hline $95-072 \mathrm{~N}$ & $-0-$ & -0 & 173.0 & 169.0 & 170.0 & 512.0 \\
\hline $95-247 \mathrm{~N}$ & $-0-$ & $-0-$ & 74.2 & $-0-$ & -0 & 74.2 \\
\hline $94-047 \mathrm{R} 1$ & $-0-$ & 179.8 & 193.1 & $-0-$ & $-0-$ & 372.9 \\
\hline $95-269 \mathrm{~N}$ & $-0-$ & $-0-$ & 99.7 & 100.0 & -0 & 199.7 \\
\hline $95-167 \mathrm{~N}$ & -0 & -0 & 68.4 & 70.0 & $-0-$ & 138.4 \\
\hline $95-241 \mathrm{~N}$ & $-0-$ & -0 & 74.2 & -0 & -0 & 74.2 \\
\hline $95-279 N$ & $-0-$ & $-0-$ & 42.1 & $-0-$ & $-0-$ & 42.1 \\
\hline $92-180 \mathrm{R} 3$ & 337.7 & 296.0 & $92.4^{6}$ & $-0-$ & -0 & $773.6^{7}$ \\
\hline $92-108 \mathrm{R} 2$ & -0 & 290.4 & 188.6 & $110.0^{8}$ & -0 & $831.1^{9}$ \\
\hline $95-176 \mathrm{~N}$ & -0 & $-0-$ & 75.6 & $-0-$ & $-0-$ & 75.6 \\
\hline $95-164 \mathrm{~N}$ & $-0-$ & $-0-$ & 70.4 & 75.0 & $-0-$ & 145.4 \\
\hline $95-183 \mathrm{~N}$ & $-0-$ & $-0-$ & 75.2 & -0 & $-0-$ & 75.2 \\
\hline $95-011 \mathrm{~N}$ & -0 & $-0-$ & 140.6 & $-0-$ & $-0-$ & 140.6 \\
\hline 93-090R2 & 284.8 & 270.8 & 162.6 & $-0-$ & -0 & 718.2 \\
\hline $95-270 \mathrm{~N}$ & $-0-$ & $-0-$ & 124.0 & 300.0 & $-0-$ & 424.0 \\
\hline 91-034R2 & -0 & 105.7 & 116.3 & -0 & -0 & $231.9^{10}$ \\
\hline $95-272 \mathrm{~N}$ & -0 & -0 & 39.1 & 465.0 & 460.0 & 964.1 \\
\hline $95-050 \mathrm{~N}$ & -0 & $-0-$ & 199.8 & -0 & -0 & 199.8 \\
\hline $95-095 \mathrm{~N}$ & -0 & -0 & 150.1 & -0 & -0 & 150.1 \\
\hline 93-094R2 & 88.9 & 201.0 & 140.5 & -0 & $-0-$ & 430.4 \\
\hline 94-167R1 & -0 & 133.6 & 137.5 & -0 & $-0-$ & 271.1 \\
\hline $95-075 \mathrm{~N}$ & -0 & $-0-$ & 244.3 & $-0-$ & -0 & 244.3 \\
\hline $95-071 N$ & -0 & $-0-$ & 180.2 & 200.0 & 200.0 & 580.2 \\
\hline
\end{tabular}




\begin{tabular}{|c|c|c|c|c|c|c|}
\hline Trioposal No. & 1993 & 1994 & 1995 & $1996^{\prime}:$ & $1997 ?$ & TOTAL \\
\hline $95-051 \mathrm{~N}$ & -0 & -0 & 198.7 & -0 & $-0-$ & 198.7 \\
\hline $95-229 \mathrm{~N}$ & $-0-$ & $-0-$ & 74.6 & -0 & -0 & 74.6 \\
\hline $95-217 \mathrm{~N}$ & $-0-$ & -0 & 74.9 & -0 & $-0-$ & 74.9 \\
\hline $95-170 \mathrm{~N}$ & $-0-$ & $-0-$ & 59.4 & $-0-$ & $-0-$ & 59.4 \\
\hline $95-191 \mathrm{~N}$ & $-0-$ & $-0-$ & 73.8 & $-0-$ & -0 & 73.8 \\
\hline 94-003R1 & $-0-$ & 128.4 & 118.4 & $-0-$ & $-0-$ & 246.8 \\
\hline $95-159 \mathrm{~N}$ & $-0-$ & -0 & 72.7 & $=0-$ & $-0-$ & 72.7 \\
\hline $94-168 \mathrm{R} 1$ & $-0-$ & 123.1 & 139.7 & 70.0 & $-0-$ & 332.8 \\
\hline $95-265 \mathrm{~N}$ & $-0-$ & $-0-$ & 104.6 & 140.0 & 200.0 & 444.6 \\
\hline . & $2,247.2$ & $6,909.6$ & $13,617.2$ & $6,664.0$ & $3,455.0$ & $32,893.0$ \\
\hline
\end{tabular}

1996 figures represent estimates and not actual expenditures

1997 figures represent projections assuming the project is renewed

Subtask under other project in 1992 and 1993

Subtask a

Subtask b (initiated in FY94)

Project completion date 6/95

Includes $\$ 47.5 \mathrm{~K}$ from FY92 (project began 7/92)

Project completion date $2 / 96$

Includes $\$ 242.1$ from FY92 (project began 1/92)

10 Includes $\$ 9.9 \mathrm{~K}$ from FY91 


\title{
95-001N -- RAPIDLY CYCLING SYNCHROTRON PERFORMANCE OPTIMIZATION
}

\author{
Associate Laboratory Director Area: Advanced Photon Source \\ Principal Investigator: \\ Y. Cho, Office of the Director \\ Funding Profile: \\ FY $1993-0-$ \\ FY $1994-0-$ \\ FY $1995 \$ 619.1 \mathrm{~K}$ \\ FY $1996 \$ 600.0 \mathrm{~K}$ \\ FY $1997 \$ 600.0 \mathrm{~K}$
}

Purpose: A great deal of progress has been made recently in the field of pulsed spallation neutron sources, both in Europe and in the US. ANL has continued to be a pioneer in the development and utilization of accelerator-based pulsed neutron sources since the 1970s. The purpose of this LDRD was three-fold:

1) To perform accelerator physics studies to optimize the design of the rapidly cycling synchrotron for the generation of $1 \mathrm{MW}$ of proton beam power. 2) To integrate previous work together with new designs and calculations and to compare the results with similar studies performed by other institutions. 3) To initiate prototyping activities for key components.

A feasibility study (ANL 95-13, April 1995) to upgrade IPNS to $1 \mathrm{MW}$ based on prior studies was reviewed by an external panel of experts in April 1995 and was very favorably received. The committee recommended that other studies be performed to ensure that the 1-MW facility can later be upgraded to $5 \mathrm{MW}$ and also suggested several improvements and additions in various areas. $\mathrm{R} \& \mathrm{D}$ on prototyping components was also to be performed. This LDRD project was initiated in anticipation of the need for these need studies.

Background: The neutron scattering scientific community has expressed a need for high-power pulsed sources in the beam power range of 1 to $5 \mathrm{MW}$. Scientists and engineers from several US laboratories as well as from Germany, the UK, Italy, Switzerland, the Netherlands, Sweden, Denmark and Austria are working on possible accelerator configurations for such high powered sources. European scientists continue to develop the 5-MW European Spallation Source (ESS), and the Austron project, designed by CERN personnel for an Austrian consortium of universities and research institutions, is still under funding consideration by the governments of Austria, the Czech Republic, Hungary and Slovakia. ANL and LANL have been studying 1-MW source designs that re-use existing infra-structure, while BNL has been working on a 5-MW greenfield source.

The ANL study team had been working on a feasibility study of a 1-MW pulsed source, based on a rapidly cycling synchrotron that would be housed in the existing buildings and infrastructure of the former ZGS. Part 1 of this year's work was to optimize the RCS performance such that it is suitable for a 1-MW source, and part 2 was to integrate and document all previously accomplished work. 
Approach: The ANL concept for the 1-MW source consists of a low energy (400 MeV) linac, a fast-cycling synchrotron, and two neutron-generating target stations. The technical facilities are to be housed in the ZGS area. The plan is that if, at some time after the 1-MW source is operational, further upgrade is warranted, the beam from the 2-GeV RCS would be further accelerated to $10 \mathrm{GeV}$ in a second RCS thus providing $5 \mathrm{MW}$ of beam power. Most of the effort has therefore been devoted to optimizing the 2-GeV, 1-MW RCS system.

Optimization work performed in FY 95 included:

1) optimization of synchrotron component layout

2) optimization of injection and extraction geometry

3) optimization of injection and capture processes

4) optimization of radio-frequency voltage programming

5) determination of synchrotron magnet apertures

6) optimization of magnet performance

7) optimization power supplies to minimize the power consumption

8) design of the linac building and low energy transport line tunnel

9) complete facility layout, including all components

10) impedance studies

11) rf system design studies

12) vacuum chamber design and vacuum services layout

13) initial ANL computer simulations to verify the AccSys linac design

To the extent this work was completed in time, it and all other previous work was integrated into a single document, IPNS UPGRADE, A Feasibility Study, ANL-95-13 (April 1995). This integrated work was reviewed by an external peer group.

One recommendation of the IPNS Upgrade peer-review was that the IPNS 1-MW source should be upgradable to $5 \mathrm{MW}$ : Work to extend the $1-\mathrm{MW}$ source to $5 \mathrm{MW}$ was begun in this fiscal year. In order to achieve $5 \mathrm{MW}$ of beam power, beam from the 1-MW source is injected into a second RCS that increases the proton beam energy from $2 \mathrm{GeV}$ to $10 \mathrm{GeV}$. Consequently, a concerted effort was made to study a $5-\mathrm{MW}, 10-\mathrm{GeV}$ proton source. An interesting result is that the performance parameters of this $5-\mathrm{MW}, 10-\mathrm{GeV} \mathrm{RCS}$ are quite similar to the proton source requirements for the $2-\mathrm{TeV}$ on $2-\mathrm{TeV}$ muon-muon collider being proposed by $\mathrm{BNL}$ and FNAL. The design under consideration by this group could, therefore, serve both purposes.

Personnel involved in the past year's work are Y.-C. Chae, E. Crosbie, H. Friedsam, K. Harkay, D. Horan, S. Kim, R. Kustom, E. Lessner, W. McDowell, D. McGhee, F. Mills, H. Moe, R. Nielsen, G. Norek, K.J. Peterson, K. Primdahl, Y.L. Qian, A. Rauchas, K. Symon, K. Thompson, D. Warner, M. White, and Y. Cho.

Technical Progress and Results: The results of the past year's work show that a 1-MW pulsed source, based on rapid cycling synchrotron technology, and utilizing existing infra-structure at the former ZGS complex is feasible. A four-sided synchrotron of some $190 \mathrm{~m}$ circumference housed in the ZGS Ring Building provides a proton beam of $2 \mathrm{GeV}$ in energy and $0.5 \mathrm{~mA}$ in average current. A $400-\mathrm{MeV}$ proton linac serves as the injector, and is housed in a new 
building designed for that purpose. Proceeding in this way allows the existing IPNS program to continue uninterrupted during most of the construction.

Accelerator hardware, such as magnets, power supplies, $\mathrm{rf}$ cavities, $\mathrm{rf}$ power sources, and vacuum chambers were investigated and refined during the past year. It was concluded that all hardware performance requirements, as defined by the physics requirements, are attainable. Refinements obtained as a result of this year's study are incorporated in the final report.

The accelerator physics studies centered around simulations to understand the injection, capture, and acccleration processes. Emphasis was on understanding and eliminating beam loss mechanisms from the injection, capture and acceleration processes, while ensuring that the beam would remain stable in longitudinal and transverse phase space during the entire acceleration cycle.

This study found that beam handling during the injection and acceleration processes is easier if the injected beam has a large energy spread and large transverse emittance, and that the synchrotron frequency has to be high enough for the beam to follow the stable phase angle.

All algorithms developed for studying the 2-GeV, 1-MW RCS are used in studying the $10-\mathrm{GeV}$, 5-MW machine.

The $10-\mathrm{GeV}$ machine is four times as large as the $2-\mathrm{GeV}$ RCS, and the $10-\mathrm{GeV}$ RCS can be sited at south side of Building 362. A detailed layout, including optimized siting of the 5-MW machine, two future target stations with long beam lines, and optimum beam transfer between the two machines is in progress.

Specific Accomplishments: The results of this year's work (five papers) were presented at the 1995 Particle Accelerator Conference in Dallas, l'exas, May 1995, and at the $2+2 \mathrm{TeV} \mu \mu$ Collider Collaboration Meeting at Fermi National Laboratory, 11-13 July 1995.

Y. Cho, et al., "Feasibility Study of 1 MW Pulsed Spallation Source," Proc. of the IEEE 1995 Particle Accelerator Conference, Dallas, Texas (May 1995), to be published.

K. Harkay, et al., "Longitudinal Instability Analysis for IPNS-II," Proc. of the IEEE 1995 Particle Accelerator Conference, Dallas, Texas (May 1995), to be published.

K. Harkay, et al., "Transverse Instability Analysis for the IPNS-II," Proc. of the IEEE 1995 Particle Accelerator Conference, Dallas, Texas (May 1995), to be published.

E. Crosbie and K. Symon, "Injecting a Kapchimskij-Vladimirskij Distribution into a Proton Synchrotron," Proc. of the IEEE 1995 Particle Accelerator Conference, Dallas, Texas (May 1995), to be published.

Y. Cho and Y.-C. Chae, "Study of Field Ionization is the Charge-Exchange Injection for the IPNS-II," Proc. of the IEEE 1995 Particle Accelerator Conference, Dallas, Texas (May 1995), to be published. 
E. Lessner, et al., "Effects of Imperfections on the Dynamics Aperture and Orbit Functions of the IPNS-II Synchrotron," Proc. of the IEEE 1995 Particle Accelerator Conference, Dallas, Texas (May 1995), to be published. 


\title{
94-057R1 -- DEVELOPMENT OF BASIC CONCEPTS AND COMPONENTS FOR THE ACCELERATION OF RADIOACTIVE BEAMS
}

\author{
Associate Laboratory Director Area: Physical Research
}

Principal Investigators:

\author{
J.A. Nolen, I. Ahmad, B.B. Back, C.N. Davids, \\ C.-L. Jiang, E.P. Kanter, R.C. Pardo, \\ K.E. Rehm, J.P. Schiffer, and K.W. Shepard, \\ Physics Division
}

Funding History:

FY $1993-0-$
FY $1994 \quad \$ 187.2 \mathrm{~K}$
FY $1995 \quad \$ 296.5 \mathrm{~K}$
FY $1996 \quad \$ 300.0 \mathrm{~K}$
FY $1997-0-$

Purpose: The goal of this project was to develop some of the critical technical concepts necessary to support a proposal for a radioactive beam facility at Argonne. There is considerable enthusiasm within the international nuclear physics community for the research that could be done with a general purpose facility capable of producing and accelerating intense beams of radioactive isotopes. A "white paper" for a new facility has been written by the North American Committee for an Isospin Laboratory, but detailed proposals for such a facility must be generated by specific laboratories. The technical feasibility of the design must be demonstrated in order to make the strongest proposal for siting a U.S. Isospin Laboratory at Argonne. During the first year of this LDRD project we identified specific components of such a facility for which an incremental effort would greatly enhance the technical soundness of the proposal. A high priority for the second year of this project was to prepare a "working paper" on the physics justification and technical concept for siting an advanced radioactive beam facility at Argonne. The goal was to present the working paper at meetings of the Nuclear Science Advisory Committee (NSAC) to justify the inclusion of such a facility in the Nuclear Physics Long Range Plan.

Approach: The benchmark facility suggested by the North American Committee for an Isospin Laboratory constructed on a "green field" is generally believed to be too costly to be pursued as a DOE construction project in the foreseeable future. We believe that during the first year of this LDRD project we have come up with a cost effective approach to achieve the physics goals of that white paper. Our efforts during 1995 were concentrated on more detailed computer simulations and hardware prototyping of those technical components which were most likely to be challenged during a technical review of our concepts. It was essential to convince NSAC that a radioactive beam facility with advanced capabilities could be constructed at a cost in the range of $\$ 100 \mathrm{M}$.

A complete radioactive beam facility consists of several major components: a "driver" accelerator with high power beams (about $100 \mathrm{~kW}$ ) to make radioactive isotopes via spallation reactions, the target complex in which the isotopes are produced and initially ionized, the preaccelcrator and isobar separator section, the main arcelerator for the radioactive beams, and the experimental facilities for research with the beams. The last two items are well covered at 
Argonne by the existing ATLAS facility. Hence, the present efforts are concentrated on computer simulation studies and hardware tests to develop and refine concepts for the following: (1) a cost effective driver accelerator to create a wide variety of radionuclides far from the stable isotopes, (2) the technology of high power targets and associated ion sources for the initial production of radioactive ions, (3) a high-acceptance mass separator with resolution of about 30,000 to separate individual isotopes of a given isobar, (4) a preaccelerator for very low velocity and low charge-to-mass ratio heavy ions, and (5) efficient methods for stripping and/or charge state enhancement of such ions. A parallel effort, supported primarily by ER programmatic funds, has involved some test runs at ATLAS to demonstrate a possible application of radioactive beams for wear analysis, and to establish credibility in the field of radioactive beams by actually accelerating a radioactive ion for a nuclear physics experiment.

The methods employed included computer simulations to quantitatively evaluate the options for the various components of the proposed facility, as well as, prototyping of some critical accelerator components. The computer codes LAHET and ISAPACE, for Monte Carlo calculations of radionuclide production induced via various primary beams, were obtained and run by Physics Division staff to support our particular selection of driver linac. To test the reliability of these calculations in some specific geometries of immediate relevance to our proposal, a collaboration of Physics Division staff with a scientist at MSU carried out some reaction yield measurements at the National Superconducting Cyclotron Laboratory. A small contract was awarded to AccSys Technology, Inc. for a physics study of the feasibility of our proposed driver linac configuration. Physics Division staff attended workshops on the production of accelerated radioactive beams and visited laboratories, such as the meson factory LAMPF at Los Alamos, to learn about techniques for shielding and remote handling at high power production targets. A summer student, J. Wendlandt, a graduate student in robotics at Berkeley, worked at ANL to help develop plans for remote handling of radioactive components. Other students, Teresa Barlow, Kevin Beyer, Mauricio Portillo, and Jared Torres, worked on a project to set up a test stand for ISOL-type ion sources. Existing apparatus in RE permitted experimental evaluation of some containment materials for liquid uranium; this work was carried out by J.L. Binder and colleagues in that division. Beam optics calculations were done for a preliminary design of a high resolution isobar separator. Considerable manpower was put into computer simulations and prototype modeling of the low $\mathrm{q} / \mathrm{m}$ injection stage of the radioactive beam post accelerator. A post doctoral appointee, J.-W. Kim, worked with PHY staff on this part of the project. Based on computer simulations and data from the literature, a technical solution for a specific highly efficient combination of target, ion source, and charge state stripping has been developed. Designs for hardware tests of some aspects of this proposal are in progress. Computer simulations on the feasibility of more advanced concepts for charge state enhancement have also continued with the help of an outside collaborator, J.C. Dooling of $\mathrm{DOE} / \mathrm{CH} / \mathrm{ESHD}$.

Technical Progress and Results: The investigations supported by this LDRD project have led to the block diagram of a radioactive beam facility based on the use of ATLAS as the post accelerator. This is shown in the attached figure. Two major Monte Carlo-based computer codes have been obtained and made operational in PHY; these are LAHET and ISAPACE, which predict yields of radionuclides for various input beam species and energies and specified target geometries. Such predictions are essential in justifying our choice of driver linac. 
A paper entitled "Concept for an Advanced Exotic Beam Facility Based on ATLAS, a Working Paper" was prepared with contributions of many Physics Division Staff members and outside collaborators. It was used, together with various oral presentations, as input for the long range plan prepared by the Nuclear Science Advisory Committee during 1995 . We feel that the cost effectiveness of the technical approach advocated in this paper combined with the physics justification was instrumental in making an "advanced ISOL-type radioactive beam facility" the highest priority of the NSAC Long Range Plan for new DOE Nuclear Physics construction.

Several technical results were also accomplished during FY 1995. Quantitative experimental data on the yields of several neutron-rich fission products were obtained in an experiment at the MSU NSCL, using a $200 \mathrm{MeV}$ deuteron beam on targets of beryllium and uranium. AccSys Technology, Inc. completed a preliminary study indicating the feasibility of our concept for a high power driver linac with multi-beam capability. A resource paper on commercially available robotics systems for remote handling in hazardous environments was prepared by a summer student supported by the LDRD project. Information on existing designs for handling activated targets and ion source components at LAMPF, TRIUMF, and ISOLDE were obtained through participation in workshops and visits to these facilities. An ISOL-type ion source was obtained from the BNL group and installed at the ion source test facility at the ATLAS tandem. That test facility was refurbished considerably since it had not been used in many years. The RE group identified an alloy, TRIBOCOR, which is potentially useful for the containment of liquid uranium under vacuum; tests of crucibles fabricated from this alloy are currently in progress. Beam optics calculations have been carried out through third order for a mass separator with a resolving power of 30,000 , and an acceptance consistent with the emittances of typical ISOLtype ion sources. Two prototype coils for the high gradient superconducting quadrupole were fabricated by Cryomagnetics, Inc. These coils were tested and reached gradients within $20 \%$ of that required by our initial simulations of the low $\mathrm{q} / \mathrm{m}$ post accelerator linac. Computer simulations of the critical very low-q/m, low-velocity RFQ were completed and a half-scale cold model was designed, fabricated and tested. The results were excellent, confirming the accuracy of the computer model.

Specific Accomplishments: A working paper on physics possibilities and our facility concept was prepared and submitted to NSAC to help ensure a place for an advanced radioactive beam facility in the Long Range Plan. Four papers (non-refereed) were presented on various aspects of our facility concept at the 1995 Particle Accelerator Conference. Three presentations were made at workshops on targets and ion sources for radioactive beams (at CERN/ISOLDE and TRIUMF). One paper (refereed) on our ion source R\&D was accepted for publication in Nuclear Instruments and Methods. Three papers on physics results and instrumentation development with the ${ }^{18} \mathrm{~F}$ radioactive beam have been accepted for publication in refereed journals. A proposal for generic R\&D for a radioactive beam facility has been submitted to DOE-ER (Nuclear Physics). 


\section{Argonne Exotic Beam Laboratory}

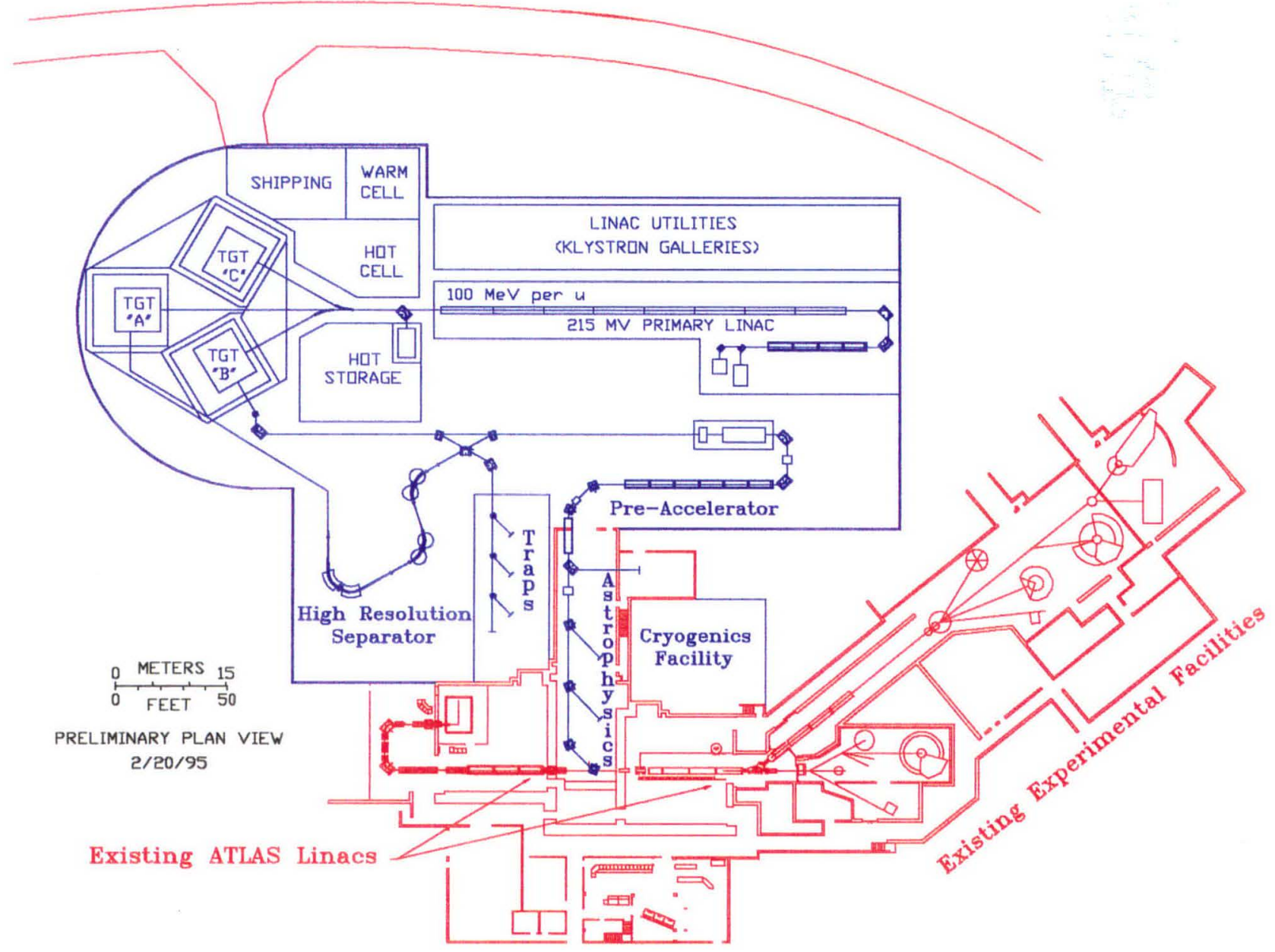

Figure 1. An overview of the present ATLAS Facility (lower part, in the present building D-203) and the conceptual layout of the proposed new components (upper part, north of D-203) to create a radioactive beam facility with advanced capabilities for nuclear physics. 


\title{
92-182R3 -- TARGET STATION STUDIES FOR THE INTENSE PULSED NEUTRON SOURCE UPGRADE
}

\author{
Associate Laboratory Director Area: Physical Research \\ Principal Investigators: \\ B.S. Brown and J.M. Carpenter, \\ Intense Pulsed Neutron Source \\ Funding Profile:

$\begin{array}{lll}\text { FY } & 1993^{1} & -0- \\ \text { FY } & 1994 & \$ 213.9 \mathrm{~K} \\ \text { FY } & 1995^{2} & \$ 202.6 \mathrm{~K} \\ \text { FY } & 1996 & -0- \\ \text { FY } 1997 & -0-\end{array}$

Purpose: To explore and document the feasibility and cost of a pulsed spallation neutron source based on a linac-synchrotron accelerator system providing a proton beam of 1-MW time-average current, having two targets and a reference set of 27 neutron scattering instruments. This work is the outgrowth of over 20 years of prior experience developing and applying lower power pulsed spallation sources at Argonne, and was pursued in response to DOE-sponsored committee recommendations for such studies.

Approach: The work on target stations and instrumentation was carried out in parallel with a separate effort on the accelerator systems under the direction of Y. Cho, Advanced Photon Source.

Technical Progress and Results: A Target Station Review Committee which met in July 1993 endorsed an early version of the target station design and made substantial suggestions for improvements which were incorporated in a reiteration of the target station reference design. That technical work was largely completed in FY 1994. In FY 1995, design of some subsystems and other details of the target station design reiteration were completed, cost and schedule estimates generated, and drawings and textual documentation compiled, edited, and prepared for reproduction.

Specific Accomplishments: The entire IPNS Upgrade program culminated in the completion of an 800-page report that was published in April 1995, entitled "IPNS Upgrade: A Feasibility Study," report number ANL-95-13. That document was reviewed by the PNS Upgrade Accelerator Systems Advisory Committee which convened in April 1995 at Fontana, Wisconsin. The Committee endorsed the project and document and offered some constructive suggestions. The report incorporated this target station work as a prominent chapter.

\footnotetext{
'This work was an undifferentiated subtask of Project 92-022 in FY92 and FY93. It began in August of 1992.

${ }^{2}$ Work concluded in July 1995 . Total duration of this task was 36 months.
} 
The total estimated cost of the upgrade will be $\$ 458 \mathrm{M}$ in 1995 dollars, and the duration of the construction project will be approximately 4.5 years. The proposed installation will make extensive use of existing buildings and infrastructure at a cost savings of about $\$ 175 \mathrm{M}$.

The proposed installation stands as a strong candidate as the nation considers prospects for its next generation neutron source facilities.

The figure shows a plan view of one target station. The details can be exhibited on the scale of the figure.

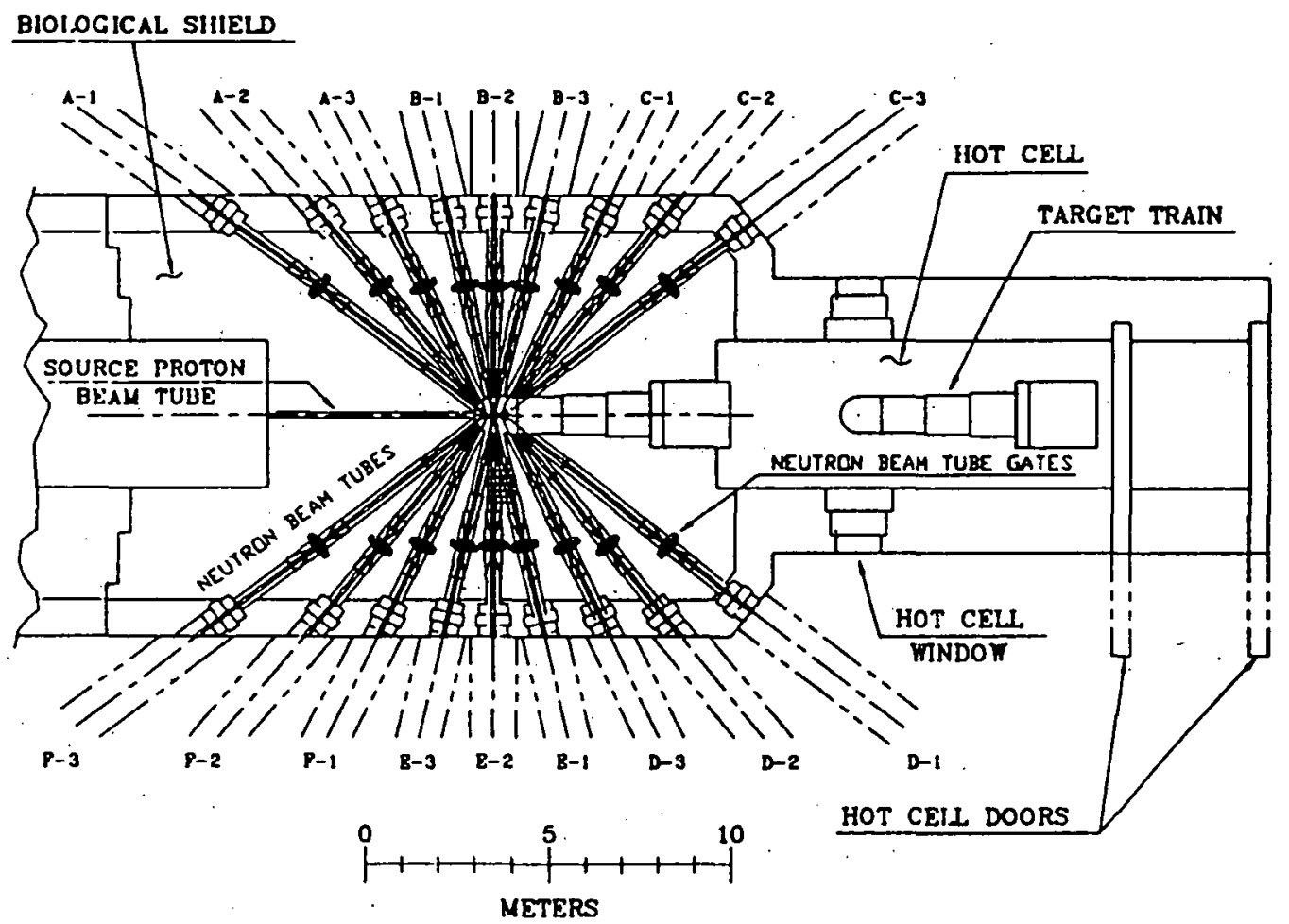




\title{
93-151R2 -- MILLIMETER WAVE LINAC AND UNDULATOR DEVELOPMENT USING MICROFABRICATION TECHNIQUES
}

\author{
Associate Laboratory Director Area: Advanced Photon Source \\ Principal Investigator: \\ R.L. Kustom, Accelerator Systems Division \\ Funding Profile: \\ FY1993 \$187.3K \\ FY1994 \$207.3K \\ FY1995 \$216.1K \\ FY 1996 -0- \\ FY $1997-0$
}

Purpose: The purpose of this project is to investigate the feasibility of building microfabricated rf cavities for mm-wave linacs and/or undulators using high-aspect-ratio micromachining techniques. This fabrication technique is a newly developing technology which could lead to fascinating applications of interest to the scientific and industrial communities. The goal is to demonstrate proof-of-principle feasibility of building $\mathrm{mm}$-wave $\mathrm{rf}$ structures for linacs, undulators, and FELs and rf amplifiers for industrial applications and secure DOE funding for a more extensive R\&D program and to attract private industry participation.

Approach: High-aspect-ratio micromachining techniques have evolved to where microcomponents can be manufactured out of copper, silver, nickel, and nickel alloys with features as small as a few microns with dimensional tolerances as good as $25 \AA$ and machining depths of a few $\mathrm{mm}$ with very little runout, thus the term high-aspect-ratio. Quite intricate structures such as submillimeter gears, motors, and actuators can be fabricated. Electric fields as high as 50 to $100 \mathrm{MV} / \mathrm{m}$ and magnetic fields as high as 0.5 to 1.0 tesla can be achieved in these small components. The possibility of making 3-dimensional structures is rapidly approaching reality. These possibilities provided the stimulus for investigating mm-wave structures for compact electron linear accelerators and undulators that could be used for vuv and x-ray synchrotrnn radiation snurces and mm-wave rf amplifiers for industrial use.

The elements of this technology that are being addressed by this project include development of $\mathrm{mm}$-wave rf structures through computer studies, oversized models for rf testing to verify computer studies, and construction and excitation of actual small-scale test sections; development and testing of masks for fabrication of structures; development of 3-dimensional fabrication, assembly, and alignment techniques; studies of beam dynamics; and, study of related technology such as cooling, vacuum, and rf sources for excitation. The work on 3-dimensional fabrication and alignment techniques is being done at the University of Illinois-Chicago under subcontract with Professor Alan Feinerman and some of the work on rf structures is being done at the Technical University of Berlin under subcontract with Professor Heino Henke.

Technical Progress and Results: During FY 1993, we performed computer calculations on a number of candidate rf structures to determine which might achieve the best rf performance consistent with the construction technique required by the high-aspect-ratio micromachining process. A muffin-tin structure with a $2 \pi / 3$ radian phase advance per cell at $120 \mathrm{GHz}$ gave a 
suitable accelerating field for a reasonable excitation power. These structures can be manufactured in $7 \mathrm{~cm}$ lengths which are compatible with present $10 \mathrm{~cm}$ disk semiconductor technology. A $10 \mathrm{X}(12 \mathrm{GHz})$ model was built with seven complete cells and input and output coupling cells for matching the rf. Tests on this oversized structure demonstrated the validity of computer calculations. An alternate structure, a side-coupled cavity arrangement, was successfully tested at the Technical University of Berlin.

$\mathrm{X}$-ray masks were made at the University of Illinois-Chicago. These masks included sample structures that have special alignment grooves for testing accuracy of alignment techniques. The masks also included sample quadrupole sections and possible magnetic mm-wavelength undulators. Techniques for testing the alignment included capacitive measurements on special sub-millimeter sized parallel plate capacitors. These capacitors were also put onto the masks.

A computer program was written for studying the beam dynamics in the rectangular geometry required for these structures. The beam dynamics equations need to account for the nonsymmetrical fields generated in the rectangular structure. These equations are quite different from those for the normal circular structures conventionally used. The program was successfully operated and used with actual fields of the muffin-tin structure.

Computer analysis of the thermal load and heating was performed on the $2 \pi / 3$ muffin-tin structure using the ANSYS code. The preliminary. results indicate that the structure can be adequately cooled. The largest temperature rise can be kept to below $40^{\circ} \mathrm{C}$ when the accelerating field is $10 \mathrm{MV} / \mathrm{m}$.

In FY 1994, we micromachined our first $2 \pi / 3$ linac structure. The high degree of precision that is possible was apparent in this structure. The rf cavity features that were machined on the die were easily within \pm 2 microns and the overall features of the surface were dominated by the surface irregularities of the substrate which was not diamond turned. Twenty diamond turned substrates that have a \pm 1 micron surface finish have been delivered for future cavity construction. This should improve the overall quality in the next units to be built. Much of this work was done by Professor Alan Feinerman and one of his students, Ted Willke, from the University of Illinois-Chicago. They prepared the optical and $\mathrm{x}$-ray masks and performed the alignment and tolerance measurements on the cavity features.

Quite a few rf and system analysis studies were performed. These studies focussed on types of rf structures, different linac assemblies and operating scenarios. The most likely options for operating parameters appear to be either, (1) operate at short pulse, 400 to $2000 \mathrm{~Hz}$ repetition rate with a $1 \%$, or less, duty factor, (2) operate with a superconducting cavity at $\mathrm{CW}$, or (3) operate with a long pulse normal conducting structure with a $10-15 \%$ duty factor.

A new constant gradient (CG) rf structure has been developed. The physical features are compatible with the deep $x$-ray lithography machining technique. The new CG structure generates a constant accelerating field and constant power distribution along the axial length, unlike the constant impedance structure. The new CG structure also has a vastly improved heat removal capability over the rectangular cell design with which we had originally started the study. 
In FY 1995, masks have been prepared for a variety of structures with different types of alignment techniques. These masks were used for polymethylmethacrylate (PMMA) exposures at the Synchrotron Radiation Center in Stoughton, the Advanced Light Source in Berkeley, and the National Synchrotron Light Source in New York. Successful exposures resulted in PMMA structures capable of being used for plating rf accelerating waveguides between 60 and $180 \mathrm{GHz}$. Attaching the PMMA films to conductive substrates for plating have not been completely successful. However, the University of Illinois-Chicago is continuing some effort on bonding techniques and in developing a plating facility as a result of the LDRD funding.

The alignment and fabrication technique that appears will work best is to plate square alignment slots in the structure, place precision-drawn quartz fibers in the slots, and clamp the structure. The desired \pm 2 micron accuracy looks achievable.

Preparations are being made to do exposures on the Advanced Photon Source when the beamline becomes available on that facility.

As a result of these studies, a new technique for making circularly symmetric structures has been conceived. It uses a flat mask and a rotating PMMA cylinder.

\section{Specific Accomplishments:}

\section{Internal Reports}

1. "Initial Beam Simulation for the mm-Wave Accelerating Structure," A. Nassiri, Internal Note, October 1993.

2. "Investigation of Planar mm-Wave RF Structures Focussing and Bunching, " S. Vaganian, H. Henke, TET-NO'TE 93/015, November 1993.

3. "Beam and Structure Misalignment Errors," A. Nassiri, Internal Note, February 10, 1994.

4. "Alternating Phase Focusing (APF) in mm-Wave Linear Accelerators," F. Mills and A. Nassiri, Internal Note, February 28, 1994.

5. "Misalignment Errors and Their Effects on the Electron Beam in mm-Wave Linac," A. Nassiri, Internal Note, February 1994.

6. "Tunable RF Undulator Using a Rectangular Waveguide," P. Matthews, Internal Note, April 1994.

7. "A mm-Wave RF Structure for Relativistic Electron Acceleration," H. Henke, et al., Internal Note, May 10, 1994.

8. "Investigation on the mm-Wave Linear Accelerator Project, " P. Matthews, Internal Note, July 7, 1994. 
9. "Effects of Quantum Excitation and Residual Gas Scattering on the Beam Lifetime in the mm-Wave Undulator for the APS Storage Ring," A. Nassiri, Internal Note, September 1994.

\section{External Reports - Conference Papers}

1. "120 GHz mm-Wave Planar Accelerating Structure Linac System for Production of Short Wavelength Synchrotron Radiation," A. Nassiri, et. al., Linear Collider Workshop 1993 (LC'93), SLAC, October 1993.

2. "50 MeV mm-Wave Electron Linear Accelerator System for Production of Tunable Short Wavelength Synchrotron Radiation," A. Nassiri, et. al., International Electron Device Meeting, Washington, DC, December 5-8, 1993.

3. "Electromagnetic Field Measurements on a mm-Wave Linear Accelerator," P. Matthews, et. al., EPAC'94, London, Great Britain, June 1994.

4. "Parallel Reflector Waveguide as Microwave Undulator," Y. Kang, R. Kustom, A. Nassiri, and P. Matthews, EPAC'94, London, Great Britain, June 1994.

5. "RF Measurements of a $12 \mathrm{GHz}$ Scale Model of a Planar Structure," Y. Kang, P. Matthews, A. Nassiri, and R. Kustom, EPAC'94, London, Great Britain, June 1994.

6. "A Constant Gradient Planar RF Cavity Structure for Linac Use," Y.W. Kang, P.J. Matthews, R.L. Kustom, IEEE 1995 Particle Accelerator Conference and International Conferences on High-Energy Accelerators, Dallas, Texas, May 1-5, 1995.

7. "RF Gun Design for a mm-Wave Linac-Based XUV FEL,". A. Nassiri, J. Foster, IEEE 1995 Particle Accelerator Conference and International Conferences on High-Energy Accelerators, Dallas, Texas, May 1-5, 1995.

8. "Design and Construction of Planar mm-Wave Accelerating Cavity Structures, " Y.W. Kang, R.L. Kustom, F.E. Mills, A. Nassir, J.J. Song, SRI'95, Argonne, Illinois, October 1995.

9. "A LIGA-based mm-Wave Undulator Microstructure," J.J. Song, Y:W. Kang, R.L. Kustom, B. Lai, A. Nassir, A.D. Feinerman, G.M. Wells, V. White, SRI'95, Argonne, Illinois, October 1995 :

\section{External Reports - Invited Conference Paper}

1. "Micro-Structures, " R. Kustom, et. al., International Linear Accelerator Conference, KEK, Japan, August 1994. 


\section{$\underline{\text { Journal Papers }}$}

1. "X-ray Lathe: an X-ray Lithographic Exposure Tool for Non-planar Objects," A.D. Feinerman, R. Lajos, V. White, D. Denton, J. Mecroelectromechanical Systems, 1995.

2. "Feasibility Studies of a Compact mm-Wave Linac FEL," A. Nassiri, R.L. Kustom, Y.W. Kang, J. Song, Journal of Nuclear Instrument Measurements, to be published in 1996.

\section{$\underline{\text { Patent Applications }}$}

1. Planar Microwave/Millimeter Wave Cavity Structure

2. Planar rf Waveguide Coupling Structure for mm-Wave Linac

3. Method of Making Planar Cavity Structures Out of Diamond 
blank page 


\title{
94-144R1 -- SHORT WAVELENGTH PULSED MAGNETIC UNDULATOR DEVELOPMENT USING MICROFABRICATION TECHNIQUES
}

\author{
Associate Laboratory Director Area: Advanced Photon Source \\ Principal Investigator: $\quad$ R.L. Kustom, Accelerator Systems Division \\ Funding Profile: $\quad$ FY $1993-0-$ \\ FY $1994 \$ 69.4 \mathrm{~K}$ \\ FY $1995 \$ 114.2 \mathrm{~K}$ \\ FY $1996 \$ 155.0 \mathrm{~K}$ \\ FY $1997-0-$
}

Purpose: The purpose of this project is to investigate the feasibility of building microfabricated pulsed magnetic undulators for mm-wave linacs and/or free electron lasers (FELs) using highaspect-ratio micromachining techniques, to determine the parameter field over which these devices could operate and be of interest to the scientific community, and to develop a proof-ofprinciple design.

Approach: High-aspect-ratio micromachining techniques have evolved to where microcomponents can be manufactured out of copper, silver, nickel, and nickel alloys with features as small as a few microns with dimensional tolerances as good as $25 \AA$ and machining depths of a few $\mathrm{mm}$ with very little runout, thus the term high-aspect-ratio. Quite intricate structures such as submillimeter gears, motors, and actuators can be fabricated. Electric fields as high as 50 to $100 \mathrm{MV} / \mathrm{m}$ and magnetic fields as high as 0.5 to 1.0 tesla can be achieved in these small components. The possibility of making 3-dimensional structures is rapidly approaching reality. These possibilities provided the stimulus for investigating micro-undulator structures for compact electron linear accelerators that could be used for vacuum ultraviolet (vuv) and x-ray synchrotron radiation sources.

The elements of this technology that are being addressed by this project include development of $\mathrm{mm}$-size pulsed magnetic structures through computer studies, oversized and actual size models for magnetic field measurements and testing to verify computer studies, and construction and excitation of actual small-scale test sections; development and testing of masks for fabrication of structures; development of 3-dimensional fabrication, assembly, and alignment techniques; studies of beam dynamics; and, study of related technology such as cooling, vacuum, and pulsed power sources for excitation. The work on 3-dimensional fabrication and alignment techniques is being done at the University of Illinois-Chicago under subcontract with Professor Alan Feinerman.

Technical Progress and Results: During FY 1994, we performed computer calculations on a number of candidate undulator structures to determine and understand magnetic-field properties which might achieve the best performance consistent with the construction technique required by the high-aspect-ratio micromachining process.

Electromagnetic field computations were performed to establish a desirable field patter for the undulator. This was solved by using counter-flowing currents in the top and bottom of the 
undulator, shifted by one pole. The problem of current flowing across the pole instead of through the conductor was partially solved by making the conductor width $0.2 \mathrm{~mm}$ and the pole width $0.3 \mathrm{~mm}$, rather than vice versa.

Other problems with current flow could not be completely resolved immediately because the 3-dimensional eddy current software appeared to have some problems enforcing current conservation. This problem is being discussed with the vendor and they will provide a version which will allow us to complete this analysis. All the simulations done so far suggest that an undulator with the proper field and current is feasible.

Two model undulators were designed and fabricated in the initial stage of this project. These devices were fabricated according to the numerical 2-D and 3-D magnetic-field simulations. The first one is a $10 \mathrm{x}$ scale model with an undulator period of $1 \mathrm{~cm}$ fabricated from a steel piece using precision electric discharge machining (EDM). This model is now being prepared for the initial magnetic tests. A second actual model with 1-mm undulator period was fabricated at the University of Illinois-Chicago by Professor Alan Feinerman which is also being prepared for magnetic measurements. To be able to measure the undulator fields in small gap, two methods have been developed. In the first case, a pick-up coil will be fabricated using the printed-circuit technology which will provide enough sensitivity for small field measurements. For very small gap size, conventional field-probe measurement techniques are not sufficient in determining the undulator field profile. A new method is being developed and implemented which employs magneto-optical techniques using a single detector crystal to measure the change of a given polarization state of a laser beam to induce information on the magnetic-field value and distribution inside the undulator.

A computer program was written for studying the beam dynamics in a 3-dimensional undulator field. An analytical analysis separating the effect on beam motion with and without parts governing the radiation aspect of the beam motion through undulators was performed. An approximate solution for the beam motion through the undulators was obtained. The effect of errors on the beam motion was analytically solved. A program using MATHEMATICA was developed to simulate the full motion through such undulators. At present, the radiative aspects in the undulators are being studied.

In FY 1994 we performed magnetic-field computer simulations to characterize the performance of both the actual (1-mm period) and the $10 x$ scale model $(1-\mathrm{cm}$ period). The initial numerical simulations have provided us with the necessary information about the physics of these shortperiod devices to conclude that proper field and current distribution is feasible. Two test models have been fabricated and are in the process of being measured. A magneto-optical field measurement system is being put together which uses a laser system along with a crystal to measure the field inside the undulator. Professor Alan Feinerman, from the University of Illinois-Chicago has fabricated the first 1-mm-period undulator using conventional machining techniques.

The field quality for the short wavelength pulsed magnetic undulator was tested and verified on the 10x scale model during FY 1995. These tests provided considerable confidence that the analytical and computational techniques that were used for predicting field quality are accurate and can be used for the mm-sized model. 
Masks for doing x-ray exposures were developed in FY 1995. These masks are of sufficient quality to be used for $\mathrm{x}$-ray exposure and subsequent micromachining of the magnets.

\section{Specific Accomplishments:}

\section{Internal Reports}

1. "A Summary of the Micro-Undulator Design for the Advanced Photon Source (APS) Electron Linac," A. Nassiri, F.E. Mills, internal note (February 1994).

\section{External Reports (unrefereed conference papers)}

1. "mm-size Micro-undulator for Production of Short Wavelength Synchrotron Radiation," A. Nassiri, F.E. Mills, and L. Turner, 6th Workshop on Advanced Accelerator Concepts, Lake Geneva, Wisconsin (June 1994).

2. "ELEKTRA Computations of a 1-mm Period Undulator Driven by Electric or Magnetic Scaler Potential," L. Turner, F.E. Mills, A. Nassiri, Vector Field Users Group Meeting, Framingham, Massachusetts (October 14, 1994).

\section{Journal Paper}

1. "A Micro-undulator Fabricated by LIGA Process," L. Turner, A. Nassiri, 14th International Conference on Magnet Technology, Tampere, Finland (11-16 June 1995); Journal paper in IEEE on Magnets (1996). 
blank page 


\section{4-191R1 -- IN-SITU SURFACE MONITORING SYSTEM FOR SYNCHROTRON OPTICS IN HIGH HEAT LOAD ENVIRONMENT}

\author{
Associate Laboratory Director Area: \\ Principal Investigators:
}

Funding History:
Advanced Photon Source

G. Srajer and D.M. Mills

Experimental Facilities Division

FY $1993-0-$

FY $1994 \$ 84.0 \mathrm{~K}$

FY $1995 \$ 122.8 \mathrm{~K}$

FY $1996 \$ 130.0 \mathrm{~K}$

FY $1997-0-$

Purpose: The project objective is the development of an opto-mechanical system for direct, real-time monitoring of thermally induced surface distortions of synchrotron radiation optical elements, particularly mirrors, subjected to high heat fluxes.

Approach: The shape or "figure" of $\mathrm{x}$-ray mirrors for third-generation synchrotron sources, such as the Advanced Photon Source (APS) is critical for the delivery of the full $\mathrm{x}$-ray brilliance to the experimental stations. However, the price for high brilliance is the unprecedented high power of the synchrotron beam. The combination of high total power and power density imposes demanding requirements on beamline components since their surfaces will be susceptible to heat induced distortions. For example, the APS undulator A will produce a total power of $1.5 \mathrm{~kW}$ and a power density of $140 \mathrm{~W} / \mathrm{mm}^{2}$, which is significantly more than the Cornell High Energy Synchrotron Source F-2 wiggler $\left(1.0 \mathrm{~kW}\right.$ and $\left.10 \mathrm{~W} / \mathrm{mm}^{2}\right)$, and the National Synchrotron Light Source X25 focused wiggler $\left(0.04 \mathrm{~kW}\right.$ and $\left.120 \mathrm{~W} / \mathrm{mm}^{2}\right)$.

Deviations from the desired shape (slope errors) less than an arc second can severely degrade the performance of mirrors for the APS. X-ray mirrors are routinely measured off-line (that is not in the beamline) in specialized laboratories for both surface roughness and figure (under ambient thermal conditions), but in-situ diagnostic, such as being proposed in this project, is much rarer. Our goal is to explore various approaches to in-situ, real-time monitoring of high-heat-load mirrors and to fabricate a prototype system (or systems) that might be suited to such measurements.

The monitoring system is essentially a metrology unit that is used to measure changes in the surface profile of an $x$-ray optical component under a thermally loaded condition. To avoid the expensive and bulky mechanical rail system that is typically associated with such system, we have chosen to only sample eight points of interest and then least-square fit the measured values to a set of orthogonal functions, allowing us map the surface in a very short time. The justification for this approach is that heat-induced deformation is most likely to be smoothly continuous. After careful analysis, we believe that the best approach for such system is the setup shown in Fig. 1, where we measure the angular deviation of the reflected probe beam to infer the mirror surface deformation. Each reference mirror forms a local resonator cavity with the surface of the high heat-load optic, enhancing the design over the simple Hartmann-type1 optical sensor in two ways: first, the probing laser beam strikes the mirror several times before 
leaving each cavity. When the different beam reflections are combined at the observation plane, sharp fringes are formed, much sharper than those from a single-pass interferometer (Fig. 2). The steeper intensity fall-off improves our ability to track the motion of the fringes and thus increases the sensitivity of the CCD camera to small positional shifts of the reflected beam. Secondly, every time the laser beam is reflected off the probing surface, it undergoes an angular deviation equal to twice the tilt of the optic's surface with respect to the beam. Applying the geometrical analysis shown in Fig. 3, one can show that when the beam is reflected $n$ times inside a resonator cavity, the angular deviation of the exit beam is equal to $2 n$ times the tilt of the optic surface with respect to the reference mirror. Hence, the position shift of the beam centroid is magnified, again leading to an increase in the sensitivity of the monitoring system. The beam splitter cubes are aligned so that the reflected beam from each "resonator" cavity falls on different corners of the CCD detector. The end mirror is then aligned so that its reflection falls near the center of the CCD detector. Each spot is subsequently image-processed and the centroid is calculated. Fluctuation in the reference center spot is subtracted from the other four to eliminate mechanical variations of the whole system, easing the vibration isolation problem in later construction. The two electronic shutters allow the user or the computer to choose the set of sample points to observe. To reduce any ambient fluctuations, the probing laser beam is spatially low-pass filtered.

The proposed monitoring system (Fig.1) consists of four sub-components:

(a) He-Ne laser with spatial filter

(b) Beam distribution/collection unit

(c) Telescope optics and CCD array

(d) Image-processing computer and software

The He-Ne laser with a spatial filter supplies a beam to a series of beam splitters in the beam distribution/collection unit, which then directs the split beam to multiple places on the particular optic (mirror or monochromator) surface. Reflected beams are collected by the same beam splitters and guided with a mirror to form multiple images on a CCD array. The image processing computer performs calculations on the multiple images and converts data to slope difference values.

Our design goals are to:

(a) develop a multi-channel version of the monitoring system

(b) achieve very high angular resolution of the order of $1 \mu \mathrm{rad}$ (or .2 $\operatorname{arcsec}$ )

(c) incorporate the monitoring system into a vibration-free support

(d) develop software for data collection and analysis

(e) construct a compact, portable and rather inexpensive system

Dr. Peter Mui has been hired to work full time on this project. Major progress has been achieved since his arrival.

Technical Progress and Results: Two competing approaches were considered for the monitoring system. One, based on the angle measurement technique, and, the other based on the interferometric technique. A comparative study was completed. While the interferometric 
technique provides better spatial resolution, its implementation is more complicated and costly. Therefore, a decision was made to build a prototype, two-element system based on the multi-pass, heterodyne2,3 angle measurement technique. The prototype system has been assembled and its performance has been tested. The results were very encouraging. Our design goal of $1 \mu \mathrm{rad}$ angular resolution was exceeded because we were able to achieve angular resolution that is less than $1 \mu \mathrm{rad}$. Furthermore, the system was kept stable to within $\pm 1.5 \mu \mathrm{rad}$ for more than 12 hours by using digital feedback technique. This is an important achievement because effects caused by slow mechanical variations that may arise due to changes in the ambient temperature, can be significantly reduced. A CCD camera was purchased along with a basic image processing package. Software for analyzing digitized images was developed. In an example, shown in Fig. 4, a reflected laser beam from a test optic piece was digitized, a centroid was calculated, and, a line scan was performed.

Specific Accomplishments: A two-element monitoring system based on multi-pass, heterodyne technique was designed, built and successfully tested. A CCD detection system and an image digitizing package was purchased. An algoritm for digitizing an image and finding the centroid of a reflected laser beam was developed and tested, and, subsequently ported onto the MS Window platform, making it easy to use.

\section{References:}

1. J.C. Fontanella, J. Optics (Paris) 2:99 (1991).

2. D. Malacara, Optical Shop Testing, John Wiley and Sons, New York (1978).

3. R.S. Sirohi, M.A. Kothiyal, Optical Components, Systems, and Measurement Techniques, Marcel Dekker, Inc., New York (1991). 


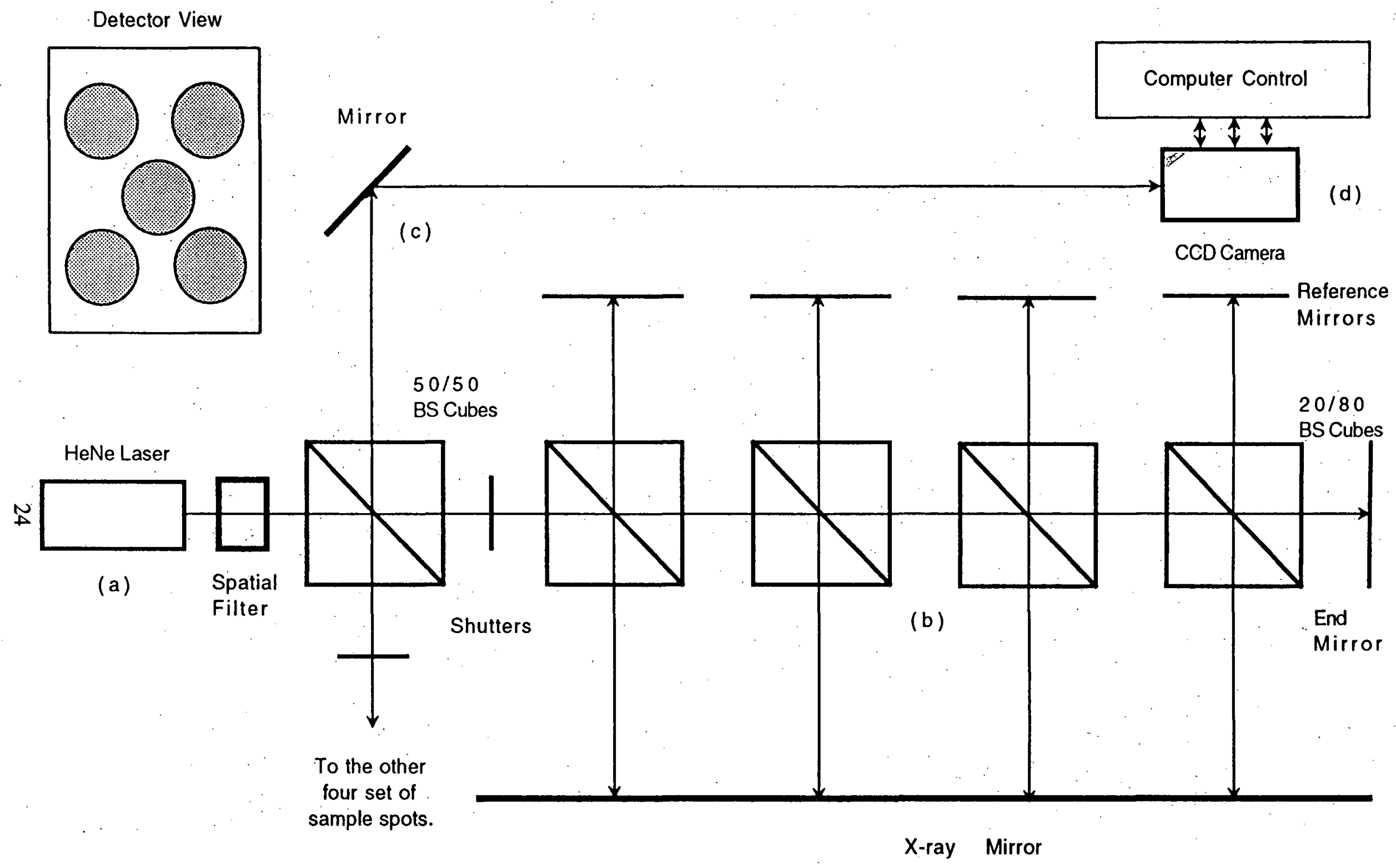

Fig. 1. A four-element surface monitoring unit. (a) He-Ne laser, (b) beam-splitter (BS) cubes, (c) collector optics, (d) detector 

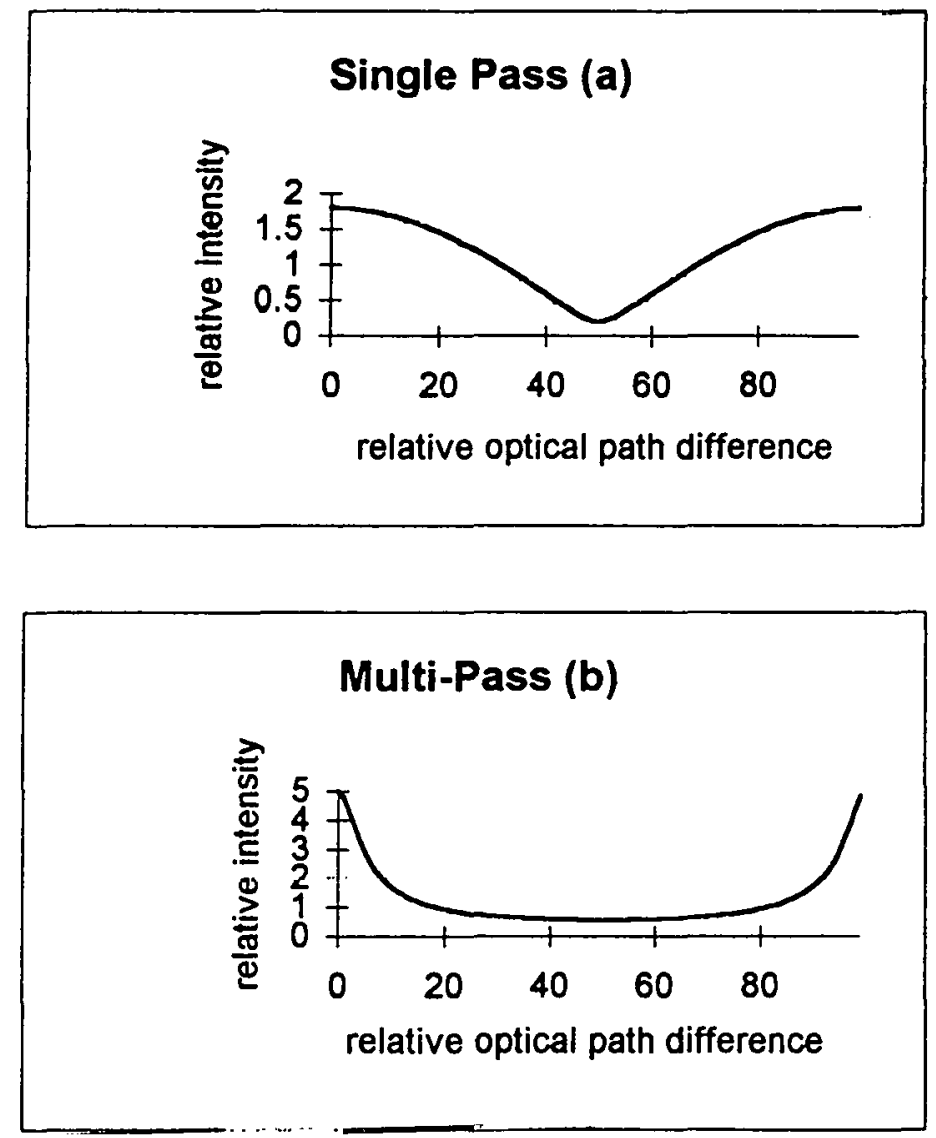

Fig. 2. Comparison between single pass (a) and multi-pass (b) fringe profiles. 


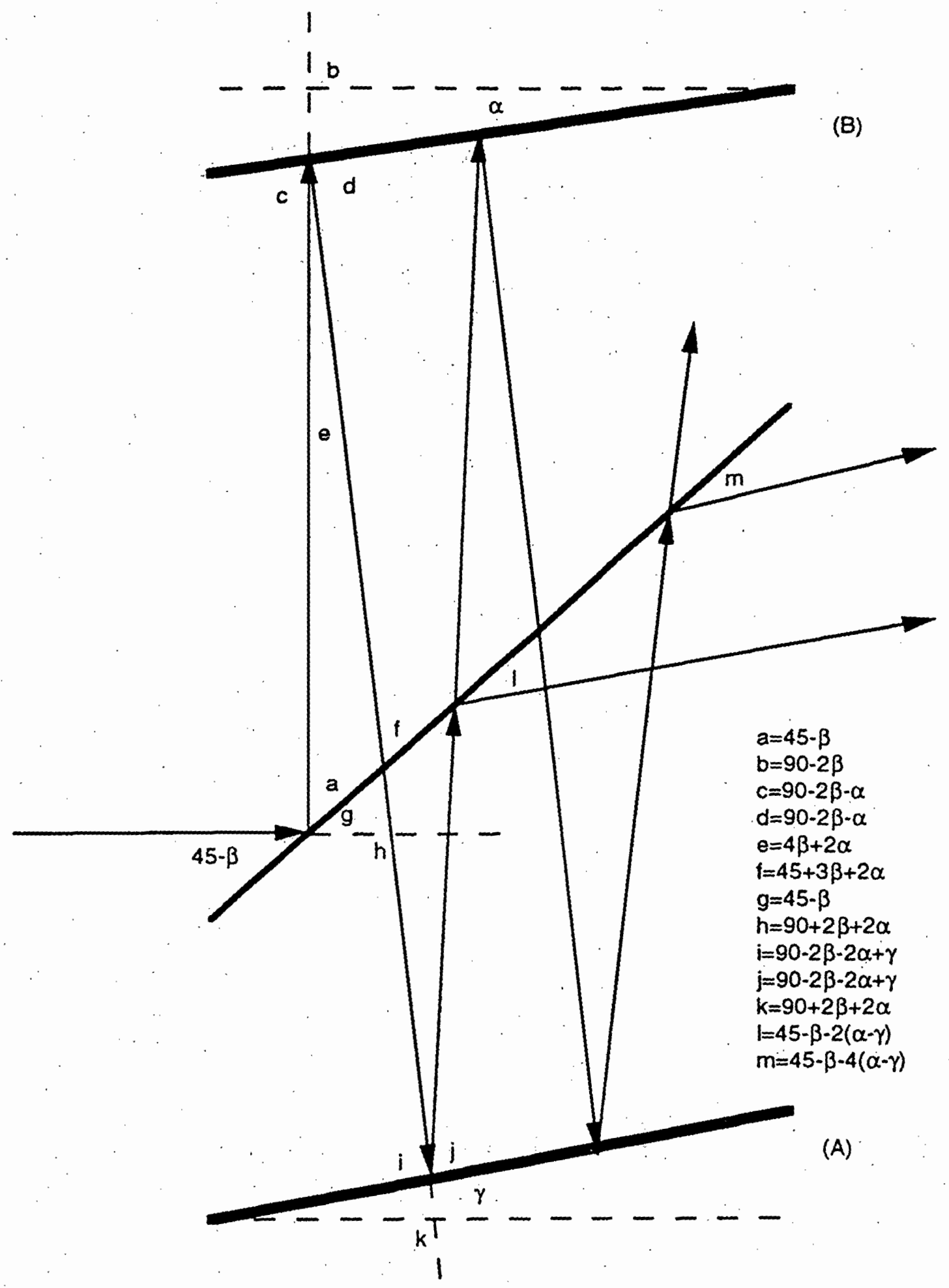

Fig. 3. Geometrical analysis showing that when the laser beam is reflected $n$ times in the cavity, the exit beam angular deviation is $2 n$ times the titt of the synchrotron mirror (A) with respect to the reference mirror $(B)$. 


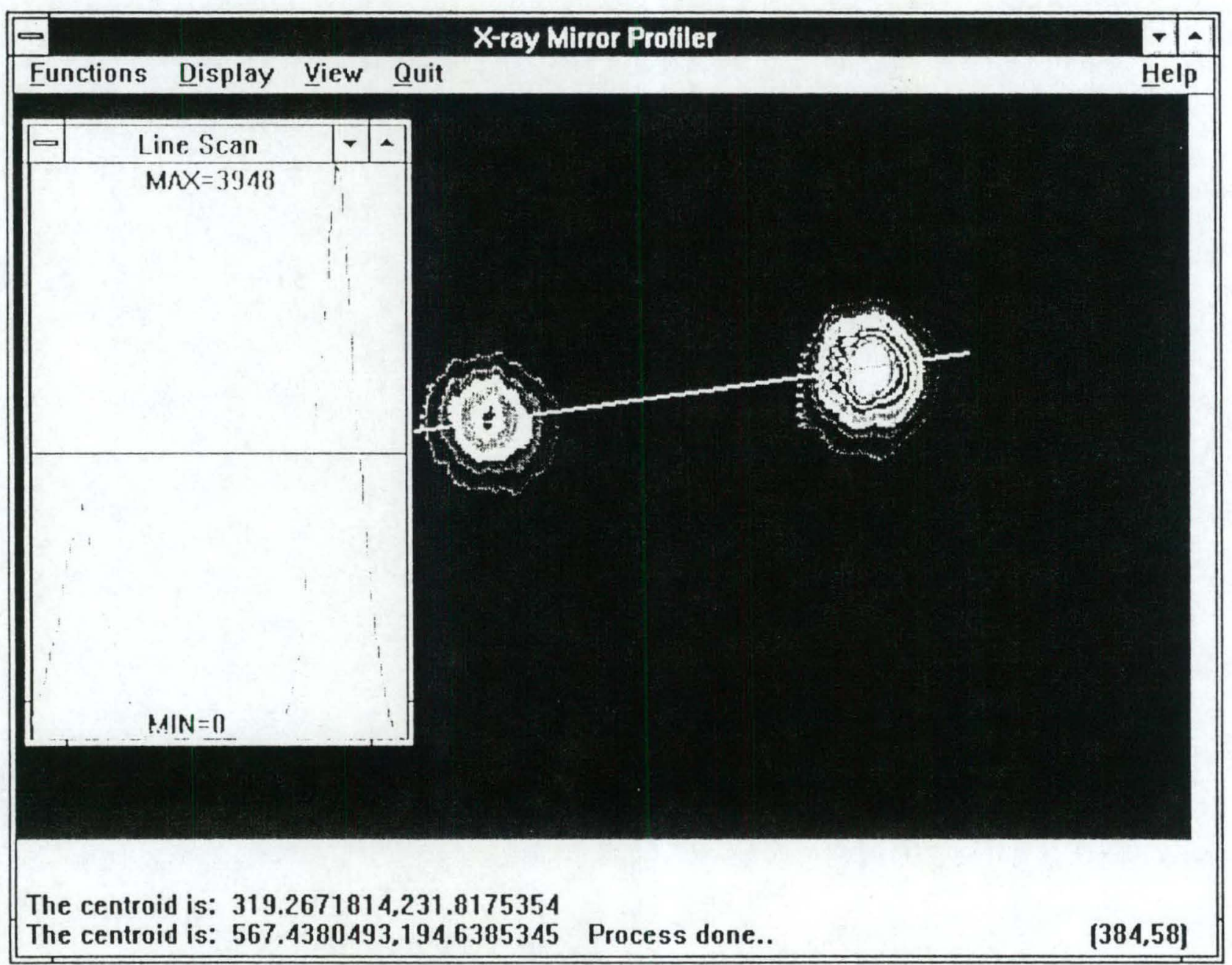

Fig. 4. Digitized image of the laser spots reflected from a test optic. The centroid was calculated using a custom-written software. In the inset, a line scan was also performed. 
blank page 


\section{4-001R1 -- AMORPHOUS SILICON SENSORS: A NEW DETECTOR TECHNOLOGY}

Associate Laboratory Director Area: Operations

Principal Investigators:

S. Ross and I Naday, Electronics and Computing Technologies Division

Funding Profile:

FY $1993-0-$

FY $1994 \$ 141.2 \mathrm{~K}$

FY $1995 \$ 189.3 \mathrm{~K}$

FY $1996-0-$

FY $1997-0-$

Purpose: The purpose of this project is to determine the feasibility of developing a new generation of large-area, high-resolution imaging detectors based on hydrogenated amorphous silicon (a:Si-H) arrays. If successful, such detectors will be extremely valuable in a wide-range of research application areas using ultraviolet and $\mathrm{x}$-ray photons. In particular, such detectors can meet the stringent requirements of research programs to be conducted using the extremely intense $\mathrm{x}$-ray beams from third-generation light sources such as the Advanced Photon Source.

Approach: Researchers engaged in experiments with third generation light sources are currently seriously handicapped because of the lack of availability of large-area high-resolution "affordable" electronic imaging detectors capable of fully utilizing the extremely intensive $\mathrm{x}$-ray beams provided. We propose to develop a new class of detectors by integrating together the amorphous silicon array technology being developed by Xerox Corporation and the thin film scintillator technology of Radiation Monitoring Devices, Inc. (RMD) in a collaborative effort.

Amorphous silicon array detectors are currently being developed by Xerox Corp. for plain paper copiers, and by the University of Michigan Department of Radiation Oncology for clinical x-ray use. ${ }^{[1],[2]}$ These developments parallel many needed for $\mathrm{x}$-ray crystallography--large area (Xerox is currently using arrays $24 \mathrm{~cm} \times 19 \mathrm{~cm}$ and $11 \mathrm{~cm} \times 14 \mathrm{~cm}$ ), high resolution (127 or 200 micron pixels), and low cost. These detectors consist of a:Si-H photodiode arrays sensitive to visible light, coupled to a:Si-H thin film field effect transistors (TFT-FET's) for pixel readout. However, for $\mathrm{x}$-ray applications these arrays need to be coated with a scintillator or phosphor to convert $\mathrm{x}$-rays to visible light. A second company, RMD has for several years been developing thin films of lead iodide $\left(\mathrm{PbI}_{2}\right)$, selenium, mercuric iodide and other $\mathrm{x}$-ray scintillators. ${ }^{[3]}$ These semiconductor films directly convert incident $\mathrm{x}$-rays into electron-hole pairs, with 5-10 times the efficiency of conventional $\mathrm{x}$-ray phosphors.

The specific approach taken in this work then is to work with Xerox Inc. to develop a working amorphous silicon imager system sensitive to visible light, and to work with RMD Inc. to develop the suitable thin film lead iodide or selenium scintillator, which can be directly and uniformly deposited over the large surface of the amorphous silicon array.

Specifically, work supported by this LDRD project has included: 
- construction of a working camera system based on the Xerox/University of Michigan 240 x 256, 450 micron amorphous silicon array;

- development of suitable electronic control systems to clock this imaging system and control its environment (e.g., temperature), and to convert the image data to digital form for computer analysis;

- measurements of preliminary performance data;

- work with RMD Inc. to deposit lead iodide and selenium semiconductor films and measure the parameters of interest;

- refinement of computer models to predict how the overall $\mathrm{x}$-ray detector will function, with particular emphasis on applications to protein crystallography at third generation light sources.

Technical Progress and Results: This work began in FY 1994 during which we designed, fabricated and performed initial tests on electronic circuitry to drive the $256 \times 240$ pixel amorphous silicon array. We developed a method to interface the glass substrate of the amorphous silicon detector to standard printed circuit boards.

During FY 1995 we finished construction of a prototype camera based on this array (figure 1). We built and tested multiple (128) readout channels and integrated 48 such channels into a VME-VAX work station-based data acquisition system similar to those used for our work for protein crystallography. We took preliminary performance data at room temperature, with emphasis on critical parameters such as noise, responsivity, image lag/latency, and sensitivity to operating conditions such as array bias. The preliminary noise measurement yielded approximately 30,000 eRMS at room temperature and is partly due to dark current noise during the 1.8 second readout. Our models predict that for this particular array, this figure should drop to an acceptable 5000-10000 eRMS when the array is cooled. Image latency was found not to be a problem with readout times of a few seconds, at room temperature. We designed and built a sealed enclosure, and partially integrated a thermoelectric cooling system, which will allow the array to be cooled to approximately $-30 \mathrm{C}$. This is motivated by a desire to reduce dark current and read noise.

As part of this work, RMD measured the resistivity (and hence given the operating bias, the leakage current), and the charge transfer characteristics of three lead iodide and selenium films. Resistivity exceeded $10^{12} \mathrm{ohm}-\mathrm{cm}$, indicating that a typical bias voltage of 20-50 volts will yield acceptably low leakage currents $\left(20-50 \mathrm{pA} / \mathrm{cm}^{2}\right)$.

We worked with Dr. Robert A. Street at Xerox PARC to obtain a 128 x 128, 200 micron pixel amorphous silicon array. This array no longer has visible light sensitive amorphous silicon photodiodes, but instead has pixel sites to collect the electron-hole pairs from direct x-ray conversion by $\mathrm{PbI}_{2}$ films, and the a:Si-H TFT-FET's for readout into the Argonne electronics. It is thus suitable for a complete test of the RMD Inc. thin films deposited onto the amorphous silicon readout arrays, which in turn are coupled to the Argonne camera electronics. 
We continued work on spreadsheet and PSPICE computer models for the crystallographic system and for analog readout with emphasis on signal to noise ratios.

Specific Accomplishments: We obtained sufficient data from both the scintillator measurements and the amorphous silicon array measurements to conclude that the integration of these two technologies will result in the development of a useful and practical $\mathrm{x}$-ray imaging detector. Furthermore, an important ongoing collaborative program has been established with Xerox and RMD.

Based upon the work performed under this program, a proposal for continued funding was developed with E. Westbrook (CMB) and submitted to the National Institute of Health (NIH). Following an extensive peer review, the proposal has been funded by NIH grant RO1 GM51488 as a multi year commitment to develop this detector into a fully operational synchrotron and rotating anode $\mathrm{x}$-ray detector.

The early results of this investigation have been presented and published. ${ }^{[4]}$

\section{References:}

1. R.A. Street, Matls. Res. Soc. Bulletin, 17, $70-76$ (1992).

2. Thin Film, Flat Panel Composite Imagers for Projection and Tomographic Imaging, L.E. Antonuk, J. Boudry, W. Huang, Weidong, K.L. Lam, E.J. Morton, R.K. TenHaken, J. Yorkston, and N.H. Clinthorne, IEEE Trans. on Medical Imaging, 13(3) (1994).

3. Electronic noise in lead iodide X-ray detectors, K.S. Shah, J.C. Lund, F. Olschner, P. Bennett, J. Zhang, L.P. Moy, M.R. Squillante, Nuc. Instruments and Methods in Physics Research, Section A, 353 p. 85-88 (1994).

4. Amorphous Silicon area detectors for protein crystallography, S. Ross, I. Naday, M. Kanyo, M.L. Westbrook, E.M. Westbrook, W.C. Phillips, M.J. Stanton, R.A. Street, Proceedings of the SPIE, Charge Coupled Devices and Solid State Optical Sensors, 2415, (February 1995). 


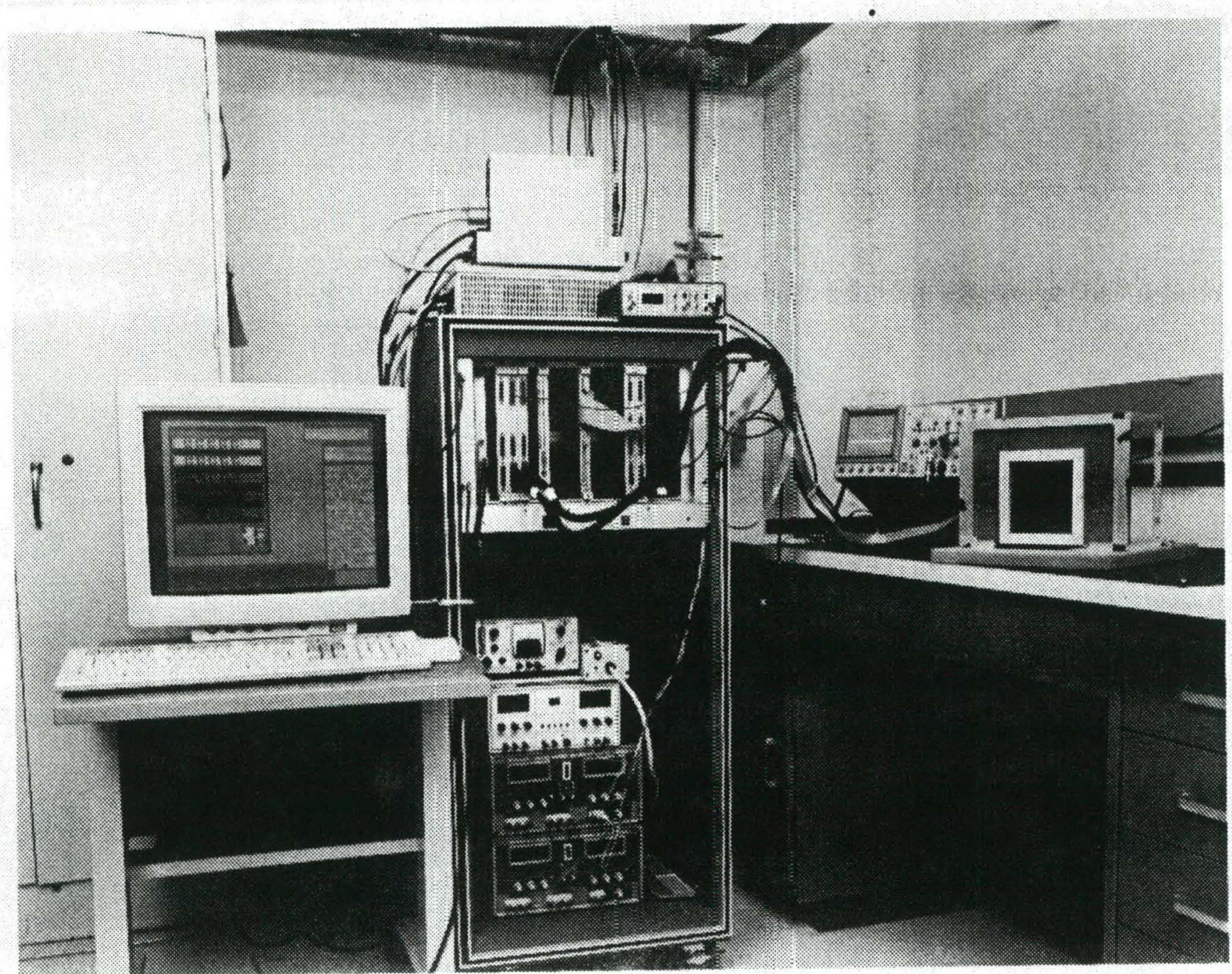

FIG 1 Prototype amorphous silicon detector system. This photo shows (from right to left) the $240 \times 256,11 \mathrm{~cm}$ amorphous silicon array in a frame with its readout electronics, test oscilloscope, the VME based data acquisition in a rack, and the controlling VAX computer. This set uf has allowed us to characterize the array and display images. 


\title{
95-271N -- BOOSTER TARGET STUDIES FOR THE INTENSE PULSED NEUTRON SOURCE
}

\author{
Associate Laboratory Director Area: Physical Research \\ Principal Investigators: \\ B.S. Brown and J.M. Carpenter, \\ Intense Pulsed Neutron Source \\ Funding Profile: \\ FY $1993-0-$ \\ FY $1994-0-$ \\ FY $1995 \$ 203.1 \mathrm{~K}$ \\ FY $1996-0-$ \\ FY $1997-0-$
}

Purpose: Conduct metallurgical and related studies to assist in determining the optimal uranium alloy and cladding for a new enriched ${ }^{235} \mathrm{U}$ Booster Target for the IPNS.

Background: IPNS operated with a Booster Target from 1988 to 1991. This resulted in a factor of 2.5 increase in the neutron flux at the neutron scattering instruments. The target developed a small leak in the cladding and was removed. Consultations with colleagues at ISIS, the British pulsed neutron source, have determined that all of the ISIS and IPNS uranium targets that have failed have failed after roughly equivalent amounts of neutron production, suggesting that the failure mechanism in all cases has been radiation-damage related, rather than depending on thermal cycling, which varies greatly at the two sources. Following the failure of the enriched uranium target, the depleted uranium target that had been used from 1981-1988 was reinserted into IPNS. After approximately one more year of operation, this target also failed and failure was sensed by the same detection of fission gases.

Approach: A thorough analysis of this failed depleted uranium target was made, primarily by means of a number of metallurgical tests at the hot cell in the Energy Technology Division at Argonne. These tests examined the cracks that had developed in the target cladding of the first disk which resulted in the release of the fission gases, which determined the end of life. An example of the crack that developed is shown in the figure below, provided by R. Strain, Technology Development Division. The first disk was cut open, which revealed cracks front to back beneath the region of failed cladding. Material from the cracks was examined by neutron diffraction, which showed the products of uranium-water corrosion. The second disk exhibited cladding deformation but remained intact. This disk was sectioned in the hot cell and examined metallographically. A system of internal cracks was revealed. No corrosion products were present in the cracks. Crack morphology indicated failure due to anisotropic crystal growth.

Technical Progress and Results. The results clearly demonstrated that the failure was due to growth of the uranium which pushed against the cladding and ultimately resulted in the crack. The swelling of the uranium is due to radiation induced growth. Since it is known that the uranium alloy exhibits anisotropic growth, i.e., larger growth in one crystallographic direction than the other, we attempted to minimize the growth by making very small grains in the uranium. This was done by various metallurgical and heat treatments for both the depleted and 
enriched uranium targets. However, since the targets failed by growth, the attempts to minimize this effect were obviously not completely effective. Therefore, it was decided for the new design to use a cubic phase of a uranium alloy, which will eliminate that part of the growth due to the anisotropic crystal structure. It is felt that this should very much reduce the growth induced by radiation damage, thereby lengthening the target's life.

Specific Accomplishments: Based on these results, the design of the replacement Booster Target has been completed. The new design used information dictated the choice of a U-10wt $\%$ Mo alloy, which has cubic crystal structure. This choice of alloy, with its thermal and neutronic properties, dictated the rest of the preliminary target design. The design was documented and underwent a technical review. The comments of the review group were accommodated in the final design. 


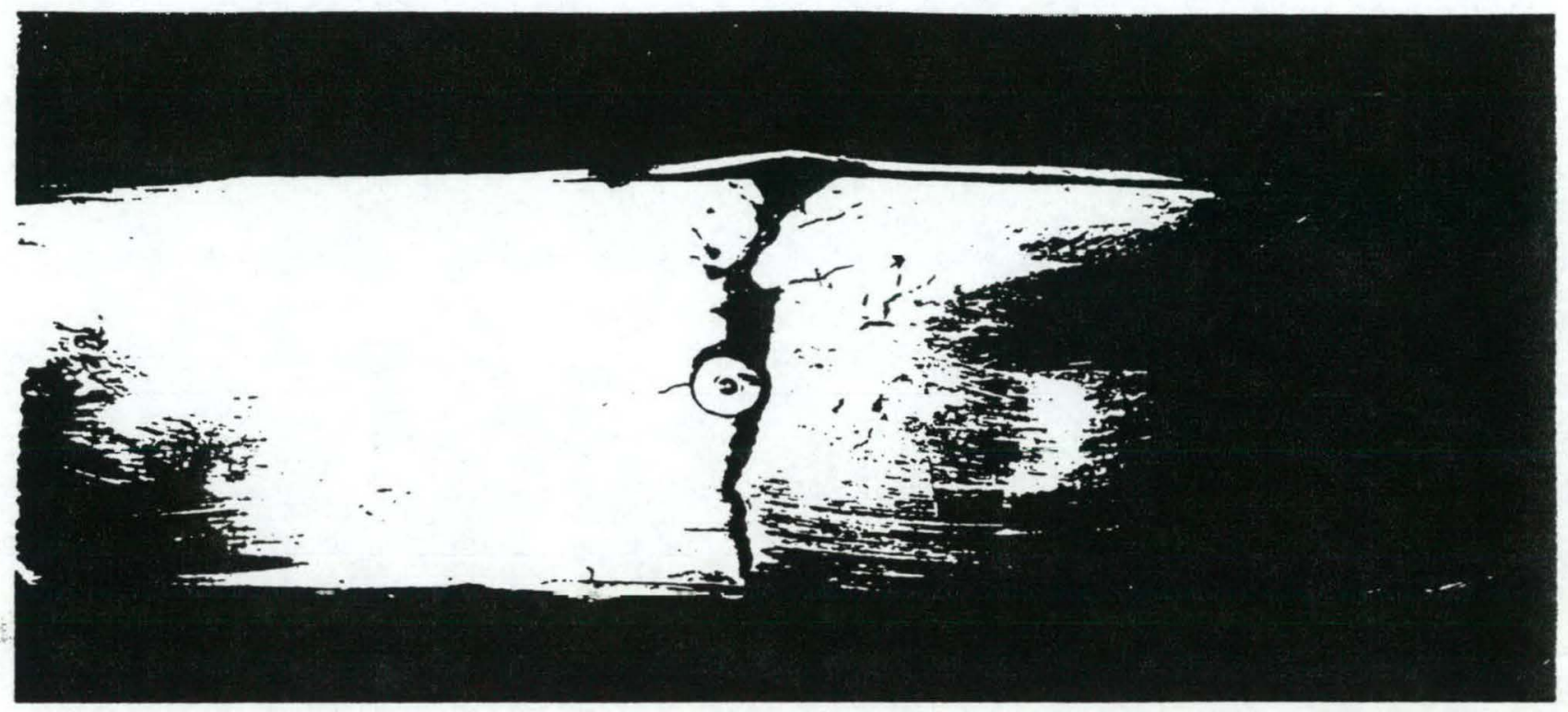

Cross-section Revealed by Cut through Disk \#1

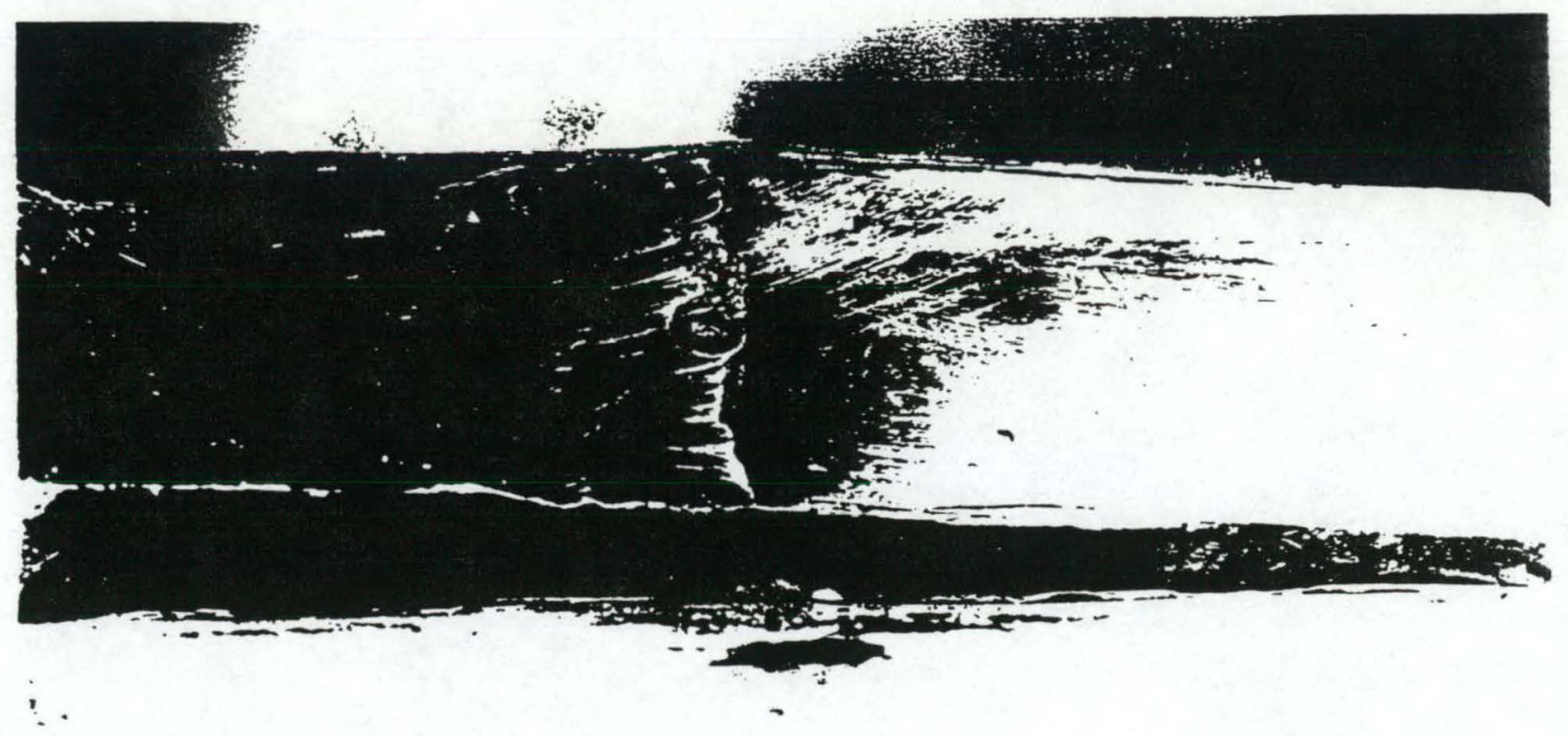

Cut Surface Tilted at an Angle. 
blank page 


\title{
94-193R1 -- DEVELOPMENT OF X-RAY SCATTERING TECHNIQUES WITH $\mu \mathrm{eV}$-meV RESOLUTION
}

\author{
Associate Laboratory Director Area: Advanced Photon Source \\ Principal Investigators: \\ E.E. Alp, W. Sturhahn, R. Röhlsberger, \\ Experimental Facilities Division \\ Funding Profile: \\ FY $1993-0-$ \\ FY $1994 \$ 84.5 \mathrm{~K}$ \\ FY $1995 \$ 136.6 \mathrm{~K}$ \\ FY $1996 \$ 180.0 \mathrm{~K}$ \\ FY $1997-0-$
}

Purpose: Development of new hard $\mathrm{x}$-ray scattering techniques with $\mu \mathrm{eV}$ to meV resolution. Currently this region is virtually unaccessible with hard $\mathrm{x}$-rays. There are many excitations in solids and liquids like soft phonons in disordered systems, magnons, librons, rotational excitations in liquids and gases which can be studied with the development of new $\mathrm{x}$-ray scattering techniques. The energy resolution attainable in the hard $\mathrm{x}$-ray regime $(\approx 5-100 \mathrm{keV})$ of the electromagnetic spectrum is limited to a few meV when higher order Bragg reflections are used. On the other hand, with the introduction of nuclear resonant scattering of synchrotron radiation, it is possible to measure interactions at neV levels via time filtering. However, there is a large gap in the $\mu \mathrm{eV}-\mathrm{meV}$ regime that cannot be measured with either diffraction or time-filtering techniques. Successful completion of this project will give us an unparalleled capability in which inelastic scattering studies from $\mathrm{eV}$ to $\mathrm{neV}$ can be pursued in one of the two experimental stations of the SRI-CAT, dedicated to high-energy-resolution x-ray scattering.

Approach: Quite recently, we have demonstrated measurement of phonon density of states via incoherent nuclear resonant scattering with $5.8 \mathrm{meV}$ resolution (Ref. 2). In order to extend this energy range from the $\mathrm{meV}$ to the $\mu \mathrm{eV}$ domain, we are proposing to develop Doppler shifting and/or interferometry techniques. This would enable one to reach $5 \mathrm{meV}$ range with $\mu \mathrm{eV}$ resolution. In the next two sections, we will discuss our approach to cover this "no man's land" of $\mathrm{x}$-rays.

High Speed Doppler Shifter - The Doppler shift experienced by photons upon scattering is described by the equation $\Delta \mathrm{E}=v \mathrm{E}_{\mathrm{o}} / \mathrm{c}$, where $v$ is the velocity along the beam direction, $\mathrm{E}_{\mathrm{o}}$ is the photon energy, and $\mathrm{c}$ is the speed of light. For $14.4 \mathrm{keV} \mathrm{x}$-rays, linear velocities up to 100 $\mathrm{m} / \mathrm{sec}$ can be obtained by a disk spinning at $10,000 \mathrm{rpm}$, which corresponds to a $10-\mathrm{meV}$ energy shift for a disk of $100-\mathrm{mm}$ radius. One advantage of the spinning disk concept is the fact that it is continuously tunable. An experimental setup is shown in Figure 1. The photon beam, after passing through the high-heat-load monochromator (not shown in the figure) hits the polarizer. Photons then scatter off the disk coated with nuclear-resonant medium (single layer, trilayer, or multilayer) to bring the bandpass to the $\mu \mathrm{eV}$ level, and to switch $\sigma$-polarization to $\pi$-polarization via nuclear transitions. The nuclear resonant portion is then analyzed by a crossed analyzer. Finally the beam hits the sample, and the scattered beam is detected by a time-discriminating detector. 
Long-coherence-length-based interferometry-Interferometry in the $1 . \AA$ wavelength $\mathrm{x}$-ray regime was first realized by Bonse and Hart. Since then many different interferometers have been designed and built. The applicability of interferometry in the $\mathrm{x}$-ray regime is limited by the lack of coherent sources. The coherent volume of the radiation source is given by the transverse coherent area of the incident beam and longitudinal coherence length. The latter is limited by the degree of monochromaticity of the $\mathrm{x}$-ray beam and is given by $\lambda_{\mathrm{c}}=\lambda^{2} / \Delta \lambda$. Typically, $\mathrm{x}$-rays are monochromatized by single crystal monochromators with $\Delta \lambda / \lambda^{\prime}-10^{-4}$. Then, the longitudinal coherence length, $\lambda_{c}$ is around $0.1 \mu \mathrm{m}$ for $\lambda=1 \AA$. When the monochromaticity is pushed to higher values by using back reflection or higher order reflection planes, it is possible to increase the $\lambda_{c}$ to $10 \mu \mathrm{m}$. This short coherence length puts severe limitations on the applicability of this technique. Further monochromatization by crystal diffraction seems to be limited by crystal imperfections at the level of $\Delta \mathrm{d} / \mathrm{d}=10^{-8}$.

In the last decade, however, tremendous progress was made in nuclear resonant monochromatization of synchrotron radiation. Experiments performed on ${ }^{57} \mathrm{Fe},{ }^{169} \mathrm{Tm}$, and ${ }^{119} \mathrm{Sn}$ and ${ }^{181} \mathrm{Ta}$ demonstrated the possibility of reaching. $\Delta \lambda / \lambda \sim 10^{-10}-10^{-14}$. This is a three-to-seven orders-of-magnitude improvement over crystal monochromators, increasing the longitudinal coherence length to several tens of meters. The new interferometric techniques we will take advantage of these long coherence lengths to measure energy difference of $\mu \mathrm{eV}$ levels.

\section{Technical Progress and Results:}

Polarizer/Analyzer Monochromator: One of the key components of the system, namely, the production of a pure nuclear resonant scattered beam via a polarizer/analyzer monochromator has been demonstrated using asymmetrically cut $\mathrm{Si}(8.4)$ for ${ }^{57} \mathrm{Fe}$ at $14.4 \mathrm{keV}$. A suppression level of 10-7 has been obtained, and results are submitted in a paper to Appl. Phys. Lett. (Ref. 1). The result of one such measurement is shown in Figure 2. It is now possible to measure nuclear decay after 1 nsec following the excitation.

Nuclear Large Energy Bandpass Mirrors: We planned to use grazing incidence geometry to take advantage of coherent speed-up for broadening of nuclear transition energy bandwidth. Three different designs were considered:

a. A single layer of ${ }^{57} \mathrm{Fe}$ on zerodur (a special glass with zero thermal expansion coefficient). The maximum bandwidth is $25 \Gamma_{0}$ at $3.6 \mathrm{mrad}$ incidence angle.

b. A trilayer system consisting of $6 \mathrm{~nm} \mathrm{Si} / 30 \mathrm{~nm}$ Pd on zerodur substrate. The maximum bandwidth is $65 \Gamma_{0}$ at $4.3 \mathrm{mrad}$ incidence angle.

c. A Mo/Si multilayer with a bilayer thickness of $4 \mathrm{~nm}$, coated with $40 \mathrm{~nm}{ }^{57} \mathrm{Fe}$. The maximum bandwidth is $53 \Gamma_{\mathrm{o}}$ at $11 \mathrm{mrad}$ incidence angle. Despite lower bandpass, the relatively larger incidence angle allows to intercept bigger portion of the beam in the vertical direction.

The angle integrated reflectivity of each system is shown in Figure 3 as a function of incidence angle. Our decision is to go ahead with option (b) first. 
The mirror will be mounted on an air lubricated bearing which is able to drive up to a rotational speed of $9000 \mathrm{rpm}$. This should allow us to tune the radiation $\pm 3 \mathrm{meV}$ around the nuclear resonance. The bearing has been ordered and is expected to arrive in August 1995.

The performance of these layers have been calculated after incorporating grazing incidence geometry into CONUSS program package, where full polarization dependence of nuclear resonant scattering is employed. The conventional Fresnel formalism is not capable of handling optically active medium. The program has been tested on $\mathrm{Fe} / \mathrm{Cr}$ multilayer system where experimental data was available and the results are published (Ref. 3).

The zerodur disks for the mirrors have been ordered from ZEISS and received. They are characterized to have $\sigma_{\mathrm{rms}}<0.3 \mathrm{~nm}$ roughness, and long range flatness of $\lambda / 4$. The sputtering system suitable to coat large disks using precious ${ }^{57} \mathrm{Fe}$ isotope has been upgraded with a second radio frequency generator to run two sputtering sources independently. The matching network between the generator and the cathode can be adjusted to provide small targets with the optimum power density. For the disk, a special holder has been developed to keep the disk in rotary motion during deposition.

\section{Experimental Results}

A variety of experiments have been carried out to characterize the sputtered films with respect to thickness, density, roughness, and magnetic properties. A $30 \mathrm{~nm}$ thick ${ }^{57} \mathrm{Fe}$ layer on a superpolished zerodur substrate has been produced, as well as several Si wafers have been coated under different sputtering conditions.

a. Measurement of electronic and nuclear reflectivity: The thickness, density and roughness of the sputtered layer has been characterized using $14.4 \mathrm{keV}$ x-rays at HASYLAB. The results are shown in Figure 4. The data evaluation yielded a microroughness of $\sigma_{\mathrm{rms}}<0.2$ $\mathrm{nm}$, and energy bandpass of $25 \Gamma_{0}$.

b. Measurement of magnetic response: The magnetic properties of the ${ }^{57} \mathrm{Fe}$ layer has been characterized with SMOKE (surface magneto-optic Kerr effect) in collaboration with E. Fullerton of Materials Science Division of ANL. The hysteresis loop of the film is shown in Figure 5. The film has a relatively high remanence and a coercivity of $35 \mathrm{Oe}$. This result has made the design of the magnetization set-up signiticantly easier.

Magnetic Assembly Design: For evaluation of magnetic assembly design, the finite element code OPERA was used. Since the coercivity of ${ }^{57} \mathrm{Fe}$ film is $35 \mathrm{Oe}$, a simple arrangement of permanent magnets like Nd-Fe-B or CoSm is sufficient to align the moments in the film parallel to the direction of photons, a condition dictated by most efficient polarization switching in resonant medium. The magnets are arranged at a distance of $10 \mathrm{~cm}$, connected with an iron yoke. The magnets are fitted with pole pieces to homogenize the field. The whole assembly is placed few $\mathrm{mm}$ above the plane of film. Figure 6 shows the field strength in the plane of film along the direction of the $\mathrm{x}$-ray beam.

Interferometry: We have started a collaboration with Prof. R. Colella of Purdue University to build a novel interferometer based on splitting the beam using multiple reflection geometry. 
Prof. Colella visited ANL in May, 1994. As a result, Mr. John Sutter, a third year graduate student at Purdue University has joined ANL full time starting January 1995. Before such crystals are cut and polished, it was necessary to calculate the intensity and the angular divergence of each beam to optimize the interferometer. Prof. Roberto Collela has developed a general purpose program, NBEAM. This program was utilized to identify a set of reflections to split the beam evenly. The schematic of these reflections are shown in Figure 7 and the reflection intensities are given in Figure 8. It is clear that $\mathrm{Si}(862)$ and (862) reflections will be suitable to split the beam. What we are working currently is to come up with a feasible scenario to scatter one branch of the beam from a sample, and recombine to measure the energy differences of $\mu \mathrm{eV}$ levels: To this end, we are inviting Dr. Walter Graeff of HASYLAB and Prof. Roberto Colella of Purdue University in August 1995 for two weeks period to study various options.

Applications: We are starting a new collaboration with a group at Louisiana State University led by Prof. P. Vashishta and Prof. R. Kalia to calculate low level excitations in solids via Molecular Dynamics simulations. The basic motivation of this work is to use phonon density-of-states measurements to form basis for development of correct two and three body potentials for computer simulations. This will not only guide the experiments, but also will calculate some of the experimental results ahead of time, and hopefully, in some cases will make the experiments unnecessary. For example, if calculations can reliably simulate momentum integrated phonon DOS, then it will be possible to obtain phonon dispersion relations in any given crystallographic direction.

Phase 1 October 1993-September 1994

Calculation and design of nuclear monochromator, polarizer/analyzer, doppler shifter, and interferometer

Phase 2 October 1994-September 1995

Manufacturing of key components and their characterization

Phase 3 October 1995-September 1996

Experimental measurements, and development of practical applications

Specific Accomplishments: Effort will continue with non-federal funding.

1. T.S. Toellner, W. Sturhahn, E.E. Alp, X. Zhang, M. Ando, Y. Yoda, S. Kikuta, "Asymmetrically Cut Polarizer/Analyzer filter for Nuclear Resonant Scattering of Synchrotron Radiation," Appl. Phys. Lett., 67:1993 (1995).

2. W. Sturhahn, T.S. Toellner, E.E. Alp, X. Zhang, M. Ando, Y. Yoda, S. Kikuta, M. Seto, C.W. Kimball, B. Dabrowski, "Phonon Density of States Measured by Inelastic Nuclear Resonant Scattering," Phys. Rev. Lett., 74:3832 (1995).

3. T.S. Toellner, R. Roehisberger, W. Sturhahn, E.E. Alp, E. Fullerton, C.H. Sowers, "Observation of Pure Nuclear Diffraction from Fe/Cr Antiferromagnetic Multilayer, "Phys. Rev. Lett., 74:3475 (1995). 


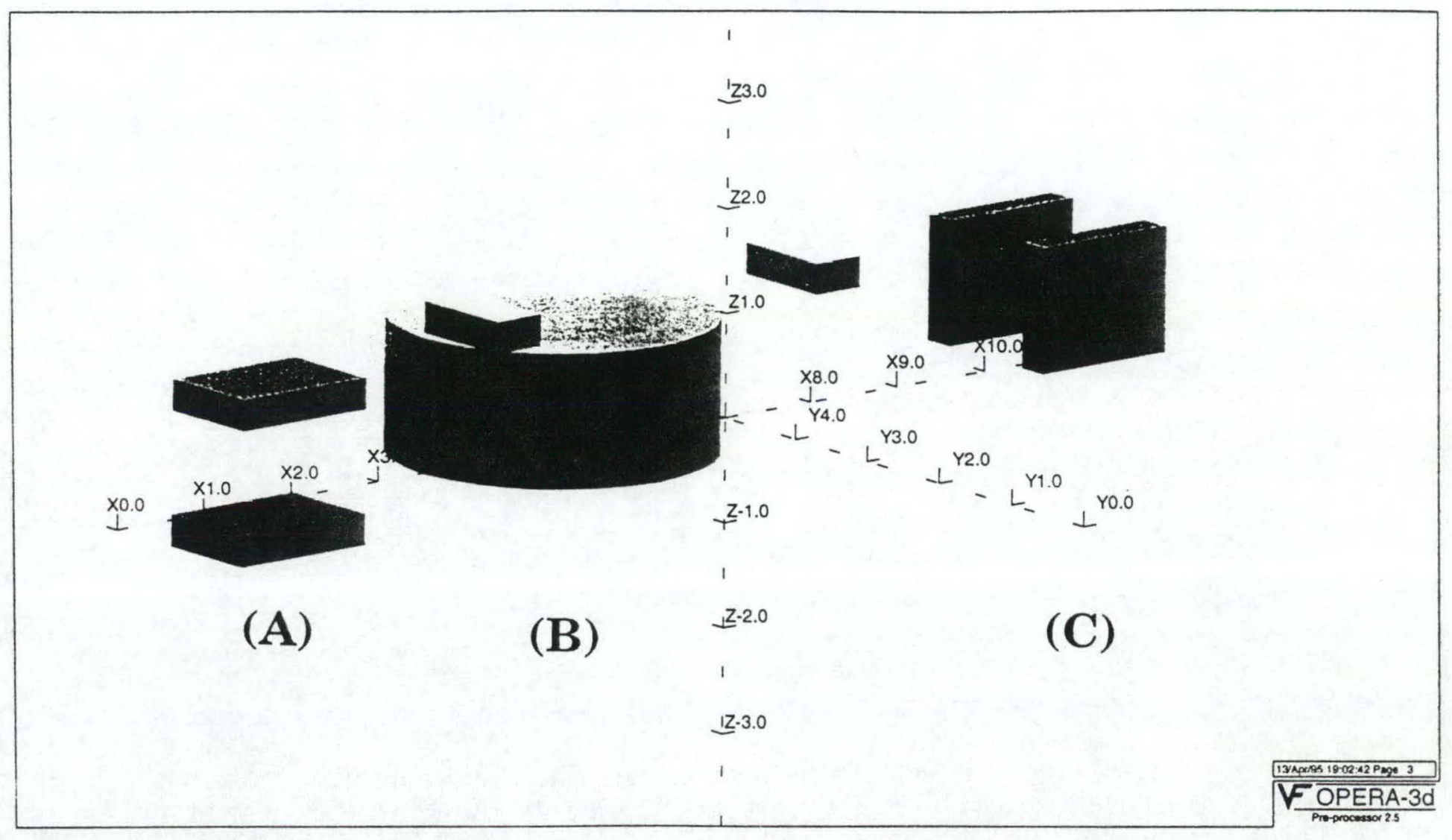

Figure 1. The schematic $\mu \mathrm{eV}$ resolution $\mathrm{x}$-ray spectrometer consisting of (A) Polarizer, (B) Doppler shifter and (C) analyzer. The resulting x-ray beam can then be scattered off a sample, and fluorescence radiation can then be collected as described in ref (1). 


\section{Fe metal}

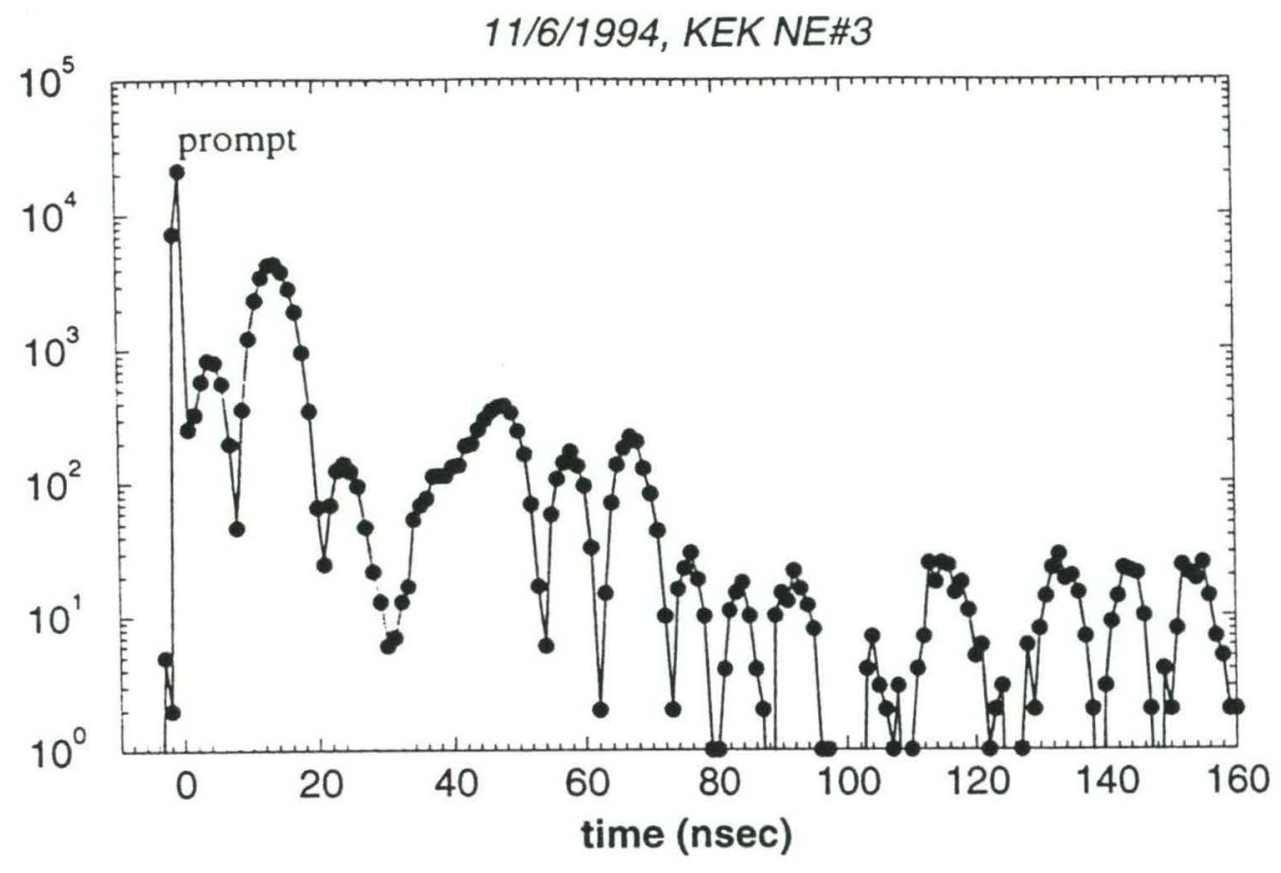

Figure 2. Time decay spectrum of iron metal. Each data point represents one nsec. It is now possible to measure nuclear decay immediately after the prompt synchrotron pulse. The energy bandwidth of the resulting beam vary from few $\mathrm{neV}$ to fraction of $\mu \mathrm{eV}$, depending on the thickness of the sample. 


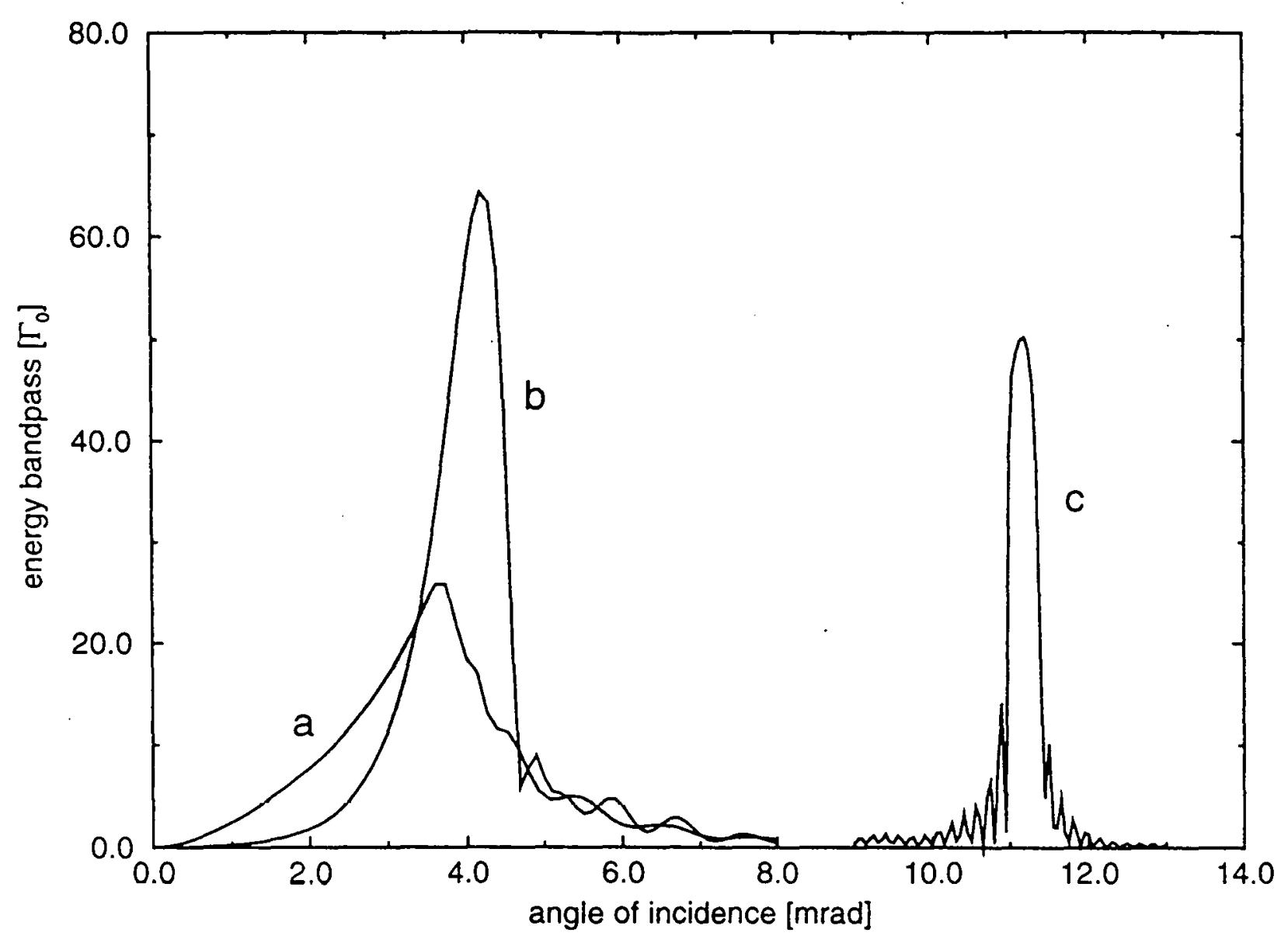

Figure 3. Energy integrated rocking curves of a) single layer of $57 \mathrm{Fe}$ on zerodur, (b) trilayer system consisting of $6 \mathrm{~nm} \mathrm{Si} / 10 \mathrm{~nm} 57 \mathrm{Fe} / 30 \mathrm{~nm} \mathrm{Pd}$, and c) $\mathrm{Mo} / \mathrm{Si}$ multilayer with a bilayer thickness of $4 \mathrm{~nm}$, coated with $40 \mathrm{~nm} 57 \mathrm{Fe}$. 


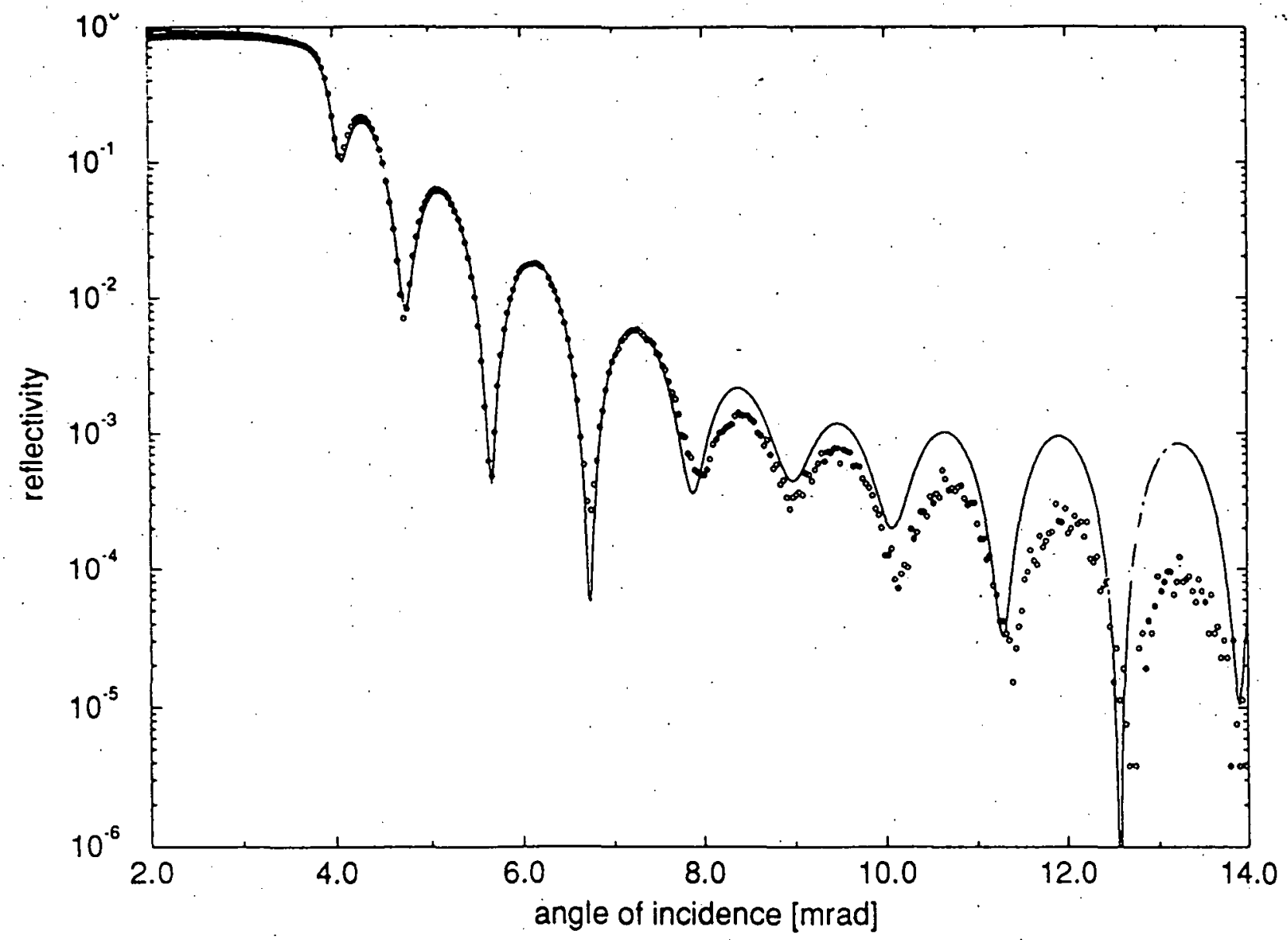

Figure 4. Electronic reflectivity of $30 \mathrm{~nm}$ Fe on ZERODUR. The fit is obtained with $2.5 \mathrm{~nm}$ iron oxide on top, and a surface roughness of $0.2 \mathrm{~nm}$. 


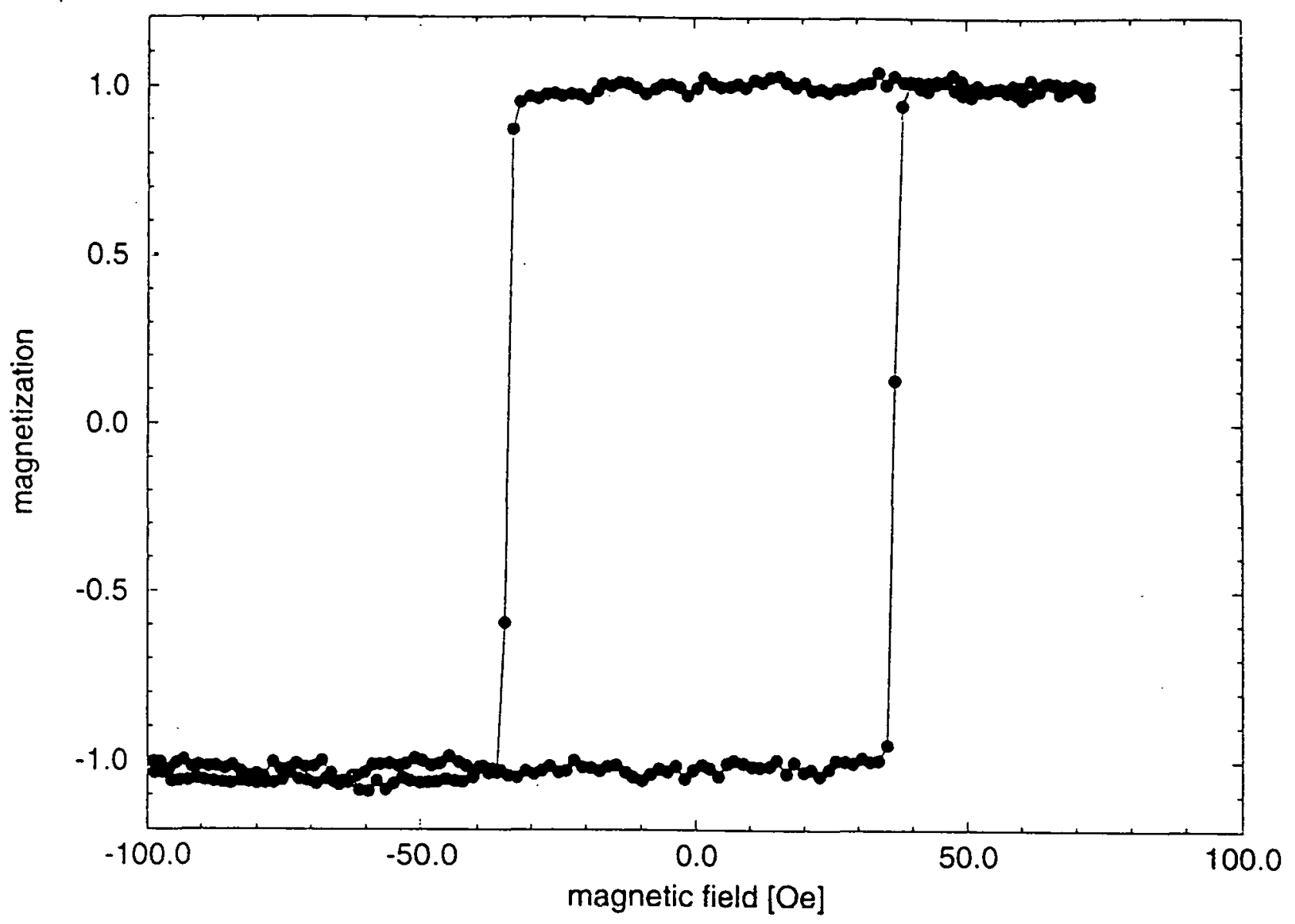

Figure 5. SMOKE (Surface Magneto Optical Kerr Effect) measurement of iron layer coated on a silicon wafer. The square shaped hysteresis loop indicates that once magnetized, it will take 35 Oe field in a reverse direction to change the magnetization direction. This information is important in magnetic assembly design. 


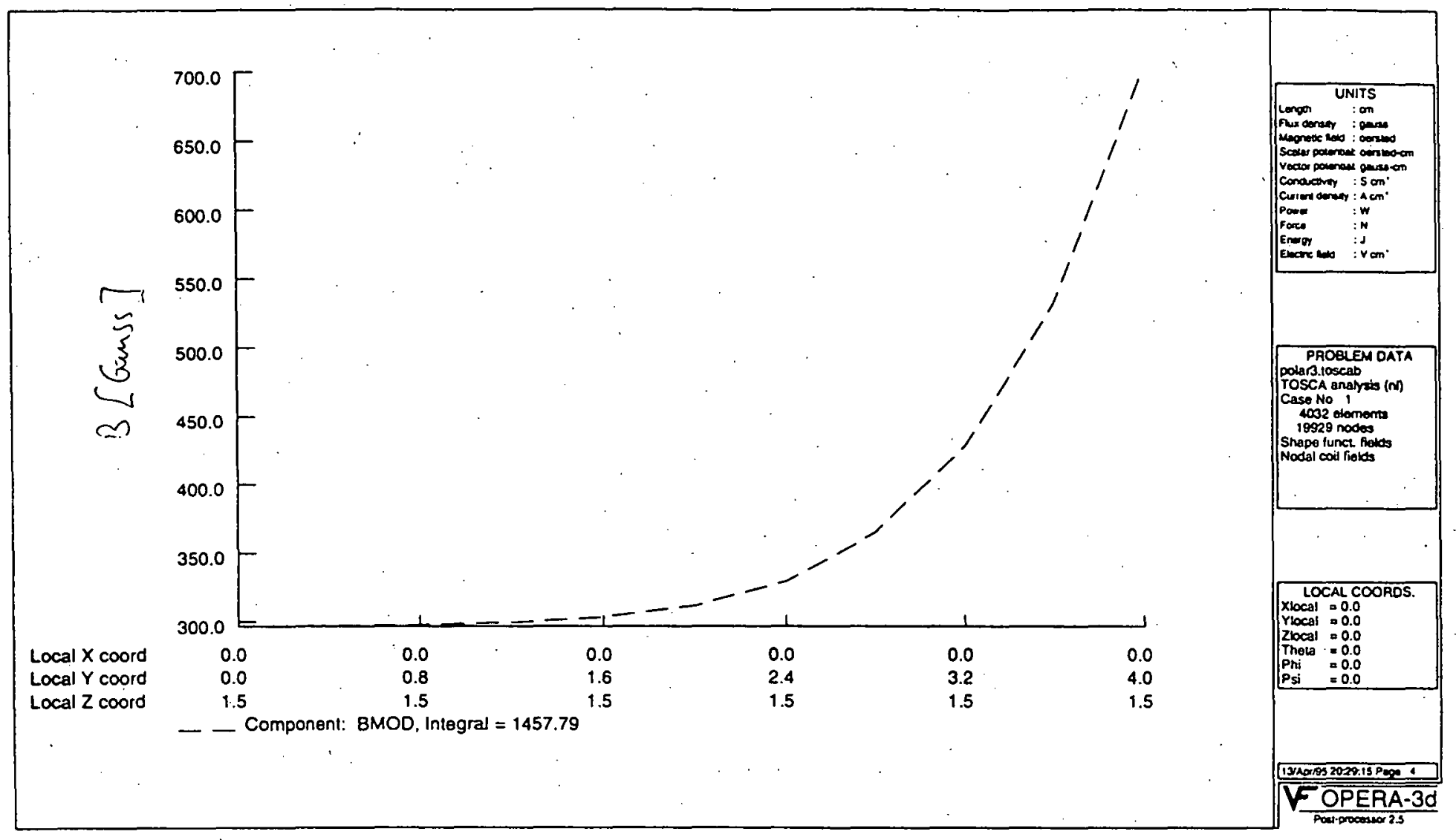

Figure 6. Magnetic field strength in the plane of the film, simulated by using the OPERA finite element analysis code. 

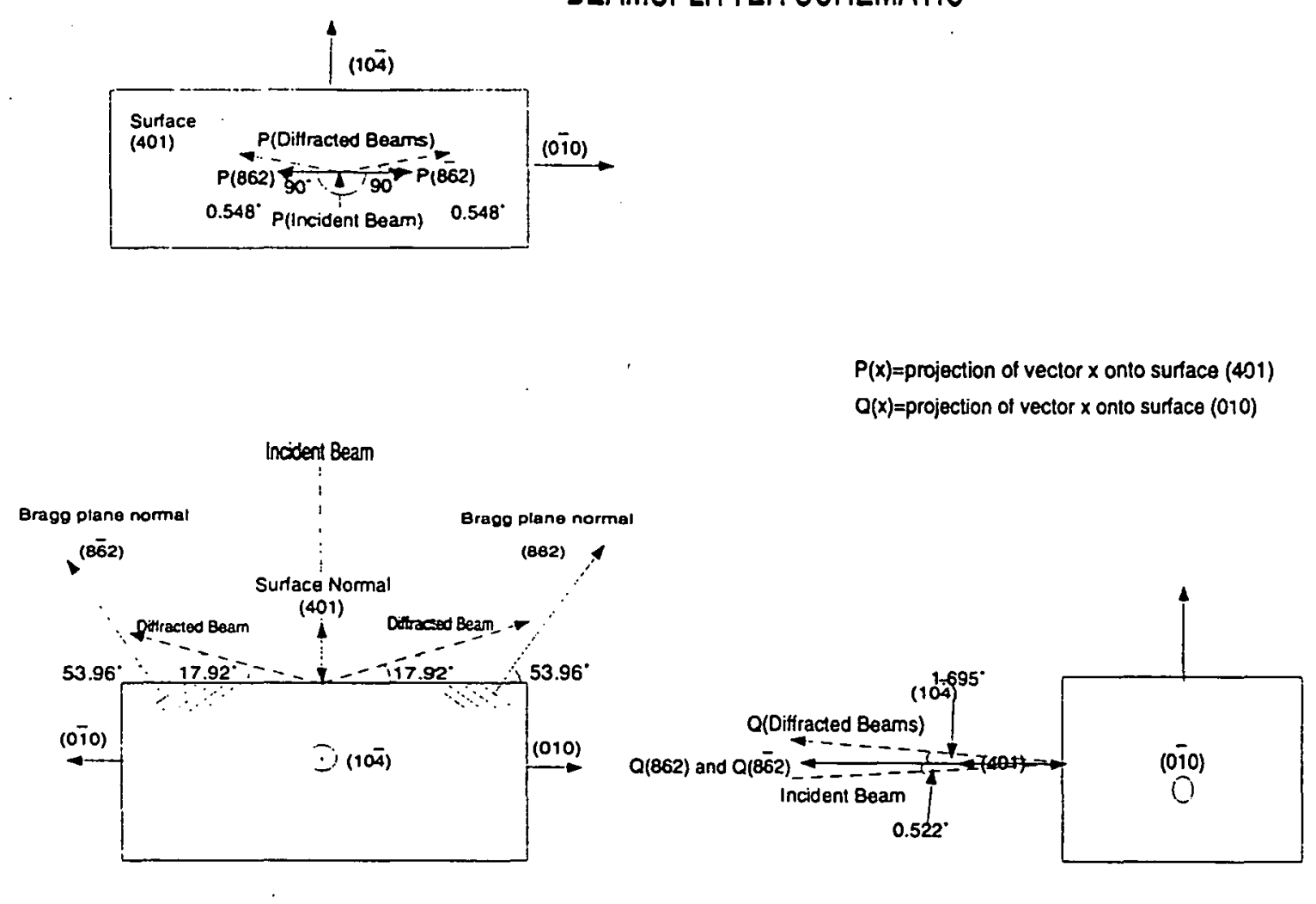

Figure 7. The schematic of beam splitter using 3-beam conditions. Side, top, and front views are given. 


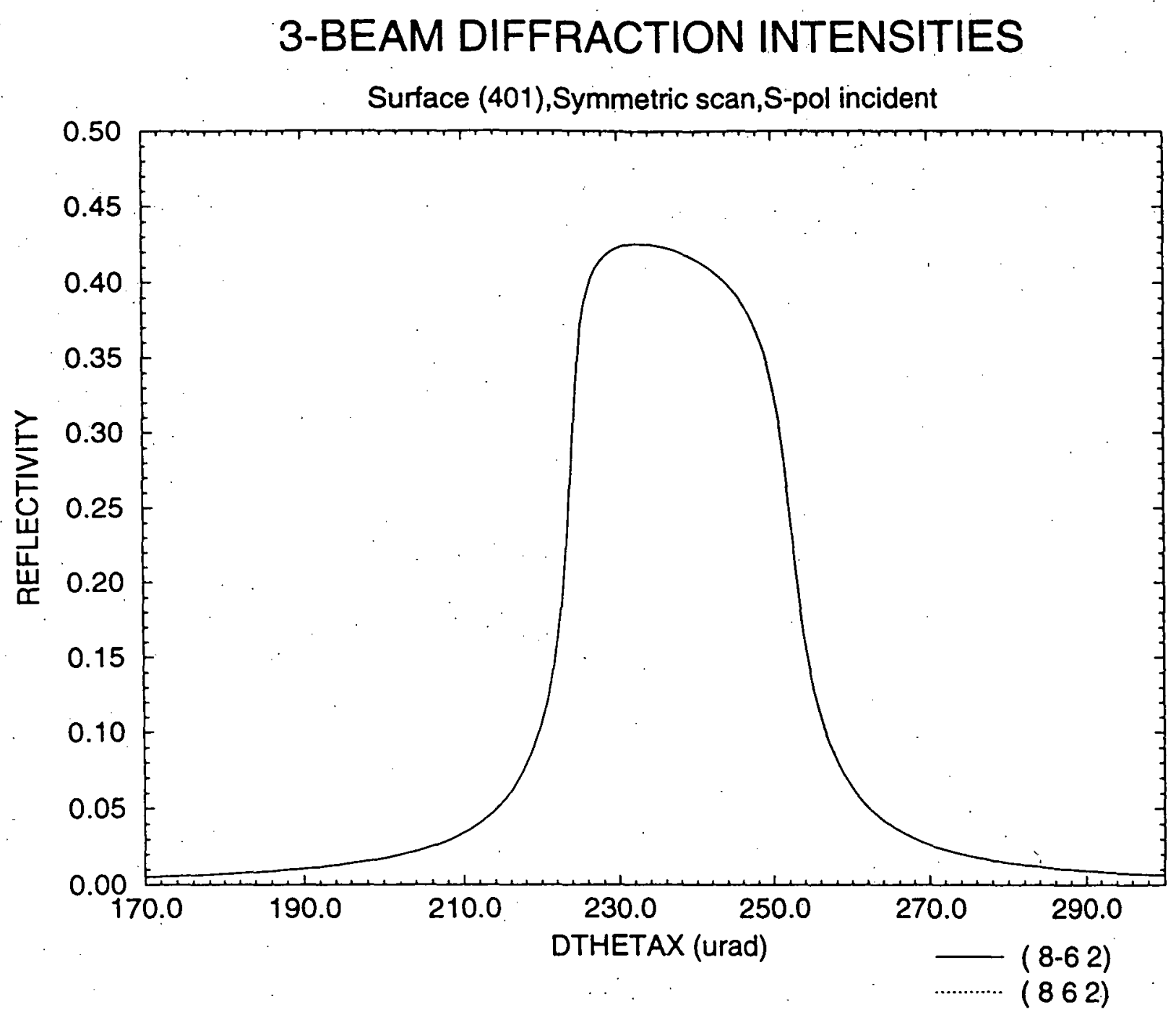

Figure 8. Reflectivity of Si (862) under 3-beam conditions. 


\title{
95-012N -- NEW SYNCHROTRON ANOMALOUS SCATTERING TECHNIQUES
}

\author{
Associate Laboratory Director Area: Physical Research
}

Principal Investigators:

Funding Profile:

\author{
M.A. Beno, P.L. Lee, and G. Jennings, \\ Materials Science Division
}

FY $1993-0-$
FY $1994-0-$
FY $1995 \$ 130.4 \mathrm{~K}$
FY $1996 \$ 100.0 \mathrm{~K}$
FY $1997 \$ 200.0 \mathrm{~K}$

Purpose: The purpose of this research is the development of new techniques for atom specific anomalous scattering diffraction experiments. Anomalous scattering is an atom selective scattering technique which is employed extensively in synchrotron single crystal and powder diffraction experiments, as well as for scattering from glass samples. This technique can be used to derive site occupancy information for complicated materials by changing the incident $\mathrm{x}$-ray energy to enhance atomic contrast. The conventional data collection procedures employed for anomalous scattering of powder and amorphous samples are extremely slow. Since the analysis of this type of data employs differences in scattering at multiple energies (i.e., small differences in large numbers), highly accurate diffraction data is required. Using new $\mathrm{x}$-ray optics, we have successfully collected anomalous scattering data for both glassy and crystalline powder samples in hours rather than the days previously required.

Approach: Anomalous scattering is a particularly important synchrotron diffraction technique. Scattering for individual constituent atoms can be isolated with this technique providing enhanced contrast between elements and giving information on atom-atom correlations at the partial structure level. Anomalous scattering results are derived from small differences in large values, therefore high count rates are necessary for the accurate measurement of diffraction data.

Conventional methods for the measurement of anomalous scattering data either employ a solid state detector or a diffracted beam monochromator to provide energy resolution. The energy resolution of solid state detectors is not sufficient to eliminate all background due to resonant Raman scattering or to limit Compton background. Although a diffracted beam monochromator provides sufficient energy resolution, the extremely high angular resolution inherent in this technique makes data rates unacceptably low. We have developed two new methods for the collection of high resolution, low background powder diffraction spectra which when applied to anomalous scattering of crystalline or amorphous materials overcome these limitations. One of these, the focusing-crystal-position-sensitive-detector method ${ }^{1-3}$ (see Figure 1) possesses both high $Q$ and high energy resolution and attains high count rates by measuring up to $4^{\circ}$ in 2 -theta simultaneously.

The second technique uses a curved crystal monochromator (polychromator crystal) has been employed to select a band of X-ray energies for X-ray absorption spectroscopy studies. However, there is no sustained effort to explore the applications of this optical geometry for diffraction studies. The X6A beamline at the National Synchrotron Light Source (NSLS) uses 
a polychromator to create a sharply convergent line image (100-120 mm) with a range of $x$-ray energies of $\sim 1 \mathrm{keV}$. The ability of this instrument to provide a microfocused beam is well suited for diffraction studies not only of powder, thin film or glass samples, but also for experiments on very small single crystal samples. The tunability ( $E=6.5 \mathrm{keV}$ to $21 \mathrm{keV}$ ) and flexibility $(\Delta \mathrm{E}=100-1000 \mathrm{eV})$ of the instrument makes this beamline ideal for energy dispersive diffraction techniques.

\section{Technical Progress and Results:}

Focusing Crystal Analyzer - The method shown in Figure 1 uses a focusing, perfect crystal analyzer which eliminates background and determines the resolution of the instrument. The crystal is curved with a 4 point bender so that its surface makes the Bragg angle with rays coming from the illuminated area of the sample over a wide range of two theta. Currently we are able to bend the crystal perfectly enough so that the Bragg condition is satisfied to within the Si 220 analyzer crystal Darwin width over a range of $4^{\circ}$ in 2 -theta. The crystal reflects all scattered $x$-rays with the proper energy that fall within a $4^{\circ}$ range into a position sensitive detector (PSD). so that the data are recorded in parallel.

The experimental setup for this method is easily accommodated on a 6-circle diffractometer. The sample is mounted on the main phi (theta) drive of the goniometer while the crystal bender and detector are positioned on the analyzer theta and 2-theta stages, respectively. The 4-point bender design is the same as that employed for the energy dispersive EXAFS beamline X6A. A $25 \mathrm{~cm}$ long Si 220 crystal is located at $\sim 30 \mathrm{~cm}$ from the sample and an ORDELA model $1050 \mathrm{X}$ detector $\sim 10 \mathrm{~cm}$ from the crystal. Crystal bending, which must be done at each energy, is quickly accomplished by tweaking each end of the crystal and observing the change in angular acceptance of the linear detector for a glass sample. Alternatively, once the crystal is bent small changes in energy may be accomplished by changing the sample to crystal distance.

Two aspects of the technique need to be improved to make this method more practical for routine use at the APS BESSRC beamlines. The first difficulty which has been encountered with this technique is the long setup time required to change energies. Methods to retune the analyzer crystal rapidly to the new energy must be developed. This technique is highly dependent on the careful alignment of the sample and on the stability of the x-ray beam. The later of these two effects should be of minimal importance at the APS but the former must be addressed. The field of view of a bent perfect crystal is determined primarily by the Darwin width of the crystal used. For the Si 220 crystals used in initial tests this field of view is only a few microns of the sample. It will be necessary to open up the Darwin width of the analyzer crystals to take full advantage of the incident $\mathrm{x}$-ray flux and also to reduce the sensitivity of this technique to sample misalignment. There a number of techniques which can be attempted to accomplish this. Simply polishing the crystal without etching has been shown to dramatically increase the Darwin width and therefore field of view. This technique along with other methods of introducing strain such as plastically bending the crystal will be studied.

Continuous Energy Diffraction - Continuous Energy Diffraction is a technique which allows the simultaneous observation of diffracted intensities for the entire energy range of the incident beam. The experimental arrangement is shown in Figure 2. 
In the second powder anomalous scattering method only one diffraction line is observed at a time but diffraction from a range of energies is observed simultaneously. As is shown in Figure 2, a curved crystal monochromator (polychromator) is used to focus a range of incident energies onto a small sample. The powder diffraction pattern which results from this type of an x-ray source provides diffracted intensity at each incident energy. This technique is much simpler than the methods previously employed since no monochromator motion is required to change beam energy. This principal has been shown to produce excellent data for single crystal samples but has limitations for powder diffraction. An additional analyzer crystal will be added to this experimental arrangement to allow the measurement of anomalous scattering in powder and amorphous samples.

\section{Specific Accomplishments:}

Focusing Crystal Analyzer. The first experiments using this technique have shown that this method for anomalous scattering produces data of high quality at data rates much higher than with conventional methods. Data derived from the Focusing Crystal Analyzer technique used for anomalous scattering experiments on a $\mathrm{Cs}_{2} \mathrm{Ge}_{4} \mathrm{O}_{9}$ glass samples ${ }^{4}$ was collected at the NSLS $\mathrm{X} 6 \mathrm{~B}$ beamline. The data were collected by stepping $2 \theta$ in small increments $\left(0.25^{\circ}\right)$ while the detector observed data over a $4^{\circ} 2$-theta window. With this data collection strategy each data point is measured many times at different points on the linear detector to eliminate errors due to detector nonlinearity. Data collection times were varied in different runs to assess the statistical accuracy attainable with this technique. Data near the $\mathrm{Ge}$ edge $(\mathrm{E}=11.100 \mathrm{keV})$ were collected with $0.25^{\circ}$ steps for $25 \mathrm{sec}$ counting time for each step giving a total data collection time of $\sim 4$ hours, while the data away from the absorption edge $(E=10.899 \mathrm{keV})$ were collected for only $8 \mathrm{sec}$ per point so that the total data collection time was only $\sim 1$ hour. Although the rapidly collected data are noisier, the essential features are present in both cases and the derived results are unaffected. Comparison of this result to that for a similar compound derived from conventional methods shows that our data collection method is far superior even though total data collection times were as much as a factor of four shorter for our method.

Continuous Energy Diffraction. We have tested this technique with single crystal and powder samples using a CCD detector ${ }^{5}$ and more recently with an image plate system. ${ }^{6}$ The further use of this method for full anomalous scattering of protein molecular structure determinations is underway. These experiments will be a definitive proof of the quality of the diffraction data obtainable from the dispersive geometry.

Refereed Publications:

P.L. Lee, M.A. Beno, C.M. Ogata, G. S. Knapp, G. Jennings, "Diffraction Applications Using the Energy-Dispersive Beamline X6A at the National Synchrotron Light Source," Rev. Sci. Instrum., 66(2):1425-1427 (1995).

P. Armand, M.A. Beno, A.J.G. Ellison, G.S. Knapp, D.L. Price, M.L. Saboungi, "Local and Intermediate-Range Order in Cesium Germanate Glass," Europhysics Letters, 29(7):549-553 (1995). 
M.A. Beno, G.S. Knapp, P. Armand, D.L. Price. M.L. Saboungi, "Application of New Synchrotron Powder Diffraction Techniques to Anomalous Scattering from Glasses, "Rev. of Sci. Instrum. 66(2): 1308-1310 (1995).

\section{References:}

1. M.A. Beno, G.S. Knapp, Rev. Sci. Instrum., 63:4134 (1992).

2. G.S. Knapp, M.A. Beno, G. Jennings, M. Engbretson, M. Ramanathan, Adv. X-Ray Analysis, 36:653 (1993).

3. G.S. Knapp, M.A. Beno, G. Jennings, M. Ramanathan, Mat. Res. Soc. Symp. Proc., 307:317 (1993).

4. P. Armand, M.A. Beno, A.J.G. Ellison, G.S. Knapp, D.L. Price, M.L. Saboungi, Europhysics Letters, 29(7):549-553 (1995).

5. M.A. Beno, G.S. Knapp, P. Armand, M.L. Saboungi, Rev. Sci. Instrum., 66(2):1308 (1995).

6. P.L. Lee, M.A. Beno, C.M. Ogata, G. S. Knapp, G. Jennings, Rev. Sci. Instrum., 66(2):1425 (1995). 


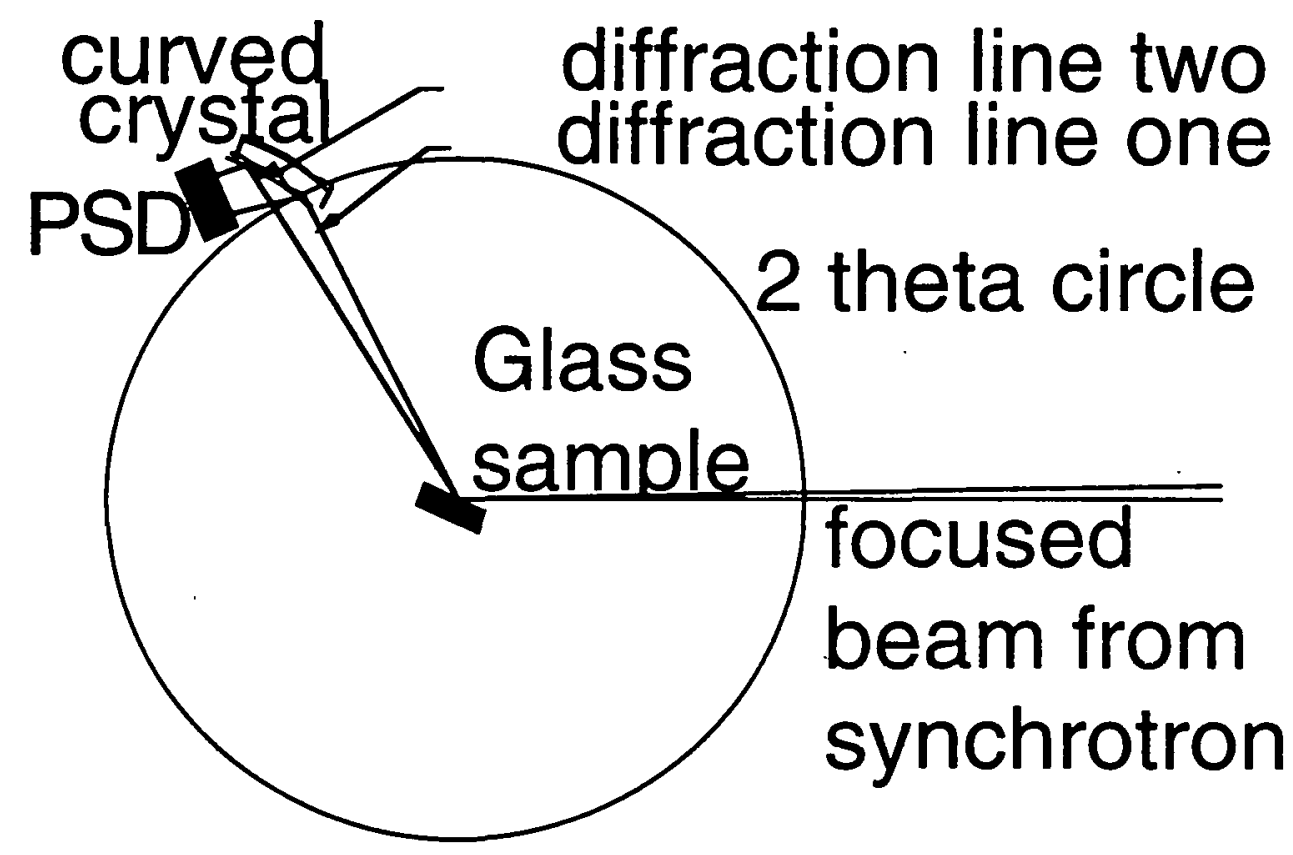

Figure 1. Schematic diagram of focusing analyzer crystal technique for anomalous scattering experiments. A focused synchrotron beam produces multiple diffraction lines which can be reflected by a bent perfect crystal analyzer. this instrument produces high data rates while retaining high angular and energy resolution.

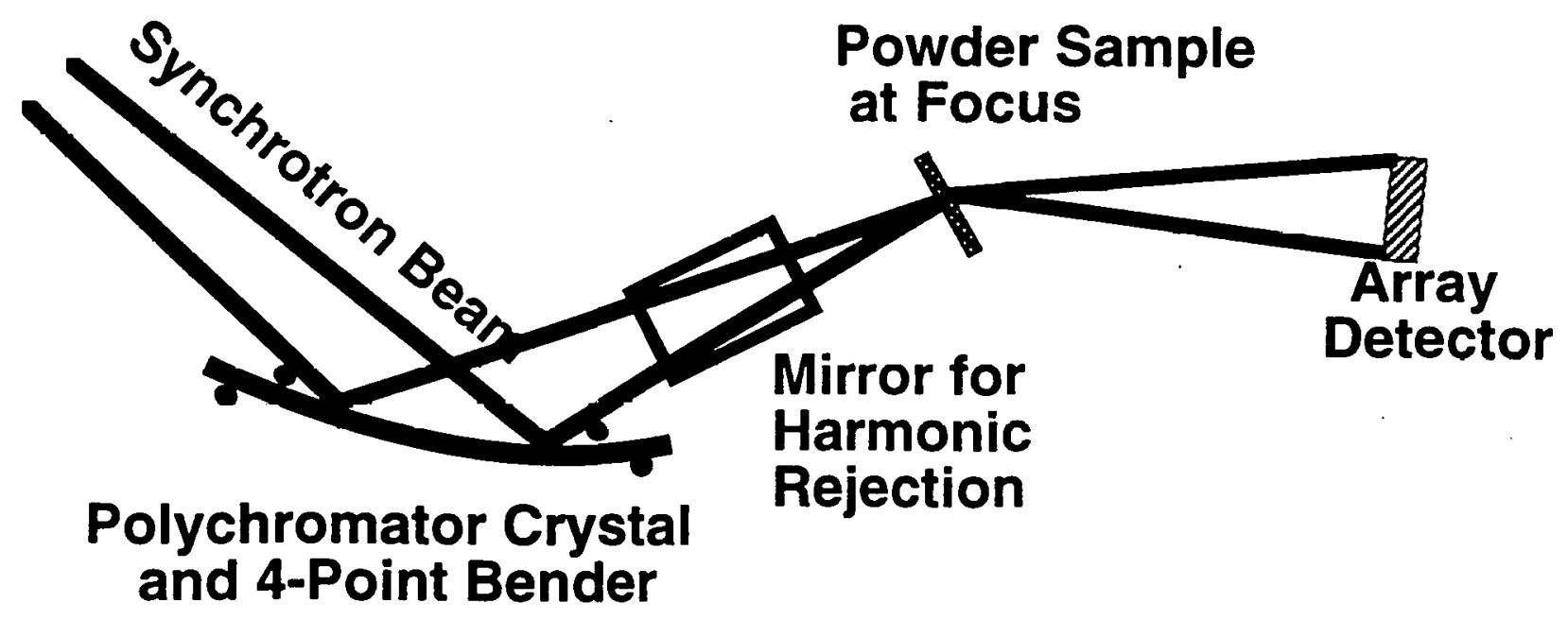

Figure 2. Anomalous scattering setup for continuous energy diffraction experiments. The polychromator focuses a range of energies on a powder sample, diffracted $x$-rays are then recorded on an image plate or array detector. 
blank page 


\title{
93-152R1 -- DEVELOPMENT OF INTERFEROMETER-BASED FOURIER TRANSFORM X-RAY TECHNIQUES
}

\author{
Associate Laboratory Director Area: \\ Principal Investigators: \\ Funding History:

FY $1993 \$ 181.3 \mathrm{~K}$
FY $1994 \$ 150.7 \mathrm{~K}$
FY $1995 \$ 148.6 \mathrm{~K}$
FY $1996-0-$
FY $1997-0-$

Advanced Photon Source

W. Yun, Z. Cai, E. Gluskin, and B. Lai, Experimental Facilities Division

Purpose: The project objective was to develop interferometer-based Fourier transform techniques for $\mathrm{x}$-rays and to explore their applications to spectroscopy and the imaging of surfaces and interfaces. The realization of these techniques will extend our spectroscopic capabilities into the $\mathrm{x}$-ray region and will significantly improve detection sensitivity of small refractive-index changes in mediums. Successful demonstration of the $x$-ray Fourier transform techniques will offer many unique capabilities for real-time imaging of surfaces and interfaces and for real-time spectroscopy, which are of major interest in the application of third-generation synchrotron sources.

Approach: The ability to study both the amplitude and the phase distributions of a signal wave offers many unique capabilities beyond simple detection of signal wave intensity. This ability can be realized by either detecting the interference of a signal wave with a mutually coherent wave or detecting the interference of several wavefronts from a signal wave through wavefront division. Techniques based on these interferometric properties have been well utilized in spectroscopy and imaging in the electromagnetic spectrum from IR to UV light.

Although some novel techniques and applications that make use of the interference in the $\mathbf{x}$-ray region have been long recognized, application of these interferometric techniques has been limited to a few spccial areas. These limitations result mainly from the difficulty of setting up an optical system to continuously scan the relative phase difference between the two interfering beams with an accuracy comparable to the wavelength of $\mathrm{x}$-rays. The scope of this project was to develop two types of Fourier transform $x$-ray interferometers. The first type is an amplitudedivision interferometer that consists of a phase shifter and a long path length LLL crystal interferometer with skew symmetry (Fig. 1). The second type is a wavefront-division interferometer (Fig. 2) in which a continuous scan of the optical-path difference can be obtained by employing one-dimensional translation. Because a phase shift is used in the interferometer of the first type and a grazing incidence geometry is used in the interferometer of the second type, mechanical accuracy for a one-dimensional translation is much less constrained, and, thus, practical applications are possible.

The project methodology involved the use of the third-generation source in the Advanced Photon Source (APS) to investigate the characteristics, such as resolving power, throughput, and sensitivity, of the wavefront-division and amplitude-division $\mathrm{x}$-ray interferometers from 
interference patterns. Novel applications to Fourier transform spectroscopy and to Fourier transform imaging of surfaces and interfaces will be also investigated. Scientific collaborators were Dr. I. McNulty and Mr. D. Legnini of the Experimental Facilities Division at ANL, and Dr. P. Siddons of Brookhaven National Laboratory.

Technical Progress and Results: The effort in the first year (FY 1993) of this project was concentrated on the preliminary design and detailed assessment of different $x$-ray interferometers, and on the development of optics and instrumentation. For the amplitudedivision interferometer, we designed a unit combining a LLL crystal interferometer with a pair of beryllium phase shifters. For the wavefront-division interferometer, we designed a novel configuration with two mirrors that reflect $x$-rays at grazing incidence for wavefront splitting and recombining and a four-mirror unit that generates optical-path differences.

During FY 1994, we completed the construction of the amplitude-division interferometer for 8$\mathrm{keV} \mathrm{x}$-rays. In order to have the split beams recombined well, the beam splitter, reflection crystals, and analyzer were made in an integrated unit from a piece of single crystal (Si 220). This interferometer was experimentally characterized. The beryllium phase shifters were also characterized. On the other hand, we conducted a more detailed analysis on the throughput, energy bandwidth, energy resolution, and the manufacturing tolerance of the wavefront-division $\mathrm{x}$-ray interferometer. It was found that the surface roughness and flatness of the beam splitter and the mirrors were highly restricted, yet, could be achieved with existing manufacturing technology.

During FY 1995, the procurement of the following components for the wavefront-division interferometer were completed: mirror substrates (less than $2 \AA$ in surface rms roughness and less than $0.5 \mu \mathrm{rad}$ in surface slope error), which, after being coated with nickel, will be used for beam splitting, beam combination, and phase-difference generation; high resolution steppingmotor-driven translation and rotation stages that carry the mirrors; high resolution DC-servomotor driven micrometers including motor drivers and control system for mirror angular alignment. We continued to develop flexure-based rotation and translation devices for positioning mirrors and translating the four-mirror unit with the accuracy required by our analysis. These stages are critical to assure the spectrum region and the resolution of the interferometric spectrometer. Our effort was also involved in the development of a higher efficiency linear zone plate that would allow us to efficiently combine two spatially coherent beams with known relative phase delays and, therefore, to generate an interferogram. In addition, we improved the spatial resolution of a high-speed $\mathrm{X}$-ray imaging system we developed in FY 1994 by reducing the thickness of a scintillation crystal $\left(\mathrm{CdWO}_{4}\right)$ to $60 \mu \mathrm{m}$, making it possible to record a hard $\mathrm{x}$-ray interferogram at the point where two coherent $\mathrm{x}$-ray beams meet. This capability may permit us to develop real-time Fourier transform techniques and other novel capabilities.

Once the imaging and coherence beamline at the APS starts commissioning, we will repeat the experiment, performed by Profs. Appel and Bonse, using the amplitude-division interferometer. Because of a continuous phase change of $2000 \pi$ and, thus, a resolving power of 2000 for $8-\mathrm{keV}$ $\mathrm{x}$-rays, our interferometer should be easily processed to obtain the spectral distribution of the copper $\mathrm{K}_{\alpha 1}$ and $\mathrm{K}_{\alpha 2}$ doublet. For the wavefront-division interferometer, we will coat mirrors, using the facility at the APS, and fabricate and characterize the flexure stages. These flexure 
stages will be made of low-expansion alloys, such as invar, in order to have thermal stability. Next, we will complete the construction, characterization, and demonstration of the wavefrontdivision interferometer. We also plan to demonstrate a hard $\mathrm{x}$-ray interferometer without moving parts, in which a spatially coherent hard $\mathrm{x}$-ray beam is reflected by two flat mirrors arranged with a small mutual angular offset. Because of the off-set angle, there will be a continuous phase shift between the two beams across the encountered area. As a result, an interferogram is formed, and it can be recorded using the $\mathrm{x}$-ray imaging system we improved in FY 1995.

Specific Accomplishments: Effort will continue under DOE-ER auspices.

1. E.N. Kaufmann, W. Yun, "Third Generation Synchrotron Radiation Source Applied to Materials Science," Advanced Materials '93, VI/Frontiers in Materials Science and Engineering, ed. S. Sōmiya et al., Trans. Mat. Res. Soc. Jpn. Volume 19B (Elsevier Science B.V., Amsterdam, 1994) p.811-819.

2. W. Yun, "Development of Interferometer-Based Fourier Transform X-ray Techniques," internal presentation in XFD, ANL. 


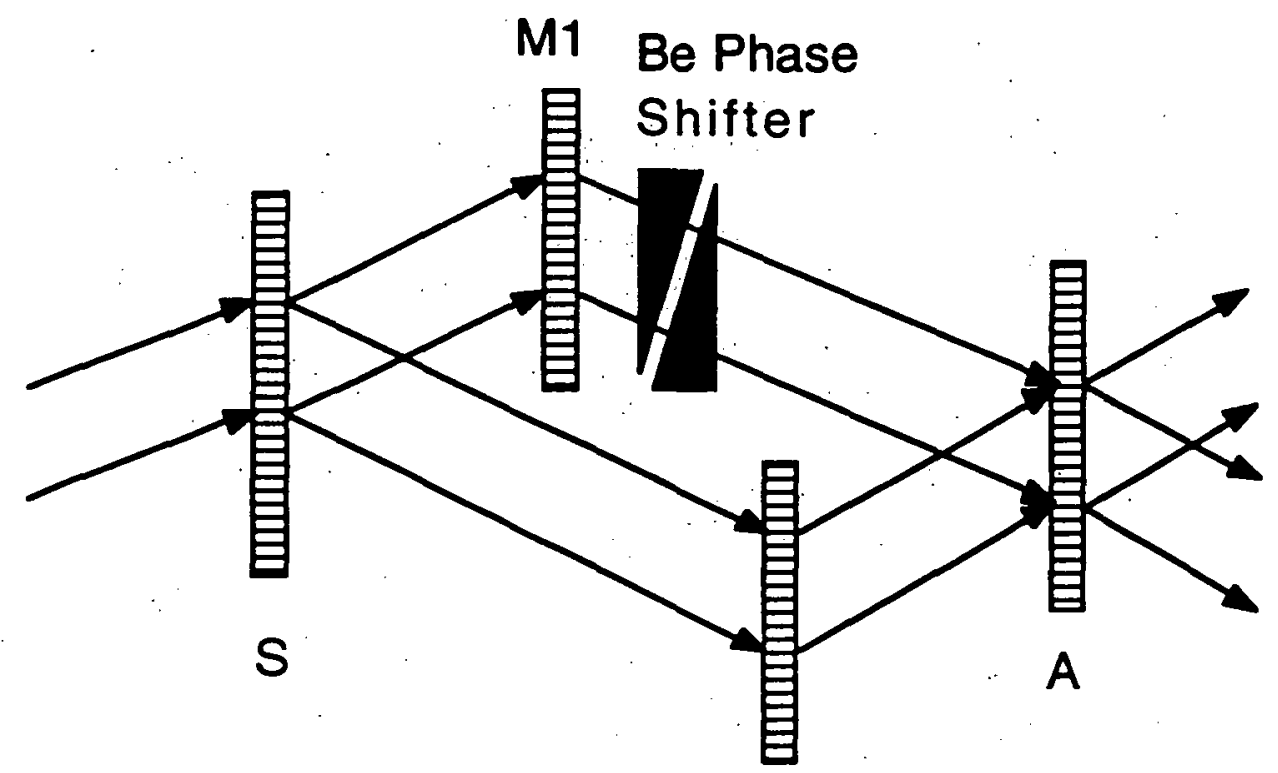

M2

Figure 1. Schematic of the amplitude-division Fourier transform interferometer to be developed. This interferometer consists of a skew symmetry LLL interferometer and a phase shifter. The LLL interferometer consists of a beam splitter $S$, two transmission mirrors/crystals M1 and M2, and an analyzer $A$. The relative phase difference between the two interfering beams split by $S$ is obtained by adjusting the thickness of the phase shifter. Real-time Fourier transform spectroscopy or Fourier transform imaging of surfaces and interfaces may be obtained by using a single wedge rotated 90 degrees from that shown above. A position sensitive detector is required to record the intensity distribution behind the analyzer crystal $\mathrm{A}$.

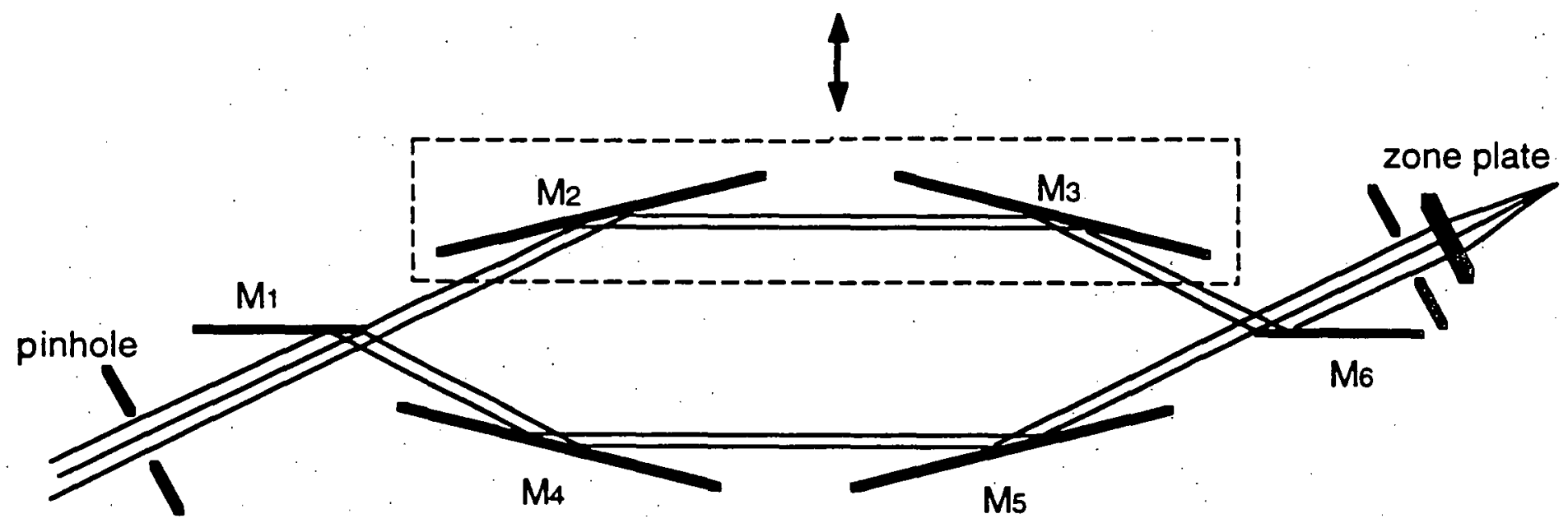

$x$-rays

Fig. 2. Schematic of a wavefront-division Fourier transform $x$-ray interferometer. A spatially coherent $\mathrm{x}$-ray beam is incident on and physically split into two beams at mirror $\mathbf{M}_{1}$. Each of the two beams is reflected by a two-mirror element. When the six mirrors are properly arranged and aligned, the two beams combine into one after the mirror M6. A variable phase delay of one beam relative to the other can be introduced by moving one of the mirror sets along the direction normal to the beam splitter. While the spatial coherence of either beam is reserved, the two beams are recombined after a linear zone plate and the resulting power of the interference of the two beams is recorded as a function of the phase delay. 


\title{
94-142R1 -- INELASTIC X-RAY SCATTERING USING HIGH ENERGY RESOLUTION
}

\author{
Associate Laboratory Director Area: Physical Research \\ Principal Investigator: \\ P.A. Montano, Materials Science Division \\ Funding Profile: \\ FY $1993-0-$ \\ FY $1994 \$ 198.8 \mathrm{~K}$ \\ FY $1995 \$ 150.4 \mathrm{~K}$ \\ FY $1996 \$ 150.0 \mathrm{~K}$ \\ FY $1997-0$ -
}

Purpose: The purpose is to design new experiments using X-ray inelastic scattering to investigate electronic excitations in solids. We will make use of the synchrotron radiation facility at the NSLS. This experiment will serve as prototypes for our research program at the Advanced Photon Source (APS).

Approach: The construction of the Basic Energy Sciences Synchrotron Radiation Center will facilitate the access by ANL researchers and general users to new fields of condensed matter science. The use of $\mathbf{x}$-ray inelastic scattering to study collective excitations in condensed matter opens new frontiers of research. This technique will play a prominent role with the arrival of the APS. The use of X-rays promises to displace other commonly available technique in the study of collective excitations in solids.

Inelastic $x$-ray scattering is an important tool to study a large number of physical properties in condensed matter systems. The advent of synchrotron radiation sources has provided enough photons to evaluate the feasibility of inelastic scattering experiments. The Advanced Photon Source (APS) will be an ideal source for X-ray inelastic scattering experiments. The high brilliance of the APS undulator will make it an optimal source of photons for inelastic scattering experiments. One advantage of $\mathrm{x}$-ray vs neutron inelastic scattering is the possibility of studying collective excitations in small samples $\left(\sim 1 \mathrm{~mm}^{2}\right)$. Inelastic $\mathrm{x}$-ray scattering (IXS) spectra are informative about a wide variety of electronic excitations. Compton scattering is a well known type of IXS that is widely applied tn determine electron momentum distributions. With the advent of modern synchrotron sources other types of IXS have become accessible and have been used to yield information not only on both single and collective excitations of electrons but also on the lattice dynamics of solids. To achieve spectra that are useful for these purposes one requires a resolution considerably higher than is typical for Compton scattering experiments. With a band pass ranging from 0.1 to $1 \mathrm{eV}$, one can study both valence band and conduction band electrons. The same energy resolution is also important for studies of collective excitations , e.g., plasmons. Electron energy loss spectroscopy (EELS) is widely used to provide information on collective electronic excitations but has the drawback that multiple scattering effects at high momentum transfers complicate the interpretation of the measurements. Because cross sections are very small, IXS measurements are devoid of multiple scattering complications and are consequently readily amenable to theoretical modeling. Furthermore hard x-rays do not require the use of ultra high vacuum conditions and bulk sampling is guaranteed. One clear advantage that IXS measurements have over the neutron inelastic scattering measurements is the fact that energy transfer and momentum transfer are nearly independent quantities and are almost 
unfettered by kinematic constraints. The additional freedom opens up new regions in the $Q, \omega$ phase space which were not accessible by any other means, where $Q$ and $\omega$ are the momentum and energy transfers, respectively. Broadly speaking, we can divide the IXS processes that involve electronic excitations into two regimes of $\omega$. Plasmons and valence band excitations occur at lower values, and inner shell excitations occur at higher values in the nonresonant Raman regime. For the excitation of core electrons in the nonresonant Raman regime IXS has the important advantage that one can change $Q$. One is not able to do so in standard $x$-ray absorption measurements. Our approach is to use IXS to study strongly bonded solids like SiC and TiC. We will make use of the NSLS facilities for our initial experiments.

Technical Progress and Results: We performed inelastic scattering measurements during last year on $\mathrm{TiC}$ and completed the analysis of the $\mathrm{SiC}$ data. EELS studies made using this TiC crystal have been reported previously. EELS studies of the SiC crystal have also been made. The orientation of the TiC crystal was $20^{\circ}$ away from [111]. We inclined the crystal to position the [111] direction along the scattering vector, $Q$. The IXS spectra were measured at beamline X21 at the National Synchrotron Light Source. This beamline has a wiggler as a source and delivers $2 \times 10^{11}$ photons per sec in a bandwidth slightly less than $1 \mathrm{eV}$. Crystal analyzers of increased collection efficiency have recently been constructed and were used for the present studies. These analyzers were $\mathrm{Ge}(444)$ at $7.6 \mathrm{keV}$ and $\mathrm{Si}(444)$ at $7.9 \mathrm{keV}$. Both of these were operated at Bragg angles in the range $86-87^{\circ}$. The spectra were obtained by scanning the incident energy with a fixed analyzer setting, i.e., at a fixed collection energy. We used both a $\mathrm{NaI}$ scintillation counter as well as a Ge solid state detector.

The IXS spectrum obtained for $\mathrm{SiC}$ at $\mathrm{Q}$ equal to $0.71 \mathrm{a} . \mathrm{u}$. is shown in Fig. 1 for comparison purposes. The spectrum reveals peaks at $25 \mathrm{eV}$ and $29 \mathrm{eV}$ as well as a shoulder at $14 \mathrm{eV}$. The peak at $25 \mathrm{eV}$ is in good agreement with EELS measurements taking into consideration a weak dispersion. Optical reflectivity measurements also shows an excitation around this energy. Optical measurements and EELS place the plasmon at $22 \mathrm{eV}$. We have also made measurements at $\mathrm{Q}$ equal to 0.25 a.u., and find a sharp plasmon peak at $23 \mathrm{eV}$ as shown in Fig. 1 . The shoulder at $14 \mathrm{eV}$ is consistent with possible excitations from the $\mathrm{C} 2 \mathrm{p}$ valence band. The occurrence of the peak at $29 \mathrm{eV}$ indicates that the critical value of the momentum transfer, Qc, had been exceeded and that plasmon decay via single particle excitations was possible. A variety of physical phenomena have been incorporated into theoretical descriptions that go beyond the random phase approximation to explain similar double peaked spectra for $\mathrm{Be}$ and $\mathrm{Li}$. The lack of a peak at $29 \mathrm{eV}$ at $\mathrm{Q}$ equal to 0.25 a.u., i.e., below Qc, support this contention.

We observed significant differences between the IXS spectra for TiC compared to that of SiC. The spectrum obtained for $\mathrm{TiC}$ is shown in Fig.2. The spectrum was obtained at $\mathrm{Q}$ equal to $0.74 \mathrm{a} . \mathrm{u}$. and shows three well resolved peaks centered at energies of $13 \mathrm{eV}, .26 \mathrm{eV}$, and $50 \mathrm{eV}$. These peaks correspond well to ones observed in EELS spectra. The $13 \mathrm{eV}$ peak was also observed in angle resolved photoemission. The $26 \mathrm{eV}$ peak is interpreted presently as the bulk plasmon and corresponds to peak $f$ of the EELS measurements which falls at an energy of 23.9 $\mathrm{eV}$. That this value is slightly lower than the observed value of $26 \mathrm{eV}$ is attributed to weak dispersion of the plasmon. We observe in addition a peak at about $50 \mathrm{eV}$ energy loss, that can not be explained by conventional excitations (i.e., plasmons, inter/intra band, core electron excitation). The $50 \mathrm{eV}$ IXS peak in the TiC spectrum does not have an analogue in the observed $\mathrm{SiC}$ spectra. This indicates an explanation involving d electrons. A quite similar peak 
was observed in the EELS spectra of TiC. The explanation for this peak put forward is that of an autoionization process, corresponding to a Ti-3p to Ti-3d transition. Autoionization occurs due to a mixing of a configuration belonging to a discrete spectrum with configurations belonging to a continuum. The significant cross section observed for the autoionization process using hard $\mathrm{x}$-rays indicates that IXS studies should be possible for many other transition metals and their compounds. A detailed analysis of the IXS measurements is in progress. [This research was performed in collaboration with A. Macrander and V. Kushnir from the APS].

\section{Specific Accomplishments:}

\section{Presentations:}

A.T. Macrander, P.A. Montano, V.I. Kushnir, R.C. Blasdell, C.C. Kao, "Inelastic X-ray Scattering from TiC Single Crystal," American Physical Society, San Jose, California (3/95).

A.T. Macrander, P.A. Montano, V.I. Kushnir, R.C. Blasdell, C.C. Kao, "Inelastic X-ray Scattering from 6H-SiC Single Crystal," American Physical Society, San Jose, California (3/95).

A.T. Macrander, R.C. Blasdell, P.A. Montano, and C.C. Kao, "Inelastic X-Ray Scattering From 6H-SiC." The Electrochemical Society, 188th Society Meeting, Chicago (October 8-13, 1995).

P.A. Montano, "Inelastic X-Ray Scattering," Materials Science Division Seminar, Argonne National Laboratory (March 10, 1995).

P.A. Montano, "Inelastic X-Ray Scattering," Colloquium, Department of Physics, University of Illinois at Chicago (April 5, 1995).

\section{Publications:}

A.T. Macrander, R.C. Blasdell, P.A. Montano, and C.C. Kao, "Inelastic X-Ray Scattering From 6H-SiC," Proceedings of The Electrochemical Society, 1995.

A.T. Macrander, P.A. Montano, D.L. Price, V.I. Kushnir, R.C. Blasdell, C.C. Kao and B.R. Cooper, "Inelastic X-Ray Scattering From TiC and Ti Single Crystals," submitted to Phys. Rev. B. 


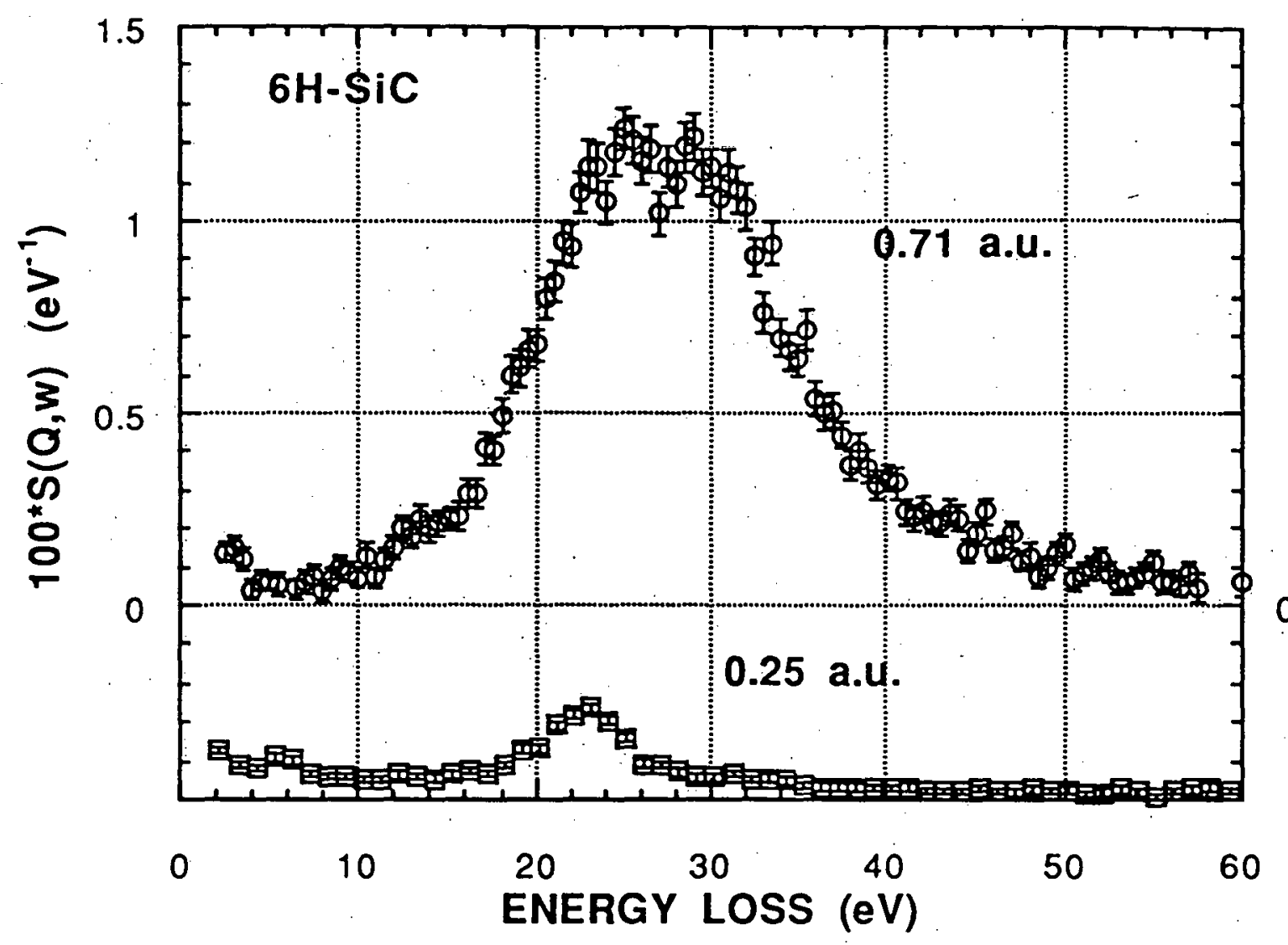

1) Inelastic $\mathrm{x}$-ray scattering spectra of $6 \mathrm{H}-\mathrm{SiC}$ for momentum transfers equal to 0.71 a.u. and 0.25 a.u. along [00:1].

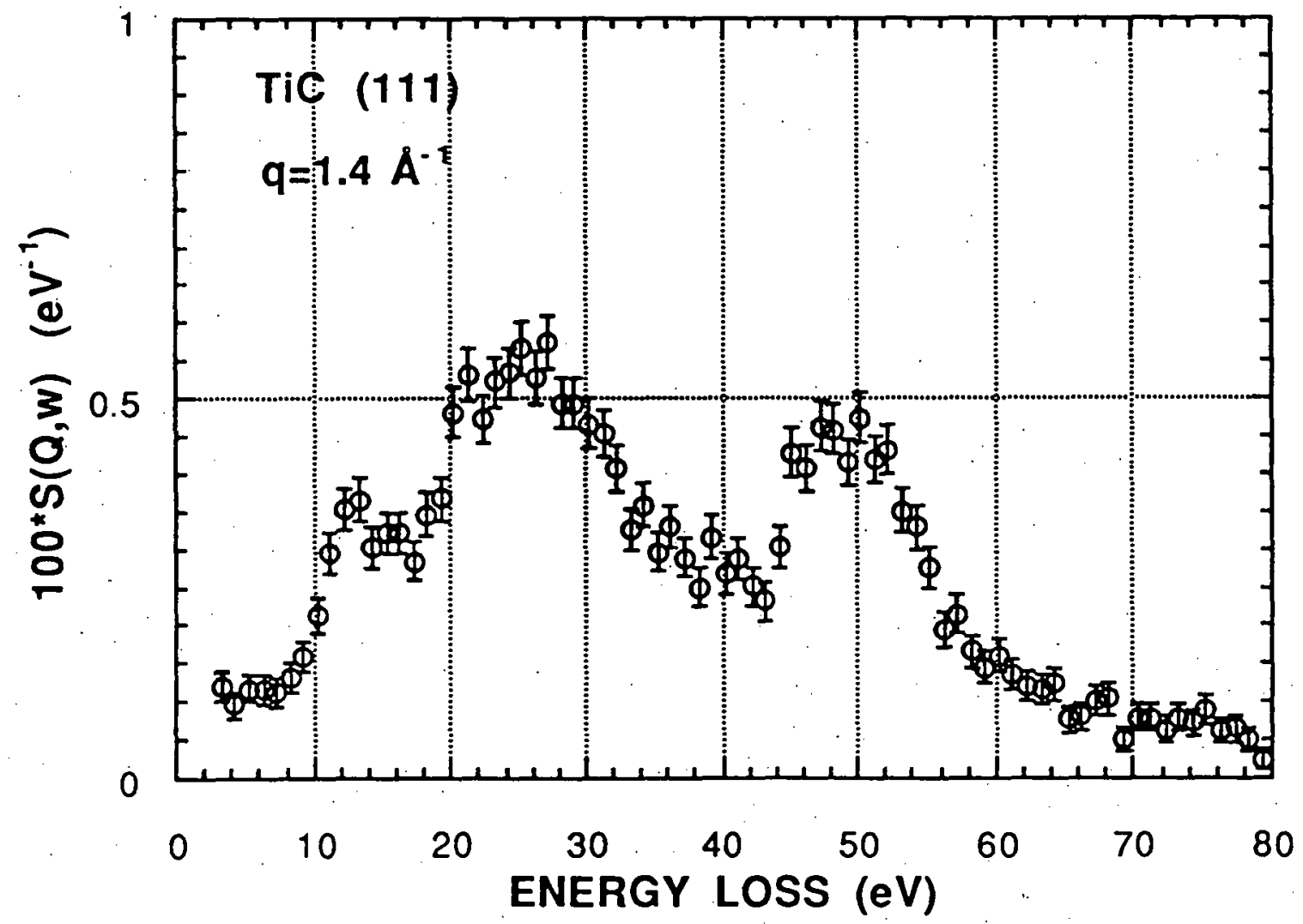

2) Inelastic $x$-ray scattering spectrum for $\mathrm{TiC}$ with $\mathrm{Q}$ equal to 0.74 a.u. along [111] 


\title{
95-007N -- DEVELOPMENT OF A MONOCHROMATOR SYSTEM FOR THE APS X-RAY BEAMLINES
}

\author{
Associate Laboratory Director Area: Physical Research
}

Principal Investigators:

Funding Profile:

\author{
M.A. Beno, G.S. Knapp, G. Jennings, \\ and P.A. Montano, Materials Science Division \\ FY $1993-0-$ \\ FY $1994-0-$ \\ FY $1995 \$ 200.9 \mathrm{~K}$ \\ FY $1996 \$ 150.0 \mathrm{~K}$ \\ FY $1997 \$ 200.0 \mathrm{~K}$
}

Purpose: The purpose of this research is the continued development of a monochromator system for use at the BESSRC beamlines at APS which provides energy scanning capabilities, allow high vacuum operations and cryogenic cooling. In a synchrotron $\mathrm{X}$-ray beam line the most important optical component is the monochromator. Typical monochromators consist of two identical crystals so that the whole system so that it is possible to vary the diffracted beam energy and maintain a fixed beam height. The scientific programs to be carried out at the BESSRC beamlines require a monochromator design which is capable of scanning energy and low energy operation. These requirements necessitate a monochromator designed for fixed exit scanning operations capable of high vacuum operation.

Approach: The first optical element in the BESSRC CAT beamlines will be a monochromator, so that a standard design for this critical component is advantageous. The monochromator we have designed is a double crystal, fixed exit scheme with a constant offset designed for UHV operation, thereby allowing windowless operation of the beamlines. The crystals are mounted on a turntable with the first crystal at the center of rotation. A mechanical linkage is used to correctly position the second crystal and maintain a constant offset. The monochromator design also includes an option for the positioning of the second crystal by a drive mechanism, which has the advantage of optionally locking the motion of the second crystal during energy scans, thereby allowing the beam to walk on the second crystal while maintaining the fixed exit beam offset. The main drive for the rotary motion is provided by a vacuum compatible Huber goniometer isolated from the main vacuum chamber. Rotary motion of the primary monochromator stage is accomplished by using two adjacent vacuum chambers connected only by the small annular opening around a hollow stainless steel shaft which connects the Huber goniometer to the turntable on which the crystals are mounted. This design allows high vacuum operation of the monochromator since it is possible to maintain $10^{-9}$ Torr on the monochromator side while maintaining only $10^{-6}$ Torr in the goniometer vacuum chamber. The central shaft also allows for passage of crystal cooling lines from UHV chamber to the low vacuum side and then into the atmosphere. The design of the monochromator is such that it can accommodate both water and liquid nitrogen cooling for the crystal optics.

Technical Progress and Results: We have designed a Bragg-Bragg geometry double crystal monochromator system to be used by the BESSRC beam lines at the APS. Figure 1 shows a geometrical representation of the two crystal monochromator scheme. The design consists of 
two matching perfect crystals mechanically linked by way of two slides mounted orthogonal to each other (a Cowan-Golovchenko linkage ${ }^{1,2}$ ). Energy tuning is achieved by a single rotary drive mechanism with the center of rotation at the first crystal (point A in Figure 1).

Figure 2 shows a top front view of the monochromator system. The whole vacuum chamber is only 24 inches in diameter and has 27 inch OD wire sealed UHV flanges. The chamber will be mounted on a kinematic mount table shown in Figure 3. A 220 1/s Ion pump will be used to pump the monochromator side and a $170 \mathrm{l} / \mathrm{s}$ ion pump for the Huber goniometer side. During initial pumping both the chambers will be pumped simultaneously by a single turbo pump. Once the ion pumps are started the two sides will be vacuum isolated.

The designs of the monochromator, monochromator chamber and mounting table are shown in Figures 2 and 3 . Initial tests of the monochromator linkage mechanism are presently underway. The parallel alignment of two mirrors mounted at the first and second crystal positions is being examined using tilt sensors and with a highly accurate autocollimator. With the second slide we have chosen for initial work the deviation from parallel alignment of the first two crystals is only acceptable to about $12 \mathrm{keV}$. A larger second slide needs to be tested to extend the operating range to higher energy. Other optical techniques being used to test the fixed exit mechanism indicate that the fixed exit operation of the monochromator mechanism is also problematic at high energies. Both problems may be related to bending of the light weight second slide originally chosen.

Further development of the BESSRC monochromator system will be required. The slides chosen for initial trials are not heavy enough for the loading of the second crystal which will occur when a sagital bender is included in the second crystal mount and/or when cooling is added to the second crystal. Larger first and second crystal slides will be fit into the current design and the testing of the monochromator continued.

Since in the initial stages of operation none of the BESSRC beam lines will have mirrors for focusing the beam, a design for a sagital focusing mechanism must be developed as an integral part of the monochromator system. This development effort will involve both the design of a bender mechanism and the determination of optimal crystal designs for each of the three BESSRC beamlines. Since the beamlines have an undulator, wiggler and bending magnet as sources, three slightly different crystal and bender designs must be developed. This development will involve a large effort in mechanical design as well as crystal cutting.

\section{Specific Accomplishments:}

Refereed. Publication: M. Ramanathan, M.A. Beno, G.S. Knapp, G. Jennings, P.L. Cowan, and P.A. Montano, "Multipurpose Monochromator for the Basic Energy Science SynchrotronRadiation Center Collaborative Access Team Beamlines at the Advanced Photon Source-X-Ray Facility, " Rev. Sci. Instrum. 66(2):2191-2193 (1995).

\section{References:}

1. J.A. Golovchenko, R.A. Levesque, P.L. Cowan, Rev. Sci. Instr. 51:509 (1981).

2. P.L. Cowan, J.B. Hastings, T. Jach, J.P. Kirkland, Nucl. Instr. \& Meth., 208:349 (1983). 


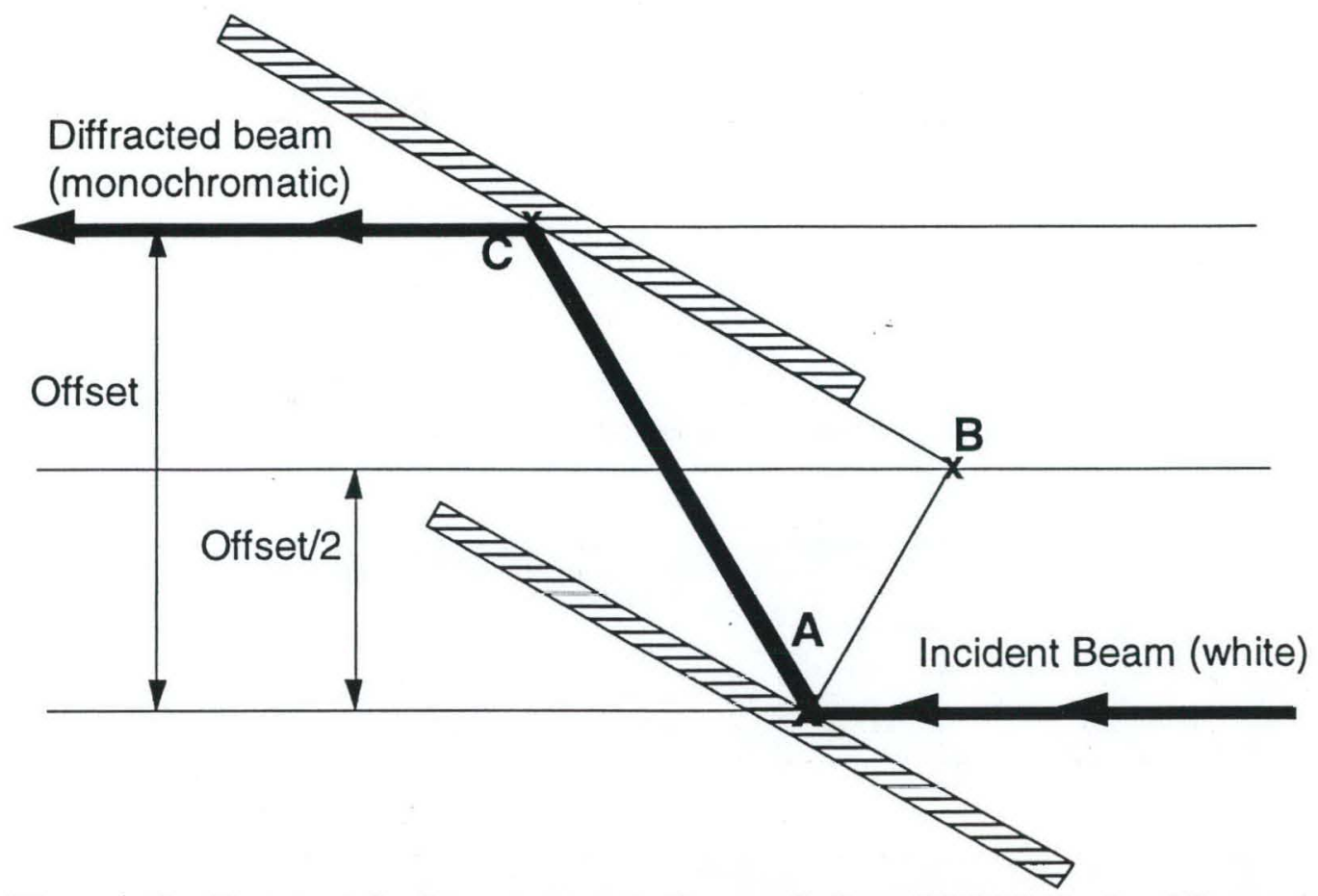

Figure 1 Geometrical representation of the BESSRC double-crystal fixed exit monochromator. The right triangle $\mathrm{ABC}$ is built of two precision slides. This linkage not only keeps the crystals parallel but produces a constant offset of the incident and diffracted beams. 


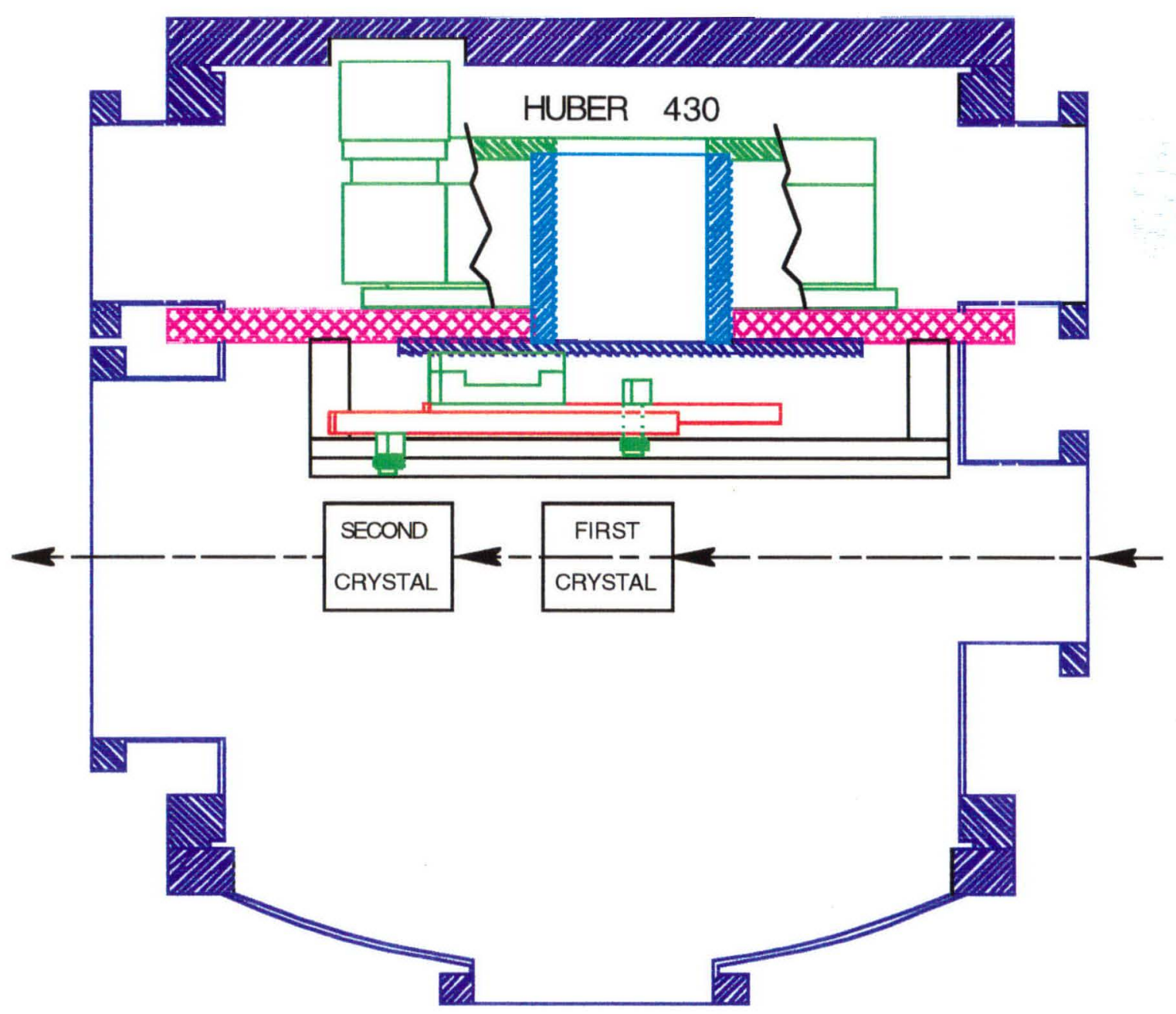

Figure 2. Top view of the BESSRC monochromator chamber. The chamber closest to the wall (at the top of the figure) contains the rotary table which drives the first crystal rotation. The larger chamber (bottom of the figure) contains the monochromator linkage mechanism, crystal mounts and crystals. This arrangement allows easy access to the crystals without disturbing the monochromator alignment. 


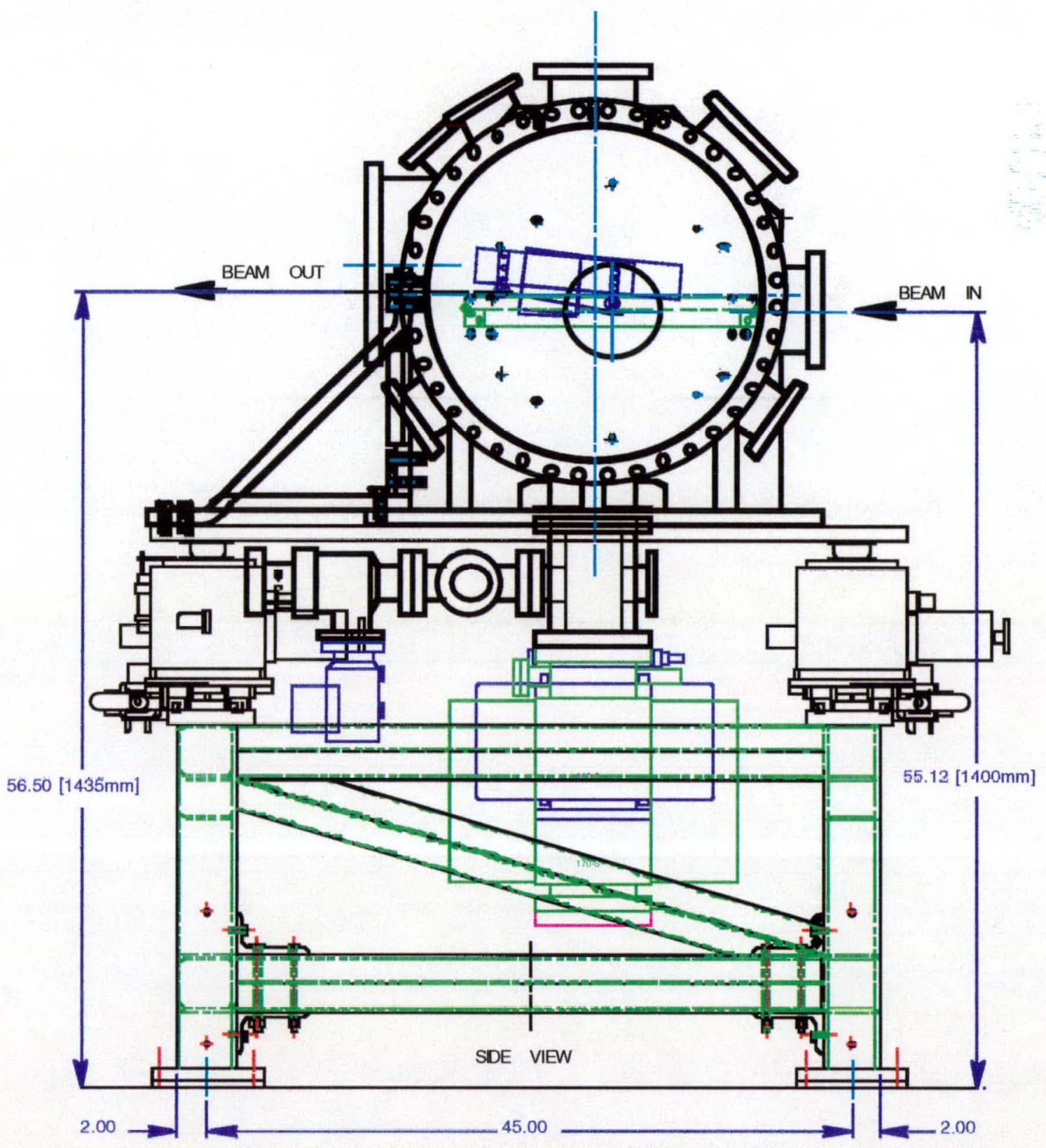

Figure 3 A front view of the BESSRC monochromator mounted on a kinematics table. The 170 and $220 \mathrm{l} / \mathrm{sec}$. ion pumps are used on the rotary drive and monochromator chambers respectively. The monochromator chamber ion pump also has a NEG insert for additional pumping. 
blank page 


\title{
95-171N -- DEVELOPMENT OF A FAST SPECTROMETER FOR STUDYING X-RAY INDUCED PROCESSES ${ }^{1}$
}

\author{
Associate Laboratory Director Area: Physical Research
}

Principal Investigators:

Funding Profile:
T.W. LeBrun, Physics Division

FY $1993-0-$
FY $1994-0-$
FY $1995 \$ 56.3 \mathrm{~K}$
FY $1996-0-$
FY $1997-0-$

FY $1994-0-$

FY $1996-0$

FY $1997-0$

Purpose: The goal of this project is to develop the facilities necessary to explore the physical and practical limits on the time resolution of fast, wide-area multichannel plate (MCP) detectors, with the aim of developing time-resolved detection methods with the highest possible time resolution.

Approach: Time resolved measurements of electrons or photons ejected from excited systems ranging from nuclei to atoms to proteins can provide a window into the dynamics of such systems on the time scale of hundreds of picoseconds or better, and are becoming more common at synchrotron facilities throughout the world. For much shorter-lived states, time resolved measurement allows the energy spectra of massive particles, such as electrons, to be recorded.

Such techniques are at the heart of a new class of electron time-of-flight spectrometers that have proven very successful in studying photon-induced processes in the soft $\mathrm{x}$-ray range (10$1000 \mathrm{eV}$ ). These spectrometers collect a wide range of energies simultaneously and combine high efficiency with good resolution at low energies. They are also ideally suited for coincidence measurements. The extension of these techniques to the measurement of higher energy electrons $(1 \mathrm{keV}-30 \mathrm{keV}+)$ could provide the same advantages for the study of $\mathrm{x}$-ray induced processes without the concomitant problems associated with the high fields or currents usually required by such measurements.

The fundamental obstacle to the application of time-of-flight spectroscopy at higher energies is the attainable time resolution. For low energies the ultimate resolution is largely limited by the ratio of the source length to the drift length, but for high energies the time resolution of the detector rapidly becomes the limiting factor. This problem is exacerbated by the need for large detectors which enhance detection efficiency but are incompatible with fast detection for a number of reasons, not the least of which is the finite propagation time $(30 \mathrm{ps} / \mathrm{cm})$ for signals to traverse the detector surface.

To address these problems, a fast detector had been developed in collaboration with John Bogaty of the Physics Division. In this project we developed the facilities necessary to fully develop these detectors so that they may be used in actual measurements. Specifically we developed the

\footnotetext{
${ }^{1}$ Individual Investigator Project
} 
pulsed excitation source, spectrometer, fast pulse electronics and mechanical support required for the study and optimization of these devices.

The success of this effort will not only allow us to push electron time-of-flight spectroscopy to higher energies, but also increase the resolution of these measurements for lower energy electrons, such as those resulting from Auger cascade processes following $\mathrm{x}$-ray excitation. In addition, the possibility exists to improve measurements of short-lived resonant states in atoms and nuclei.

Technical Progress and Results: To assist in the tasks associated with development described here, a summer student, Dan McKinsey, was supported and trained in the techniques required for this project including vacuum systems, mechanical design, drawing, and construction, electron gun operation, nuclear electronics, etc. Specific tasks accomplished were the assembly of the required vacuum system, design and construction of the gas target and slit assembly, and the construction of an electron gun. The gun was also commissioned and operated as a pulsed source. In addition a time-of-flight spectrometer and detector were assembled and the electronics required for fast timing measurements acquired. Finally, the spectrometer was commissioned and electron spectra obtained using the electron gun excitation source.

Specific Accomplishments: Prior to this LDRD project, we developed a series of fast detectors which were described in invention report \#ANL-IN-93-103. The time resolution of these detectors is known to be better than $150 \mathrm{ps,} \mathrm{but} \mathrm{accurate} \mathrm{characterization} \mathrm{was} \mathrm{precluded} \mathrm{by} \mathrm{the}$ test equipment available. In this project we have developed the facilities necessary to characterize these detectors on time scales down to tens of picoseconds, enabling us to explore the limits to the time resolution on very fast time scales. This effort should open the door for the use of these detectors for fast timing measurements at synchrotron facilities and expand the range of time-resolved measurements currently feasible. 


\title{
93-041R2(b) ${ }^{1}$-- DEVELOPMENT OF SYNCHROTRON RADIATION ANALYTICAL CAPABILITIES AT THE CENTER FOR ADVANCED MICROSTRUCTURES AND DEVICES
}

\author{
Associate Laboratory Director Area: Engineering Research Program Administration \\ Principal Investigators: \\ M.C. Petri, Engineering Division \\ L. Leibowitz, Chemical Technology Division \\ Funding Profile: \\ FY 1993 \$137.9K (subtask a) \\ FY $1994 \$ 223.6 \mathrm{~K}$ (subtask b) \\ FY $1995 \$ 343.1 \mathrm{~K}$ (subtask b) \\ FY 1996 \$150.0K (subtask b) \\ FY $1997-0$ -
}

Purpose: The purpose of this work is to develop a micro-analytical facility that will allow simultaneous $\mathrm{X}$-ray diffraction and $\mathrm{X}$-ray fluorescence examination of materials on a microscopic scale. For this work, a dedicated beam line is being developed at the synchrotron radiation source at the Louisiana State University (LSU) Center for Advanced Microstructures and Devices (CAMD). This work will result in a unique facility for the microscopic X-ray examination of a wide range of technologically important systems.

Approach: A vast array of technologies face fundamental materials issues related to small-scale structures. In particular, chemical interactions between bonded materials can degrade the materials over time and can cause local precipitation of phases that can weaken bonds. Alternatively, improved bonds can be made by controlling local interactions. This control, however, can only come about from an understanding of the kinetics and the thermodynamics of the chemical interactions. The present work employs a synchrotron source to extract structural and compositional information from bonded materials that can lead to a better understanding of the processes involved. In contrast to scanning electron microscopy, synchrotron radiation sources can reveal both composition (via fluorescence) and structure (via absorption spectroscopy and diffraction), providing an ideal tool for studying materials and their interactions on a microscopic scale.

This work involves developing high-resolution collimated $\mathrm{X}$-ray beams and focused $\mathrm{X}$-ray beams for micro-diffraction and micro-fluorescence analyses. These capabilities for synchrotron radiation analytical techniques will be developed at CAMD, a facility owned by the state of Louisiana and operated by LSU. CAMD, which began operations in 1992, consists of a 1.5 $\mathrm{GeV}$ electron storage ring with $16 \mathrm{X}$-ray beam line ports. Up to three insertion devices can be installed for higher energy and higher intensity radiation. Earlier work by us at the National Synchrotron Light Source (NSLS) at Brookhaven National Laboratory (BNL) has shown that these novel techniques have great promise, giving results unobtainable by other means. Separate beamlines are required at the NSLS, however, since no single beamline allows simultaneous diffraction and fluorescence work. The proposed project will adapt the methods used at NSLS

\footnotetext{
${ }^{1}$ subtask b was initiated in FY94.
} 
to the CAMD facility, providing a unique experimental station that combines several X-ray analysis techniques in a single beamline. CAMD offers distinct advantages to the development of these microscopic X-ray capabilities. In particular, the ready availability of beam time and a dedicated working space are attractive features of working at CAMD.

The kinetics and thermodynamics of material interactions can be studied by conducting isothermal diffusion couple experiments. These experiments consist of bonding two materials under controlled conditions and examining the interdiffusion of the chemical elements. In such a study, a collimated or focused synchrotron X-ray beam can be translated in steps across the diffusion zone. At each step, diffraction patterns or fluorescence spectra can be collected to provide structure and composition profiles along the diffusion path. Similar techniques can be applied to other interfaces of interest such as welds, brazes, and battery or fuel-cell connections, to name just a few.

Technical Progress and Results: Since FY1994 Argonne has designed a experimental station at CAMD that allows simultaneous fluorescence and diffraction on a microscopic scale. In addition, the synchrotron beamline will also allow X-ray absorption spectroscopy (XAS) for local element-specific coordination information.

The micro-analytical beamline is now capable of performing $X$-ray fluorescence studies using a collimated beam of white $\mathrm{X}$-rays. A unique four-circle goniometer with an $\mathrm{X}-\mathrm{Y}-\mathrm{Z}$ translation stage with $1-\mu \mathrm{m}$ stepping motors allows samples to be manipulated and scanned in many orientations. An intrinsic germanium detector for collecting characteristic X-rays from samples was designed with a 12 -inch-long by 1 -inch-diameter snout that can be placed close to the sample to maximize collection efficiency. (A duplicate detector has since been procured by researchers at the APS.) A special X-ray collimator was also designed for the ANL/CAMD project to provide a thin line of X-rays either $50 \mu \mathrm{m}, 20 \mu \mathrm{m}$, or $6 \mu \mathrm{m}$ wide. This collimator is now being marketed for other synchrotron users.

All table and diffractometer motors have been successfully tested remotely through a DEC Alpha computer. Software provided by Mark Rivers (University of Chicago) allows "virtual motors" to be defined, so that several motors can be activated simultaneously. For example, a single command can pitch the table to a given angle by operating four motors at the same time. Software was also set up to collect X-ray spectra from three detectors and to analyze the results. A linear fluorescence scan was performed by collecting spectra as a copper and titanium sample was stepped through a collimated X-ray beam. The spectra were then analyzed to produce a qualitative profile of the copper and titanium concentrations in the sample (see figure). Quantitative fluorescence work will require that reference standards with known concentrations be used. Additional software will be needed to account for cross-absorption effects between elements.

During FY 1996-the third year of the LDRD project-LSU will add a fixed-exit, double-crystal monochromator to tune the beam to a single $X$-ray wavelength. In a collaboration with University of Chicago researchers, focusing optics will be designed to intensify the X-ray flux. These upgrades will allow X-ray diffractometry and XAS for structural analyses. In a typical study, fluorescence at high energies can be used to locate specific microstructural features on 
a sample. The monochromator can then be tuned to a lower X-ray energy for diffraction and XAS of that feature.

\section{Specific Accomplishments:}

\section{$\underline{\text { Outside Presentations }}$}

M.C. Petri, Current ANL Activities at CAMD, ANL/CAMD Synchrotron Application Workshop, Argonne National Laboratory, Argonne, Ill. (November 1994).

M.C. Petri, A Micro-Analytical Synchrotron X-ray Beamline for the Study of Interfaces, 1995 Workshop on Science and Technology at CAMD, Louisiana State University, Baton Rouge, La. (March 1995).

M.C. Petri, A Micro-Analytical Synchrotron X-ray Beamline for the Study of Interfaces, Intl. Symposium on Optical Science, San Diego, Calif. (July 1995).

$\underline{\text { Publication }}$

M.C. Petri, L. Leibowitz, and P. Schilling, "Hard X-ray Microanalytical Beamline at the CAMD Synchrotron," in X-ray Microbeam Technology and Applications, Wenbing Yun, editor, Próc. SPIE 2516, 180-84 (1995). 


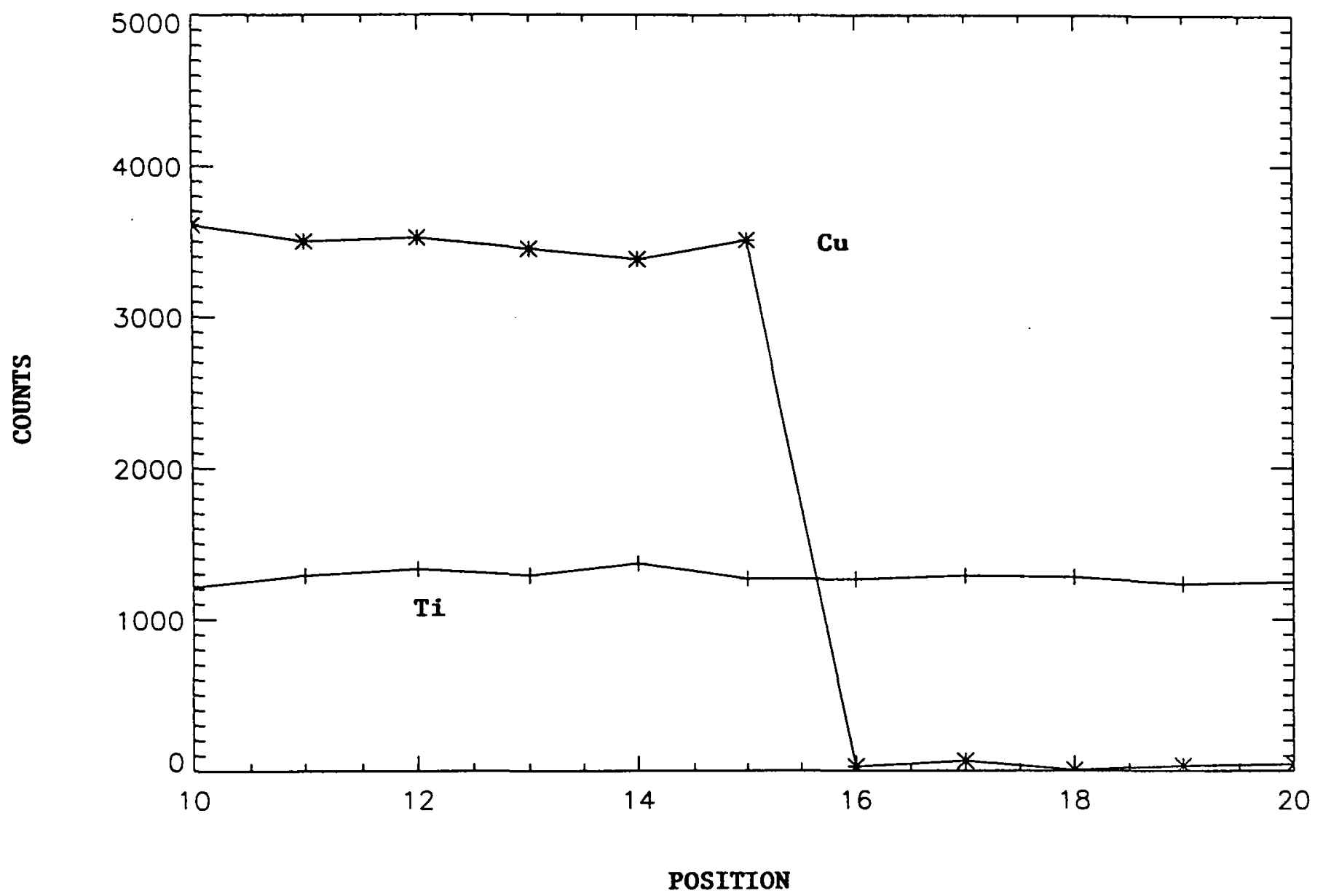

Qualitative X-ray Fluorescence Profiles of Cu and Ti Concentration Across a Sample 


\title{
95-013N -- OPTICAL LUMINESCENCE X-RAY ABSORPTION FINE STRUCTURE: A NEW METHOD TO PROBE THE COORDINATION AND VALENCE OF f-ELEMENTS IN AMORPHOUS MATERIALS
}

\author{
Associate Laboratory Director Area: Physical Research \\ Principal Investigator: \\ M.R. Antonio, Chemistry Division \\ Funding Profile: \\ FY $1993-0-$ \\ FY $1994-0-$ \\ FY $1995 \$ 81.5 \mathrm{~K}$ \\ FY $1996 \$ 50.0 \mathrm{~K}$ \\ FY $1997-0$ -
}

Purpose: The purpose of this LDRD is the site selective structural analyses of amorphous f-ion materials, such as glasses, polymers, catalysts and nuclear wastes. Such analyses will be facilitated by the development of X-ray excited optical luminescence (XEOL) X-ray absorption fine structure (XAFS) methods. XAFS detected by optical luminescence provides the element specificity of conventional XAFS but with an added advantages of increased sensitivity, as well as site selectivity through luminescence filtering in the frequency and time domains. The geometric and electronic information to be obtained through this novel approach is not available through the conventional methods of either laser spectroscopy or XAFS alone. The combination of these two techniques provides a unique entry to structure studies of amorphous materials with luminescent centers, and should eventually lead to a predictive understanding of an f-ion's site preference in amorphous materials, and the resulting influence on optical properties.

Approach: Optical luminescence from selected f-ions, which emit strongly in the visible, has been extensively studied. The luminescence occurs between localized f-states that are represented well by crystal-field theory. Detailed characteristics of the luminescence are dependent strongly on the environment of the central-ion. The crystal field slightly alters the splitting of the terms generated from the f-configuration by electron-electron repulsion, thus influencing the energy of the emitted radiation. In addition to variations in the relative energy of a fluorescing state, the lattice in which the ion is imbedded can substantially effect the lifetime of the emitting state. Lifetimes can range from nanoseconds through seconds. The combination of energy and lifetime dependencies on spectral features permits optical spectroscopists to do site-specific studies of fluorescing ions in complex, multiple-site samples. Unfortunately, whereas extremely detailed optical and crystal-field information can emerge, there is no straightforward way to relate these results to any crystallographic information that may exist. For example, a crystalline sample with two rare-earth sites exhibits two separable and interpretable spectra, but it is very difficult to confirm which optical spectrum originates from which of the two sites.

The focus of this proposal is to examine the possibility of extending the concepts employed by optical spectroscopists into X-ray absorption spectroscopy. We propose that, by monitoring selected wavelengths, some of the techniques developed by optical spectroscopists can be used to obtain site specific XAFS. 
$\mathrm{XEOL}$ has been previously reported in select systems such as $\mathrm{CaF}_{2}$, in which the luminescing states are thought to be localized, excitonic states trapped in the energy gap, or transition metals in inert hosts such as $\mathrm{ZnS}$. The fluorescing states in these materials are not well understood. We hypothesize that the decay of the excited state created by absorption of an X-ray by an f-ion will, through cascade processes not well understood, result in some population of excited f-states. These excited states will undergo luminescent decay similar to that observed by optical spectroscopists pumping those same states with lasers. This hypothesis is somewhat borne out in the literature of X-ray phosphors, from which it is observed that some of the best available current phosphors involve $\mathrm{Eu}$ or $\mathrm{Tb}$ as the active material. These are the two rare earths with the strongest optical luminescence.

Technical Progress and Results: The first experiments have yielded mixed results. Monitoring energies from $5000-24,000 \mathrm{~cm}^{-1}$ (slightly broader than the optical window) using a photomultiplier tube and associated optics, we obtained EXAFS, shown in Figure 1, from $\mathrm{Eu}_{2} \mathrm{O}_{3}$ at room temperature. An analysis of these data, including a comparison with transmission data taken simultaneously, is encouraging. The EXAFS obtained using optical detection reproduce nicely those taken using transmission detection, and the fits are indistinguishable. However, we also encountered some very unusual results. For example, the EXAFS from $\mathrm{TbCl}_{3} \bullet 6 \mathrm{H}_{2} \mathrm{O}$ obtained in transmission geometry are compared with those detected optically in Figure 2. These optical results were reproducible, and to the best of our knowledge are NOT an experimental artifact. Needless to say, the distances determined from the optical data do not match those from a fit to the transmission data, and the coordination number cannot be fit at all. There is clearly some process(es) occurring in this particular Tb compound that interfere with the optical decay pathway. Unfortunately, in these first experiments we could not monitor the energy of the optical response. A knowledge of the exact energy of the luminescence is crucial, even for these early experiments, if we are to understand the luminescing process, and whether we are monitoring the transitions expected from the optical-spectroscopy literature. In addition to these data, we found evidence that the optical response is sensitive to sample preparation and the angle of the incident X-rays. These results have been previously reported for XEOL. We also noted a dependence on scan rate and scan DIRECTION. This may be related to lag effects observed for X-ray phosphors, but has not been investigated.

Specific Accomplishments: Initial proof-of principal has been accomplished, although several questions have been raised. Further work is necessary before these data can be published. 

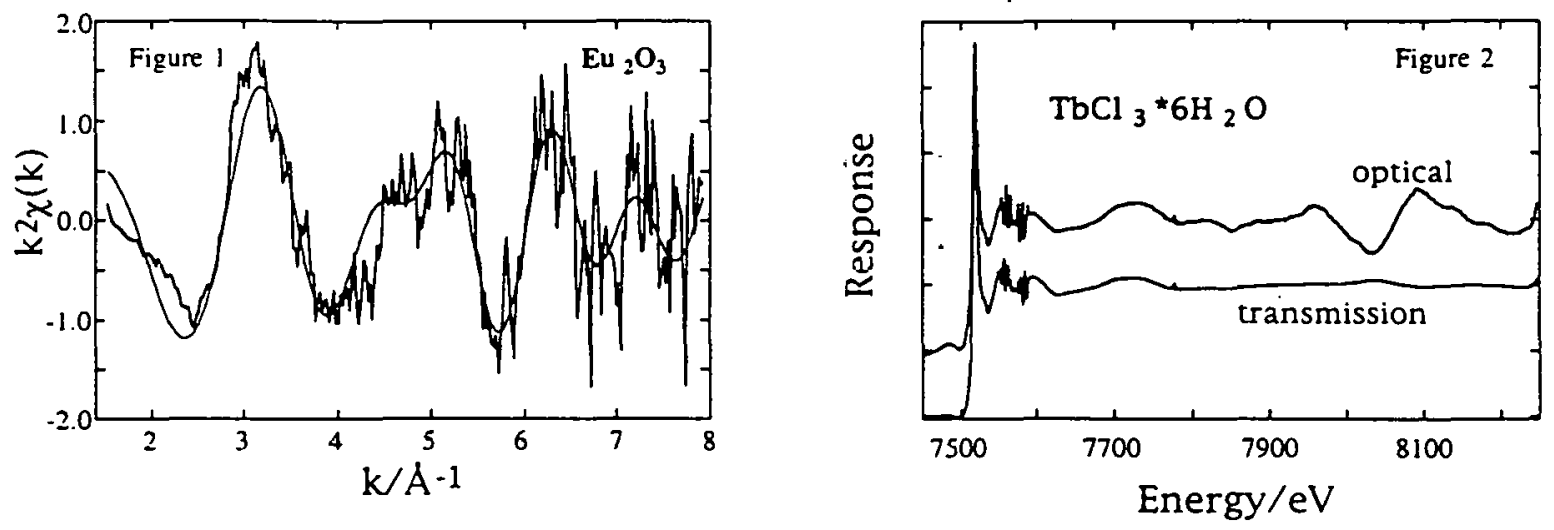

Figure 1. XEOL detection of EXAFS from $\mathrm{Eu}_{2} \mathrm{O}_{3}$. Solid line is the fit, which agrees well with known structure.

Figure 2. Normalized XEOL response is compared with transmission data from $\mathrm{TbCl}_{3} \cdot 6 \mathrm{H}_{2} \mathrm{O}$. Unusual response above $7900 \mathrm{eV}$ is reproducible. 
blank page

$$
3
$$




\section{3-150R2 -- NOVEL APPLICATIONS OF UNDULATOR RADIATION: HOLOGRAPHY, INTENSITY INTERFEROMETRY, AND SPECKLE}

Associate Laboratory Director Area:

Principal Investigators:

Funding Profile:
Advanced Photon Source

Ian McNulty, Barry Lai, Wenbing Yun, and Efim Gluskin, Experimental Facilities Division

FY $1993 \$ 145.3 \mathrm{~K}$

FY $1994 \$ 81.5 \mathrm{~K}$

FY $1995 \$ 130.3 \mathrm{~K}$

FY $1996-0-$

FY $1997-0$ -

Purpose: The main thrust of the program was to develop novel applications that exploit the unprecedented brilliance of the 0.1 to $1-\mathrm{nm} \mathrm{x}$-ray beams that will be produced by undulator sources at third-generation synchrotron facilities currently under construction (e.g., APS, ESRF, and SPring8). The three applications, $x$-ray holography, interferometry, and speckle methods, were determined to be of particular significance and having high long-term potential. These techniques offer substantial advantages over conventional, non-brilliance-dependent x-ray techniques currently in use and are likely to impact substantially on the future direction of applications of high brilliance $\mathrm{x}$-ray beams.

Approach: Three techniques were selected for this project. X-ray holography enables imaging of microstructures in three dimensions at higher spatial resolution than is possible using visible light, with less radiation damage than using electrons. X-ray intensity interferometry is promising as a coherence and source diagnostic technique that is capable of greater angular resolution with much less sensitivity to phase instabilities than amplitude interferometry. X-ray speckle methods are useful for characterizing disordered states, inhomogeneities, and fluctuations in matter at submicron down to atomic length scales. To take full advantage of these novel applications of undulator radiation, it is essential to study their feasibility, develop the necessary $\mathrm{x}$-ray optics, and conduct proof-of-principle experiments.

Technical Progress and Results: This R\&D program has made significant experimental and theoretical achievements in FY 1995 in these three areas. Zhonghou Cai, Yiping Feng, Zhongde $\mathrm{Xu}$, and Lisheng Yang of the Advanced Photon Source Experimental Facilities Division, and Kegang Huang of the ANL Materials Science Division, contributed substantially to this program.

1. X-ray holography - Progress toward high resolution 3D x-ray holography and imaging in FY 1995 included a soft $x$-ray scanning microtomography experiment using actual biological specimens, further development of the x-ray holographic/scanning microscope, and a hard x-ray imaging microscope experiment using a resolution test object.

The scanning microtomography experiment was conducted at the X1A beamline at the National Synchrotron Light Source ${ }^{1}$ in collaboration with Dr. Waleed Haddad, Dr. Heung-Rae Lee, and Dr. James Trebes of Lawrence Livermore National Laboratory, Dr. Andrej Zalenski of the University of California at Davis, and Dr. Erik Anderson of Lawrence Berkeley Laboratory. 
A series of scanned projections were taken of fixed, gold-labeled human sperm cells, both dried and re-hydrated, over a $\pm 60^{\circ}$ angular range (fig. 1). The imaging resolution was about $100 \mathrm{~nm}$. The specimens were supported on silicon nitride membranes and surrounded by an opaque gold order-sorting aperture to minimize the background signal from unwanted zone plate diffraction orders. The apparatus was similar to that which we used previously to perform scanning soft $x$-ray microtomography of a microfabricated $3 \mathrm{D}$ test object. ${ }^{2}$ As in that work, we will apply an algebraic reconstruction technique (ART) code to reconstruct the projection data. In addition, we have had preliminary success with reconstructions performed using a new quantized iterative method developed by Dr. Lee. Both reconstruction algorithms show good results with limited sets of tomographic data, which is crucial for low radiation dose microtomography. ${ }^{3}$

We also conducted a high resolution hard $\mathrm{x}$-ray direct imaging microscope experiment in which a zone plate was used as an $\mathrm{x}$-ray lens to project a magnified image of a test pattern onto a CCD camera (fig. 2). ${ }^{4}$ The x-ray energy was $8 \mathrm{keV}$. The test object consisted of a microfabricated spoked pattern made of gold with 0.3 - to $1.0-\mathrm{mm}$-wide and 2 -mm-thick features When combined with a precision sample rotation stage and numerical reconstruction using tomographic methods, this approach is similarly capable of very high resolution $3 \mathrm{D}$ imaging.' In addition to serving as a control experiment in which to test the same reconstruction codes that we will use to reconstruct data obtained by 3D x-ray holography and scanning tomography, this technique is. well suited to $\mathrm{x}$-ray sources with limited coherent power.

Development of the $\mathrm{x}$-ray holographic/scanning microscope included design and procurement of the precision multiaxis zone plate and sample stages, analysis of the effect of the beamline optics on the microscope performance, and quantitative calibration of the CCD camera system with hard $\mathrm{x}$-rays. ${ }^{5}$

2. Intensity Interferometry - Our main goal for FY 1995 was demonstration of the intensity interferometer using partially coherent $0.3-0.5 \mathrm{keV}$. undulator radiation. The X13A undulator beamline at the National Synchrotron Light Source was completed on schedule in FY94, providing an ideal opportunity to test the interferometer with a high-brightness second-generation undulator source. ${ }^{6}$ The completed instrument ${ }^{7}$ was tested through FY 1995. The interferometer optics proved to be straightforward to align to the $\mathrm{X}$-ray beam at X13A, and fast oscilloscope and autocorrelation measurements of the detectors and correlator electronics showed the interferometer time resolution was better than $1 \mathrm{~ns}$. We also developed a new profilometer to measure the undulator beam shape and intensity. Using the interferometer, correlation data were taken under various source-size conditions. The results are presently being analyzed. ${ }^{8}$

In FY 1995 we developed a new theory to describe the influence of the undulator source asymmetry $^{9}$ and pulsed synchrotron radiation ${ }^{10}$ on the signal-to-noise ratio of the intensity interferometer: The new models not only account for these effects on coherence measurements made with interferometer, it also has direct impact on other time-correlation experiments with high brightness undulator beams, especially those utilizing coherent $\mathrm{x}$-rays. The effort to model as well as characterize the coherence properties of beams produced by high brightness $\mathrm{x}$-ray undulators and free-electron lasers is timely in view of rapidly growing interest in these properties for coherence-dependent applications. 
3. X-ray Speckle - In FY 1995 we proceeded with detailed analysis of speckle data obtained in FY 1994 using a copolymer sample, ${ }^{11}$ and conducted further experiments on this and other samples. Polystyrene and polymethylmethacrylate cololymers, which form micron-size surface islands and holes upon annealing, are model systems for studying $\mathrm{x}$-ray speckle. Timedependent experimental data obtained with coherent $6 \mathrm{keV} \mathrm{x}$-rays indicate that evolution of the size and distribution of these features depends critically on the annealing time and temperature in a dynamic as well as static fashion.

By contrast, bimetallic alloys can exhibit interesting fluctuations near order-disorder phase transitions. Additional progress with x-ray speckle methods in FY 1995 included participation in a experiment at the European Synchrotron Radiation Facility in Grenoble, in which static and time-varying speckle data were obtained with a single crystal of $\mathrm{Fe}_{3} \mathrm{Al} .{ }^{12} \mathrm{~A}$ series of 10 -second exposures acquired over the course of several hours as the sample was heated through a secondorder phase transition demonstrated the existence of correlated fluctuations at near-atomic lengthscales. These experiments constitute some of the first time-resolved results obtained via speckle methods. They clearly point the way to time-correlation experiments using high brilliance undulator sources.

Specific Accomplishments: Effort will continue under the auspices of DOE-ER.

1. W.S. Haddad, J.E. Trebes, D.M. Goodman, H.-R. Lee, I. McNulty, E.H. Anderson, and A.O. Zalensky, "Ultrahigh-resolution soft x-ray tomography," SPIE Proc. 2516, 102 (1995).

2. I. McNulty, W.S. Haddad, J.E. Trebes, and E.H. Anderson, "Soft x-ray scanning microtomography with submicrometer resolution," Rev. Sci. Instrum. 66, 1431 (1995).

3. W.S. Haddad, I. McNulty, J.E. Trebes, E.H. Anderson, R.A. Levesquc, and L. Yang, "Ultrahigh-resolution x-ray tomography," Science 266, 1213 (1994).

4. B. Lai, W. Yun, Y. Xiao, L. Yang, D. Legnini, Z. Cai, A. Krasnoperova, F. Cerrina, E. DiFabrizio, L. Grella, and M. Gentili, "Development of a hard x-ray imaging microscope," Rev. Sci. In.strum. 66, 2.2.87 (1995)

5. I. McNulty, Y.P. Feng, W.S. Haddad, and J.E. Trebes, "Tomographic scanning microscope for 1-4 keV x-rays", SPIE Proc. 2516, 217 (1995).

6. Z. Xu, I. McNulty, K.J. Randall, L. Yang, E. Gluskin, E.D. Johnson, and T. Oversluizen, "X13A: A versatile soft x-ray undulator beamline," Rev. Sci. Instrum. 66, 1827 (1995).

7. L. Yang, I. McNulty, and E. Gluskin, "An intensity interferometer for soft x-rays," Rev. Sci. Instrum. 66, 2281 (1995).

8. I. McNulty, Y.P. Feng, E. Gluskin, L. Yang, Z. Xu, K.J. Randall, and E. Johnson, "An experiment to measure the Hanbury Brown and Twiss effect with $\mathrm{X}$-rays," in preparation. 
9. - Y.P. Feng, I. McNulty, Z. Xu, and E. Gluskin, "Signal-to-noise ratio of intensity interferometry experiments with highly asymmetric $\mathrm{x}$-ray sources," submitted to $J$. Opt. Soc. $B$.

10. Y.P. Feng, I. McNulty, and E. Gluskin, "Correlation signals of x-ray intensity interferometry experiments with pulsed synchrotron sources, " in preparation.

11. Z.H. Cai, B. Lai, W.B. Yun, I. McNulty, K.G. Huang, and T.P. Russell, "Observation of X-ray Speckle by Coherent Scattering at Grazing Incidence," Phys. Rev. Lett. 73, 82 (1994).

12. G. Grubel, D. Abernathy, G.B. Stevenson, S. Brauer, I. McNulty, S.G.J. Mochrie, B. McClain, A. Sandy, M. Sutton, E. DuFresne, I.K. Robinson, R. Fleming, R. Pindak, and S. Dierker, "Intensity Fluctuation Spectroscopy Using Coherent X-rays," ESRF Newsletter 23, 14 (1995). 

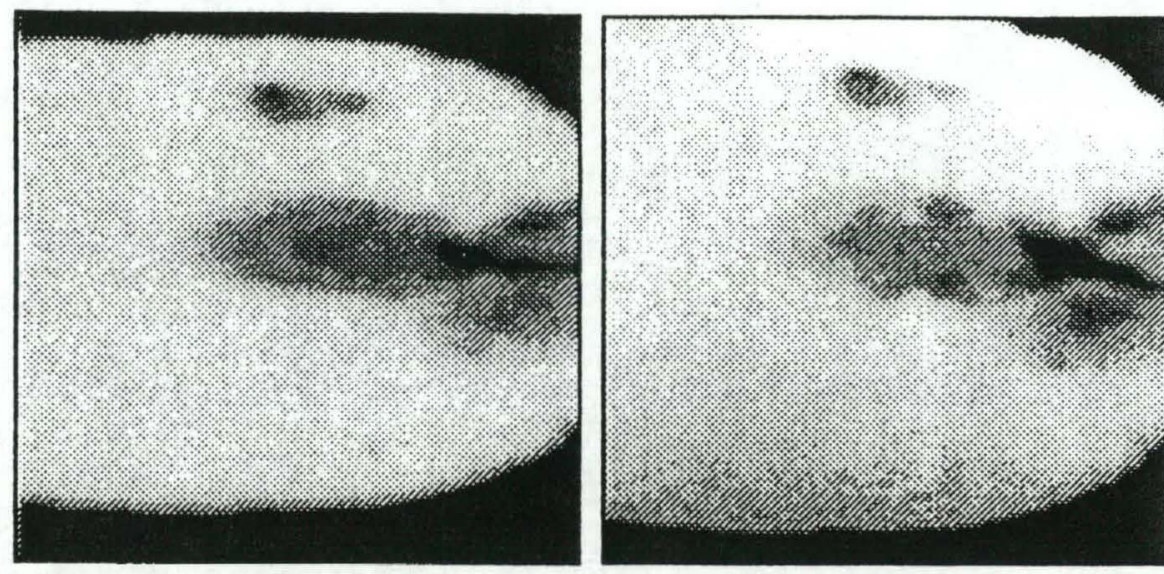

ํㅗㅇ
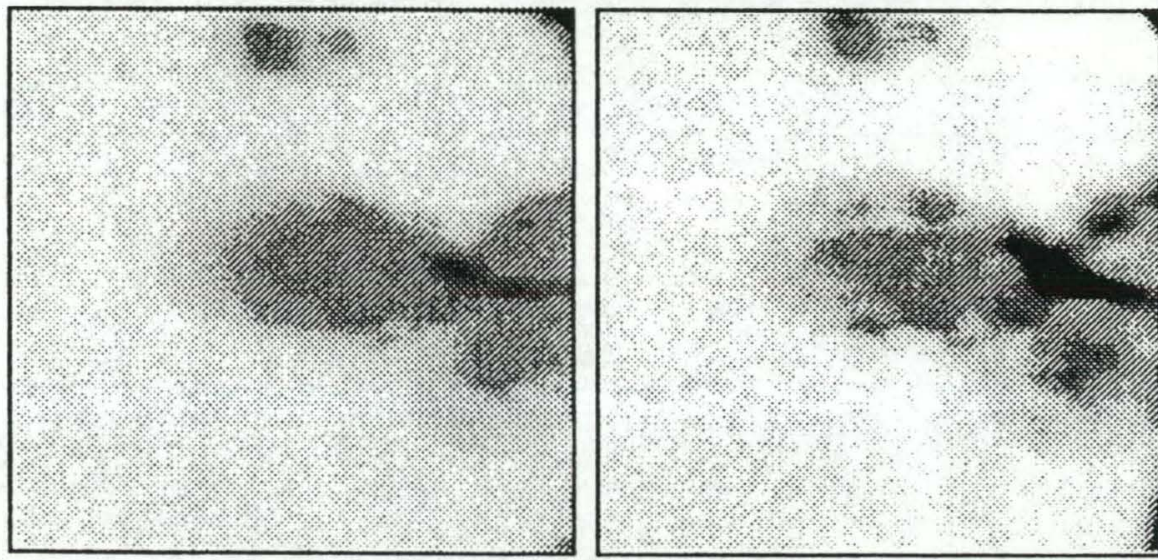

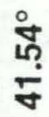
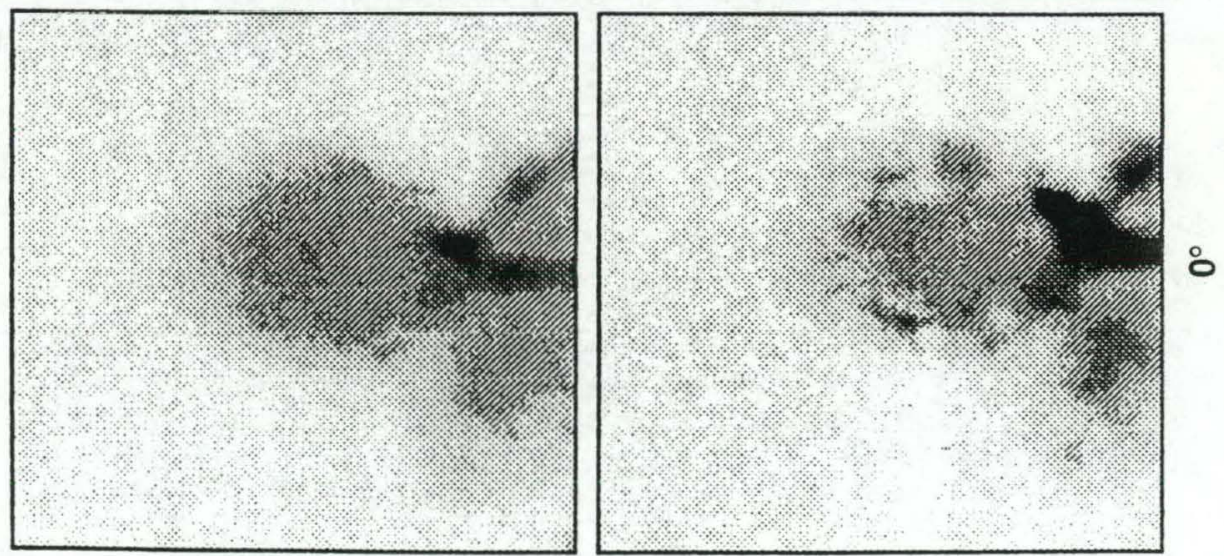

(a)

(b)

Fig. 1. Three tomographic projections through a gold-labeled human sperm cell, as recorded with $2.5 \mathrm{~nm} x$-rays in the scanning tomographic $\mathrm{x}$-ray microscope. (a) Projections taken with dry sample. (b) projections taken through the same sample, after re-hydration. 


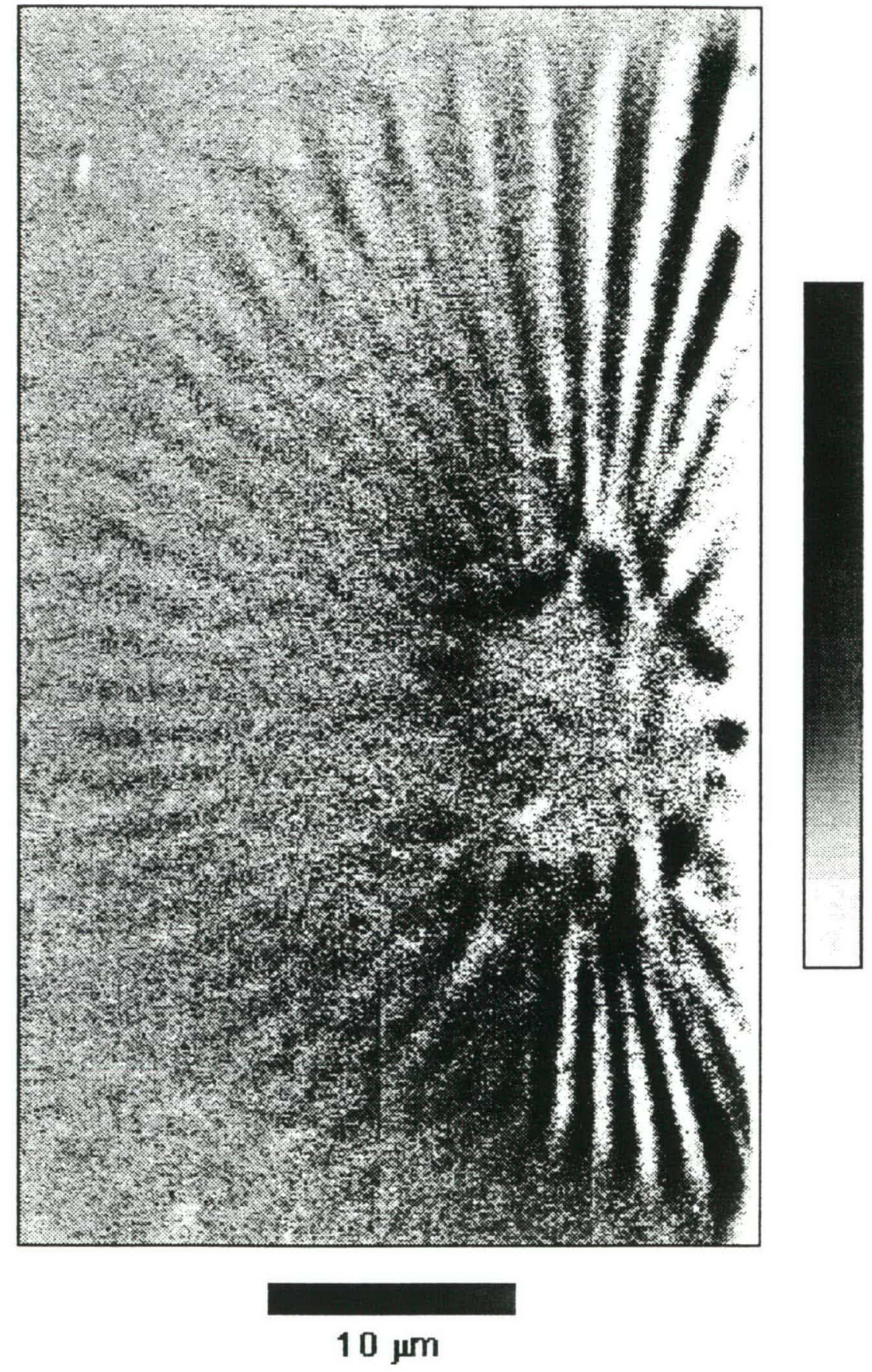

Fig. 2. Image of high resolution microfabricated gold "spoke" test pattern, obtained by imaging microscope using $8 \mathrm{keV} x$-rays and high resolution zone plate lens. The finest spokes resolved near the center of the pattern are $0.3 \mathrm{~mm}$ wide. 


\title{
95-003N -- HIGH POWER X-RAY INDUCED MODIFICATION OF MATERIALS
}

\author{
Associate Laboratory Director Area: $\quad$ Advanced Photon Source \\ Principal Investigators: $\quad$ R. Rosenberg, J. Noonan, \\ G. Goeppner, and W. Farrell \\ Accelerator Systems Division \\ Funding Profile: \\ FY $1993-0-$
FY $1994-0-$
FY $1995 \$ 43.3 \mathrm{~K}$
FY $1996 \$ 100.0 \mathrm{~K}$
FY $1997 \$ 130.0 \mathrm{~K}$
}

Purpose: The APS will produce unprecedented power densities of hard X-rays. Interaction of these X-rays with materials poses power management challenges for accelerator and beamline engineers, but at the same time may open up new materials' processing options. In the accelerator this power will be handled by specially engineered photon absorbers and in the beamlines by optical and front end components made from copper alloys, silicon, beryllium, and other materials. The effect of high powered laser and electron irradiation on the mechanical properties of these materials has been previously examined, to some extent. However, there has been no serious effort devoted towards understanding the effects of high powered X-rays on the materials' properties. It is our intent to gain insight into the mechanisms by which the $\mathrm{X}$-rays interact with such materials and thereby establish a basis for predicting their long term stability. While the high power X-rays may cause undesired effects to accelerator components they may also find beneficial use as novel materials processing options. Two applications which we wish to pursue, and that have piqued the interest of industrial concerns, are specialized welding, and surface modification of metals by the formation of metallic glasses. The unique penetration and atomic specificity capabilities of X-rays may lead to new approaches for such transformations. We propose to study the feasibility of using high power X-rays for both welding and inducing metallic glass formation. In order to form a basis for understanding our results we will also perform fundamental studies aimed at understanding the interface formation in metal-matrix composites (MMCs) using both conventional and synchrotron radiation based electron microscopies. The X-ray work will be performed on an appropriate SRI CAT beamline, while the characterization studies will be performed in the laboratory, at SSRL, ALS, and APS.

\section{Approach:}

Welding. The inherent potential of the high energy photon beam for materials processing resides in its ability to penetrate deeply into the light alloys at a selected power density. Electron beam and laser energy sources extensively used for structural welding can also penetrate deeply, but only if the incident beam is focused to a power density of approximately $10^{6} \mathrm{watts} / \mathrm{cm}^{2}$. At this very high specific power violent vaporization occurs and a small hole is formed through the thickness of the target material, giving the beam access to the full joint thickness.

Metal matrix composites (MMC) generally consist of light weight metal alloys reinforced with particulates or whiskers. Alumina and silicon carbide are among the reinforcing materials 
utilized. The MMC materials exhibit higher specific strength or stiffness, higher operating temperature and wear resistance as compared with the un-reinforced matrix alloy. Potential MMC applications include structural components of the space shuttle, fuel tank and structure for rocket launchers, high temperature aircraft structures and engines, National Aerospace plane skin and engines, high speed mechanical systems and electronic packaging. These potential applications have not been realized to date because it is necessary to join the MMC elements to produce an integrated structure, and conventional welding technology is inadequate for this purpose.

It is reasonable to expect that a high energy photon beam adjusted to a power density selected to melt the aluminum matrix but not the silicon carbide, will produce a square butt weld in which the reinforcing whiskers are present at the same density as in the base MMC, in the weld metal. This should produce a welded joint with properties comparable to those of the parent MMC.

Irradiation Induced Structural Changes. Many critical components in both the APS storage ring and beamlines will be subjected to high power densities of X-rays. Although there is an ongoing research effort into the manner in which high power electron or laser beams cause thermally induced modifications to such components, there have been no systematic studies into changes brought about by high power X-rays. Besides causing thermal transients X-rays may also induce defects by electronic means. As the absorption coefficient of the $\mathrm{X}$-rays is atom and energy dependent, it is reasonable to expect vastly different behavior from electron or laser irradiation. In order to understand the mechanisms by which X-rays induce defects and the manner in which such defects cause failure in accelerator components, it is imperative that systematic studies be undertaken on representative samples.

High power X-rays may also induce beneficial changes in materials. In particular they may cause crystalline-to-amorphous (CA) transitions in alloys, an area commanding a high degree of current research interest, particularly in Japan. ${ }^{1}$ The cause for this interest is that amorphous materials often have superior materials properties over their crystalline counterparts. ${ }^{1}$ In particular they may have high specific strength and corrosion resistance. In addition new types of amorphous magnetic materials with superior properties have recently been developed (Y. Sakurai in Ref. 1, p. 199). Normally amorphous materials are produced by rapid quenching from the liquid. However, it has been demonstrated that irradiation by both ions and electrons can produce CA transitions. It is thought that the amorphization is brought about by a process in which the simplest type of crystal defects play a role. Most of the electron-induced CA transition studies were performed using $2 \mathrm{MeV}$ electrons in a high voltage electron microscope with flux densities of approximately $1 \times 10^{18} \mathrm{e} / \mathrm{mm}^{2} \mathrm{sec}$. These flux densities are of the same order of the X-ray flux densities that will be incident on APS absorber components and transmitted by insertion device beamlines. It is therefore reasonable to expect CA transitions to occur either under uncontrolled (absorbers, beamline components, etc.) or controlled conditions. It is our hope that by performing research on well characterized alloys we can gain

Current Topics in Amorphous Materials: Physics and Technology, edited by Y. Sakurai, Y. Hamakawa, T. Matsumoto, K. Shirae, and K. Suzuki (1993, Elsevier Science Publishers, Amsterdam). 
insight into the mechanisms by which radiation induced CA transitions occur, which will enable us to predict possible failure avenues in accelerator components and new avenues for producing superior materials.

Technical Progress and Results: In the first year of the project, progress was made in two areas. First of all we found that the feasibility of using high power X-rays for welding has been demonstrated previously. This work was performed by Prof. Daryl Crozier of Simon Frazer University who utilized the wiggler radiation from beamline 10 at SSRL to join two silica tubes together. We initiated a collaboration with Prof. Crozier who was agreeable to working with us both at SSRL and at APS. Further feasibility studies were thwarted by the inability to obtain beamtime at SSRL, even though proposals were highly rated. Significant progress was made in the area of materials and interfacial studies of MMCs. Such knowledge is critical if we hope to be able to analyze the perturbations caused by high power X-ray interactions in these materials. Collaborations were formed with Prof. Brian Tonner, University of Wisconsin (photoelectron microscopy), Prof. Piero Pianetta (photoelectron microscopy), Stanford, and Prof. Jian Ku Shang (MMC characterization), University of Illinois. As a result of the efforts of Prof. Tonner's group, we obtained the first photoelectron micrographs of MMCs (see figure).

In addition, a post-doc, Dr. Qing Ma joined the group in July, 1995 to begin experiments on $\mathrm{x}$-ray modification of materials and photoelectron microscopy of MMCs. LDRD funds were also used to modify existing equipment to incorporate efficient transfer of samples between atmosphere and UHV. These modifications accelerate data acquisition used in the analysis of the X-ray modified materials.

\section{Specific Accomplishments:}

1. "Synchrotron Radiation Photoemission Study of Interfacial Bonding in Metal Matrix Composites," Q. Ma and R.A. Rosenberg, in preparation.

2. "Synchrotron Radiation Spectromicroscopy and Scanning Auger Microscopy Study of Interfacial Reactions in Ti/SiC Metal Matrix Composites," R.A. Rosenberg, Q. Ma, T. Dunham, and B. Tonner, in preparation. 


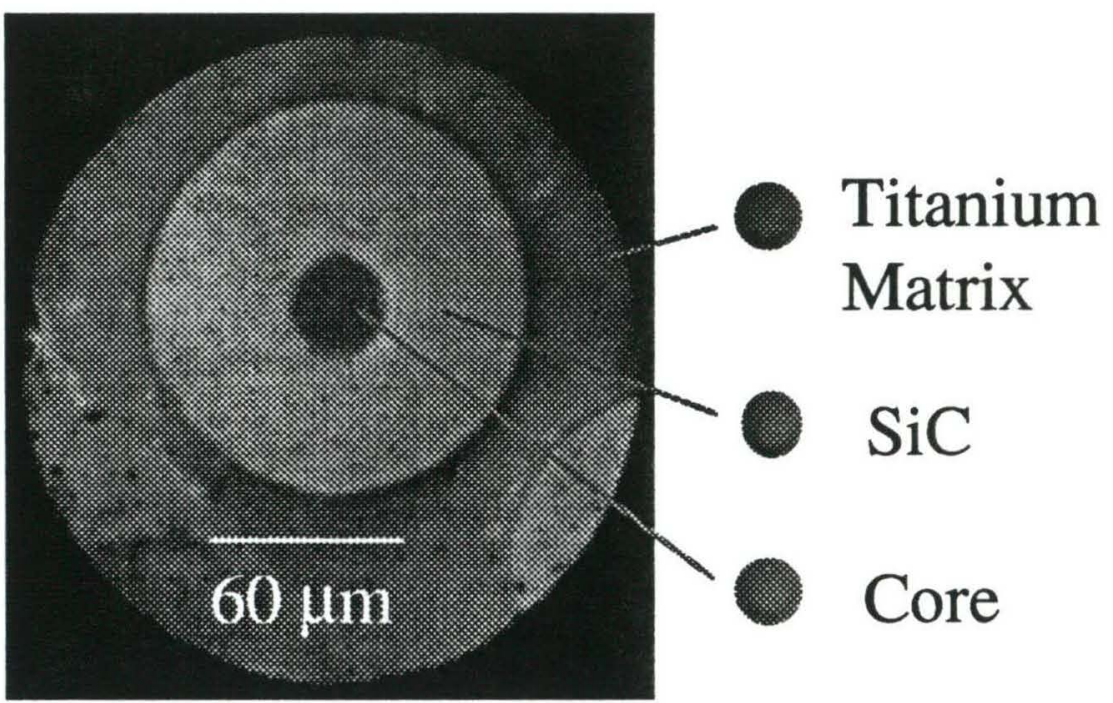




\title{
95-006N -- DEVELOPMENT OF CRYOGENICALLY-COOLED MONOCHROMATOR CRYSTALS FOR AN APS UNDULATOR-A BEAMLINE
}

\author{
Associate Laboratory Director Area: Physical Research \\ Principal Investigators: \\ G.S. Knapp and C.L. Wiley, \\ Materials Science Division \\ Funding Profile: \\ FY $1993-0-$
FY $1994-0-$
FY $1995 \$ 220.9 \mathrm{~K}$
FY $1996 \$ 220.0 \mathrm{~K}$
FY $1997-0-$
}

Purpose: To develop a monochromator system capable of handling the extremely high heat loads generated by the standard APS undulator, Undulator A. This effort is necessary for the development of the BESSRC-CAT and of the APS. Many other groups are planning to use the cryogenic thin-crystal method so this work is very important to the entire synchrotron radiation science community.

Approach: One of the most difficult design problems for a beamline developer is how to handle the extremely high heat loads generated by the standard APS undulator, undulator A. Of the many approaches have been tried, cryogenically cooled Si seems to be one of the best. Earlier designs would handle high heat loads, but not as high as APS undulator -A generates. Therefore the Basic Energy Sciences Synchrotron Radiation Center (BESSRC) is developing a new type of cryogenically-cooled monochromator system which will be able to handle the heat loads that will be generated. We are developing designs of crystals, designs of manifolds and strain free mounts and methods of handling liquid nitrogen. This work should lead to a design for a cryogenically-cooled monochromator system which employs a thin-crystal strategy that is capable of handling the central-cone power of the Advanced Photon Source's Undulator A at closed gap and the full-design current of $300 \mathrm{~mA}$.

Technical Progress and Results: We have been developed new design for a cryogenicallycooled crystal monochromator system which will employ a thin crystal that is capable of handling the central-cone power of the Advanced Photon Source's Undulator A at machine currents up to $300 \mathrm{~mA}$ which is three times the initial current of the APS. The key to the proposed design was to machine two opposing narrow channels in a large, directly-cooled block of Si crystal, leaving a thin, $\sim 0.6-\mathrm{mm}$-thick strip of Si. The thin section absorbs only a fraction of the incident-beam power so that the operating temperatures of the crystal are in the range where Si has both a very high thermal conductivity and a very small or slightly negative thermalexpansion coefficient. The fact that the crystal is operated in the negative range of thermal expansion means that the crystal surface illuminated by the $\mathrm{x}$-ray beam is always under tension and therefore will not buckle. By making the crystal thin, the power absorbed drops to 120-130 Watts from the 300 Watts of the central cone of the undulator output, producing a temperature rise in the Si strip which is small enough not to induce unacceptable strains. 


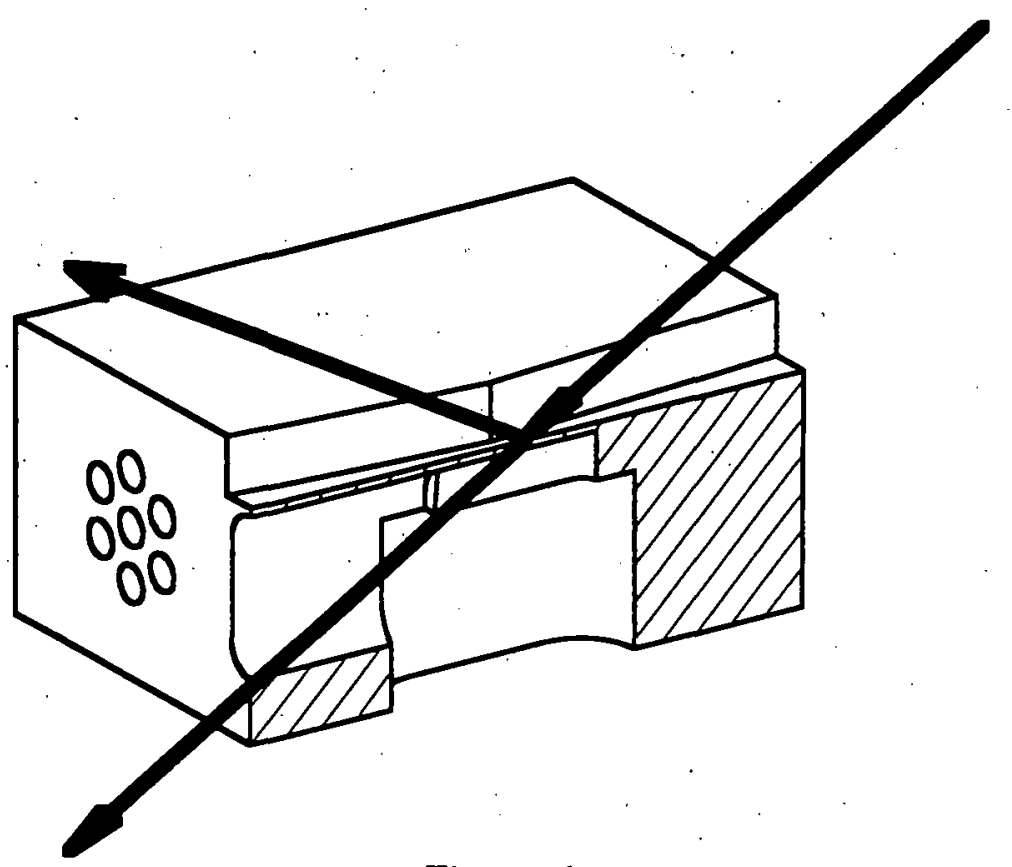

Figure 1

Figure 1 shows a half view of the first crystal of a monochromator for undulator A. Two narrow channels, each $2.4 \mathrm{~mm}$ wide, were machined into opposite sides of a Si block. The thin strip of $\mathrm{Si}$ which diffracts the incident beam is approximately $15 \mathrm{~mm}$ long and $0.6 \mathrm{~mm}$ thick. For clarity Fig. 1 shows only one half of the Si crystal block together with the incident, reflected, and transmitted x-ray beams. The crystal strip is almost strain free since it is supported by the square section in back and the small block in front. The cooling channels are machined into the Si and are coupled to invar manifolds clamped around the Si block. The seal between the invar and the Si is made with In wire seals. Much of the development of the manifolds and seals is being carried out by the staff at the APS. They have learned how to make these joints almost completely strain free. The x-rays hit the monochromator crystal near the center of the channel.

Experiments on the first models of the crystal were done at the European Synchrotron Radiation Facility (ESRF) in cooperation with the APS and SPring-8, the Japanese advanced synchrotron facility. These experiments subjected the crystal to higher powers and higher power densities than will be present at the APS, and no noticeable thermal strain was observed. The results showed that the thin crystal cryogenically-cooled monochromator will solve the high heat load problem at advanced synchrotron sources.

\section{Specific Accomplishments:}

\section{Publications:}

Solution to the High Heat Loads from Undulators at Third Generation Synchrotron Sources: Cryogenic Thin Crystal Monochromators, G.S. Knapp, M.A. Beno, C.S. Rogers, C.L. Wiley, P.L. Cowan, Review of Scientific Instruments, 65(9):2792-27.97 (September 1994). 
Dealing With Heat Loads From Undulators at Third Generation Synchrotron Sources:

The Cryogenic Thin Crystal Monochromator Solution, G.S. Knapp, C.S. Rogers, M.A. Beno, C.L. Wiley, P.L. Cowan, Review of Scientific Instruments, 66(2):2138-2140 (February 1995).

Performance of a Liquid-Nitrogen-Cooled, Thin Silicon Crystal Monochromator on a HighPower, Focused Wiggler Synchrotron Beam, C.S. Rogers, D.M. Mills, W.K. Lee, G.S. Knapp, J. Holmberg (European Synchrotron Radiation Facility, France), M. Wulff (ESRF, France), M. Rossat (ESRF, France), M. Hanfland (ESRF, France), H. Yamaoka (JAERI-RIKEN, Japan), Review of Scientific Instruments, 66 (6):3494-3499 (June 1995).

Further tests on liquid-nitrogen-cooled, thin silicon-crystal monochromators using a focused wiggler synchrotron beam, C.S. Rogers, D.M. Mills, P.B. Fernandez, G.S. Knapp, M. Wulff, M. Hanfland, M. Rossat, A. Freund, G. Marot, J. Holmberg, H. Yamaoka; submitted to Review of Scientific Instruments.

Invention Disclosure: ANL-IN-94-146 Improved Cryogenic Monochromator for High Heat Loads, G.S. Knapp and C.S. Rogers. 
blank page 


\title{
93-007R2 -- DEVELOPMENT AND CHARACTERIZATION OF TRANSITION METAL CLUSTER BEAMS FOR X-RAY ABSORPTION STUDIES
}

\author{
Associate Laboratory Director Area: Physical Research \\ Principal Investigators: \\ S.J. Riley and M.B. Knickelbein, \\ Chemistry Division \\ Funding Profile: \\ FY $1993 \$ 58.3 \mathrm{~K}$ \\ FY $1994 \$ 98.8 \mathrm{~K}$ \\ FY $1995 \$ 92.5 \mathrm{~K}$ \\ FY $1996-0$ \\ FY $1997-0-$
}

Purpose: A method for producing a high flux, nearly continuous molecular beam of transition metal clusters is needed in order to perform in-flight X-ray studies of these transient species at APS. Currently available methods of gas phase metal cluster generation employ high peak power, low-repetition rate $(<100 \mathrm{pps})$ pulsed lasers for the metal vaporization process. This project investigates the use of a special high-repetition rate laser for vaporization of transition metals as a means of quasi-continuous production of a high-density metal cluster beam.

Approach: There is slowly emerging an understanding of the relationships between metal cluster structure and chemical reactivity. Such understanding is vital to the eventual design of more efficient and selective heterogeneous catalysts. What little we now know has been derived from chemical probes of cluster structure, but these are inadequate to provide detailed information as to metal-metal coordination and the location of adsorbate binding sites. Calculations have shown that if stable, high-flux cluster beams can be produced, X-ray absorption studies at APS will be able to provide such information.

The scope of this project is to develop and refine a method of producing a stable, quasi-continuous molecular beam of metal clusters, adapting existing cluster source technology for use with a high average power, high repetition rate pulsed copper vapor laser, and to characterize the number density and size distribution of the clusters so produced.

A high repetition rate $(5 \mathrm{kHz})$ copper vapor laser has been used in conjunction with a continuous-flow cluster source to produce continuous metal cluster beams. Various transition metals have been investigated in order to gauge the generality of the method. Detection and mass analysis of the neutral cluster distribution has been accomplished via laser ionization time-of-flight mass spectrometry in an existing cluster beam apparatus.

Technical Progress and Results: The project centers around a Plasma Kinetics copper vapor laser that is on loan from another group. In the first year an existing laboratory was modified to accommodate this laser, and the laser was prepared for operation. In the second year, considerable effort and time were spent before we could get the laser to work reliably, but we were finally able to do so. Metal target vaporization tests demonstrated the production of an aluminum atom beam, but the laser's fluence (photons $/ \mathrm{cm}^{2}$ ) was too low to produce sufficient atom density for cluster formation. Therefore unstable resonator optics for the laser were 
procured and installed on the laser which greatly increased its beam quality and allowed focusing to higher fluences. With this arrangement, in the third year we successfully made cluster beams of iron, nickel, rhodium, and platinum. In addition, we demonstrated that the beams are indeed continuous, in the sense that there is no intensity modulation due to the $5 \mathrm{kHz}$ vaporization cycle. We observed no dependence of cluster signal intensity on the relative timing of the vaporization laser and the ionization laser. However, the cluster beams were not truly continuous. We found substantial shot-to-shot variations in cluster intensity, presumably due to variations between vaporization laser shots. Three observations suggest that these variations are related to the extremely high power that is concentrated in a small spot on the surface of the metal target rod: 1) Cluster signal is best (i.e., most steady) when the rod is rotated as fast (0.6 rps) as our target manipulation system allows; 2) Signal is best at the highest inert gas pressure and flow that our vacuum system can accommodate; 3) Large clusters are not seen under conditions (inert gas pressure, clustering time) that at lower vaporization rates produce extensive cluster distributions. These observations suggest that the volume of inert gas over the metal target is being perturbed in some manner so that it is less effective in cooling the vaporized metal plume. Such cooling is essential for efficient cluster growth. Presumably the very high average laser power that is deposited in a small area on the metal target surface is locally heating the surrounding gas and "blowing it away." While such a process also occurs at lower (typically $40 \mathrm{~Hz}$ ) vaporization rates, the time between vaporization pulses is sufficiently long that the inert gas can be replenished before the next pulse. This replenishment does not fully occur at a vaporization rate of $5 \mathrm{kHz}$.

Specific Accomplishments: These results were presented in invited talks at the International Workshop on Clusters and Nanostructured Materials, Puri, India, December 29, 1994-January 4, 1995; at the University of Chicago review of the Metal Cluster Chemistry program in May 1995; and at the Metal and Semiconductor Cluster Gordon Research Conference in August 1995. 


\title{
93-004R2 -- ATOMIC, MOLECULAR, AND OPTICAL PHYSICS WITH SYNCHROTRON RADIATION ${ }^{1}$
}

\author{
Associate Laboratory Director Area: Physical Research \\ Principal Investigators: \\ D.S. Gemmell, E.P. Kanter, T. LeBrun, and \\ L. Young, Physics Division \\ H.G. Berry, Notre Dame University \\ P.L. Cowan, deceased
}

Funding Profile:

FY $1993 \$ 312.0 \mathrm{~K}$

FY $1994 \$ 353.1 \mathrm{~K}$

FY $1995 \$ 257.9 \mathrm{~K}$

FY $1996-0-$

FY $1997-0-$

Purpose: Initiate research in atomic, molecular and optical (AMO) physics using synchrotron radiation. Prepare for atomic physics studies at the APS.

Approach: There are two principal components in our effort to establish a research program in AMO physics with synchrotron radiation: (1) performance of experiments at the National Synchrotron Light Source (NSLS) at Brookhaven National Laboratory, and (2) preparations to conduct atomic physics research at the Advanced Photon Source (APS) at Argonne.

(1) Work at the NSLS - We shared with staff from NIST the responsibility for operation of the X24A beamline at the NSLS. We provided half the financial support for the beamline technician (B. Karlin) and we performed several measurements at the beamline with objectives that included developing expertise and research goals for work at the APS. Our research received important theoretical guidance in this period from the presence in the Physics Division of Dr. M. Ya. Amusia as an Argonne Fellow.

(2) Preparations for the APS - The APS is expected to commence operations for research early in 1996. The Basic Energy Sciences Synchrotron Research Center (BESSRC) is likewise expected to start its research programs at that time. As members of the BESSRC CAT (Collaborative Access Team), we began planning, together with scientists from the University of Western Michigan and the University of Tennessee, a series of atomic physics experiments that will exploit the unique capabilities of the APS, especially its high brilliance for photon energies extending from about $3 \mathrm{keV}$ to more than $50 \mathrm{keV}$.

Personnel - During the past year we experienced several changes in personnel: Dr. P.L. Cowan died in August, 1994. Dr. H.G. Berry left Argonne for a post at Notre Dame University in Dec. 1994. Dr. S.H. Southworth (from NIST) joined our staff in April, 1995. In addition, two excellent postdocs (Drs. Jung and Krässig) were appointed early in FY 1995.

\footnotetext{
${ }^{1}$ Project title in FY93 and FY94: Absorption, Fluorescence and Coincidence Experiments Using Synchrotron Radiation
} 


\section{Technical Progress and Results:}

(1) At the NSLS - Several measurements (including some in collaboration with NIST) were completed at the X24A beamline over the past year. They included the following:

Non-Dipolar Asymmetries of Photoelectron Angular Distributions - Current understanding of atomic photoionization phenomena is largely based on the dipole approximation of the photon/atom interaction. However, non-dipolar interactions have been predicted to strongly modify photoelectron angular distributions due to interference between dipolar and non-dipolar amplitudes. These effects have generally been ignored in experimental studies. We measured the non-dipolar contributions to the $\operatorname{Ar} 1 \mathrm{~s}, \mathrm{Kr} 2 \mathrm{~s}$, and $\mathrm{Kr} 2 \mathrm{p}$ photoelectron angular distributions using $2-5 \mathrm{keV}$ polarized $\mathrm{x}$-rays. The non-dipolar interaction results in a forward/backward asymmetry with respect to the photon beam and this was measured by rotating an electron spectrometer about the polarization vector with a fixed polar angle of $54.7^{\circ}$, the "magic angle" which eliminates the angular dependence on the dipolar asymmetry parameter $\beta$. The nondipolar asymmetries for different atomic subshells vary with energy in different ways; at certain energies the photoelectron intensity is enhanced in the backward direction, while at other energies the asymmetry vanishes or is enhanced in the forward direction. In all cases, the measured asymmetries are in good agreement with recent non-relativistic calculations which include interference between the electric-dipole and electric-quadrupole photoionization amplitudes. Future experiments at APS beamlines will investigate photoelectron angular distributions at higher energies in heavier atoms where relativistic effects are also expected to play a significant role.

Auger Resonant Raman Spectroscopy - Photoionization of an atomic inner-shell electron produces a highly-excited vacancy. state which can relax by the emission of an Auger electron. At excitation energies far above threshold, the Auger-electron spectrum displays peaks at characteristic kinetic energies determined by the energies of the initial (one-hole) and final (twohole) states involved. However, when the excitation energy is tuned near and below threshold, the inner-shell electron is excited to bound states or low-energy continuum states for which the Auger decay process cannot be described using a two-step model. Instead, the energies and lineshapes depend on the precise energy and bandwidth of the incident photons, and the excitation and decay must be treated as a one-step, resonant inelastic-scattering process. In this case, the Auger spectrum displays resonant peaks with positive-linear dispersion and lineshapes which correspond to resonant Auger Raman scattering. The continuum peak displays negative dispersion and asymmetric broadening characteristic of post-collision interaction with the photoelectron. We have recorded such spectra at both the $\mathrm{Ar} \mathrm{K}$ and $\mathrm{Xe} \mathrm{L}_{3}$ edges. The $\mathrm{Ar} \mathrm{KLL}$ Auger resonant Raman spectrum, for example, clearly reveals features due to bound and continuum excitations of the $\mathrm{K}$ shell which can only be inferred from the $\mathrm{x}$-ray absorption spectrum.

Electron/X-Ray Coincidence Studies of Inner-Shell Processes - A new coincidence technique has been used to disentangle the complex processes involved in the decay of the 1s vacancy in $\mathrm{Ar}$ following x-ray absorption. After excitation of the 1 s electron to a bound or continuum state the atom relaxes rapidly by Auger-electron emission or $\mathrm{x}$-ray fluorescence. Most of these decay processes lead to unstable states which decay further, creating a cascade of decay processes and an array of final charge states, $\mathrm{Ar}^{1+}$ to $\mathrm{Ar}^{8+}$. The new technique uses coincident detection of 
$\mathrm{x}$-rays and electrons to select decay pathways that involve emission of both an $\mathrm{x}$-ray photon and electrons. The kinetic energy spectra of photoelectrons and Auger electrons produced in coincidence with fluorescent $\mathrm{X}$-rays are selectively recorded. In a simple demonstration of this technique, we recorded the Ar 1s photoelectron spectrum in coincidence with $\mathrm{X}$-ray fluorescence to eliminate the asymmetric broadening and shifting of the energy distribution due to postcollision interaction (PCI) with $\mathrm{K}$-Auger electrons. We have also applied this technique to the complex problem of understanding the L-Auger electron spectra produced by vacancy cascades. The Auger-cascade spectra recorded in coincidence with $\mathrm{x}$-ray fluorescence are relatively simple to interpret and have given information on inner-shell processes which have not previously been observed. These effects include the observation of resonant L-Auger spectra produced via initial resonant excitation of the K-shell and PCI between 1s photoelectrons and L-Auger vacancycascade electrons.

X-Ray Resonance Raman Scattering - At incident photon energies far above threshold, photoionization of an atomic inner-shell electron leads to an $x$-ray fluorescence spectrum having characteristic energies, intensities, and line shapes. The characteristic fluorescence spectrum is well-described by the energies and lifetime widths of the inner-shell and final hole states within a two-step model. However, when the photon energy is lowered to the region near threshold and subthreshold resonances, the energies, intensities, and line shapes of the emitted x-ray spectrum are modified and are sensitive to the precise energy and bandwidth of the incident $x$ rays. In this case, the process is described as a one-step, resonant inelastic scattering process known as resonance Raman scattering (RRS). The inner-shell-excited states participate as intermediate states in the RRS process, and the spectra display line width narrowing, asymmetric peak shapes, and dispersion. We have recorded comprehensive data sets across the $\mathrm{Xe} \mathrm{L}_{3}$ edge which demonstrate the evolution of RRS to characteristic fluorescence, and the measured RRS features are in good qualitative agreement with theory. In the $\mathrm{Xe} \mathrm{L}_{3}$ study, both bound and continuum intermediate states contribute to the RRS. Similar measurements were made at the $\mathrm{Ag} \mathrm{L}_{3}$ edge of solid $\mathrm{Ag}$ to study the case of continuum-only intermediate states.

(2) Preparations for the APS - The Basic Energy Sciences Synchrotron Research Center (BESSRC) has been allocated two out of the 34 sectors available for research at the APS. Each sector contains a bending-magnet source and an insertion-device source. Initially three beamlines will be constructed: a bending magnet line (expected to be ready for use in January, 1996), an undulator line (March, 1996), and an elliptical-motion wiggler line (June, 1996). Much of the atomic physics research conducted at the APS will use these beamlines. Indeed, a large hutch designed primarily for atomic physics and capable of containing sizable items such as a small accelerator and/or various lasers, is part of the planned installation at the undulator beamline. Our group has undertaken responsibilities in such areas as hutch design, interfacing and equipment, sagittal focussing in the cryogenically cooled monochromator systems, evaluation of undulator performance, etc.

The initial experimental program, being planned in collaboration with atomic physicists from the University of Western Michigan, the University of Tennessee, and the University of Notre Dame, is beginning to take shape. We expect to exploit the high $\mathrm{x}$-ray energies and intensities to measure photoattenuation and photoionization cross sections in the rare gases in the $1-100$ $\mathrm{keV}$ energy range. Then, as the beamlines and associated apparatus come into operation, we plan to refine these measurements and to conduct research on the basic physics of photon/atom 
interactions in the hard $\mathrm{x}$-ray regime where currently few data exist and where the theory remains largely untested.

Specific Accomplishments: Effort will continue under DOE-ER auspices. The following publications resulted from this project in FY1995:

1. Attenuation of Photons at $3-\cdot 14-\mathrm{keV}$ Energies in Helium, Y. Azuma, H:G. Berry, D.S. Gemmell, J. Suleiman, M. Westerlind, I.A. Sellin, J.C. Woicik, and J.P. Kirkland, Phys. Rev. A 51, 447 (1995).

2. Evolution of X-Ray Resonance Raman Scattering into X-Ray Fluorescence from the Excitation Of Xenon Near the $\mathrm{L}_{3}$ Edge, M.A. MacDonald, S.H. Southworth, J.C: Levin, A. Henins, R.D. Deslattes, T. LeBrun, Y. Azuma, P.L. Cowan, and B.A. Karlin, Phys. Rev. $A$ 51, 3598 (1995).

3. Solution to the High Heat Loads from Undulators at Third Generation Synchrotron Sources: Cryogenic Thin-Crystal Monochromators, G.S. Knapp, M.A. Beno, C.S. Rogers, C.L. Wiley, and P.L. Cowan, Rev. Sci. Instrum. 65, 2792 (1994).

4. Electron-Ion-X-Ray Spectrometer System, S.H. Southworth, M.A. MacDonald, T. LeBrun; and R.D. Deslattes, Nucl. Instrum. Methods Phys. Res. A 347, 499 (1994).

5. Electron Spectroscopy Studies of Argon K-shell Excitation and Vacancy Cascades, S.H. Southworth, M.A. MacDonald, T. LeBrun, Y. Azuma, and J.W. Cooper, Proceedings of the Workshop on Atomic Physics at High Brilliance Synchrotron Sources (Argonne National Laboratory Report ANL/APS/TM-14, 1994), p. 205.

6. Non-dipolar Asymmetries of Photoelectron Angular Distributions, B. Krässig, M. Jung, D.S. Gemmell, E.P. Kanter, T. LeBrun, S.H. Southworth, and L. Young, Phys. Rev. Lett. (accepted for publication). 


\section{4-046R1 -- SODIUM IMAGING SYSTEM, PHASE II}

Associate Laboratory Director Area: Engineering Research

Principal Investigators:

S.A. Brown-VanHoozer, R.W. King, and H.P. Planchon, Engineering Division

Funding Profile:

FY $1993-0-$

FY $1994 \$ 89.7 \mathrm{~K}$

FY $1995 \$ 152.3 \mathrm{~K}$

FY $1996-0-$

FY $1997-0-$

Purpose: Liquid metals are known to be transparent to ultra-violet (UV) light under ideal circumstances. ${ }^{1}$ The purpose of this research is to determine the feasibility of using UV lasers and optical technology in liquid sodium to detect equipment damage, and to determine the position and movement of equipment submerged in the liquid sodium. The central question is: "Can we use the transparency of sodium to UV to 'see through the sodium' under reactor type conditions?"

Approach: The approach for the project was as follows:

1. Through literature search and fundamental analysis determine range of liquid metals transparency and relate this to practical applications.

2. Develop a bench test for testing the basic concepts: (i) proof of transparency at the optical wavelength determined earlier, and (ii) simulation test with a sample with crack and flaws, immersed in liquid sodium. This will determine the validity of the technology concept of overall imaging through liquid sodium and determination of flaws, cracks, etc., in particular.

3. Develop a prototype laser, ultrasonic imaging detector system from the results gathered from the bench test and mathematical model. The laser ultrasonic technique will use short, energetic laser pulses to generate ultrasound in a material and use laser-based optical techniques to detect ultrasonic displacements at the surface of the material.

4. Investigate the potential for transmitting the signals detected by the prototype device to a three dimensional or virtual reality (VR) image of the primary tank and internal equipment. A VR and 3D image of the EBR-II reactor and fuel handling equipment has been generated.

Technical Progress and Results: In Phase I a literature search and initial feasibility calculations were completed. The evaluations were based on a theoretical free electron model as initially developed by Drude, Zener and Kronig. The theoretical conclusions were that transmission of ultra-violet light at wavelengths below $0.21 \mu \mathrm{m}$ in liquid sodium is feasible.

${ }^{1}$ M. Born and E. Wolf, Principles of Optics, Pergamon Press, New York (1959). 
Phase II focused on experimental verification and determining the possibility of detecting cracks in steel structures submerged in sodium. The initial planning for the sodium imaging system bench tests was initiated. Informational exchange meetings with experts from NIST, John Hopkins, and EG\&G were held.

Parallel research (alkali metal laser doppler velocimetry) was being conducted by Dr. Jon McWhirter. The aim of this parallel research was to develop methods of measuring turbulent velocity in liquid metals using an ultra-violet laser. McWhirter's research found measurement data of high energy optical properties of the alkali metals for high frequency radiation. The graph below shows the measured skin depth for different alkali metals. The skin depth is the distance into the medium over which the intensity will be reduced by a factor of e. As shown the skin depth for ultra violet radiation increases almost an order of magnitude between wavelengths of 200 and $300 \mathrm{~nm}$. However, even at the optimum wavelength, the skin depth of $10^{3} \mathrm{~nm}(1 \mu \mathrm{m})$ is still quite small for reactor applications.

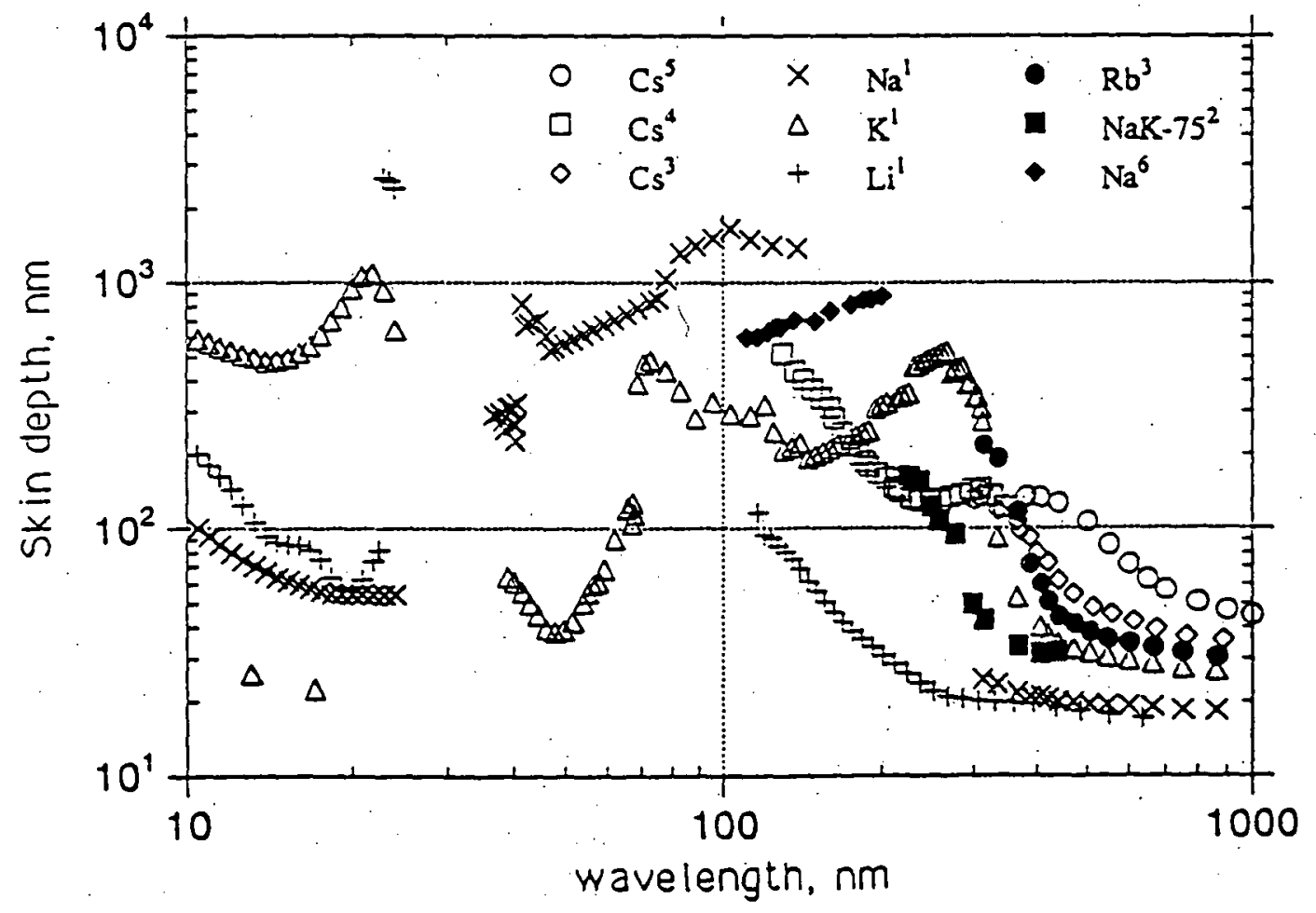

Skin depth versus wavelength for alkali metals. 
For component detection applications over meter ranges (d), the intensity reduction would be

$$
\frac{I}{I_{0}}=e-\frac{d}{\delta}=e^{-10^{6}}
$$

where $\delta=$ skin depth

For transmission over a millimeter for crack detection the intensity would be reduced by

$$
\frac{l}{I_{0}}=e^{-10^{3}}
$$

As result of this work we concluded that although the alkali metals are theoretically "transparent" in a UV wavelength range; the intensity of the radiation is exponentially reduced to very low levels in very short distances from the UV source. This makes the UV laser impractical for a velocimeter. The high attenuation also makes under-sodium viewing highly impractical. Plans for further experimental work are not recommended. 
blank page 


\section{5-040N -- OPTIMIZATION OF FUEL HANDLING AND PROCESSING AT ANL- WEST}

$\begin{array}{ll}\text { Associate Laboratory Director Area: } & \text { Engineering Research } \\ & \\ \text { Principal Investigators: } & \text { H.E. Garcia, Engineering Division } \\ & \text { A. Houshyar, Western Michigan University } \\ & \\ \text { Funding Profile: } & \text { FY } 1993-0- \\ & \text { FY } 1994-0- \\ & \text { FY } 1995 \$ 158.2 \mathrm{~K} \\ & \text { FY } 1996-0- \\ & \text { FY } 1997-0-\end{array}$

Purpose: The goal of this work was to analyze and characterize the fuel handling and processing tasks performed at ANL-W and then propose operational strategies to improve current and future operations. The expected technical results include: (i) formal description of fuel handling and processing operations performed at different facilities at ANL-W; (ii) characterization of this system both at the level of its separate components as well as at an integrated, system wide level; (iii) evaluation of the behavior of the system under current operational policies; (iv) verification whether existing resources and policies can meet the directed missions; (v) evaluation of the system under different operational scenarios; (vi) identification of means to improve current operations; and (vii) differentiation among alternate engineering solutions.

Approach: The installations at ANL-W are equipped with operational resources and facilities that include those especially suited for handling, examination, characterizing, and conditioning irradiated material. Specifically, several facilities at ANL-W are involved to complete these tasks including the Experimental Breeder Reactor (EBR-II), the Hot Fuel Examination Facility (HFEF), the Fuel Conditioning Facility (FCF), and the Radiative Scrap and Waste Storage Facility (RSWF). Some of these operations involve simple tasks that follow single operational procedures while others are more complex involving diverse resources and completed under several operational scenarios. Although there has been a certain degree of success in improving the rate of fuel handling transfer at ANL-W, there has not been a formal study suggesting on how to use the existing resources in order to improve safety, productivity and efficiency. In principle, fuel handling can be achieved without carefully analyzing this process under formal techniques such as those utilized in manufacturing engineering. However, feasible operational states and changes may be far more difficult to characterize, correctly interpret, and respond whenever executing fuel conditioning tasks. The ability to formally simulate diverse operational situations and thus identify ways to make the entire system perform more efficiently has been identified to be most helpful under actual operations or during training exercises.

The scope of the investigation encompasses several tasks that include: (i) conducting a literature review on previous and current research involving discrete event simulation; (ii) identification of the architecture, constituents, responsibilities and interactions of the fuel handling tasks at ANL-W; (iii) development of discrete event models of critical activities, resources and scenarios; (iv) development of dynamic computer models to simulate constituent functionality, interactions, 
and fuel flow paradigms under different operational scenarios; (v) identification of operational bottlenecks and engineering solutions; (vi) recommend modifications or upgrading approaches to current operational strategies and equipment to improve system performance.

To carry out this work, methods from discrete event techniques, operational research, and manufacturing engineering were employed. In particular, discrete event and operational research approaches were utilized to characterize the ANL-W capabilities for fuel handling and conditioning as a fuel processing ensemble constituted of manifold cells of limited resources intended to function together in a safe, productive, and a cost-effective manner. To analyze and characterize the fueling operations, discrete event models of several components and activities were then developed. Given these discrete models, dynamic simulation models were created to identify critical operations, allocated limited resources, and propose appropriate planning schedules for assumed scenarios. In particular, simulation models were used to reveal several undesirable system characteristics and production problems, such as bottlenecks, before they appear on actual implementations. Simulation models were also utilized to identify fuel handling and conditioning configurations to guarantee a safer, more economical, and more efficient system. Finally, manufacturing engineering techniques were used to suggest more efficient ways to execute critical fuel processing tasks.

Technical Progress and Results: The contributions of this research can be categorized into two areas, namely, development of simulation models and recommendation of engineering solutions to improve current and future fuel conditioning operations. In particular, dynamic simulation models were developed of several fuel handling and conditioning activities performed at ANL-W. Simulation was utilized for several purposes such as: (I) an explanatory device to characterize the system; (ii) an analysis vehicle to determine critical issues; (iii) a design evaluator to analyze and synthesize proposed solutions, and (iv) a predictor to forecast and aid in future planning. In brief, two major simulation models were developed utilizing acquired knowledge of relevant fuel handling activities that included inherent time constraints and policies of each stage of the process. These two models are the Cask Transfer Model and the Fuel Conditioning Model. Both of these models can run in different computer platforms including Macintosh and PC compatibles. The Cask Transfer Model models several routes for the removal of subassemblies from EBR-II. In particular, three routes for drivers and three routes for blankets were considered in this research. In modeling this fuel transfer system, a series of hierarchical blocks was developed to provide a better representation of the system. The model is complex, consisting of more than 40 hierarchical blocks at a maximum of eight layers with a total of more than 1000 blocks. It occupies about 3 megabytes and using a 90-MHZ Pentiumbased computer it takes 45 minutes to complete a three-year simulation. Several tests were completed. For instance, simulating experiments were conducted to: (i) estimate additional cask transfer and defueling time required under different routing scenarios; (ii) evaluate importance of RSWF availability; (iii) evaluate sensitivity of the system to number of fuel transfer casks available, HFEF storage capability, and FCF startup time; and (iv) observe system behavior when increasing number of subassemblies introduced in the systems. The particulars of these tests, outcomes, and conclusions can be found in the given publication list. In summary, the results implicate that (1) the "normal" transfer strategy, as defined in Ref. 4 is the preferable choice; (2) RSWF dependency on weather conditions must be minimized; (3) defueling does not significantly improve with an increase of fuel transfer casks, HFEF capacity, or EBR-II fuel delivering rates; however, it does improve with an increase of operative schedule or decrease 
of delay for opening FCF. The Fuel Conditioning Model was developed to model the operations performed at FCF for conditioning spent fuel. In particular, it employs process flows and estimated processing time for the Vertical Assembler Dismantler and aircell processes, the Element Chopper, the Electrorefiner, the Cathode Processor and the Casting Furnace. The flow of material inside of FCF is rather complex involving several processing stations, equipments and repositories. An initial model was developed that describes these conditioning processes at a basic level of detail. Initial testing showed that the chopping process is not the limiting factor but the cathode task is. In addition, studies were performed to identify the chopping requirements under different operational scenarios in order to meet current missions. Further refining of this model is in progress to qualify and quantify planned production operations at FCF. On the other hand, several engineering analysis and solutions were proposed to improve current and future fuel conditioning operations. For instance, to speed up the unloading rate, an analysis was performed to demonstrate the adequacy of passive cooling during transfer of depleted uranium subassemblies in both the FUM and IBC. To assure safety of fuel handling operations, a study on the reactivity effects to loading changes was also conducted to assure these unloading operations maintain the reactor deeply subcritical. A study was performed (currently under further investigation) to evaluate the use of laser technology in hot cells for cutting metallic structures. The idea is to use a laser beam to cut subassemblies (and irradiated metallic structures, in general) inside hot cells to increase flexibility and production rates by reducing two current operations, i.e., disassembling and chopping, into one operation. In this respect, the usage of YAG Pulse technology to devise power lasers was identified as a promising solution. With this technology, a laser beam generated outside of a hot cell is transferred inside by fiber optic which, at the frequency of the laser, becomes transparent to the beam. Finally, an analysis of the FCF waste streams and their form was performed for possible disposal of material in the reactor vessel.

\section{Specific Accomplishments:}

\section{Publications and Presentations:}

1. H.E. Garcia, A. Houshyar, and G.R. Imel, "Planning and Supervision of Reactor Defueling using Discrete Event Techniques," Proceedings of the INRIA/IEEE Conference on Emerging Technologies and Factory Automation, Paris, France, October 10-13, 1995.

2. G.R. Imel and A. Houshyar, "System Modeling of Spent Fuel Transfers at EBR-II," DOE Spent Nuclear Fuel - Challenges and Initiatives Conference, Salt Lake City, UT, Dec. 1994.

3. G.R. Imel, et al., "System Modeling to Support Accelerated Fuel Transfer Rate at EBR-II," British Nuclear Energy Society Conference on Fuel Management and Fuel Handling, Edinburgh, UK, March 1995.

4. H.E. Garcia and A. Houshyar, "Discrete Event Simulation of Fuel Transfer Strategies for Defueling a Nuclear Reactor," submitted to Intl. Journal of Computer and Industrial Engineering. 
5. A. Houshyar and G.R. Imel, "A Simulation Model of the Fuel Handling System in a Nuclear Reactor," accepted for publication in Intl. Journal of Computer and Industrial Engineering.

6. H.E. Garcia, "Analysis of Current Operational Policies and Solutions for Meeting Directed Defueling Objectives," Argonne National Laboratory Internal Technical Report, in preparation.

7. H.E. Garcia, "In Hot Cell Cutting Laser Technology for Improving Conditioning of Spent Fuel," Argonne National Laboratory Internal Technical Report, in preparation. 


\title{
95-027N -- USE OF SPENT EXPERIMENTAL EBR-II FUELS TO ADDRESS POTENTIAL MATERIALS PROBLEMS UNDER DRY STORAGE CONDITIONS
}

\author{
Associate Laboratory Director Area: Engineering Research \\ Principal Investigators: \\ Engineering Division \\ Funding Profile:

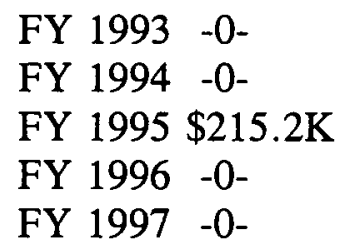

R.G. Pahl and J.D.B. Lambert,

Purpose: The purpose was to re-examine spent EBR-II fuels which had been dry stored at the Radioactive Scrap and Waste Facility (RSWF) at ANL-W. The intent was to utilize existing post-irradiation examination data as a baseline and to assess material degradation mechanisms that may have developed during the storage period (up to 25 years). Data obtained on any observed mode of degradation would be fed back to the national special nuclear fuel (SNF) program to guide the safe storage of SNF at DOE facilities.

Approach: The RSWF is one of very few facilities in the DOE complex where significant quantities of SNF have been dry stored for any length of time. Interaction with other contractor personnel in the SNF program in 1994 indicated a great need for information on the condition of such fuels because the majority of DOE fuels were in water-filled basins and dry storage was an option being considered to mitigate degradation in the basins. Accordingly, the approach was to choose from the RSWF inventory of stored elements those whose immediate post-irradiation condition had been characterized prior to prolonged storage in the RSWF; these would be retrieved and re-examined in the Hot Fuel Examination Facility (HFEF) for evidence of physical deterioration.

In March 1995 this approach was broadened to include: (a) examination of the effects of wet storage on spent EBR-II fuel (and canisters), in collaboration with Lockheed Idaho Technologies Corporation (LITCO); and (b) the use of SNF that had been dry stored in HFEF to evaluate degradation mechanisms which had been postulated for sodium-exposed fuel stored in an inerted hot cell (so called "hot cell rot"). This new approach better complemented the Laboratory's redirected program, which emphasized the treatment of DOE spent fuel.

Technical Progress and Results: The first task was to establish a comprehensive inventory of SNF in the RSWF by rationalizing existing partial (but overlapping) databases from EBR-II, HFEF and RSWF. Although for accountability purposes the fissile inventories for these facilities were available from the restricted special nuclear material (SNM) database, additional information needed for the present work, like fuel form, element design parameters, and storage configurations, were contained in the other less well maintained records. The organization of this additional information on RSWF into a spreadsheet format was largely completed. In FY96 information from this database will be subjected to QA and put into the national SNF database. 
Table 1 summarizes the container configurations, and the number and contents of the liners used to store fuel elements in the RSWF, as of August 31, 1995.

Table 1. Storage Configurations at the RSWF

\begin{tabular}{clllc}
\hline Storage Date & \multicolumn{1}{c}{ Liner Type } & Inner Container & Main Contents & Number \\
\hline $7 / 65-8 / 77$ & $\begin{array}{l}16 " \text { diam. } \\
\text { inside 24" } \\
\text { diam. }\end{array}$ & $\begin{array}{l}\text { Single (crimped) } \\
\text { "paint" can* }\end{array}$ & $\begin{array}{l}\text { EBR-II blanket } \\
\text { fuel }\end{array}$ & $104 \#$ \\
$7 / 78-11 / 89$ & $16 "$ diam. alone & $\begin{array}{l}\text { HFEF-5 double } \\
\text { can } \\
\text { (welded/bolted)** }\end{array}$ & $\begin{array}{l}\text { Experimental } \\
\text { fuels: oxides, } \\
\text { carbides, nitrides }\end{array}$ & 41 \\
$5 / 92-$ Now & $16 "$ diam. alone & $\begin{array}{l}\text { HFEF-5 double } \\
\text { can } 9 \\
\text { (welded/bolted)** }\end{array}$ & $\begin{array}{l}\text { Mark III EBR-II } \\
\text { driver fuel }\end{array}$ & 90 \\
\hline
\end{tabular}

*Loose elements with hot cell waste

**New basket holder, no co-mingled waste

\#There are three additional liners (16" in 24") that contain HFEF-5 double cans

Approximately two metric tonnes heavy metal (MTHM) of EBR-II fuel are in interim wet storage at the Idaho Chemical Processing Plant in the CPP-603 and CPP-666 basins. LITCO personnel have observed that 9 out of the 3,636 canisters containing the sodium-bonded fuel are leaking, calling into question the integrity of all the canisters. Improper seating of the compression seal is the likely cause of the leaks. Several canisters will therefore be retrieved from the basins to perform examinations in HFEF to determine if this is so, and what is the extent of element deterioration. Similar examination at ANL in 1976-77 on non-canistered EBRII subassemblies B3272 and B3274S after 350 and 540 days of water storage, respectively, showed 23 elements with visible breaches. The cause of failure was probably the high chloride content of the basin water $(\sim 780 \mathrm{ppm})$ which gave rise to stress corrosion cracking of the austenitic stainless steel cladding. In contrast, chloride levels are currently $\leq 50 \mathrm{ppm}$ in the CPP-666 basin and $\leq 200$ ppm in the CPP-603 basin.

Although examination of. RSWF-stored fuel remained a project goal, manpower limitations delayed retrieval of material from RSWF until required by the EBR-II spent fuel treatment. Work therefore began on possible in-cell degradation with re-examination of elements from subassemblies X068A(B), X071H and B7274S. The X068 and X071 elements had been stored in inerted containers in HFEF since examination in the late 60s and early 70s; the B7274S elements (retrieved from the CPP basins) were similarly stored in HFEF since examination in 1977. Comparison of the two sets of elements will help establish the relative degradation in HFEF versus that occurring in the water basins. A neutron radiography sampling plan was submitted to HFEF. Photographs of X068 elements were obtained. Handling tools for neutron radiography were designed and submitted for manufacture. Destructive examinations will continue through FY96 to establish the extent of degradation of EBR-II fuel during dry and wet 
storage. The usefulness of this work in identifying the "path forward" for Na-bonded fuel has been clearly established (see Specific Accomplishments).

Specific Accomplishments: This effort will continue under DOE-NE auspices.

- R.G. Pahl, R.S. Wisner, R.L. Parks and J.D.B. Lambert, "Spent Fuel Characterization Capabilities at the Hot Fuel Examination Facility," Proc. of the ANS, DOE Spent Nuclear Fuel--Challenges and Initiatives, 579-581 (1994).

- Participated in ANL/LITCO/WHC/DOE-EM meeting on path forward plan for sodiumbonded fuel; see memo, D.L. Porter and H.F. McFarlane to Y.I. Chang, "Summary of the John Baker Meeting on Na-Bonded SNF (7/26/95 in Idaho Falls), " DLP-95-014, August 7, 1995.

- R.G. Pahl and M.A. Ebner, "Technical Assessment of Continued Wet Storage of EBR-II Fuel." Non-refereed summary to DOE Spent Nuclear Fuel and Fissile Material Management Meeting, Reno, Nevada, June 16-20, 1996.

- Presentation at the National SNF Program QA and Data Review Meeting, Idaho Falls, October 26-27, 1995. 
blank page 


\title{
95-054N -- REMOVAL OF LITHIUM OXIDE AND LITHIUM FROM LITHIUM CHLORIDE-BASED PROCESS SALTS
}

\author{
Associate Laboratory Director Area: Engineering Research \\ Principal Investigator: \\ C.C. McPheeters, Chemical Technology Division \\ Funding Profile: \\ FY $1993-0-$ \\ FY $1994-0-$ \\ FY $1995 \$ 294.4 \mathrm{~K}$ \\ FY $1996-0-$ \\ FY $1997-0-$
}

Purpose: The proposed project is intended to develop techniques and devices for the removal of residual $\mathrm{Li}_{2} \mathrm{O}$ and lithium from reduction salts used in pyrochemical processes.

Approach: The lithium process has been developed in the Chemical Technology Division of Argonne National laboratory for the treatment of spent oxide nuclear fuel. In this process, the oxide fuel is reduced by lithium metal in a molten salt medium $(\mathrm{LiCl})$ at $650^{\circ} \mathrm{C}$. The resulting $\mathrm{Li}_{2} \mathrm{O}$ is dissolved in the process salt $(\mathrm{LiCl})$ which also contains some lithium. The salt is recycled to the reduction step after electrochemically reducing the $\mathrm{Li}_{2} \mathrm{O}$ to lithium metal and oxygen gas. The reduced metal is separated from the salt phase and transferred to an electorefiner where the uranium is separated from the fission products and transuranic elements. Some amount of entrained salt, which contains lithium and $\mathrm{Li}_{2} \mathrm{O}$, accompanies the reduced fuel to the electrorefiner. The presence of $\mathrm{Li}_{2} \mathrm{O}$ is undesirable in the electrorefining operation because it reacts with $\mathrm{UCl}_{3}$ to form $\mathrm{UO}_{2}$ which must be filtered out of the salt. In addition, it is desirable to recover and regenerate all the lithium metal in the entrained salt. Hence there is a need to remove the $\mathrm{Li}_{2} \mathrm{O}$ and lithium from the entrained process salt before the electrorefining operation. It has been postulated that the use of aluminum which reacts with the dissolved $\mathrm{Li}_{2} \mathrm{O}$ and lithium, may offer a means of eliminating or reducing the concentration of dissolved $\mathrm{Li}_{2} \mathrm{O}$ and lithium. The expected reactions are:

$$
\begin{aligned}
& \mathrm{Li}(\text { salt })+\mathrm{Al}(\mathrm{c})=\mathrm{AlLi}(\mathrm{c}) \\
& 2 \mathrm{Li}_{2} \mathrm{O}(\text { salt })+\mathrm{Al}(\mathrm{c})=\mathrm{LiAlO}_{2}(\mathrm{c})+3 \mathrm{Li}(\text { salt })
\end{aligned}
$$

In FY 1994, a preliminary experiment was performed under program support where $\mathrm{Li}_{2} \mathrm{O}$ dissolved in $\mathrm{LiCl}-\mathrm{KCl}$ salt was contacted with a mixture containing aluminum and lithium. At the end of the experiment, the salt was found to contain $0.27 \mathrm{wt} \% \mathrm{Li}_{2} \mathrm{O}$, almost a factor of six lower than that charged $(1.27 \mathrm{wt} \%)$. This LDRD study has focused on laboratory-scale experiments to determine the equilibrium phases and phase compositions following the aluminum-treatment of process salt containing $\mathrm{Li}_{2} \mathrm{O}$ and lithium. Typical experiments involve equilibration of known amounts of salt, $\mathrm{Li}_{2} \mathrm{O}$ and lithium with a measured amount of aluminum. Filtered samples were drawn from the molten salt and analyzed by Inductively Coupled Plasma Atomic Emission Spectrometry (ICP-AES) for aluminum concentration. The filtered samples were also titrated against $0.1 \mathrm{~N} \mathrm{HCl}$ acid to determine the $\mathrm{Li}_{2} \mathrm{O}$ concentration. Further, the salt at the end of the experiment was washed with water to dissolve the salt and the water-insoluble portion examined by $\mathrm{x}$-ray diffraction(XRD) to determine the equilibrium phase. 
Technical Progress and Results: In this report period, experiments were performed with both aluminum and $\mathrm{Al}_{2} \mathrm{O}_{3}$ to gain some insight into the equilibrium phases that might exist in the system $\mathrm{Li}-\mathrm{Cl}-\mathrm{O}-\mathrm{Al}$ and the results for some of these experiments are presented in Tables 1 and 2 respectively. In UR-114, the $\mathrm{Li}_{2} \mathrm{O}$ concentration decreased after the aluminum treatment, however, the reduction in $\mathrm{Li}_{2} \mathrm{O}$ concentration was similar (0.45-0.62 wt \%) even though the $\mathrm{Li}_{2} \mathrm{O}$ concentration varied from 0.66 to $3.00 \mathrm{wt} \%$. The water-washed residue was found to contain aluminum and $\mathrm{LiAlO}_{2}$ as expected from reaction (2) and in addition a hydrolyzed product which probably resulted from the water-wash. Experiment UR-120 was a variation of UR-114 in that an aluminum rod was dipped into the melt instead of using aluminum particles and low $\mathrm{Li}_{2} \mathrm{O}$ concentrations were used. The experiment was performed at a slightly lower temperature of $630^{\circ} \mathrm{C}$ to prevent melting of the aluminum rod. As the $\mathrm{Li}_{2} \mathrm{O}$ concentration is progressively increased reaction (2) should take place with the formation of $\mathrm{LiAlO}_{2}$, which, due to its low concentration, is expected to be dissolved in the salt. Within experimental uncertainty there does not appear to be any significant reduction of $\mathrm{Li}_{2} \mathrm{O}$ in this experiment, however, the slight increase in aluminum (as $\mathrm{LiAlO}_{2}$ ) in the salt phase suggests some reaction took place. An important result obtained in these experiments is that significant concentrations of soluble aluminum in $\mathrm{LiCl}$ were obtained. The concentration of aluminum in $\mathrm{LiCl}$ increased with increasing $\mathrm{Li}_{2} \mathrm{O}$ concentration, showing a strong functional dependence on the $\mathrm{Li}_{2} \mathrm{O}$ concentration in $\mathrm{LiCl}$. It appears that $\mathrm{LiAlO}_{2}$ has significant solubility in $\mathrm{LiCl}$ which was unexpected. A few additional experiments were performed with $\mathrm{Al}_{2} \mathrm{O}_{3}$ to better understand the $\mathrm{LiAlO}_{2}$ solubility.

In the experiments with $\mathrm{Al}_{2} \mathrm{O}_{3}$, summarized in Table 2 , the important reactions are considered to be:

$$
\begin{aligned}
& \mathrm{Li} 2 \mathrm{O} \text { (salt) }+\mathrm{Al}_{2} \mathrm{O}_{3}(\mathrm{c})=2 \mathrm{LiAlO}_{2} \text { (salt) } \\
& \mathrm{LiAlO}_{2} \text { (salt) }=\mathrm{LiAlO}_{2} \text { (c) }
\end{aligned}
$$

It is clear that the equilibrium concentration of aluminum (i.e., aluminum-bearing species) is significant and the formation of $\mathrm{LiAlO}_{2}$ (salt), which is assumed to be the soluble species, is an important consideration in this study. In the experiments with $\mathrm{Al}_{2} \mathrm{O}_{3}$, again, the measured concentration of aluminum in the salt is strongly dependent on the $\mathrm{Li}_{2} \mathrm{O}$ concentration of the salt, with a maximum aluminum concentration of $0.19 \mathrm{wt} \%$. This would be equivalent to $0.46 \mathrm{wt} \%$ $\mathrm{LiAlO}_{2}$ and is assumed to be the solubility limit of $\mathrm{LiAlO}_{2}$ in $\mathrm{LiCl}$ at $650^{\circ} \mathrm{C}$.

In conclusion, although aluminum can reduce the $\mathrm{Li}_{2} \mathrm{O}$ concentration in $\mathrm{LiCl}$ process salts, the unexpected high solubility of $\mathrm{LiAlO}_{2}$ in these salts defeats the ability to remove oxygen from the system.

Specific Accomplishments: A patent had been submitted by the Chemical Technology Division (Invention Report ANL-IN-93-133) covering the use of aluminum in the removal of $\mathrm{Li}_{2} \mathrm{O}$ and lithium from $\mathrm{LiCl}$-base salt. 
Table 1. Results of Experiments with Aluminum

\begin{tabular}{|c|c|c|c|c|}
\hline Experiment & $\begin{array}{l}\text { Total Amount of } \\
\mathrm{Li}_{2} \mathrm{O} \text { added } \\
(\mathrm{wt} \%) \text { before } \\
\text { Al-treatment }\end{array}$ & $\begin{array}{c}\mathrm{Li}_{2} \mathrm{O} \\
\text { concentration in } \\
\mathrm{LiCl}(\mathrm{wt} \% \text { ) after } \\
\text { Al-treatment }\end{array}$ & $\begin{array}{l}\text { Al concentration } \\
\text { in } \mathrm{LiCl}(\mathrm{wt} \%)\end{array}$ & $\begin{array}{l}\text { Phases present in } \\
\text { water-insoluble } \\
\text { residue }\end{array}$ \\
\hline $\begin{array}{c}\text { UR- } 114 \\
(150 \mathrm{~g} \mathrm{LiCl} \\
0.15 \mathrm{~g} \mathrm{Li} \\
3.00 \mathrm{~g} \mathrm{Al} \\
\left.650^{\circ} \mathrm{C}\right)\end{array}$ & $\begin{array}{l}0.66 \\
1.32 \\
1.96 \\
3.00\end{array}$ & $\begin{array}{l}0.06 \\
0.70 \\
1.42 \\
2.55\end{array}$ & $\begin{array}{l}0.0044 \\
0.0207 \\
0.0626 \\
0.1440\end{array}$ & $\begin{array}{c}\mathrm{Al}+\mathrm{LiAlO}_{2}+ \\
\mathrm{LiAl}_{2}(\mathrm{OH})_{7} \cdot \mathrm{xH}_{2} \mathrm{O}\end{array}$ \\
\hline $\begin{array}{c}\text { UR-120 } \\
(150 \mathrm{~g} \mathrm{LiCl} \\
0.15 \mathrm{~g} \mathrm{Li} \\
\mathrm{Al} \text { rod } \\
\left.630^{\circ} \mathrm{C}\right)\end{array}$ & $\begin{array}{l}0.00 \\
0.07 \\
0.20 \\
0.33\end{array}$ & $\begin{array}{l}0.00 \\
0.10 \\
0.16 \\
0.34\end{array}$ & $\begin{array}{l}0.001 \\
0.004 \\
0.003 \\
0.006\end{array}$ & - \\
\hline
\end{tabular}


Table 2. Results of Experiments with $\mathrm{Al}_{2} \mathrm{O} 3$

\begin{tabular}{|c|c|c|c|c|}
\hline Experiment & $\begin{array}{c}\text { Total Amount of } \\
\mathrm{Li}_{2} \mathrm{O} \text { added (wt } \% \text { ) } \\
\text { before } \\
\mathrm{Al}_{2} \mathrm{O}_{3} \text { treatment }\end{array}$ & $\begin{array}{c}\mathrm{Li}_{2} \mathrm{O} \\
\text { concentration in } \\
\mathrm{LiCl}(\mathrm{wt} \%) \text { after } \\
\mathrm{Al}_{2} \mathrm{O}_{3} \text { treatment }\end{array}$ & $\begin{array}{l}\mathrm{Al} \text { concentration } \\
\text { in } \mathrm{LiCl}(\mathrm{wt} \%)\end{array}$ & $\begin{array}{c}\text { Phases present in } \\
\text { water-insoluble } \\
\text { residue }\end{array}$ \\
\hline $\begin{array}{c}\text { UR-110 } \\
(150 \mathrm{~g} \mathrm{LiCl} \\
0.6 \mathrm{~g} \mathrm{Li} \\
3.8 \mathrm{~g}-\mathrm{Al}_{2} \mathrm{O}_{3} \\
\left.650^{\circ} \mathrm{C}\right)\end{array}$ & 3.00 & 2.66 & 0.19 & $\mathrm{LiAlO}_{2}+$ \\
\hline $\begin{array}{c}\text { UR- } 111 \\
(150 \mathrm{~g} \mathrm{LiCl} \\
0.6 \mathrm{~g} \mathrm{Li} \\
3.8 \mathrm{~g} \mathrm{Al}_{2} \mathrm{O}_{3} \\
\left.650^{\circ} \mathrm{C}\right)\end{array}$ & $\begin{array}{l}1.64 \\
4.76\end{array}$ & $\begin{array}{l}1.57 \\
2.89\end{array}$ & $\begin{array}{l}0.067 \\
0.190\end{array}$ & $\begin{array}{c}\mathrm{LiAlO}_{2}+ \\
\mathrm{LiAl}_{2}(\mathrm{OH})_{7}, \mathrm{xH}_{2} \mathrm{O}\end{array}$ \\
\hline $\begin{array}{c}\text { UR-118 } \\
(150 \mathrm{~g} \mathrm{LiCl} \\
12.0 \mathrm{~g} \mathrm{Li} \\
3.8 \mathrm{~g} \mathrm{Al}_{2} \mathrm{O} 3 \\
\left.650^{\circ} \mathrm{C}\right)\end{array}$ & 0.00 & - & $\cdot$ & - \\
\hline
\end{tabular}




\title{
93-043R2 -- IMPLEMENT RELIABILITY CENTERED MAINTENANCE AND CONDITION MONITORING AT EBR-II
}

\author{
Associate Laboratory Director Area: Engineering Research \\ Principal Investigators: \\ Funding History: \\ FY $1993 \$ 248.2 \mathrm{~K}$ \\ FY $1994 \$ 225.2 \mathrm{~K}$ \\ FY $1995 \$ 223.9 \mathrm{~K}$ \\ FY $1996-0-$ \\ FY $1997-0-$
}

J.C. Braun and R.E. Nietert,

Reactor Analysis Division

H.P. Planchon, Engineering Division

Purpose: Expand the practices used in the maintenance activities at the ANL-W site to become as sophisticated as the best practices in the civilian industry today and then advance the state of the art by introducing innovative data collection, coordination and computer technology. Develop processes that can be used to expand the state of the art in maintenance practices throughout the DOE complex as well as other civilian industries.

Approach: The aviation industry has made major improvements in the areas of maintenance and condition monitoring of critical components. The nuclear industry has followed these practices slowly, and is now only beginning to implement some of the practices that are appropriate to maintain complex, expensive systems in top operating condition without performing unnecessary maintenance. The military has realized significant benefits and cost savings by implementing reliability centered maintenance (RCM) and condition monitoring techniques. It is very appropriate for a national laboratory to assume a position of leadership in the advancement of this technology.

The first phase of this study identified a number of promising areas where the application of advanced monitoring practices, such as vibration monitoring, can substantially enhance the performance of important equipment. We also studied the twelve ANL-W emergency diesel generators (EDGs). These studies examined the maintenance practices carried out at ANL-W. This involved a review of the types of equipment maintained, the procedures utilized in the effort, national consensus standards, and the identification of worthwhile improvements.

In addition to hardware improvements, we judged that major improvements at ANL-W could be made by adding, modifying, and upgrading software to automate such things as the trending of data taken from preventive maintenance inspections, procedures, procedure review, spare parts identification and ordering, and maintenance planning and scheduling.

In the second year of the project, the scope was expanded to involve not only the EBR-II facility, but also the entire ANL-W site; the software development mentioned above was also added. 
In the second phase of the study we designed a modern client/server database for maintenance management of large facilities. We then examined various software packages and selected and procured a package that was most appropriately suited to our present activities and to future plans for this project. A software development laboratory was established at ANL-E and similar equipment and software, which forms the initial building blocks of a complete site wide system, was deployed at ANL-W.

We demonstrated prototypes of some of these automation tools; specifically we demonstrated an on-line paperless procedure via wireless-networked notebook computer, and implemented preventive maintenance planning and scheduling modules for use by the work control department of the ANL-W site.

In this final phase, we advanced the development of the automation tools initiated in the second year of the project and developed a methodology for transferring the entire cadre of analytical data base tools to U.S. industry.

By using probabilistic risk analysis (PRA) techniques, we assessed the impact on overall safety and reliability resulting from maintenance improvements. We developed a technique for feeding maintenance information into the relevant PRA analyses to determine the new risks associated with the new maintenance practices. This technique permits us to calculate the risk reduction or increase that results from improved or degraded maintenance practices, and enables us to perform a benefit vs. cost analysis on various maintenance practices or changes in these practices.

Although it was our intent, we were unable to expand the original scope to develop intelligent algorithms for optimizing spare parts inventories. The amount of work involved in the earlier steps proved to be much greater than we anticipated.

Finally, the title of this study has become somewhat of a misnomer. When we originally developed this program, we imagined that we would use the ANL-W site and one of the systems from the EBR-II as a test bed for this study. The subsequent shut-down of the EBR-II motivated us to seek another plant. We formed a collegial relationship with a group of researchers from the Commonwealth Edison Company (ComEd) whose offices are located near the Argonne East site and used the Chemical Volume and Control System (CVCS) from the Braidwood Nuclear Power Station as the basis for our PRA studies.

Technical Progress and Results: The main thrust of this year's activities has been the development of analytical tools related to performance of an "economic" probabilistic risk assessment of the Braidwood Nuclear Power Station CVCS.

We developed a computerized model of the CVCS and ran it on the CAFTA computer program, which is owned by the Electric Power Research Institute (EPRI) and loaned to us, with EPRI's permission by ComEd Edison for specific use on this project. The first part of this task was to define the events which could lead to a shutdown of the power plant. Next, we performed a ranking of the top 50 to 100 components of the 300 available in the CVCS system that were most critically influenced by maintenance activities. 
Components were ranked using the Risk Achievement Worth (RAW), by assuming in the PRA that only a particular component fails and also by the Risk Residual Worth (RRW), to which we assumed that the component had a failure probability of 0 , i.e., that the component is perfect. These techniques as well as linear variations of component failure probability near the normal operating band gave us information about the relative importance of various components.

The next step included the estimation of economic impact. ComEd Edison laid the foundation in a paper entitled, "An Algorithm for the Assessment of Nuclear Power Plant Preventive Maintenance Impacts on System/Component Unavailability". This unpublished paper developed formulas for characterizing unavailability of rotating equipment, specifically pumps and diesel generators. We used some of the concepts proposed by ComEd and developed additional formulations of our own to estimate the economic impact of individual component failures, and changes in component reliability.

ComEd has developed computer software called ReCOM. Because of the extensive effort required in the PRA, we were not able to obtain the kind of detailed information that we were seeking from the preventive and corrective maintenance records from ComEd. We were, however, able to use general information produced by ReCOM to obtain differential maintenance costs with respect to component reliability. Finally, we were able to prepare curves of riskrelated costs and maintenance-related costs vs. various component parameters, most typically component reliability. Using these indicators, we were able to develop curves of total costs (risk costs + maintenance costs) as a function of a parameter such as component reliability. These curves have a minimum point, and we were then able to identify whether the extent of maintenance performed on various components was reasonable. We were able to identify some components which were being over maintained, and hence, these become candidates for maintenance program reductions. We found no components in the CVCS to which additional maintenance would reduce risks. The tendency in this particular system was toward over maintenance.

We held discussions and continued to hold discussions with ComEd regarding this information. ComEd is very interested in these results and wishes to perform some additional studies of their own using ReCOM, and perform additional comparisons with our results to evaluate the feasibility of using these techniques to change maintenance programs in an operating plant. We have been dealing with personnel from the corporate office. They wish to continue the application of this technology to all (about 40 ) of the emergency diesel generators in the ComEd system.

We believe that the next step in this development will involve an actual application of this study and our findings to maintenance programs on the Braidwood CVCS. We are hopeful that this study will be funded as a Work-for-Others (WFO) project for ComEd.

\section{Specific Accomplishments:}

1. Demonstrated the utility of this technique by applying it to a practical system - the CVCS of the Braidwood Nuclear Generating Station.

a. Ranked top 50-100 components in CVCS with regard to "economic impact." 
b. Estimated economic impact of component failures.

2. Combined economic and reliability data to generate curves of shutdown risk and maintenance cost to begin the process of evaluating the effectiveness of maintenance practices.

These accomplishments, plus the accomplishments of earlier phases of this program, have led to a demonstration of all of the components of the overall program. 


\title{
95-037N -- INVESTIGATION OF FAST NEUTRON RADIOGRAPHY
}

\author{
Associate Laboratory Director Area: Engineering Research \\ Principal Investigator: \\ R.T. Klann, Engineering Division \\ Funding Profile:

FY $1993-0-$
FY $1994-0-$
FY $1995 \$ 35.7 \mathrm{~K}$
FY $1996-0-$
FY $1997-0-$

Purpose: To investigate the feasibility of fast neutron radiography. Fast neutron radiography has been studied in the past, and offers several advantages over thermal neutron radiography and $\mathrm{X}$-radiography. Specifically, cadmium and other thermal neutron absorbers are transparent to fast neutrons, therefore, materials that contain cadmium and boron can be examined. Other radiography techniques cannot probe through these materials. Fast neutron radiography also offers several other benefits over other radiographic methods, some of which are the ability to probe thicker objects, the ability to probe objects surrounded by metals and hydrogenous materials, and the ability to discern metallic objects and low atomic number objects simultanenusly. The objective of this project was to demonstrate the benefits and determine the weaknesses of fast neutron radiography. Specific applications for this process after development include probing waste cans and waste drums, probing crucibles and containers which contain cadmium, and probing larger objects unaccessible by thermal neutron radiography.

Approach: Historically, radiography was first invented in 1895 with the discovery of X-rays by Roentgen. Radiography utilizing neutrons was first developed in the 1930s and 1940s by Kallman and Kuhn using a small accelerator source. The methods and processes developed by these researchers led to a great number of patents and the basic detection techniques outlined by Kallman in a 1948 publication are still being used today to perform thermal neutron radiography. The first radiography performed with a nuclear reactor was in 1956 by Thewlis and Derbyshire at Harwell. The technique developed slowly after 1956 but it was principally the work of Berger at Argonne National Laboratory that led to the commercial applicability of thermal neutron radiography. Although, thermal neutron radiography has been thoroughly developed and is now commercially available, very little research with fast neutrons has been performed. It was reported by Berger that fast neutron radiography utilizing a neutron generator with activation foils is possible. Since that time several researchers in the U.S. and Japan have investigated fast neutron radiography using activation foils, converter screens, and scintillator methods. Most of these studies have involved neutron energies at or below $1 \mathrm{MeV}$. Richardson was able to obtain reasonable radiographs utilizing $14.5 \mathrm{MeV}$ neutrons with calcium tungstate scintillator screens. With the advent of stronger neutron sources and better scintillator and converter screens in recent years, fast neutron radiography can now be examined and enhanced to the point of being useful for industrial radiographic applications.

The work scope of the project is to develop the method of performing fast neutron radiography and to perform radiography to demonstrate the benefits and drawbacks of fast neutron radiography. Specifically, the project was divided into several logical steps: (1) characterize the 
neutron source; (2) perform scoping studies to determine foil characteristics; (3) develop cassettes and foil handlers; (4) obtain foils, converter screens, and film to perform radiography; (5) perform direct and indirect radiography, and (6) iterate on the radiographic method.

The first stage of the project includes characterizing the neutron source. Characterization of the neutron source involves performing several irradiation experiments with copper, nickel, and aluminum foils to determine the average neutron energy and peak neutron output available. Once these are known, the generator and radiography cassette configurations will be modelled. These studies will investigate the various foil and converter screen options and provide insight into the optimum foil and converter screen arrangements. The second stage of the project includes designing the foil and cassette holders, developing the radiographic process, and obtaining the necessary foils, converter screens, and film. Included in the design tasks are the design of the film and foil cassette, design of the cassette housing, development of the cassette transfer method, and development of the film exposure method. The final stage of the project is to perform fast neutron radiography, iterate on the radiographic method, and demonstrate the benefits of fast neutron radiography through a comparative study with thermal neutron radiography and $\mathrm{X}$-radiography. The comparative study includes performing radiography of all three types (X-ray, thermal neutron, and fast neutron) on identical configurations of objects and observing the differences among the three types.

\section{Technical Progress and Results:}

Progress was made in almost all areas of the project. First, the neutron source was characterized as to neutron average energy, neutron source strength, and useful neutron flux field around the generator source. Second, numerous activation foils were obtained and tested for fast neutron radiography potential. Both indirect and direct processes were tested and background densities measured.

Scintillation screens were then obtained and tested. It was found that the scintillation method offered the best images in the shortest amount of exposure time, therefore scintillation screens were used exclusively to perform radiography. The radiographic process was then examined and many iterations were performed before suitable radiographs were produced. Once suitable radiographs were produced, the benefits and drawbacks of fast neutron radiography were investigated.

The greatest benefit of fast neutron radiography is in its penetrating power. Radiographs of samples were produced through as much as 1 inch of lead, 4 inches of steel, 4 inches of aluminum, 4 inches of water, 6 inches of borated polyethylene, 6 inches of polyethylene and 2 inches of cadmium. These are not necessarily the limits of penetration for this process but reflect actual material thicknesses tested. Most notably, the cadmium was completely transparent to the fast neutrons. For all the other materials, the edges of the shields were observed, whereas the edge of the cadmium sheets was not observed. The biggest drawback has been in the resolution of the radiographs. The images appear grainy or fuzzy and not in focus. This has been postulated to be a result of the film speed and possibly the scintillating screen light emission, but this has not been confirmed. 
It was hoped that a comparative study could be performed to demonstrate the differences among the three types of radiography - X-ray, thermal neutron, and fast neutron. However, due to budget and time constraints, no comparative samples were prepared and no comparative test shots were performed.

\section{Specific Accomplishments:}

Publications in preparation as a result of FY95 work performed under this LDRD:

Refereed Publications:

1. Measuring the $14.5 \mathrm{MeV}$ n,p Cross-Section for Ytterbium-174 - will be submitted to Nuclear Science and Engineering.

2. Proton Radiography Using A Neutron Generator - will be submitted to Nuclear Instruments and Methods in Physics Research or the Fifth World Conference on Neutron Radiography, Berlin, Germany, June 17-20, 1996.

Non-refereed Publications:

1. Determining the Average Neutron Energy and Flux from a Neutron Generator - will be submitted to the American Nuclear Society Annual Meeting, Reno, NV, June 16-20, 1996.

2. Fast Neutron Radiography Utilizing Activation Foils - will be submitted to the Fifth World Conference on Neutron Radiography, Berlin, Germany, June 17-20, 1996.

3. Fast Neutron Radiography Utilizing Scintillation Screens - will be submitted to the Fifth World Conference on Neutron Radiography, Berlin, Germany, June 17-20, 1996. 
blank page 


\title{
94-187R1 -- NUCLEAR DECONTAMINATION AND DECOMMISSIONING CUTTING TECHNOLOGY DEVELOPMENT
}

\author{
Associate Laboratory Director Area: Engineering Research \\ Principal Investigators: \\ V.J. Novick and A.E. Knox, \\ Technology Development Division \\ Funding Profile: \\ FY $1993-0-$ \\ FY $1994 \$ 226.0 \mathrm{~K}$ \\ FY $1995 \$ 201.7 \mathrm{~K}$ \\ FY $1996 \$ 200.0 \mathrm{~K}$ \\ FY $1997-0$ -
}

Purpose: The long-term objective is to establish a decontamination and decommissioning (D\&D) program at the Laboratory for evaluating and developing hazardous material removal and cutting technologies. The primary effort focuses on Laboratory based R\&D to evaluate existing cutting technologies in order to recommend or suggest methods to decrease the risk of cutting hazardous material. Further efforts will be made to enlist industrial participants to provide equipment and access to facilities to obtain additional test data and broaden the database for a hazardous materials cutting handbook. The information and test data acquired would increase the efficiency of DOE/EM D\&D and other clean-up efforts that involve cutting hazardous materials. No such program currently exists in the United States and no other organization or facility is as uniquely qualified to pursue such research because of the combination of the Laboratory's ongoing D\&D efforts, research programs in the areas of aerosol characterization and mitigation, waste stream analysis, pollution control and recent experience in evaluating cutting technologies.

Approach: It is estimated that more than 400 radioactively-contaminated facilities such as nuclear plants, manufacturing facilities, commercial and government laboratories, are scheduled for decontamination and decommissioning in the next decade in the U.S. In order to minimize costs and effort, most D\&D operations resort to cutting structural materials to facilitate handling and to compact the waste. The choice of cutting tool is determined by the material composition, space or geometry considerations and thickness as well as speed, cost and concerns for workers and environmental safety. While a significant body of knowledge is generally available to aid in tool selection based on material, speed, cost, etc., there is a definite lack of information on many of the safety issues, particularly the quantity and size of particles formed during the cutting process. Recent incidents at the EBWR facility have demonstrated the need for this type of information. Airborne particles or aerosols are particularly important because they can be inhaled and deposited in the workers' air passages or they can be transported great distances across site boundaries. Therefore, in order to quantify the level of risk included in a particular D\&D operation and to properly design protective or control systems, the aerosol size distribution and quantity that is generated by a particular cutting technique must be known.

This need is approached in three phases. The first phase is to develop a data base of information on aerosol production from laboratory controlled cutting operations. The second phase is to compare the controlled methudology with field sampling to broaden the applicability to the end 
users. Part of this task would also include measurements of particles released into the atmosphere during underwater cutting operations. Finally, a compilation of the data will be presented in a reference handbook format allowing the user easy access to the information to aid in the design of pollution control and waste minimization strategies as well as appropriate tool selection and personal protective devices.

Technical Progress and Results: An experimental test chamber was designed and fabricated. The chamber allows cuts up to one meter in length on structural pieces weighing as much as $75 \mathrm{~kg}$. The material being cut travels under a fixed cutting tool to allow the aerosol source to be stationary and directly below the aerosol sampling port. The motor driving the material being cut is computer controlled to precisely define the cutting speed and length of the cut. The chamber is presently instrumented with a cascade impactor for particle size determination and High Efficiency Particulate Air (HEPA) filters for mass quantification.

A 100 amp plasma torch was installed in the test chamber and a series of cuts were made on various thickness of stainless steel, carbon steel and aluminum. Results indicate that the mass median aerodynamic diameter (MMAD) of the stainless steel aerosol produced by the plasma torch is $0.36 \mu \mathrm{m} \pm 0.09 \mu \mathrm{m}, 0.52 \mu \mathrm{m} \pm 0.16 \mu \mathrm{m}$ for carbon steel and $0.48 \mu \mathrm{m} \pm .16$ for aluminum. The MMAD does not appear to be a function of cutting speed, thickness, length or kerf width. While a linear relationship exists between the quantity of aerosol produced and the length of the cut, for all of the materials tested, only carbon steel exhibits a single linear relationship between the aerosol mass and length of cut times material thickness. Aluminum and stainless steel both exhibit linear relationships, but the slope of the line depends on the thickness of the material.

An aerosol field sampling unit was designed and utilized to collect aerosols resulting from plasma torch and oxyacetylene torch cuts in air at the EBWR facility. Another sampling unit was designed and tested to collect aerosols resulting from laser cutting operations. An extensive sampling program of the laser cuts is planned for next fiscal year.

A user guide has been developed consisting of a database and combined with a program that calculates certain aerosol and cutting parameters based on specific user input. To date, this user guide only contains information on the plasma torch. The database portion is a compilation of pertinent data, with appropriate references, from our laboratory tests, field tests and data in the literature. The program portion uses the laboratory data to calculate the expected aerosol mass produced for a specified cut, the aerosol production rate and the minimum filtration area needed to collect the expected aerosol without exceeding a pressure increase of 10 inches of water. In addition, suggested parameters such as cutting speed and torch power are provided based on standard cutting guides and laboratory experience. Communication continues with the Commission of European Communities (CEC), but most of the information in the literature does not contain sufficient detail to be included in the calculational portion of the user guide.

Specific Accomplishments: An overview of the work and a demonstration of the user guide was presented to the DOE review of ANL D\&D programs on May 31, 1995.

An abstract has been submitted to the 1996 ANS topical meeting on the "Best of D\&D" to be held in Chicago in April 1996. 
Preliminary work was reported at the Western Regional Air and Waste Management Association Conference, May 13, 1995, in Los Angeles, CA. And the National Air and Waste Management Association Conference, June 18-23, 1995 in San Antonio, TX. The paper won first place in the B.S. Division student competition at the Western Regional and second place at the national meeting. 
blank page 


\section{5-221N -- ALKALI METAL LASER DOPPLER VELOCIMETRY ${ }^{1}$}

Associate Laboratory Director Area: Engineering Research

Principle Investigator:

Funding Profile:
J.D. McWhirter, Engineering Division

FY $1993-0-$

FY $1994-0-$

FY $1995-\$ 57.1 \mathrm{~K}$

FY $1995-0-$

FY $1997-0-$

Purpose: Provide a means of obtaining nonintrusive point velocity information in conducting fluids. The conducting fluid of choice was the alkali metals. Application to EBR-II sodium flowrate calibration was anticipated. The work was to be mostly analytical, but some experiments could have been required.

Approach: The alkali metals are described in the optics and solid state physics literature as being "transparent" in the ultraviolet below some wavelength dependent upon the particular element (Kittel, 1986, Introduction to Solid State Physics, Wiley). If the alkali metals were "transparent," then Laser Doppler Velocimetry (LDV) could be performed. Such a capability would represent the first nonintrusive point velocity probe for use with conducting fluids. This capability would allow precise measurement of the effects of the magnetic body force on the turbulence characteristics in a flowing fluid. Many ways of expressing optical data exist in the literature, such as the extinction coefficient, the real and imaginary parts of the dielectric constant, and the optical conductivity. The approach taken was to accumulate experiment results from the literature, and transform the data into a common format, specifically, the extinction coefficient. Data for all alkali metals (lithium, sodium, potassium, rubidium and cesium) and a sodium-potassium alloy were covered. The skin depth (distance at which light intensity is reduced by a factor of "e") and the skin depth per wavelength were then computed versus wavelength from 10 to $1000 \mathrm{~nm}$ (visible light is from 400 to $700 \mathrm{~nm}$ ).

Technical Progress and Results: The results of the investigation showed that the skin depth of light in alkali metals was exceedingly short, with the deepest penetration of around three micrometers (this means that, over a distance of three millimeters, a signal would be attenuated by factor of $e^{-1000}$; very, very large attenuation). (This would seem to be a far cry from "transparency;" however, considering that most metals strongly reflect incident electromagnetic radiation, the fact that some radiation was transmitted through very thin layers of alkali metals was quite astonishing for the researchers.) It was therefore concluded that LDV was not feasible in alkali metals.

An alternative approach toward the goal of LDV in conducting fluids was then undertaken. The electrical conductivity of a mixture of fine copper powder suspended in water or electrolyte was calculated, with encouraging results. At reasonable concentrations (around ten volume percent),

\footnotetext{
${ }^{1}$ Individual Investigator Project
} 
the conductivity of the suspension exceeded that of many liquid metals. (Sodium has the highest electrical conductivity of the liquid metals at the melting point. Copper, at room temperature, has an electrical conductivity. of roughly three times that of sodium.)

These results then encouraged an assessment of the optical characteristics of such a suspension. The results of this analysis show that such a suspension can have a skin depth of the order of tens of centimeters if the particle diameter is of the order of ten nanometers. Copper powder is sold by several chemical distributors, but the smallest size available is nominal one micrometer in diameter. A process which produced gold colloidal suspensions with around ten nanometers was identified from the literature. Application of this process to copper appears feasible but has not been attempted to date.

The thermal transport properties of the suspension were calculated, revealing perhaps the most promising features of the suspension. The thermal conductivity is, as expected, equal to or above that of several liquid metals, due to the high conductivity of the copper, but with an extremely high specific heat compared with liquid metals. Previously, the gap between the heat transfer properties of liquid metals (high thermal conductivity, low specific heat) and water (low thermal conductivity, high specific heat) appeared unavoidable. The suspension blends the desirable characteristics of liquid metals and water into a single fluid.

Specific Accomplishments: A patent disclosure has been submitted for the application of the copper powder-water suspension to heat transfer systems where improved performance is needed. Examples are computer chip cooling systems and crewed spacecraft cooling systems. A paper entitled "Feasibility of Laser Doppler Velocimetry in Alkali Metals" was submitted for consideration as a Technical Report to Optics and Lasers in Engineering. The paper was rejected as a Technical Report. This paper will be resubmitted as a Technical Note. A paper entitled "Model for Inertialess Magnetohydrodynamic Flow in Packed Beds" was written, with application to the flow of the copper powder in water suspensions through a magnetic field. This paper is being reviewed by its co-authors (two at The University of Texas at Austin, one at Sandia National Laboratory) and will be submitted to the Journal of Fluid Mechanics. A paper entitled "Electrical, Optical, Fluid Mechanical and Thermal Properties of Water-Copper Powder Suspensions" is currently under preparation. A target journal has not yet been identified. 


\title{
94-015R1 -- DEVELOPMENT OF REAL TIME X-RAY EXAMINATION FOR HOT CELL EXAMINATION
}

\author{
Associate Laboratory Director Area: Engineering Research \\ Principal Investigator: \\ F.L. Yapuncich, Technology Development \\ Division
}

Funding Profile:

FY $1993-0-$
FY $1994 \$ 111.3 \mathrm{~K}$
FY $1995 \$ 99.9 \mathrm{~K}$
FY $1996-0-$
FY $1997-0-$

Purpose: To investigate real time NDA systems for nuclear and non-nuclear applications.

Real time Nondestructive Analysis (NDA) allows for direct measurement of specimens with concise results immediately. In the area of nuclear applications, a NDA system that does not create its own waste while examining the contents of various waste streams would be beneficial to the remediation field. Specifically, a NDA system will be designed to determine its suitability in determining Pu content of EBR-II blanket pins. If suitable for this purpose, the technique could be used to help verify fissile material content in other reactor blankets of interest and other heterogeneous waste forms. In the area of non-nuclear applications, NDA systems that could determine quality control parameters of agricultural products would benefit the grower, buyer, and consumer.

Approach: The examination of metal fuel sodium levels by real time $x$-ray systems was introduced by Argonne for the Fuel Cycle Facility in 1993. Because of this initial success with real time NDA systems it was decided to attempt to evaluate the sodium bond area between the metal fuel and fuel jacket. It became evident that these type of NDA systems could have applications in other nuclear and nonnuclear areas. Therefore the foundation was laid for NDA examinations of agricultural items and the examination of nuclear waste streams. This work led to the award of a CRADA between University of Idaho and ANL-W and the initial design of a neutron generator based NDA system for assaying various nuclear waste streams.

The technique chosen to accomplish the NDA objective for nuclear waste forms involves a combination of passive neutron counting (neutron emitted primarily from spontaneous fissions in ${ }^{240} \mathrm{Pu}$ ) and cyclic irradiation/delayed neutron counting of the material of interest. Initial experiments to investigate issues involving the use of the proposed methodology will be conducted in the NRAD-NRS cell. Lead bricks will be stacked in a rectangular array in the cell within which the material will be contained in a small stainless steel can. A cadmium liner surrounds the can containing the material of interest. A $14 \mathrm{MeV}$ neutron generator is used as a source of fast neutrons while delayed neutron detection is accomplished using $40^{3} \mathrm{He}$ detectors dispersed in a polyethylene matrix surrounding the lead brick array. The polyethylene matrix containing the $40{ }^{3} \mathrm{He}$ detectors is located on 3 sides and the bottom of the lead brick array with the neutron generator occupying the fourth side of the array. Located directly above the lead 
brick array is an elevator access port which will be used to lower the material cans into place at the center of the array. List-mode data acquisition is used.

Several computational techniques for calculating sample isotopics from the experimental data will be tested. Among these are neural networks, least squares, Kalman filtering, genetic algorithms, and nonlinear programming. As data analysis techniques such as these have very broad applicability, techniques developed and/or refined for the NDA data analysis may have broad application for other purposes such as system diagnostics and process monitoring.

Various techniques were evaluated for use in the agricultural area. Initial imaging was accomplished with a $160 \mathrm{~V}$ "microfocus" $\mathrm{X}$-ray imaging system. This system was constructed by Dr. W.A. Ellingson with the Energy Technology Division. The system features a microfocus $\mathrm{x}$-ray tube that produces a 6 micron spot size. The $\mathrm{x}$-rays are detected with a 9" image intensifier optically coupled to a CCD array. Both Nuclear Magnetic Resonance (NMR) and neutron assay were also evaluated for the use in detecting damaged potatoes and determining specific gravity.

Technical Progress and Results: In the area of nuclear waste assaying, all the electronics (amplifiers, preamplifiers, time base, and time tag units) were designed, constructed, installed and currently in the process of being checked. Several data analysis techniques were tested using simulated data including neural networks, least squares, Kalman filtering, genetic algorithm, and nonlinear programming. Results from these initial studies look promising. Computational modeling of the NDA system was initiated using the VIM monte carlo code with results giving insight into optimal detector placement, expected detector response, and detection efficiency profiles for various system arrangements. Progress was also made in the development of a user-friendly personal computer interface for data acquisition.

In area of nonnuclear applications, magnetic resonance was found to be able to measure specific gravity in potatoes. Initial results indicate accuracy should be as good or better than the standard method. Also the measurement can be made rapidly which is essential to a on-line sensor. MR was also able to detect certain types of internal defects in potatoes. MR has the potential of being competitive with $\mathrm{x}$-ray imaging for defect determination and may provide additional information than currently used $\mathrm{x}$-ray systems. Real time $\mathrm{x}$-ray systems showed excellent results for pressure and shatter bruise defects. However, due to the length of time currently involved with obtaining these results, this system is better suited for further basic research.

Specific Accomplishments: The initial design of a neutron generator based NDA system for examining various waste streams is complete. Of the various types of NDA systems evaluated for use in the agricultural sector, the Magnetic Resonance system has shown to have excellent potential for the development of an in-line low-cost sensor for the determination of specific gravity of potatoes.

S.E: Aumeier, "Noise Analysis Techniques for FCF NDA Program," Intra-Laboratory Memo to G.R. Imel and R.W. Schaefer, March 21, 1995. 


\title{
93-040R2 -- LASER BASED HOT CELL ELEMENTAL AND ISOTOPIC ANALYSIS SYSTEM
}

\author{
Associate Laboratory Director Area: Engineering Research \\ Principal Investigators: \\ Engineering Division \\ Funding Profile:

$\begin{array}{lll}\text { FY } 1993 & \$ 216.2 \mathrm{~K} \\ \text { FY } 1994 & \$ 124.4 \mathrm{~K} \\ \text { FY } 1995 & \$ 132.5 \mathrm{~K} \\ \text { FY } 1996 & -0- \\ \text { FY } 1997 & -0-\end{array}$

S.G. Johnson and S.M. Frank,

Purpose: The objective of this proposal is to introduce laser based analytical methods for direct elemental quantification at trace, minor and major levels in a hot cell environment. The ultimate goal being to reduce the need for complex labor intensive sample dissolution techniques, while providing accurate, rapid analyses of irradiated fuel, salt, cadmium and various waste samples. Direct chemical analysis of solid samples, without the need for dissolution and separations, would reduce the waste generation in the Analytical Laboratory, and demonstrate that the same could be done in future applications, i.e., hot cell based spent fuel or waste characterization facilities. Moreover, the end product of this effort would be to demonstrate techniques that could be adapted directly to the production floor of a DOE radiological waste characterization facility. This concept would reduce facility construction costs and provide for more timely analysis for input to process and material balance controls.

Approach: Sample analysis procedures used currently in the Analytical Laboratory (AL) hot cells at ANL-W require the sample be converted into solution form. Further chemical separations are necessary in order to obtain accurate elemental and isotopic analysis by Inductively Coupled Plasma Atomic Emission Spectroscopy (ICP-AES) and Mass Spectrometry (MS). This is due to the extreme spectral congestion for ICP-AES and isobaric interferences for MS of the actinide and lanthanide elements present in these samples. Additionally, the removal of radioactive fission products is required for MS analysis outside the hot cells. Procedures presently used to prepare the samples for ICP-AES and MS analyses generate a variety of waste forms including mixed hazardous waste which presents problems in terms of waste disposal.

Laser ablation has the following advantages. First, the use of organic solvents and concentrated acids during the separation procedure to prepare a sample for ICP-AES or MS analysis will be eliminated. This reduces the amount of contaminated liquid waste generated by the AL and decreases the error in estimating the amount of special nuclear material (SNM) distributed in the respective waste streams. During laser ablation approximately $1 \mathrm{mg}$ of the total sample may be used for both elemental analysis and isotopic assay thus greatly reducing the quantity of sample necessary and therefore the waste generated. Second, the material can be surveyed for its isotopic and elemental composition entirely within the hot cell environment reducing the risk of contamination involved with removing a sample from the hot cells. Additionally, the laser ablation technique can be performed on a sample in minimal time relative to present methods 
for isotopic assay and trace elemental determinations. This has tremendous potential for the decreasing the turn-around time necessary for an analysis. Determination of fission products in spent oxide reactor fuels using the technique of laser ablation-ICP-MS is currently underway at the Institute for Transuranium Elements in Karlsruhe, Germany.

The application of glow discharge sampling technologies can yield isotopic information and depth resolution directly for conductive solid samples. This additional capability can be obtained at a very nominal expense yet yield a great deal of information when coupled to a time of flight mass spectrometer (TOFMS) system which is already part of the direct solid sample analysis system. For these reasons this technology will be investigated and incorporated part of the system.

Technical Progress and Results: Progress was noted on two fronts in the year just past. The progress achieved in the field of laser ablation ICP-AES will be covered first and then the subject of application of time of flight mass spectrometry to direct solid sampling and analysis. LA-ICP-AES was applied to the analysis of salt samples emanating from the electrorefiner for the determination of lanthanides and uranium. The first elemental determination confronted was that of uranium in the $\mathrm{KCl} / \mathrm{LiCl}$ salt. The chemical form of uranium in the electrorefiner is $\mathrm{UCl}_{3}$. This is unobtainable commercially so a search for alternative forms of uranium for doping into a series of standards was conducted. $\mathrm{U}_{3} \mathrm{O}_{8}, \mathrm{UO}_{2}\left(\mathrm{NO}_{3}\right)_{2}-6 \mathrm{H}_{2} \mathrm{O}$ and $\mathrm{UCl}_{4}$ were explored for their usefulness as potential sources of uranium in a series of salt standards. Also, several matrices were explored for use in assembling this collection of standards. The $\mathrm{LiCl} / \mathrm{KCl}$ eutectic salt from the electrorefiner is not ideal for use as a standard matrix due to its hygroscopic nature. $\mathrm{KCl}$ and a mixture of $\mathrm{NaCl} / \mathrm{KCl}$ were used in combination with actual eutectic salt from the electrorefiner. By conducting an extensive series of experiments using the three different salt matrices, four different uranium compounds (including $\mathrm{UCl}_{3}$ from the electrorefiner), three different ablation wavelengths $(1064,532$ and $355 \mathrm{~nm})$ and varying other parameters of the ICP such as: carrier gas flow, forward power and auxiliary gas flow, the optimal conditions and optimal standard compositions were determined. The conclusion was that the series of standards will have to be prepared using diluted samples from the electrorefiner since the chemical form of the uranium is important and the matrix needs to be $\mathrm{LiCl} / \mathrm{KCl}$ eutectic salt. In all of these experiments either $\mathrm{Li}$ or $\mathrm{K}$ was used as an intrinsic internal standard for the uranium. The effect of typical "expected" concentrations of rare earths was determined to be negligible. Work is progressing on development of the standards with both uranium and lanthanides present.

LA-ICP-AES and conventional sample introduction were applied to the issue of applicability of high resolution ICP-AES to obtain isotope specific information for uranium and in the near future plutonium. The preliminary studies focused on the ${ }^{235} \mathrm{U}$ and ${ }^{238} \mathrm{U}$ ratios for a series of isotopic standards and actual analysis of MKIII and MKIV alloys with $67 \%$ and $69.5 \%$ enrichment of the ${ }^{235} \mathrm{U}$ content with respect to total uranium. The $424: 4 \mathrm{~nm}$ emission line of uranium is isotopically split and spectrally resolvable into two components separated by $25 \mathrm{pm}$; one component for each ${ }^{235} \mathrm{U}$ and ${ }^{238} \mathrm{U}$, see Fig. 1 for MKIII, dashed line, and MKIV, solid line. The results of our preliminary study are summarized in the paper listed below (J. Anal. Atom. Spect.) and can be briefly summarized here: 1) the technique is workable for solutions yielding RSD's of $<0.3 \%$ with a predictable bias, 2) the application of laser ablation is viable for sheared pieces of uranium/zirconium alloy with no sample preparation, although the RSD's are 
typically $1-2 \%$ and 3 ) the application of zirconium or uranium as an intrinsic internal standard is necessary in order to improve the RSD's to the level quoted above. The application of an installed fiber optic cable into the ICP present in the analytical laboratory's hot cells will allow for use of the high resolution spectrometer for isotopic determinations of uranium and plutonium in the future. There are several transitions of plutonium that are amenable to isotope specific spectroscopy and these will be explored in the year to come.

Work involving TOFMS has proceeded with a focus on the installation of the system into the analytical laboratory's hot cells. The installation has been successfully accomplished with complete calibration using gas standards. Testing with the solid sampling apparatus is on-going.

Bench top experiments have been conducted during the past year using simulated binary metallic fuels and salt samples doped with lanthanides in order to obtain the appropriate operating parameters and analytical figures of merit for the hot cell based laser ablation ICP-AES system. These have been conducted primarily by Dr. Phillip S. Goodall a Post-Doctoral Fellow. Parallel studies have been performed on the TOFMS system using both laser ablation and glow discharge sampling methodologies with a series of standards to determine operating parameters and analytical figures of merit. Additionally, portions of the TOFMS system have been installed into the renovated hot cells in the Analytical Laboratory.

Collaborators for the accomplishments presented are Prof. Sandra Kimbrell of University - Alaska-Fairbanks, Dr. Phillip Goodall, and Mr. Elon Wood.

Specific Accomplishments: Research will continue under the auspices of DOE-NE.

1. Oral Presentation and Paper entitled "Analysis of Metallic Fuels by Laser Ablation," presented at SPIE conference Advanced Optical Methods for Ultrasensitive Detection, February 6-7, 1995, San Jose, California, 2385, p. 68.

2. Oral presentation entitled "Laser Ablation and High Resolution ICP Spectrometry for the Determination of Lanthanides and Selected Actinides," presented at The Winter Plasma Conference 1995, January 9-11, 1995, Cambridge, England.

3. Paper, P.S. Goodall, S.G. Johnson, "Application of Laser Ablation Inductively Coupled Plasma Atomic Emission Spectroscopy (LA-ICP-AES) to Selected Analysis Needs in the Nuclear Industry," submitted to Spectroscopy (October 1995).

4. Paper, P.S. Goodall and S.G. Johnson, "Isotopic Uranium Determination by Inductively Coupled Plasma Atomic Emission Spectrometry Using Conventional and Laser Ablation Sample Introduction," Journal of Analytical Atomic Spectroscopy (October 1995), in press.

5. Paper, P.S. Goodall, S.G. Johnson, E.L. Wood, "Laser Ablation Inductively Coupled Plasma Atomic Emission Spectrometry of a Uranium-Zirconium Alloy: Ablation Properties and Analytical Behavior," Spectro Chimica Acta B (1995), in press. 


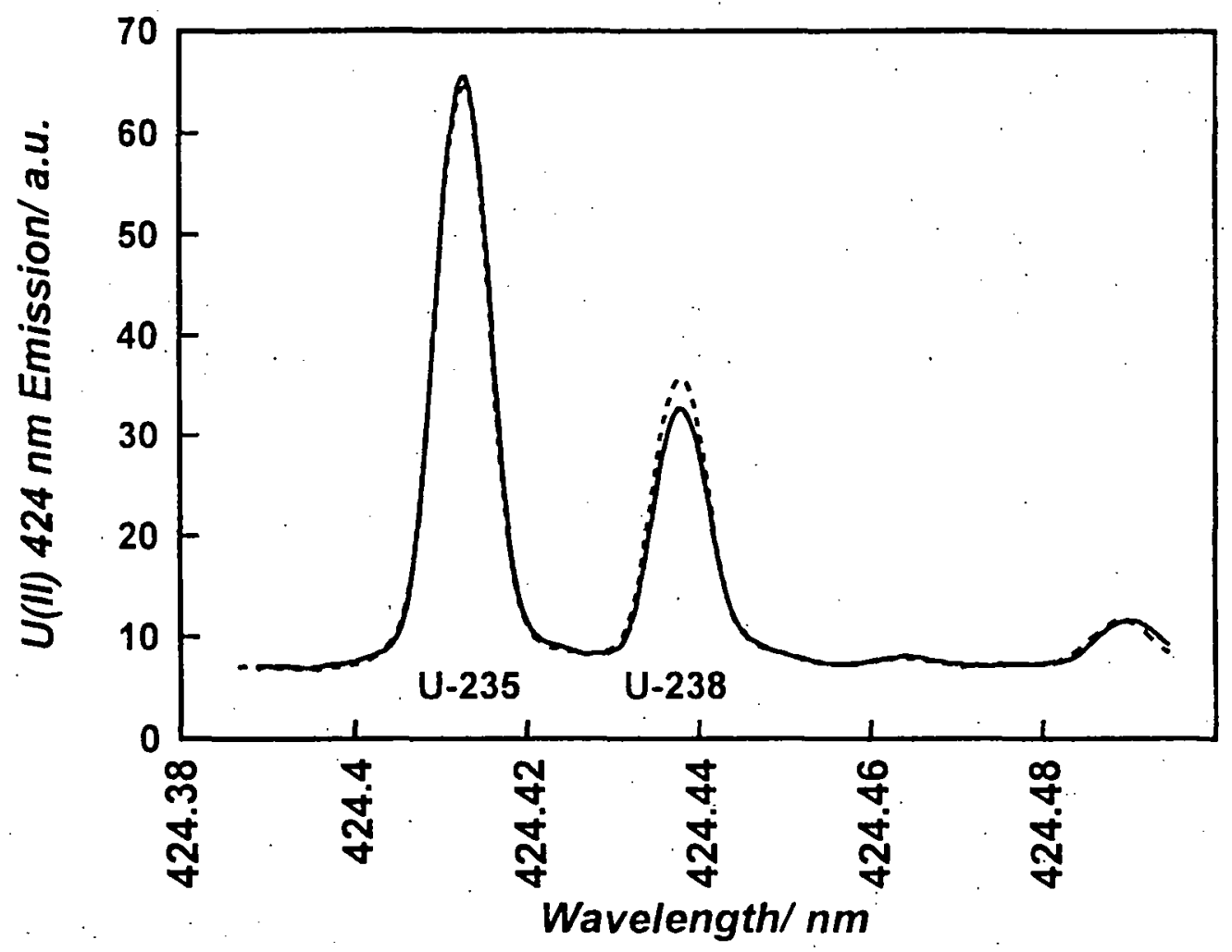




\section{5-041N -- UTILIZATION OF WASTE HARDWARE}

\section{Associate Laboratory Director Area: Engineering Research}

\section{Principal Investigators:}

Funding Profile:

\author{
G.R. Imel and C. Trybus, \\ Engineering Division
}

Purpose: The disposal of reactor hardware (e.g., stainless steel) at the present time creates a large volume of waste that is presently occupying valuable storage space in near-surface storage sites. This is a problem in both the present nuclear reactors, and is a main concern in future fusion reactor designs. This project consists of two parts: a calculational effort to characterize the waste hardware in terms of the best quantitative values for isotopic composition, and a metallurgical effort. The metallurgical effort will study the recycling potential of the waste hardware, study casting methods to reduce the volume of the hardware waste, and investigate advanced storage forms. The calculational effort will provide the tools to separate the waste stream such that ground disposal when necessary can be performed in the most efficient manner, and it will provide the best estimate of the composition of the waste stream to determine the feasibility of recycling or advanced storage.

Approach: The approach is as follows:

- Calculational methods to improve the characterization. The objective was to improve the calculations of stainless steel activation through three methods: obtain better estimates of impurity concentrations through chemical analysis of archival EBR-II material; use modern cross-section data sets (e.g., ENDF-VI); and use the best available calculational techniques (two-dimensional transport).

- Metallurgical studies, including potential use of "scrap" material. The decommis-sioning of EBR-II will create two large classifications of waste, the fuel and the subassembly hardware and other various stainless steel components. The fuel is clearly a high level waste (HLW). The stainless steel components on the other hand consist of alpha-free activated metal. Their waste classification is less obvious. Uncertainties exist in the impurity content and in the calculations, mainly the cross sections of some isotopes of concern. Finally, if EBR-II stainless steel hardware is greater than class $\mathrm{C}$ low level waste (GTCC-LLW) strategies are investigated to mitigate the problem through metallurgical processing and/or waste management. Initially, this LDRD was limited in scope to these two issues however, upon examination of the material inventory, initial results of the calculations, and acquisition of knowledge of LWR aging issues it became apparent that EBR-II materials would be valuable in a study of LWR aging. The irradiation conditions, spectrum and temperature in the reflector region of EBR-II compare quite favorably with those of LWR core internals. 
- Advanced storage methods. The objective in this part of the LDRD is to study the potential of advanced storage methods such as the conversion of the mineral and metal waste streams from FCF to a material and form that could be stored in the EBR-II reactor vessel.

\section{Technical Progress and Results:}

- Calculational methods. An "average" elemental composition for stainless steels used to fabricate EBR-II structural and experimental subassemblies was determined by chemical analysis of archival specimens. This average composition was used as the input composition for ORIGEN calculations. ENDF/B VI cross sections were produced for the nuclides present in stainless steels. S8-P1 two-dimensional transport theory calculations were done to provide scalar fluxes. These scalar fluxes were used to collapse the cross-sections to one group for input to ORIGEN. Higher order scattering transport calculations were completed, but did not yield increased accuracy. The results of these calculations show that the ${ }^{94} \mathrm{Nb}$ activity in reflector and blanket subassemblies irradiated to the fluence limit is over the 10 CFR 61.55 limit by at least an order of magnitude. ${ }^{59} \mathrm{Ni}$ and ${ }^{63} \mathrm{Ni}$ activity vary to a maximum of -0.5 of the allowed Class $\mathrm{C}$ limit, high enough to be of concern but not high enough to preclude a Class $\mathrm{C}$ determination individually. Chemical analysis of actual reflector and blanket S/A is necessary to benchmark these calculations.

- Metallurgical studies. The GTCC-LLW classification of EBR-II hardware is driven by the impurity $\mathrm{Nb}$, in the stainless steels. A chemical analysis of 35 heats of reactor grade austenitic (304 and 316) stainless steels was completed in an effort to determine the baseline level of $\mathrm{Nb}$ in EBR-II steels. The results were inconclusive because of the scatter in the data no confidence that these heats were "typical" of EBR-II steels was established. However, the results indicated that the $\mathrm{Nb}$ content was extremely low, $-300 \mathrm{ppm}$ wt. Coupling the low $\mathrm{Nb}$ content with the low chemical activity of $\mathrm{Nb}$ in stainless steels, yields a minuscule chemical potential of $\mathrm{Nb}$ in these steels, making it impossible to separate $\mathrm{Nb}$ from the steels via a chemical metallurgical method. Zone refining was explored as a potential route for separation but it would require numerous (on the order of 6!) remelting passes to obtain an acceptable (i.e., the "other constituents" would then be class $\mathrm{C}$ waste) separation of $\mathrm{Nb}+\mathrm{Cr}+\mathrm{Mo}$ from the other constituents. This scheme is totally impractical for remote operation. Thus this area of research has been abandoned.

- Advanced storage methods. In this part of the research, the feasibility of storing all of the EBR-II irradiated hardware produced since beginning operation was studied. Calculations using the ORIGEN code determined a characteristic spent composition for a Mark IIIA driver element. From this, an estimate of the volume, mass, heat load, and activity of the waste streams were determined. The waste canister would be a sub-assembly type structure that could be moved in and out of the reactor vessel using existing fuel handling hardware.

A candidate waste form for the mineral waste is a pressed glass of fission product loaded zeolite and frit. These could be fashioned into waste pins to be loaded in the waste sub-assembly. The metal waste form would be used to construct the sub-assembly hardware. A $25 \mathrm{~kW}$ remote metal casting induction furnace has been tested at the Utah State University in an air atmosphere. This type of technology will be investigated to 
prepare the sub-assembly hardware using the metal waste form. A higher density waste form for the mineral wastes will be investigated to lower the volume of that form.

The heat load is of very little consequence. The largest centerline temperature due to decay heat in the new waste form is on the order of 120 degrees $\mathrm{C}$ above that of the storage gas (air or argon) circulating in the vessel. A complete analysis of the thermodynamics of this system must be completed in order to determine the temperature of the hottest waste pin in the system and the flow of heat out of the reactor vessel.

The next step is to do the same analysis for the blanket sub-assemblies and thoroughly investigate the mechanical structure of the vessel to determine the design basis accident. Various calculations based on this model will be needed to support a safety analysis to determine if the material will be safe for the 300 to 500 year life of the waste.

\section{Specific Accomplishments:}

A perusal of the surveillance subassembly data and a substructure examination of stainless steels irradiated at dramatically different rates led to a paper and an invited proposal which is currently being negotiated. In addition, there is very limited data from neutron irradiated materials on irradiated stress corrosion cracking of stainless steels. EBR-II materials are prototypic of many LWR core internals at end-of-life, having experienced a neutron environment at a low damage rate to a total damage of $60 \mathrm{dpa}$. Because of these attributes EBR-II irradiated stainless steels would have microstructures characteristic of LWR core internals at end-of-life and thus in turn possess similar mechanical properties. This is the basis for proposals which have been made to NRC, EPRI, CRIEPI, JAPIC and the Japan Atomic Power Company. Effort will continue under the auspices of a non-federal program.

Listed below are the presentations, publications in progress, and the current proposal which is under negotiation as result of this LDRD:

1. "The Effect of Neutron Exposure Rate on Tensile Behavior and Substructure of Type 304 Stainless Steel" presented by C.L. Trybus in February 1995 at the Annual TMS Meeting, Las Vegas, Nevada. This paper is currently being prepared for submission to Metallurgical Transactions $A$.

2. "Evaluation Of Austenitic Stainless Steels From The EBR-II Surveillance Test Program" invited paper to the Annual Meeting of the Japan Institute of Metals to be presented by D.L. Porter in December 1995 in Honolulu, Hawaii. Publication planned concurrent with meeting in Transactions of the Japan Institute of Metals.

3. "Utility Of EBR-II Hardware To The Light Water Reactor Industry" invited paper to the Annual Meeting of the Japan Institute of Metals to be presented by C.L. Trybus in December 1995 in Honolulu, Hawaii. Publication planned concurrent with meeting in Transactions of the Japan Institute of Metals.

4. "Calculation of Displacement Rate in EBR-II Reactor Materials" to be presented by S.L. Hayes at the ANS Winter Meeting in San Francisco, Calif., November 1995. 
5. "Waste Classification And Management Of Irradiated EBR-II Hardware" to be presented by K.A. Bunde at the Joint ASM-TMS meeting in Cleveland, Ohio, November 1995. Publication to be determined.

6. "Electron Microscopy and Microstructural Analyses of 304 Stainless Steel Specimens" proposal (no. P-95128) approved by DOE. Work sponsored by The Institute of Nuclear Safety Systems, Kyoto, Japan through Battelle Seattle Research Center, C.L. Trybus and D.L. Porter, principal Investigators. 


\section{5-030N -- DEVELOPMENT OF ANALYTICAL METHODS TO PERFORM WASTE CHARACTERIZATION OF ACTIVATED METALS}

\author{
Associate Laboratory Director Area:
}

Principal Investigators:

Funding Profile:

\author{
Engineering Research
}

S.M. Frank, J.R. Krsul, C.D. Morgan, and T.P. Zahn, Engineering Division

$\begin{array}{lll}\text { FY } & 1993-0- \\ \text { FY } & 1994-0- \\ \text { FY } 1995 & \$ 198.9 \mathrm{~K} \\ \text { FY } & 1996 & \$ 150.0 \mathrm{~K} \\ \text { FY } & 1997 & \$ 150.0 \mathrm{~K}\end{array}$

Purpose: The objective of this project is to develop rapid and economical chemical characterization methods for the determination of radionuclides in activated metals. Determination of radionuclide content in activated metals waste is mandated before ultimate disposal of these waste streams. New separation and solid sampling schemes, being investigated in this proposal, will significantly reduce the man power and cost involved with the measurement of specified radionuclides. In addition to the development of direct chemical measurement techniques, surrogate analytes will be identified that can be measured non-destructively and, with the use of scaling factors or empirical calculations, be used to estimate the content of difficult to measure radionuclides. These indirect methods will allow rapid and accurate characterization of activated metal waste streams.

Approach: Argonne-West is currently faced with the difficult task of identifying and characterizing its activated waste metal inventory. These sources of activated metal come from past hardware waste located in the Radioactive Waste Management Complex (RWMC) and from the decommissioning of the EBR-II reactor. Characterization of activated metal waste, particularly for the determination of class $\mathrm{C}$ waste, is difficult, expensive and labor intensive. The difficulty arises with the measurement of specific, federally listed, radionuclides which tend to be relatively low energy, beta emitting species hidden beneath an intense, high energy gamma emission background. Three methods exist to characterize activated metal waste for listed radionuclide content, including direct chemical analysis of specific radionuclides, indirect analysis of surrogate nuclides which can then be used to estimate listed radionuclide content and estimation by computer neutronic balance codes. Both direct and indirect analysis methods of class $\mathrm{C}$ waste identification are being investigated under this project.

For direct identification of listed radionuclides, chromatographic separation methods are being developed, for hot cell application, in which radionuclides of interest are separated from the more active gamma emitting background. Once separated, the listed nuclides are measured with high precision and accuracy using traditional radiochemical methods. For indirect determination, surrogate gamma emitting radionuclides will be identified which can be measured in a more efficient manor and that can give an accurate estimate of the listed radionuclide. Development of indirect analysis techniques will first identify possible surrogate nuclides, to be used for the estimation of listed radionuclides by gamma-ray spectroscopy and solid sampling time of flight mass spectrometry (TOFMS). Initial analyses of irradiated steel samples will involve a complete 
gamma-ray spectral survey of the sample. Following the gamma-ray survey, the sample will be analyzed by solid sampling TOFMS to determine ratios of surrogate analyte to listed analyte. These ratios will be used to formulate the scaling factors or empirical calculations that would be applied to the gamma-ray survey to estimate the concentration of listed radionuclides. Possible surrogates will be investigated using solid sample techniques and TOFMS or inductively induced plasma atomic emission spectroscopy (ICPAES). These techniques greatly reduce sample preparation time and offer rapid sample component determination. These detection methods also provide ratio measurements that can be used to compare possible surrogates to listed radionuclide content. The results obtained from direct and indirect analysis of radionuclide content of activated metal waste streams will provide valuable input data and validation of neutronic computer codes. These codes will ultimately provide the most cost effective estimation of radionuclide content of activated metal waste streams. Finally, a comprehensive survey of activated metal waste at the ANL-W site is being performed which includes waste type, irradiation history and storage location. This database survey will be used in conjunction with neutronic codes to classify the waste dependent on its radionuclide content. The results from the neutronics estimation will routinely be confirmed by direct chemical or surrogate radionuclide analysis.

Technical Progress and Results: The major technical progress for the first year of this project has been to confirm the chemical separation and analysis schemes, procure the instrumentation and install the instrumentation in to the ANL-W Analytical Laboratory hot cell. Two distinct schemes have been developed. The first involves ion chromatographic (IC) separation of dissolver solution containing National Institute of Science \& Technology (NIST) traceable radionuclides of interest. Work this past year on the IC system involved optimization of elution parameters using a Dionex Model 4000i ion chromatograph with gradient pump and a Dionex CS 5A column using citrate-oxalate and sulfuric-oxalic acid eluent. In addition, a flow through liquid scintillation detector (B-Ram), down stream from the IC column, was shown to be effective in detecting radionuclides at class $\mathrm{C}$ levels in the eluent. This work has demonstrated that an activated metal can be dissolved, the various components separated automatically in an IC system and 10 CFR 61.55 listed radionuclides can be detected accurately in a time frame of tens of minutes. Figure 1 shows the IC separation of transition metals and lanthanides from an alloy matrix. Figure 2 shows counts versus retention time for tritium and nickel-63 standards eluted from the IC flow-through liquid scintillation detector. In addition, the hot cell IC equipment has been installed and tested. When the hot cells are operational, actual irradiated metal samples can be analyzed for lited radionuclide content.

The second analysis scheme involves solid sampling, by either laser ablation or glow discharge sputtering, followed by TOFMS of metal samples. To date, the major effort has been directed at laser ablation sampling TOFMS. This has been performed using $1064 \mathrm{~nm}$ radiation from a Nd:YAG, Q-switched laser (Continuum Corporation). The ionized, ablated material is then entrained into a carrier gas and expanded into the source region of the TOFMS. The ionized material is then pulsed down the flight tube where it is detected relative to its flight time. Sample sizes range from $0.6 \mathrm{~cm}$ to $1.3 \mathrm{~cm}$ in diameter. The ablated region is approximately 200 micrometers in diameter with nanogram quantities of sample material ablated for every laser plulse. Investigation of nonirradiated metals has been performed using the laboratory TOFMS instrument and NIST traceable steel samples. Figure 3 shows a laser ablation time-of-flight mass spectrum of 304 stainless steel. This work will determine mass spectral resolution, detection 
sensitivity and possible interfering species. Irradiated samples will be analyzed using the hot cell TOFMS instrument when the cells are operational.

Computer database inventories of Radioactive Scrap \& Waste Facility (RSWF) liners containing EBR-II activated metal have been completed. These investories contain estimates of activated metal class $C$ waste, but will have to be verified by one of the above mentioned methods. These databases will next be updated with irradiation histories of the various metal components.

Specific Accomplishments: None for this period. 


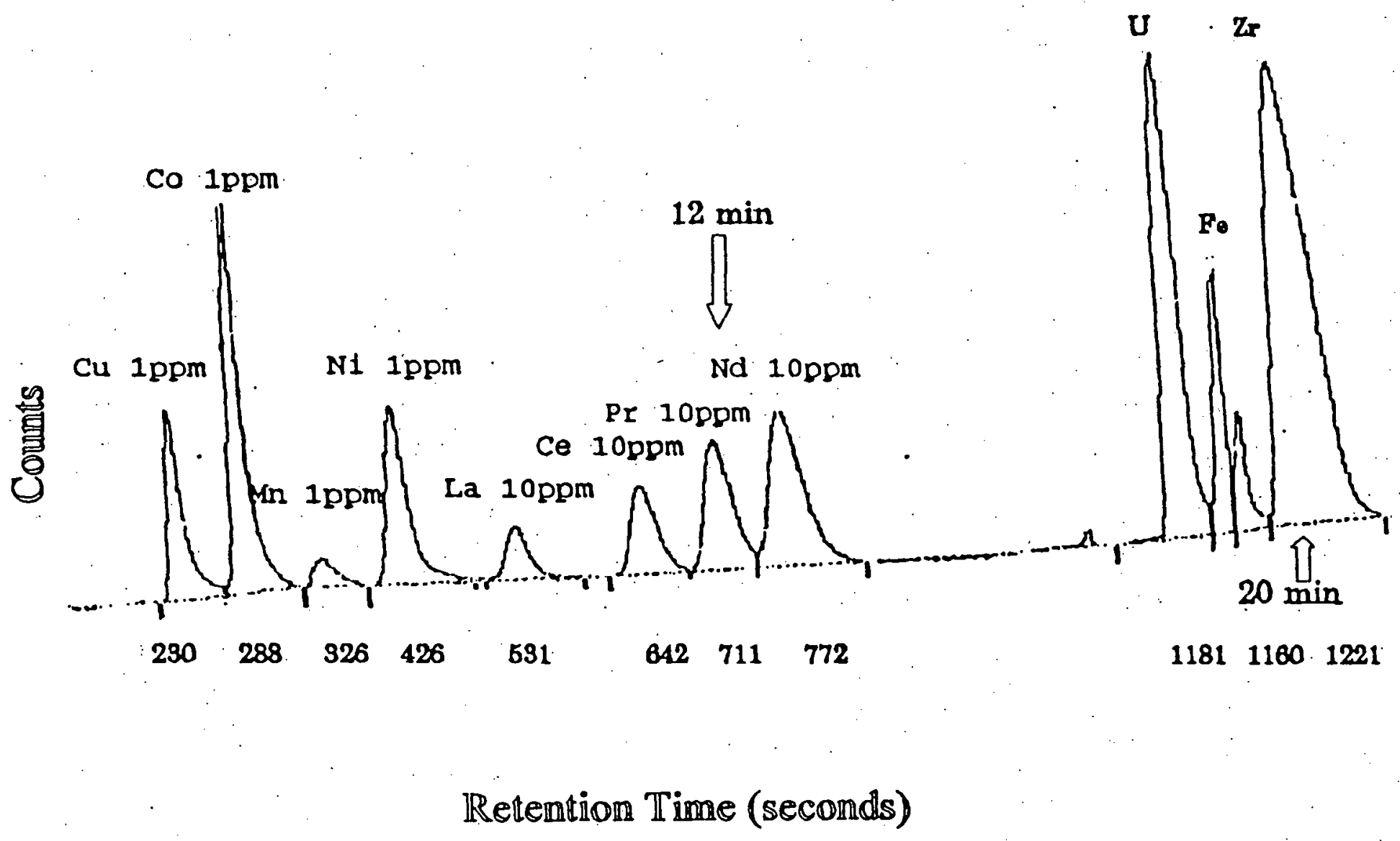

Figure 1. IC separation of transition metals and lanthanides from alloy matrix. 


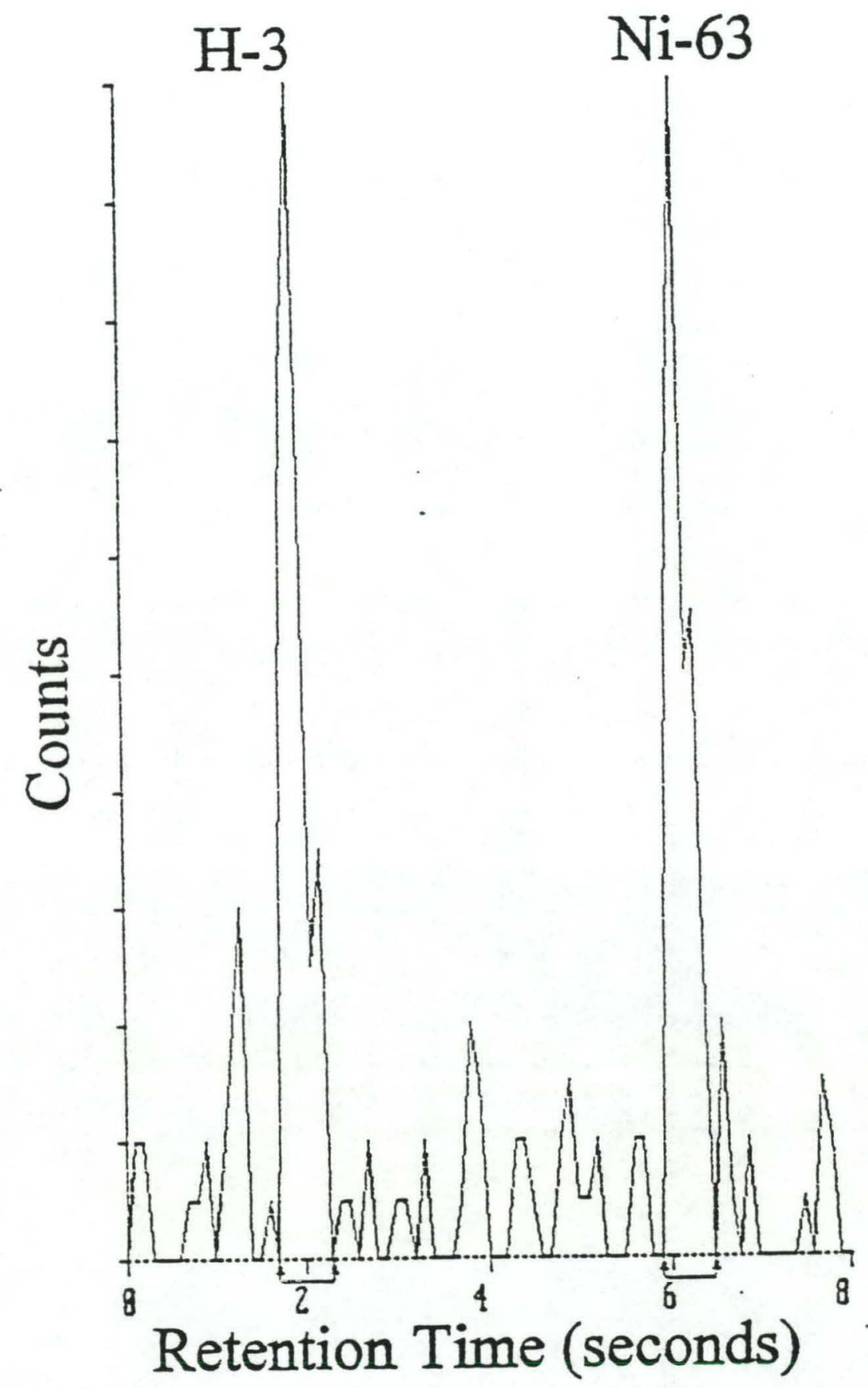

Figure 2. IC separation of $\mathrm{H}-3$ and Ni-63. Flow through liquid scintillation detector. 


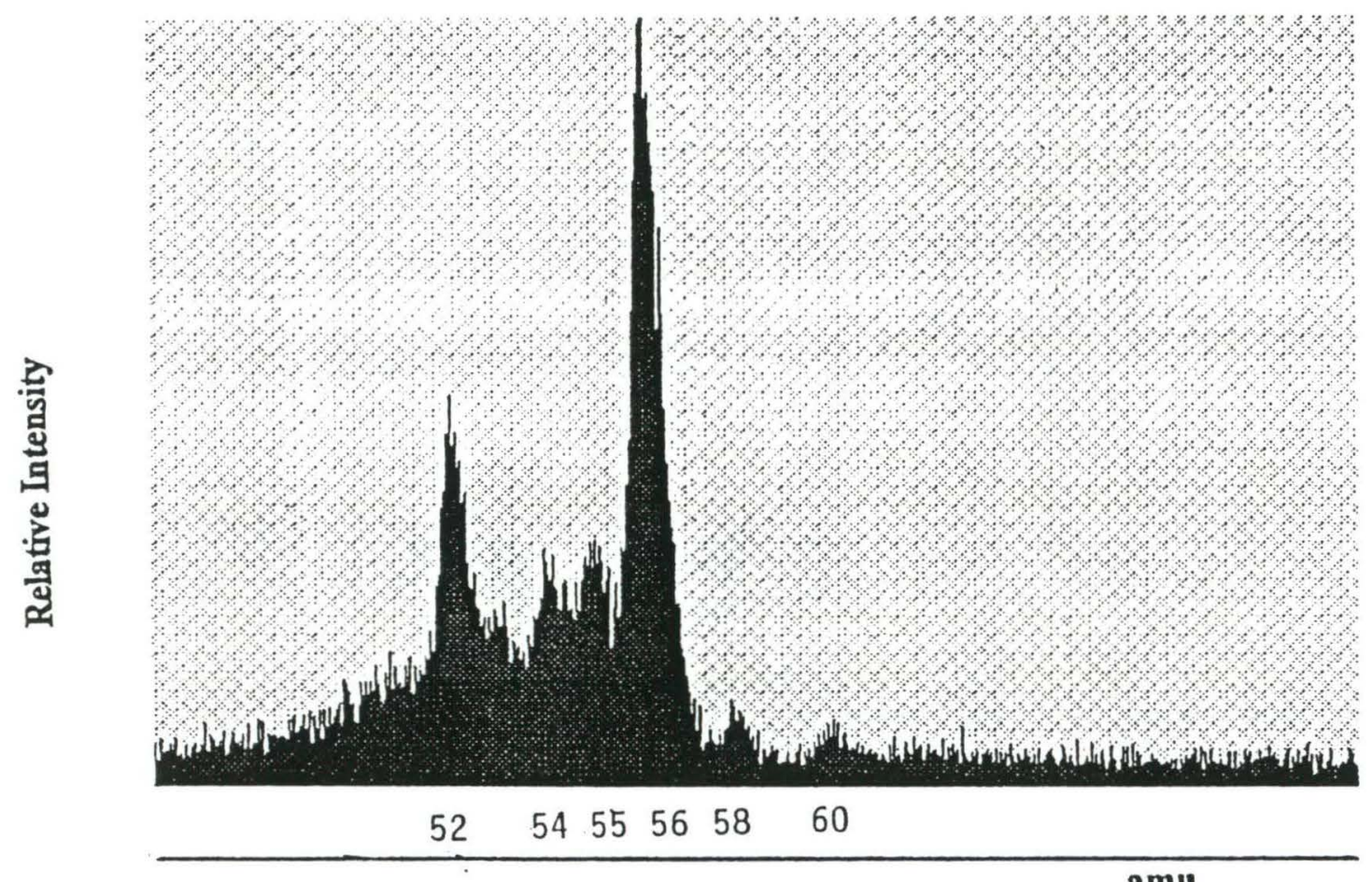

amu

Figure 3. Laser ablation TOFMS of stainless steel. 

$\begin{array}{ll}\text { 95-049N -- } & \text { HYDROCLONES FOR REMOVAL OF SUSPENDED MATERIAL FROM } \\ \text { FUSED SALTS }\end{array}$

Associate Laboratory Director Area:

Principal Investigator:

Funding Profile:
Engineering Research

C.C. McPheeters, Chemical Technology Division

FY $1993-0-$

FY 1994 -0-

FY 1995 \$298.6K

FY $1996-0-$

FY $1997-0-$

Purpose: The objective of this project was to develop devices incorporating the principles of cyclonic flow for the removal of suspended material or solids from liquid salt streams involved in pyrochemical processes.

Approach: The process streams in pyrochemical processing often contain entrained material that needs to be separated. This entrained material may be the desired product to be recovered, a waste stream to be discarded, or a material to be recycled to a process step. A simple device that removes entrained material would find many applications in pyrochemical processing. This project investigated the use of hydroclones for removing suspended material from molten salt streams. Particular interest was the metal suspended in molten salt that is generated in the lithium reduction of spent oxide fuel. Also of interest is a device that could effectively separate the sludge that has accumulated in the Hanford storage ponds from the cooling water.

This project included: (1) The design and fabrication of a centrifugal filter and a submersible centrifuge for the recovery of entrained material in a salt stream. (2) Testing of these devices with water and suspended solids that have a density similar to that of the heavy metal produced in the lithium reduction process in a container that has similar dimensions to the reduction vessel in the engineering-scale glovebox. (3) The design of an improved device to be fabricated and tested for separating suspended solids from a molten salt stream. (4) The demonstration of an effective separation of sludge-like material from water by a top-loading submersible centrifuge. Co-investigators of this work included Drs. R.D. Pierce and L.S. Chow.

Technical Progress and Results: Two apparatus were designed and fabricated to recover material entrained in the molten salt in a reduction vessel. Particular attention was for the recovery of uranium-transuranic product from a reduction crucible used for the lithium reduction of spent oxide fuel. The first device was a centrifugal filter depicted in Figure 1. The internal filter was designed to simulate an electrorefiner anode basket. The concept was that this filter containing the reduced metal would be transferred into the electrorefiner where separation of the uranium and transuranic elements could be conducted electrochemically. The filter was made of 325 mesh screen and was surrounded by a cylindrical shroud during the recovery of entrained solids. The device is $14.6 \mathrm{~cm}$ in diameter and $11.4 \mathrm{~cm}$ high. A $43-\mathrm{cm}$ diameter tank, similar in size to the engineering-scale reduction vessel, was fabricated. Tests of the centrifugal filter and the submersible centrifuge were performed in the tank filled with water to a depth of $33 \mathrm{~cm}$. Several inlet nozzles were tested. The best results for particle recovery were obtained with an 
inlet nozzle that was $1.0 \mathrm{~cm}$ in diameter and $2.3 \mathrm{~cm}$ in length. In a few tests, radial flow vanes were installed on the vessel bottom. The presence of the radial flow vanes significantly improved the particle collection efficiency under the same operating conditions.

The second device was a submersible centrifuge depicted in Figure 2. The unit was $10 \mathrm{~cm}$ high with an upper diameter of $10 \mathrm{~cm}$ and a lower diameter of $7.5 \mathrm{~cm}$. It had replaceable nozzles and mixing blades. The conical riser tube was intended to promote upward flow of particles. The conical body was intended to assist particle-electrolyte contact should the device be used as an anode during electrorefining. Liquid entered the unit from the bottom and exited from the openings near the top. Most of the tests were performed to determine the collection rate using $500 \mathrm{~g}$ of $0.8 \mathrm{~mm}$ diameter copper shot $(\sim 8.9 \mathrm{~g} / \mathrm{mL}$ density). A few tests were made with $500 \mathrm{~g}$ of 0.4 to $0.5 \mathrm{~mm}$ lead particles ( $\sim 11.3 \mathrm{~g} / \mathrm{mL}$ density). In these tests, four different nozzles and three different gaps between the nozzle of the centrifuge and the vessel bottom were compared and the effect of the mixing blades was examined. Results at about $600 \mathrm{rpm}$ indicate that a $13 \mathrm{~mm}$ I.D. x $25 \mathrm{~mm}$ long nozzle provided the best recovery of the particles when the gap between the nozzle and the vessel bottom was $7 \mathrm{~mm}$ or less.

A larger submersible centrifuge ( $15 \mathrm{~cm}$ diameter $\times 19.7 \mathrm{~cm}$ high) as shown in Figure 3 was designed and fabricated. The device was first tested to determine its applicability to collect entrained material from the simulated reduction vessel. Then it was tested to assess its applicability to the removal of sludge from Hanford ponds. The device consisted of four equal quadrants separated by four blades. At each quadrant, two openings were made on the center tube to allow the flow of liquid into the device. Covers were fabricated to close these opening when needed. A pair of horizontal disks were installed near the top exit openings. Liquid entered the unit from the bottom inlet tube and exited the unit from the openings near the top. In the first test, copper pellets were recovered from the water tank. The optimum configuration was obtained when the device was spinning at $520 \mathrm{rpm}$, all the openings at the center tube were opened, and the inlet tube was $7 \mathrm{~mm}$ from the tank bottom. It took $1 \mathrm{~min} .45 \mathrm{sec}$. to collect all the copper shot. In the second test, zeolite pellets $(\sim 1.5 \mathrm{~g} / \mathrm{mL}$ density) were used to simulate the Hanford pond sludge. It took $1 \mathrm{~min}$. to recover all of the zeolite pellets with the optimum centrifuge speed at $260 \mathrm{rpm}$.

Because of its size, many centrifuge units would be needed in order to effectively collect sludge material entrained in a storage pond. Alternatively, a more economical and efficient method would be to pump the sludge material directly to a device such as a top-loading centrifuge as shown in Figure 4. Tests were conducted with the top-loading centrifuge to collect the following matarials: (1) calcined powder with an average particle size of 0.9 micron with a density of $\sim 3.7 \mathrm{~g} / \mathrm{mL}$, (2) paste forming powder of 200 mesh with a density of $\sim 3.9 \mathrm{~g} / \mathrm{mL}$, and (3) wax powder with a density of $\sim 0.9 \mathrm{~g} / \mathrm{mL}$, respectively. About $90 \%$ of the 0.9 micron powder was recovered in one minute. About $90 \%$ of the paste forming powder was also recovered in one minute. In the tests with the low-density $(0.9 \mathrm{~g} / \mathrm{mL})$ powder, the test configuration was modified such that the exit openings for the liquid were located near the bottom of the device. With a rotational speed between 200 to $250 \mathrm{rpm}$, about $70 \%$ of the powder was collected at the top of the device in one minute. The top-loading submersible centrifuge was demonstrated to be very versatile and effective at collecting different types of materials. 
In summary, the centrifugal filter performed well when radial flow vanes were installed at the bottom of the process vessel. The additional flow vanes may, however, complicate the design and adversely affect the performance of the process vessel. The submersible centrifuges performed well and could be easily modified to collect material entrained in a molten salt within a process vessel. When the vessel or storage pond is too large, pumping the liquid into a toploading centrifuge would be a more suitable choice.

\section{Specific Accomplishments:}

- Designed, fabricated, and tested a centrifugal filter. Effective recovery of entrained material was demonstrated.

- Designed, fabricated, and tested two versions of submersible centrifuge. Effective recovery of entrained material was demonstrated.

- Designed, fabricated, and tested a top-loading submersible centrifuge. Effective recovery of entrained material was demonstrated. 


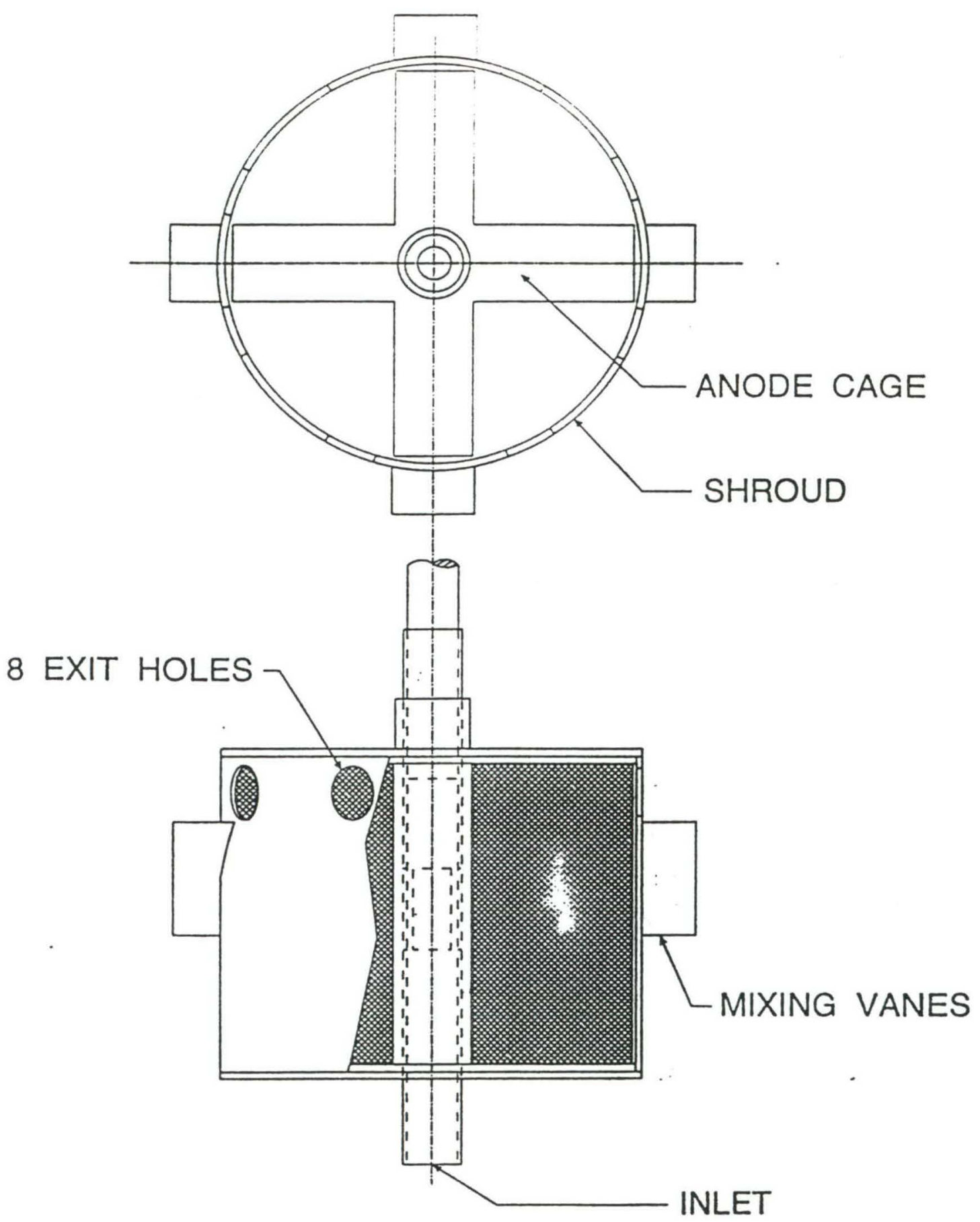

Fig. 1. Centrifugal Filter 


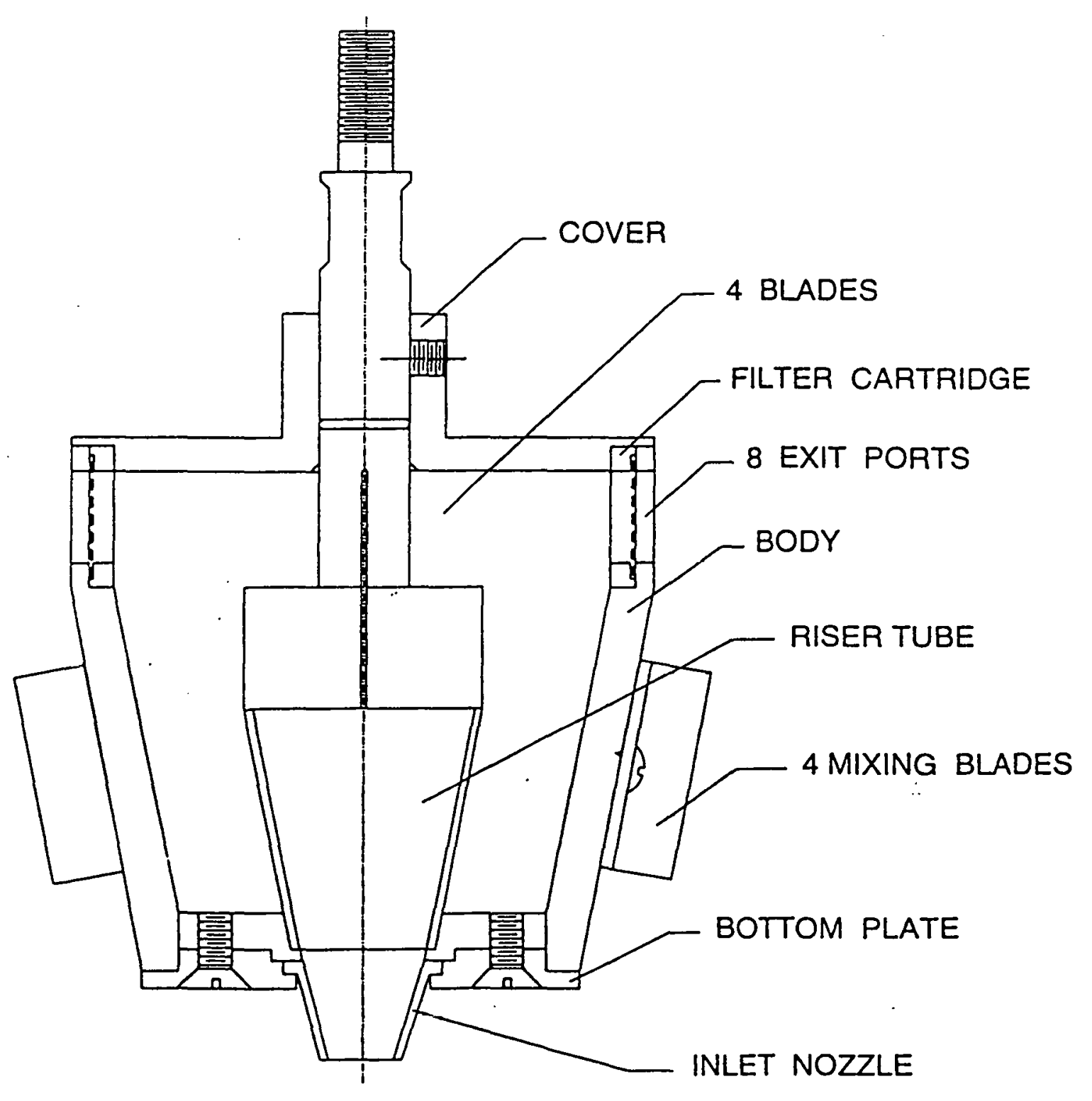

Fig. 2. Submersible Centrifuge. 


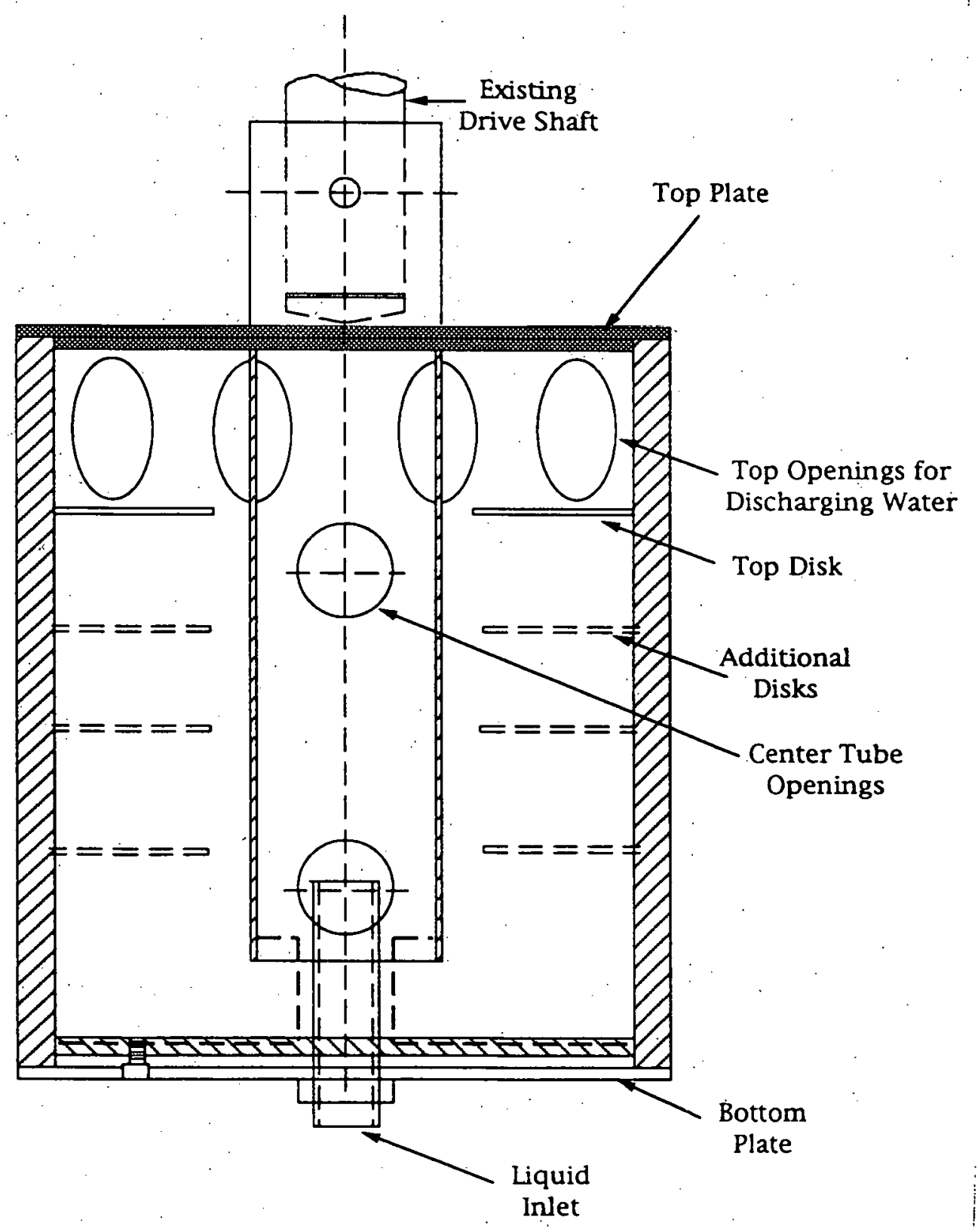

Fig. 3. Submersible Centrifuge. 


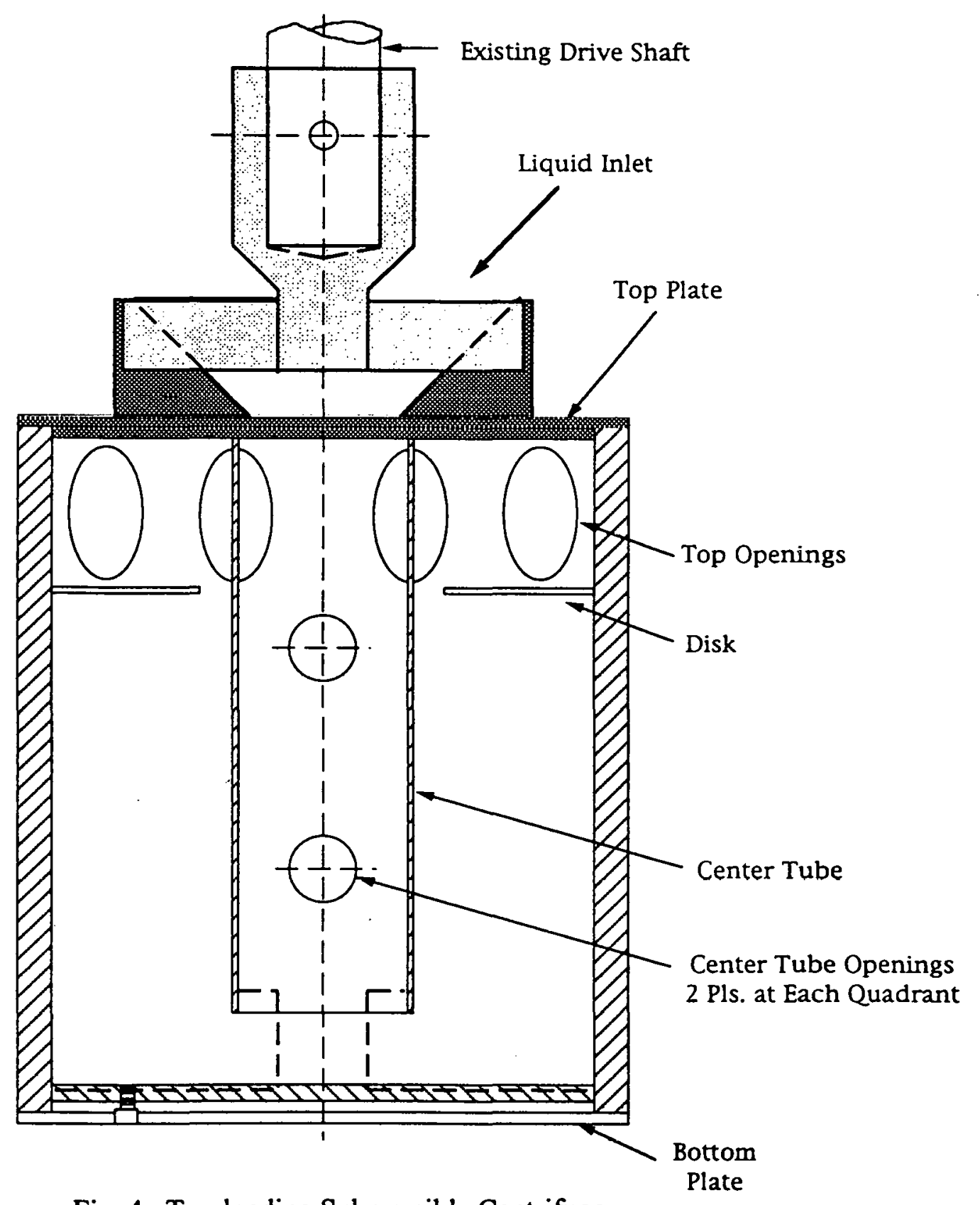

Fig. 4. Top-loading Submersible Centrifuge. 
blank page 


\title{
94-114R1 -- WEAPONS PLUTONIUM DISPOSITION - AN EVALUATION OF FUEL CYCLE AND TECHNOLOGY OPTIONS
}

\author{
Associate Laboratory Director Area: Engineering Research \\ Principal Investigators: $\quad$ R.N. Hill, H.S. Khalil, J.J.R. Liaw, and \\ G. Palmiotti, Reactor Analysis Division \\ Funding History: \\ FY $1993-0-$ \\ FY $1994 \$ 314.7 \mathrm{~K}$ \\ FY $1995 \$ 148.8 \mathrm{~K}$ \\ FY $1996-0-$ \\ FY $1997-0-$
}

Purpose: The project objective is to evaluate a variety of weapons plutonium disposition options. The comparison of alternative technologies and fuel cycle strategies is expected to indicate potential refinements of proposed disposition systems; and to identify the waste forms associated with alternative weapons plutonium disposition strategies. The primary focus in FY 1995 was the evaluation of plutonium immobilization concepts where the weapons material is rapidly blended with (existing) spent fuel.

Approach: Large quantities of weapons plutonium currently exist in US and CIS weapons stockpiles. Arms reduction treaties call for the disassembly and disposition of many of these weapons. The disassembled weapons material must be closely safeguarded to prevent its diversion to produce new nuclear weapons. For US arms reduction policy, the short-term goal is to quickly transform the weapons-grade material into a form which is not readily usable for weapons. This goal is accomplished by making the weapons material extremely difficult to handle. In most proposed disposition techniques, the weapons material is denatured by dilution and the introduction of radioactivity; thus, chemical separation and remote handling would be required to recover the weapons-grade material.

Many different strategies can be employed to achieve this denaturing of the weapons material. In FY 1994, the introduction of weapons material into a closed fuel cycle was investigated; in that case, the weapons material would be diluted during fuel fabrication and radioactivity introduced as the fuel is fissioned in a reactor. The FY 1995 investigation focused on nonreactor disposition options. In non-reactor strategies, the radioactivity is obtained from an existing source (e.g., spent nuclear fuel); and this radioactive material is blended with the weapons material. Blending strategies can be implemented more rapidly because the entire denaturing is accomplished in a single step and subsequent reactor irradiation is not required. However, destruction of the weapons material (by fission) is not achieved in non-reactor options. Therefore, active safeguarding of the denatured weapons product is required; and its proliferation characteristics are crucial.

The project methodology involves the construction of computer simulations modeling the various weapons plutonium disposition proposals. The calculations are designed to perform detailed tracking of weapons material (plutonium) during its transmutation and/or chemical processing. In addition, other parameters which impact the proliferation characteristics, such as radiological 
impact (dose), must be evaluated. The impact of key design variables is evaluated in a parametric manner.

Technical Progress and Results: The main focus. in FY 1995 was the evaluation of a metal waste form designed to immobilize weapons plutonium. The plutonium composition is diluted by mixture with "dirty" plutonium and radioactivity is introduced by including fission and activation products: . Fission products, transuranic elements, and cladding are recovered from LWR spent fuel by electrometallurgical treatments and mixed with weapons plutonium in the form of a corrosion-resistant $\mathrm{Zr}$-Fe-Pu alloy. Several additives which increase the self-protecting radiation field were also investigated: specifically, the incorporation of Cs-137 to increase the photon dose, and beryllium to enhance the neutron dose.

Several self-protective (proliferation resistant) characteristics of the direct disposal metal alloy were estimated:

- the maximum neutron and photon dose rates on the outer surface and at a 1 meter distance from a typical disposition ingot;

- the relative contributions of the various sources of neutrons and photons to the total dose as a function of time

- the neutron/photon shielding requirements for ingot transportation purposes

- the heating rates and temperature distributions in the shielded ingot

Computational techniques were developed to model the production and transport of neutrons created by the beryllium $(\alpha, n)$ source. In addition, the decay of fission products and transport of their decay particles was tracked. Computer codes were developed to interface accurate decay distributions to state-of-the-art Monte Carlo transport and dose calculations.

In addition to the plutonium immobilization evaluations, studies of reactor options were continued in an international context. These studies included:

- participation in the NEA benchmark for plutonium recycling

- evaluation of MOX fuel usage in Russian fast and thermal reactors

- evaluation of accelerator-driven concepts for plutonium destruction

The proposed focus for FY 1996 continuation of this project is to evaluate other proposed technology options' (e.g., disposition in existing light-water reactors and disposition in a thoriumbased fuel cycle) in more detail. Alternative disposition strategies will be analyzed in a consistent manner; and the relative merits of each option will be evaluated. In addition, the proliferation resistance of the resulting denatured weapons material and waste forms will be assessed. 
Specific Accomplishments: A technical paper "Physics Studies of Weapons Plutonium Disposition in the IFR Closed Fuel Cycle" summarizing the comparisons of conventional, moderate burner, and pure burner IFR core designs was published in the September 1995 issue of Nuclear Science and Engineering. An internal memo "Investigation of the Self-Protective Characteristics of Conceptual Alloy Ingots Composed of a Mixture of LWR Waste, Weapons Plutonium, and Beryllium" was completed on August 31, 1995. Contributions were made to an internal ANL report "Russian Capability to Utilize Mixed-Oxide (MOX) Fuel" SPO-94/2. In addition, significant contributions were produced for the NEA plutonium recycling benchmark summary reports. 
blank page 


\title{
95-061N -- PRE-CONCEPTUAL DESIGN OF A FACILITY FOR CONDITIONING SPENT NUCLEAR FUEL USING PYROPROCESS ${ }^{1}$
}

\author{
Associate Laboratory Director Area: Engineering Research \\ Principal Investigators: \\ Funding Profile: \\ FY $1993-0$ \\ FY $1994-0-$ \\ FY $1995 \$ 297.2 \mathrm{~K}$ \\ FY $1996-0-$ \\ FY $1997-0-$
}

L.W. Deitrich, Reactor Engineering Division

J.J. Laidler, Chemical Technology Division

Purpose: The purpose of this study was to define key elements of a pre-conceptual design for an Electrometallurgical Treatment Facility (EMTF) to treat $\mathrm{N}$ Reactor fuel which constitutes roughly $80 \%$ of the total DOE inventory of spent fuel. The facility would be installed at Hanford, either as part of new construction or within the existing FMEF building. Currently, the spent fuel is stored in aging and leaky $\mathrm{K}$ Basins that constitute a potential threat to the nearby Columbia river. It is planned to remove and dry this deteriorated fuel and place it in interim storage. Additional conditioning of the spent fuel in dry interim storage is necessary for final disposal. Utilization of the electrometallurgical process would be a cost-effective means to separate uranium from fission products. The relatively clean uranium produced could be stored for eventual utilization or disposal as a low-level waste; whereas, the plutonium would be contaminated with higher actinides and fission products, making it diversion resistant. Immobilization of the fission products with a zeolite-based process minimizes waste volume for disposal in a geologic repository.

Approach: The thruput requirements of an electrometallurgical conditioning system for Hanford fuel are a direct function of the desired cleanup time for the current inventory. On the assumption that a time span approximately 10 years is reasonable, a plant capacity around $1000 \mathrm{Kg} \mathrm{HM}$ per day is adequate for the estimated $2100 \mathrm{MT}$ of spent fuel on site. This report gives a brief description of the key features of a pyroprocess facility sized to condition, at that rate, Hanford fuel. A process flow sheet and material balance is developed for an Electrometallurgical Treatment Facility that receives wet or dry $\mathrm{N}$ Reactor fuel that has been removed from the $\mathrm{K}$ basins and placed in interim storage. An equipment layout, starting with a fuel pin shredding operation on through to waste packaging, is established that defines the dimensions of an inerted cell to contain the overall process. The output products include decontaminated uranium, as low-level waste, and zeolite-glass ingots containing TRU elements mixed with fission products to satisfy non-proliferation requirements.

'In FY 1994, project 94-186N treated a similar topic as it related to Argonne's Idaho facilities. In FY 1995, facilities in Hanford, Washington, were considered which made the content of this project sufficiently different from that in 1994 to consider this a new start. 
Technical Progress and Results: An initial pre-conceptual design study for an EMTF installed at Hanford to treat $\mathrm{N}$ Reactor fuel shows that a new full-scale plant can be constructed and operational by the year 2003 with dedicated financial support. This option appears less expensive than using the FMEF. A cell of approximately $2500 \mathrm{sq} \mathrm{ft}$ will be required to house the basic process elements. Based on current experience with key process elements, no technical roadblocks to successful and timely development are indicated. Present equipment scaleup to treat a daily load of one tonne per day of $\mathrm{N}$ Reactor fuel requires only straightforward extrapolation of current designs. Although this larger equipment does not pose a criticality hazard, more study is needed to determine whether multiple smaller units would be more cost effective from a plant availability standpoint.

Specific accomplishments: A draft report outlining key features of an Electrometallurgical Facility to treat $\mathrm{N}$ Reactor fuel has been completed. The attached flowsheet shows material movement in the EMTF. An included drawing shows the process equipment layout within the new facility. 


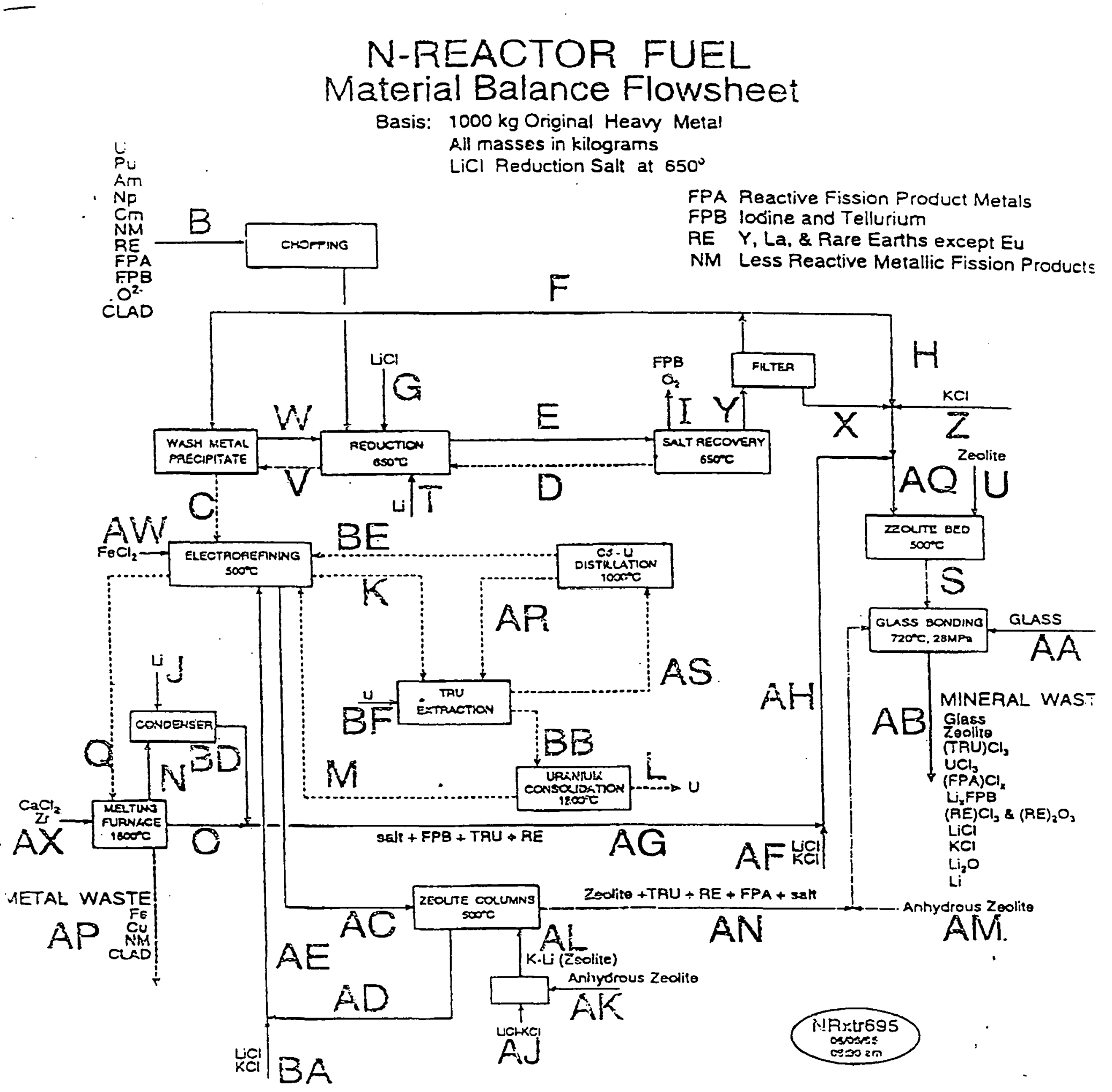




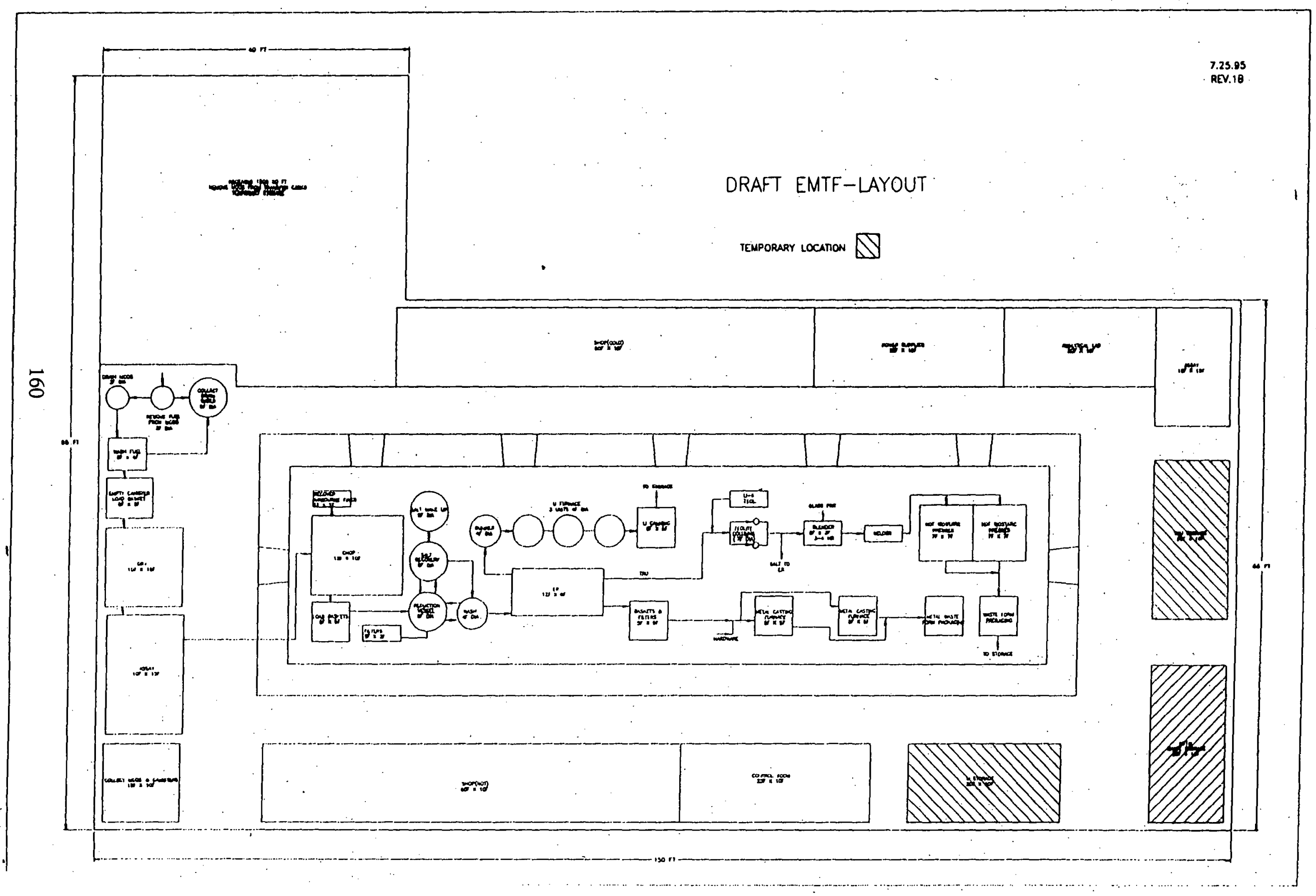




\title{
95-280N -- ENGINEERING EVALUATION OF UTILIZATION OF ANL-W FACILITIES TO SUPPORT BORON NEUTRON CAPTURE THERAPY (BNCT)
}

\author{
Associate Laboratory Director Area: Engineering Research \\ Principal Investigators: \\ G.R. Imel and H.P. Planchon, \\ Engineering Division \\ Funding Profile: \\ FY $1993 \$-0-$ \\ FY $1994 \$-0-$ \\ FY 1995 \$25K \\ FY $1996 \$ 200 \mathrm{~K}$ \\ FY 1997 \$275K
}

Purpose: Boron neutron capture therapy (BNCT) is a promising cancer therapy treatment in which tumor cells are laden with boron compounds; upon neutron irradiation, the resultant $(\mathrm{n}, \alpha)$ reaction deposits a large amount of energy locally to the tumor without large amounts of whole body radiation. The purpose of this study is to evaluate the feasibility of using of ANL-W facilities to perform tests important to BNCT development and/or to actually provide this therapy. Two facilities in particular will be the primary focus: NRAD and TREAT. The evaluation will address the areas in which the specific capabilities of these facilities can contribute uniquely to the medical research in BNCT. For example, TREAT is unique in its ability to provide large depositions of energy over a wide range of time scales (other reactor types such as TRIGA's can have high powers, but for much shorter time durations leading to inadequate total energy deposition). This capability may allow studies of rate effects in treatment. NRAD is a TRIGA reactor, and this class of reactor is potentially viable due to the development of new high density neutron filters to yield the correct epithermal spectrum to a target. NRAD could be used for filter evaluations, and potentially cell biology studies.

Approach: The approach is as follows:

1. Create design concepts of filters and collimators for NRAD and TREAT needed to achieve the required flux and spectrum at the proposed target position.

2. Perform a study of utilizing the facilities for BNCT research, with comparisons to utilization of facilities off site. Focus on the unique features of the facilities, particularly regarding contributions to this medical research. Obtain contacts in the medical and biological communities to determine the feasibility of using TREAT or NRAD for cell biological research or treatment of live subjects.

3. Perform experiments in NRAD to verify the calculations of filtered neutron flux for TREAT and NRAD.

4. Design and perform advanced dose deposition experiments in NRAD to verify calculated doses to targets. 
Technical Progress and Results: A total neutron fluence of about $5 \times 10^{12} \cdot \mathrm{n} / \mathrm{cm}^{2}$ between the energies of $10 \mathrm{eV}$ and $10 \mathrm{keV}$ is required for BNCT, with rapid falloff below $10 \mathrm{eV}$ and above $10 \mathrm{keV}$. Calculations have demonstrated that this is achievable with a $500 \mathrm{~kW}$ TRIGA reactor and a 15 minute treatment time. Discussions with medical people have indicated that there may be a rate effect, a shorter treatment times could be desirable.

The work to date has concentrated on the feasibility of using TREAT for BNCT, although calculations have also been performed for TRIGA reactors such as NRAD. The results are encouraging, and can be summarized as follows: based on our calculations to date, TREAT is capable of producing at least as high a beam purity factor as any other reactor studied, at a higher intensity. Additionally, since TREAT is air-cooled, the cost of modification to include neutron filters and collimators is expected to be much less than in a water-cooled reactor.

Specific calculations have been performed comparing three reactor types: TREAT, PBF, and a TRIGA, using several filter types $\left(\mathrm{AlF}_{3} / \mathrm{Al}, \mathrm{Al} / \mathrm{Al}_{2} \mathrm{O}_{3}, \mathrm{Al} / \mathrm{D}_{2} \mathrm{O}\right.$, with and without bismuth filtering. One dimensional transport calculations were utilized for the comparisons (ONEDANT) with the BUGLE-80 cross-section sets (with a few isotopes from BUGLE-93). In this way, the effects of reactor spectrum and filter material are isolated from specific geometrical effects of the reactors. The PBF reactor, and more recently, a TRIGA reactor, have been the "favored" reactors in the BNCT community regarding intensity and beam purity, so relative comparisons were made with TREAT against these other two reactor types. The main points of the analyses are:

- The TREAT spectrum is relatively flat compared to the other reactors---there are plenty of neutrons in the proper energy band.

- Regardless of filter material type, a TRIGA has the highest fast neutron component, followed by PBF; TREAT has the lowest. As the inverse of the fast neutron component dominates the beam purity factor, TREAT has the highest calculated beam purity.

The results of the one-dimensional analyses demonstrate that, based on reactor type and filter type alone, TREAT will provide a superior beam purity factor. Further analyses provide the bridge to a more realistic geometry by doing two-dimensional transport calculations. In order to derive the same conclusions in the two-dimensional analysis, it is necessary to make the filter material essentially cover the core face. Otherwise, fast neutrons entering the collimator from the sides contaminate the beam and degrade the beam purity. It was demonstrated that high beam purities are possible in TREAT if the filter material completely covers the core face---thicknesses of $65 \mathrm{~cm}$ are near optimal. . Filters of $35 \mathrm{~cm}$ of Al followed by $30 \mathrm{~cm}$ of $\mathrm{Al}_{2} \mathrm{O}_{3}$ or $65 \mathrm{~cm}$ of $\mathrm{AlF}_{3}$ were studied; the more exotic $\mathrm{AlF}_{3}$ provided the highest purities and intensities.

Specific Accomplishments: The calculational methods for computing beam purities consistently with other researchers in BNCT have been installed and checked. Calculations have been performed that show TREAT is a feasible facility for performing BNCT research. When finalized, the calculations will be submitted to the next Reactor Physics topical meeting in Saratoga Springs, and possibly at PYSOR 96. Some contacts have been made with the medical community to determine what role TREAT could play in BNCT research. 


\title{
94-032R1 -- INTERPRETING CRACK PROPAGATION IN CRITICAL COMPONENTS OF MECHANIZED SYSTEMS
}

\author{
Associate Laboratory Director Area: Engineering Research \\ Principal Investigators: \\ J.M. Kramer and R.J. DeMelfi, \\ Reactor Engineering Division \\ Funding Profile: \\ FY $1993-0-$ \\ FY $1994 \$ 160.8 \mathrm{~K}$ \\ FY $1995 \$ 137.5 \mathrm{~K}$ \\ FY $1996 \$ 141.0 \mathrm{~K}$ \\ FY $1997-0$ -
}

Purpose: The objective of this research is to develop a scientific methodology for sensing and gauging the remanent life of flawed mechanically-loaded components critical to the operation of mechanized systems. The development of this capability would provide the basis for replacing time-based maintenance schemes with safer and more economical condition-based maintenance procedures in many technologies, including transportation systems and power-plant components. The scientific understanding developed in this research can also be used to devise methods to mitigate conditions that produce system failures. Researchers from both the Navy and railroad $R \& D$ communities have expressed explicit interest in this work with regard to helicopter gearboxes and railroad rails, respectively.

Approach: In recent years, many key industries, e.g. the transportation and power generation industries, have expressed a desire to switch from time-based maintenance to condition-based maintenance of their machinery and structures. The latter implies the ability to electronically track the condition of critical components in service, and to understand the meaning of detected signals with regard to remaining component life. For the mechanically loaded components that are of interest to this research, life-ending failure occurs when initiated flaws (i.e. cracks) propagate to some catastrophic extent at which the component can no longer sustain nominal design loads.

A reliable condition-based maintenance scheme consists of three interdependent parts: sensing the existence of a flaw, "knowing" the nature of the sensed defect, and interpreting this information in terms of remanent life expectancy. This last part is lacking in attention, yet is the stated most pressing issue recognized by prospective sponsors. It is this part of the puzzle that is the focus of this research project. Interpreting the underlying meaning of sensed flaws and their growth relative to component life involves performing both the mechanical analyses that relate crack configuration and size to vibrational spectra and the material property investigations that allow the prediction of crack propagation rate and component failure. The main focus of this research is the extraction and formulation of the mechanical properties of component materials in relation to component life prediction models. Secondarily, necessary work on the mechanical analysis of flawed components has been planned for this project so that the connection can be made between flaw growth and vibrational mode changes. Inasmuch as it is our desire to investigate the behavior of material extracted from real components and relevant systems, and since railroad rails are a readily available source of material fabricated into 
a simple geometry, we decided to perform our mechanical property analyses on railroad rail steels. Samples of as-fabricated rail and rail that was heavily deformed in service were provided by the Association of American Railroads (AAR).

In ductile materials, like railroad steels, crack initiation and propagation occurs because large plastic strain, microscopically localized at flaws within the steel's microstructure, becomes so intense that the material separates at the flaw. Therefore, in order to understand crack propagation in rail steel, it is necessary to know its flow behavior to large values of plastic strain. A rail failure mode typical of what we propose to monitor through the results of this research involves the nucleation and growth of cracks ("shell defects") that start roughly parallel to and $1 / 4$ " below the rail top surface, running in the direction of travel. We have hypothesized that flaw development results from the interaction of the initial mechanical state in the rail head, produced during forming, with strains produced during service loading including localized plastic strain in the presence of irregularities in the rail steel microstructure itself. Since cracks evolve from intense localized plastic flow, it is necessary to have a full model of the flow behavior of the material. The project plan was to measure the flow behavior of specimens extracted from rail samples. To this end, we planned mechanical testing, including microhardness testing, of both virgin rail material and material from heavily serviced rail. Dr. John W. Holland has been our collaborator this year with regard to microhardness testing and microstructural examination of rail samples. We further planned to formulate from this data a large-strain flow law to be incorporated into a model for crack propagation in rail material.

In our research last year we uncovered the fact that, in addition to normal loading during service, shear stresses produced by rail/wheel friction make a significant contribution to crack nucleation in rails. We therefore planned this year, as an additional thrust, to test the feasibility of mitigating this process by reducing friction at the rail gage corner via laser surface treatment. Drs. Keng Leong and Boyd Hunter were our collaborators for this work. A prospective sponsor, the AAR, has expressed considerable interest in this new aspect of the research.

Technical Progress and Results: Last year, with LDRD support, we completed a series of tensile tests on virgin rail material provided by the AAR and extracted a large-strain deformation model for rail in this condition. The same flow law was found applicable within $10 \%$. to samples from all regions of the unused railhead. Microhardness tests performed on a cross-section of the same railhead similarly indicated uniform properties. We used this flow law in connection with a crack propagation model suitable for life prediction calculations. With LDRD support in FY 1995, we performed a series of mechanical tests on used rail samples also provided by the AAR: As in the earlier experiments, testing consisted of micro-hardness scans of the surfaces of railhead cross-sections exposed by cuts perpendicular to the travel direction along the rail, and tensile tests on standard ASTM (small) flat samples cut so that the tensile axis was parallel to the travel direction. The same mathematical formalism that was used to describe flow in virgin material was also found applicable in this case for most regions in the railhead. The exception to this finding was for samples taken.from just below the surface of the upper gage corner, where the flow behavior was observed to be significantly different in the used rail. This observation was verified by additional microhardness measurements made this year on the used railhead cross-section. The used rail sample experienced severe plastic flow at the upper gage corner during service, which was visible to the naked eye. The effect that this deformation 
has on the flow behavior of subsurface material is significant with respect to crack nucleation and propagation.

The Figure shows the results of tensile tests performed on used rail steel specimens. The data (marked by symbols) taken from all across the rail head can, for the most part, be fit (within $10 \%$ ) by a single equation. The fit, derived last year, to the flow behavior of virgin rail is also shown for comparison. The behaviors represented by the two fits are clearly the same, but shifted from one another, as the virgin and used rail are of slightly different steels. The flow law (stress, $\sigma$ vs strain, $\varepsilon$ ) for these two materials is of the form:

$$
\sigma=\sigma_{\mathrm{S}}-\left(\sigma_{\mathrm{S}}-\sigma_{1}\right) \exp \left(-\varepsilon / \varepsilon_{\mathrm{c}}\right),
$$

where $\sigma_{1}$ is the yield stress, $\sigma_{\mathrm{S}}$ is the stress associated with intinite strain (strain exhaustion), and $\varepsilon_{\mathrm{c}}$ is a parameter associated with the hardening rate of the material. The Figure also shows that the flow behavior of the hardened layer in the used rail is such that this region need only deform elastically even when the neighboring material has reached its saturation level $\sigma_{s}$. This can induce severe stress concentrations at the interface between the hardened and "normal" material during service loading and cause crack nucleation to occur there.

Last year we expressed the crack propagation rate per cycle $(\mathrm{da} / \mathrm{dN})$ in terms of $\sigma_{\mathrm{s}}$ :

$$
\mathrm{da} / \mathrm{dN} \propto\left(\sigma_{\mathrm{S}}-\sigma^{*}\right)^{-2}
$$

(see last year's progress report for details). This formula can be used to determine crack advance and to make predictions of remanent life as indicated above. We have added the term $\sigma^{*}$ here to represent the idea that, especially in used rail, the state of the material may be closer to the saturation stress once a crack has nucleated. This would increase the crack propagation rate.

Virgin rail samples were laser "glazed" under various beam conditions to test the feasibility of this process to reduce friction. Under appropriate conditions, a multilayered surface consisting of a very hard [HV (Vickers) 840)] heat effected zone just beneath a surface glaze (HV 800) was formed on the rail substrate (HV 300). There is good reason to believe that these hard, yet ductile, layers will serve to reduce friction and flaw-nucleating shear stresses.

Specific Accomplishments: A mechanistic flow model that was developed last year for virgin rail steel that is useful in failure predictions was also found to be applicable to the case of used rail. However, a hardened layer was discovered in the used rail with significantly different flow properties. This discovery has important ramifications with regard to crack nucleation and propagation and needs to be explored in the final year of this research. The reduction of friction at rail gage corners by laser glazing has been shown to be feasible, and future funding from the AAR to pursue this aspect of the project is likely. The underlying hypothesis for this successful new thrust was a direct result of earlier research findings in this LDRD project. 


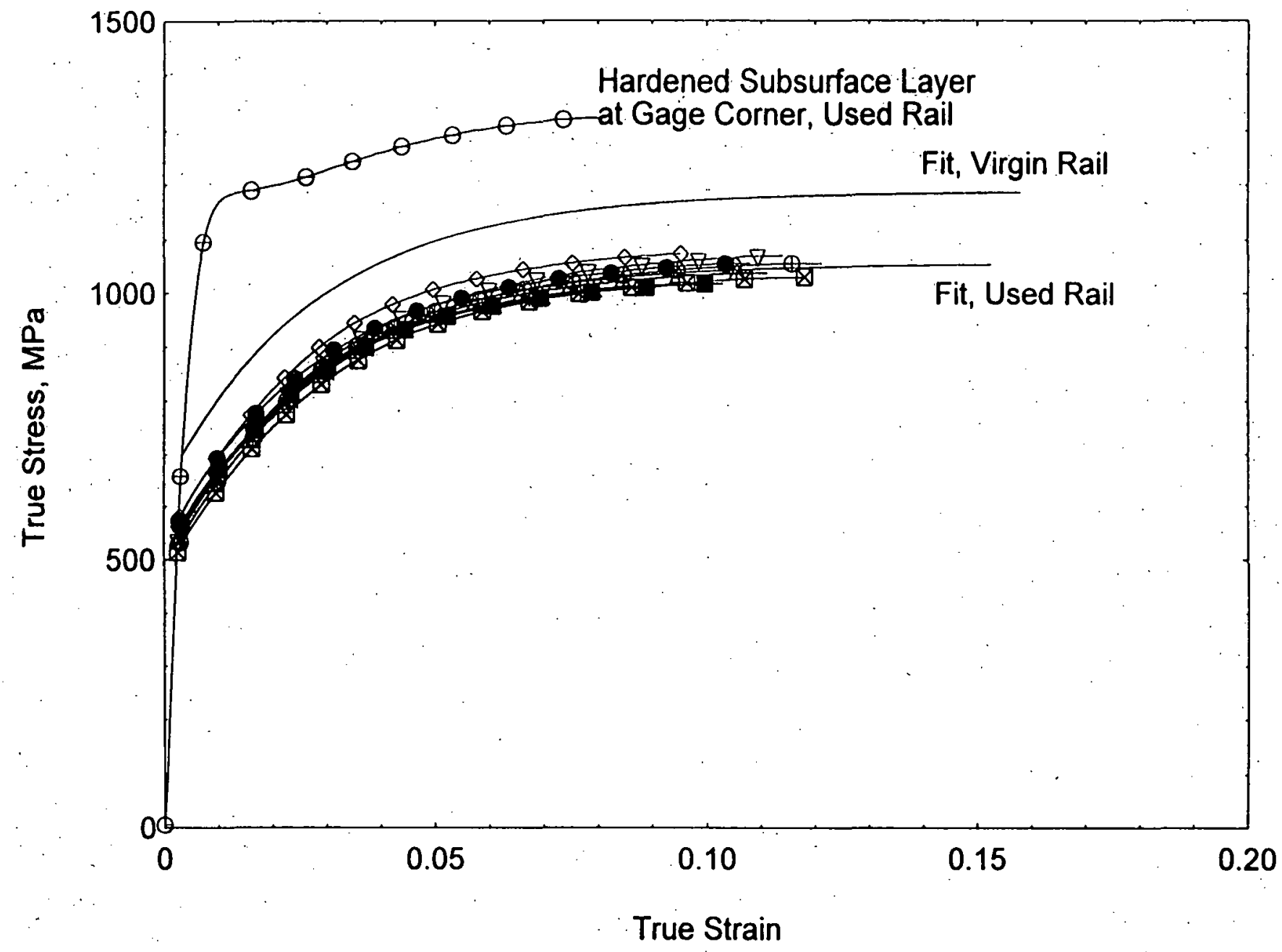




\title{
94-055R1 -- NEW MATERIALS SYNTHESIS STRATEGIES FOR HIGH- TEMPERATURE SUPERCONDUCTORS COMPOSED OF CU-O PLANES SEPARATED BY SILICATE-BASED LAYERS
}

\author{
Associate Laboratory Director Area: Physical Research \\ Principal Investigators: \\ K.A. Carrado and L. Soderholm, \\ Chemistry Division \\ D. Hinks, Materials Science Division \\ Funding Profile: \\ FY $1993-0-$ \\ FY $1994 \$ 155.1 \mathrm{~K}$ \\ FY $1995 \$ 25.2 \mathrm{~K}$ \\ FY $1996-0-$ \\ FY $1997-0-$
}

Purpose: This initiative explores new synthetic strategies for the assembly of the superconducting $\mathrm{Cu}-\mathrm{O}$ planes that are essential to the class of so-called 1-2-3 high temperature superconductors. The unique link underpinning these strategies is the use of silicate-based layers to separate the $\mathrm{Cu}-\mathrm{O}$ planes. The use of silicate-based layers allows for control of electron dopants and flexibility in spacing between the planes. This research represents a distinctly new approach to preparing materials of potential as high-temperature cuprate superconductors. The initiative offers the opportunity to explore alternative mechanisms to the formation of high- $T_{c}$ compounds, an area in which any advancement could have critical technological importance.

Approach: High-temperature cuprate superconductor materials are most often prepared by tradilional ceramic methods. These "shake and bake" techniques involve mixing and grinding of the component oxides, carbonates or other salts, and heating the mixture at a very high temperature. While the procedures are relatively simple, the products suffer the disadvantages of inhomogeneity with a loss of chemical control over the reaction mechanism. The exploration of strategies that offer more direct ways to monitor and control the synthesis is therefore warranted.

All known copper oxide superconductors contain two-dimensional $\mathrm{CuO}_{2}$ layers. These layers are separated by metal oxygen layers, $\mathrm{AO}$, which form $\mathrm{AO}-\mathrm{CuO}_{2}-\mathrm{AO}$ structural blocks. When large amounts of gallium or aluminum are incorporated, the new single-layer copper compounds $\mathrm{IaSrCuAlO}_{5}$ and $\mathrm{LaSrCuGaO}_{5}$ are formed. These structures reflect the preference for tetrahedral coordination by the group 13 elements. The occurrence of oxide tetrahedra as "spacer" blocks has been demonstrated by the gallate materials, which serves as a precedent for the consideration of silicate tetrahedra.

There are many naturally-occurring layered silicate minerals for which laboratory synthetic procedures have been developed. The scope of this work is to create two-dimensional arrays of pre-existing minerals composed of $\mathrm{SiO}_{2}$ tetrahedra in sheets that are modified with the precursors to superconducting oxides. Such an approach allows for variability between superconducting $\mathrm{Cu}-\mathrm{O}$ planes since minerals consisting of one, two, or more silicate sheets can 
be used for separation. The proper firing conditions for creating a $\mathrm{Cu}-\mathrm{O}$ plane must then be determined, followed by characterization of the resulting materials physical properties.

The project methodology involves the systematic application of known clay modification procedures to precursors of superconducting oxides. Once materials are made, they are characterized by $\mathrm{X}$-ray powder diffraction, scanning electron microscopy with energy dispersive $\mathrm{X}$-ray analysis, thermal gravimetric analysis, differential thermal analysis, and microanalysis. Further, X-ray absorption spectroscopy (XAS) can be employed to examine the short-range structural and electronic information about the copper ions, especially after changes due to thermal treatments.

Technical Progress and Results: Suitable materials critical to the development of this program were prepared in the previous year. To be suitable, a large amount of copper ions $(>10 \%)$ had to be introduced within the gallery space of layered silicate minerals. Two methods were found to be successful. One was the use of a high charge density called fluorohectorite, which loaded up to $20 \%$ copper by standard ion-exchange techniques (Cu-hect). Another strategy involved using a $\mathrm{Cu}$-acetate precursor species in order to favor the successful incorporation of Cu-hydroxy complexes into a montmorillonite clay ( $\mathrm{CuAcOH}-\mathrm{mont})$.

Thermal gravimetric analysis and differential thermal analysis data were collected in order to determine the conditions necessary for creating a $\mathrm{Cu}-\mathrm{O}$ plane within the layered silicate materials. Copper metal clusters were made under reducing conditions and copper oxide clusters were made under oxidizing and inert atmospheres. Such ideas are very similar to those in the field of heterogeneous catalysis wherein metals on supports are sintered to form catalytically active centers.

Innovative X-ray absorption spectroscopy (XAS) experiments were carried out at SSRL to probe the short-range ordering around the $\mathrm{Cu}$ centers before, during, and after thermal treatment. Samples were sintered in a controlled atmosphere while monitoring the oxidation state and coordination of copper within the silicate layers. By changing the gas environment over the clay from reducing to oxidizing after $\mathrm{Cu}$ metal clusters are formed, the growth of $\mathrm{Cu}-\mathrm{O}$ sheets is possible. Indeed, the clustering of $\mathrm{Cu}$ ions in the reducing atmosphere was observed: A wealth of structural information can be gleaned from such XAS experiments as copper aggregates form and potentially become sheet-like. Special equipment was designed and assembled in order to probe, in situ, the effects of heating on the copper coordination sphere.

Preliminary data analysis has revealed differences in the copper data throughout the progression of the above described experiment. However, due to the limited amount of funding allotted to this program, complete data analysis was not possible during FY95. The data collected to date will be analyzed, and a manuscript will be prepared, in FY96.

What couldn't be done due to limited funding: The next step in terms of thermal characterization is to determine the stability of the copper metal clusters within the clay layers, and to inhibit their migration to the outside surfaces. 
As a complement to the XAS experiments, the process of metal cluster to oxide sheet formation can also be monitored in part by in situ X-ray powder diffraction methods, a capability already in-house.

The use of smectite-type minerals also allows for the incorporation of other transition metal ions within the interlayer. Ions of such metals as $\mathrm{Ca}, \mathrm{Y}, \mathrm{Ln}$, etc., are all known to be stable within this region, and can be added to vary the electronic properties of the $\mathrm{Cu}-\mathrm{O}$ plane. These dopant ions also provide the benefit of compensating the charge on the silicate layers once the $\mathrm{Cu}(\mathrm{II})$ ions become incorporated into a $\mathrm{Cu}-\mathrm{O}$ sheet and are no longer capable of doing so. In all of the synthetic scenarios, the silicate mineral acts as a pre-existing spacer group, and also a template for $\mathrm{Cu}-\mathrm{O}$ plane formation.

Specific Accomplishments: Nothing to report. 
blank page 


\title{
95-063N -- MODELING AND APPLICATIONS OF COMPOSITE STRUCTURES
}

\author{
Associate Laboratory Director Area: Engineering Research \\ Principal Investigators: \\ D.Y. Pan, E.J. Plaskacz, \\ and R.A. Uras, \\ Reactor Engineering Division \\ Funding Profile: \\ FY $1993-0-$ \\ FY $1994-0-$ \\ FY $1995 \$ 188.0 \mathrm{~K}$ \\ FY $1996 \$ 200.0 \mathrm{~K}$ \\ FY $1997-0-$
}

Purpose: The goal of this project is to investigate innovative ways of using composites and to extend the current ANL structural modeling capability into the forefront of composite structural simulation.

Approach: Modern composite materials have been used in a wide variety of structures. A well-known example is the fuselage of the Stealth bomber aircraft. Although composite materials were mostly used in the aerospace industry initially, recent interest has resulted in many new ways of using composite material. The National Institute of Science and Technology (NIST) has identified automobile, oil drilling and infrastructure as three main areas for composite application in the Advanced Technology Program (ATP). Thirteen multi-year projects have been funded with a total budget of about 103 million dollars. Other agencies are also funding composite materials $\mathrm{R} \& \mathrm{D}$. The most ambitious project in infrastructure to date is a 450 feet composite bridge to be built by the University of California at. San Diego at a cost of 55 million dollars.

Composite materials have many advantages. First, a reinforced plastic component does not corrode. Second, it can be snapped together and be bonded by glue to simplify the construction. Third, the fibcrs in the composites can he arranged to give strength in the desired direction which may increase survivability and reduce weight. The payoff may be large in some areas which can use the unique features of composite materials. Much of the technology developed appears to be applicable to nuclear safety or decontamination and decommission (D\&D) work. For example, composites column repair technology can be used to strengthen the structure to improve seismic survivability of an aging nuclear plant or to prepare a decontaminated structure for new use.

The simulation and prediction of macroscopic behavior of metallic structural components in both the linear and nonlinear material behavior range has been extensively studied and is present in a large majority of finite element packages. In sharp contrast, models for the prediction of structures and components made of plastics and fiber-reinforced composites are still emerging. This project will develop constitutive models and finite elements for composites capable of simulating large structures. The activity will give ANL the capability to simulate a variety of composite structures. The project will then take a step further to link neural networks and the ANL codes to characterize and predict composite behavior. This new approach has the potential 
ability to capture causality and trends in the input data as well as being less costly. Parallel to the modeling effort, the project identifies areas for potential application of composite materials. Potential areas should have high pay offs by utilizing the unique properties of composite materials.

Technical Progress and Results: The project was funded in FY95 with the following accomplishments.

Finite Element Developments. A finite element formulation which utilizes the directional reduced integration (DRI) concept was developed for simulating thin layered structures. The methodology eliminates the difficulties of shear locking and non-physical modes which reduce efficiency and accuracy. The developed finite element code was validated against available linear elastic analytical solutions. Finite element models of beams under various loadings were developed. The results of finite element models using the DRI are compared with those using the full integration (FI) scheme. The advantage of the DRI is clearly demonstrated in Table 1 , where the ideal value is 1 .

Table 1. Comparison of the Directional Reduced Integration (DRI) with the Full Integration (FI)

\begin{tabular}{|c|c|c|c|c|}
\hline & \multicolumn{2}{|c|}{ FI } & \multicolumn{2}{c|}{ DRI } \\
\hline Case* $^{*}$ & $4 \times 4$ Grid & $8 \times 4$ Grid & $4 \times 4$ Grid & $8 \times 4$ Grids \\
\hline A & $0.0050^{* *}$ & 0.0196 & 0.9845 & 0.9962 \\
B & 0.0045 & 0.0195 & 0.9379 & 0.9848 \\
C & 0.0047 & 0.0193 & 0.9009 & 0.9754 \\
D & 0.7211 & 0.9046 & 0.9802 & 0.9921 \\
\hline
\end{tabular}

*Case A: Cantilever beam with a concentrated load at the end.

Case B: Simply. supported beam with a concentrated load at the end.

Case C: Simply supported beam with a uniformly distributed load.

Case D: Thick beam with parabolic shear.

**Maximum deflection normalized by the analytical solution.

Neural Network Based Constitutive Model: Literature search on neural network modeling of constitutive relations was conducted. The theoretical formulation of an eight-node hexahedral element was examined and implemented in a nonlinear explicit transient finite element code. A neural network module has been added to the finite element code.

Composite Structure Research: Two main areas have been identified for further study after extensive literature search. In the area of infrastructure, composite wrapped concrete beams and columns have been identified. The joints at the beam to column intersections are critical areas which have received little attention in the literature thus far. In the area of offshore oil drilling, high pressure tubular structures such as risers, coiled tubing and spoolable flowlines are of current industrial interests. In FY95, unwrapped concrete beams and concrete beams wrapped 
with carbon fiber reinforced plastics (CFRP) have been tested to demonstrate the effectiveness of the composite.

Four square columns (labeled 1A, 1B, 2A, and 2B) were fabricated and tested to failure. All specimens were cast from the same batch of concrete and have the dimensions shown in Fig. 1. The difference in transverse reinforcement between Sets 1 and 2 was chosen so that the columns of Set 1 would fail in flexure, and the columns of Set 2 would fail in shear.

Specimens $1 \mathrm{~A}$ and $2 \mathrm{~A}$ were used as controls and had no CFRP reinforcement. Specimens 1B and $2 \mathrm{~B}$ were externally reinforced using unidirectional CFRP tow sheets. To improve flexural performance (strength and stiffness), column 1B was externally reinforced by two layers of CFRP sheets on the tension face (fibers oriented longitudinally). Both column 1B and column 2B had two layers of CFRP sheets wrapped transversely around the shear spans to improve shear strength. The external CFRP reinforcement schemes for columns $1 B$ and $2 B$ are shown in Fig. 2.

All columns were tested in 4-point bending using a 200,000 lb capacity Tinius Olsen universal testing machine. The midspan load-deflection behavior for the four columns is presented in Fig. 3. The results of the testing have demonstrated that externally bonded CFRP sheets can effectively improve both the flexural and shear performance of the reinforced concrete columns. The limited tests performed showed that significant increases in shear capacity (greater than $76 \%$ in this case) can be achieved by wrapping CFRP sheets around the circumference of square columns. The tests also showed how columns susceptible to brittle shear modes of failure can be externally reinforced such that more ductile flexural failures occur. With regard to flexure, moderate increases in stiffness $(12.5 \%)$ and strength $(17 \%)$ were achieved by applying two layers of CFRP sheets on the columns tensile face.

The positive results of this study suggest that further testing is warranted. Some aspects that should be investigated in future work include: the effect of axial load on shear and flexural capacity, the effect of column shape on retrofit effectiveness, the number of CFRP sheets that can be effectively used, the ability of passive and active confinement to enhance column compression ductility and strength, the development of robust analytical models capable of predicting all possible modes of failure and, therefore, usable for design, and the long-term durability of the CFRP-concrete bond. Finally, the lack of bond failure of the CFRP sheets under such large loads is quite reassuring. This implies that the reinforcement can occur by bonding flat CFRP sheets on four sides without wrapping continuously around the column. This would result in easier application at the beam to column intersections.

Specific Accomplishments: An internal report entitled "Modeling and Applications of Composite Structures" is in preparation. Detailed results are available in the report. 


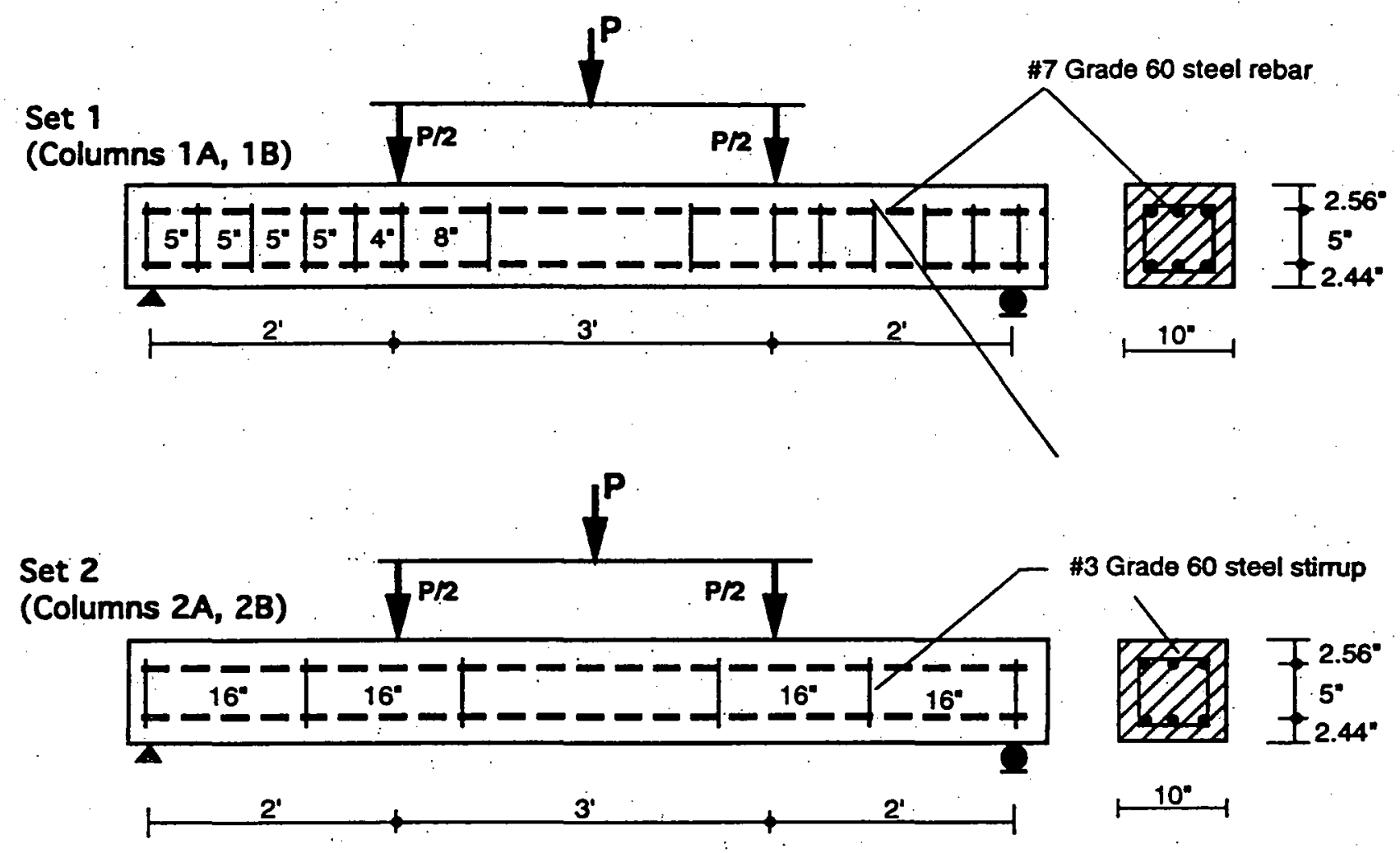

Fig. 1 Dimensions and Loading Configuration of Test Specimens 


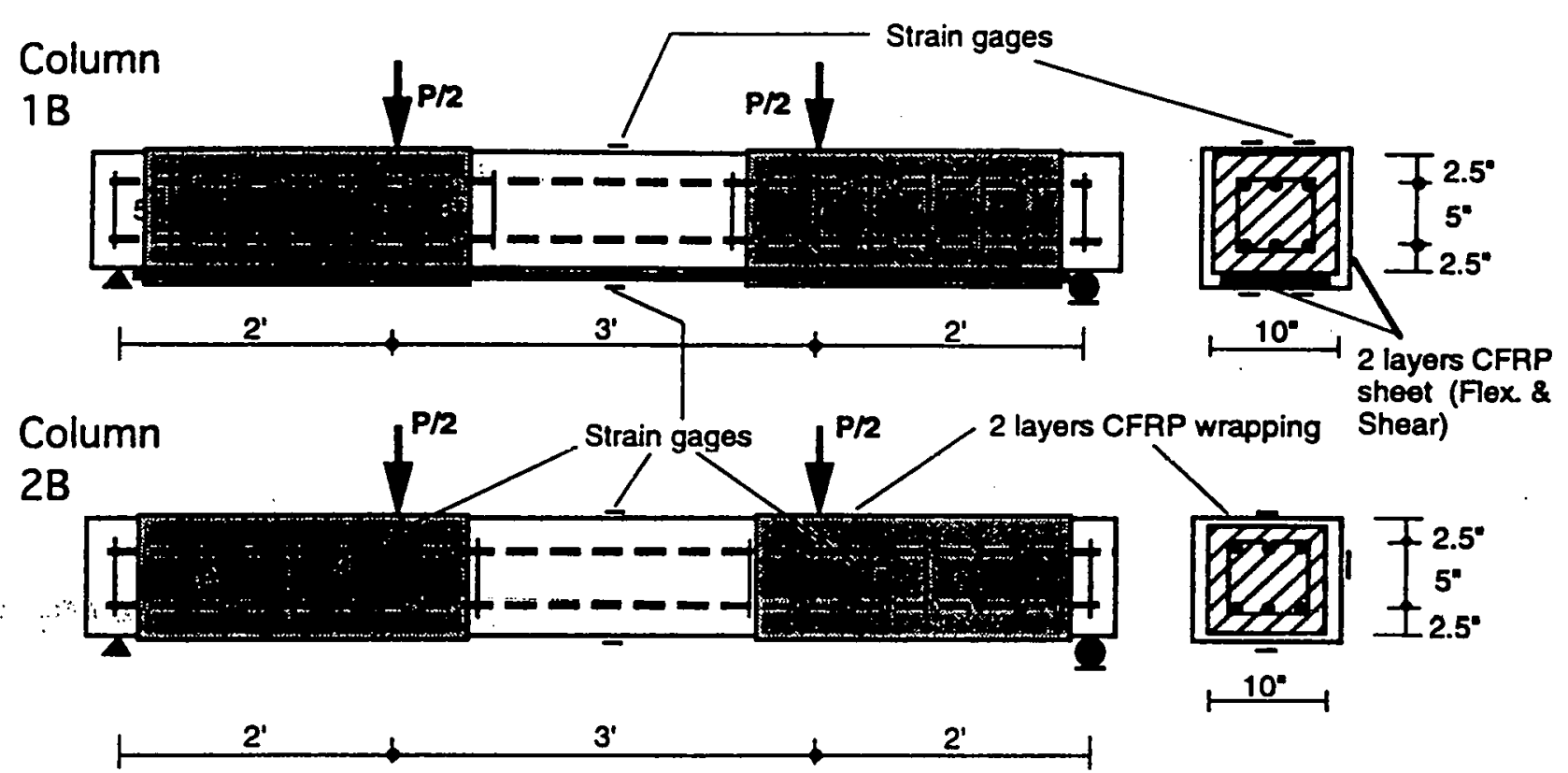

Fig. 2 CFRP External Reinforcement Scheme for Columns 1B and 2B 


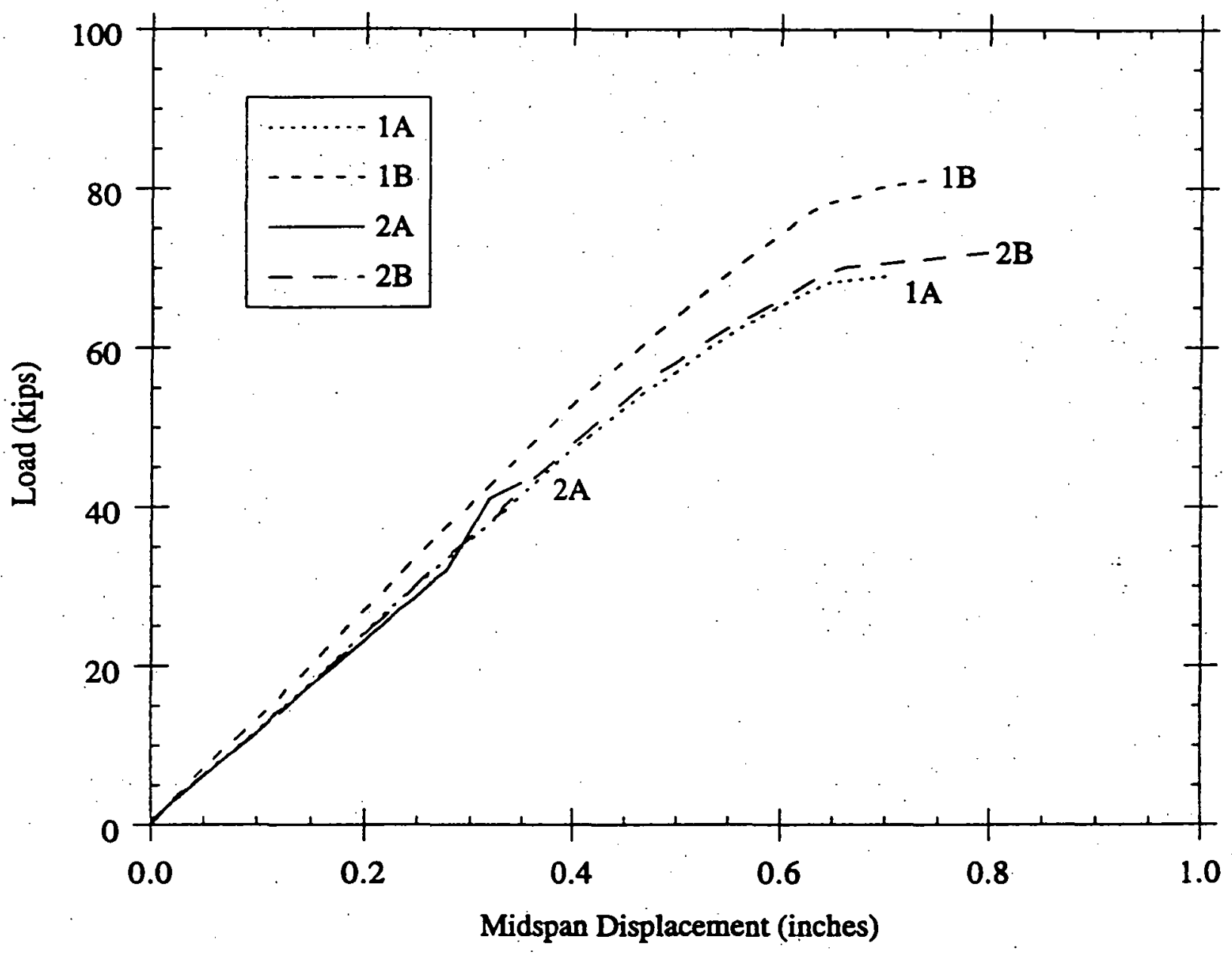

Fig. 3 Load versus Midspan Displacement (Sets 1 and 2) 


\title{
95-160N -- $\quad$ PLASMA SYNTHESIS OF NOVEL CATALYSTS ${ }^{1}$
}

\author{
Associate Laboratory Director Area: Physical Research \\ Principal Investigator: \\ Funding Profile: \\ FY $1993-0$ \\ FY $1994-0-$ \\ FY $1995 \$ 75.2 \mathrm{~K}$ \\ FY $1996-0-$ \\ FY $1997-0$
}

M.B. Knickelbein, Chemistry Division

Purpose: The goal of this effort is to produce and characterize novel, high-dispersion, catalytic materials by exploiting the microwave-driven plasma dissociation of gas phase inorganic and organometallic precursors. This novel strategy is aimed at producing high activity heterogeneous catalysts in which the active metal particles and an inert carbon nanofiber support matrix are generated simultaneously in a dry, single-step synthesis. Introduction of more active and selective catalysts into processes such as hydrodesulfurization of sour crude and catalytic reforming of transportation fuels will benefit the refiner directly by lowering operating expenses, and will contribute to the strategic national goals of pollution remediation and decreasing energy consumption. This exploratory work is targeted toward establishing a larger, long range collaborative effort with a suitable industrial partner such as Amoco or Exxon.

Approach: In 1994, a German group reported ${ }^{1}$ that dispersions of nanometer-size platinum and palladium particles could be generated by laser photolysis of vapor phase organoplatinum and organopalladium complexes, namely bis HFAA platinum and bis HFAA palladium. ${ }^{2}$ The nearly pure platinum and palladium particles, formed by rapid gas-phase aggregation of atoms produced in the plasma, remained segregated within a carbonaceous nanofiber matrix. This matrix, simultaneously produced from condensation of the organic ligand fragments, actually served as an inert, high surface area support for the metal particles. Most notably, these metal particle dispersions were up to a factor of 60 times more active than conventionally prepared catalysts employing these same metals in a model catalytic reaction, the hydrogenolysis of ethane. The potential advantages of employing a microwave discharge over ultraviolet laser light in this application are its scalability, simplicity, and significantly lower cost of operation. In order to assess the feasibility of this scheme, exploratory experiments were conducted in collaboration with John Harkness of Energy Systems Division. Using his fast-flow microwave discharge reactor apparatus, pure organometallic liquids (or solutions of solids in an appropriate solvent such as toluene) were atomized using an ultrasonic atomizer. This organometallic-containing aerosol was injected into a flow steam of argon carrier gas (» 50 Torr total pressure) and the resulting mixture passed through an electrodeless discharge driven by 500-1000 watts of 2450 $\mathrm{MHz}$ microwave power. The soot-like material resulting from the plasma decomposition of the organometallic precursors and solvent (if any) was collected on glass or quartz wool a few inches downstream of the plasma zone, and removed for analysis.

'Individual Investigator Project 
Technical Progress and Results: Our preliminary studies focussed on on three prototypical metal particle systems: iron, cobalt and cobalt/molybdenum ("Co-Mo", the metal combination in a widely used hydrodesulfurization catalyst). The particle size distribution of the samples were characterized using transmission electron microscopy and the effective surface areas were measured using liquid nitrogen physisorption by postdoctoral associate Jim Brenner of the Chemistry Division. These preliminary experiments have demonstrated that high surface area dispersions of iron, cobalt and cobalt/molybdenum can be produced by this method.

Using (neat) iron pentacarbonyl as the precursor, we generated iron particles in the $2-3 \mathrm{~nm}$ diameter range, with surface areas ranging from 50 to $90 \mathrm{~m}^{2} \mathrm{~g}^{-1}$. As demonstrated in the accompanying electron micrograph (Fig. 1), the iron particle size distribution is quite narrow, a desirable attribute for catalysis applications. Similarly, using a mixture of cobalt carbonyl and molybdenum carbonyl precursors in toluene solvent, we have produced dispersions of $\mathrm{Co} / \mathrm{Mo}$ particles with surface area of up to $125 \mathrm{~m}^{2} \mathrm{~g}^{-1}$. The $\mathrm{Fe}, \mathrm{Co}$, and $\mathrm{Co} / \mathrm{Mo}$ samples generated from carbonyl precursors desolved in toluene or tetrahydrofuran were found to contain an amorphous carbon nanofiber matrix, similar to those found in the German laser photolysis study. These results confirm that the metal atoms and carbonaceous fragments condense into distinct, segregated microstructures in these aggregates.

In the production of these samples, a rapid buildup of carbon on the quartz reactor walls occurred, particularly for those samples containing an organic solvent carrier. This carbon film selectively absorbed the microwave power in the cavity, reducing the power delivered to the plasma, and hence limiting dissociations yields to a few percent or less. A straightforward approach to solving this problem involves injecting the organometallic precursor aerosol through a concentric inner injector tube whose exit orifice is slightly downstream of the plasma production zone. Time and expense limitations prevented these modifications from being accomplished during the course of these preliminary experiments, however.

Having successfully demonstrated the feasibility of metal nanoparticle production by this method, we will (pending continuation of funding) focus our next efforts on improving the process yield through apparatus modifications and ultimately on the production and testing of actual catalysts. We will target key catalysis areas including hydrodesulfurization of sour feeds and reforming of transportation fuels. Production of novel bimetallic catalysts, formed from combination of transition metals with known promoters/stabilizers (e.g. tin), will be explored using mixed precursors. The chemical composition, surface area and microstructure of the collected aggregates will be examined. Promising candidates will be tested for catalytic activity and selectivity using simple model reactions such as the hydrodesulfurization of dibenzothiophene and the hydrogenolysis of ethane. These studies will be performed in the recently completed plug-flow reactor catalysis pilot plant located in Building 200 by coinvestigators Chris Marshall and Jim Brenner.

\section{References:}

1. H. Willwohl, J. Wolfrum, V. Zumbach, P. Albers, and K. Seibold, "Production and Characterization of Highly Dispersed Catalytic Active Platinum and Palladium Powders by Excimer Laser Photolysis," J. Phys. Chem. 98:2242 (1994).

2. $\mathrm{HFAA}=1,1,1,5,5,5$-hexafluroacetylacetone $\left(\mathrm{C}_{5} \mathrm{HF}_{6} \mathrm{O}_{2}\right)$.

Specific Accomplishments: At this time there are no specific accomplishments. 


\title{
95-062N -- DEVELOPMENT AND TESTING OF A SIMULATION SYSTEM TO ASSESS AND PREDICT THE STRUCTURAL PROPERTIES OF WELDS
}

\author{
Associate Laboratory Director Area: Engineering Research \\ Principal Investigators: $\quad$ R.F. Kulak, J.L. Binder, \\ Funding Profile: \\ $\begin{array}{lll}\text { FY } 1993-0- & - \\ \text { FY } 1994 & -0- \\ \text { FY } & 1995 & \$ 172.6 \mathrm{~K} \\ \text { FY } & 1996 & \$ 200.0 \mathrm{~K} \\ \text { FY } & 1997 & \$ 200.0 \mathrm{~K}\end{array}$
}

J.M. Kramer, J.J. Sienicki,

E.J. Plaskacz, and B.W. Spencer,

Reactor Engineering Division

Purpose: The purposes of the project are (1) to develop numerical simulation software on parallel computing platforms to model the multidisciplinary aspects of the welding process, (2) to develop an experimental arc welding and visualization laboratory to perform welding research and generate data for use in software validation, and (3) to develop the capability to determine material properties at temperatures ranging from room temperature up to the melting point, which will be used as input for numerical simulations.

Approach: The modern welding process started about 100 years ago and, although much progress has been made, remains a black art that relies on the costly trial-and-error approach for new weldment designs. With today's recent advances in parallel-distributed computing, the numerical simulation of the multidisciplinary welding process could provide optimized weld designs to U.S. industries.

Modern computing algorithms for solving the field equations of continuum mechanics, structural mechanics, and heat conduction are being developed for use on high performance parallel computers (e.g., IBM SP-2) and a network of distributed workstations. In this research project, we expect to make significant contributions to the development of a validated welding simulation environment. Simple arc welding experiments are being performed to obtain accurate data during welding and for post welding distortion measurements. Using the latest in thermalmechanical testing equipment, experimental procedures for finding elevated-temperature material properties are being developed.

Technical Progress and Results: This project is divided into three sub-tasks: (1) the development of a parallel welding computer code, (2) the development of an experimental welding and visualization laboratory, and (3) the determination of the mechanical properties of common metals used in weldments. The progress and results from each of the subtasks are described below.

Parallel Welding Computer Code. For the parallel finite-element code development work of this project, a modular "welding" code is under development that contains a "library" of submodels for weld zone simulation (as developed from experimental work performed below), 
microstructure evolution, heat transfer, and continuum/structural mechanics. The first requirement of thermal stress analysis of weldments is an accurate description of the temperature distribution within the weldment. Since thermal history plays a key role in determining the nature and extent of the microstructural effects and resulting residual stresses and distortion, a thermal analysis module was the first to be developed and incorporated in the preliminary version of the code.

The thermal analysis module was coded to solve the transient heat conduction equation by using explicit integration in the finite element format. Several simple problems were exercised as part of the verification procedure. A finite element model of a plate that is being welded to a second plate along its long edge was simulated. Calculated temperature distribution in the plate with a moving heat source (i.e., the welding rod) were computed for the entire weld length.

Experimental Arc Welding and Visualization Laboratory. The Weld Group took the first steps in setting up an arc welding investigative laboratory by building two rigs using inexpensive, offthe-shelf components. The first rig was a welding rig capable of reproducibly making short butt welds in steel plates. The single axis rig was built using a commercial MIG (Metal-Inert Gas) welder consisting of a 300 A constant voltage power supply, a variable speed wire feeder (5 $30 \mathrm{~cm} / \mathrm{s}$ ), and a welding gun. MIG welding was selected as it is the most common industrial welding process. The welding gun is held in a fixture above a moving table driven by a.ball screw and a stepping motor. The table can move $35 \mathrm{~cm}$ at rates between 0.1 and $10 \mathrm{~cm} / \mathrm{s}$. Motion is controlled by a PC that also records the motion and the voltage and current of the welding process. Modifications were made to the welder to allow independent control of the inert gas supply.

The second rig was a scanning rig made up of a commercial $x-y$ table driven by toothed belts and stepper motors carrying a non-contact displacement sensor. The $\mathrm{x}$ - $\mathrm{y}$ table is supported about $30 \mathrm{~cm}$ above a bench on which the scanning sample is placed. A PC controls the scanning motion in one mm increments and records the position of the displacement sensor and its output, which is the z-distance at each step. Results of a scan are displayed on the operator's PC in real time using pseudo color indications of $z$-distance on an $x-y$ grid. The complete array of measurements is recorded for later analysis.

Mechanical Properties of A36 Steel. Accurate simulations of the welding process, weld distortion and weld integrity will require properties data for the materials of interest at temperatures ranging from room temperature up to the melting point. The material selected for this LDRD study was ASTM A36 steel. The selection was based primarily on the importance of this steel, and similar low alloy steels, in welded structures for the heavy equipment industry. The flexible chemical requirements for A36 steel, which depend on both form and thickness, would be expected to produce a significant heat-to-heat variation in material behavior, especially those properties related to mechanical deformation. Because of this, sufficient material (three 4'x 8 'x 5/16" sheets) was procured to fabricate both the welding samples and the properties samples from the same heat.

The properties work during FY95 concentrated on initiating baseline A36 mechanical properties testing at room temperature. The test samples were cut from the plate in the form of "dog bones" that were machined to a uniform thickness of $1 / 4$ in. with a gauge section that is 3 in. 
long and $1 / 2$ in. wide. The choice of the elongated gauge section and the holes machined in the ends is to facilitate future high-temperature testing where the specimens will have to be gripped by clevis couplings with the extensometer measuring the change in length between the clevis pins. The low temperature testing performed in FY95 used hydraulic wedge grips and a straingauge extensometer clipped to the reduced section. Sample A36W01r was cut from the plate with its axis aligned with the long (rolling) dimension of the plate. Sample A36W02p was cut perpendicular to the rolling direction.

FY 1996 Continuation. This work is expected to continue as follows: (1) heat conduction and continuum mechanics will be coupled and a constitutive model with phase transition will be added to the parallel welding code, (2) temperature and deformation will be obtained from highly controlled welding tests along with weld pool visualization via flash X-rays, and (3) high temperature material response data will be obtained from materials tests.

Specific Accomplishments: Journal publications are anticipated during FY 1996. 


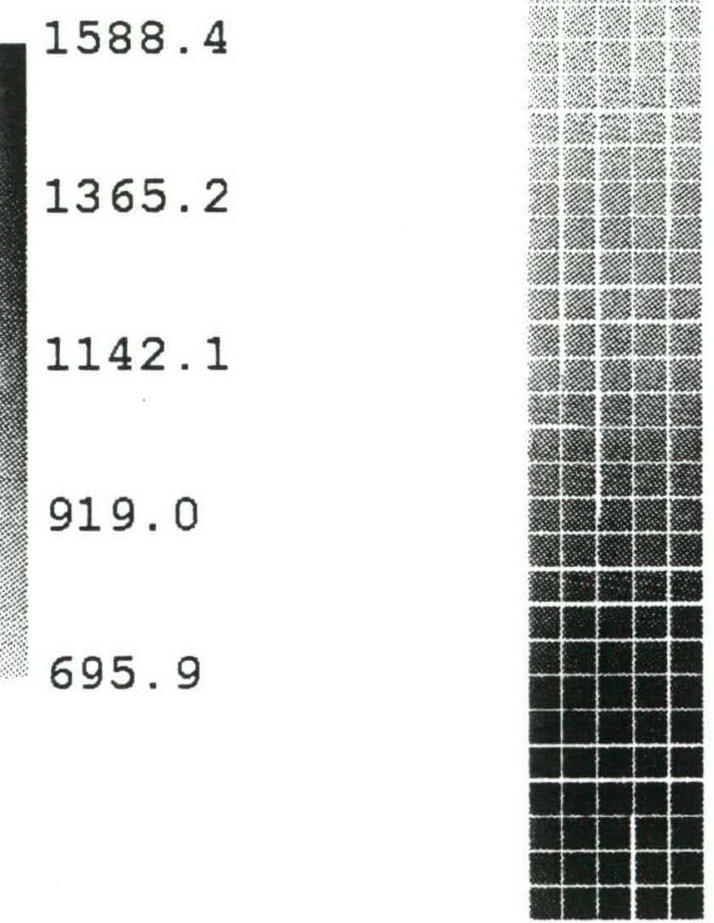

Figure 1. Finite element mesh with temperature profile during welding. 


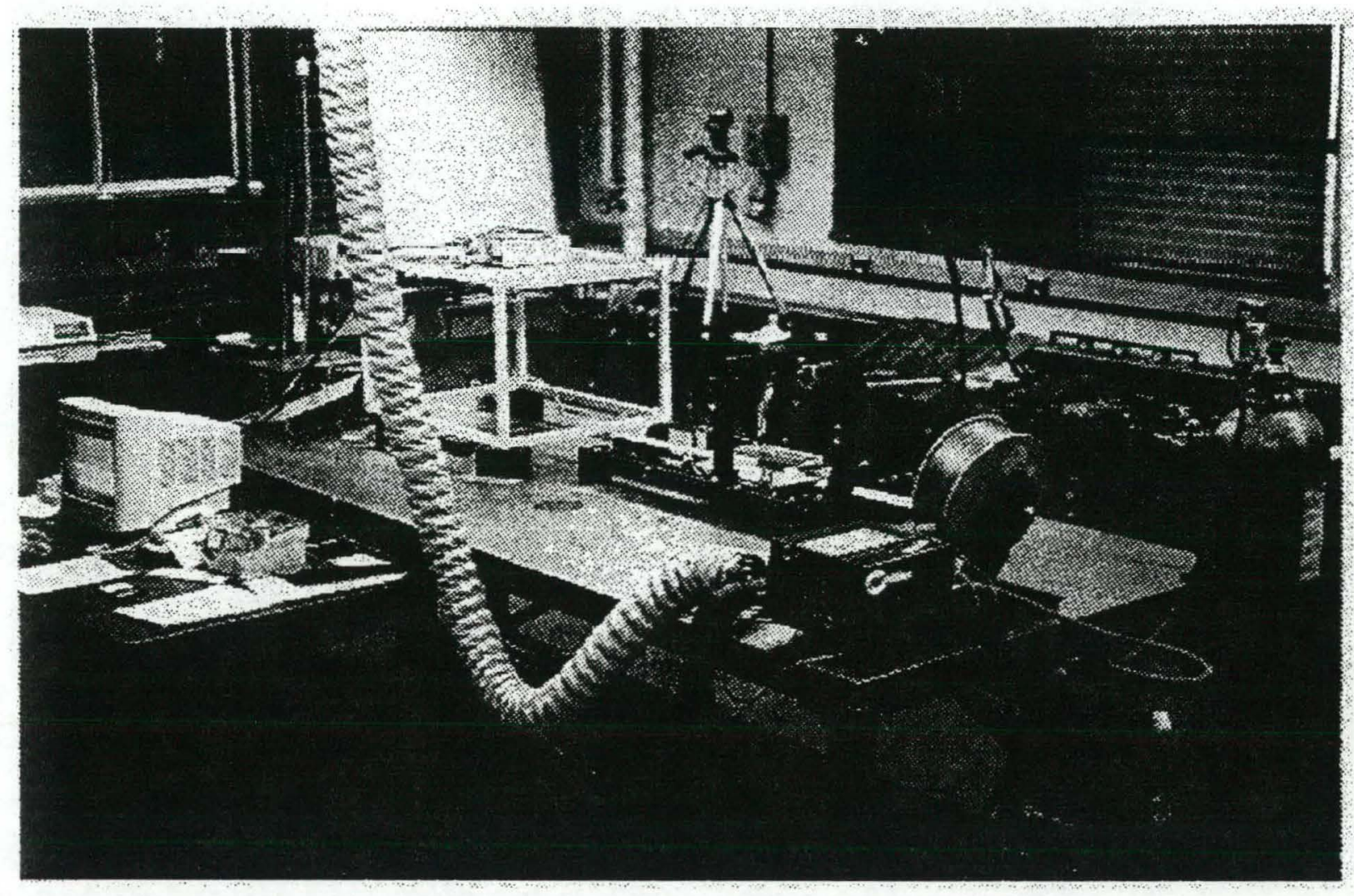

Figure 2. Experimental welding setup. 


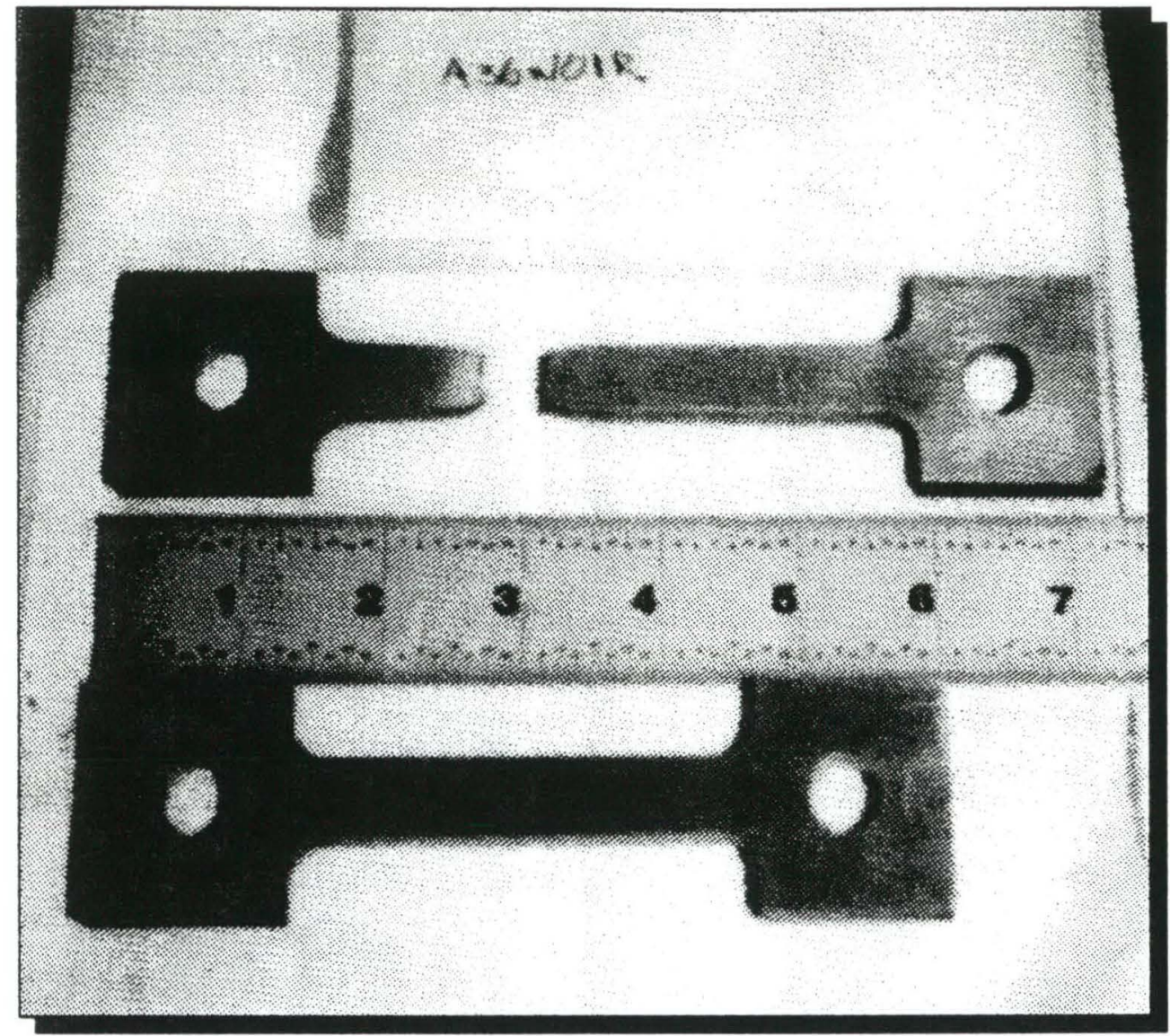

Figure 3. Dog bone shaped test specimens.

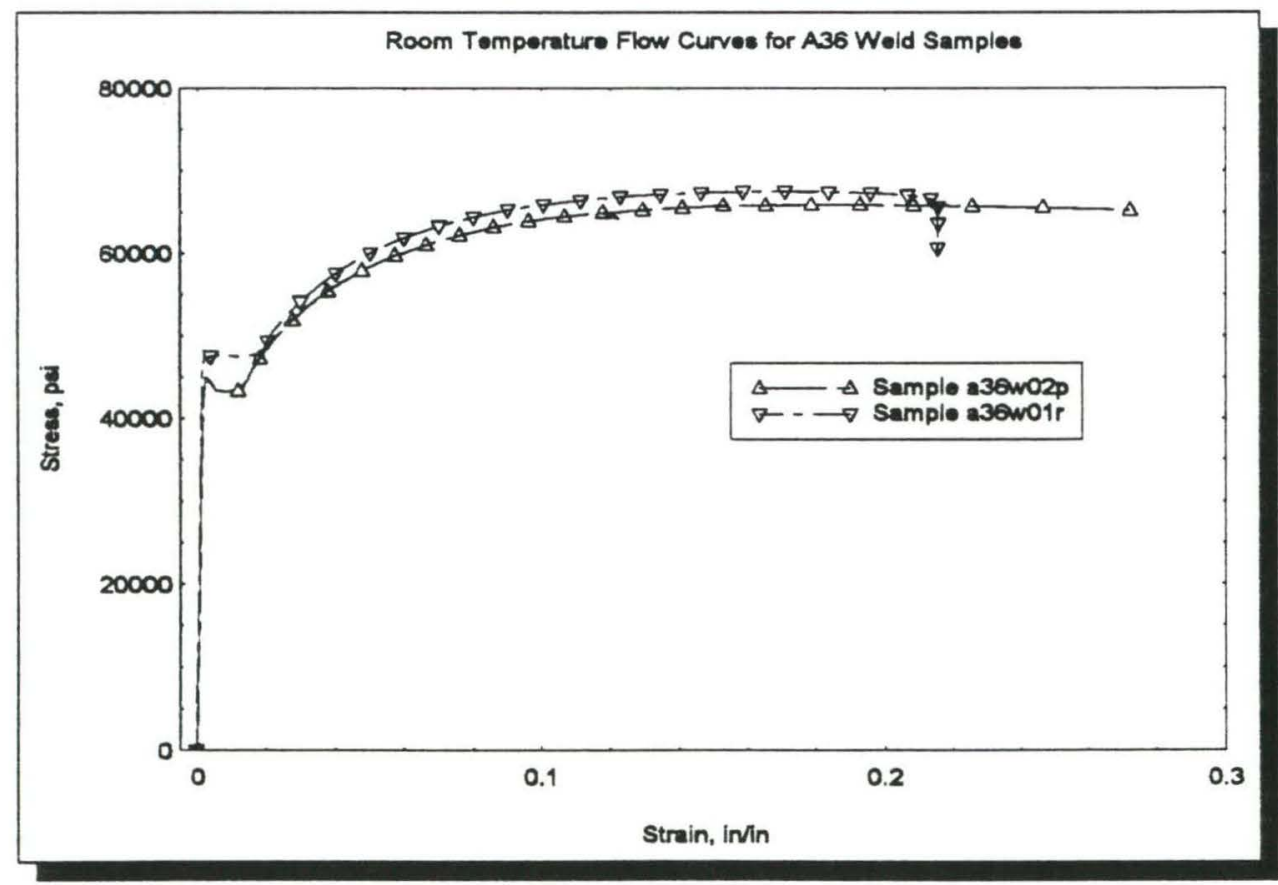

Figure 4. Stress-strain relationship for A36 steel. 


\title{
94-169R1 -- PLASMA MODIFICATION AND CHARACTERIZATION TO IMPROVE MICRO-MACHINING AND ETCHING TECHNOLOGIES ${ }^{1}$
}

\author{
Associate Laboratory Director Area: Physical Research \\ Principal Investigators: \\ Funding Profile:

FY $1993-0-$
FY $1994 \$ 162.4 \mathrm{~K}$
FY $1995 \$ 141.4 \mathrm{~K}$
FY $1996 \$ 70.0 \mathrm{~K}$
FY $1997-0-$

K.R. Lykke and M. Pellin, Chemistry Division

D. Spence, Technology Development Division

Purpose: Building on pioneering work at ANL which allows modification of oxygen, hydrogen and other plasmas to produce high concentrations of reactive neutrals, this proposal seeks to use a unique ability to independently control the plasma ion/neutral ratio as a platform for an increased understanding of anisotropic plasma etching. Key to this effort is an ability to analyze both the gaseous plasma and reactive-etched substrates rapidly and with exquisite sensitivity. The strategic purpose of this work is to develop both uniquely capable and more environmentally-benign manufacturing processes.

Approach: Micromachining and etching technologies involve the production of structural components that have dimensional tolerances of $1 \mu \mathrm{m}$ or less. Most microsystems are currently made from silicon or similar semiconductors for which advanced etching techniques from the microelectronics industry can be applied. Key to the production of these devices is the ability to etch materials with high aspect ratios. That is, to produce narrow, deep trenches.

The microscopic mechanism responsible for high-aspect ratio etching is termed ion-neutral synergy. Although not quantitatively understood, the mechanism is basically one in which etching is performed by reactive neutral atoms that have been directed perpendicular to the surface of the material to be etched by collisions with ions that have been accelerated by a potential applied perpendicular to the working surface. Such anisotropic etching enables microstructures to be fabricated with the large depth-to-width ratios that are essential for the high-density component integration.

At ANL, pioneering work has been made on the modification of oxygen, nitrogen, and other plasmas (similar to those used in etching), such that the degree of dissociation (producing reactive neutral radicals) can be varied at will to give yields from a few percent to more than 50 percent by the addition of environmentally-benign additives such as $\mathrm{N}_{2}$ and $\mathrm{H}_{2} \mathrm{O}$ in concentrations less than one percent. Concomitantly, the production of ionic species can be varied independently by the control of power or gas flow to the reactor.

${ }^{1}$ CCST Project 
Thus, our proposed technique provides independent control of ions and reactive neutrals, which will enable ready optimization of plasma conditions to then achieve optimal etching conditions. Our methods remove the "Edisonian approach" to etching which is the present art in industry.

Technical Progress and Results: All of our measurements are carried out on a source test stand incorporating a high-brightness electron cyclotron resonance (ECR) source and large diagnostic chamber that is located in Building 208. In FY94, this test stand was modified to accept the special diagnostics required for these studies, including a high resolution quadrupole mass spectrometer for plasma diagnostics which was commissioned in FY94. In addition, proofof-principle of our plasma modification technique was demonstrated in $\mathrm{O}_{2}$ plasmas.

Progress and Results in FY95 include:

- Demonstration of significant enhancement in the proton and deuteron fractions of beams extracted from our ECR source by the addition of about $1 \%$ of $\mathrm{H}_{2} \mathrm{O}$ or $\mathrm{D}_{2} \mathrm{O}$ to the hydrogen or deuterium in the source. Enhancements of the proton fraction from about $75 \%$ (which is state-of-the-art) to greater than $95 \%$ has resulted in a major advance in high-current, highpurity, high-emittance $\mathrm{cw}$ proton beam product which has applications to gallium arsenide (GaAs) etching by $\mathrm{H} 2 / \mathrm{CH} 4$ plasmas.

From the point of view of semiconductor manufacture, this breakthrough in proton beam purity has immediate applications in high energy implantation of protons into silicon for the production of insulated gate bipolar transistors (IGBPs), gated turn offs (GTO), and solid state thyratrons. Proton implantation produces trapping centers that reduce the minority carrier lifetime and increases switching speeds. Proton implantation is a much superior technology for the production of trapping centers than use of electron beams (in which case the trapping centers may be annealed out at fairly low temperatures) or chemical diffusion processes (which are difficult to control on a production line). These devices have switching applications ranging from control of small motors to control of the electrical output of entire generation plants, and any increase in efficiency has obvious energy conservation implications.

- In FY95 we completed construction of a novel Ion Scattering Spectroscopy (ISS), which was designed and modeled in FY94 as our chosen technique for the in situ diagnosis of etching and growth of the chip surface.

Our new Ion Scattering Spectrometer (ISS) incorporates a unique ion source for the study of the etching process in real time. The ion source in the ISS is based on laser desorption of metals and other species. The major improvement over all existing ion sources for ISS is that the source incorporates a high-rep-rate Nd:YLF laser which is inherently short-pulsed ( $T<10$ nsec) providing good time (mass) resolution and generates extremely high intensity ion beams (space-charged limited).

This will allow a careful examination of the elemental composition of the surface to be probed. One of the extraordinary features of this technique is the capability to detect elemental constituents on the surface in real time and at relatively high (ambient) pressure, a very elusive capability for the plasma-etch and growth community to the present. 
- We have constructed an $x y z \theta$ sample stage that will allow positioning the sample in front of the plasma modified ECR source. This stage also allows for heating of the substrates.

- We have designed and constructed an all-reflecting microscope objective that will allow for high-resolution imaging $(1<\mu \mathrm{m}$, in situ $)$ of the surface. This feature will be useful in studying the ion-neutral synergy in forming steep walls with high aspect ratios.

In FY96, we expect to fully characterize plasma etching using our advances in plasma modification.

\section{Specific Accomplishments}

1. David Spence and Charles L. Fink, "Production of High Proton Fractions from ECR Sources by Plasma Modification," Proceedings 1994 International Linac Conference. Aug 21-26, Tsukuba, Japan. Vol. 1. pp. 396-397. (FY94 data, unrefereed).

2. David Spence and Keith R. Lykke, "Generation of High Purity cw Proton Beams from Microwave Driven Sources," Proceedings of 1995 International Particle Accelerator Conference (PAC). May 1-5. Dallas, TX. To be published by IEEE. (FY95 results, refereed).

3. D. Spence and K.R. Lykke, Argonne National Laboratory, and J.D. Schneider, J. Sherman, R. Stevens Jr. and D. Hodgkins, Los Alamos National Laboratory, "Production of High Brightness cw Proton Beams with Very High Proton Fractions," Proceedings of 1995 International Conference on Ion Sources (ICIS) Sept. 10-16, Whistler, B.C. Canada and Rev. Sci. Instrum. (In press, FY95, refereed).

4. J. Sherman, J. Bolme, C. Geisik, D. Gilpatrick, K. Hansborough, D. Hodgkins, P. Lara, E. Meyer, M. Needes, J. Power, C. Rose, D. Sandoval, P. Schastall, J.D. Schneider, M. Stettler, R. Stevens, Jr., M. Thout, R. Wright, and T. Zaugg, Los Alamos National Laboratory and D. Spence and G. McMichael, Argonne National Laboratory, "Microwave Proton Source Development for a High-Current LINAC Injector, " invited paper, Proceedings of 1995 International Conf. on Ion Sources (ICIS), Sept. 10-16, Whistler, B.C. Canada and Rev. Sci. Instrum. (In press, FY95, refereed).

5. U.S. Patent applied for by USDOE. David Spence and Keith Lykke, "Improved Method for the Production of Atomic Ion Species from Plasma Ion Sources." 
Fig. 1 Example of plasma modification by additives: Modification of microwave generated $\mathrm{H}_{2}$ plasma by $\mathrm{H}_{2} \mathrm{O}$ additive.
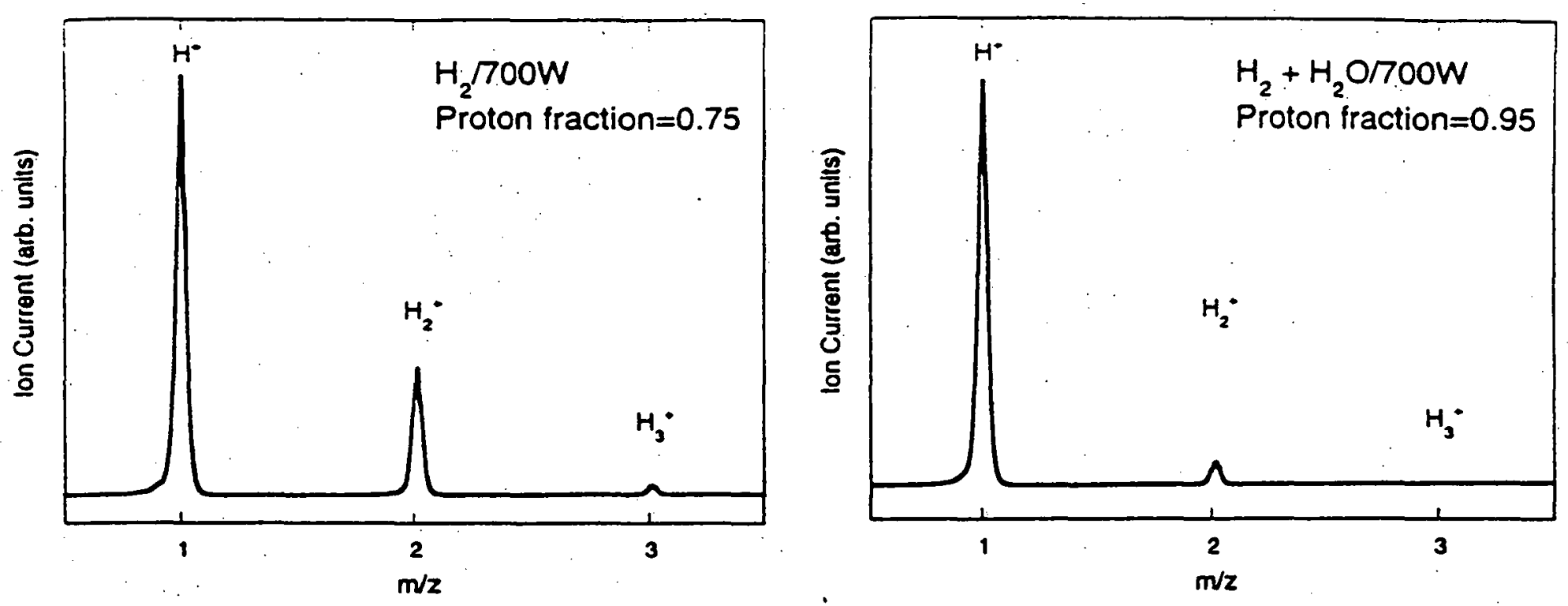

a)

Sampling of microwave generated pure $\mathrm{H}_{2}$ plasma (left) and same plasma with $<1 \% \mathrm{H}_{2} \mathrm{O}$ additive (right). (Spence \& Lykke, ANL)
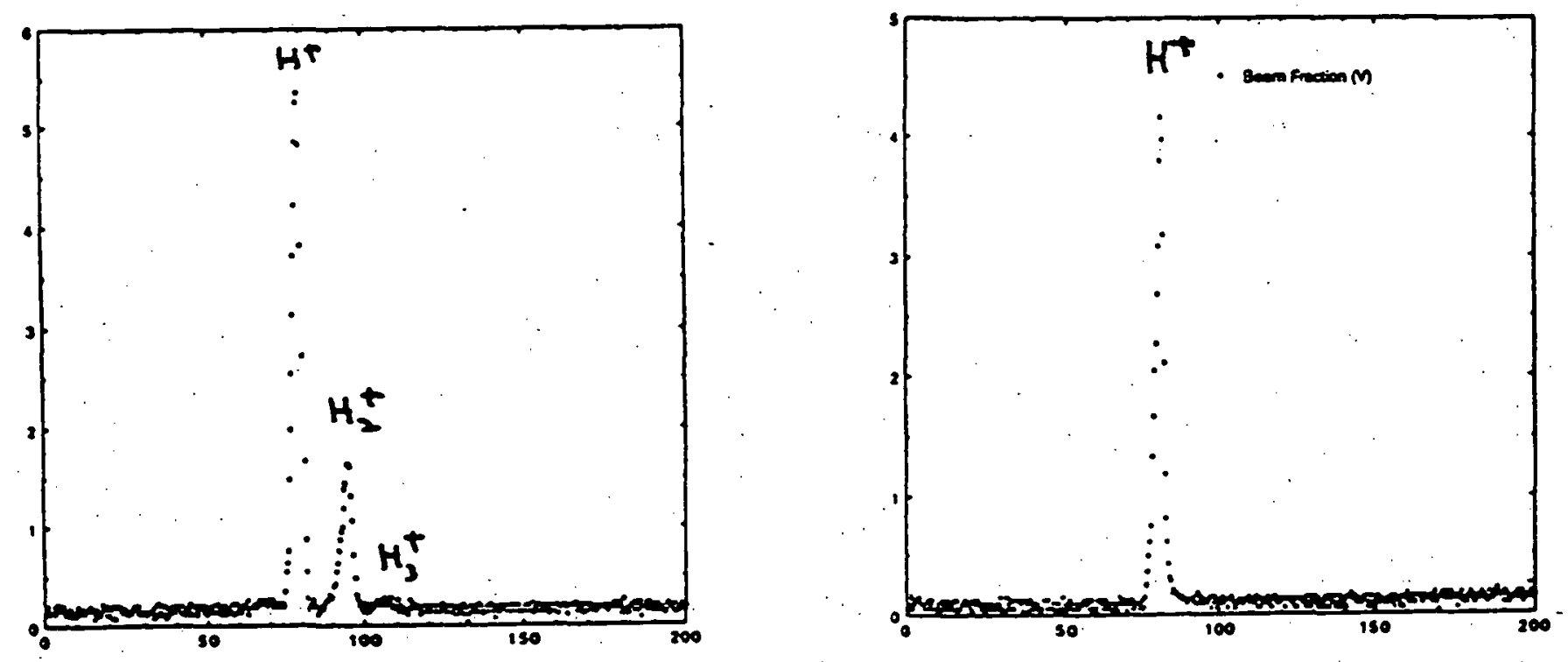

b) Spectrum of high energy (45 kV), high current ( $45 \mathrm{~mA}$ ) $\mathrm{cw}$ ion beam from microwave generated plasma. Pure $\mathrm{H}_{2}$ plasma (left) and same plasma with - $1 \% \mathrm{H}_{2} \mathrm{O}$ additive. Additive produces almost pure proton beam. Joint ANLLANL experiments. 


\title{
95-178N -- HIGH CRITICAL CURRENTS AND PATTERNING BY SPLAYED AND COLUMNAR DEFECTS IN SUPERCONDUCTORS ${ }^{1}$
}

\author{
Associate Laboratory Director Area: · Physical Research \\ Funding Profile:

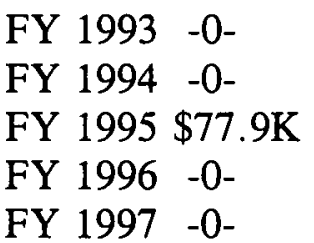

Principal Investigators: $\quad$ W.K. Kwok, G.W. Crabtree, V.M. Vinokur, Materials Science Division

B.G. Glagola, Physics Division

Purpose: The technical objective is to significantly enhance the critical currents in high temperature superconductors in the regime just below their superconducting transition temperature, thus enabling their technological use at these high temperatures. This is achieved via introduction of defects with high energy heavy ion irradiation. This includes amorphous 'columnar' track defects and 'splayed' track defects created from the passage of heavy ions through a high temperature ceramic superconductor. In addition, a sophisticated device to pattern sub-micron sized defect 'channels' is fabricated to study vortex dynamics in the vortex liquid state. This enables the creation and study of model pinning systems with a hydrodynamic model to extract the shear viscosity and friction components of the vortex liquid.

Approach: Since the advent of high temperature superconductors, a new magnetic flux structure, the vortex liquid, has been recognized as the principle barrier to the development of technological applications. This vortex liquid state encompasses a large portion of the magnetic phase diagram in the high temperature-high field region. The characteristic absence of a shear modulus in this state prevents effective flux pinning and the development of high superconducting critical currents for applications. So far, the only available means of effectively pinning the vortex liquid are the columnar defects which are amorphous tracks created by the passage of a high energy heavy ion traveling through the superconductor. The dimensional matching of long flux lines with these line defects serves to effectively trap the vortices within these columnar defects. Although individual vortices may be pinned by a single columnar defect, the high thermal energies at $\mathrm{T}>90 \mathrm{~K}$ permit these vortices to hop out of their pin sites via a creep mechanism. Moreover, the effect of pinning is reduced at high magnetic fields when the density of vortices exceeds the density of columnar defects.

In order to gain insight into an effective means of localizing the vortices in the liquid state, we initiated an investigation with two approaches. First, we studied the geometrical interaction of the vortices with these columnar defects. In order to prevent dissipation from such vortex creep motion, a splayed columnar defect geometry has been proposed to enhance the localization of the vortices. Secondly, measuring the shear viscosity of a vortex bundle can lead to a better

${ }^{1}$ Individual Investigator Project 
understanding of vortex pinning dynamics in the liquid state. The viscosity can be measured by investigating the dynamic flow of vortices within channels created by several rows of columnar defects. For the first initiative, we fabricated a two degree of freedom sample holder which enabled us to insert a sample into the beam line at Argonne's Tandem Linear Accelerator through their beam diagnostic port and to rotate the sample, thereby creating splayed tracks with a variety of spay angles. For the second initiative, we built a sophisticated sub-micron slit and sample translation device to create channels of columnar defects (Figure 1).

Technical Progress and Results: A preliminary working version of both the splayed-defect creation device and the sub-micron channel creation device has been completed. Transport measurements on two crystals of the $\mathrm{T}_{c}>90 \mathrm{~K}$ superconductor $\mathrm{YBa}_{2} \mathrm{Cu}_{3} \mathrm{O}_{7-d}$, prepared with no intrinsic twin boundaries and irradiated with $680 \mathrm{MeV}$ Xenon and $1 \mathrm{GeV}$ Uranium to induce columnar tracks, are shown in Fig. 2. The sharp drop in the angular dependence of the resistivity for fixed field and temperature when the magnetic field is aligned with the columnar defects demonstrates the dramatic pinning capability of the columnar defects in the vortex liquid state. A side issue which arose during our investigation is the pronounced difference between the $\mathrm{Xe}$ and $\mathrm{U}$ irradiations, with the latter showing a much larger pinning accommodation angle. We plan to pursue this effect in our future work. A sophisticated data acquisition program for ac and dc transport measurements using a Macintosh computer graphical interface has been completed. We will look into the possibility of copyrighting the software.

The irradiation experiments were carried out at Argonne's ATLAS facility which requires the submittal of a formal proposal for experiments scheduled in six months intervals. This long time scale has limited the testing of our device to only two times during the fiscal LDRD '95. Experiments to test the patterning device have been scheduled for FY96. In order to obtain additional beam time, we have submitted a proposal to the National Superconducting Cyclotron Laboratory (NSCL) accelerator at Michigan State University. The proposal was recently approved, and we are scheduled to receive beam time for two days in February 1996.

The initial research funded by the LDRD has garnered interests from several other institutions. Among them, Harvard University (Prof. Charles Lieber), University of Chicago (Prof. Tom Rosenbaum, Prof. Heinrich Jaeger), Iowa State University (Prof. Doug Finnemore), University of Kentucky (Prof. Lance DeLong), Western Michigan University (Prof. Lisa Paulius), and University of Madrid-Complutense (Prof. Jose-Luis Vicent) have all requested and sent samples for irradiation studies, resulting in a wide collaborative effort.

\section{Specific Accomplishments:}

Follow-on funding has been obtained from the National Science Foundation through the Science and Technology Center for Superconductivity to continue this work under its "Vortex Dynamics and Critical Currents" theme.

\section{Refereed Journals:}

"Columnar defect pinning in untwinned single crystal $\mathrm{YBa}_{2} \mathrm{Cu}_{3} \mathrm{O}_{7}$, " Physica $C, 235-240$ (1994) 2643-2644. 
"Vortex Localization in Single Crystals of $\mathrm{Tl}_{2} \mathrm{Ba}_{2} \mathrm{CuO}_{6+\mathrm{d}}$ with Columnar Defects, " submitted to Physical Review B.

\section{External Presentations:}

"Ion Track Pinning in $\mathrm{YBa}_{2} \mathrm{Cu}_{3} \mathrm{O}_{7-\mathrm{d}}$ Single Crystals," 1995 March Meeting of the American Physical Society, San Jose, California, March 20-24, 1995.

"Heavy Ion Irradiation in Superconductors, " Workshop on Accelerators in Non-Nuclear Physics, Michigan State University-National Superconducting Cyclotron Laboratory, November 22, 1994. 


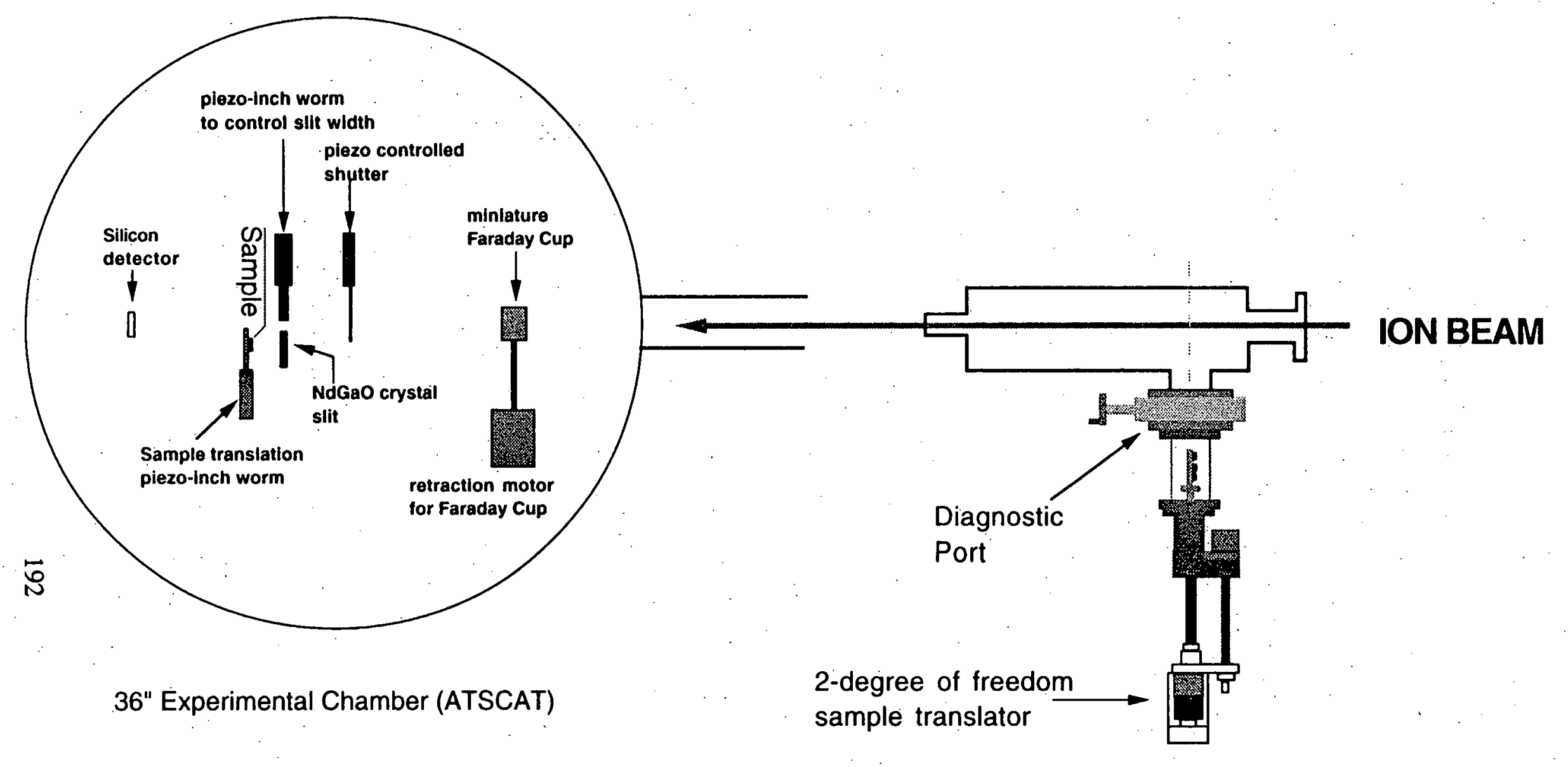

Fig. 1 Experimental Set-up for sub-micron patterning of columnar defects and 'splayed' columnar defects at ATLAS 


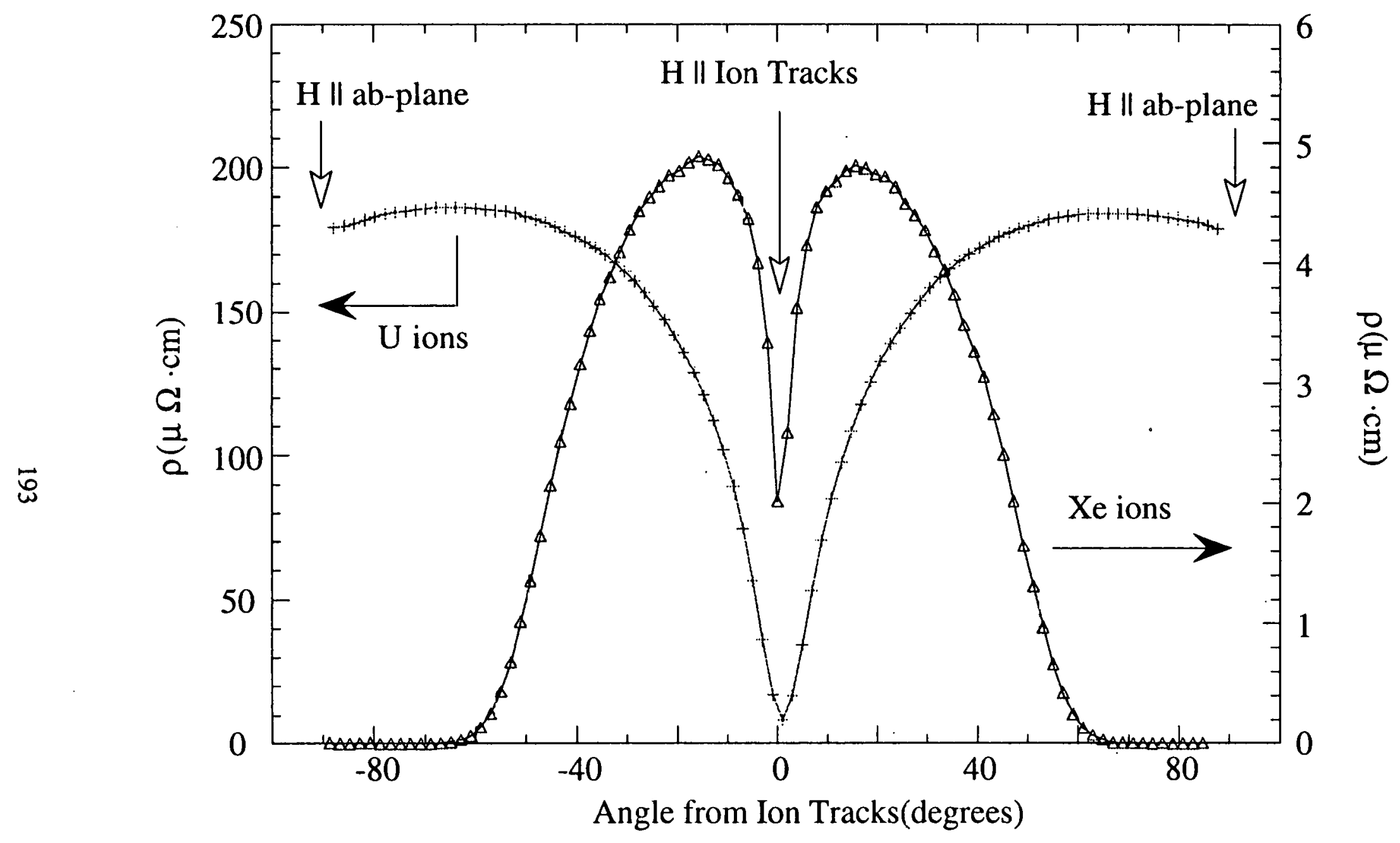

Fig. 2 Angular dependence of the resistivity of $800 \mathrm{MeV}$ Xenon and $1 \mathrm{GeV}$ Uranium irradiated untwinned crystals of $\mathrm{YBa}_{2} \mathrm{Cu}_{3} \mathrm{O}_{7-d}$ 
blank page 


\title{
95-182N -- SYNTHESIS AND CHARACTERIZATION OF NANOCRYSTALLINE BARIUM TITANATE ${ }^{1}$
}

\author{
Associate Laboratory Director Area: Physical Research \\ Principal Investigator: \\ J.A. Eastman, Materials Science Division \\ Funding Profile: \\ FY $1993-0-$ \\ FY $1994-0-$ \\ FY $1995 \$ 75.4 \mathrm{~K}$ \\ FY $1996-0-$ \\ FY $1997-0-$
}

Purpose: Grain size is known to have a strong effect on the dielectric properties of ferroelectric materials. For example, the room temperature dielectric constant is known to typically increase substantially as grain size is decreased below approximately $1 \mathrm{~mm}$. This effect has been exploited to enhance energy storage density and to lower the impedance characteristics for decoupling capacitors applications. The results of previous studies of fine-grained ferroelectrics with grain sizes of 0.1-5 mm have shown considerable scatter and irreproducibility, most likely due to the inability to produce clean, reproducible materials with the wet chemistry synthesis techniques commonly used. The primary purpose of this project was to synthesize ultrafinegrained (nanocrystalline) $\mathrm{BaTiO}_{3}$ with average grain sizes an order of magnitude smaller than previously produced, and to do this using an inherently clean synthesis technique. A secondary objective was to provide theoretical insight into the nature of grain- or particle-size effects on ferroelectricity.

Approach: Barium titanate $\left(\mathrm{BaTiO}_{3}\right)$ is a dielectric material used in numerous technologically important applications, many of which have direct impact on current DOE mission priorities. For example, the development of advanced multi-layer capacitors with high energy-storage density could have significant impact in the current advanced automobile program. More importantly, in the advanced electronics industry there is an urgent need for materials with high local capacitance. This need is exemplified in rapidly evolving multichip module systems, as well as in digital/analog mixed mode electronics. The expected unique high dielectric permittivity of nanocrystalline $\mathrm{BaTiO}_{3}$ makes it potentially desirable for these types of applications. In addition, improvements in selective gas sensors, humidity sensors, and positivetemperature-coefficient resistors are needed for many environmental applications involving site contaminant evaluation, remediation, and monitoring. In order to enhance the performance of these devices, advances in the synthesis and processing of the active materials are required along with improved understanding of the relationship between materials microstructure and properties. Reducing grain size to the $1 \mathrm{~mm}$ range is known to cause a significant increase in the dielectric constant of $\mathrm{BaTiO}_{3}$, but the reasons for this increase are not well understood.

The scope of this investigation involved primarily experimental efforts to produce nanocrystalline $\mathrm{BaTiO}_{3}$ powder, consolidate this powder into a clean, high-density bulk material, determine

${ }^{1}$ Individual Investigator Project 
phase and elemental compositions, and characterize the dielectric behavior of the material. In addition, a theoretical analysis of the effects of dimensionality and size on ferroelectric properties was developed and published. The majority of the work completed to-date on this project has been carried out by $\mathrm{Dr}$. S. Li, a post-doctoral appointee in Argonne's Materials Science Division. Dr. Li was supported by the funds provided through this project and devoted $100 \%$ of his efforts to this experimental study.

A number of experimental methods were employed in the study. Nanocrystalline powders were produced in a state-of-the-art gas-condensation nanocrystalline materials powder preparation system. One of the major advantages of gas-condensation processing is that it is an inherently clean process, involving no solution chemistry. The cleanliness afforded by the gas-condensation process is believed partially responsible for improved properties such as reductions in sintering temperatures of nanocrystalline oxides compared to conventional coarser-grained materials. Powders were sintered to high density under vacuum conditions in an attached compaction unit. Rutherford backscattering (RBS) techniques were employed to characterize the elemental composition of the materials produced. The RBS experiments were performed courtesy of P. Baldo of Argonne's Materials Science Division. X-ray diffraction techniques were used to provide a phase analysis of the powders produced. Transmission electron microscopy was used to determine particle size. Dielectric property measurements are planned, but not yet completed.

Technical Progress and Results: This study presented several experimental challenges. The primary challenge was to determine the proper conditions for fabricating nanocrystalline $\mathrm{BaTiO}_{3}$ while controlling both the grain size and chemistry. While many single-component oxides have been produced previously in Argonne's nanocrystalline materials processing facilities, this was the first attempt to produce a two-component oxide. The vastly different melting temperatures and vapor pressures of barium and titanium make evaporating these two materials in a controlled ratio a difficult task. While barium-titanium-oxide multicomponent materials with grain sizes below $10 \mathrm{~nm}$ have been produced and significant progress has been made towards this milestone, to-date pure single-phase $\mathrm{BaTiO}_{3}$ has not been produced. As seen in the attached Figures 1 and 2 , the material produced so far has been Ti-deficient and consists of a phase mixture of the desired $\mathrm{BaTiO}_{3}$ phase and $\mathrm{Ba}_{2} \mathrm{TiO}_{4}$. While the material produced in the early stages of this study was produced by evaporating $\mathrm{BaO}$ and $\mathrm{Ti}$ simultaneously from separate crucibles, recent studies have shown that the ratio of Ti-to-Ba can be improved by evaporating material from a single evaporation source containing a mixture of coarse-grained $\mathrm{BaTiO}_{3}$ and $\mathrm{Ti}$ metal. It is anticipated that nanocrystalline $\mathrm{BaTiO}_{3}$ powders will soon be obtained if we continue to increase the amount of $\mathrm{Ti}$ in the starting materials.

Materials produced to date have also been found to be oxygen deficient. This problem was solved, however, by annealing powders ex situ in air or oxygen. Little or no grain growth was found to accompany these annealing treatments. If further funding is forthcoming, we plan to produce nanocrystalline $\mathrm{BaTiO}_{3}$ by evaporating in an oxygen environment rather than the helium environment used to-date. It is expected that single phase $\mathrm{BaTiO}_{3}$ would be produced within the next few months. Initial dielectric constant measurements would also be done.

The ferroelectric behavior of nanocrystalline materials was also studied theoretically as part of this project. Since the issue investigated is of general interest, a phenomenological model was adapted to describe dielectric behavior in nanocrystalline ferroelectrics by considering interfacial 
interactions rather than an explicit microscopic model or computing simulation. This analysis has shown that the phase-transition behavior in nanocrystalline systems are controlled by the grain size and the presence of surrounding interfacial boundaries. An elastic interaction can substantially influence the features of the phase transition, resulting in both a shift and a broadening of the transition temperature. The transition temperature of $\mathrm{BaTiO}_{3}$ was found to shift towards lower temperatures than the Curie temperature of conventional coarse-grained material. Furthermore a substantial increase in dielectric permittivity below the Curie temperature was predicted and attributed to the same shift and broadening of the phase transition point arising from the finite size effect in fine-grained materials. The results of this theoretical study were recently submitted for publication in an archival journal.

\section{Specific Accomplishments:}

A refereed external publication has been submitted for publication: S. Li, J.A. Eastman, Z. Li, C.M. Foster, R.E. Newnham, and L.E. Cross, "Dimension and Size Effects in Ferroelectrics," submitted to Phys. Rev.B (1995).

An abstract submitted to the Fall Materials Research Society Meeting in November 1995 was accepted for presentation and will be published in a refereed external publication.

A third refereed external publication emphasizing the experimental studies completed in this project is in preparation and will be submitted to an archival journal. 


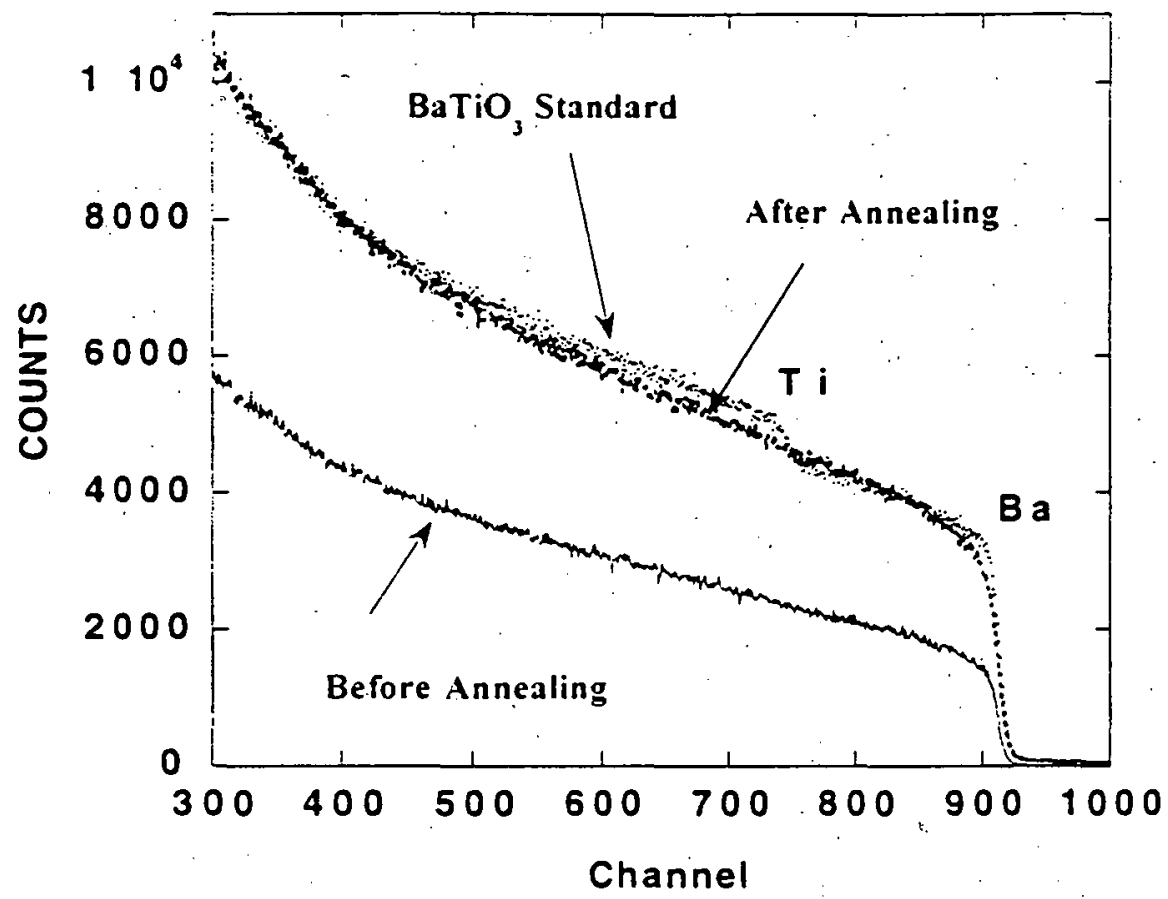

Fig. 1 RBS data showing the effects of low temperature annealing on oxygen concentration for nanocrystalline powders.

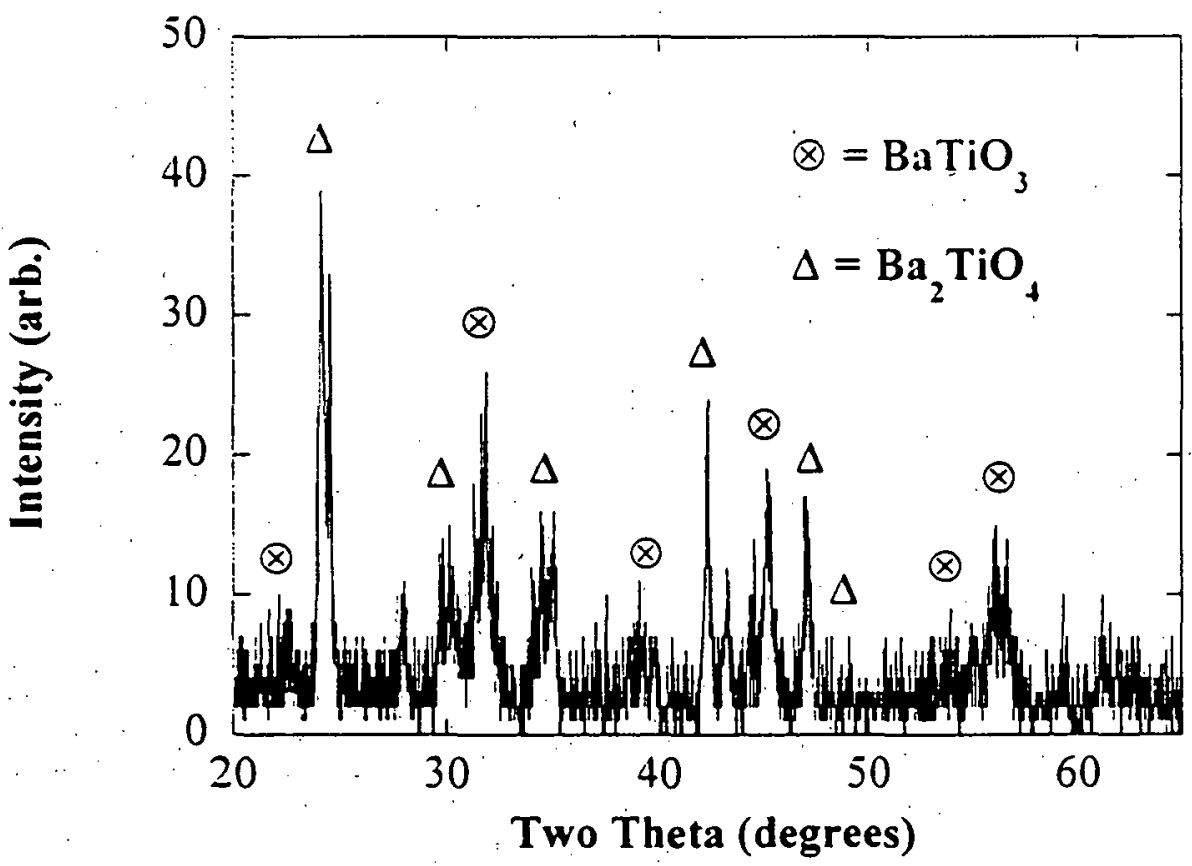

Fig. 2 X-ray diffraction data showing that the nanocrystalline powder produced to-date is a two-phase mixture. 


\title{
95-184N -- EXPLORATORY DEVELOPMENT OF SULFIDE FIBER GLASS MAT ${ }^{1}$
}

\author{
Associate Laboratory Director Area: Energy and Environmental Science and \\ Technology
}

Principal Investigator(s):

T.D. Kaun and M.C. Hash, Chemical Technology Division

Electrochemical Technology Program

Funding Profile:

$\begin{array}{lll}\text { FY } 1993 & -0- \\ \text { FY } 1994 & -0- \\ \text { FY } 1995 & \$ 74.3 \mathrm{~K} \\ \text { FY } 1996 & -0- \\ \text { FY } 1997 & -0-\end{array}$

Purpose: Our LDRD addressed the technical feasibility of producing fiber from newly discovered sulfide ceramic materials. Fibers from sulfide ceramic are inherently stable to extremely corrosive environments of molten salts with lithium and sulfur activity. Therefore, this material is an outstanding prospect for separators in molten salt batteries. The battery's cost and performance relies upon separator properties. The resilient nature of a fiber separator is a great advantage for long cycle life over the conventional $\mathrm{MgO}$ powder separator. In this effort, fiber separator boosted cell power $40 \%$. Additionally, ceramic fiber remains of interest for development as high-strength ceramic composites and as filtration media for use in extremely corrosive environments, such as nuclear waste processing. The sulfides have a better-thanaverage stability to radioactive disintegration. These fibers present a new level of chemical and thermal stability for fibrous materials in high-temperature corrosive environments.

Approach: Ceramic developers are generally interested in the fiber form for a variety of applications. Ceramics with the required chemical stability (e.g., AlN) have quite high melting points, about $2000^{\circ} \mathrm{C}$, and are not available as fibers. At this time, only whiskers of these materials have been made, and they are not commercially available. The opportunity for chemically stable sulfide ceramic fibers arises out of their much lower melting points (850$1050^{\circ} \mathrm{C}$ ), which are in the temperature range of fiberglass technology.

Our initial approach to fiber production examined techniques typical of the fiberglass industry: liquid drooled into fiber, or fiber drawn by elongating a solid bar of ceramic. Several moltenceramic delivery systems and furnace designs were considered. The chill rate of the molten ceramic was a significant issue. Generally, fiber formation was prevented by insufficient glassy transition temperature of the $\mathrm{CaAl}_{2} \mathrm{~S}_{4}$ and by the high surface tension of the molten $\mathrm{CaAl}_{2} \mathrm{~S}_{4}$. These were properties newly identified for this material. From this effort, we learned to make very nice ceramic spheres, which may have applications of their own.

Our ultimate goal is to produce a fiber mat by a process that can be expanded for mass production. We shifted our approach to a process with a more rapid chill rate. A cool spinning

${ }^{1}$ Individual Investigator Project 
wheel pulling a fiber from a molten pool of ceramic was a possible solution. (In a similar processing approach, metal ribbon is produced by a rotating wheel. By this process, tin solder, another material having high molten surface tension, can be made into ribbon by sequential solidification onto a wheel.) We set up a small tungsten arc-melter with a TIG welding torch within our glovebox (Ar). With this arrangement, we formed a small molten pool of sulfide ceramic within a graphite tray. A cool spinning wheel was brought down into the melt. As the wheel touched the molten ceramic, it would pick up and flash chill strands of molten ceramic fiber. The fiber was thrown out from the wheel and accumulated in a collection tray. Fiber properties relied upon the type of metal for the wheel, contour of incident surface, rotation speed of the wheel, and depth of the molten pool. Fibers were subsequently examined by scanning electron microscope (SEM) with their composition verified by energy dispersive spectroscopy (EDS).

Technical Progress and Results: The technical feasibility of creating fiber from sulfide ceramic has been demonstrated. Scanning electron microscopy (SEM) reveals fibers whose length varies up to approximately $3 \mathrm{~mm}$, and whose average diameters varied from 0.02 to $0.05 \mathrm{~mm}$. The thicker fibers tended to show large amounts of grain growth at the surface of the fiber, suggesting a slightly slower cooling than that experienced by the thinner fibers. These thinner fibers show little or no grain growth which implies rapid, homogenous cooling was achieved. From these results, it appears that we can control the morphology of the fibers by varying the drawing/cooling rates. Both types of fiber exhibit the same $\mathrm{CaAl}_{2} \mathrm{~S}_{4}$ composition: that is, no phase separation occurs. In our present process, the rotation speed of the cool wheel that pulls fibers from the melt enables us to control both the diameter, and the surface morphology of the fiber. The smooth, thin fiber morphology would lend itself well to the fabrication of woven components that require good flexibility. The coarser, thicker fibers would lend well for filtration applications.

Our process for producing crystalline fiber employs a unique wheel design. The wheel has a 1-mm-deep groove machined into its surface, with a notch in the surface contour that takes a molten bead from the melt pool and pulls it into a fiber. The fiber forms as the elongated bead is airborne. Some fiber is formed as a "string of pearls" is elongated. In another mechanism, very-uniform fibers are formed as molten ceramic is drawn between two molten ceramic beads. This process has obvious differences from the rotating wheel, ribbon-production process. With a much slower wheel rotation, the ribbon forms by sequential solidification at the chilled wheel surface. On the other hand, the crystalline fiber process, identified in the LDRD, creates fiber from molten material while airborne. A well-rounded fiber diameter is evidence of this fiberformation mechanism.

A primary application of the new fiber material is for separators in bipolar $\mathrm{Li} / \mathrm{FeS}_{2}$ cells for electric vehicle batteries. To assess the performance of such a cell, we used an $\mathrm{Al}_{2} \mathrm{O}_{3}$ fiber substitute. The $\mathrm{Al}_{2} \mathrm{O}_{3}$ has marginal chemical stability in our battery system, but it provided a realistic test of the anticipated fiber separator. The separator properties affects battery power through internal impedance values. Whereas the conventional $\mathrm{MgO}$ powder separator cell produced $1.05 \Omega / \mathrm{cm}^{2}$ impedance, the fiber separator cell impedance was significantly lower at $0.75 \Omega / \mathrm{cm}^{2}$. This reduction in impedance increased the power $40 \%$, (by $\mathrm{V}^{2} / \mathrm{R}$ ) for a thinner, more conductive separator. 
These initial results provide a basis from which to refine the fiber process to develop selective properties. Further characterization would proceed with interaction with other organizations (K. Goretta, Energy Technology Division). The process must be scaled up to produce a significant volume of fiber to proceed with the next development step of mat production. The crystalline nature of the fiber promotes high temperature application: that is, bonded mat production can be approached by a simple sintering operation. The ultimate goal is to supply high quality samples of the sulfide-fiber mat to end-users. Interaction with end-users will specifically define the desired mat properties, which is best approached as an iterative process.

Specific Accomplishments: This LDRD gave us an opportunity to develop a unique material, a sulfide ceramic fiber that has outstanding chemical and thermal stability. This achievement is another mark of ANL's continuing leadership in materials process development. We will provide an invention report and publications to document this advancement.

The sulfide fiber has a specific application for advanced battery separators. Improved power performance was demonstrated in cell tests. The sulfide-fiber production process serves as a foundation for development of resilient mat materials. In addition to high-performance battery separators, the sulfide mat may be useful in process development of electrochemical treatment of radioactive wastes (E.C. Gay, Chemical Technology Division). A mat of sulfide fiber presents interesting filtration prospects.

Unexpected characteristics of the sulfide fiber may provide some unexpected applications. The high-surface-area form of the fiber would be of interest to heterogeneous catalysis, where gas/liquid interfaces are required. (This property is specifically required in fuel cell electrodes.) Lastly, our experience in producing crystalline fiber may be extended to other ceramics with similar physical properties. 


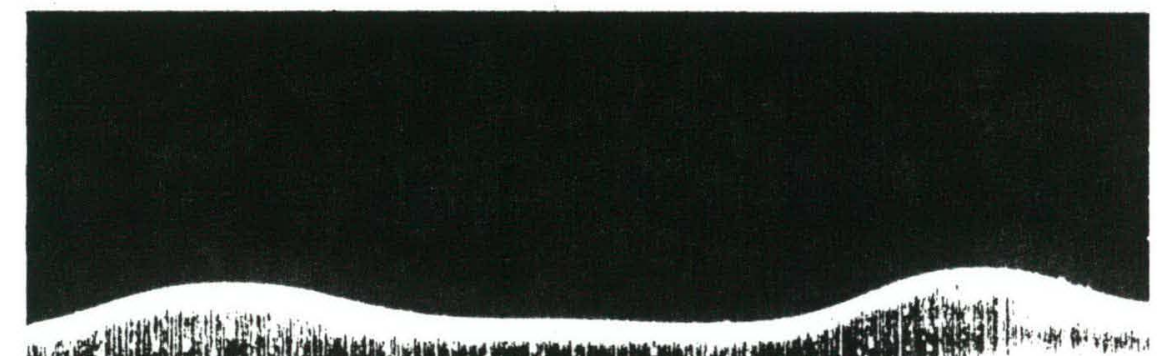

How
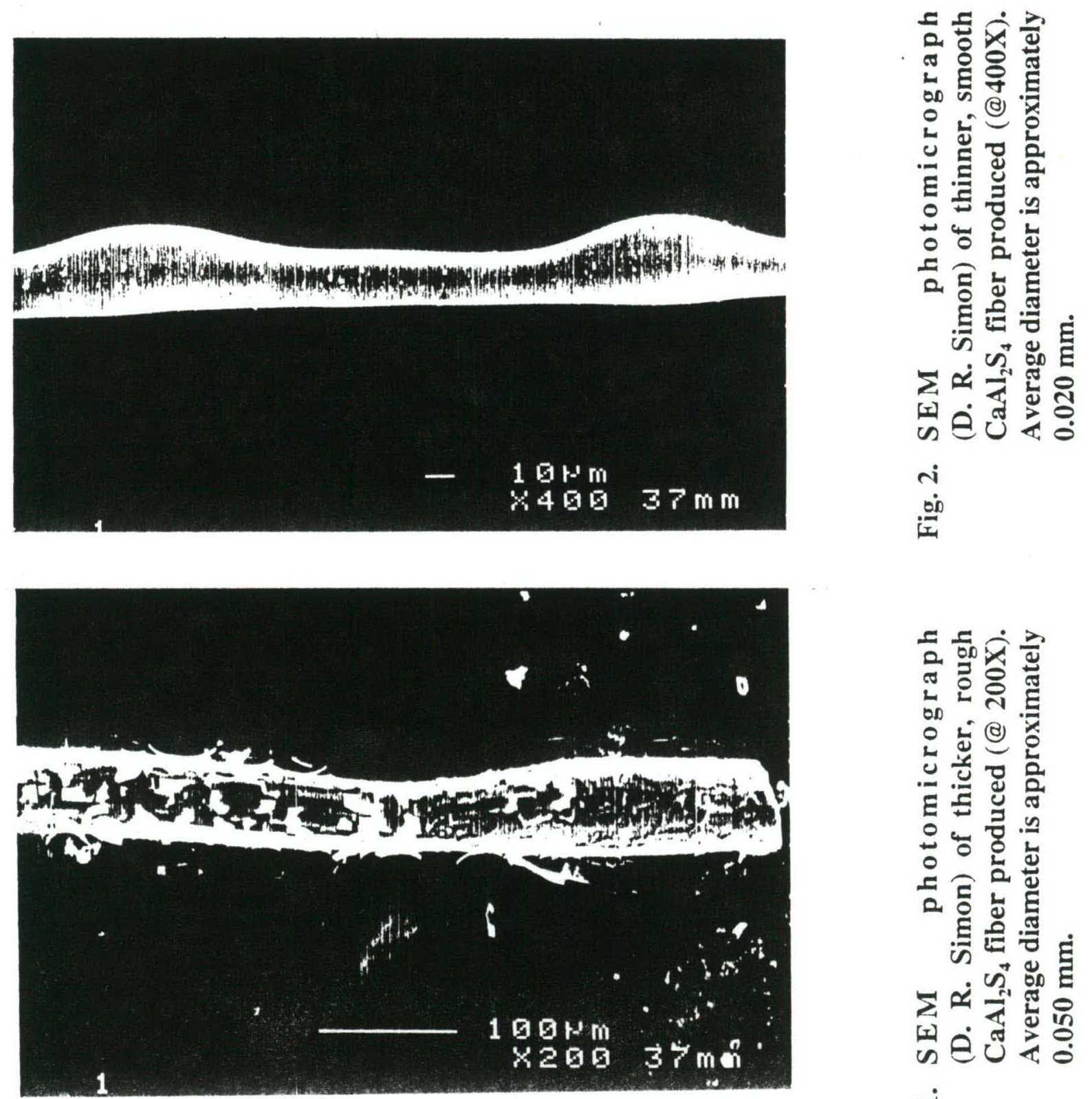

$\dot{-1}$

章 


\title{
95-163N -- USE OF SELF-ASSEMBLED MONOLAYERS AS TEMPLATES FOR GROWTH OF NOVEL MICROPOROUS MOLECULAR SIEVE FILMS ${ }^{1}$
}

\author{
Associate Laboratory Director Area: Physical Research \\ Principal Investigator(s): \\ M.J. Pellin, Chemistry and \\ Materials Science Divisions \\ L. Iton, Materials Science Division \\ Funding Profile: \\ FY $1993-0-$ \\ FY $1994-0-$ \\ FY $1995 \$ 73.7 \mathrm{~K}$ \\ FY $1996-0-$ \\ FY $1997-0-$
}

Purpose: This project explored a new approach to the growth of microporous materials. Selfassembled monolayers (SAMs), consisting of tertiary amine functionalized thiols bound on gold substrates, have been employed as template arrays for the crystallization of adherent zeolite films. This approach to the growth of molecular sieve materials represents a promising new avenue in the enterprise to develop thin films of an important class of materials that might be suitable for molecular shape selective chemical sensors, membrane filters, and optoelectronic applications.

Approach: Two unique chemistries have been combined in this effort. The first, microporous molecular sieve materials (e.g zeolites), are now well established as critical materials in many major industries. These industries include commercial ion exchange, water treatment and detergent manufacture, petroleum refining (as catalysts) and fine, commodity and bulk chemical synthesis. The second chemistry, molecular self assembly, is both relatively new and undergoing intensive research efforts. SAMs provide a means to fabricate microstructures through the stepwise formation of multilayered nano-structured films. Applications are envisaged in areas ranging form electronic devices to controlled delivery of therapeutic drugs.

Zeolites are usually synthesized by hydrothermal; crystallization of aluminosilicate gels. Mineral analogs exist for many zeolite structures; however, synthesis of almost all novel synthetic zeolite structures require the use of structure directing or promoting template molecules. These template molecules are typically organic bases such as tertiary amines which are irreversibly occluded in the pores of the growing zeolite. These molecules are ultimately removed by calcining (burning) in air. The template molecules serve to stabilize an open zeolitic phase which would otherwise be unstable with respect to dense crystalline or amorphous phases. The relative instability of the open crystalline phase has made growth of large single crystals generally not feasible. Similarly, growth of thin, single component oriented membranes of zeolite materials has not been very successful. Zeolitic membranes 20-250 $\mu \mathrm{m}$ thick, composed of continuous intergrowths of 10-100 $\mu \mathrm{m}$ crystallites of ZSM-5 zeolite, have been synthesized without surface funtionalization. Thin molecular sieve films would find a wide range of

${ }^{1}$ Individual Investigator Project 
industrial uses in chemically selective sensors, optoelectronic devices and efficient gas separation technologies, among others.

SAMs can allow molecular monolayer structures to be fabricated in a routine inexpensive manner. Yet in many cases the films are of high quality -- free of pores, dense and often crystalline. In this technology, the strength of the gold - sulfur bond is exploited by introducing long chain alkane thiols to a gold surface. The resulting molecular film is dense and close packed with the alkanethiols preferentially and strongly bond to the Au surface via their sulfur atom. The surface is so completely covered that water and polar molecules are excluded from the gold surface. Recently thiols have been used to form an alkyl diphosphonate film on which a zincophosphate molecular sieve was grown. This multilayer film was oriented, but not continuous. This is a specific example of a more general biomimetic approach to controlled crystallization by the use of Langmuir-Blodgett films as templates for the nucleation of inorganic crystals.

In this effort, we have used thiols functionalized with tertiary amino groups to form SAMs on gold surfaces. These molecules (e.g. 2-diethylaminoethanethiol) are readily available and inexpensive; moreover, amines (primary, secondary, and tertiary) and quaternary ammonium ions act directly as templates for aluminosilicate zeolite growth. The novel concept is to choose the molecules for the self-assembled film so that they resemble molecules that are known to work as templates for the target zeolite. By this design molecules in the SAM could become occluded in the first layer of growing zeolite molecular sieves. To date we have produced adherent zeolite films of densely arrayed 1-2 $\mu \mathrm{m}$ zeolite crystals. At present the films are not coherent, however much work remains to explore the growth conditions including concentration, temperature and type of growth solution.

\section{Technical Progress and Results:}

1. Substrate development. Initially, substrates were prepared by evaporative deposition of a gold films over an adhesion layer of chromium on a quartz disc. (Chromium is required for the gold to adhere to the quartz.) These substrates proved susceptible to chemical attack during the zeolite syntheses. Improvements in substrate smoothness and durability were obtained by means of ion beam-assisted deposition of the gold film on a silicon wafer; no chromium layer is required for adhesion of the gold to the silicon. Finally, a multiple-celled block machined from teflon was designed to allow the crystallization of up to ten samples simultaneously. This device was formed with a top seal made of teflon while the gold-coated silicon wafer formed the bottom seal.

2. Growth of silicalite molecular sieve from silicate synthesis solutions. The SAM of 2diethylaminoethanethiol was deposited on the gold from alcohol solution and its molecular integrity on the substrate was confirmed using laser desorption mass spectroscopy. However, the exact geometry and the intermolecular spacing of the thiol have not been directly determined. A synthesis procedure for the molecular sieve material was chosen that could be carried out at moderate temperatures $(100 \mathrm{C})$ from a synthesis solution rather than from a synthesis gel. Synthesis solutions (Type A) were prepared from sodium silicate solution and tetrapropylammonium (TPA) bromide with the following molar composition: $1.0 \mathrm{SiO}_{2}: 0.297 \mathrm{Na}_{2} \mathrm{O}: 0.03$ TPA-Br : $40.0 \mathrm{H}_{2} \mathrm{O}$. We have previously studied the nucleation and crystallization of solutions 
with this composition at $150 \mathrm{C}$, and have established that the nucleation is heterogeneous with an amorphous gel precursor being first formed; crystallites subsequently develop from this gel. In the crystallization experiments with the SAM-modified substrates, we have established that a precursor film material is present on the substrate after one day of heating at $100 \mathrm{C}$. Raman microscopy measurements establish however that this precursor film is a polymerized silicate but does not incorporate the TPA template. This had not previously been established for the studies which did not utilize substrates for crystallization. It is reasonable to suppose that in the presence of the SAM-modified substrate, the deposition of this thin amorphous silicate film occurs under the influence of the amine molecules in the SAM, and the subsequent nucleation of the silicalite involves TPA ions assembling from the solution at the film-solution interface. This corresponds to our original conceptual construct, and micron-sized silicalite crystals are formed after 2-3 days of heating, but they tend to be dispersed on the substrate in cluster aggregates that are not very extensive.

A new synthesis solution was formulated by reducing the silica content so as to make the solution less aggressive toward the substrate. The molar composition of this solution (Type $\mathrm{B}$ ) was: $1.0 \mathrm{SiO}_{2}: 0.297 \mathrm{Na}_{2} \mathrm{O}: 0.12 \mathrm{TPA}-\mathrm{Br}: 120.0 \mathrm{H}_{2} \mathrm{O}$. In contrast to the type A solution, this solution was found to undergo homogeneous nucleation with no evidence of a gel intermediate being formed during the crystallization process. This solution is much less damaging to the substrate, and undamaged substrates could be obtained for silicalite growth in the multiple-celled crystallization bomb. Extended dense arrays of closely spaced micron-sized crystals were obtained on the substrate using this synthesis solution, but continuous films have not yet been achieved. The accompanying figure shows examples of scanning electron micrographs of such silicalite crystal arrays and an identifying spectrum of a typical sample obtained using Raman microscopy. The Raman spectrum shows the primary framework vibration at $380 \mathrm{~cm}^{-1}$ as well as the bands corresponding to the encapsulated TPA template.

3. Growth of ZSM-5 zeolite molecular sieve from an aluminosilicate gel. ZSM-5 is the aluminosilicate zeolite with the same crystal structure (MFI) as silicalite. The gel crystallization was performed initially at $170 \mathrm{C}$ for four days in a conventional preparation in an autoclave. This was very destructive to the $\mathrm{Au} / \mathrm{Si}$ substrate. We have now established that $\mathrm{ZSM}-5$ can be obtained by heating at $120 \mathrm{C}$ for only 3 days using a gel with composition: $47.4 \mathrm{SiO}_{2}: 1.0$ $\mathrm{Al}\left(\mathrm{NO}_{3}\right)_{3}: 14.4 \mathrm{Na}_{2} \mathrm{O}: 4.94 \mathrm{TPA}-\mathrm{Br}: 10.3 \mathrm{H}_{2} \mathrm{SO}_{4}: 1850 \mathrm{H}_{2} \mathrm{O}$. This preparation will be attempted using the multiple-celled crystallization bomb. We anticipate a higher likelihood for formation of thick films of intergrown crystallites by this method.

4. Prospects. In addition to exploring the wide range of composition space and template structure available for molecular sieve syntheses, the results obtained to date suggest that one promising route to thin film formation is to use a substrate on which has already been deposited an array of micron-sized crystals from a homogeneously nucleated solution. The second step of crystal growth could be from either a solution or from a gel. In the case of the silicalite synthesis solution of type B (above), the growth of the crystallites can be controlled to the 20 $100 \mathrm{~nm}$ range. 
Specific Accomplishments: Research will continue under DOE-ER auspices.

Presentation: Microscopic Photopatterning and Laser Desorption/Fourier Transform Mass Spectrometry of Alkanethiols, Annual Spring Meeting of the AVS, Illinois Chapter, University of Wisconsin-Milwaukee, Wisconsin, May 25, 1995. 


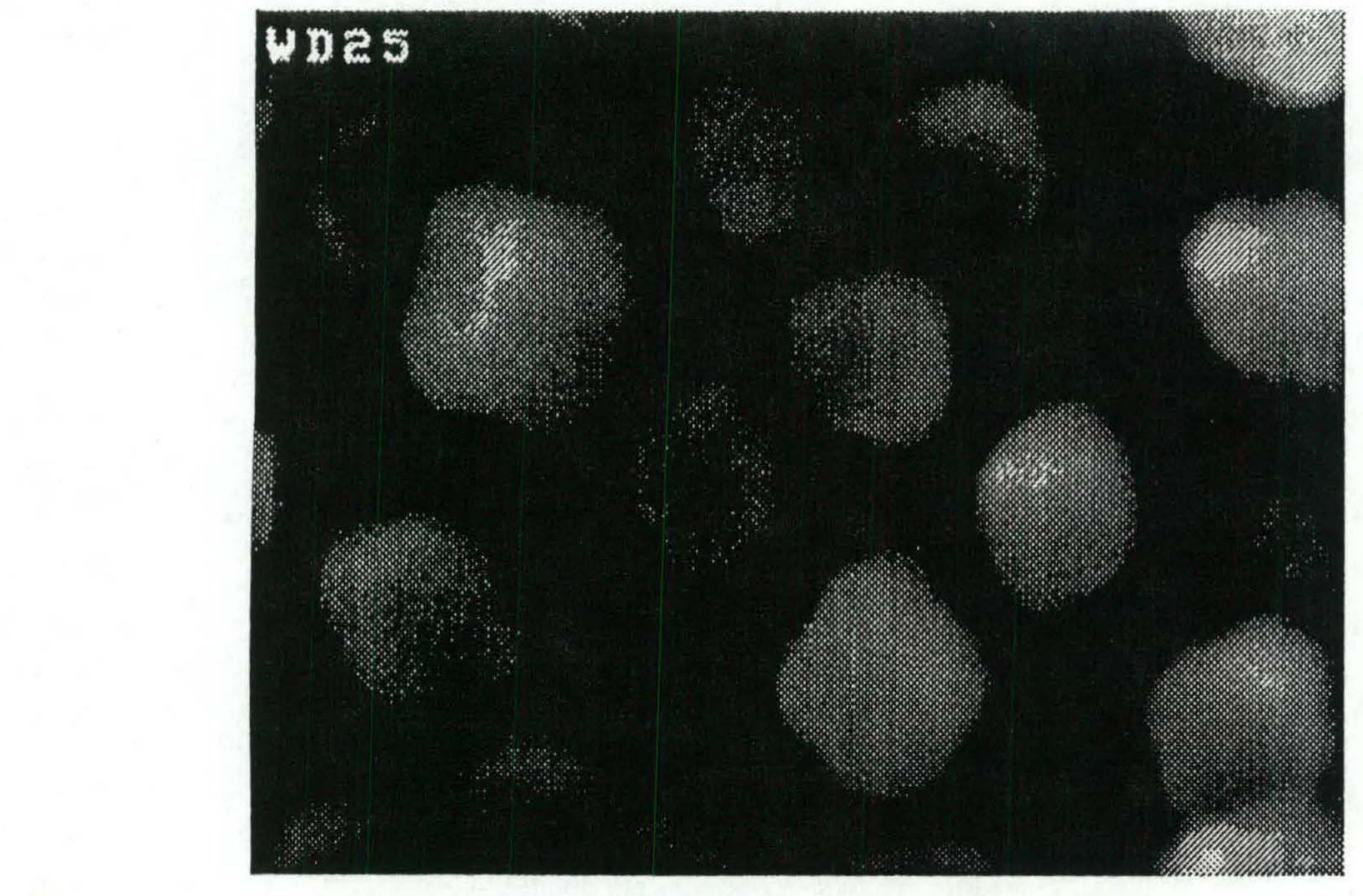

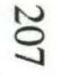

\section{Zeolite Crystals (x20K)}

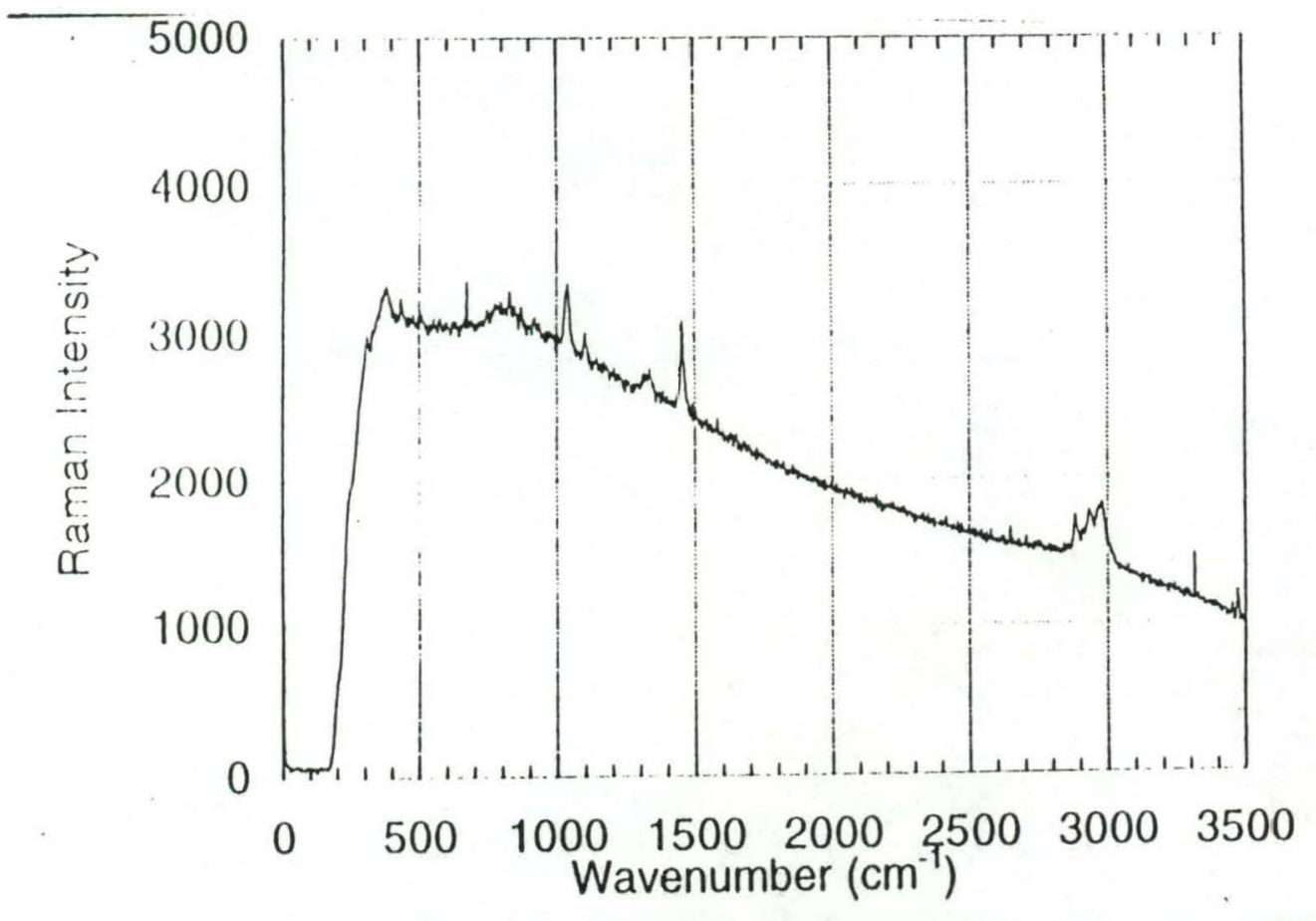

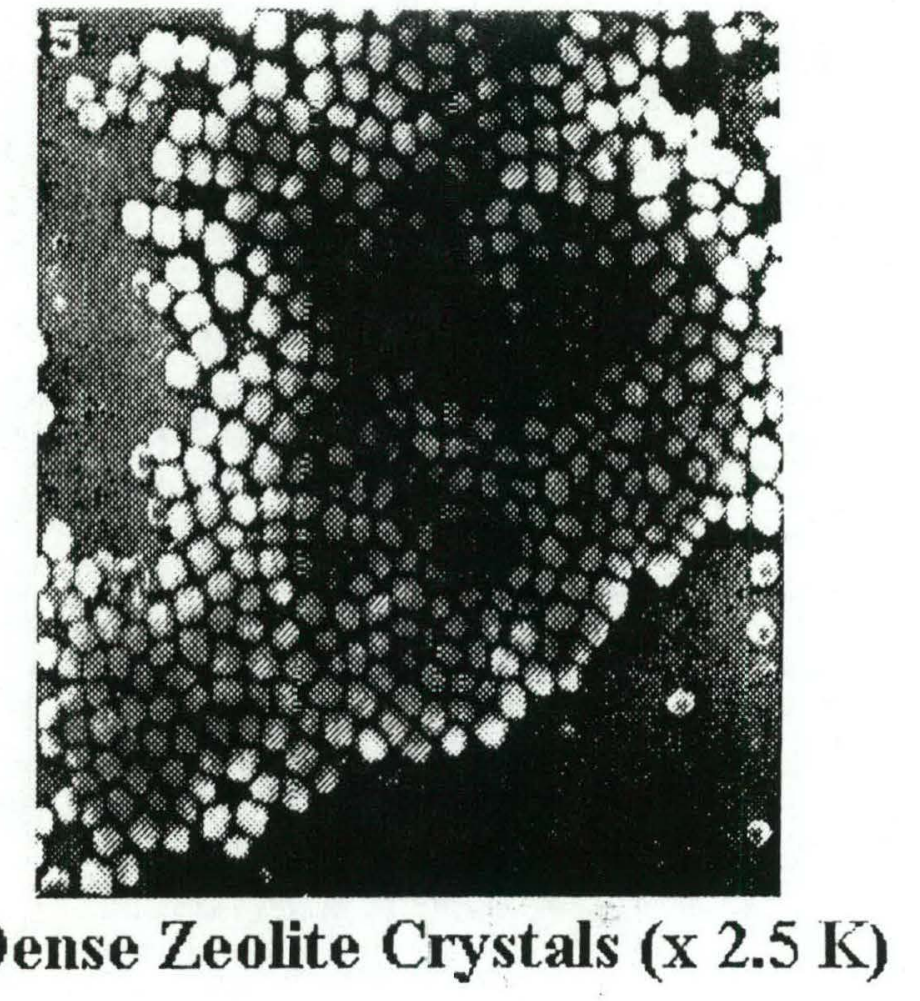

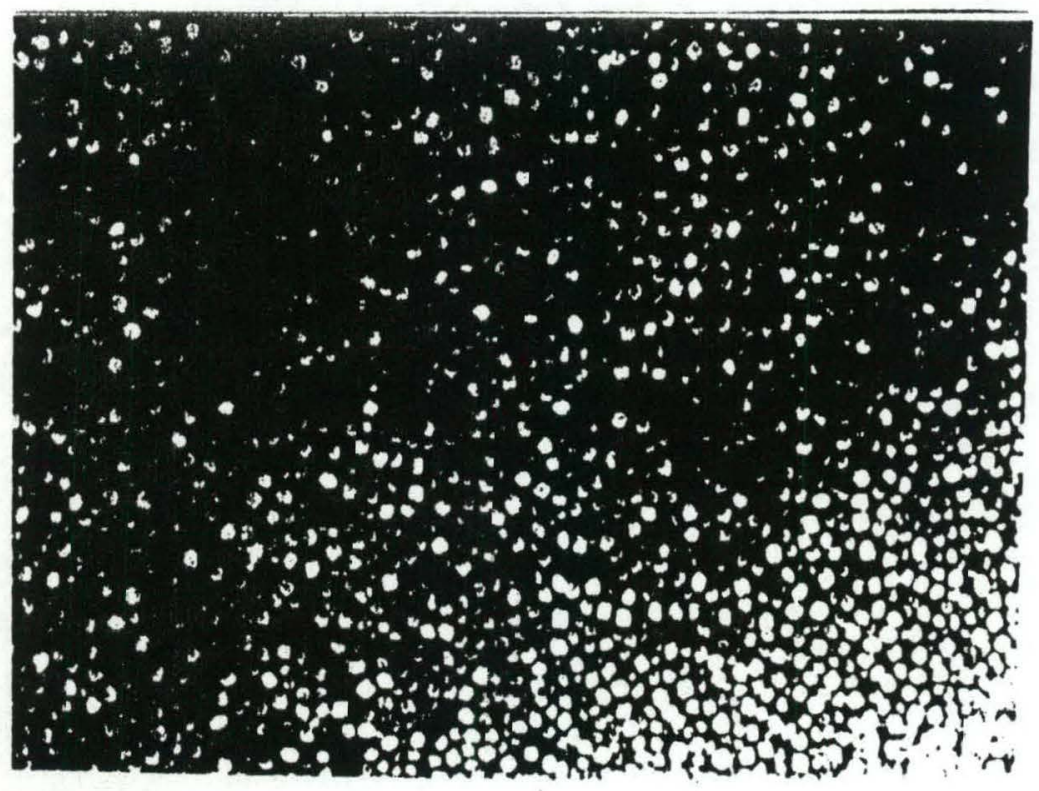


blank page 


\section{5-196N -- FEASIBILITY STUDIES FOR APPLICATION OF PHOSPHATE-BASED COMPOSITES IN LIGHTWEIGHT CONSTRUCTION MATERIALS ${ }^{1}$}

\author{
Associate Laboratory Director Area:
}

Principal Investigators:

Funding Profile:
Energy and Environmental Science and

Technology

D. Singh and A. Wagh,

Energy Technology Division

FY $1993-0-$

FY $1994-0-$

FY $1995 \$ 72.2 \mathrm{~K}$

FY $1996-0-$

FY $1997-0-$

Purpose: The proposed project will demonstrate the feasibility of using novel chemically bonded phosphate ceramic binders for fabrication of inexpensive, value-added, and lightweight construction materials using a variety of high-volume benign wastes. This project addresses the need to recycle high-volume benign wastes generated from domestic and industrial activities and to prevent pollution. Specifically, we will investigate the fabrication of pumpable insulating materials and particle boards from phosphate ceramics and high-volume wastes such as styrofoam and lumber wastes. The relevant properties of the fabricated composites will be evaluated to establish their applicability as lightweight, fire-resistant, and environmentally friendly construction materials. Development of these novel composite products is critical for the housing industry for construction of energy-efficient buildings at low cost. This project will establish ANL's role in both building materials development and waste recycling/minimization initiatives of DOE. In addition, this project will complement ANL's efforts in the residential rehabilitation projects via Argonne-Bethel New Life partnership and under the proposed Empowerment Zone initiative by the Department of Housing and Urban Development (HUD).

Approach: As part of the Mixed Waste Integrated Program (EM-50), ANL's Energy Technology Division has developed various room-temperature-setting phosphate ceramics for the stabilization and disposal of hazardous and radioactive wastes. Phosphate binders can be fabricated by acid-base reactions between inexpensive oxides, such as magnesium oxide $(\mathrm{MgO})$, and either phosphoric acid or acid phosphate solutions. The slurry formed by these reactions sets under ambient conditions into a dense, hard, and durable phosphate material. The intention of this study was to investigate the use of magnesium phosphate ceramics to bind various waste products and fabricate value-added construction materials. This effort addressed two specific needs in the building industry: (a) development of novel pumpable/blowable and fire-resistant insulation and (b) fabrication of wood particle boards.

The need for an innovative insulating material and its application technology is key for rehabilitation of existing homes. It is envisioned that by using a pumpable/blowable insulation, it will be possible to fill the wall cavities of moderate rehab homes. This novel technology will

${ }^{1}$ Individual Investigator Project 
not only provide fire-resistant insulation to the home but also reduce air infiltration within the wall cavities and conserve energy. Also, such novel insulation has potential application in newhome construction.

Current bonded structural materials such as wood particle board use polymers (e.g., urea formaldehyde resins) as binders for wood fibers. Fabrication of such material requires heating the polymer until it melts, and then mixing it with the wood fibers. Subsequently, the mix is pressed into the requisite geometry. These binders pose a major safety hazard because of their high flammability and production of toxic fumes in the event of fire. Furthermore, resin-bonded particle boards creep during extended service, even under moderate loads. In this regard, roomtemperature-setting nonflammable phosphate binders have the potential for binding wood fibers to make particle boards with improved load-bearing capacity and creep resistance.

The processing steps used in this investigation to fabricate blowable insulation and particle board are similar to one another. Calcined $\mathrm{MgO}$ powder is mixed with powdered boric acid and the mixture is reacted with dilute phosphoric acid. Subsequently, waste particles (shredded styrofoam and wood fibers for insulation and particle board applications, respectively) are mixed with and thoroughly coated by the binder. For particle board, the mixture is molded into desired shapes under pressure $(\approx 1,000 \mathrm{psi})$. In the case of insulation, mix consistency is maintained so that it can been blown into cavities by air pressure. After allowing the composite to cure for several days, a variety of tests (strength, thermal conductivity, air permeability, flammability, durability) are performed to assess their applicability as a building material.

Marcin Tlustochowicz, a Science and Engineering Research Semester student from the University of Illinois-Chicago, provided experimental assistance during the entire course of this project.

Technical Progress and Results: During FY 95, a novel blowable insulation system based on magnesium phosphate binder and shredded recycled styrofoam was developed. Processing parameters such as particle size of shredded styrofoam and binder content were optimized by improving density and strength properties of the insulation. In the optimized product, density was $2 \mathrm{lb} / \mathrm{ft}^{3}$ and the weight loading of styrofoam was $7.5 \%$, which corresponded to $85-90 \%$ by volume. In addition, no air permeance was observed in the insulation when air at 20 psi was applied across a 4-in.-thick piece.

Thermal conductivity measured with a modified radial hot-wire technique in an independent laboratory (Anter Laboratories, Pittsburgh) showed that thermal resistance ( $\mathrm{R}$ value) of this novel insulation system was 4.5 for 1 -in.-thick piece. This value is significantly higher than that of typical currently used insulation such as fiberglass $(R \approx 2-3)$ and cellulose $(R \approx 3-3.5)$.

ASTM Standard E 84-94 was employed to determine the burning characteristics of the blowable insulation product. The insulation did not ignite when brought into contact with an open flame, thus proving its nonflammable nature. Moreover, the insulation material was determined to be extremely durable in humid environments. Tests conducted per the ASTM D 2126-87 Standard showed less than $2 \%$ change in dimensions of the insulation and less than $4 \%$ water absorption on exposure to $95 \%$ humid environment at $38 \mathrm{C}$. This is especially important because insulation is expected to remain rigid and not degrade with time. The nonflammability and 
durability of the insulation product is due to the inorganic phosphate binder that coats the waste particles and protects them from external hazards such as fire, chemical attack, humidity, and other weathering conditions.

A laboratory-scale system was designed and constructed to demonstrate the application of blowable insulation in a 4' $x$ 3' demonstration wall. A photograph of the applied insulation in the demo wall is shown below. Installation of insulation is simple and requires only air pressure to carry the premixed styrofoam-binder mixture at a particular consistency. After application, the insulation sets in place within 2-3 hours. Because small pieces of shredded styrofoam are used in this process, it is possible to completely fill wall cavities, even around obstructions, thereby resulting in a superior insulation system.

In this project, fabrication of $3 \times 3$ in. particle boards using wood fibers has been demonstrated. Process optimization has resulted in boards with flexural strengths of $\approx 1500$ psi with 50 wt. $\%$ magnesium phosphate binder content. Similar to insulation, these particle boards are nonflammable.

Based on our efforts in FY 95, we have clearly demonstrated that magnesium phosphate can be used for binding various high-volume wastes to produce value-added structural products. The next step is to scale-up the technology to a pilot-scale level, test its commercial viability, and ultimately transfer this technology to the field. In this regard, several industrial contacts have been established.

Specific Accomplishments: One invention report titled, "Phosphate-bonded value added structural products from high-volume wastes" has been filed. A seminar, "Ceramic Binders for Insulation from Recycled Materials," was presented at an Argonne-Illinois Institute of Technology collaborative seminar on Affordable Housing and Sustainable Urban Development. Several demonstrations of the technology have been conducted for various ANL staff and industrial personnel. Contact has been made with Illinois Department of Energy and Natural Resources which is involved in the development of energy conservation related technologies. In addition, a proposal was submitted to DOE for a pilot study to incorporate energy-efficient technologies in large-scale community development projects. 


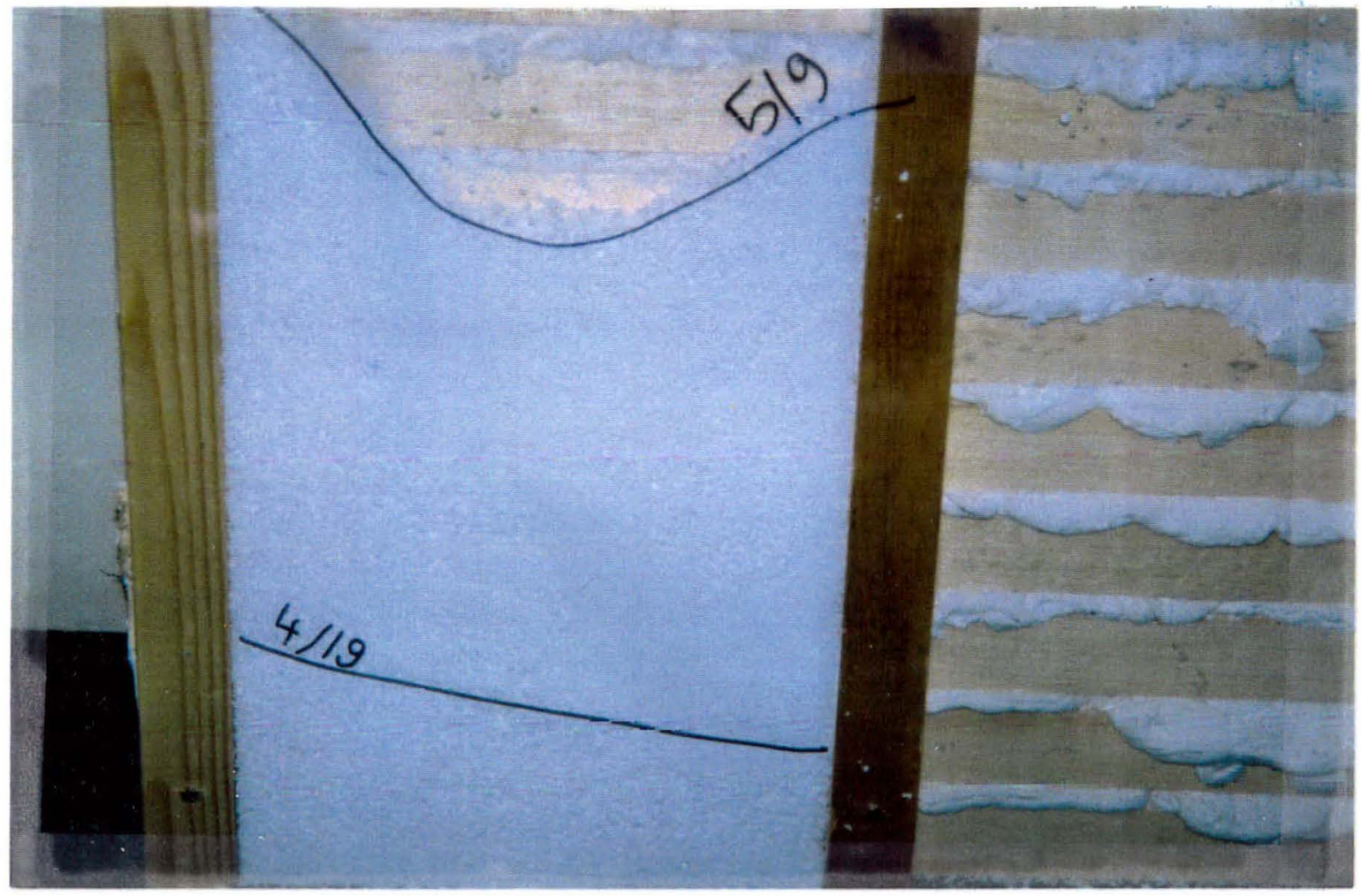

Photograph of blown styrofoam-magnesium phosphate insulation in a demonstration wall. 


\title{
94-058R1 -- X-RAY SYNCHROTRON RADIATION STUDIES OF SEMICONDUCTOR/ SEMICONDUCTOR HETEROPHASE EPITAXY
}

\author{
Associate Laboratory Director Area: Physical Research \\ Principal Investigators: \\ M.J. Bedzyk and K.-G. Huang, \\ Materials Science Division \\ Funding Profile: \\ FY $1993-0-$ \\ FY $1994 \$ 209.5 \mathrm{~K}$ \\ FY $1995 \$ 142.5 \mathrm{~K}$ \\ FY $1996 \$ 100.0 \mathrm{~K}$ \\ FY $1997-0-$
}

Purpose: The project objectives are to study the structure and growth mechanism of surfactant mediated heteroepitaxial growth of $\mathrm{Ge}$ on $\mathrm{Si}^{1}$ with the $\mathrm{X}$-15A NSLS synchrotron $\mathrm{x}$-ray beamline and its UHV surface science chamber. The surfactants in this heterophase epitaxial strained layered structure are to be group V metals $\mathrm{As}, \mathrm{Sb}$ and $\mathrm{Bi}$. Here we are interested in developing insights into how the above surfactants are incorporated in the Si surface structure before Ge thin film growth commences and in the Ge strained film during the growth process. This would improve our understanding of how to grow lattice mismatched epitaxial films and thus avoid 3D island nucleation.

Approach: Prior to achieving the final goal of studying surfactant mediated growth in this strained layer heteroepitaxial structure, we need to lay the ground work of studying the individual constitucnts of this complicated structure in a step-by-step manner. Our approach has been to first study the surface structure of individual pentavalent adlayers of $\mathrm{As}, \mathrm{Sb}$, and Bi on $\mathrm{Si}(001)$ with $\mathrm{x}$-ray standing waves. Next we would study epitaxial Ge thin film growth on $\mathrm{Si}(001)$ without a surfactant. And then study it with $\mathrm{Sb}$ or $\mathrm{As}$ or $\mathrm{Bi}$ as a surfactant.

The UHV surface preparation and $\mathrm{x}$-ray measurements are made with collaborators $\mathrm{Y}$. Qian (Northwestern/ANL graduate student ), P.F. Lyman (Northwestern postdoc), G.E. Franklin (Harvard postdoc), and J.C. Woicik (NIST). Theoretical cluster calculations of the surface structures are made by our NU/Physics collaborators S. Tang and A.J. Freeman.

Technical Progress and Results: We have continued to develop our UHV surface science MBE capabilities for deposition of and in-situ $\mathrm{x}$-ray characterization of highly ordered monolayer and submonolayer phases of metal atoms on semiconductor surfaces.

In FY94 we completed the first stages of this research program by solving the surface adlayer structures for one monolayer coverage of $\mathrm{As}^{4} \mathrm{Sb}^{2}$ and $\mathrm{Bi}^{3}$ on $\mathrm{Si}(001)$. All of these group $\mathrm{V}$ adsorbate species form saturated ordered adlayers on $\mathrm{Si}(001)$ which remove the $2 \times 1$ dimerized reconstruction of the clean $\mathrm{Si}(001)$ surface and reform a $1 \times 2$ surface structure with group $\mathrm{V}$ addimers aligned orthogonal to the preexisting $\mathrm{Si}$ dimers. The $\mathrm{Si}(001) \cdot 2 \times 1-\mathrm{As}$ (or $\mathrm{Sb}$ or $\mathrm{Bi}$ ) surface is non reactive in comparison to clean $\mathrm{Si}(001) 2 \times 1$ surface due to the fact that unlike the quadravalent $\mathrm{Si}$ atoms, the pentavalent $\mathrm{As}, \mathrm{Sb}$ and $\mathrm{Bi}$ atoms in this dimerized structure lead to a filled shell electronic configuration, leaving no unsaturated surface dangling bonds. Upon 
these group V element adsorbed Si(001) surfaces, we have undertaken XSW measurements using the Si (004) and (022) Bragg reflections. To summarize the structural results for the group V metals (As, $\mathrm{Sb}$ and $\mathrm{Bi}$ ) adsorption on $\mathrm{Si}(001)$, Table 1 tabulates values of ad-dimer bond lengths (L) and ad-dimer heights (h') measured by our XSW experiments and calculated by Tang and Freeman's cluster calculations, as well as covalent radii for $\mathrm{As}, \mathrm{Sb}$ and $\mathrm{Bi}$. As can be seen from Table 1, the XSW measured ad-dimer bond length of group V elements matches their covalent bond length (except for As). . This suggests that As dimer is under significant tensile stress $\left(\mathrm{L}_{\mathrm{As}}>2 \mathrm{r}_{\mathrm{As}}\right.$ by $7 \%$ ) while $\mathrm{L}_{\mathrm{Sb}} \approx 2 \mathrm{r}_{\mathrm{Sb}}$ and $\mathrm{L}_{\mathrm{Bi}} \approx 2 \mathrm{r}_{\mathrm{Bi}}$. The height of the group $\mathrm{V}$ ad-dimer shows an increase with respect to the size of the element as well. The XSW measurements also have good agreement with theoretical predictions.

In FY94 we also solved the related and relevant structure of group III Ga on Si(001) ${ }^{6.7 .8}$ This surface saturates at one-half monolayer, forming a much less stable and more reactive $2 \times 2$ structure which has $\mathrm{Ga}$ dimers lined up parallel to the underlying Si dimers which remain unbroken in a $2 \times 1$ surface reconstruction.

We also used x-ray standing waves to measure the ad-atom's thermal vibrational amplitude on the $\mathrm{Si}(001)$ surface. The thermal vibrational amplitude of surface ad-atoms is rather important since it contains valuable information about surface bonding chemistry. Precise measurement of thermal vibrational amplitude at room temperature is also a necessity for determining the addimer bond length with higher accuracy. We studied the temperature behavior of the As thermal vibrational amplitude on $\mathrm{Si}(001)$ and indirectly determined its value $(0.14 \AA)$ at room temperature ${ }^{4}$ In FY94 and FY95, we directly measured the thermal vibrational amplitude of $\mathrm{Sb}^{9}(0.16 \AA), \mathrm{Bi}^{10}(0.13 \AA)$ and $\mathrm{Ga}^{8}(0.14 \AA)$ on the $\mathrm{Si}(001)$ surface at room temperature by combining the fundamental $\mathrm{Si}(004)$ reflection with the 2 nd-order harmonic $(008)$ reflection.

In FY95, we used $x$-ray standing waves to measure the surface structure and to observe a thermally activated irreversible phase transition on a low coverage $\mathrm{Bi} / \mathrm{Si}(001)$ surface. The $(2 \times 2)$ Bi phase, predicted by theory ${ }^{11}$ to be the low coverage structure, was found to be a metastable phase that undergoes an irreversible phase transition to the stable $(1 \times 2)$ phase upon annealing (see Figure 1). The thermal activation energy and exponential prefactor of the transition were determined from the measurement. This experimental work demonstrates a new approach for investigating the surface kinetics of adsorption.

In the last half of FY95, we initiated XSW/MBE studies of the $\mathrm{Ge} / \mathrm{Si}(001)$ and $\mathrm{Bi} / \mathrm{Ge}(001)$ surface structures. This will lead us in FY96 to our goal of XSW studies of Ge heteroepitaxial growth on $\mathrm{Si}(001)$ with $\mathrm{Bi}$ as a surfactant.

Specific Accomplishments: We have combined the synchrotron radiation based $\mathrm{x}$-ray standing wave method with convention surface science techniques (LEED, Auger, and MBE) to study epitaxial surface structure systems. The XSW method affords high structural resolution and chemical sensitivity for solving surface and heteroepitaxial strained-layer thin film structures. The XSW $0.01 \AA$ spatial resolution not only allows us to pin-point the location of ad-atoms with respect to the substrate lattice, but also allows us to measure the thermal vibrational amplitude of the ad-atoms. We have applied this experimental methodology to a series of metal/semiconductor surface structures that are relevant to our goals of describing surfactantmediated epitaxy of semiconductor/semiconductor heteroepitaxial structures. In particular, we 
have made measurements for various coverages of $\mathrm{Sb}, \mathrm{Bi}$ and $\mathrm{Ge}$ on $\mathrm{Si}(001)$ as well as, $\mathrm{Bi}$ on $\mathrm{Ge}(001)$. These measurements lay the ground work for studying the more complicated surfactant mediated growth structures of $\mathrm{Ge}$ on $\mathrm{Si}(001)$ with $\mathrm{Sb}$ or $\mathrm{Bi}$ as the surfactant. In the course of our $\mathrm{Bi} / \mathrm{Si}(001)$ work we also demonstrated how one can use the XSW probe for following surface kinetics by observing a thermally induced irreversible phase transition from a metastable $(2 \times 2)$ to a stable $(1 \times 2)$ phase.

\section{References:}

1. F.K. LeGoues, M. Copel, and R.M. Tromp, Phys. Rev. Lett. 63 (1989) 1826; M. Copel, M.C. Reuter, M. Horn von Hoegen, and R.M. Tromp, Phys. Rev. B42 (1990) 682.

2. "Adsorbate Structure and Substrate Relaxation for the $\mathrm{Sb} / \mathrm{Si}(100)-(2 \times 1)$ Surface," P.F. Lyman, Y. Qian, and M.J. Bedzyk, Surf. Sci. 325, L385 (1995).

3. "A High-Resolution Structural Study of Bi on Si(001)," G.E. Franklin, S. Tang, J.C. Woicik, M.J. Bedzyk, A.J. Freeman, and J.A. Golovchenko, Phys. Rev. B. 52, R5515 (1995).

4. "Thermal Vibration Amplitudes and Structure of As on Si(001)," G.E. Franklin, E. Fontes, Y. Qian, M.J. Bedzyk, J.A. Golovchenko, and J.R. Patel, Phys. Rev. B 50, 7483 (1994).

5. S. Tang, and A. J. Freeman, Phys. Rev. B 47, 1460(1993).

6. "Resolving the Ga Ad-Dimer Location and Orientation on the Si(100) Surface," Y. Qian, M.J. Bedzyk, S. Tang, A.J. Freeman, and G.E. Franklin, Phys. Rev. Lett. 73, 1521 (1994).

7. "Combined Theoretical and Experimental Investigations of the Adsorption Geometry of Ga on Si(100) at Low Coverage," S. Tang, A.J. Freeman, Y. Qian, G.E. Franklin, M.J. Bedzyk, Phys. Rev B 51, 1593 (1995).

8. "Structure and Ad-Atom Thermal Vibrational Amplitude of the Ga/Si(001) $2 \times 1$ Surface," Y. Qian and M.J. Bedzyk, J. Vac. Sci. Technol. A 13, 1613 (1995).

9. "Thermal Vibration Amplitudes and Structure of Sb on Si(001)," Y. Qian, P.F. Lyman, T. Lee, and M. J. Bedzyk, accepted by Physica $B$.

10. "Adsorbate structure and vibrations on the $\mathrm{Bi} / \mathrm{Si}(001)-(1 \times 2)$ surface, " P.F. Lyman, Y. Qian, T.-L. Lee, and M.J. Bedzyk, accepted by Physica $B$.

11. S. Tang and A. J. Freeman, Phys. Rev. B 50, 1701 (1994). 
Table 1. Our XSW measured, and Tang and Freeman's theoretically calculated dimer bond length $L$ and height $h$ ', as well as, the covalent radius $r_{\text {cov }}$ for group $V$. elements adsorbed on $\mathrm{Si}(001)$. The corresponding (1x2) ad-dimer surface structure is depicted in Fig. 1.

\begin{tabular}{cccccc}
\hline & $\begin{array}{c}2 \mathrm{r}_{\text {cov }} \\
(\AA)\end{array}$ & $\begin{array}{c}\text { XSW } \\
\mathrm{L}(\AA)\end{array}$ & $\begin{array}{c}\text { Theory } \\
\mathrm{L}(\AA)\end{array}$ & $\begin{array}{c}\text { XSW } \\
\mathrm{h}^{\prime}(\AA)\end{array}$ & $\begin{array}{c}\text { Theory } \\
\mathrm{h}^{\prime}(\AA)\end{array}$ \\
\hline \hline $\mathrm{As}$ & 2.40 & 2.58 & 2.52 & 1.40 & 1.37 \\
\hline $\mathrm{Sb}$ & 2.76 & 2.75 & 2.93 & 1.68 & 1.64 \\
\hline $\mathrm{Bi}$ & 2.92 & 2.96 & 3.16 & 1.80 & 1.73 \\
\hline \hline
\end{tabular}

\section{(2x2) Phase (1x2) Phase}
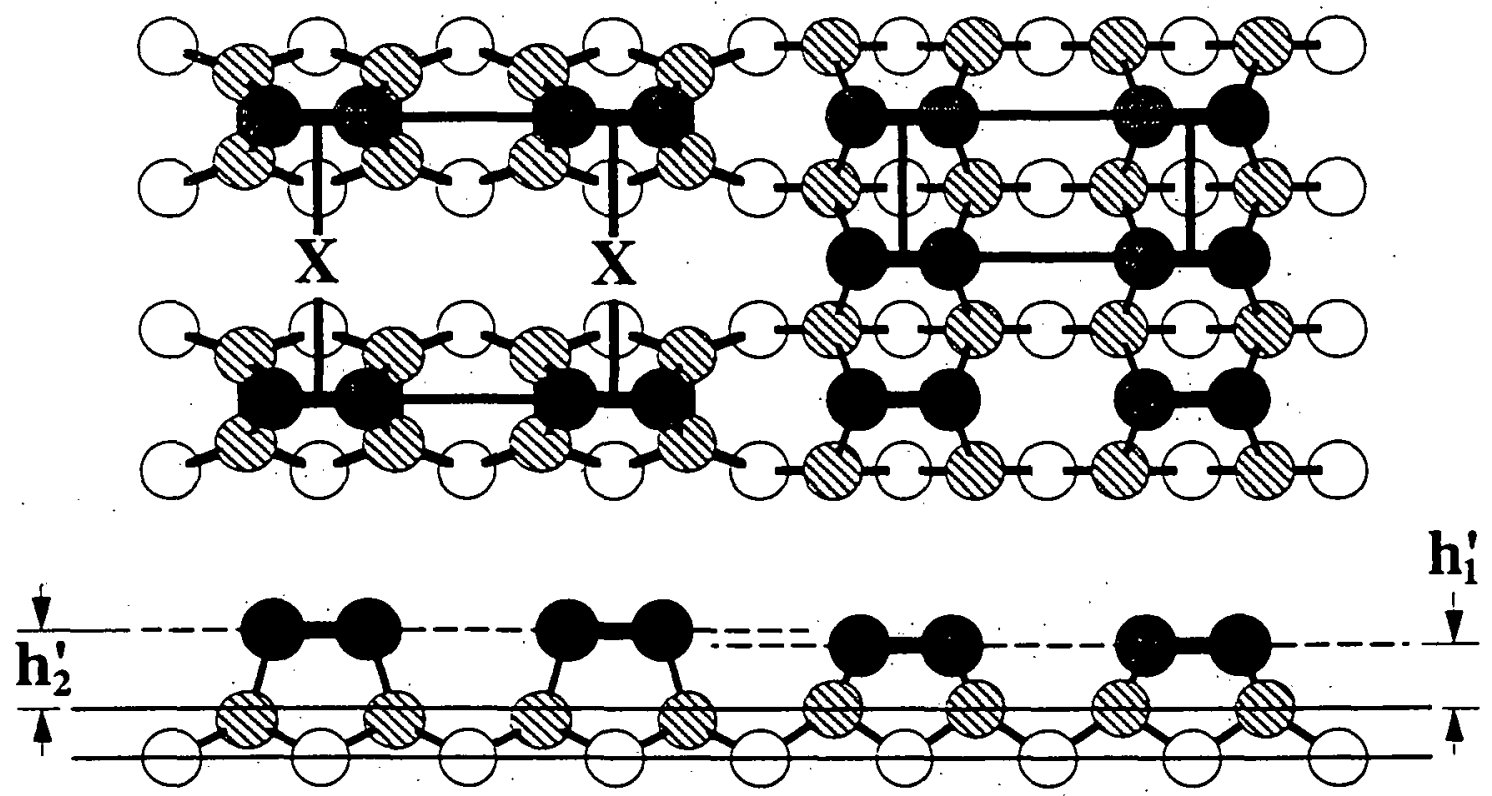

Figure 1. Top view and side view of the $(2 \times 2)$ (left side) and (1×2) (right side) Bi phases on the Si(001) surface proposed by Tang and Freeman. ${ }^{11}$ The open circles are bulk Si atoms, the hatched circles are top layer Si atoms and the dark circles are Bi atoms. $h_{1}^{\prime}$ and $h_{2}^{\prime}$ are the height of the $(1 \times 2)$ and $(2 \times 2)$ phase $\mathrm{Bi}$ dimers above the $\mathrm{Si}(004)$ bulk-extrapolated lattice planes. 


\section{4-060R1 -- INVESTIGATIONS OF TIME-RESOLVED AND ANOMALOUS SAXS STUDIES FOR CRITICAL PROBLEMS IN ADVANCED MATERIALS RESEARCH}

Associate Laboratory Director Area:

Principal Investigators:

Funding Profile:
Physical Research

\author{
K.A. Carrado and R.E. Winans, \\ Chemistry Division \\ P. Thiyagarajan, Intense Pulsed Neutron Source
}

Purpose: This initiative seeks to explore the mechanism of formation of technologically important disordered systems. Synchrotron X-ray scattering techniques have been employed to begin to gain an understanding of the critical structural features present. Such understanding will lead to fundamental knowledge regarding structure-activity relationships and also help to make the synthesis of these materials more precise, systematic, and predictable. Time-resolved small angle X-ray scattering (SAXS) studies are suited for monitoring the mechanism of formation in situ of such disordered materials as clays because of the inherent crystallization times for this system. Anomalous SAXS (ASAXS) allows a heavy metal ion to be tracked in terms of its disposition within a disordered framework. This feature is also exploited to track the solvation and diffusion of heavy metal ions within clay interlayers. These approaches will provide pioneering information in the near term, and set the stage for more detailed studies of many classes of materials in the long term.

Approach: Disordered systems constitute some of the most technologically and industrially important materials. This disorder, which is in most cases essential for the materials' activity, inherently precludes their detailed characterization by conventional techniques like X-ray powder diffraction. Current limitations towards understanding the important structural features of disordered systems can now begin to be overcome by using synchrotron radiation scattering methods. These techniques are of the ideal length scales to probe the size, shape, and morphology of particles. Initially, layer silicate clays will be examined because of their microcrystalline nature. Although these minerals are crystalline, their inherent disorder is so high that their particle size is extremely small. Of special interest is their mechanism of formation, which is as yet unknown but of relevance to their use as catalysts and supports.

We have recently developed a new procedure for preparing clays under mild hydrothermal conditions. The gels can also contain a wide variety of organic and organometallic spacer molecules. Among the direct methods available for structural characterization of these colloidal systems, small angle scattering proves to be among the most powerful. Small angle neutron scattering (SANS) studies of the gels quenched at several different reaction times revealed that within the first few hours the repulsive interactions between the colloidal silica particles decrease and larger colloidal systems are formed. It is, however, not possible to monitor the growth and 
formation of the hectorite in situ by SANS because the signals are too weak. In addition, the growth of the particles can be seen only in a small part of the low q-region of SANS spectra. Monitoring these reactions thus requires high-flux sources and powerful small angle scattering instruments that can access much lower q-regions, which points to the use of synchrotron small angle X-ray scattering (SAXS).

The presently available SAXS instruments at NSLS (National Synchrotron Light Source) and SSRL (Stanford Synchrotron Radiation Laboratory) have very high fluxes, but they do not access q-regions smaller than what one can achieve at the SANS facilities. However, SAXS experiments done at these facilities help us to develop and test the equipment necessary for studying the slurries at high temperature. This is done while information on the type of scattering signals at various times of the synthesis is obtained. In addition, we have begun to develop anomalous SAXS (ASAXS) methodology for studying various clay systems. When the energies of $\mathrm{X}$-rays are in the immediate vicinity of an absorption edge of a heavy scattering atom, the scattering becomes strongly wavelength dependent. With a tunable intense X-ray source, it is possible to selectively alter the contribution of a particular element to the SAXS scattering profile, and thus derive information on the disposition of those elements. The variation of distances between, and disposition of, metallic centers during crystallization can be examined using ASAXS. We have also applied this technique to probing the solvation of rare earth ions within the interlayers of a clay. This provides additional insight into the short-range information derived from $\mathrm{X}$-ray absorption spectroscopy studies on $\mathrm{Cu}$-clays obtained earlier in our group.

Technical Progress and Results: The SAXS instruments at SSRL and NSLS are capable of yielding adequate SAXS signals in the very low q-region $\left(0.01\right.$ to $\left.0.2 \AA^{-1}\right)$ within a five-minute time frame. Attempts were made to follow the structural aspects of the clay colloids during their synthesis in situ on these beamlines. Within just a few hours, the colloids were observed to grow enough in size from the initial sol-gel to settle to the bottom of the reaction vessel. Hence, a specially-designed sample cell was developed (with the aid of Dr. S. Henderson of ORNL) in an attempt to insure that a representative sample stayed in the beam during the entire synthesis. Experiments performed at SSRL showed that there was very little change in morphology after reaction at $95^{\circ} \mathrm{C}$ for 18 hours in situ. This temperature is slightly lower than the $100^{\circ} \mathrm{C}$ temperature usually employed, wherein layers have been seen within 24 hours by SANS. The fragile glass sample cells used are not able to hold the pressures generated at $100^{\circ} \mathrm{C}$, hence the need to use a slightly lower temperature. The scattering signals indicated the presence of only colloidal particles. It will be necessary to repeat these experiments using other sample cell designs and perhaps longer crystallization times. These studies will yield kinetic information in terms of both structure and morphology of the reactive colloids during the onset, growth, and the final formation of layered silicate materials.

We are also interested in delineating information on the disposition of $\mathrm{Ni}(\mathrm{II})$ incorporated within the inorganic framework. Ni(II)-containing synthetic montmorillonite clays, which are hydroprocessing catalysts, were first prepared and examined. Clay gels, and powders from these gels, were isolated and quenched at several different reaction times. Excellent ASAXS measurements were made at SSRL. Collection of X-ray absorption spectroscopy data was also done in order to derive the structural environment within the immediate vicinity of the metal ion. 
This complements the longer range distance information provided by SAXS and ASAXS. Bulk crystallization data was obtained by X-ray powder diffraction (XRD).

ASAXS data were measured at 5 different energies near the $\mathrm{K}_{\mathrm{a}}$ edge of $\mathrm{Ni}$ at three different reaction times: unreacted, 4 hours, and 15 hours. After 15 hours the crystallization is essentially complete as deemed by XRD. The ASAXS data are presented in Figure 1. The data for the unreacted sample showed no correlations for a lamellar particle, while after 4 hours the evolution of lamella were indicated, and the crystallized sample after 15 hours exhibited much larger lamellar correlations. Systematic variations were observed in the data for the 4 hour and 15 hour samples that are due to the anomalous scattering from the ordered $\mathrm{Ni}$ atoms in the layered silicates. The variation of these signal intensities as a function of the energy of the Xrays indicates the presence of $\mathrm{Ni}-\mathrm{Ni}$ correlations in the layered particles. Were nickel ions to remain in solution, there would be no order and therefore no specific, observable $\mathrm{Ni}-\mathrm{Ni}$ correlations. The observable changes in intensity (ASAXS) for the unreacted sample are due to the fact that $\mathrm{Ni}$ exists here as $\mathrm{Ni}(\mathrm{OH})_{2}$. The XRD pattern shows sharper peaks, indicating the longer-range crystalline order that was deduced from the ASAXS investigation, and a dspacing of $10 \AA$. This work represents the first application of ASAXS to a catalytically relevant materials issue.

ASAXS was also applied to another issue in clay science, one involving the incorporation of heavy metals in trapping media. We probed $\operatorname{Er}(\mathrm{III})$ ions that were ion-exchanged into natural clays. Systematic energy-dependent variations in the signals near the $L_{\text {III }}$ edge of erbium were observed for a hydrated sample, but not for a dried sample.

The specifics of this experiment, which were done in collaboration with Drs. S. Wasserman and $\mathrm{K}$. Song (CHM), are as follows. In order to examine how the distribution of metal ions changes as solvent is added to a clay interlayer, we introduced erbium(III) cations by standard ionexchange techniques. A lanthanide ion was used because the metal loading in an ion-exchanged clay are on the order of only a few weight $\%$, and the cross-sectional scattering area of these atoms is relatively large. Dry and wet samples of this material were examined using ASAXS and XRD. Figure 2a presents the ASAXS modified Guinier plot for a dry sample of Er-clay. XRD shows (001) interlamellar spacing of $15.0 \AA$. Unlike the Ni-containing synthetic clays (Fig. 1), the curves in Figure 2 dn not tend to decrease in the low $q^{2}$ region. This is because the natural clay has a much larger aspect ratio than the synthetic material discussed above. As the energy of the X-rays is changed, there is no variation in the intensity of scattering. This result implies that the $\operatorname{Er}(\mathrm{III})$ ions are not located at specific, regularly ordered sites on the clay lattice.

The ASAXS data for the Er-clay sample after addition of excess water are shown in Figure $2 b$. XRD shows an increased d-spacing of $19.5 \AA$. Unlike the "dry" sample, the ASAXS signals for the wet sample systematically vary as a function of the incident $\mathrm{X}$-ray energy. This result indicates that there are correlations between $\operatorname{Er}($ III) cations in the swelled clay which are not present in the dry material. ASAXS thus gives unique information about the ability of the solvent (water) to solvate $\mathrm{Er}(\mathrm{III})$ ions within the clay interlayer. More experiments will be done to elucidate why the correlations are not observed for the dried material. 
In general, these studies have provided the first SAXS and ASAXS measurements in two areas: (1) the mechanism of formation of disordered materials such as clays that can be useful in the developing new synthetic strategies, and (2) heavy metal ion solvation and migration in clays, which has implications for both catalysis and environmental issues.

\section{Specific Accomplishments:}

Publication of FY 1995 results - submitted and in review:

P. Thiyagarajan, K.A. Carrado, S.R. Wasserman, K. Song, and R.E. Winans, "Anomalous Small Angle X-ray Scattering Study of Layered Silicate Clays Containing Ni(II) and Er(III)," in Reviews of Scientific Instrumentation.

Poster Presentation:

P. Thiyagarajan, K.A. Carrado, S.R. Wasserman, K. Song, and R.E. Winans, "ASAXS of Layered Silicate Clays Containing Ni(II) and Er(III),"

National Conference on Synchrotron Radiation Instrumentation 1995 (SRI '95), in conjunction with the 7th Users Meeting for the Advanced Photon Source, Argonne National Laboratory, October 1995. 

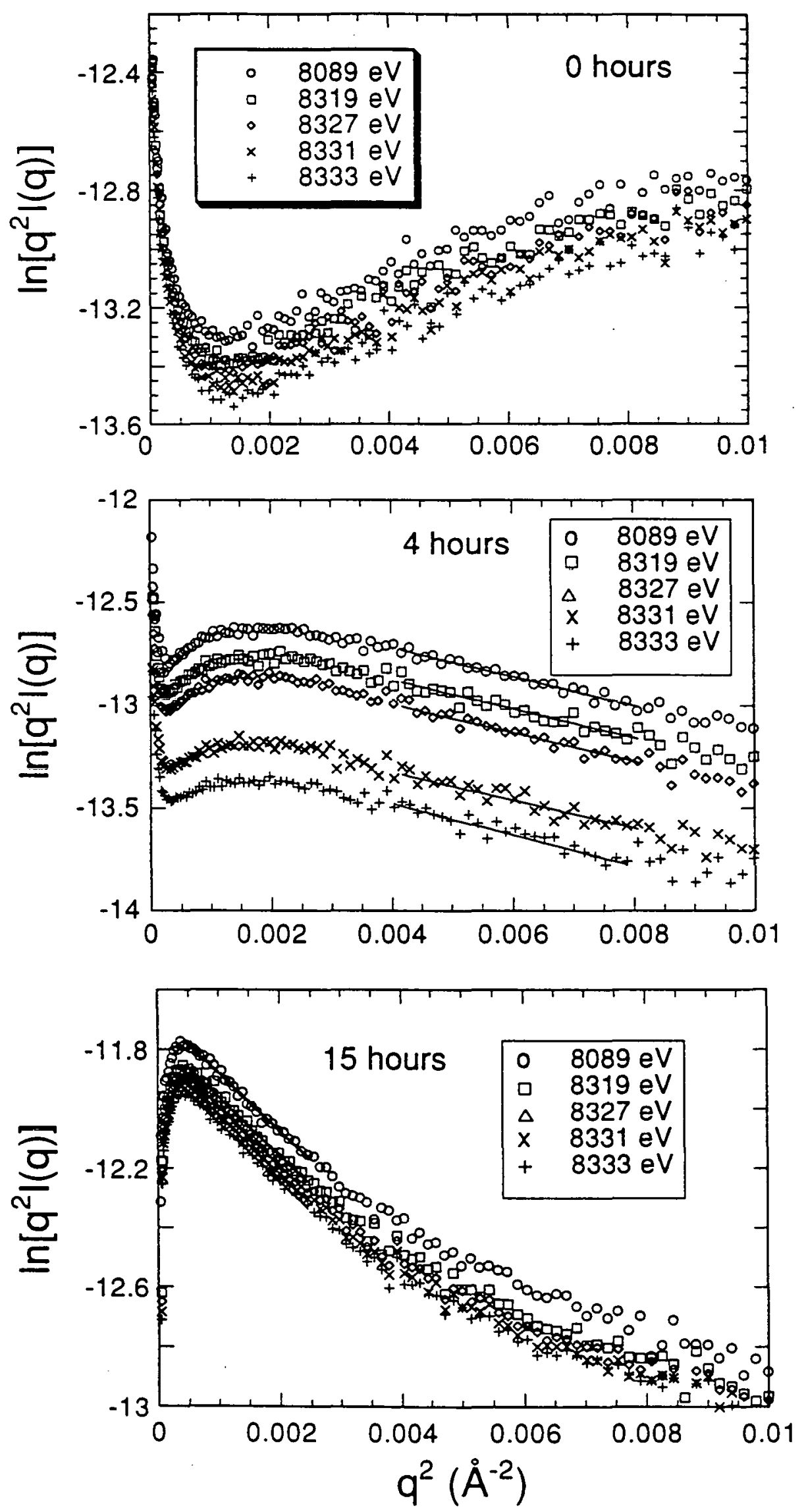

Figure 1. ASAXS measurements of synthetic $\mathrm{Ni}$-clay system. 

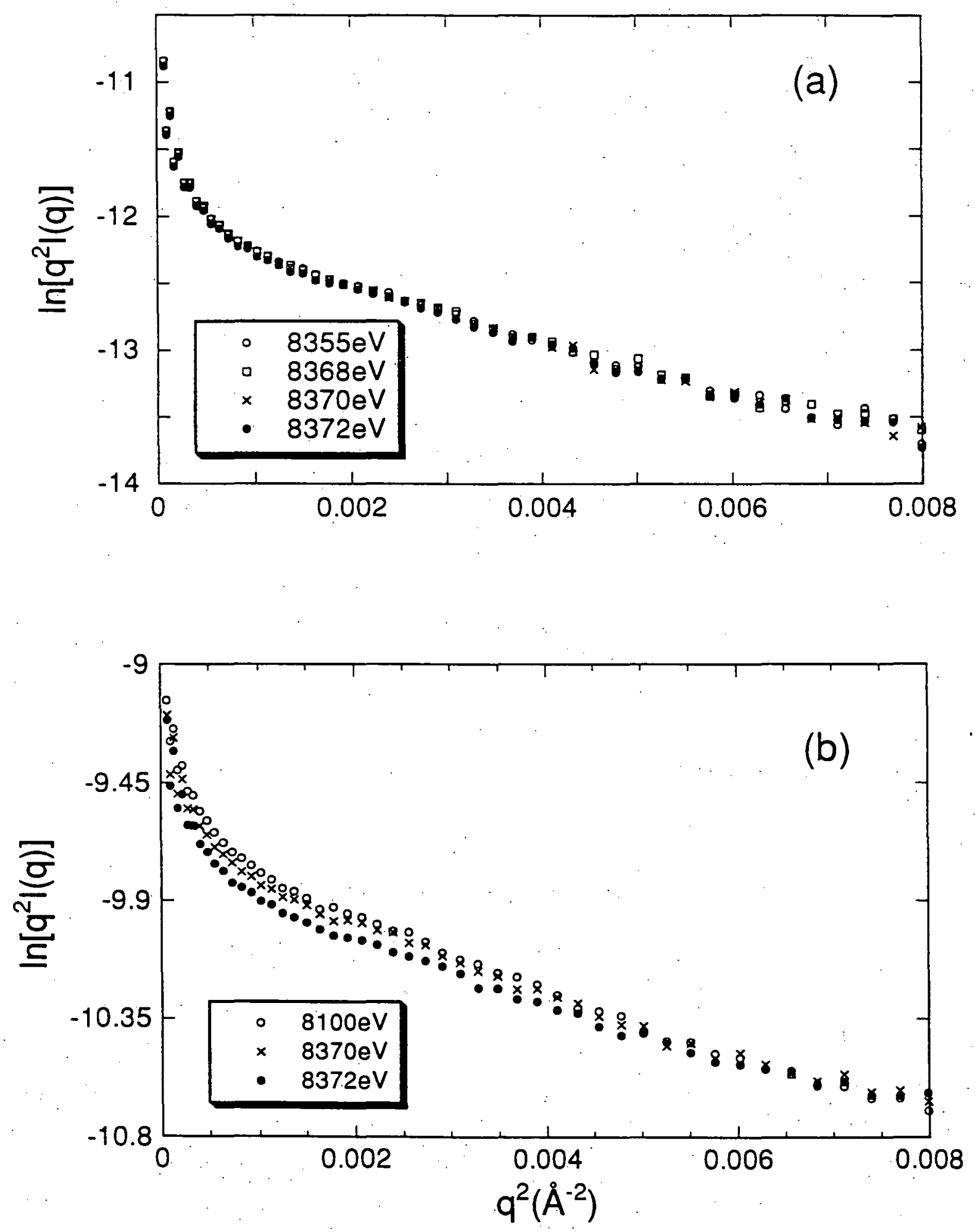

Figure 2. ASAXS measurements of $\operatorname{Er}(\mathrm{III})$-clay system (a) dry and (b) hydrated. 


\title{
94-103R1 -- DEVELOPMENT OF A CODE FOR SIMULATING WELDING PROCESSES
}

\author{
Associate Laboratory Director Area: Energy and Environmental Science and \\ Technology
}

\section{Principal Investigators:}

Funding History:

\author{
R.A. Valentin and H.M. Domanus, \\ Energy Technology Division
}

FY $1993-0-$

FY $1994 \$ 218.4 \mathrm{~K}$

FY $1995 \$ 131.0 \mathrm{~K}$

FY $1996 \$ 100.0 \mathrm{~K}$

FY $1997-0-$

Purpose: Modify Argonne's recently developed casting simulation code, CaPS (Casting Process Simulator), to model gas-tungsten-arc (GTA) and gas-metal-arc (GMA) welding. Both GTA and GMA welding are used extensively in the heavy equipment fabrication industry. Creation of a simulation tool that could quickly evaluate how variations in process variables such as current and voltage, fill-wire feed speeds, and component motion alter final weld geometry, residual strain, and distortion would have great value in improving weld quality and reducing process time. Because a major component of such a simulation concerns formation, motion, and solidification of the weld pool, the CaPS code is a natural starting point. In particular, the convective fluid dynamics of the pool and its solidification are already available through CaPS. This project concentrates on modifications needed to add mass transfer, evaporation, surface tension, and electrodynamic effects. Also, because the process of adding molten material to build the weld involves a constantly changing computational region, it is necessary to make certain basic changes in the structure of the existing code. At the end of FY 96, development is to be at a point where a convincing demonstration of feasibility could be made to those industrial concerns that have already indicated interest in the project.

Approach: In developing the CaPS code and applying it to various problems of mold filling and solidification, it became evident that many of the technical issues being addressed for casting did not differ greatly from those associated with the weld process. In particular, the formation and behavior of the weld pool could certainly be modeled by an extension of the simulation techniques employed when developing CaPS.

In discussing the subject of weld simulation with various industrial contacts, it became clear that there are two coupled but distinct areas of concern. The first concerns local effects, that is, physical processes that are local to the weld pool, its solidification, and to evolution of final material properties and local stresses. The second area concerns more global features such as distortion of an entire structure due to the strains induced during welding. Because CaPS was well suited to the study of local effects, it was decided that only weld pool dynamics would be considered during the early stages of this feasibility study. A link to global structural concerns and perhaps to other computer codes is to be left for later development if an eventual industrial partner is found after the feasibility work has been completed. 
While the methods used in developing CaPS will be those used in the welding code, the later will not be a simple extension of CaPS. Instead, a new code will be built on lessons learned during that development, together with a modified code structure needed to simulate the complexity associated with the dynamics of the evolution of the weld pool.

The welding process is simulated by computing detailed transient, three-dimensional distributions of volume of fluid, velocity vectors, pressure, temperature, solid mass fraction, and most recently, solute mass fraction. The spatial variable distributions are computed for a large number of hexahedral cells arranged in an Eulerian structured mesh framework. Integral forms of the conservation of mass, momentum, energy, and species equations along with temperaturedependent physical properties are used. The finite volume formulation involves approximating the various terms of the integral conservation equations with respect to a cell and its six adjacent neighbors.: The finite-volume approach gives rise to a set of algebraic equations, one per variable for each cell. The coefficients of the linearized algebraic equations are assembled into matrices. A segmented-solution algorithm employing sparce matrix solvers is then used to determine the cell values. The terms of the algebraic equations can be identified as transient terms, flux terms (convective and diffusive), and source terms. Transient results are obtained by repeating a time-stepping algorithm where each variable distribution is advanced over a time increment.

The geometry is described by a collection of parametric cubic volumes (hyperpatches) which are an extension of Hermite curves. It is assumed that the model space is completely described by this set of hyperpatches. Commercial software is used to define the geometry and obtain the set of hyperpatches. Physical characteristics such as boundary and initial conditions are assigned directly to the geometric description and are, therefore, mesh independent. An automatic mesh generator is used to render the geometry into hexahedral cells.

\section{Technical Progress and Results:}

FY 1994: Development of the welding process simulator software was ported to an HP 735 with 112 megabytes of memory. Benchmark comparisons indicate a factor of 13 increase in computation speed over the SUN SPARCstation.

The momentum and energy routines in the simulator module were restructured to accommodate the addition of new source terms. The matrix coefficients are now built modularly to facilitate implementation of new physical models. In the momentum routines, there are now options for new source terms to model the forces due to surface tension and electromagnetic effects. Heat loss from the weld pool by evaporation and heat added from the arc can now be considered as sources in the energy routines.

Practical welding process simulations can be expected to involve more than 1 million computational cells. While the algorithms and storage schemes were designed to (at least theoretically) automatically scale to any size problem, practical limits were observed in test cases. A 1-million-cell rendering took more than $35 \mathrm{CPU}$ hours to determine. Several modifications to the automatic mesh generator module were therefore made. These sped up the mesher so that a 1-million-cell rendering can now be done in less than 11 minutes and a 2million-cell rendering in less than 25 minutes. The simulator module was found to be even more 
constrained. A major revision was made to the storage allocation scheme that explicitly distinguishes between potential fluid/phase change cells and cells that will always be solid throughout the simulation. The new storage scheme had implications for virtually every subroutine in the simulator module, so the modifications were extensive. A 1-million-cell simulation was demonstrated.

FY 1995: It has been observed that occasionally a hyperpatch face doesn't match the adjacent neighboring hyperpatch face. Thus, a concave gap may be present in the geometry. In mesh of fine enough resolution, cells are excluded from the gap region. This is an unacceptable situation and several methods to accommodate such poor geometric models coming from commercial software were attempted. While these modifications improved the situation, no method was found to universally fix a flawed geometry coming out of commercial software. An alternative geometric description based on the stereo lithography (STL) file format is currently believed to be more reliable. Development of an STL alternative is for future work.

Due to cost, slowness, difficulty of use, and size limitations of the commercial package, PATRAN, pre- and post-processing alternatives have been investigated and developed. A simple preprocessor, CPRE, was developed to generate a geometric description from free form user input. While this initial version can only handle geometries that are made up of straight edged hexahedra, it is adequate for our immediate needs. Visualization of the geometry, as it is being constructed, uses the AVS commercial software package.

Implicit in the welding process simulator software is the need to visualize the results. Alternative results presentation schemes have been investigated and a new postprocessor, CPOST, developed, using AVS to visualize the results. CPOST can do everything that the CaPS postprocessor, which used PATRAN, could do and more, and is much faster. The newly developed CPRE and CPOST software have established a viable alternative to PATRAN.

Most weld materials are alloys or mixtures of several constituents. While the constituents are uniformly distributed in the object part and filler wire, they are usually of different compositions. As the filler wire melts and combines with the molten portion of the object part and begins to solidify, a separation of the alloying constituents occurs due to solubility differences between liquid and solid phases. Macroscopic segregation refers to this process which creates an inhomogenous distribution of the constituents in the final weld. Inhomogenous distributions of alloy constituents result in variations of the mechanical properties which, in turn, effect the performance of the weld. An initial, binary macrosegregation model has been developed and implemented.

During FY 1996, efforts will continue to develop and implement various physical models needed to accurately simulate the weld pool dynamics. The STL alternative will also be investigated. Sufficient capability will exist by the end of FY 1996 to demonstrate code feasibility to potential industrial sponsors.

Specific Accomplishments: Participated in the benchmark exercise of the "Modeling of Casting, Welding and Advanced Solidification Processes" conference held in London, England from September 10-15, 1995. This included performing a blind simulation of an experiment and presenting a paper titled "Computer Simulation Using CaPS of an Aluminum Plate Casting" at 
the conference. Our simulation did quite well in comparison to the actual experiment and relative to the nine other submissions. Figures 1 and 2 show the mold cavity just as the liquid aluminum starts to enter it and the solid/liquid interface before the plate completely solidifies, respectively. A video was prepared showing the complete mold filling and solidification sequences.

To. broaden the potential interest in such models, interactions with staff in other program areas have continued to investigate the coupling of the welding process simulator with structural codes capable of modeling global and local strain and distortion. 


\section{CaPS-3D}

$t=0.73 \mathrm{~s}$

Figure 1. Filling of the benchmark plate 


\section{CaPS-3D}

$$
t=35.00 \mathrm{~s}
$$

Figure 2. Solidification of the benchmark plate 


\section{5-020N -- DEVELOPMENT OF LASER TECHNOLOGY FOR THE DECONTAMINATION OF SURFACES}

\section{Associate Laboratory Director Area:}

Principal Investigator(s):

Funding Profile:

\author{
Engineering Research
}

K.H. Leong and D.R. Henley, Technology Development Division

\author{
FY $1993-0-$ \\ FY $1994-0-$ \\ FY $1995 \$ 207.1 \mathrm{~K}$ \\ FY $1996-0-$ \\ FY $1997-0-$
}

Purpose: This project is an investigation of the engineering and processing parameters required for optimal effectiveness of lasers as a tool for decontamination of surfaces. The first major area of investigation is the beam delivery system, i.e., the fiber-optic system and the optical system used to transmit, shape and control the laser intensity. The second major area is the effluent collection system. The effluent collection system must prevent any of the material removed from the surface from being released into the environment. Methods will be developed to quantify the success of these techniques and compare them with other decontamination methods. This project directly supports ANL's efforts to develop a lead role in the U.S. Decontamination and Decommissioning (D\&D) program by investigating a technique that offers great promise for reducing the volume of contaminated material that must be stored. This reduction of volume results in both economic and safety benefits.

Approach: There are many techniques for cleaning a contaminated surface. Some are as simple as soap and water. Many techniques remove contaminants by sandblasting, ablation with frozen $\mathrm{CO}_{2}$ pellets, or high-pressure/abrasive water jets. These techniques are effective, but have many drawbacks: they often generate mixed wastes, they can damage the surfaces, and they often drive some fraction of the contaminants deeper into the material, particularly if the material is porous. Laser ablation or stripping avoids these difficulties because the contaminated surface material is vaporized, yielding a smaller volume of contaminated waste. The surface can be protected from damage by precise control of the laser energy. The vaporization process does not drive the contaminants into the material.

The work done in this project exploits the existing facilities of the Laser Applications Laboratory (LAL) to investigate material removal using a laser. The laser system used for this work is a $1600 \mathrm{~W}$ pulsed Nd:YAG laser. This laser was chosen because the Nd:YAG laser, operating at $1064 \mathrm{~nm}$, is well suited for transmission through standard high-power optical fibers. The use of an optical fiber to transmit the laser power from the laser to the workpiece allows the laser energy to be transmitted into hazardous or hard-to-access locations via a small, flexible cable. The laser beam is shaped and targeted on the surface by the optical system. The optical system may be mounted on a CNC motion system, such as a robotic arm or a 5-axis workstation. This concept is schematically illustrated in Figure 1 . The existing 5-axis workstation in the LAL was used. 


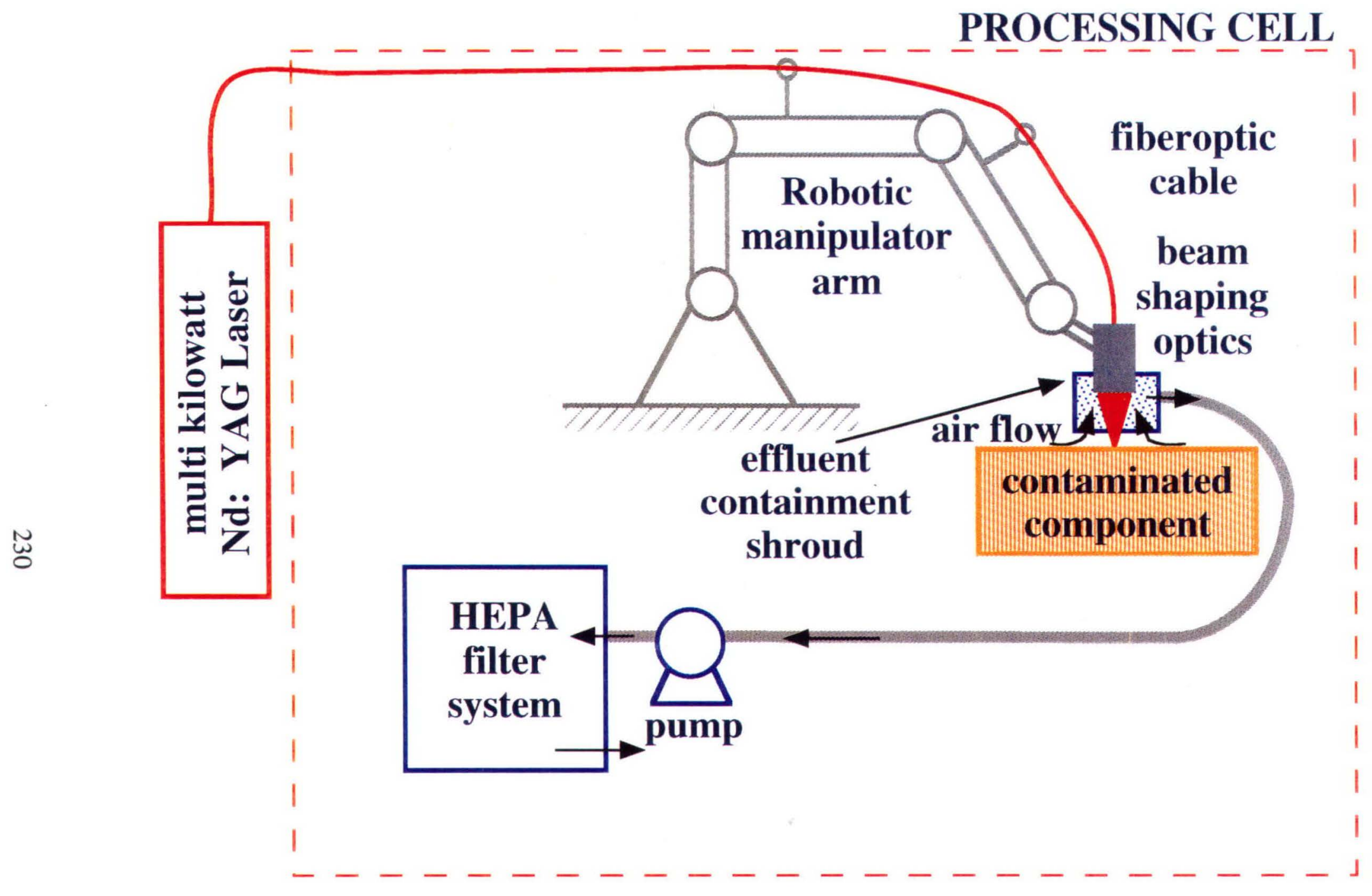

Figure 1. Schematic diagram of robotic decontamination concept. 
The effluent collection system uses a shroud and suction to contain the particles generated by the ablation or combustion of the contaminated surface. The particles are passed through a HEPA filter to remove the hazardous aerosols. The design of the shroud, which also must incorporate some protection for the optical system, is a key consideration in the effectiveness of the effluent collection and the efficiency of material removal. Various shrouds and variants were investigated to better understand the aerodynamics in the shroud so that constraints for an optimal design can be determined.

The effectiveness of the shrouds was determined by using standard aerosol counting techniques to monitor the total number of particles and gather information about the size distributions of particles. This aerosol monitoring was one method used to assess the containment effectiveness of the shrouds. Additionally, based upon knowledge of the paint composition, mass-balance measurements provided a second vehicle for determining collection efficiency. Painted surfaces were stripped during this portion of the project. The experimental setup used for some of these experiments is shown in Figure 2.

Finally, the beam delivery system must be amenable to use with a remote system. A system was designed for robustness and flexibility that used commercially available components and a highpower fiber-optic cable that were mechanically rugged and offered some damage tolerance to back reflections, as might be encountered when cleaning an aluminum surface. The optical system also had to be designed to provide adequate irradiances and good coverage. Improvements to the beam delivery system: more rugged fiber connectors, better lenses, and easier optics changes, were investigated.

Jeff Grace, a postdoctoral appointee, collected the data for this project, including all of the aerosol diagnostics. He also selected the hardware for the beam delivery and effluent collection systems. Boyd Hunter, a postdoctoral appointee, did some shroud and optical system design and review activities, including the work on the evaluation of new lens technologies. He also coordinated the implementation of a diagnostic interface for the Nd:YAG laser.

Technical Progress and Results: The most important technical result of this effort was that the shroud collection system successfully captured the effluent. By mass balance, at least $99 \%$ of the paint was collected. The aerosol data showed that at least $99.97 \%$ of the particles smaller than $10 \mu \mathrm{m}$ were collected. The collection efficiency improves for smaller particles; at least 99.999999\% of the particles smaller than $1 \mu \mathrm{m}$ were collected. Thus, the shroud provided the requisite capture efficiency for removing radioactive contaminants.

The tests showed that the shroud aerodynamics strongly influences the efficiency of paint removal. The shroud dimensions, flow rates, and flow patterns can reduce the overall process efficiency by encouraging soot formation and redeposition. This soot must be removed by an additional cleaning pass. This soot formation is more of a problem with this laser because peak irradiances achieved are orders of magnitude lower than the irradiances used by some researchers to strip paint. This irradiance difference is because our laser is not Q-switched. At this point, we expect that the shroud aerodynamics can be substantially improved with additional effort. 
The data showed that the particle distributions are measurably different between different paint compositions (latex and oil-base). This is illustrated in Figure 3, which shows impactor data for the different paints. Data obtained with a Particle Measurement System aerosol spectrometer, which gives particle size data, have shown that the $10 \mathrm{mil}$ and 14.5 mil latex have the same particle distributions. These paint thicknesses are considered representative of the D\&D application. Figure 4 suggests that the selection of laser parameters is driven most strongly by paint thickness, rather than

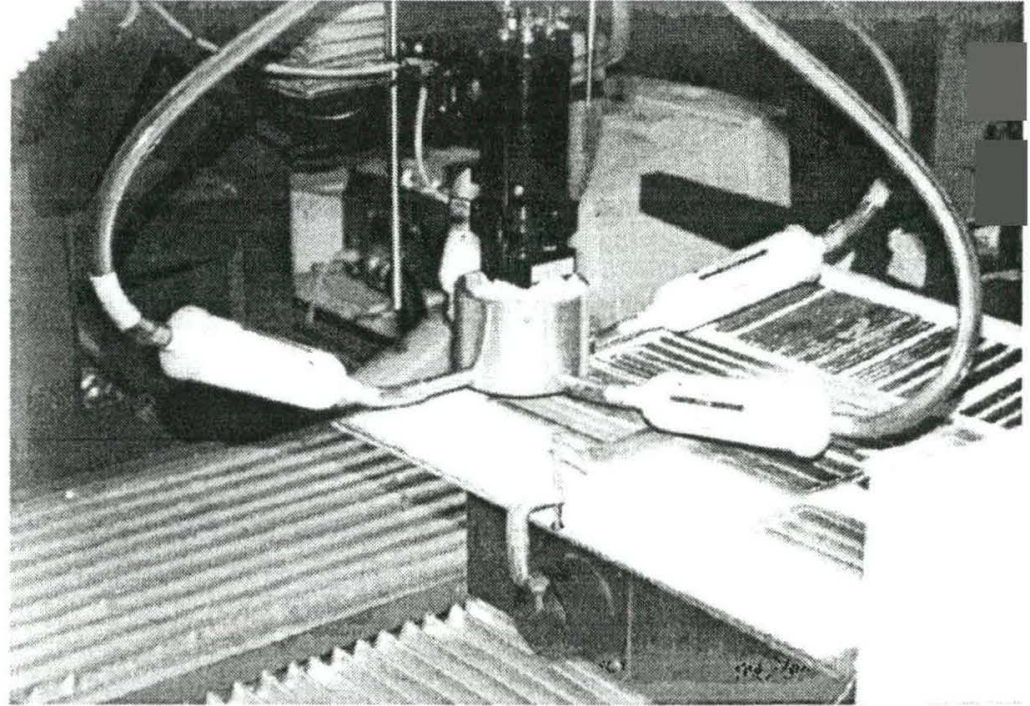

Figure 2. Paint stripping using fiber-optic delivered Nd:YAG laser. This photo shows the new beam delivery system, a shroud, and HEPA filters used during the mass-balance tests. peak power or repetition rate.

The removal rate is relatively insensitive to peak power level; for this average power level, 10 mils is approximately the optimal thickness. Note that Figure 4 plots the volumetric removal rate, not the surface area removal rate; volumetric rates were considered more appropriate because of the thick paint layers in this application. If the paint is too thin, energy is wasted by heating the metal plate and a low removal rate results. If it is too thick, too little paint is removed and several passes are required to remove the paint, yielding a low rate. The removal rate takes into account the volume of paint removed, the feed rate, and number of passes required to clean the surface effectively. There are several possible reasons for this relative insensitivity to peak power: (1) the range of peak power levels did not vary enough to change the removal mechanism, (2) the process appears to be energy limited, and (3) soot formation forces multiple passes to be made. Preliminary tests indicated that soot formation can be eliminated by a cross-jet; however, containment of effluent is not possible because the high

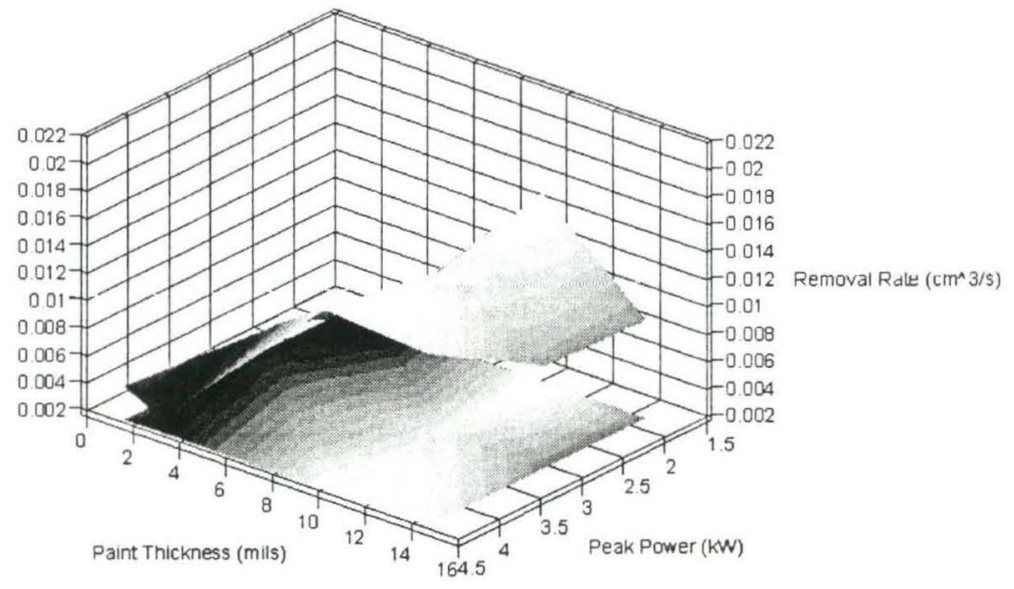

$\begin{array}{lllllllllll}0.022 & 0.02 & 0.018 & 0.016 & 0.014 & 0.012 & 0.01 & 0.008 & 0.006 & 0.004 & 0.002\end{array}$

Figure 4. Latex paint removal rates as a function of paint thickness and peak laser power. The laser average power ranged from $730 \mathrm{~W}$ to $890 \mathrm{~W}$. The surface is a fit to the data. Each location on the surface had to account for multiple data points, each representing different feed rates and number of repeated passes. The "floor" of the figure is a contour map of the $3 \mathrm{D}$ surface above it. 
velocity jet blows the aerosol out underneath the shroud.

The beam delivery system design yielded a robust system with highpower connectors that are tolerant of back-reflections from the workpiece and allowed easy maintenance of lenses and protective fixtures. Work was begun to investigate improvements possible when replacing standard transmissive optics with Gradium ${ }^{\mathrm{TM}}$ (axial-gradient) optics. Because the axial-gradient lenses produce very little spherical aberration, smaller spot sizes and longer stand-off distances are expected than can be produced with comparable BK7 glass lenses. The Nd:YAG laser controls were retrofitted to interface with diagnostic instruments in the LAL.

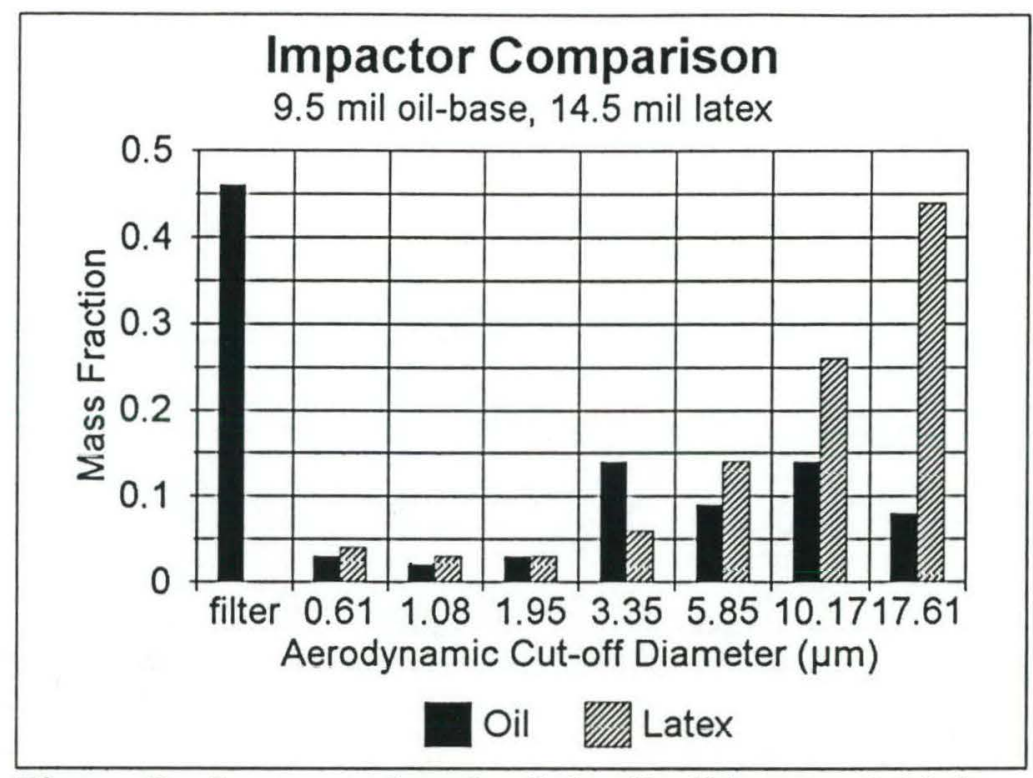

Figure 3. Impactor data for 9.5 mil oil-base and 14.5 mil latex paint which shows the marked difference between the combusted/ablated material. The large fraction of the oil-base paint captured by the filter indicates that combustion is a significant process for the oil-base paint.

In FY 1996, we plan to refine the shroud design to reduce or eliminate the soot redeposition. As demonstrated by the cross-jet experiments, avoiding the soot redeposition will increase (up to a factor of two) the paint removal rate. We would like to expand our efforts to include paint removal from surfaces other than metals. We intend to continue making improvements to the beam delivery system as problems or new technologies are identified. We intend to develop the cross-flow shroud design further.

Specific Accomplishments: An internal report documenting the results of this effort is in preparation. The work performed under this project was the subject of a presentation, "Experimental Study of an Aerosol Collection System for Laser Processes," at the October 1995 AAAR Conference; an additional (refereed) article is being prepared. The work with the axial gradient lenses will be presented in articles under preparation for Industrial Laser Review (trade magazine), and Applied Optics (refereed). A preliminary design for a cross-flow shroud system, which will be required to ablate contaminated metal surfaces, has been prepared and is illustrated in Figure 5. 


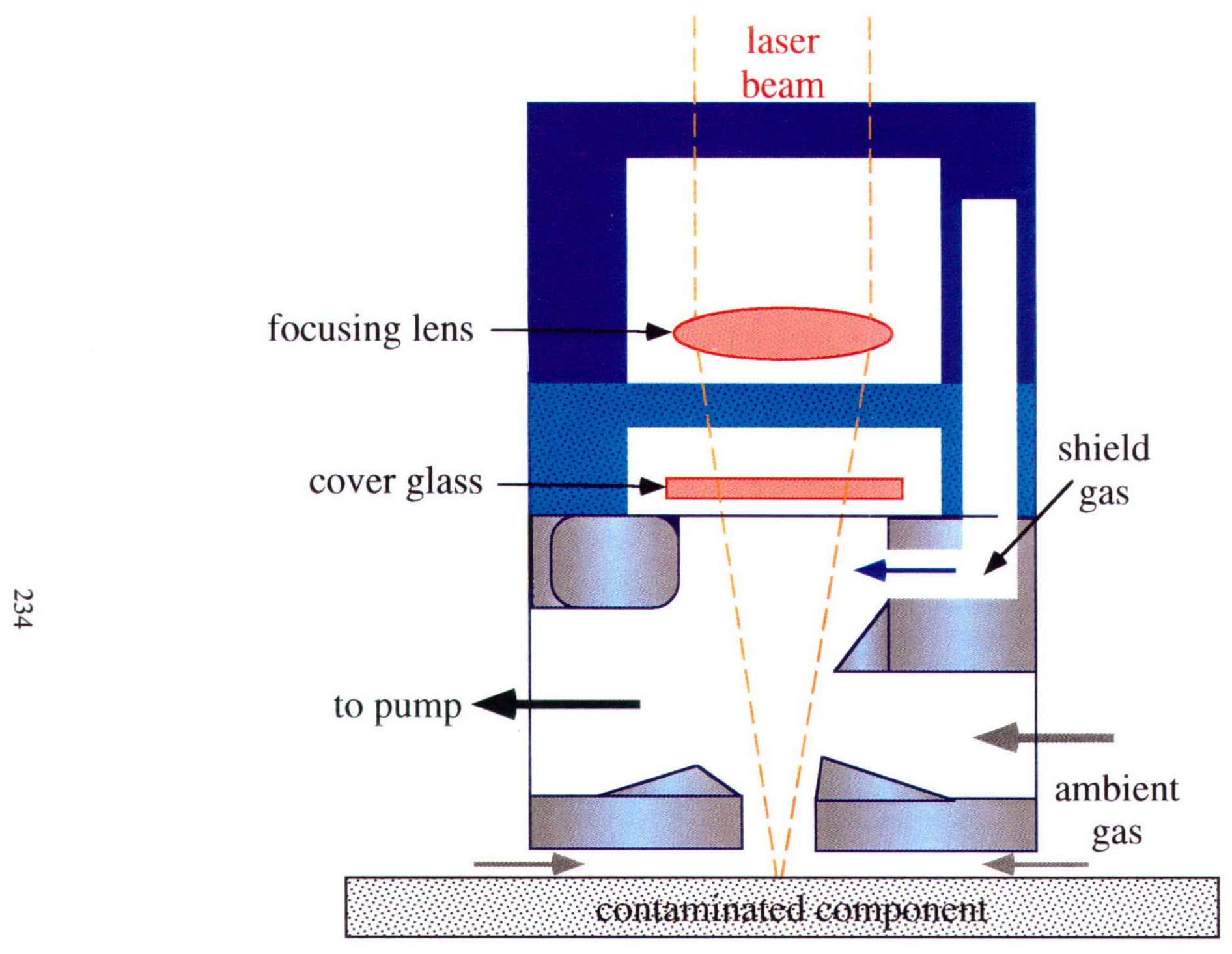

Figure 5. Preliminary cross-jet design for lens protection and effluent removal. 


\section{4-182R1 -- REMOVAL OF CONTAMINANT CU FROM REMELTED STEEL SCRAP ${ }^{1}$}

Associate Laboratory Director Area: Physical Research

Principal Investigators:

M. Blander, Chemical Technology Division

D.R. Diercks and A.B. Cohen, Energy Technology Division

Funding Profile:

FY $1993-0-$

FY $1994 \$ 144.4 \mathrm{~K}$

FY $1995 \$ 148.2 \mathrm{~K}$

FY $1996-0-$

FY $1997-0-$

Purpose: The purpose of this science and technology LDRD-funded project is to examine an approach which uses aluminum-sulfide or alumino-silicate based slag for the removal of copper from molten scrap. Copper is a major contaminant of steel scrap, and its presence in steels at levels greater than $0.10 \mathrm{wt}$. \% can adversely affect the mechanical and fabrication properties of the resulting steel. No economically viable process for $\mathrm{Cu}$ removal is currently available, and the demand for high quality scrap is increasing as mini-mills expand into the production of high grade steel sheet. The development of a practical method for removing copper from scrap would make it possible to beneficiate a significant portion of our nation's uniquely large (approx. 750 million ton) scrap pool and provide the raw material for expanded domestic production of high quality products such as steel sheet at reduced cost. In addition, the increased use of scrap would result in significant environmental benefits and energy savings.

Approach: Slagging of molten scrap to remove copper would be the method preferred by steel manufacturers because it is consistent with methods used to remove other contaminants. However, the thermodynamics for reactions such as:

$$
\mathrm{FeS}+2 \mathrm{Cu}=\mathrm{Fe}+\mathrm{Cu}_{2} \mathrm{~S}
$$

are not favorable for oxides $\left(\mathrm{K}=7.8 \mathrm{E}-4\right.$ at $\left.1600^{\circ} \mathrm{K}\right)$ and only slightly favorable for sulfides $\left(\mathrm{K}=2.9\right.$ at $\left.1600^{\circ} \mathrm{K}\right)$. The distribution coefficient for copper between a slag phase and the molten steel can be written as:

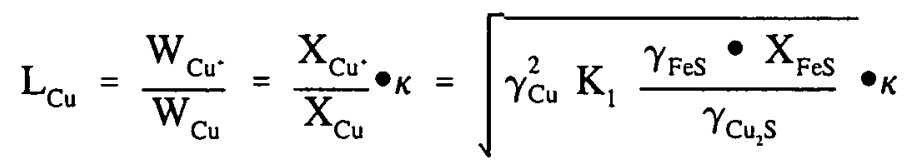

where $\kappa$ is a constant to convert mol. \% to wt. $\%, \mathrm{~K}_{1}$ is the equilibrium constant for eq. $1, \mathrm{X}_{\mathrm{i}}$ is the mole fraction of component $i$, and $\gamma_{i}$ is the activity coefficient of component $i$. Several

${ }^{1}$ CCST Project 
factors have small effects on increasing $\mathrm{L}_{\mathrm{Cu}}$; however, a decrease in the activity coefficient of cuprous-sulfide has the potential for a large increase to $\mathrm{L}_{\mathrm{Cu}}$. Experimental work in the field of molten salts has shown that the activity coefficients of a given salt can be significantly decreased in binary mixtures, and the change is a function of both relative cation size and charge. The activity coefficient of a given monovalent solute (e.g., $\mathrm{Cu}^{+}$) can be decreased in a binary mixture by increasing the charge and decreasing the cation radius of the solvent. To make cuprous-sulfide more stable relative to ferrous-sulfide, use of a binary slag containing aluminumsulfide would lower the activity coefficient of cuprous-sulfide. The $\mathrm{Cu}^{+}$ion has a radius of about $0.96 \mathrm{~A}, \mathrm{Fe}^{+2}$ has a radius of $0.75 \mathrm{~A}$, and $\mathrm{Al}^{+3}$ has a radius of $0.45 \mathrm{~A}$.

A natural extension of the above discussion suggests that the use of a solvent with a polyvalent, small radius cation could make the use of an oxide-based slag favorable for the removal of copper from steel. In other words, the use of alumina and silica as a solvent may make the formation of cuprous-oxide more favorable than iron-oxide. In addition, there is a charge compensation effect in silicates in which $\mathrm{Al}^{+3}$ ions bond strongly (i.e., forms a complex) with a monovalent cation in the presence of a silicate network in acid solutions. Estimates have been made that indicate the activity coefficient of cuprous-oxide may be quite low. Further complexing by the addition of a complexing anion, such as $\mathrm{S}^{2-}$, to the silicate melt could also help make the oxide slags favorable.

Experiments are designed to systematically study the effects of compounds with polyvalent cations on the removal of copper from molten steel; they are conducted by Adam Cohen, who is a full-time staff employee in Energy Technology Division and is pursuing a $\mathrm{PhD}$ in Material Science on a part-time basis at Northwestern University. The experiments involve heating a crucible with copper-bearing steel and the slag composition of interest to the desired testing temperature and maintaining the temperature until equilibrium between the metal and slag phases is reached, usually with the aide of mechanical agitation. The sulfide slag experiments are run in a controlled, low oxygen environment under an inert gas (argon) atmosphere to minimize the adverse effects of oxygen on the sulfide slagging process. The furnace tube assembly for the alumino-silicate slag experiments is less elaborate because the potential for oxygen contamination does not exist.

Technical Progress and Results: The project builds upon preliminary results obtained by ANL during the USDOE Steel Initiative in 1987 during which a distribution coefficient for copper in a binary $\mathrm{Al}_{2} \mathrm{~S}_{3}$-based slag as high as 31 was found. The use of $\mathrm{Al}_{2} \mathrm{~S}_{3}$-based slags to remove copper from steel was patented in 1990 (U.S. Patent No. 4,925,488). The new work on the sulfide-based slags was designed to eliminate oxygen interference. Thirty tests were completed with sulfide melts; fifteen with a binary slag mixture, and the remainder with $\mathrm{CaS}$ or $\mathrm{MgS}$ added. While higher distribution coefficients were theorized, experiments showed a maximum distribution coefficient of 31 . for a slag with 30 at. \%. FeS. The effect of additives has not been clearly defined. The highest distribution coefficient with a ternary slag was 40 , with a composition of $\mathrm{Al}_{2} \mathrm{~S}_{3}-14 \mathrm{wt}$. \% FeS-4wt. \% MgS; however, the result was not repeated and is now questionable.

Fifty four tests were completed using an alumino-silicate based slag; thirty seven of the tests had sulfide additives, while the remainder were pure oxide slag. Alumino-silicate based slag experiments without sulfides added showed a maximum distribution coefficient of $\sim 0.5$. While 
the copper content was not reduced below $0.1 \mathrm{wt} \%$ and the distribution coefficient is significantly below that necessary for a viable process, the result can be considered somewhat successful. The "high value" for $\mathrm{L}_{\text {cu }}$, considering the extremely low equilibrium constant for the ferrous-oxide - cuprous-oxide reaction $\left(\sim 10^{-4}\right)$, indicates that the activity coefficient for $\mathrm{Cu}_{2} \mathrm{O}$ can be made very low.

The sulfide additive was included in the slag to improve the distribution coefficient by promoting the formation of cuprous-sulfide while still employing the advantages of the alumino-silicate slag. The highest distribution coefficient achieved was 6.8, with a slag composition of $\mathrm{SiO}_{2}-23$ wt. \% $\mathrm{Al}_{2} \mathrm{O}_{3}-1$ wt. \% CaO-3 wt. \% FeS-3 wt. \% MgS. It was discovered late in the project that the slagging in the oxide system with sulfides added works best on low carbon steel, as opposed to the pure sulfide slags which are optimal in carbon saturated steel. The presence of carbon increases the activity coefficient of copper in the steel, which in turn increases the distribution coefficient for copper. However, in the oxide system there is a competing effect; carbon reacts with the iron-oxide species to form carbon-monoxide and iron. The iron-oxide species is necessary to drive the reaction with copper. While other tests explored variations in the amount of calcia, iron-sulfide, magnesium-sulfide and calcium-sulfide, only two of the tests used low carbon steel. More tests should be run to optimize the slag composition and maximize the distribution coefficient.

\section{Specific Accomplishments:}

One publication, "Removal of Copper from Carbon-Saturated Steel with an Aluminum Sulfide Iron Sulfide Matte," has been written, but not yet submitted. It will be submitted to Metallurgical Transactions $B$ during the early part of FY 1996.

An invention report on the alumino-silicate (oxide-based) slag for the removal of copper from steel has been submitted to the Laboratory's Industrial Technology Development Center (ITDC). 
blank page 


\title{
95-162N -- ION-SPECIFIC SENSORS BASED ON CONDUCTIVE ORGANIC POLYMERS ${ }^{1}$
}

\author{
Associate Laboratory Director Area: Physical Research \\ Principal Investigator: $\quad$ M.R. Wasielewski, Chemistry Division \\ Funding Profile: $\quad$ FY $1993-0-$ \\ FY $1994-0-$ \\ FY $1995 \$ 79.9 \mathrm{~K}$ \\ FY $1996 \$ 60.0 \mathrm{~K}$ \\ FY $1997-0-$
}

\begin{abstract}
Purpose: The purpose of this project is to develop new conductive organic polymers that will incorporate ion-selective binding sites for use as sensors. Exposure of thin films of these materials to metal ions results in significant changes in both the conductivity and in the electroluminescent properties of these films. These new polymers are of potential use in the detection of trace amounts of specific ions in the presence of other contaminants. Cheap, sensitive, ionselective sensors are very valuable for quantitation of hazardous materials in waste streams and in efforts to gauge the effectiveness of environmental remediation strategies. The ease with which polymers can be synthesized and modificd to obtain desirable properties makes this approach to sensors appealing.
\end{abstract}

Approach: Poly(phenylenevinylene) (PPV) polymers have emerged as promising new materials for possible use in display technologies. This family of polymers exhibits metal-like conductivity and luminesces strongly when subjected to a bias voltage. The conductivity and luminescence properties of these polymers are known to depend on the detailed structure of the polymer backbone. The introduction of functional groups onto the polymer backbone that modify this structure results in large changes to both the conductivity and luminescence of the polymers. Synthetic strategies for producing organic structures that specifically recognize metal ions is well-developed. However, the introduction of molecular recognition sites into conductive polymers, such as PPV, for the purpose of controlling their structure, and thus their properties, is just beginning to be researched. In this project we have developed new PPV-type polymers that are soluble in organic solvents and have appropriate ligands for binding metals. This is a significant advance over the use of soluble precursor polymers to produce intrinsically insoluble final polymer products. The combined material makes use of the unique electrical and optical properties of the polymer to indicate whether a particular type of metal ion is bound to a thin film of the polymer.

A series of polymers based on the PPV structure have been synthesized. These polymers have been derivatized with side chains that make these polymers soluble in organic solvents, and thereby make processing of these materials easy. In addition, we have incorporated side chains on the PPV polymer bearing ligands that can bind metal ions. We have made a fundamental advance by preparing final conductive polymers that are themselves soluble in organic solvents,

'Individual Investigator Project 
and are thereby easily processed. This improves substantially on our original idea that the final polymer films would be made by synthesizing a soluble precursor polymer, preparing a film from it, then converting the precursor polymer film by further chemical treatment into an insoluble final polymer film. This basic improvement makes it much easier to process the final polymeric sensor material into whatever form applications require, e.g. films, strips, sheets, etc. We have already produced the requisite polymers bearing alkoxy side chains for enhanced solubility and processability. We have demonstrated that metal binding sites within the polymer can select between transition metal ions. We have also investigated both the conductivity and electro-luminescence of these polymer films in the presence of these metal ions. Conductivity measurements can be made on the films themselves, while electro-luminescence measurements depend on light produced by the film, when electrical charge is injected into the film. Metal ions can quench the resultant luminescence, usually by an electron transfer mechanism, again resulting in a quantitative probe of ion concentration. The stability and longevity of these films will be investigated in chemical environments that approximate those found in chemical waste streams.

Technical Progress and Results: New conductive polymers based on a poly(phenylvinylene) structure with ion-binding sites were synthesized. Several problems were addressed: 1) Which molecular structures are most likely to selectively bind a particular kind of ion to the polymer, e.g. alkali metals, lanthanides, transition metals? 2) How does the conductivity and electroluminescence of these polymers depend quantitatively on ion binding and structure of the binding site? 3) Can a practical, inexpensive sensor material be developed from these molecular systems?

Dr. Bing Wang developed the soluble PPV polymers which incorporate metal binding ligands. The strategy that we applied successfully to this problem was to produce a monomer with fullyfunctionalized side chains that bear organic groups that not only impart solubility to the final polymer, but in addition, incorporate the appropriate ligands for metal binding. We found that bipyridyl ligands are useful for binding metals to the polymer. The requisite monomers that go into making the polymer are depicted in Figure 1.
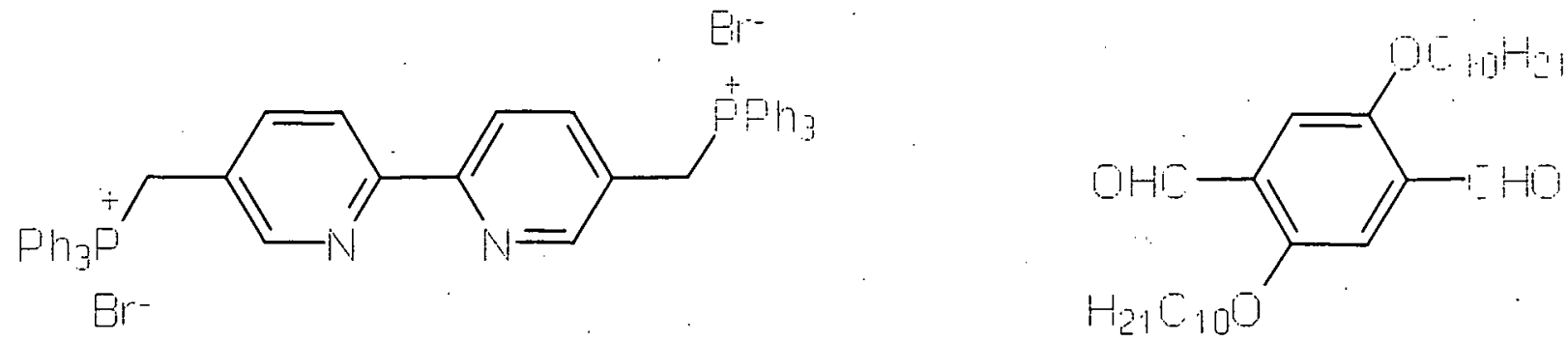

$$
\text { Figure } 1
$$

The polymer synthesis uses the Wittig olefin coupling reaction to yield a clean product, the structure of which is depicted in Figure 2. Dr. Martin Debreczeny characterized the polymer by measuring its optical properties, such as absorption and fluorescence, and its redox properties in solution and in thin films. These films were produced on indium-tin-oxide (ITO) conductive 
glass using a spin-coating technique. The fluorescence from these films responds in a characteristic manner to various heavy metal ions, such as transition metals that are brought into contact with the film by dipping the film coated onto ITO into aqueous solutions of transition metal salts. The results that were obtained give us confidence that sensors can be developed from these new materials. Continued funding under the Individual Investigator LDRD program has been secured for FY 1996 to finish this preliminary study. During FY 1996 macrocyclic ligands that offer better selectivity toward different metals will be incorporated into these conductive polymers. It is hoped that differentiation between most transition metal ions and between lanthanides and transition metals can be achieved.

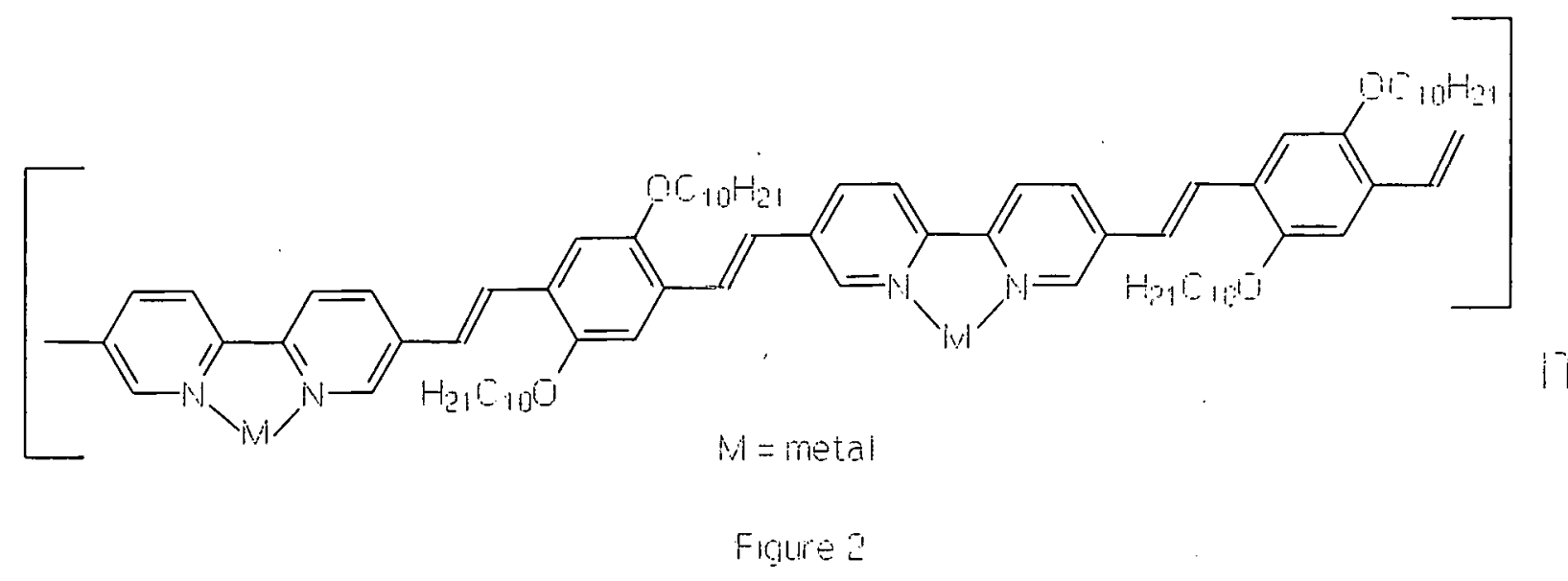

\section{Specific Accomplishments:}

1. This project has been selected for continued LDRD support for FY 1996 during which time we will complete the project.

2. One paper on these new materials is in preparation: "Selective Metal Binding to a Soluble PPV Bipyridyl-Containing Polymer," Bing Wang, Martin Debreczeny, and Michael R. Wasielewski. 
blank page 


\section{4-165R1 -- DEVELOPMENT AND APPLICATIONS OF NOVEL BIOMIMETIC $\mathrm{TiO}_{2}$ PHOTOCATALYSTS FOR SELECTIVE HEAVY METALS REMOVAL ${ }^{1}$}

\author{
Associate Laboratory Director Areas:
}

Principal Investigator(s):

Funding Profile:
Energy and Environmental Science and Technology and Physical Research

M.C. Thurnauer, D.M. Tiede, and D. Meisel, Chemistry Division

N.K. Meshkov, R.W. Peters, and

J.M. Wu, Energy Systems Division

FY $1993-0-$

FY $1994 \$ 176.9 \mathrm{~K}$

FY $1995 \$ 149.5 \mathrm{~K}$

FY $1996 \$ 140.0 \mathrm{~K}$

FY 1997 -0-

Purpose: The purpose of this project is to develop and test advanced, new photocatalysts that have the unique capability of both sequestering and converting heavy metal ions from aqueous solution to their less toxic, readily recoverable metallic forms. This project exploits a strategy to enhance the reactivity of semiconductor photocatalysts for selected toxic compounds by derivatization of the photocatalyst surface with biomimetic polymers. This study will result in identification and development of potential biomimetic photocatalysts for simultaneous heavy metal recovery and organic destruction, in identification of treatment conditions which minimize the residual metal concentration(s) contained in the effluent (even in the presence of complexants and interferences), in development of appropriate scale-up criteria, and in determination of system performance including an economic analysis which will be compared to conventional technologies (such as pump-and-treat using metal hydroxide precipitation or ion exchange).

Approach: Oxidative degradation of pollutants by photocatalysis using semiconductor particles such as $\mathrm{TiO}_{2}$ has been the subject of numerous recent studies. The principle behind semiconductor-assisted photocatalysis in the aqueous phase containing organic pollutants and semiconductor particles involves the photoexcitation of the semiconductor particles by ultraviolet (UV) light causing the energy state of the electrons to change from the valence band of the solid to the conduction band, resulting in the formation of electrons and holes at the surface of the semiconductor particle which can either recombine, producing thermal energy, or interact with other molecules. The radicals generated can either oxidize organic pollutants or reduce heavy metals at the solid/liquid surface. The focus of the majority of laboratory studies involving $\mathrm{TiO}_{2}-$ assisted photocatalysis to date has been on the destruction of organic pollutants in solution using photocatalytic oxidation, with little attention devoted to recovery of heavy metals.

\footnotetext{
${ }^{1}$ CCST Project
} 
The current study seeks to enhance the performance of $\mathrm{TiO}_{2}$-assisted photocatalysis to sequester and recover heavy metal ions in their metallic form while simultaneously destroying organic compounds, potentially in a single step. To achieve our goal a dichotomous approach was taken: (1) the development and design of the photocatalytic system and (2) their application to contaminated waste streams. The approach of the development and design work is to use multifunctional ligands which bind to the colloid surface and the heavy metal ions. The surface of colloidal $\mathrm{TiO}_{2}$ was modified with a series of bidentate and tridentate compounds that have the mercapto, carboxyl and amino groups in different relative position and hydrocarbon chain lengths. The structural, dynamic and redox potential analyses were performed by using IR spectroscopy, EPR spectroscopy and pulse radiolysis, respectively in order to design catalysts that are specific and efficient. The influence of the surface structure on the mechanism and efficiency of the photochemical reduction of metal ion and/or decomposition of organic wastes is being investigated. The application task involves batch, bench-scale studies including parametric testing.

The experimental approach of this project is based on the methods developed and studied by Dr. O.I.Micic (Boris Kidrich Institute - Vinca, Belgrade, Yugoslavia) during 1992 at Argonne National Laboratory as a Maria Goeppert Mayer Scholar for synthesis of small semiconductor particles. In collaboration with Dr. Micic the techniques of EPR were applied to study the photochemistry of colloidal semiconductors. This method allowed direct observation of both reduced and oxidized intermediate species formed during semiconductor illumination.

Technical Progress and Results: The most significant result of this FY is the demonstration that within detectable limits, lead ions could be completely removed from the solution using thiolactic acid modified $\mathrm{TiO}_{2}$. The choice of thiolactic acid as a surface modifier was based on the "selection rules" obtained by investigation of structural (IR spectroscopy), dynamic (EPR spectroscopy) and redox (pulse radiolysis) properties of photocatalytic system.

It was found by IR spectroscopy that the surface modifier must contain the carboxyl group to bind the colloid surface. Heavy metals such as $\mathrm{Pb}^{2+}$ and $\mathrm{Cd}^{2+}$ bind to the other free oxygen of the same carboxyl group. In this way, the carboxyl group bridges the semiconductor particle and the metallic ion, and photogenerated electrons can be easily transferred to the metal ions through delocalized $\pi$ orbital of the carboxyl group. It was found also that $\alpha$ substituted mercapto carboxylic compounds bind to the colloid surface with both the carboxyl and mercapto group with the formation of a five membered ring around surface $\mathrm{Ti}$ atoms. The five membered ring formation stabilizes the complex and the surface modification results in a formation of strong charge transfer complexes of these compounds with small particle $\mathrm{TiO}_{2}$ colloids.

On the other hand it was shown in our dynamic studies using EPR spectroscopy that the efficient separation of photogenerated charges is established when the holes are trapped on the modifier, while the electrons are trapped on $\mathrm{TiO}_{2}$ particles, or in other words, the modifier has to be an electron donating substance. The separation is efficient when the modifiers provide a trap that removes the holes away from the colloid surface. In monodentate bound modifiers the mercapto group acts as the hole trap while in bidentate bound modifiers the holes are trapped on the hydrocarbon side group. Thus, the surface modifier must have a mercapto group either at the 
end of the hydrocarbon chain to scavenge a hole or in $\alpha$ position to bidentately bind to a colloid surface and provide a side hydrocarbon chain as a hole trapping center. Thus, if the $\alpha$ substituted mercapto - carboxylic modifier contains $\mathrm{RCH}_{2}-$ group in $\alpha$ position the photogenerated charges are separated by large distances. Therefore, using an $\alpha$ substituted mercapto -carboxylic acid that has $\mathrm{CH}_{3}$ group in $\alpha$ position - thiolactic acid - we have obtained the highest yield of $\mathrm{Pb}^{2+}$ reduction and unique capability of reducing $\mathrm{Cd}^{2+}$.

Investigation of the redox properties of this system suggests that the redox potential of $\mathrm{TiO}_{2}$ (conduction band) is significantly enhanced by surface modification. It is a known fact that redox potential of metal ions is enhanced after complexation. We have used this approach to modify the redox potential of small particle colloids also. Thus we have found with pulse radiolysis that strong adsorption of electron donating modifiers enhances the reduction potential of photogenerated electrons. The consequence of this shift of the conduction band of surface modified $\mathrm{TiO}_{2}$ colloids is the unique capability of the reduction of cadmium ions $\left(\mathrm{E}^{0}\left(\mathrm{Cd}^{2+} / \mathrm{Cd}\right)=\right.$ $-0.445 \mathrm{~V}$ vs NHE).

It was also found using EPR spectroscopy that in the presence of methanol, holes were converted into methanol radicals which can inject electrons into $\mathrm{TiO}_{2}$. Therefore, the presence of methanol can potentially double the yield of reduced metal. Meanwhile degradation of methanol occurred simultaneously. These results suggest that the reduction of metal ions can occur simultaneously with degradation of some organics on surface modified $\mathrm{TiO}_{2}$.

Testing in the ES Division demonstratcd that the removal of $\mathrm{Pb}^{+2}$ ions in the system using thiolactic acid modified $\mathrm{TiO}_{2}$ photocatalysts was complete within the detectable limits of the atomic absorption analyzer (Figure 1). The adsorption of lead $\left(\mathrm{Pb}^{+2}\right)$ ions occurred in the systems using either untreated (virgin) or treated (modified using cysteine or thiolactic acid) $\mathrm{TiO}_{2}$. However, deposition of metal occurred only in the illuminated systems (Figure 2). $\mathrm{Pb}^{+2}$ concentrations in the irradiated solutions decreased as the UV irradiation time increased; greater metal removals were achieved at longer irradiation times.

Experiments were also performed in which organics (naphthalene) and heavy metals $\left(\mathrm{Pb}^{2+}\right)$ were treated with the $\mathrm{TiO}_{2}$ photocatalysts. The presence of lead did not interfere with removal (destruction) of naphthalene from solution. The presence of naphthalene resulted in "lag-time" effect on the photoreduction of lead from solution; after the majority of naphthalene had been removed, the lead was then removed by photoreduction. In both cases (lead and naphthalene), the cysteine-modified $\mathrm{TiO}_{2}$ photocatalyst resulted in removal rates which were faster and more effective than the untreated $\mathrm{TiO}_{2}$ system. This technique has resulted in the simultaneous photocatalytic removal of naphthalene and lead.

LDRD funding has been requested to continue this research during FY96. This study seeks to enhance the performance of $\mathrm{TiO}_{2}$-assisted photocatalysts to sequester and recover heavy metal ions in their metallic form while simultaneously destroying organic compounds in a single step. The future work will be performed in two directions: improvement of the catalysts and improvement of the process. 
Improvement of the photocatalysts will include following tasks:

Task 1: Determine the effect of the hydrocarbon chain length of surface modifier on the efficiency of charge separation.

Task 2: Investigate surface modifier which would lead to the efficient selective reduction of other metals $\left(\mathrm{Cu}^{2+}, \mathrm{Zn}^{2+}, \mathrm{Cr}^{6+}, \mathrm{U}^{6+}, \mathrm{Pu}^{6+}\right.$, etc $)$.

Task 3: $\quad$ Perform structural and dynamic studies using SERS and EXAFS.

Task 4: $\quad$ Further investigate concurrent removal of heavy metal ions and organics.

Improvement of the process will include following tasks:

Task 5: Investigate the methods for metal and catalyst recovery (e.g. acid treatment, low energy sonication).

Task 6: Design and develop a continuous flow process including design of supports for $\mathrm{TiO}_{2}$ colloids (e.g. magnetic ceramics, glass beads).

Task 7: $\quad$ Perform experiments with visible light for the photocatalysts with the absorption threshold in visible range.

In addition efforts will be made to secure one or more industrial partners to conduct a field demonstration in the third year of this project.

Specific Accomplishments: Three papers have been presented at national technical conferences during this year and two are submitted to the relevant journals, based upon the results obtained during, FY94 and 95 . These papers are listed below:

"Use of Cysteine-Modified $\mathrm{TiO}_{2}$ Photocatalysts for Treatment of Combined Organic/Inorganic Wastestreams," by R.W. Peters, J.-M. Wu, N. Meshkov, M.C. Thurnauer, and A.E. Ostafin, presented at the 5th International Symposium on Chemical Oxidation: Technology for the Nineties, Nashville, Tenn., February 15-17, 1995.

"Conversion of Holes into Reducing Species on Surface Modified Small-Particle $\mathrm{TiO}_{2}$," by O.I. Micic, A.E. Ostafin, T. Rajh, J.J. Sabelko, M.C. Thurnauer, and D.M. Tiede, presented at the 5th International Symposium on Chemical Oxidation: Technology for the Nineties," Nashville, Tenn., February 15-17, 1995.

"Combined Photooxidation/Photoreduction Using $\mathrm{TiO}_{2}$ Photocatalyses to Treat Organic/Heavy Metals-Laden Wastewaters," by R.W. Peters, J.-M. Wu, N. Meshkov, M.C. Thurnauer, and A.E. Ostafin, presented at the Waste Management 95 Symposium on HLW, LLW, Mixed Wastes, and Environmental Restoration--Working Towards a Cleaner Environment, Tucson, Ariz., February 26-March 2, 1995.

"Surface Modification of Small Particle $\mathrm{TiO}_{2}$ Colloids with Cysteine for Enhanced Photochemical Reduction: An EPR Study," T. Rajh, A.E. Ostafin, O.I. Micic, D.M. Tiede, M.C. Thurnauer, submitted to J. Phys. Chem. 
"Surface Modification of $\mathrm{TiO}_{2}$ Nanoparticles with Bidentate Ligands Studied by EPR Spectroscopy," T.Rajh, D.M. Tiede, M.C. Thurnauer, submitted to J. Non-crystalline Solids, presented at 9th International Conference on Liquid and Amorphous Metals, Chicago, Ill., August 27-September 1, 1995. 
Figure 1. Removal of lead ions with surface modified and unmodified $\mathrm{TiO}_{2}$

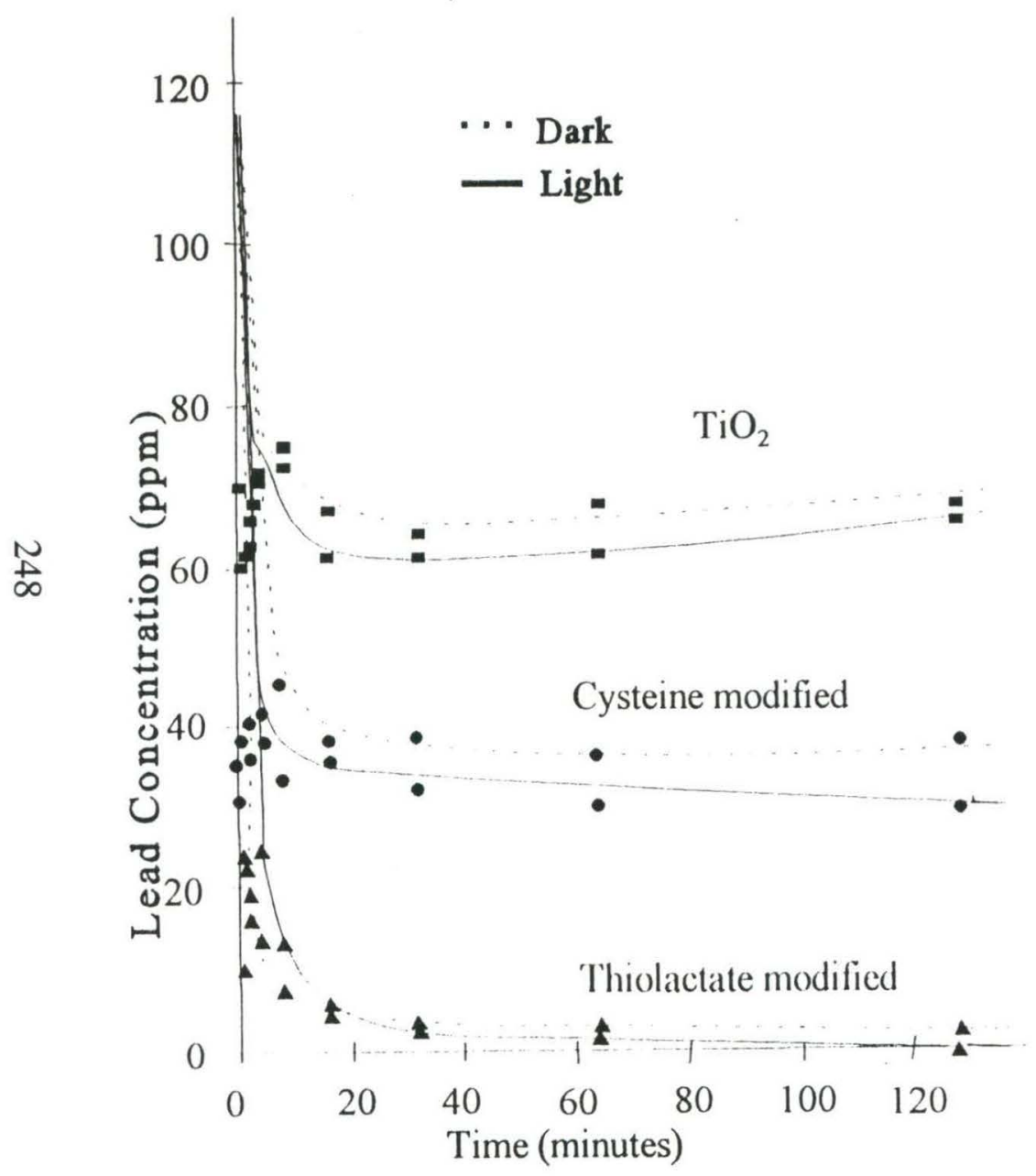

Figure 2. Metal deposition on the filter paper samples.

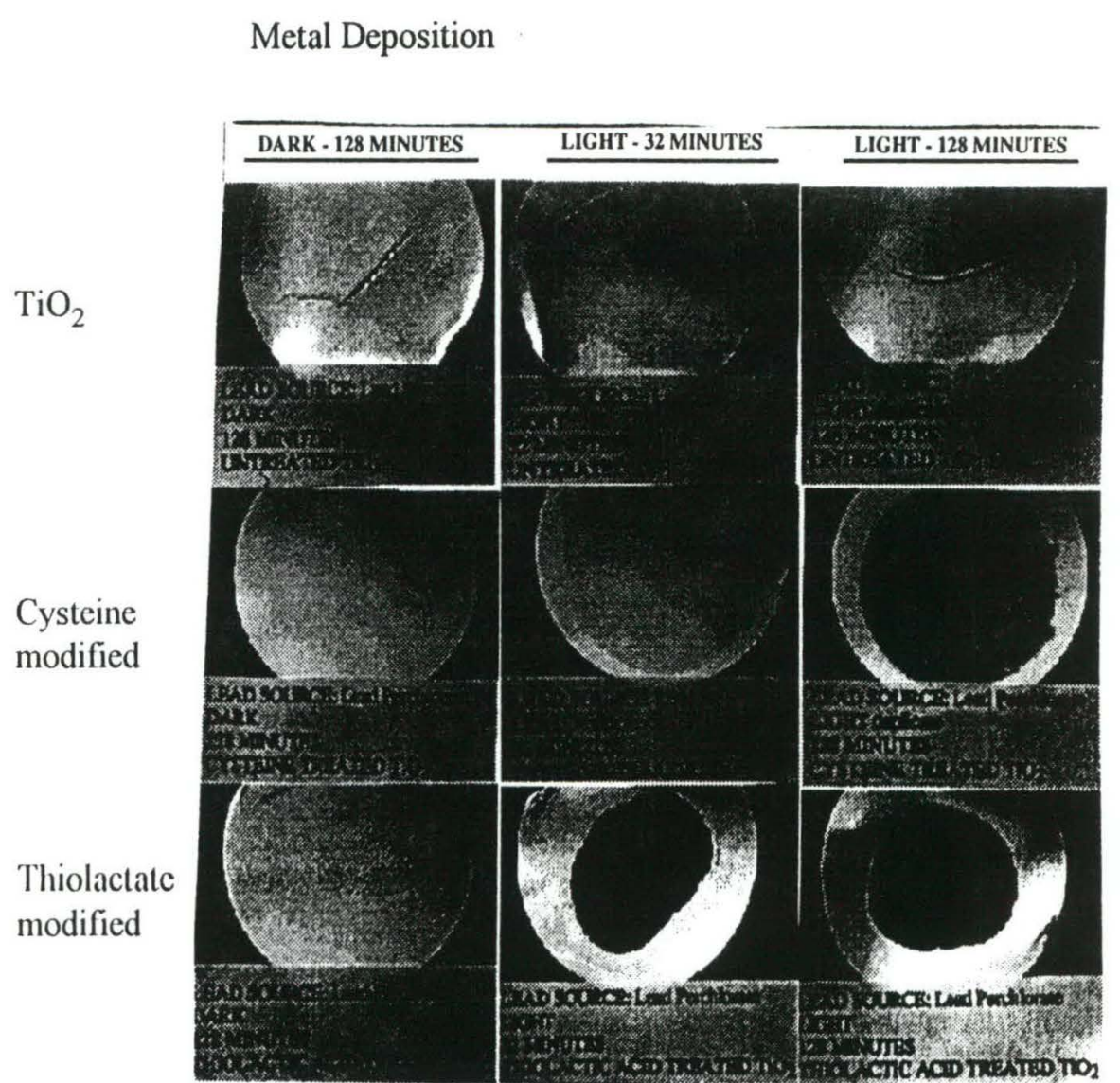




\section{4-179R1 -- APPLICATION OF ADVANCED MATERIALS AND METHODS TO CERMET GAS SENSOR DEVELOPMENT ${ }^{1}$}

\author{
Associate Laboratory Director Area:
}

Principle Investigators:

Funding Profile:
Energy and Environmental Science and Technology

E.L. Shoemaker and M.C. Vogt, Energy Systems Division C. Foster and J. Vetrone, Materials Science Division

Purpose: The extensive research in advanced materials at Argonne National Laboratory (ANL), along with its exploration in applied computer science, can be focused to make marked improvements in existing gas sensing technology. This expertise can be used to produce unique gas sensing capabilities by developing a series of advanced cermet devices. These improvements in current gas sensing technology are invaluable to industry as new, strict EPA regulations demand continuous monitoring of waste streams and cleanup of previously contaminated sites. Current gas micro-sensing technologies suffer from drift, cross-sensitivity and lack of robustness. In addition, most gas sensors are selectively limited to detect only one type of gas.

Recently, new thick-film cermet gas microsensors have been produced at ANL that employ special materials and intelligent (AI) computer analysis methodologies. In addition, recent advances in the growth of high-quality oxide thin-film materials have been made at ANL based on Metal Organic Chemical Vapor Deposition (MOCVD). In the FY94 LDRD program, these two techniques were brought together to produce a hybrid thick-film/thin-film electrocatalytic cermet gas microsensor with improved response and sensitivity. By building on this proven earlier work and tying the improvement of the current sensors more closely to specialized advanced materials and processes development, new and more capable microsensors can be constructed and can serve as an additional development platform for further gas sensor research. The results of this effort can be shown in the new ANL electrocatalytic gas (ECG) sensor and in the potential improvements that ANL's advanced materials can make in the tin oxide $\left(\mathrm{SnO}_{2}\right)$ sensor area.

Approach: Typical sensors in the current market and under development focus on a narrow and dated method for processing their signals. They produce an electrical signal, usually in the form

${ }^{1} \mathrm{CCST}$ project 
of a voltage, proportional to some physical phenomena (i.e. temperature, pressure, gas concentration, etc.). The burden of development of these sensors has been constrained by these dated signal processing systems. This is especially true of gas sensors. A variety of sensor technologies are currently available for gas-sensing applications. Some of these sensors are highly accurate for the single gas they are designed to detect but are unsuitable for other gases or multi-gas mixtures because of interference or lack of sensitivity: Many of the sensors are also affected by temperature and humidity. The selectivity of these individual sensors make them less attractive for many applications.

The electrocatalytic gas (ECG), multi-gas microsensor under development at ANL has significant potential advantages over some of the other gas-sensor technologies.

- Small size - current prototype micro-sensor is $2 \times 3 \mathrm{~mm}$;

- Rugged cermet materials, suitable for high-temperature operation $\left(300^{\circ} \mathrm{C}-500^{\circ} \mathrm{C}\right)$;

- Potential remote "flash" forced overheating to vaporize organic contaminants;

- Demonstrated multi-gas monitoring potential;

- Demonstrated proof-of-concept in detecting $\mathrm{O}_{2}, \mathrm{~N}_{2}, \mathrm{CO}_{2}, \mathrm{CO}, \mathrm{CH}_{4}$, and $\mathrm{NO}$ as well as a variety of other organic vapors including acetone, hexane, xylene and dichloroethane.

- Interfacing with artificial intelligence (AI) neural signal processing techniques for potential complex mixture recognition and drift compensation.

For these reasons it is desirable to pursue research for further advancement of the prototype $\because$ sensors. Thin-film versions of the sensors. made by applying MOCVD methods could produce a much smaller, much more sensitive device with a quicker response time and dramatically reduced power requirements.

Solid-state gas sensors that employ tin dioxide $\left(\mathrm{SnO}_{2}\right)$ have been available commercially since the 1960s. The applications for that sensor include methane, hydrogen, and carbon monoxide detection, as well as hydrogen sulfide and humidity sensing. The sensors are relatively nonselective, yielding a response to most reducing gases. These sensors tend to be cross-sensitive to other gases and are very temperature dependent, with the response drifting without controlled temperature. Alternative measurement techniques may provide more information from the $\mathrm{SnO}_{2}$ sensors and may eliminate some of their current drawbacks. The measurement techniques employed in testing the ECG sensors (voltammetry) could be applied to the $\mathrm{SnO}_{2}$ sensors, possibly yielding more qualitative and quantitative information. The current industrial tin oxide sensors are bulk formed and by film. standards are very crude. . This new work will include producing lithographed sensors using ANL thick-film and thin-film methods.

Technical Progress and Results: The Energy Systems Division, under LDRD FY95, investigated additional alternative solid electrolyte materials as well as the sensing properties of tungsten stabilized bismuth oxide sensors and thick film tin dioxide devices. Work was also done to integrate the sensor with the neural network signal processing algorithms and miniature sensing support system. 
Previous LDRD work investigated alternative solid electrolytes, metal oxides, and catalyst materials for improved sensor response. It was found that sensor response could be "tailored" by modifying one or all of these materials. A new solid electrolyte material, tungsten stabilized bismuth oxide, exhibited potential for improved sensor response and extensive film tests were conducted to attempt to make a operational sensor from the material.

SOLID ELECTROLYTES - Lanthanum fluoride $\left(\mathrm{LaF}_{3}\right)$ was investigated as an alternative solid electrolyte material. This solid electrolyte has shown to conduct ions at near room temperatures. Incorporating this electrolyte into the current ECG prototype could yield ambient temperature (dramatically reduced power requirement), multi-gas microsensors. Sensors were constructed and tested. Initial problems related to the difficulty in sintering the $\mathrm{LaF}_{3}$ led to sensors with a much thicker solid electrolyte layer than previous prototypes. These $\mathrm{LaF}_{3}$ sensors showed unique responses to different gases but required tcmperatures of $>200^{\circ} \mathrm{C}$. This is thought to be due to the thickness of the layer. New films incorporating sintering agents are being currently studied.

SENSOR CHARACTERIZATION - Tungstcn stabilized bismuth oxide (WBO) sensors were successfully constructed by incorporating a yttria stabilized zirconia (YSZ) buffer layer between the WBO and the upper platinum electrode. The buffer layer was necessary to keep the Pt electrode from diffusing through the WBO during fabrication and shorting the sensor. It also provides additional film interfaces for gases to react at. The WBO sensors were tested extensively for drift, reproducibility, and gas sensitivity especially $\mathrm{O}_{2}$ and $\mathrm{CO}_{2}$. It was found that these sensors offer a high degree of stability over the prototype YSZ sensors. In addition, the WBO sensors exhibit very unique responses to $\mathrm{O}_{2}, \mathrm{CO}_{2}$ and mixtures of the gases. An invention disclosure for these sensors has been filed.

Thick-film tin dioxide $\left(\mathrm{SnO}_{2}\right)$ sensors were constructed and tested. The sensors consist of a thick film of $\mathrm{SnO}_{2}(\approx 20 \mu \mathrm{m})$ on an alumina substrate with two inter-digitated platinum electrodes on the surface. Cyclic voltammetry was applied. Successful results from this measurement technique could yield $\mathrm{SnO}_{2}$ sensors which are more stable than current $\mathrm{SnO}_{2}$ sensors and could eliminate problems due to material saturation. The $\mathrm{SnO}_{2}$ sensors were tested in a variety of gases. Rcsults show voltammograms which reflect a change in $\mathrm{SnO}_{2}$ resistivity with applied voltage. Different gases appear to effect the resistivity with respect to voltage uniquely (this was expected as $\mathrm{SnO}_{2}$ changes conductance when exposed to hydrocarbons). Required temperatures remained high compared to commercial $\mathrm{SnO}_{2}$ sensors $\left(600^{\circ} \mathrm{C}\right.$ as opposed to $\left.400^{\circ}\right)$. This is thought to be due to the condition of the $\mathrm{SnO}_{2}$ thick-film. The thin $\mathrm{SnO}_{2}$ films are not homogeneous and contain holes and cracks where the alumina substrate sticks through. This areas are highly resistive and high temperatures must be attained before these gaps can be overcome. This problem could be rectified by applying multiple films of $\mathrm{SnO}_{2}$ to make a continuous film with no interference from the insulative alumina.

INTEGRATION OF SENSING SUPPORT SYSTEM - The first functional (trained) neural network, running on a single 18 pin DIP IC, was tested. The code is capable of being upscaled to identify the gas patterns generated by new experiments. A new set of data acquisition code was written to mimic the actual electronics that will control the sensor in the miniature sensing 
support system. Since it was not possible to cycle an analog sawtooth voltage between $-1 \mathrm{~V}$ and $+1 \mathrm{~V}$ through the sensor and measure current simultaneously with the microelectronics, a cycled "discrete" stepped voltage was implemented. The response from the discrete voltage was similar to the cycled analog voltage response and reproducible.

The first prototype miniature sensing support system was designed and built on a palm sized circuit board. This board contains instrumentation to heat and drive the sensor, as well as the 40 pin neural net IC chip for response identification.

The Materials Science Division, under LDRD FY95, investigated the production of complete thin-film versions of the current working ECG.sensors using MOCVD methods as well as thickfilm/thin-film hybrids and characterized the films using different microscopy techniques. The MSD also investigated interactions between the solid electrolyte and catalytic electrode to investigate the complicated kinetics involved in the sensor.

DEVELOPMENT OF THICK-FILM/THIN-FILM HYBRID SENSORS - While several approaches were attempted to integrate thin and thick film techniques, all have failed due mainly to the porous, rough nature of the YSZ thick film layer. Severe shorting for Pt evaporated even at room temperature on thick film YSZ was observed. Shorting may occur by both internal cracks and micron size voids, both yisible in SEM pictures of the YSZ film. The lower thick Pt film is prohibitively rough to permit a continuous $\mathrm{YSZ}$ thin film overcoat at a reasonable thickness $(<5 \mu \mathrm{m}$ thick).

DEVELOPMENT OF ALL THIN-FILM MOCVD ECG SENSORS - An all thin film structure was fabricated from an MOCVD grown YSZ layer (1-2 $\mu \mathrm{m}$ thick) having MOCVD grown $\mathrm{RuO}_{2}$ electrodes. The structures produce gas-sensitive current-voltage curves similar to those generated by standard thick film sensors. MSD has completed extensive studies of MOCVD growth conditions for films deposited on a variety of polished substrates including silicon, sapphire, magnesium oxide, and polycrystalline alumina. The smoothest, and most textured $\mathrm{RuO}_{2}$ films were deposited at very low temperatures with conductivities on the order of $10^{-3}$ $\mathrm{S} / \mathrm{cm}$. YSZ films (containing 8 mole $\% \mathrm{Y}_{2} \mathrm{O}_{3}$ ) were grown over a wide temperature range. It was found that the best method is to grow YSZ layers at very low temperatures which increases film density by increasing the initial density of nucleation sites; avoids electrode diffusion, and reduces incorporation of large gas phase particles. Although the growth rate is slow (.08 $\mu \mathrm{m} / \mathrm{hr}$ ), a complete sensor can be fabricated much sooner than the typical 20 hours needed for thick-film sensors. The MOCVD system can accommodate 2 large samples $\left(1 / 2{ }^{\sim} \times 1 / 2^{\sim}\right)$ or $201 / 8^{\sim}$ $\mathrm{x} 1 / 4^{\sim}$ micro-size sensors. Very recently films have been grown in a much larger, vertical MOCVD reactor which can handle $4^{\sim}$ wafers, i.e. approximately 44 minisensors or 440 microsensors. In contrast to the YSZ layer, the air-exposed working electrode film should be highly porous to facilitate gas-surface reactions. Porous electrodes were successfully fabricated by both sputtering $\mathrm{Pt}$ and by MOCVD growth of $\mathrm{RuO}_{2}$ at high temperatures.

THIN-FILM CHARACTERIZATION - The A/C. conductivity of thick film sensors was studied as a function of temperature and frequency in order to understand how gases affect the sensor current-voltage behavior. The thick film sensor has a single activation energy of $0.9 \mathrm{eV}$ between 
$100^{\circ} \mathrm{C}$ and $350^{\circ} \mathrm{C}$. Activation energies near $1 \mathrm{eV}$ are consistent with oxygen ion transport, although minor electronic contributions cannot be ruled out. The conductivity is on order of $10^{-3}$ $\mathrm{S} / \mathrm{cm}$ which is consistent with literature data of YSZ at low temperatures. It was found that the gas most strongly affects low frequency transport, which is often associated with charge transfer reactions at electrical double layers (i.e. ionic/electrical interface). Hence, these initial results suggest the gas-sensing mechanism is associated with changes in the capacitance of the electrode/electrolyte interface via chemical reduction of oxygen ions at the surface.

Specific Accomplishments: Awarded patent for the prototype microsensor entitled "Electrocatalytic Cermet Gas Detector/Microsensor," U.S. Patent 5,429,727, July 4 1995, by M.C. Vogt, E.L. Shoemaker and A.V. Fraioli.

Filed an invention disclosure (ANL-IN-95-136) for the new WBO sensors titled "Improved $\mathrm{O}_{2} / \mathrm{CO}_{2}$ Electrocatalytic Cermet Sensor," by Erika L. Shoemaker, and Michael C. Vogt.

Filed an invention disclosure (ANL-IN-95-064) for a "Modular Neural Network" by Michael Vogt and Daniel MacShane.

Awaiting publication of four journal articles to Solid State Ionics, Sensors and Actuators, NASA Tech Briefs, and SENSORS magazine.

Papers presented describing current effort by Dr. J. Vetrone at the American Vacuum Society Symposium and the Meeting of the Electrochemical Society.

This sensors work was highlighted in ANL's display for the Technology 2005 convention in Chicago, October 1995.

Continued collaboration with staff at Idaho National Engineering Laboratory (INEL) to do further testing of the microsensor with selective gas coatings under the auspices of the Department of Defense. 


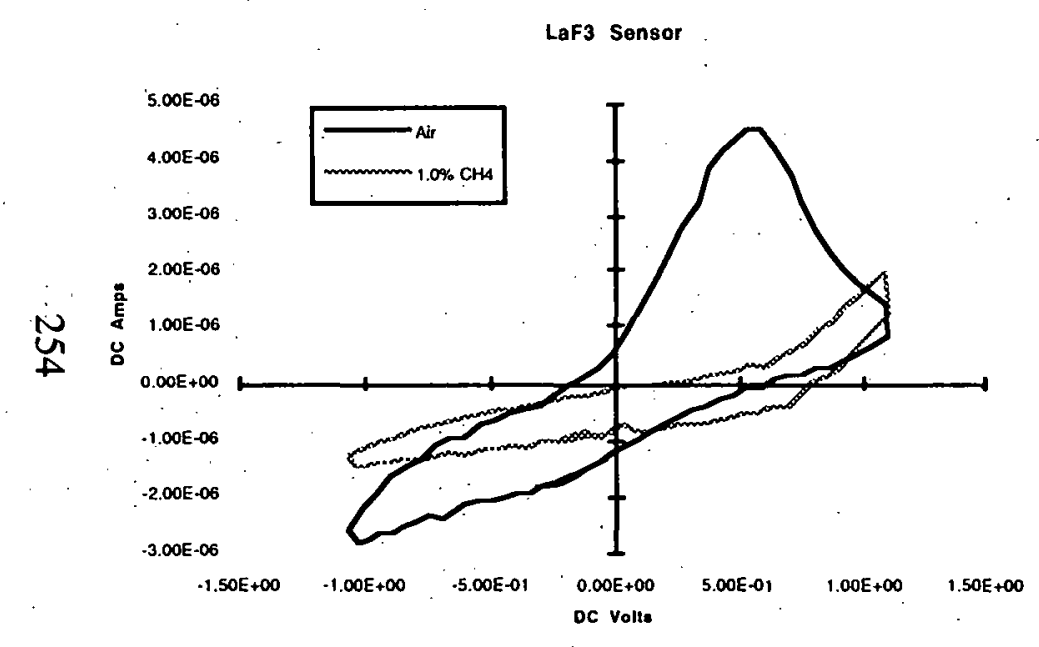

Figure 1. Lanthanum fluoride sensor response to methane.

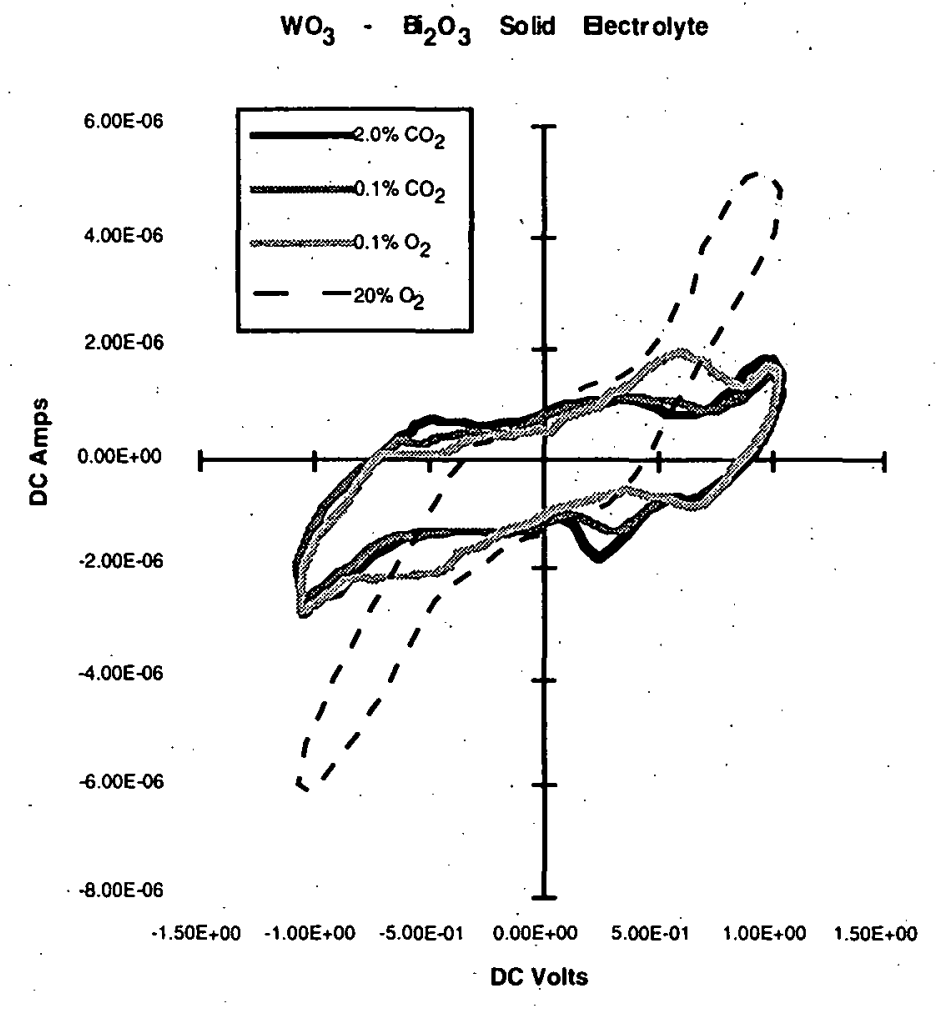

Figure 2. WBO sensor response to $\mathrm{O}_{2}$ and $\mathrm{CO}_{2}$. 
wBO Sensor

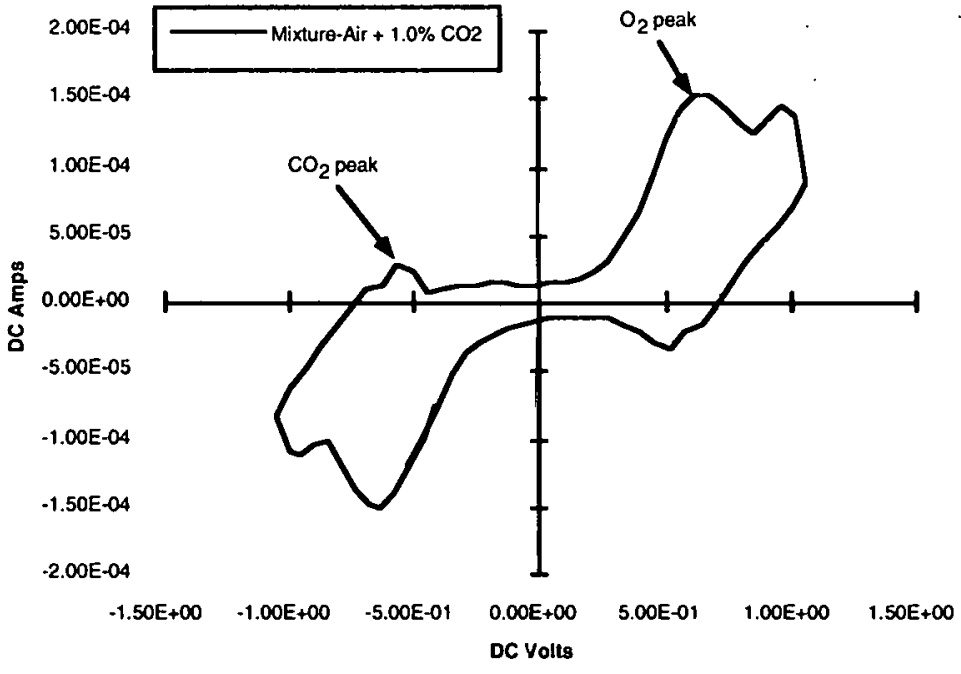

Figure 3. WBO sensor response to an $\mathrm{O}_{2} / \mathrm{CO}_{2}$ mixture.

㭊

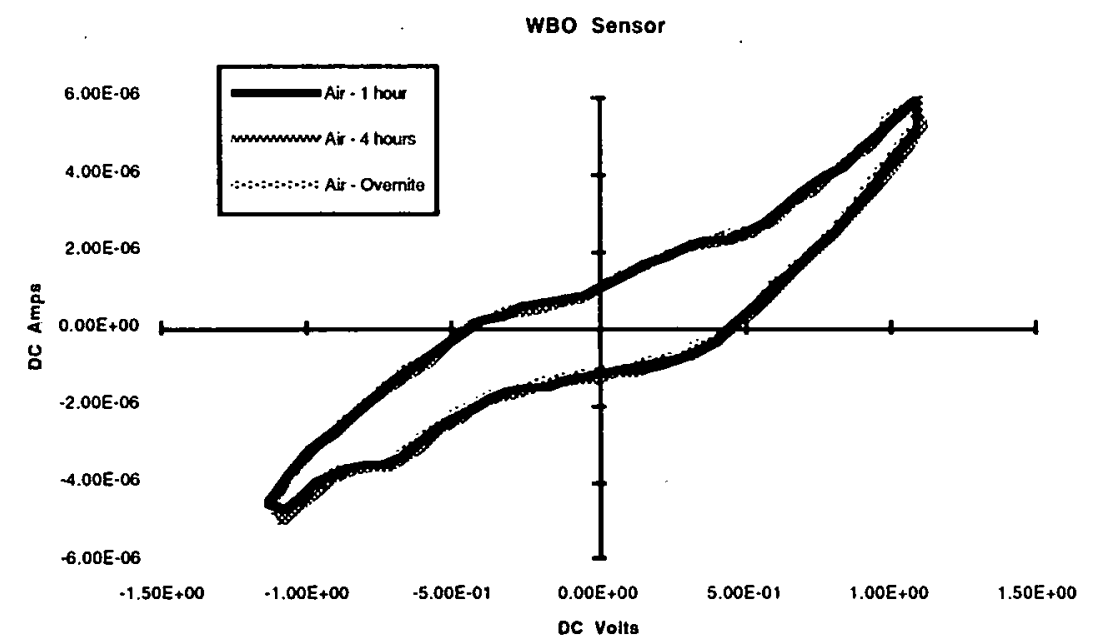

Figure 5. WBO sensor drift.

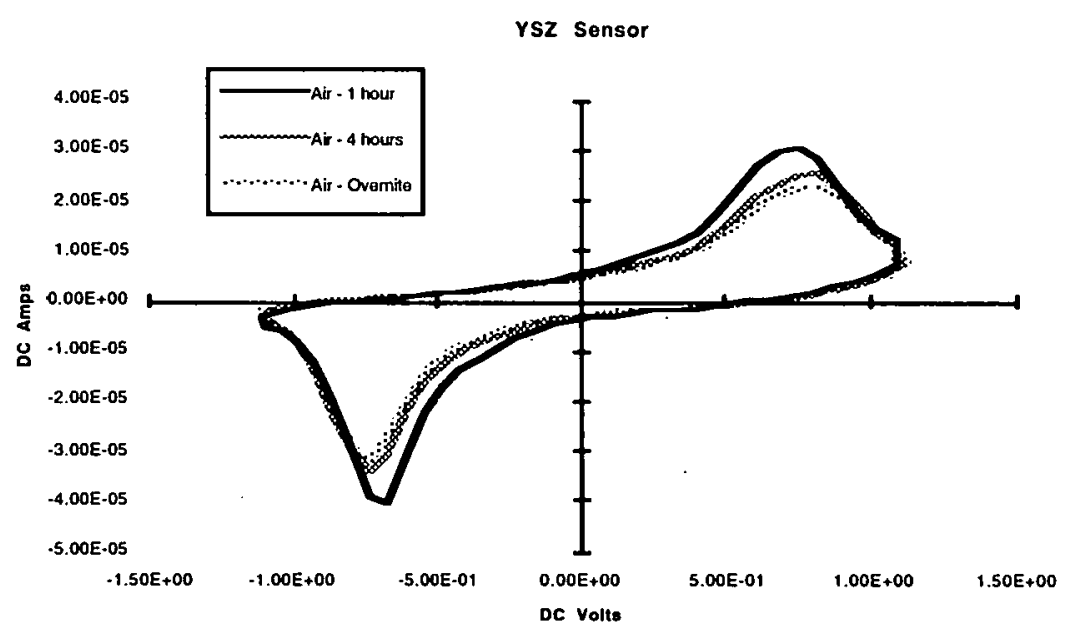

Figure 4. YSZ sensor drift.

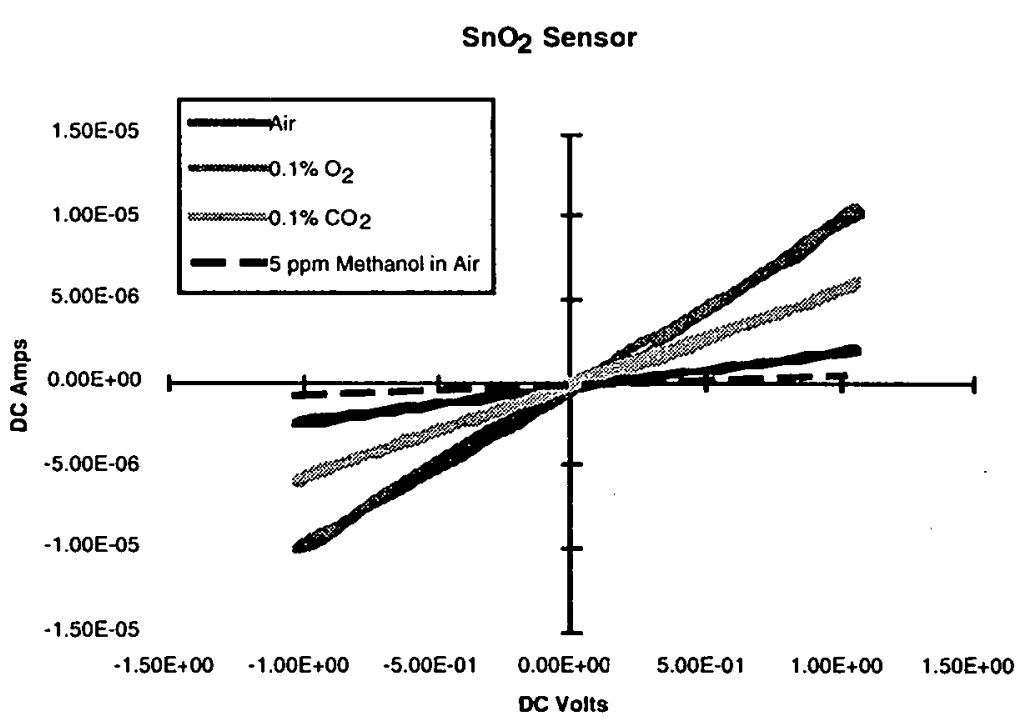

Figure 6. $\mathrm{SnO}_{2}$ sensor response to different gases 


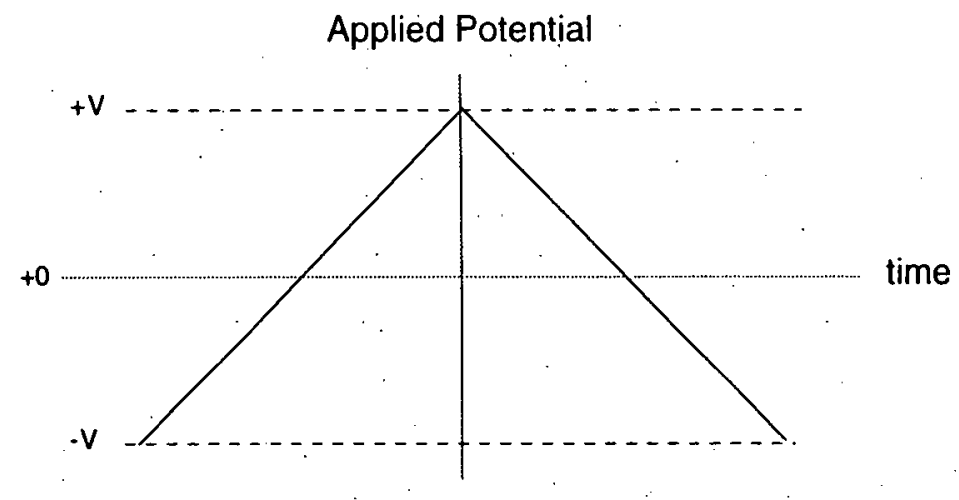

Figure 7. Applied cyclic voltage - analog.

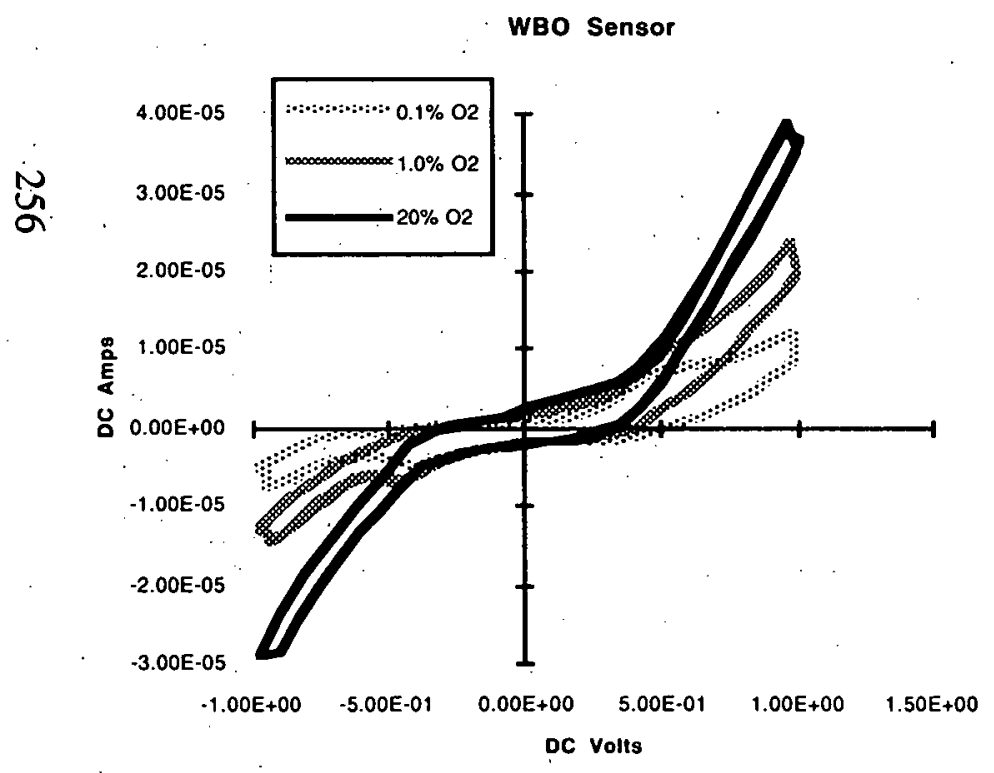

Figure 9. WBO sensor response to oxygen - analog.

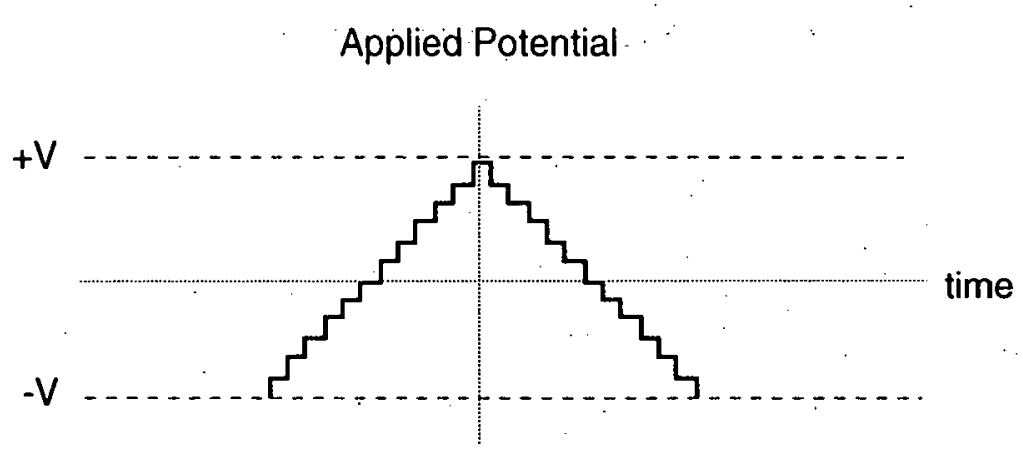

Figure 8. Applied cyclic voltage - discrete.

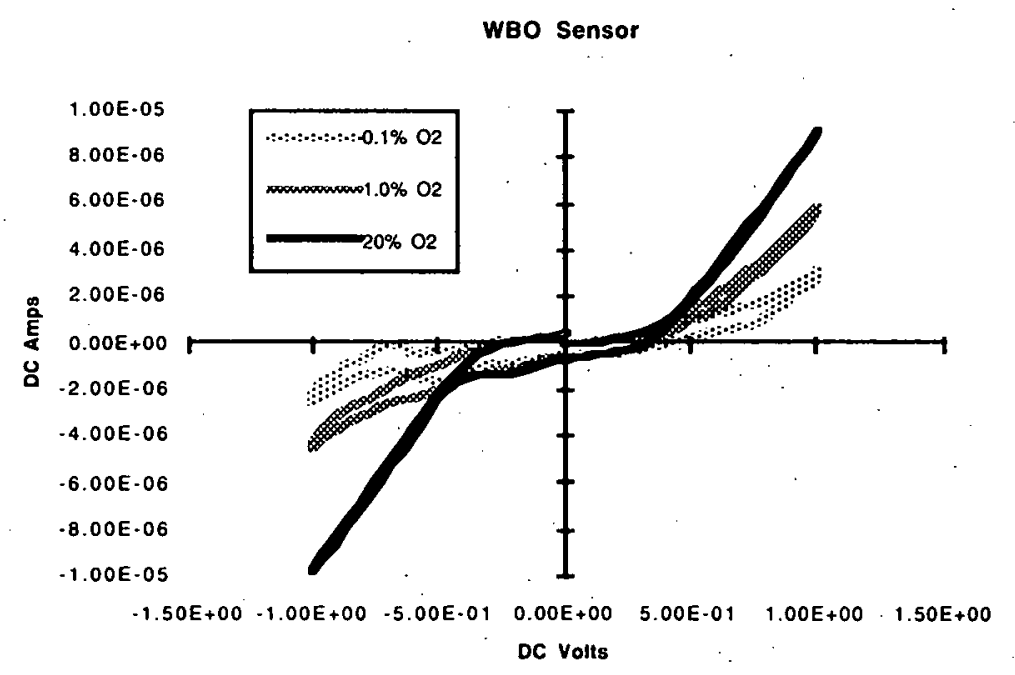

Figure 10. WBO sensor response to oxygen - discrete. 


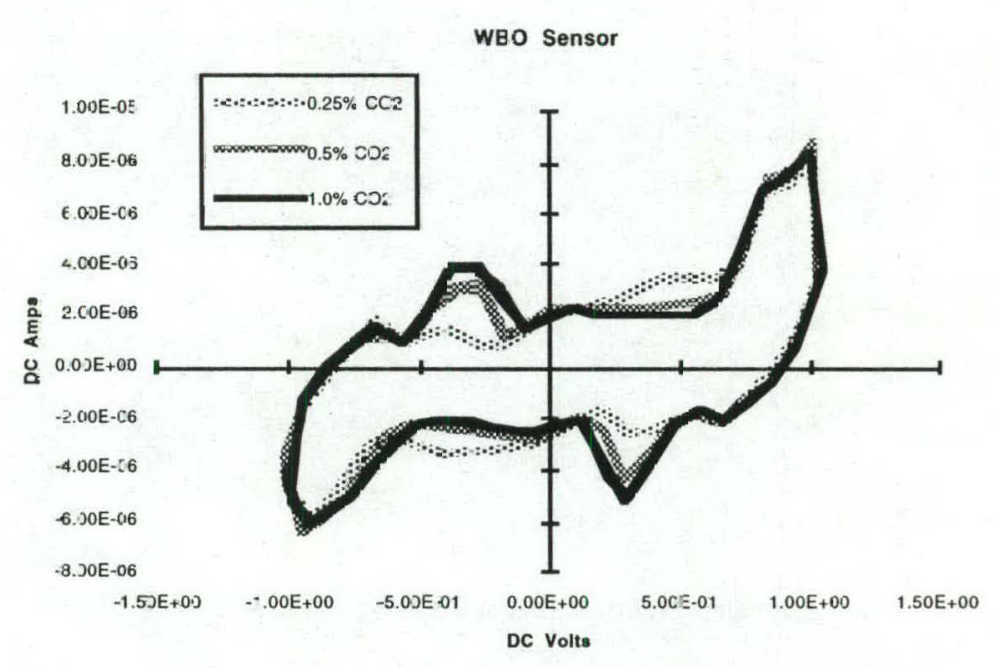

Figure 11. WBD sersor response to carbon dioxide - analog U

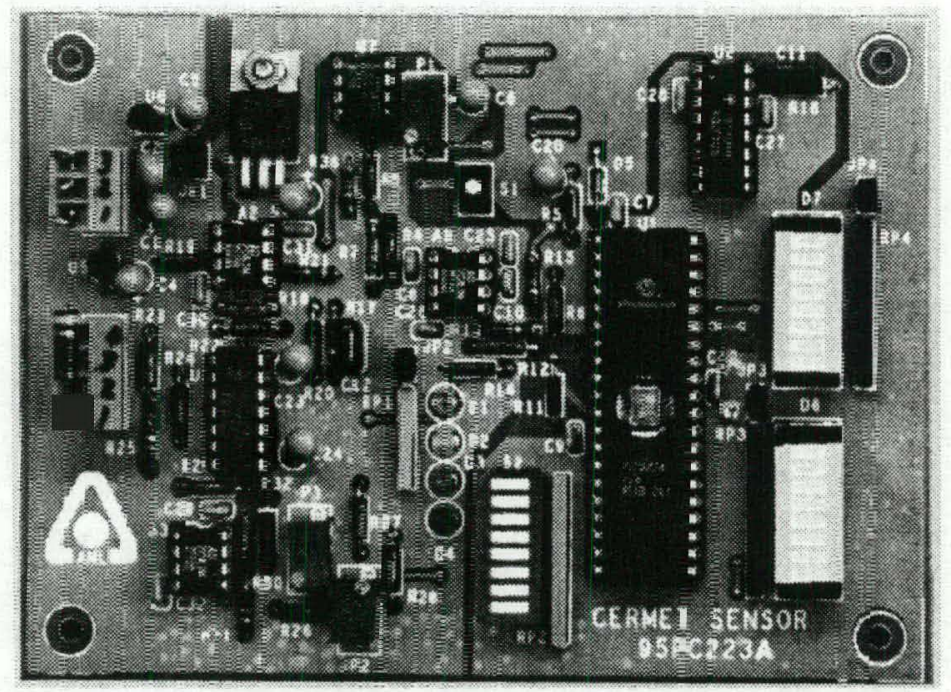

Figure 13. Sensing support electronics

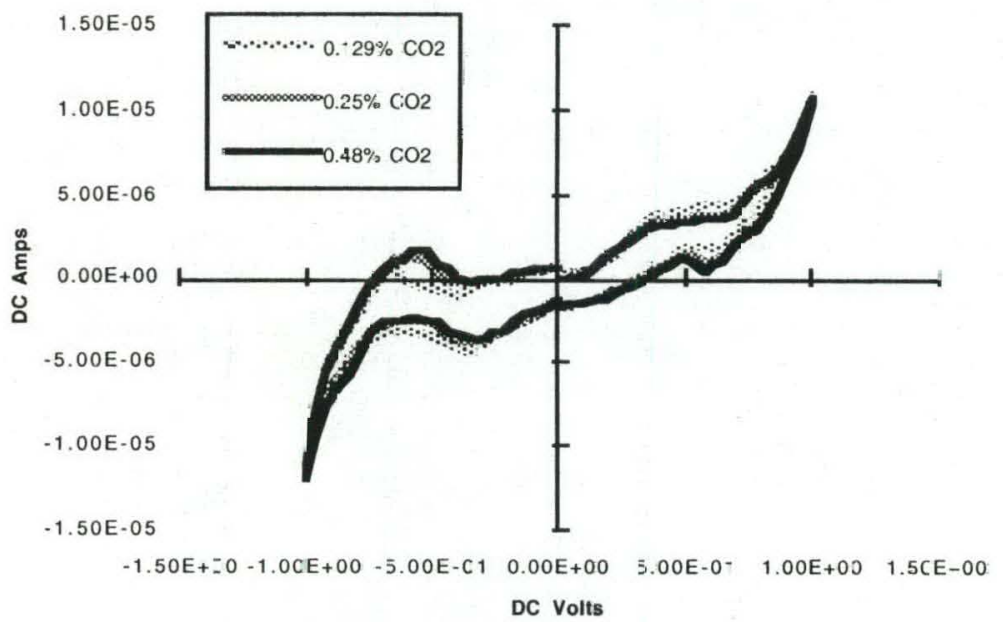

Figure 12. WBO sensor response to carbcn dioxice - discrete

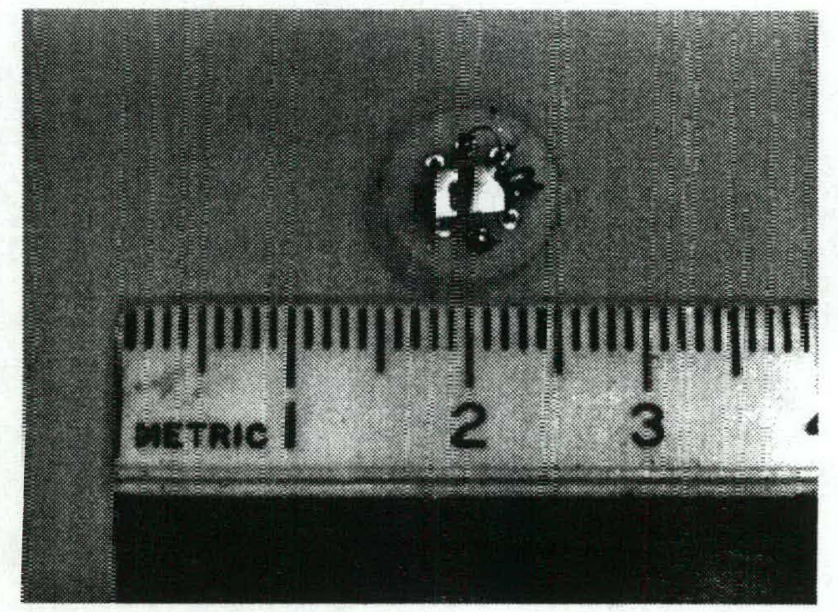

Figure 14. Thin-film MCCVD deposited ECG sersor. 
Response to air and dirty $\mathrm{N}_{2}$

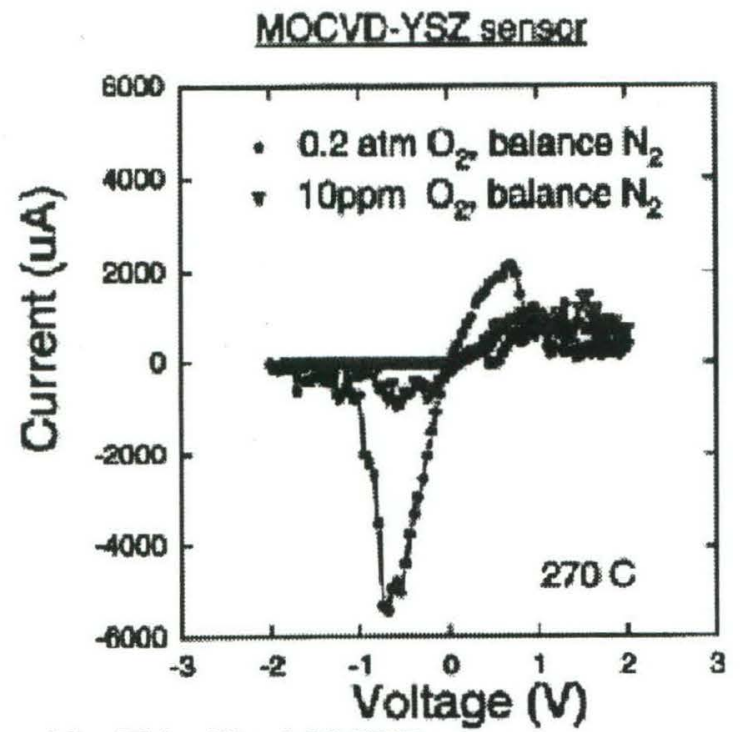

Figure 15. Thin-film MOCVD sensor response to nitrogen and air.

$\stackrel{N}{\infty}$

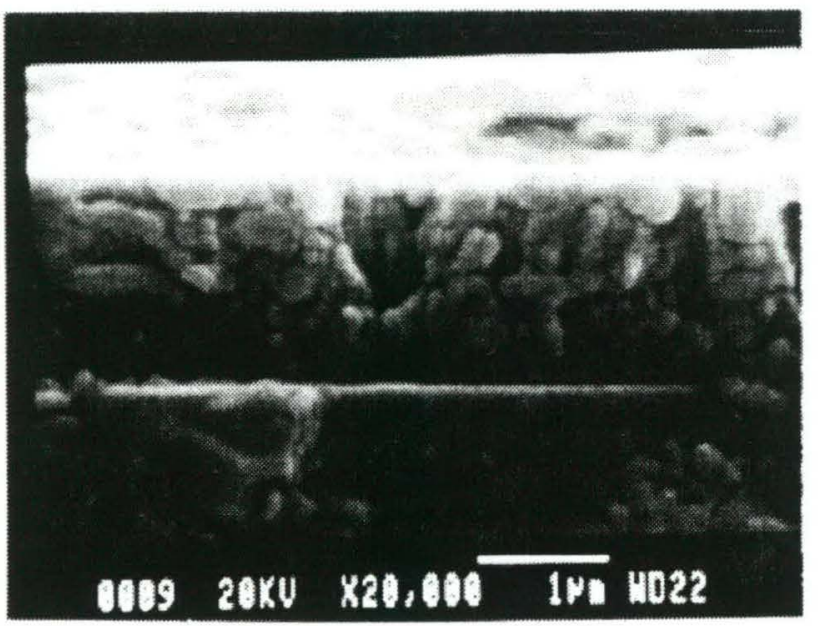

Figure 17. High density, MOCVD grown YSZ film.

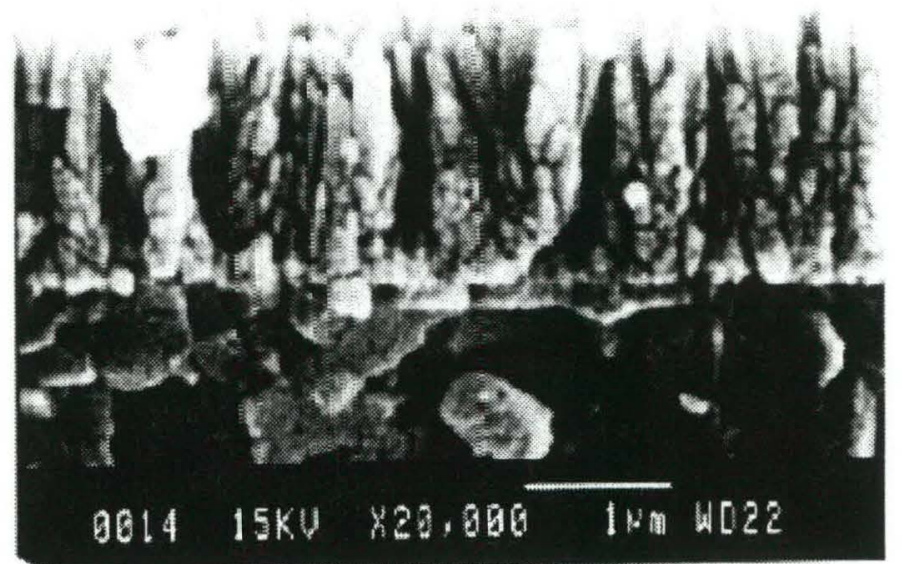

Figure 16. Low density, MOCVD grown YSZ film.

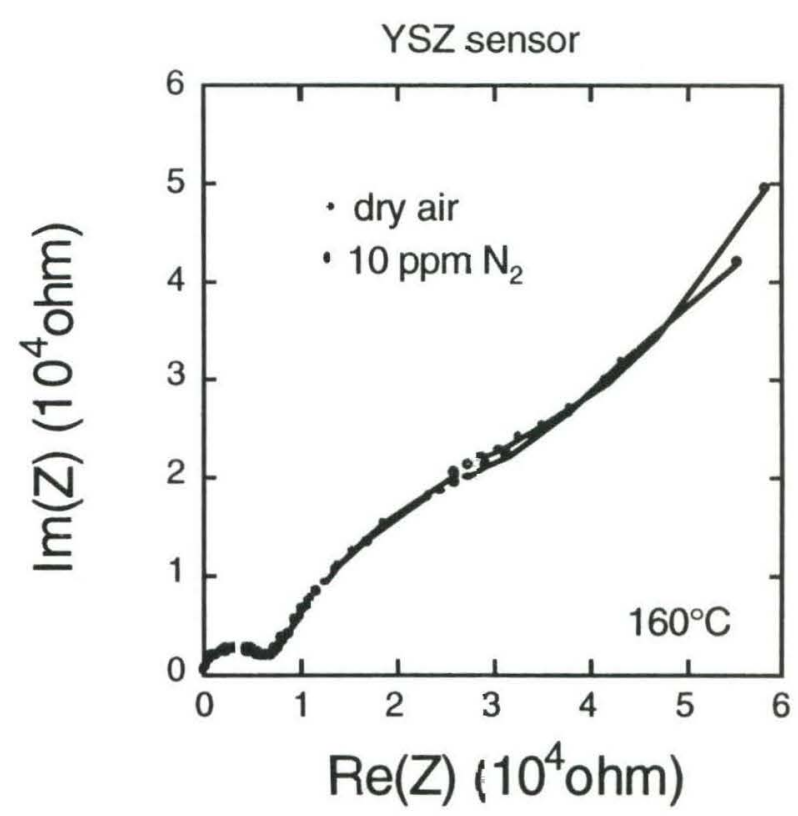

Figure 18. Complex impedance of thick-film YSZ sensor. 


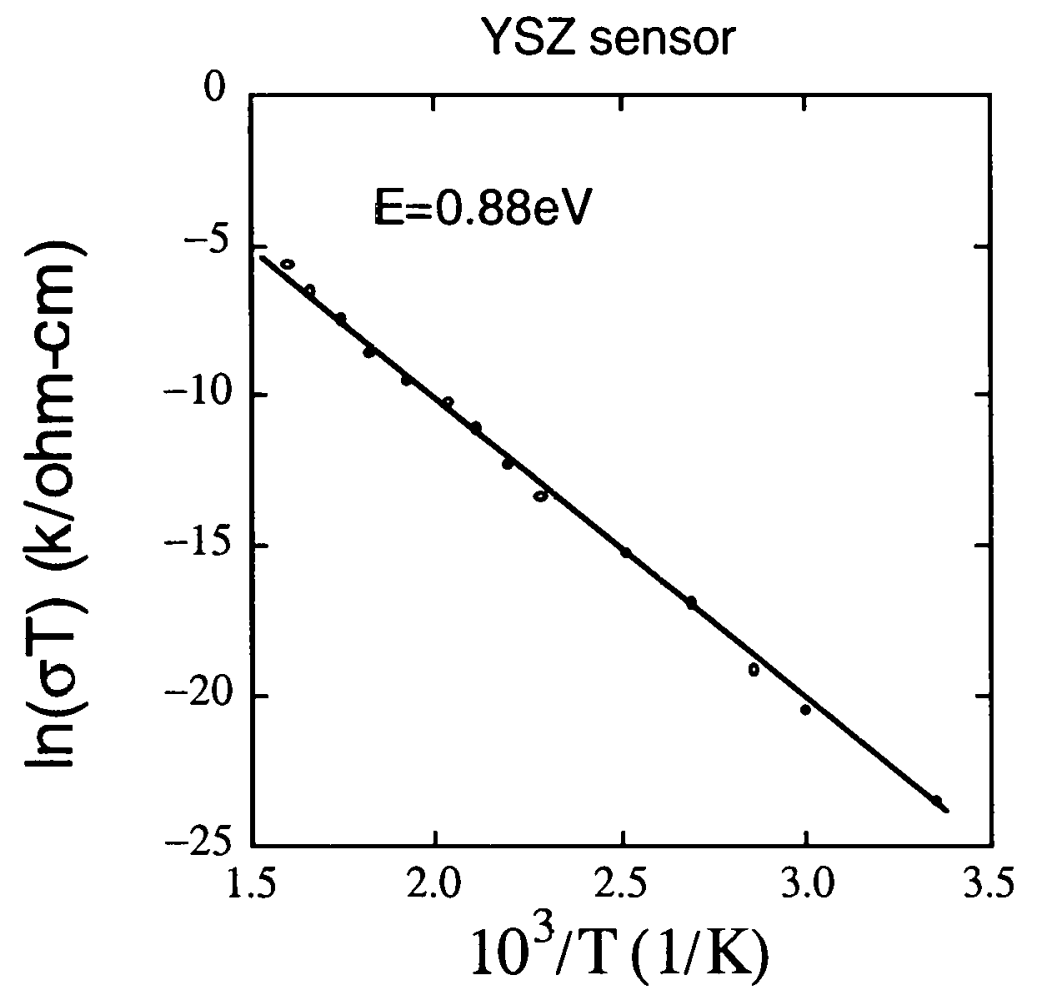

Figure 19. Ionic conductivity of a thick-film YSZ sensor. 
blank page 


\section{5-253N -- APPLICATIONS OF NANOPARTICLE TECHNOLOGY TO THE DEVELOPMENT OF A NEW CLASS OF ENERGY-EFFICIENT INDUSTRIAL HEAT TRANSFER FLUIDS ${ }^{1}$}

Associate Laboratory Director Area:

Principal Investigators:

Funding Profile:

\author{
Physical Research
}

\author{
J.A. Fastman, Materials Science Division \\ S.U. Choi, Energy Technology Division
}

$\begin{array}{lll}\text { FY } & 1993 & -0- \\ \text { FY } & 1994 & -0- \\ \text { FY } & 1995 & \$ 100.5 \mathrm{~K} \\ \text { FY } & 1996 & \$ 140.0 \mathrm{~K} \\ \text { FY } & 1997 & \$ 150.0 \mathrm{~K}\end{array}$

Purpose: Low thermal conductivity is a primary limitation in the development of energyefficient heat transfer fluids required in many industrial applications relevant to current mission priorities of DOE and other federal agencies. The purpose of this research project is to demonstrate the feasibility of engineering an innovative new class of heat transfer fluids by suspending ultrafine metallic particles of nanometer dimensions in traditional heat transfer fluids. The resulting "nanofluids" are expected to possess extremely high thermal conductivities compared to currently existing heat transfer fluids and consequently are expected to dramatically improve energy efficiency of traditional heat transfer fluids such as water, ethylene glycol, or oil.

Approach: In spite of considerable previous rcsearch and development efforts focusing on industrial heat transfer requirements, major improvements in cooling capabilities have been held back because of a fundamental limit in the heat transfer properties of conventional fluids. It is well known that metals in solid form have orders-of-magnitude larger thermal conductivities than fluids. For example, the thermal conductivity of copper or silver at room temperature is about 700 times greater than that of water and abnut 3000 times greater than that of engine oil. Therefore, fluids containing suspended solid metallic particles are expected to possess significantly enhanced thermal conductivities compared to conventional heat transfer fluids. In fact, numerous theoretical and experimental studies of the effective thermal conductivity of dispersions containing particles have been conducted since Maxwell's theoretical work was published more than 100 years ago. However, all of the studies on the thermal conductivity of suspensions have been confined to mm- or micron-sized particles. Maxwell's model shows that the effective thermal conductivity of suspensions containing spherical particles increases with the volume fraction of the solid particles. It is also known that the thermal conductivity of suspensions increases with the ratio of the surface area to volume of the particle. Therefore, the much larger relative surface areas of nanocrystalline powders compared to conventional

${ }^{1}$ CCST Project 
powders should result in markedly improved heat transfer capabilities, and also are expected to increase the stability of the suspensions.

The scope of this investigation involves primarily experimental efforts producing nanofluids and characterizing their thermal conductivities. This involves nanocrystalline powder synthesis, powder dispersion in liquids, and measurement of heat transfer properties. In addition, a theoretical analysis of the benefits of adding nanoparticles to conventional heat transfer liquids is required.

The approach taken to characterize the thermal conductivities of nanofluids involves several steps. First, nanocrystalline powders must be produced and the dispersion behavior of this material in heat transfer fluids such as water and ethylene glycol must be characterized. Portions of these studies were done in collaboration with S. Bhowmik of Iowa State University. Characterization of the powder sizes and agglomeration behaviors is carried out using transmission electron microscopy. The heat transfer behavior of the nanofluids is then characterized using a special transient hot wire cell designed and constructed specifically for this project. The latter work is partially carried out in collaboration with Professor Bock C. Pak of the Department of Mechanical Engineering and Design at Chonbuk National University.

Technical Progress and Results: In the first year of this program, several significant milestones were attained and the research progressed to the point that successful completion is likely within the expected three-year duration of the project. Specifically, nanocrystalline powders were produced, the particle size, agglomeration behavior, and suspension properties of these powders in several types of liquid were characterized, an apparatus for measuring thermal conductivities of nanofluids was designed and built, and a theoretical analysis of the expected heat transfer behavior of nanofluids was completed and published. Specific results and technical milestones are described in the following paragraphs.

Nanocrystalline copper with an average particle size less than $20 \mathrm{~nm}$ and silver powder with an average particle size less than $50 \mathrm{~nm}$ were produced in state-of-the-art gas-condensation nanophase materials preparation facilities. Characterization of particle size and agglomeration behavior was done using transmission electron microscopy techniques. Technology for the production of nanoparticle suspensions was successfully developed and suspensions of these powders in water and ethylene glycol were produced. Both ultrasonic vibration and centrifuging techniques were used in an attempt to break powder agglomerations and disperse powders in liquids. While we still intend to develop a new apparatus that will allow us to directly evaporate non-agglomerated nanocrystalline powders directly into flowing low vapor pressure liquids as was proposed in our original second- and third-year work plan, we were able to make satisfactory progress this year in dispersing materials produced with existing equipment by experimenting with dilute acidic solutions. Conditions for breaking agglomerations by dissolving the neck regions between particles without dissolving the particles themselves were identified. Transmission electron microscopy of powders distilled from nanofluid solutions was used to characterize the effects of various dilute acid solutions on agglomeration behavior. A typical electron micrograph of non-agglomerated $\mathrm{Cu}$ nanoparticles is shown in the attached Figure 1. 
Suspensions of nanophase copper and silver particles were produced by dispersing the powders in water and ethylene glycol at room temperature using a Premier Mill Model $90 \mathrm{High}$ Speed Disperser. A 1.5 in. diameter Hi-Vis mixing blade was used to break up the agglomerated nanoparticles for one hour at a constant speed of $2000 \mathrm{rpm}$. The well stirred suspensions were then put in transparent graduated cylinders to monitor the sedimentation behavior of dispersed particles as a function of time. The sedimentation behavior can be qualitatively characterized optically into three zones: a clear zone containing little or no particles, an optically-dimmed zone containing a low concentration of particles, and a well-dispersed zone with a high concentration of uniformly dispersed particles. These dispersion experiments demonstrated that nanocrystalline metal powders can be suspended in industrial heat transfer fluids such as water or ethylene glycol to produce stable suspensions of nanoparticles. Particularly in ethylene glycol solutions, it was found that static solutions were stable with respect to settling of the nanoparticles even after timcs of more than seven days without agitation, as seen in Figure 2. This figure shows the sedimentation behavior of the copper/water system and the copper/ethylene glycol system. The clear zone for the copper/water system is more than two times greater than that of the copper/ethylene glycol system. This difference may be due to the greater viscosity of ethylene glycol compared to water. Unlike copper particles, silver particles settled in both water and ethylene glycol within several minutes and more aggregates were observed at the bottom of the cylinder. This fast settling could be due mainly to : (a) the average grain size of the silver powders tended to be $\geq 50 \mathrm{~nm}$, which is significantly larger than our goal of producing material with sizes $\leq 10-20 \mathrm{~nm}$, and (b) the agglomeration behavior of these powders suggested some difficulty dispersing this material successfully in liquids. This emphasizes the importance of using powders with the smallest possible particle size.

The fabrication of a transient hot wire cell for the measurement of the effective thermal conductivities of nanofluids was recently completed. A photograph of this apparatus is shown in Figure 3. The effective thermal conductivities of copper and silver nanofluids are currently being measured with this apparatus and the measured thermal conductivities of nanofluids will be compared with those of conventional fluids. Tests demonstrating the expected dramatic improvement in energy efficiency of nanofluids compared to conventional heat transfer fluids are underway and will be completed in FY 1996 and 1997.

A theoretical analysis of the effective thermal conductivity of the copper-water system was completed this year using the Hamilton and Crosser model for three values of sphericity, which is defined as the ratio of the surface area of a sphere with a volume equal to that of the particle to the surface area of the particle. The results of this study clearly show that the thermal conductivity of the fluid-particle system depends on both the particle volume fraction and the shape. Assuming that the sphericity of copper nanoparticles is 0.3 , the thermal conductivity of water can be enhanced by a factor of 1.5 at the low volume fraction of $5 \%$ and by a factor of 3.5 at the high volume fraction of $20 \%$. This study demonstrates that metallic nanoparticles are capable of significantly increasing the thermal conductivity of conventional heat transfer fluids. Furthermore, Masuda et al. recently showed experimentally that $\mathrm{g}-\mathrm{Al}_{2} \mathrm{O}_{3}$ particles at a volume fraction of $4.3 \%$ can increase the effective thermal conductivity of water by $\approx 30 \%$. The agreement between the estimated and measured conductivities is satisfactory. Therefore, our 
nanofluids are expected to possess extremely high thermal conductivities compared to currently existing heat transfer fluids.

Development of a successful dispersion technique is the first major milestone of this project because it represents the underlying technology for the production of nanofluids. There remain two more major milestones to complete this project: a) to measure the effective thermal conductivities of nanofluids and show that, as predicted by the Hamilton and Crosser model, nanofluids possess high thermal conductivities compared to those of currently used heat transfer fluids and b) to demonstrate the expected dramatic improvement in energy efficiency of nanofluids compared to conventional heat transfer fluids. These milestones are expected to be met in the second and third years of this project.

Specific Accomplishments: A refereed external publication highlighting the theoretical justification of the concept of nanofluids was completed this year:

S. Choi and J. Eastman, "Enhancing Thermal Conductivity of Fluids with Nanoparticles," submitted to the Proceedings of the ASME International Mechanical Engineering Congress and Exposition, 1995.

A second publication for external publication in a refereed journal summarizing the experimental findings of the first year of this project is in preparation. 


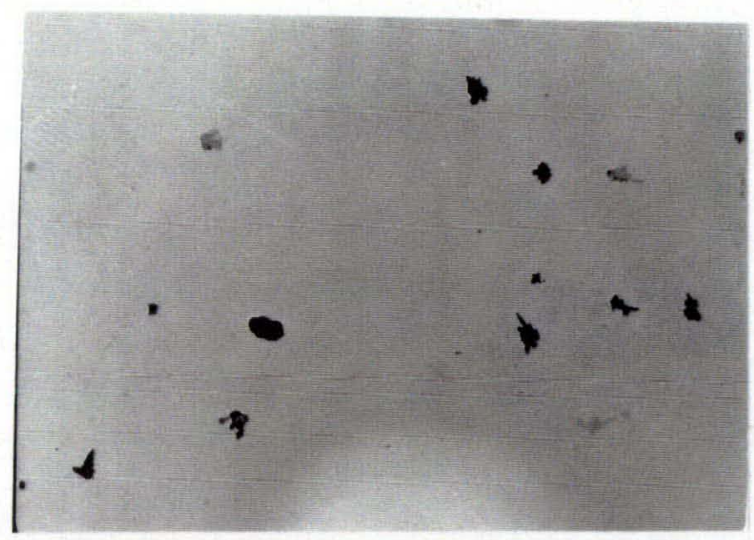

$1 \mu \mathrm{m}$

Fig. 1 Transmission electron micrograph of nanocrystalline copper powder after dispersion in a dilute acid-distilled water solution, followed by drying on a C-coated TEM grid.

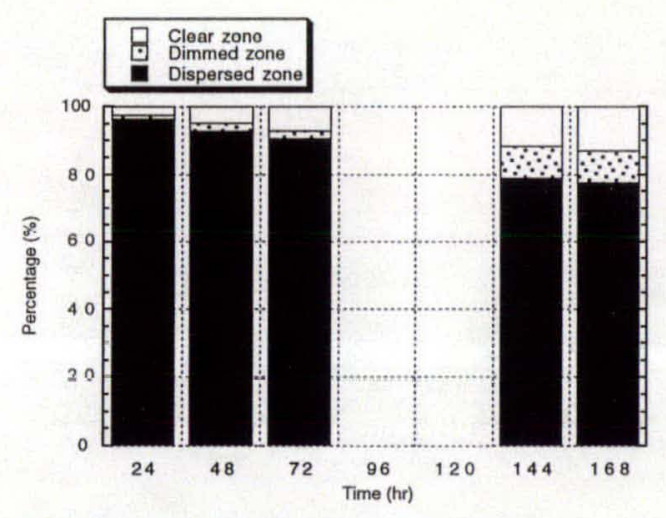

(a)

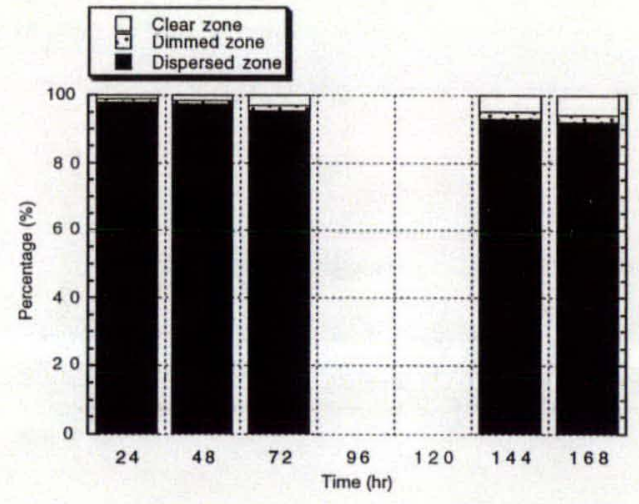

(b)

Fig. 2 Sedimentation behavior of (a) the copper/water system and (b) the copper/ethylene glycol system.

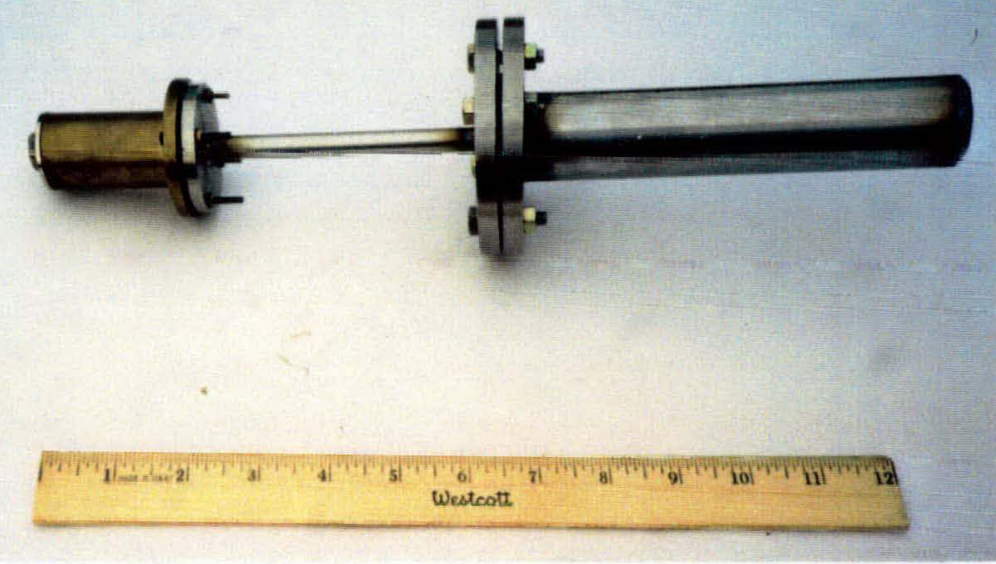

Fig. 3 Photograph of an actual thermal conductivity cell. 
blank page 


\title{
95-260N -- DEVELOPMENT OF SMOOTH DIAMOND FILMS FOR COST- EFFECTIVE MACHINING AND WEAR APPLICATIONS ${ }^{1}$
}

\author{
Associate Laboratory Director Area: Physical Research \\ Principal Investigators: \\ Funding Profile: \\ $\begin{array}{lll}\text { FY } & 1993 & -0- \\ \text { FY } & 1994 & -0- \\ \text { FY } & 1995 & \$ 100 \mathrm{~K} \\ \text { FY } & 1996 & \$ 140 \mathrm{~K} \\ \text { FY } & 1997 & -0-\end{array}$
}

D.M. Gruen and A.R. Krauss, Materials Science and Chemistry Divisions

A. Erdemir and G.R. Fenske, Energy Technology Division

Purpose: The main objective of this project is to demonstrate an efficient process for the deposition of smooth, low-friction, low-wear, and adherent diamond films on metallic and ceramic substrates for cost-effective machining and wear applications. The combination of these qualities in a diamond film is essential for commercial exploitation for cutting tools, bearing surfaces, and mechanical seals.

Approach: The diamond films produced by conventional processes consist of large diamond grains with non-diamond precursor material between them and their surfaces are very rough. When used in machining or sliding wear applications, these films cause high frictional losses and equally rough surface finishes on the mating surfaces. In order to overcome such deficiencies, we have developed a new deposition process producing high-quality, smooth diamond films. Our process uses a modified microwave chemical vapor deposition (CVD) system. Specifically, unlike conventional CVD processes, our technique utilizes a quartz transpirator where $C_{60}$ is evaporated from a fullerene-rich soot and carried to the microwave plasma by an Ar gas flow. The diamond films produced by our process are high-quality and exceptionally smooth as veritied by a variety of analytical tools. Furthermore, using a number of tribotest machines, we cvaluated the adhesion, friction, and wear properties of these diamond films on $\mathrm{WC}, \mathrm{SiC}$, and $\mathrm{Si}_{3} \mathrm{~N}_{4}$ substrates. Test parameters explored included wear of counterface materials, wcar of diamond films, load and temperature capability, and lifetime. A series of long-duration sliding tests were also performed to assess the lifetime and true wear rates of smooth diamond films.

Technical Progress and Results: Work carried out in FY95 has shown a high degree of success in producing films with the desired properties (improving on previously published friction and wear results by as much as two orders of magnitude). We have characterized the smooth diamond films by synchrotron radiation, electron diffraction, Raman spectroscopy, $\mathrm{x}$-ray diffraction (XRD), electron energy loss spectroscopy (EELS), scanning electron microscopy

${ }^{1}$ CCST Project 
(SEM), atomic resolution transmission electron microscopy (TEM), atomic force microscopy (AFM), and infrared spectroscopy (IR). The results of these studies verified that using the $\mathrm{C}_{60}$ precursor in an argon microwave discharge produces high-quality, nanocrystalline diamond films without the addition of hydrogen or oxygen to the plasma. Furthermore, the kinetics of incorporation of carbon into the diamond lattice were vastly improved during the first year of this program, leading perhaps to a more cost-effective deposition process. In fact, the growth rates achieved using our process are comparable with those of the conventional microwave plasma-assisted CVD methods. The growth rate is a key factor in determining cost.

The films have excellent adhesion to a variety of industrially important substrates and are exceptionally smooth. The combination of these qualities in a diamond film is crucial for commercial exploitation in applications where smooth surface finishes reduce friction, frictional heating, and thus minimize wear.

Work performed during the first year of this program has identified a second set of growth conditions leading to the formation of smooth nanocrystalline diamond films with high growth rates. An Ar-rich microwave plasma using a $\mathrm{CH}_{4}$ carbon precursor with a very low plasma hydrogen content has been found to produce films with properties similar to the films produced in the Ar-fullerene plasmas. Both the $\mathrm{C}_{60}$ and $\mathrm{CH}_{4}$ precursors result in carbon dimer-rich plasmas, although the $\mathrm{C}_{60}$ seems to be a more prolific source of carbon dimers and results in a higher growth rate. X-ray photoabsorption measurements carried out by Lawrence Berkeley Laboratory on twelve films produced under this LDRD have shown an equally high degree of $\mathrm{sp}^{3}$ electron bonding as conventional CVD-grown diamond films, and the films. were found in most cases to be indistinguishable from natural diamond.

TEM studies show the grain size of the films deposited from the $C_{60}$ precursors to range from 3 to $103 \mathrm{~nm}$, with an average size of $15 \mathrm{~nm}$ as shown in a micrograph in Figure 1. Renucleation occurs continuously during growth, with the result that grain coarsening does not occur with increasing film thickness as it does with conventional diamond film CVD growth. Nucleation is not restricted to discrete sites, resulting in what appears to be layer-by-layer growth rather than discrete site nucleation and growth from a point. Such growth behavior opens the doors to a number of applications requiring ultra thin coatings and interfaces with material having the outstanding physical, thermal, and electrical properties of diamond.

During past year, we performed numerous tests on these films using our extensive friction, wear, and adhesion test capabilities. Specifically, we determined the wear rates of counterface materials, load and temperature capability, and lifetime of diamond films, all of which are important for cutting tools, bearing surfaces, and mechanical seals. We also performed a series of long-duration sliding tests to assess the lifetime and true wear rates of smooth diamond films on $\mathrm{Si}$ substrates. Overall, the smooth diamond films deposited on Si substrates performed exceptionally well. They provided some of the lowest friction coefficients (e.g., 0.05 to 0.1 ) in open air as well as in dry $\mathrm{N}_{2}$ reported to date.

Figure 2 shows a comparison of the measured friction coefficients against a $\mathrm{Si}_{3} \mathrm{~N}_{4}$ ball in $\mathrm{N}_{2}$ for a conventional $\left(\mathrm{H}_{2}-\mathrm{CH}_{4} \mathrm{CVD}\right)$ diamond film with an rms roughness of $300-700 \mathrm{~nm}$, and a 
smooth $\left(\mathrm{Ar}-\mathrm{C}_{60}\right)$ nanocrystalline diamond film with an rms roughness of 30-40 nm. After an initial conditioning period, the friction coefficient for the nanocrystalline film drops to 0.04 , essentially indistinguishable from the natural diamond data as highlighted in Figure 2 and very close to the value obtained for Teflon. The friction coefficient remained nearly constant throughout the test. For the $\mathrm{H}_{2}-\mathrm{CH}_{4}$ film, the friction coefficient was more than an order of magnitude higher, and continuously varied as individual grains were torn loose from the film. In air, (Figure 3) the friction coefficient of the nanocrystalline diamond film again stabilized at a value close to that of natural diamond, while the friction coefficient of the conventional diamond film remained high and unstable. Highly abrasive nature of $\mathrm{H}_{2}-\mathrm{CH}_{4}$ diamond films resulted in very high wear rates on the counterface material. The absence of this behavior in the nanocrystalline diamond film is reflected in the measured wear of the counterface ball subjected to long duration tests as described below. A number of papers/presentations have been prepared based on these initial results.

Quite recently, we successfully deposited and ran a long-duration friction and wear test on a 6 $\mu$ m-thick smooth diamond film grown on a SiC substrate. $\mathrm{A} \mathrm{Si}_{3} \mathrm{~N}_{4}$ ball sliding against this film provided friction coefficients of $0.1-0.15$ for over 2,500,000 sliding passes ( 9 days of sliding time) (see Figure 4). The depth of the wear groove on the diamond film was determined by a high-resolution stylus profilometer to be only $0.5 \mu \mathrm{m}$. The RMS roughness (determined by AFM) of the wear track formed on the diamond film was 2-3 nm. The wear rate of the counterface $\mathrm{Si}_{3} \mathrm{~N}_{4}$ pin was $1.4 \times 10^{-8} \mathrm{~mm}^{3} / \mathrm{N}$-m (this is two orders of magnitude lower than values reported by other researchers on conventional diamond films).

Undoubtedly, such high-quality diamond films with smooth surface finish can have tremendous impact on a large variety of industrial applications. For example, machine tools coated with these films can be used at much higher speeds and feed rates, resulting in higher productivity and reduced unit cost. Currently, a variety of cutting fluids are used in large quantities in machining to dissipate heat and to reduce adhesion and/or friction between tools and work pieces. They represent an environmental hazard, mainly because most of them contain complex chemical additives including chlorine, phosphorus, and sulfur which are environmentally unsafe and potentially hazardous. Some of the fluids are also very flammable and pose fire hazards during machining. Because of their very high thermal conductivity and inherently low-friction character, smooth diamond films can substantially reduce and possibly eliminate the use of these potentially hazardous lubricants in friction, wear, and machining applications.

In short, tribological work carried out during first year of this program has successfully demonstrated that we can produce very smooth diamond films affording some of the lowest wear rates and coefficients of friction reported to date (improving on previously published wear results by as much as two orders of magnitude). Furthermore, it gave us the confidence that large-scale industrial participation will be feasible upon completion of a similarly successful second year which will concentrate on $\mathrm{Si}_{3} \mathrm{~N}_{4}$ and WC tool inserts and $\mathrm{SiC}$ wear parts. Several companies (John Crane International, Advanced Material Process Corp., Norton Corp., GTE Velanite, Durametallics, Inc., and ASTeX Inc.) have expressed interest in CRADA participation, if the anticipated properties of these films are demonstrated for their specific applications. 


\section{Specific Accomplishments:}

Patent Applications:

93-111. "Diamond Growth from Fullerene Precursors, " filed 10/27/93

93-132. "Particle Suppression of Particulates in Arc Cathodic Thin Film Deposition Processes"

94-124 "Improved Method for the Preparation of Nanocrystalline Diamond Thin Films, " filed $4 / 4 / 95$

94-020 "Improved Method for the Preparation of Nanocrystalline Diamond Thin Films, " filed $10 / 11 / 95$

94-127 "A Method of Improving the Field Emission Characteristics of Diamond Thin Films," in preparation

95-020 "Diamond Field Emission Thin Film Coatings on Discrete Field Emitter Arrays," filed 4/4/95 as CIP to ANL-IN-94-124

95-111 "Smooth Diamond Films as Low Friction, Long Wearing Surfaces," in preparation

\section{Publications:}

D.M. Gruen, X. Pan, A.R. Krauss, S. Liu, J. Luo, and C.M. Foster, "Deposition and Characterization of Nanocrystalline Diamond Films," J. Vac. Sci. Technol. A 12(4):1491-1495 (1994).

D.M. Gruen, "Diamonds from Dust," Mater. Technol. 9, Technological Advances, 149-151 (1994).

D.M. Gruen, A.R. Krauss, J. Luo, X. Pan, and S. Liu, "Turning Soot into Diamonds with Microwaves," Proc. 29th Microwave Power Symp., Chicago, IL, July 25-27, 1994, pp. 79-83 (1994).

D.A. Horner, L.A. Curtiss, and D.M. Gruen, "A Theoretical Study of the Energetics of Insertion of Dicarbon $\left(\mathrm{C}_{2}\right)$ and Vinylidene into Methane $\mathrm{C}-\mathrm{H}$. Bonds," Chem. Phys. Lett. 233:234-248 (1995).

C.D. Zuiker, D.M. Gruen, and A.R. Krauss, "Laser-Reflectance Interferometry Measurements of Diamond-Film Growth;" MRS Bulletin XX(5):29-31 (1995).

D.M. Gruen, C.D. Zuiker, A.R. Krauss, and X. Pan, "Carbon Dimer, $\mathrm{C}_{2}$, as a Growth Species for Diamond Films from Methane/Hydrogen/Argon Microwave Plasmas," J. Vac. Sci. Technol. A 13(3): 1628-1632 (1995). 
R. Csencsits, D.M. Gruen, A.R. Krauss and C.D. Zuiker, "Characterization of Diamond Thin Films using Transmission Electron Microscopy," Extended Abstract: Optical Engineering Midwest, 2622(1):405 (1995).

C.D. Zuiker, D.M. Gruen and A.R. Krauss, "In Situ Surface Roughness Measurements during PECVD Diamond Film Growth," Extended abstract published in the Proceedings of the Symposium on "Diamond Materials" 187th Meeting of the Electrochemical Society, Reno, Nevada, May 21-26, 1995.

D.M. Gruen, J.S. Luo, R. Csencsits, C.D. Zuiker and A.R. Krauss, "Microstructure of CVD Diamond Films Grown from Fullerene Precursors," Extended abstract published in the Proceedings of the Symposium on "The Fullerenes: Chemistry, Physics, and New Directions," 187th Meeting of the Electrochemical Society, Reno, Nevada, May 21-26, 1995.

Submissions:

C. Zuiker, A.R. Krauss, D.M. Gruen, X. Pan, J.C. Li, R. Csencsits, A. Erdemir, C. Bindal and G. Fenske, "Physical and Tribological Properties of Diamond Films Grown in Argon-Carbon Plasmas," Thin Solid Films in press.

A.R. Krauss, J. Im, J.A. Schultz, V. Smentkowski, K. Waters, C.D. 7uiker, D.M. Gruen, and R.P.H. Chang, "Pulsed Ion Beam Characterization of CVD Diamond Surfaces under Thin Film Deposition Conditions," Thin Solid Films in press.

D.M. Gruen, C.D. Zuiker and A.R. Krauss, "Diamond Films Grown from Fullerene Precursors, " to be published in the Proceedings of the Fullerenes and Photonics II Conference, Part of SPIE's 1995 International Symposium on Optical Science, Engineering and Instrumentation, San Diego, California, July 9-14, 1995.

R. Csencsits, C.D. Zuiker, D.M. Gruen, and A.R. Krauss, "Grain Boundaries and Grain Size Distributions in Nanocrystalline Diamond Films Derived from Fullerene Precursors," Proceedings of the Internuliunal Conference POLYSE '05, "Polycrystalline Semiconductors - Physics, Chemistry, and Technology, "Villa Feltrinelli, Gargnano, Italy, September 9-14, 1995 - in press.

D.M. Gruen, C.D. Zuiker, and A.R. Krauss, "Nanocrystalline Diamond Films from FullereneArgon Microwave Plasmas," Proceedings of the International Conference POLYSE '95, "Polycrystalline Semiconductors - Physics, Chemistry, and Technology," Villa Feltrinelli, Gargnano, Italy, September 9-14, 1995 - in press.

D.M. Gruen, A.R. Krauss, C.D. Zuiker, R. Csencsits, L.J. Terminello, J.A. Carlisle, I. Jimenez, D.G.J. Sutherland, D.K. Shuh, W. Tong, and F.J. Himpsel, "Characterization of Nanocrystalline Diamond Films by Core-Level Photoabsorption," submitted to Applied Physics Letters. 
C.D. Zuiker, D.M. Gruen and A.R. Krauss, "In Situ Laser Reflectance Measurements of Film Properties," submitted to Journal of Applied Physics.

A. Erdemir, G. Fenske, C. Bindal, C. Zuiker, A.R. Krauss and D.M. Gruen, "Friction and Wear Properties of Smooth Diamond Films Grown in Fullerene-Argon Plasmas, " submitted to Diamond and Related Materials.

\section{Presentations:}

A. Erdemir, G.R. Fenske, F.A. Nichols, C. Bindal, C. Zuiker, A.R. Krauss and D.M. Gruen, "Sliding Friction and Wear Mechanisms of Smooth Diamond Films Grown in Fullerene-Argon Plasmas," Poster presentation at the 10th International Conf. on Wear of Materials, Boston, Mass., April 9-13, 1995.

A.R. Krauss, D.M. Gruen, C.D. Zuiker, Z. Yi, A. Erdemir, C. Bindal, G. Fenske, and R. Csencsits, "Growth Mechanism and Properties of Nanocrystalline Diamond Films Grown in Carbon Dimer-Rich, Hydrogen-Deficient Microwave Plasmas, " Abstract for invited talk -- Physics Department, Purdue University, West Lafayette, Indiana, September 22, 1995.

D.M. Gruen, "Nanocrystalline Diamond Films from Fullerene Precursors," Abstract for invited talk -- International Symposium on the Science and Technology of Atomically. Engineered Materials, Richmond, Virginia, October 30-November 4, 1995.

A.R. Krauss, D.M. Gruen, C.D. Zuiker, Z. Yi, A. Erdemir, C. Bindal, G. Fenske, and R. Csencsits, "Growth Mechanism and Properties of Nanocrystalline Diamond Films Grown in Carbon Dimer-Rich, Hydrogen-Deficient Microwave Plasmas," Abstract for invited talk -- XV Congress of the Mexican Society of Surface Science and Vacuum (SMCSV), Mexico City, Mexico, November 13-16, 1995.

J.C. Li, A.R. Krauss, D.M. Gruen, C.D. Zuiker, and R. Csencsits, "AES, XPS, and Raman Characterization of Surface and Interfacial Properties of Diamond Films," Abstract for poster -"Diamond for Electronic Applications" Symposium, Materials Research Society 1995 Fall Meeting, Boston, Massachusetts, November 27-December 1, 1995.

R. Csencsits, D.M. Gruen, A.R. Krauss, and C. Zuiker, "TEM Study of Diamond Films Grown from Fullerene Precursors," Abstract for poster -- "Polycrystalline Thin Films II - Structure, Texture 1995 Materials Research Society Fall Meeting, Boston, Massachusetts, November 27December 1, 1995.

D.M. Gruen, C.Z. Zuiker, A.R. Krauss, R. Csencsits, A. Erdemir, C. Bindal, L.A. Curtiss, D.A. Horner, P.C. Redfern, L. Terminello, and J. Carlisle, "Characterization and Properties of Diamond Films from Fullerene Precursors," Abstract for talk -- "Fullerenes, Buckytubes, and Related Materials -Science and Applications" Symposium, Materials Research Society 1995 Fall Meeting, Boston, Massachusetts, November 27-December 1, 1995. 
L.A. Curtiss, D.A. Horner, P. Redfern, and D.M. Gruen, "Theoretical Studies of Reaction Mechanisms for Growth of Diamond Surfaces from Buckyball Precursors," Abstract for invited talk -- Symposium on "Computational Quantum Chemistry: A Viable Partner to Experiment in Chemical Research," 1995 International Congress of Pacific Basin Societies, Honolulu, Hawaii, December 17-22, 1995.

C. Zuiker, A.R. Krauss, D.M. Gruen, R. Csencsits, A. Erdemir, C. Bindal, and G. Fenske, "Physical Properties of Diamond Films Grown from Fullerene Precursors," Abstract for talk -1996 International Conference on Metallurgical Coatings and Thin Films Symposium on the Synthesis and Characterization of Diamond and Related Materials, San Diego, California, April 22-26, 1996. 
Figure 1. TEM image of a 600 grown smooth diamond film showing the nanocrystalline structure.

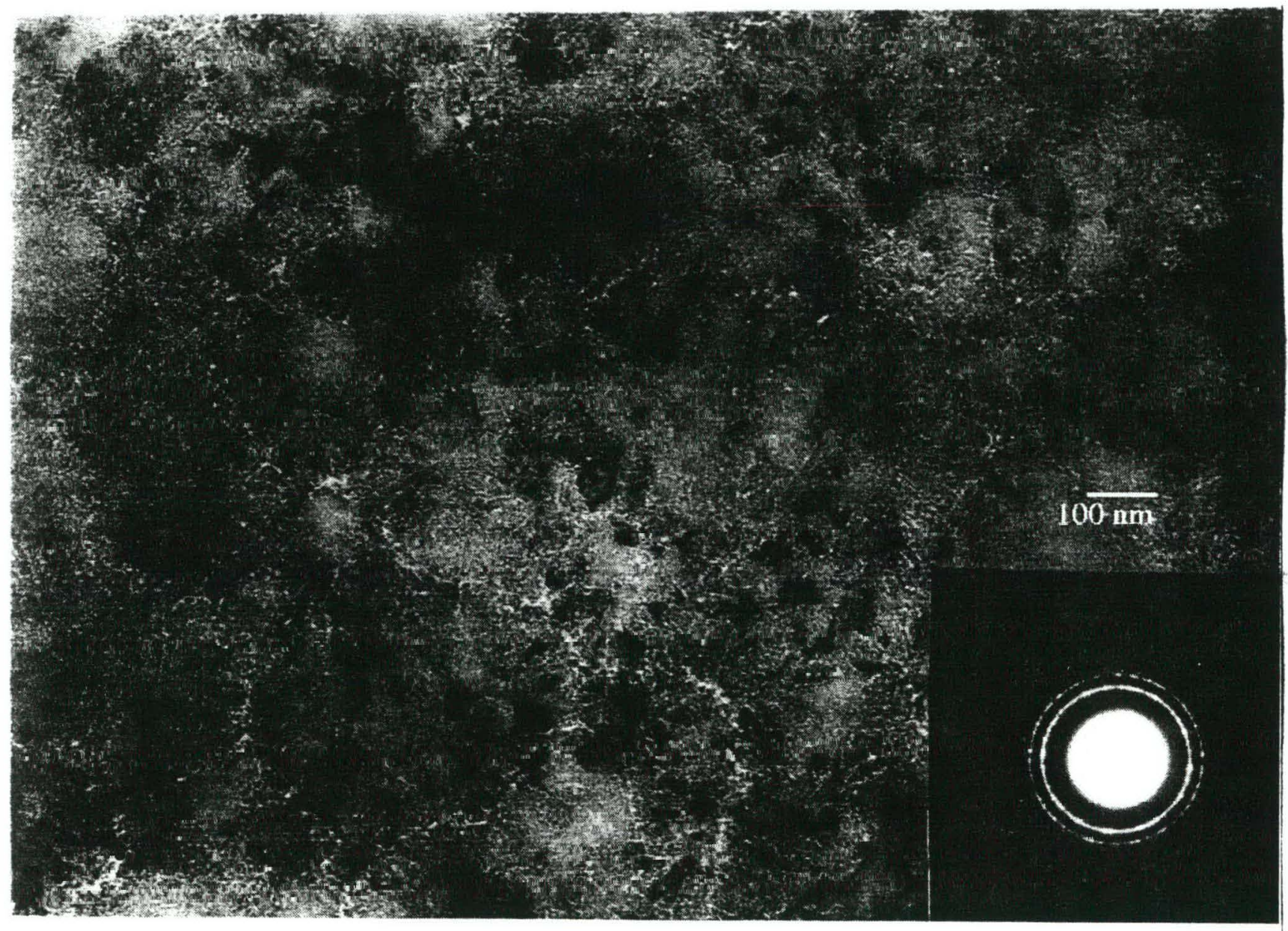


Figure 2. Frictional Performance of Rough and Smooth Diamond Films in Dry Nitrogen

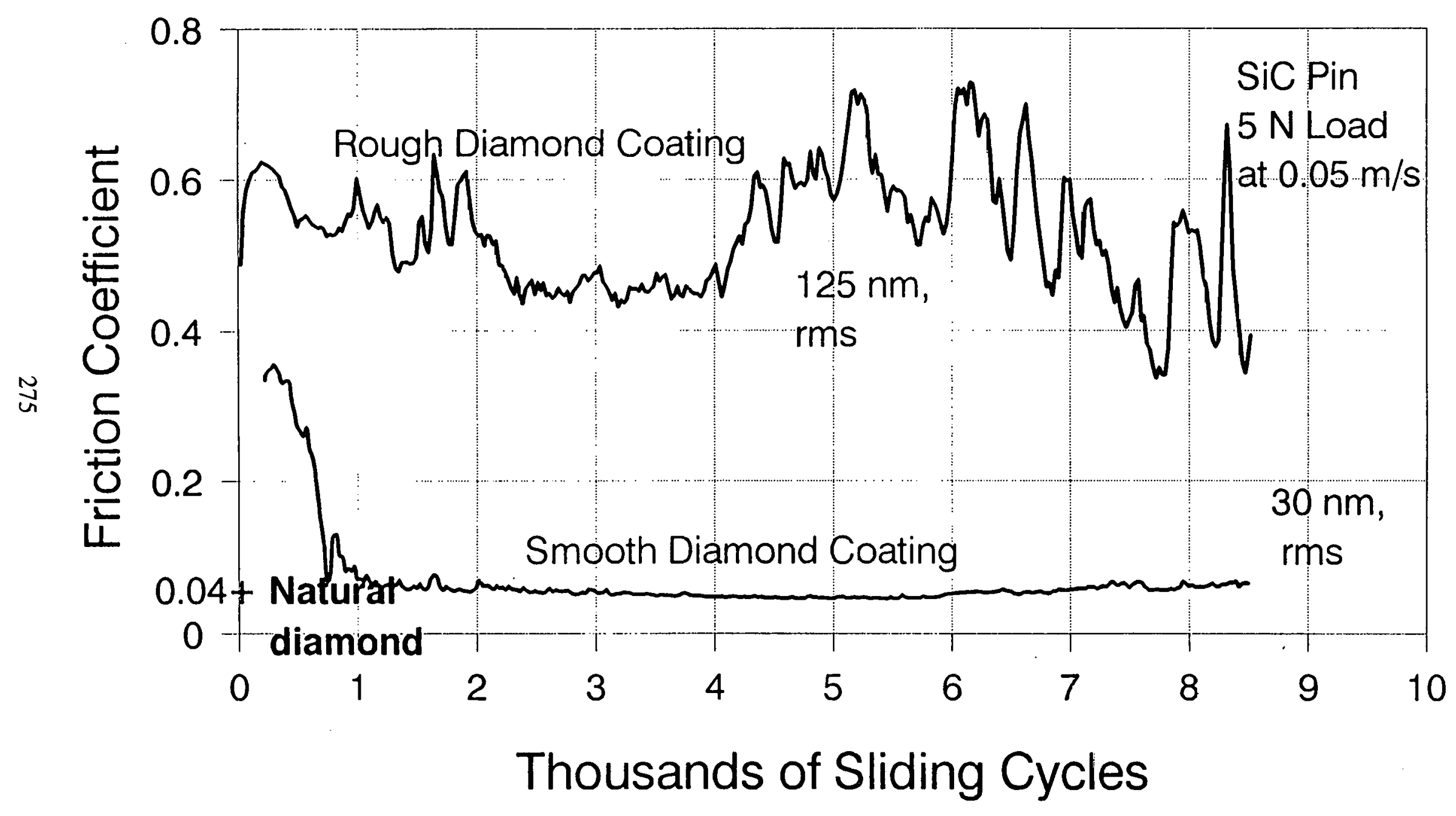


Figure 3. Frictional Performance of Rough and Smooth Diamond Films in Air

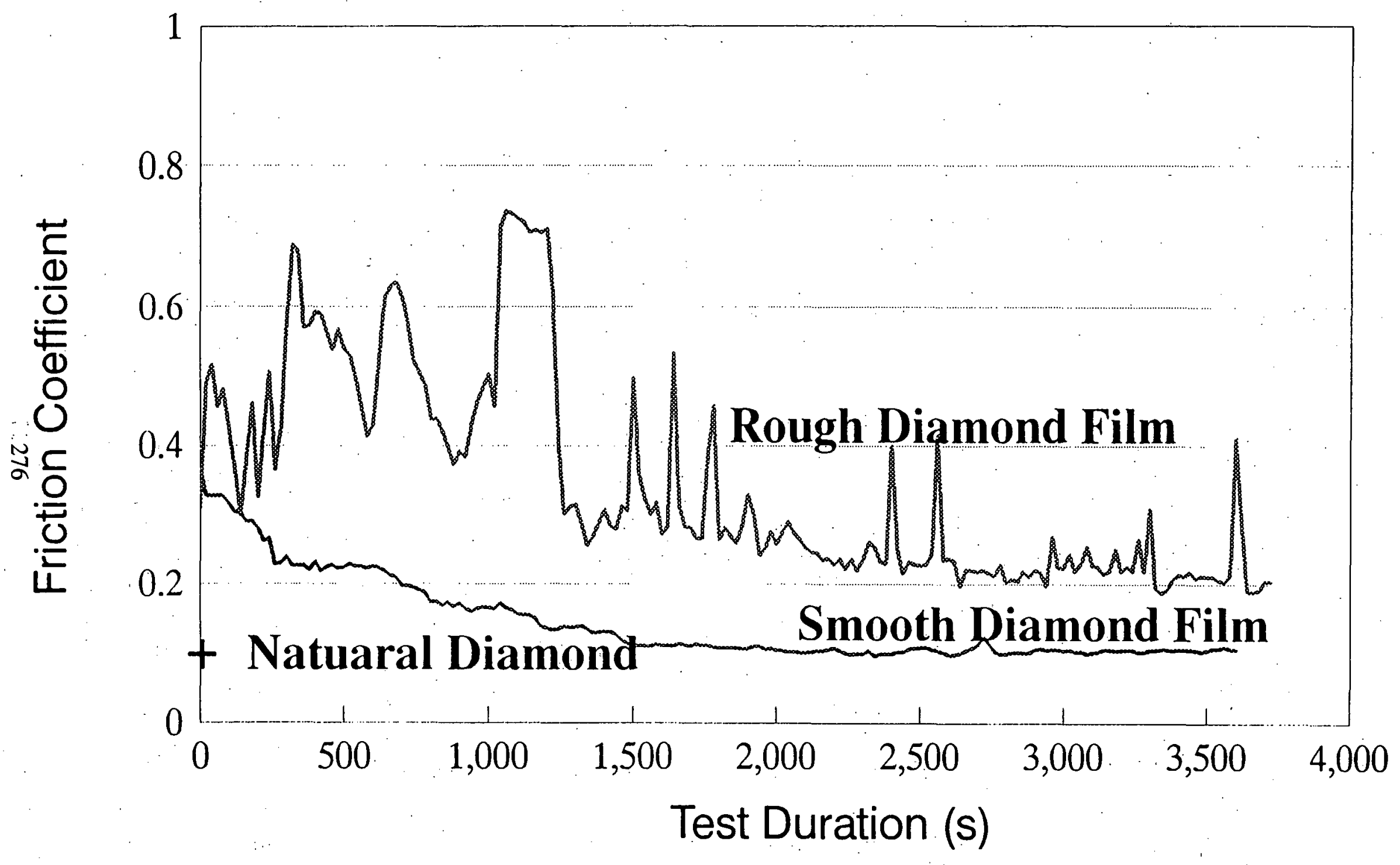


Figure 4. Long-term Friction and Wear Performance of Fullerene-Drived Smooth Diamond Film

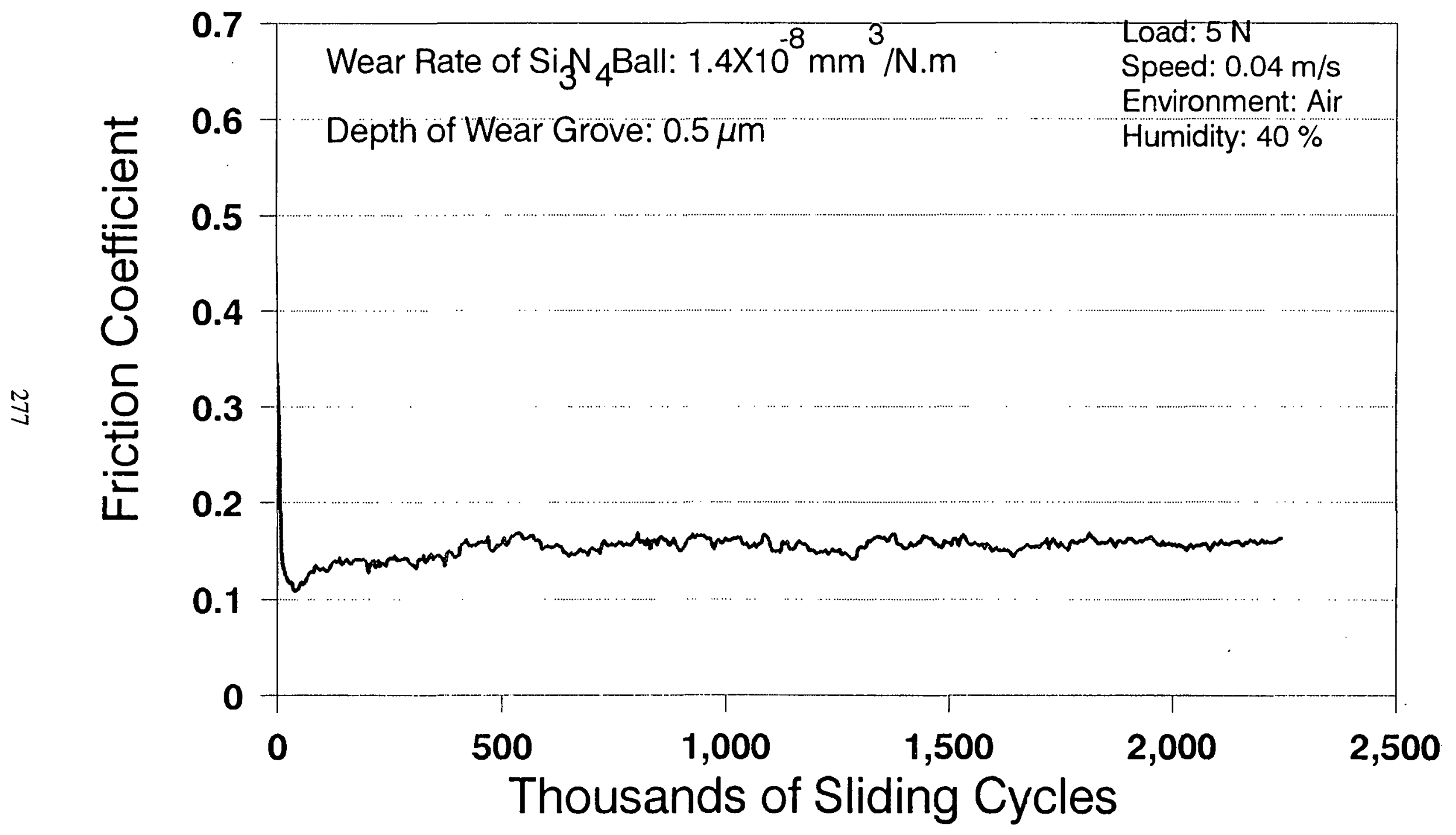


blank page 


\title{
95-305N -- THEORY OF MACROSCOPIC QUANTUM PHENOMENA AND DISORDERED MATERIALS
}

\author{
Associate Laboratory Director Area: Physical Research \\ Principal Investigator: \\ Funding Profile: \\ A. Abrikosov, Materials Science Division \\ FY 1993 \$ -0- \\ FY $1994 \$-0-$ \\ FY $1995 \$ 53.3 \mathrm{~K}$ \\ FY $1996 \$ 60.0 \mathrm{~K}$ \\ FY $1997 \$ 80.0 \mathrm{~K}$
}

Purpose: This project took advantage of visiting, well-known scientists from republics of the former Soviet Union (FSU), to work on projects close to experimental efforts in the MSD and thus enhance theoretical support of these programs. This program allowed us also to engage people with a higher level of capability and reputation in theoretical work than we could normally attract to ANL, and create new programmatic foundations. The idea was also to continue the new developments with our existing theoretical staff, as the new methods were taught to the existing staff. Funds were also used to support the postdoctoral appointee for the Laboratory's Distinguished Scientist, Dr. A.A. Abrikosov.

Approach: The success of the previous three-year LDRD-funded program led to a decision to continue it in some form. At the same time it was decided to change the accents. Whereas, the main goal of the previous program was learning new analytical methods, and thus to help the Theory Group the transition from mostly computational to analytical activity, the new program put the center of attention on support of experimental programs existing in MSD. This, on the other hand, could help the staff of the Theory Group to be more involved in experimental programs (M. Grimsditch, S. Bader, E. Fullerton, G. Crabtree, U. Welp, W. Kwok). In FY95 the invited physicists were Dr. A.I. Larkin, L.D. Landau, Institute for Theoretical Physics, Moscow (6 months); Dr. L.P. Pitaevskii, P.L. Kapitza, Institute for Physical Problems, Moscow; Dr. V.L. Gurevich, A.F. Ioffe Physico-Technical Institute, St. Petersburg (both one month). The Postdoctoral appointec was C. Sa de Melo.

\section{Technical Progress and Results:}

A. Superconductivity. Motion of Abrikosov vortices was studied in the high temperature superconductors (HTSC). High temperatures make fluctuations of the vortices strong and this leads to many effects which are difficult to observe in usual superconductors. Existence of a wide temperature interval where the pinning is absent, or small, permitted to observe the Hall- effect in superconductors, and the change of its sign was found. The microscopic theory of this phenomenon in superconductors with a small concentration of magnetic impurities was constructed with I.N. Ovchinnikov and published in Phys. Rev. B. In the work with Geshkenbein, Feigelman and Vinokur a phenomenological theory of the Hall-effect was proposed and the change of its sign was explained. The general picture of the pinning and creep of vortices in HTSC was constructed 
in earlier works but in experiment new effects appeared. A peak-effect was found in the motion of vortices along the twin boundaries. In a work with Marchetti and Vinokur the theory of pinning at such boundaries was proposed and the peak-effect was explained as a consequence of softening of the vortex lattice.

The melting theory of the vortex lattice predicts its existence not only in strong magnetic fields but in weak fields as well. This was not confirmed experimentally. In the work with Vinokur (accepted to Phys. Rev. Lett.) it was shown that in this region an unusual type of glass appears, where the vortices are pinned in bundles.

The small value of the coherence length permits to hope that not only thermal but also quantum fluctuations of vortices can be discovered. In the work with Bulaevsky and Vinokur the quantum tunneling of a vortex was calculated of a vortex, trapped by a columnar defect.

The HTSC are layered substances with a small current along the c-axis. In the work with Glazman and Koshelev it is shown how defects can destroy this current.

In experiments by K.Gray a paramagnetic effect was found during the cooling of a superconductor in a magnetic field. In the work with Koshelev (accepted to P.hys. Rev. B) the distribution of the magnetic field in a superconducting sheet was found and it was shown that a paramagnetic effect can appear, if the magnetic field is pressed into the sample.

Magnetic flux quantization was studied in exotic geometries. If an isolated superconducting wire makes a short-circuit coil the magnetic flux in it can be an integer times $\mathrm{Fi} / \mathrm{N}$, where $\mathrm{Fi}$ is the usual flux quantum and $\mathrm{N}$ - the number of windings. In the Little-Parks type experiment the periodicity of critical temperature variation with magnetic field should exhibit also a $\mathrm{Fi} / \mathrm{N}$ periodicity. A short paper was sent to Phys. Rev. Lett., and a longer one is under preparation for Phys. Rev. B.

The idea of "vortex cooling and heating" for superconductors in a magnetic field was proposed similar to laser cooling of a gas and based on resonant absorption of external electromagnetic radiation by electrons in the vortex cores. The article was published in the Phys. Rev. Lett.

B. Mandelstam-Brillouin scattering. Work on the theoretical interpretation of experiments on light scattering in substances undergoing a liquid-glass transition, performed by $\mathbf{M}$. Grimsditch was finished and is prepared for publication. In this work the ideas of elasto-viscous theory by S.M. Rytov were used.

C. Microstructures. A theory of non-Ohmic ballistic resistance in quantum wires was developed further. A variation of ballistic current was calculated through a spatially uniform conductor with a weak electron-phonon scattering. A non-Ohmic regime takes place, if $\mathrm{eV}>\mathrm{kT}$ due to the dependence of the rate of electron-phonon collisions on the electron distribution which is dependent on voltage under a ballistic regime. 
D. Magnetic multilayers. The work concerning magnetic multilayers with a semiconducting spacer was continued. In experiment the coupling between magnetic layers changed from ferromagnetic to antiferromagnetic at a definite thickness of the spacer. An explanation was proposed based on the idea of bound excitonic states in the spacer. Previously interactions of localized spins imbedded in a semiconductor were considered. This time real multilayers were studied. A paper was published in the Phys. Rev. B.

F. Ferroelectricity. In connection with the use of ferroelectric particles as memory elements a study was performed of the quantum tunneling switching of its polarization as function of its size with the goal to establish the limits of miniaturization. The problem was studied, at which temperatures quantum switching becomes important and also the influence of phonons and various defects: impurities, vacancies and surfaces.

Specific Accomplishments. The results of the work were published or submitted to scientific journals. Here is the list of them:

1. Hall-Effect in Superconductors, by A.I. Larkin and I Ovchinnikov, Phys. Rev. B 51 (1995).

2. Creep through Surface Barriers and the Irreversibility $\mathrm{L}$ in High-Temperature Superconductors, by L. Burlachkov, V. Geshkenbein, A. Koshelev, A. Larkin and V. Vinokur, Phys. Rev. B 50, 16770 (1994).

3. Sign Change of the Flux Flow Hall effect in HTSC, by M. Feigelman, V. Geshkenbein, A. Larkin and V. Vinokur, submitted to Phys. Rev. Lett.

4. Peak-Effect in Twinned Superconductors, by A. Larkin, C. Marchetti and V. Vinokur, accepted by Phys. Rev. Lett.

5. Disorder-Induced Decoupling of Pancake Vortices in a Layered Superconductor, by A. Koshelev, L. Glazman and A. Larkin, submitted to Phys. Rev. B.

6. Theory of Vortex- and Bose-Glass Transitions, by A. Larkin and V. Vinokur, submitted to Phys. Rev. Lett.

7. Quantum depinning in Layered superconductors, by L. Bulaevsky, A. Larkin, M. Maley and V. Vinokur, submitted to Phys. Rev. B.

8. Paramagnetic Effect in Field Cooled Superconductors, by A. Koshelev and A. Larkin, accepted by Phys. Rev. B.

9. Nonlinear conductivity of a Layered Metal, by A. Larkin, M. Randeria and A. Varlamov, manuscript in preparation.

10. Vortex Cooling, by C. Sa de Melo, Phys. Rev. Lett. 73, 1978 (1994). 
11. Vortex Cooling: A New Photovoltaic Effect, by C. Sa de Melo, submitted to Phys. Rev. B.

12. Magnetic Exchange Coupling Mediated by Bound States, by C. Sa de Melo, Phys. Rev. B 51, 8922 (1995).

13. Fractional Flux Quanta in Superconducting Wires, by C. Sa de Melo, submitted to Phys. Rev. Lett.

14. Quantum Limits on Classical Memories, by C. Sa de Melo, submitted to Phys. Rev.. Lett.

15. Quantum Switching of Polarization in Mesoscopic Ferroelectrics, by C. Sa de Melo, manuscript in preparation. 


\section{4-054R1 -- LARGE-SCALE WAVELET-BASED SIGNAL PROCESSING}

Associate Laboratory Director Area: Physical Research

Principal Investigator(s):

M.K. Kwong and P.T.P. Tang, Mathematics and Computer Science Division

Funding Profile:

FY $1993-0-$

FY $1994 \$ 160.6 \mathrm{~K}$

FY $1995 \$ 168.0 \mathrm{~K}$

FY $1996-0-$

FY $1997-0$

Purpose: The objective of this project is to explore the application of wavelet techniques in large-scale signal processing. Advances in high-definition television technology, military surveillance technology, scientific computation, and medical imaging have dramatically increased the need for improved algorithms and computational techniques for efficient image management, storage, and retrieval. The project will focus on the development of new still and motion image compression algorithms and their implementation on high-performance computer architectures. The new algorithms will be of use in the new multimedia laboratory under development at Argonne.

Approach: Signal processing is a crucial component in the information infrastructure, especially in the age of high-speed communication networks. Although signal processing is a well-established field, two major new areas are opening. The first is the emergence of wavelet techniques as a new fundamental tool in signal processing that rivals, and surpasses in some cases, Fourier techniques. The second is the area of large-scale image processing, mainly in the form of compression of image data of the order of gigabytes.

The project has several components.

- Design of improved wavelet transforms that generalize and outperform classical wavelet transform

- Mathematical analysis of the approximation theory of wavelets

- Development of new motion estimation techniques exploiting the hierarchical property of wavelets for superior video compression

- Construction of parallel image-processing software

- Exploration of parallel wavelet pyramid decomposition techniques for motion estimation

Our method principally involves experimentation with alternative wavelet transforms and investigation of new numerical analytic techniques, with the goal of ensuring the convergence of the approximation schemes and the accuracy of the encoded signals. Based on our 
preliminary work on devising a parallel MPEG decoder on Argonne's IBM SP computer, we are devising fast and efficient algorithms that render video encoding practicable for large-scale problems. We also explore parallel MPEG encoding schemes and compare our results with those using standard MPEG techniques. Finally, we are incorporating our new algorithms and software into a parallel image-processing library. The project is conducted in collaboration with Argonne postdoctoral researchers B. Lin and S. Yau. We also collaborate with scientists from the University of Chicago on medical imaging; Purdue University on real-time parallel video transmission and networking; Brookhaven National Laboratory on 3D tomography; David Sarnoff Research Center on HDTV processing; and AWARE Inc. on wavelet techniques and seismic data analysis.

Technical Progress and Results: We improved the performance of our parallelization of the MPEG decoder, to achieve almost the peak rate allowed by the frame buffer. We worked on the new W-transform and released our new W-matrix multiresolution analyses toolbox to the public through the World Wide Web. The transform has been applied to design image compression and edge-detection algorithms. Using the transform, we designed a feature-oriented medical image retrieval system. We obtained good performance on a test prototype.

\section{Specific Accomplishments:}

Refereed Publications:

Tomasz Bieleck, Li M. Song,.. Stephen S. T. Yau, and Man Kam Kwong, "Random Wavelet Transforms, Algebraic Geometric Coding, and Their Applications in Signal Compression and De-Noising," Proc. International Conference on Control and Information (ICCI95), Chinese University Press, Hong Kong (1995), pp. 283-289.

J. Chen, J. Aarsvold, C.T. Chen, M. Griem, P. Davies, T. Disz, I. Foster, R. Hudson, M: Kwong, B. Lin, and P. Tang, "High-Performance Image Analysis and Visualization for Three-dimensional Light Microscopy," Proc. LASTED Conference, to appear.

M.K. Kwong, "Orthogonally Compensate W-Multiresolution Analysis and Signal Processing," Proc. ICIAM 95, Hamburg, Germany, to appear.

M.K. Kwong and B. Lin, "W-Transform Method for Feature-oriented Multiresolution Image Retrieval;" Proc. SPIE Conference on Wavelet Applications, Orlando, April 17-21, 1995, to appear.

M.K. Kwong, P.T. Peter Tang, and B. Lin, "A Real-Time MPEG Software Decoder Using a Portable Message-Passing Library," Proc. SPIE Conference on Wavelet Applications, Orlando, April 17-21, 1995, to appear.

Elwood T. Olsen and Biquan Lin, "A Wavelet Phase Filter for Statistical Image Restoration," Proc. SPIE Conference on Wavelet Applications, Orlando, April 17-21, 1995, to appear. 


\section{5-010N -- CAVE VIRTUAL ENVIRONMENT TECHNOLOGY DEVELOPMENT}

\author{
Associate Laboratory Director Area: \\ Principal Investigator(s):
}

Funding Profile:
Physical Research

T. Disz, W. Nickless, and R. Stevens, Mathematics and Computer Science Division

Purpose: The purpose of this project is to develop new technology for 3D immersive virtual environmental displays and to apply this technology in the Argonne CAVE. The CAVE has become a centerpiece of virtual environments research at Argonne. Substantial technology development is needed to improve the ability to use the CAVE virtual environment as a high-performance peripheral to the IBM SP parallel computer. The project will focus on improvements in display technology, recording and playback of virtual experiences, improved texture mapping, new methods for navigation and control, and new tracking methods.

Approach: At Argonne in mid-1994, we installed a CAVE (Cave Automatic Virtual Environment) based on a design developed by the Electronic Visualization Laboratory at the University of Illinois at Chicago. The CAVE creates a large field of view by projecting images onto two walls and the floor of a ten-foot cube. Stereo cues are provided by displaying images, sequentially showing the left-eye view followed by the right-eye view, coupled with LCD shutter glasses that switching the view each eye receives. The correct viewer-centered perspective is accomplished by an electromagnetic tracking system that constantly updates the user's location in the CAVE. The user interacts with the data by means of a wand, a three-dimensional equivalent to the traditional workstation mouse. The wand has three buttons that can be programmed to interact with the data in application specific manners.

The CAVE has provided an exciting new means for scientists to explore the behavior of multidimensional physical and biological phenomena. To ensure effective use of the CAVE for these important applications, however, we must significantly enhance the basic CAVE capability. Of particular interest is hardware development for communicating among multiple CAVEs and for linking the CAVE to multiple supercomputers; technology to permit archiving of CAVE experiences; and improved display technology, for example, to support multiple active viewers.

This project focuses on technology research issues, including secure network protocols, archiving, integration of virtual environments and mass storage systems, interfacing of multimedia with massively parallel processors, and computer-human interaction. Specific areas under investigation include (1) CAVE-to-CAVE communications, (2) recording and playback of CAVE experiences, (3) improved linkage of the CAVE to SP supercomputers, (4) performance analysis of distributed CAVE applications, and (5) sonification and sonic imaging. 
Technical Progress and Results: Progress was made in several challenging research areas.

- We compared stereo compression algorithms, as well as a variety of motion compensation ideas and hybrid algorithms. Using new compression techniques, we successfully captured, recorded, and played back compressed image data from the CAVE. This is a first step in being able to archive CAVE interactions.

- We developed and prototyped models to support CAVE-to-CAVE communication. For example, we explored techniques for incorporating measured, on-site data into the computational model and real-time transmission of the tremendous amount of scientific data from the computational model. The results have been put on the Web and will be demonstrated as part of the I-WAY project.

- We created a Futures Lab communication library, called CAVEcomm, for connecting CAVEs and ImmersaDesks to each other.

Specific Accomplishments: Effort will continue under the auspices of DOE-ER.

A. Choudhary, I. Foster, and R. Stevens, "Guest Editors' Introduction: Multimedia Applications and High-Performance Computing," IEEE Parallel \& Distributed Technology: Systems \& Applications, 3(2):2-3 (summer 1995).

T. L. Disz, R. Evard, M. W. Henderson, W. Nickless, R. Olson, M. E. Papka, and R. Stevens, "Designing the Future of Collaborative Science: Argonne's Future Laboratory," IEEE Parallel \& Distributed Technology: Systems \& Applications, 3(2):14-21 (summer 1995).

T. Disz, M. Papka, R. Stevens, M. Pelligrino, and V. Taylor, "Virtual Reality Visualization of Parallel Molecular Dynamics Simulations," High-Performance Computing '95, Phoenix, Arizona, April 12-13, 1995 (MCS preprint MCS-P502-0395).

T. Disz and M. Papka, "Sharing Visualization Experiences among Remote Virtual Environments," Proc. International Workshop on HPC for Computer Graphics and Visualization, Swansea, July 3-4, 1995.

V. Taylor, R. Stevens, and M. Kandaswamy, "Performance Models of Interactive, Immersive Visualization for Scientific Applications," Proc. International Workshop on HPC for Computer Graphics and Visualization, Swansea, July 3-4, 1995.

S. Bradshaw, T. Canfield, J. Kokinis, and T. Disz, "An Interactive Virtual Environment for Finite Element Analysis, " Proc. High-Performance Computing '95, Phoenix, 1995 (also preprint MCS-P501-0395).

M. Huang, M. E. Papka, T. DeFanti, D. Levine, L. Turner, and L: Kettunen, "Virtual Reality Visualization of Accelerator Magnets," Proc. High-Performance Computing '95, Phoenix, 1995 (MCS preprint MCS-P496-0295). 


\title{
95-070N -- A HUMAN-COMPUTER EXPERT SYSTEM FOR AUTOMATED HIGHWAY SYSTEM
}

\author{
Associate Laboratory Director Area: Engineering Research \\ Principal Investigators: \\ A.M. Tentner, Reactor Analysis Division \\ T.F. Ewing, Technology Development Division \\ Funding Profile: \\ FY $1993-0-$ \\ FY $1994-0-$ \\ FY $1995 \$ 198.0 \mathrm{~K}$ \\ FY $1996 \$ 200.0 \mathrm{~K}$ \\ FY $1997 \$ 200.0 \mathrm{~K}$
}

Purpose: The Intelligent Transportation System (ITS) program of the U.S. Department of Transportation (U.S. DOT) is designed to use advanced computing and communications technologies for proactive control and management of traffic flow and transportation facilities in order to improve traveler's safety and mobility, reduce congestion, minimize energy consumption and negative environmental impact, and promote economic competitiveness of the U.S. industry. One of the ITS initiatives, Automated Highway Systems (AHS), deals with the development of automated navigation systems for vehicles and highway systems.

Building on the existing ANL ITS simulator, our broad objective is to build a suitable simulation testbed to support the study of emerging AHS concepts, technologies and architectures. The ITS simulator provides a comprehensive, large-scale simulation of an intelligent highway system running on distributed or parallel computer systems. This continuing work focuses on developing automated vehicle and highway prototype models for the ITS simulator to provide a capability to simulate an automated intelligent vehicle-highway system, as well as accommodate human factors studies in support of safety and operational research.

Approach: The project requires the development of an intelligent system called the Expert Driver Model (EDM) describing the behavior of a vehicle operating under both AHS and conventional highway system designs. Two modules will be developed for the existing ITS simulator:

- : Human Driver Model (HDM) describing the behavior of the human driver in conventional vehicles

- Automated Driver Model (ADM) describing the behavior of a fully automated (driverless) vehicle operated in an AHS environment

Both models will be developed in two phases. In the first phase, both HDM and ADM will describe vehicle behavior in the absence of the Traffic Management Center (TMC) component (representing an early AHS deployment). The second phase will provide 2-way communications capability with a TMC model. The vehicles will act as probes, sending traffic data to the TMC, and receiving advisories and AHS state information. This information would provide vehicles 
with information about global traffic conditions, road conditions, and other information that would otherwise not be available.

Technical Progress and Results: During the first project year FY95 a vehicle-to-vehicle interaction model has been defined to support conventional and AHS vehicle simulations in the ITS simulator. Information regarding the immediate environment of a vehicle, such as speed limit, weather and road conditions, proximity to other vehicles (current position, velocity, and acceleration of nearby vehicles) is maintained in a "link manager" process, which acts as a central clearing house for relevant data on a particular region of road. Furthermore, a threedimensional data matrix is stored by the link manager containing speed variations of the vehicles from posted speeds. These speed variations are functions of the type of driver (3 types considered), weather condition (13 types considered), and road condition (6 types considered).

Vehicles exchange messages with link managers to receive information about other vehicles and conditions on the road link, and to provide the link manager with requested information. The link manager is to some extent an artifact of simulation -- it allows simulated vehicles to gather information real drivers obtain by "looking out the window". Thus, every AHS vehicle will have enough information about neighboring vehicles (location, speed, acceleration, and vehicle type). Such information is needed for the AHS vehicle to make maneuvering decisions. However, the link manager can also model features which represent elements of an intelligent highway system, such as communications between traffic lights and emergency vehicles on the road link to facilitate preferential routing of the emergency vehicle.

Also in the current year, we have initiated the development of new controls for the ITS simulator vehicle module user interface to support human factors studies and define the functionality of a smart cruise control (SCC). The SCC provides new AHS functions to the conventional cruise control. Our SCC design would provide two levels of automation enhancement: Mode 1 - maintain selected speed in current lane, but reduce speed to maintain safe separation from a forward vehicle; and Mode 2 - maintain selected speed with an option to pass a vehicle under certain conditions (multi-lane road with well-spaced traffic and clear passing lane, forward vehicle below current speed limit) In order to develop and test these AHS concepts, a specific scenario has been devised representing a straight, multi-lane stretch of rural highway in the Chicago-Gary-Milwaukee Priority Corridor.

Specific Accomplishments: We have developed contacts with the National Automated Highway Consortium (NAHSC) lead by. General Motors. ANL has become, through the ANL ITS Initiative, an associate member of NAHSC. NAHSC has been awarded the DOT contract for the development of the national automated highway system. We are discussing with NAHSC the possibility of including the ANL Expert Driver/ITS Simulator in the 1997 AHS Demonstration. 


\title{
94-190R1 -- INTEGRATING MULTIMEDIA WITH HIGH-PERFORMANCE COMPUTING AND COMMUNICATIONS
}

\author{
Associate Laboratory Director Area: Physical Research \\ Principal Investigators: \\ Funding Profile: \\ FY $1993-0-$ \\ FY $1994 \$ 158.9 \mathrm{~K}$ \\ FY $1995 \$ 140.8 \mathrm{~K}$ \\ FY $1996-0$ - \\ FY $1997-0-$
}

R. Olson and R. Stevens, Mathematics and Computer Science Division

Purpose: The project objective is to explore the close coupling of multimedia technology (images, sound, and text) and high-performance computing architectures and applications. By enabling systems such as the IBM SP to process voice and image data along with scientific data resulting from computations, we hope to expand the types of applications suitable for parallel computing (e.g., digital libraries). Additionally, we are creating new collaborative environment technologies that combine advanced networking, virtual space technologies, and high-end virtual environments.

Approach: Current parallel supercomputer systems like the IBM SP are well suited for "traditional" scientific computing (i.e., rapidly evolving a simulation described by a set of numerical partial differential equations forward in time and periodically capturing the state of the system). Future uses of supercomputers will include not only numerical computations but also the reduction of output data to meaningful statements about reality (virtual or otherwise). Specifically, in the future one would like to use the supercomputer as a general-purpose tool for computational science, including the use of the system for supporting visualization, collaborative development of systems, collaborative exploration and analysis of experimental and computational data, and the storage and archiving of such data and collaborative experiences. These uses of massively parallel processing systems require additional hardware and software support. One of the purposes of this project is to evaluate ways of expanding the capabilities of the IBM SP system.

The project involves exploration and development of the features and architectures needed to support digital libraries and interactive multimedia use of parallel supercomputers. Six subprojects are being investigated: audio/video storage and retrieval, support for parallel image processing and analysis, support for remote visualization via high-speed fiber channel networks, development and testing of a multimedia node concept for future IBM SP systems, demonstration of multimedia to enhance scientific applications, and demonstration of integration of wireless communications technology with high-performance computing and communications. It is anticipated that one of the outcomes of this project will include software and architectures that will enable Argonne to successfully compete for federally supported work on the National Information Infrastructure. 
Technical Progress and Results: Under earlier LDRD funding, a multimedia laboratory was established in the Mathematics and Computer Science Division. Video tunneling from the CAVE was demonstrated, and early experiments were conducted with the SP as a video archive machine.

Building on this work, during FY 1995 we achieved several accomplishments:

- Enabled large number of CAVE applications projects

- Secured funding of collaborative environment

- Secured funding of video voyager

- Added integrated imaging to applications

- Began exploring use of wide-area ATM networks to support multimedia and virtual computing

- Designed prototype experimental local-area networks to explore virtual reality demos and large-screen projection systems

- Began work on high-speed wireless LANS

- Prototyped Argonne video server architecture

- Enhanced insight into diverse applications, including interactive unstructured mesh computation, visualization of casting processes, and interactive molecular modeling.

Specific Accomplishments: Effort will continue under the auspices of DOE-ER.

\section{Refereed Publications:}

Alok Choudhary, Ian Foster, and Rick Stevens, "Guest Editors' Introduction: Multimedia Applications and High-Performance Computing," IEEE Parallel \& Distributed Technology: Systems \& Applications, 3(2):2-3 (summer 1995).

Terrence L. Disz, Remy Evard, Mark W. Henderson, William Nickless, Robert Olson, Michael E. Papka, and Rick Stevens, "Designing the Future of Collaborative Science: Argonne's Futures Laboratory," IEEE Parallel \& Distributed Technology: Systems \& Applications, 3(2):14-21 (summer 1995).

Terrence L. Disz, Michael E. Papka, Michael Pellegrino, and Rick Stevens, "Sharing Visualization Experiences among Remote Virtual Environments, " Proc. International Workshop on HPC for Computer Graphics and Visualisation, Swansea, July 3-4, 1995.

T. Disz, M. Papka, R. Stevens, M. Pellegrino; and V. Taylor, "Virtual Reality Visualization of Parallel Molecular Dynamics Simulation," High-Performance Computing '95, Phoenix, Arizona, April 12-13, 1995. (MCS preprint MCS-P502-0395)

Valerie E. Taylor, Rick Stevens, and Thomas Canfield, "Performance Models of Interactive, Immersive Visualization for Scientific Applications," Proc. International Workshop on HPC for Computer Graphics and Visualisation, Swansea, July 3-4, 1995.

Shannon Bradshaw, Thomas Canfield, John Kokinis, and Terrence Disz, "An Interactive Virtual Environment for Finite Element Analysis." 
Milana Huang, Michael E. Papka, Thomas DeFanti, David Levine, Larry Turner, and Lauri Kettunen, "Virtual Reality Visualization of Accelerator Magnets."

\section{Presentations:}

R. Olson, "Foraging the Web: A Futures Laboratory Talk," February 23, 1995.

R. Stevens, "Fast Forward: Where 100 Mbps Is Not Enough Today," Networld Interop '95, Las Vegas, Nevada, March 29, 1995.

R. Stevens, "LabSpace: Developing the National Electronic Laboratory Infrastructure," Carnegie Mellon University, Pittsburgh, Penna., December 2, 1994.

R. Stevens, "Building the I-WAY," I-WAY Workshop, Argonne National Laboratory, April 18-20, 1995. 
blank page 


\title{
95-072N -- RECURRENT ARTIFICIAL NEURAL NETWORKS FOR MODELING OF NONLINEAR SYSTEMS
}

\author{
Associate Laboratory Director Area: Engineering Research \\ Principal Investigators: \\ J. Reifman, J.E. Vitela, \\ and T.Y.C. Wei, \\ Reactor Engineering Division \\ Funding Profile: \\ FY $1993-0-$ \\ FY $1994-0-$ \\ FY $1995 \$ 173 \mathrm{~K}$ \\ FY $1996 \$ 169 \mathrm{~K}$ \\ FY $1997 \$ 170 \mathrm{~K}$
}

Purpose: The purpose of this project was to perform feasibility studies in the application of recurrent artificial neural networks (RANNs) for modeling the dynamics of nonlinear systems. The effectiveness of the developed RANN algorithm was first tested with data generated from analytical expressions (where the state variables needed to represent the model were known) and then applied to industrial process plant data (where the state variables needed to adequately represent the model were not known and only a few variables were available). Successful demonstration of the RANN to capture (from data) and model the dynamics of nonlinear systems when analytical models are unknown or unavailable can be readily transferred to the modeling of complex systems in different areas.

Approach: The efficiency of complex dynamic industrial processes is highly dependent on how well the system is understood and controlled. The key basic element for performing these two functions is to be able to accurately model the process. With an appropriate model of the process, sensitivity analysis can be performed to provide a better understanding of the importance of each one of the process variables and allow for more sophisticated and effective control strategies. However, the complexity of real-world industrial processes add to the difficulty of developing dynamic models based on physical principles. Great economical benefits would result from slight increases in process efficiency.

An alternative approach for modeling complex and highly nonlinear dynamic systems when the exact analytical model is unknown, unavailable or impractical is to use RANNs. Recurrent multilayer neural networks are prime candidates for system modeling and identification due to their ability to approximate large classes of nonlinear functions sufficiently accurately in cases where the dynamic system is known only in terms of its inputs and outputs. This is indeed the case for emissions generated in coal-firing fossil power plants where only input variables and parameters (coal rate, inlet air rate, burner tilt position) into the process and emissions (or process output) such as Nitrogen Oxide $\left(\mathrm{NO}_{x}\right)$ and Carbon Monoxide $(\mathrm{CO})$ are known.

Unlike static feedforward multilayer neural networks, in which the output depends solely on the present input, recurrent neural networks contain recurrent connections that together with time-delays allow for the representation of time-dependent information. Hence, recurrent networks can be used to map ordered sequences of inputs into ordered sequences of outputs 
which are characteristic of dynamic systems. The scope of this investigation was to first develop RANNs capable of modeling the dynamics of nonlinear systems, test the algorithm with analytical data and then apply RANNs to model the emissions of a fossil power plant boiler using plant data. This work has close ties to another FY 1995 funded LDRD ("Neural Network $\mathrm{NO}_{x}$ Emission Controller for Fossil Plants" 95-229N) where neural network-based controllers were developed.

Earl E. Feldman from the Reactor Analysis Division collaborated in the training and testing of RANNs to model the emissions of a fossil power. plant boiler from plant data. Kent E. Wanninger, Jim S. Herzau and Ron G. Kubik from Commonwealth Edison (ComEd) provided the proprietary plant data.

Technical Progress and Results: The research conducted under LDRD auspices during FY 1995 was composed of four tasks: analysis of RANNs, code development, neural network training and neural network testing. In the first task, various recurrent neural network architectures and learning algorithms were analyzed. From the analyzed RANNs, one was selected and implemented into software in the second task. The last two tasks, neural network training and testing, were performed for data generated from an analytical model and from plant data. We first validated the developed software with data generated from an analytical model, where the state variables needed to represent the model were known, and then applied the software to model the emission of $\mathrm{NO}_{x}$ from a coal-firing fossil power plant, where the state variables needed to adequately represent the model were not known and limited input-output data were available.

Results from both validation tests are presented here. In the first test, we applied the developed RANN software for predicting the chaotic time-series $x_{k+1}=\left[2 x_{k}-x_{k-1}+0.5 \sin x_{k}\right]$ module $2 \pi$. Given $x_{k-1}$ and $x_{k}$, this discrete-time nonlinear dynamical model calculates $x_{k+1}$. In this test, the training data consisted of the set of input pairs $x_{k-1}$ and $x_{k}$ (for $k=2,3, \ldots, 102$ ), generated from arbitrary initial conditions $x_{1}=0.0$ and $x_{2}=1.0$, and associated target output $x_{k+18}$ (for $k+18=20,21, \ldots, 120)$. In other words, given the input values $x_{k-1}$ and $x_{k}$, the RANN was trained to predict the value of $x_{k+18}$. The results of this training phase is illustrated in Fig. 1a which shows a good agreement between the analytical values and network trained values. The validation sequence consisted of the values of $x_{k}$ over the interval $[120,138]$ which was used to predict the values of $x_{k}$ over the interval $[138,156]$. Figure $1 b$ illustrates the validation results which indicate a good agreement between the analytical values and network predicted values.

In the second test, we applied the RANN software to model $\mathrm{NO}_{\mathrm{x}}$ emissions from one ComEd. fossil power plant using actual plant data. A total of forty data points were provided representing eighty minutes of plant maneuvers where the plant load and a small set of control variables (the neural network input) were varied and the $\mathrm{NO}_{\mathrm{x}}$ (the neural network output) was measured. The first twenty input-output pairs of the time sequence were used to train the RANN to model the time-discrete dynamical system and the subsequent twenty input-output pairs were used to test the capability of the trained network to predict $\mathrm{NO}_{\mathrm{x}}$ levels given the input.

Figure 2 shows the results obtained for this test. The continuous line represents time-discrete measured $\mathrm{NO}_{\mathrm{x}}$ data sampled at two-minute intervals for eighty minutes. The circles with the plus sign inside corresponding to the first twenty minutes of data, represent the neural network 
predicted values which were used for training. The empty circles corresponding to the last twenty minutes of data represent the network predicted values. Although the network predicted $\mathrm{NO}_{x}$ can be off by as much as $15 \%$, with such a limited data set and small number of input variables, it does show a correct overall trend. In addition, the RANN allowed us to perform sensitivity analysis of the system (if the model is taken to represent the actual system). From numerous tests of the model where each control variable was changed independently in a step change, instantaneous change, and sinusoidal change, the following properties were captured:

- the trained network behaved as a dynamic physical model for all test cases,

- the internal time constant of the system is approximately six minutes, and

- the system seems to have a linear response to small perturbation amplitudes for the set of initial states and frequencies tested.

The overall results indicate that RANNs provide an excellent tool for better understanding and modeling of dynamical nonlinear systems when exact analytical models are unknown or unavailable.

Additional LDRD funding was requested for FY 1996. If support is granted, work will be extended to model other dynamical systems where models exist but are much slower than real time. We will test the capability of using RANNs as faster-than-real-time computer programs for modeling complex systems. From the liaison established with ComEd, we also plan to pursue other funding opportunities to continue this work and include the control part of the problem by coupling a neural network model of the plant with a neural network controller.

Specific Accomplishments: An internal report entitled "Modeling of Nonlinear Dynamical Systems with Neural Networks" is being prepared, an external presentation of the results (to ComEd) will occur after October 1, 1995, and we plan to summarize the results and publish them as a refereed conference paper. 


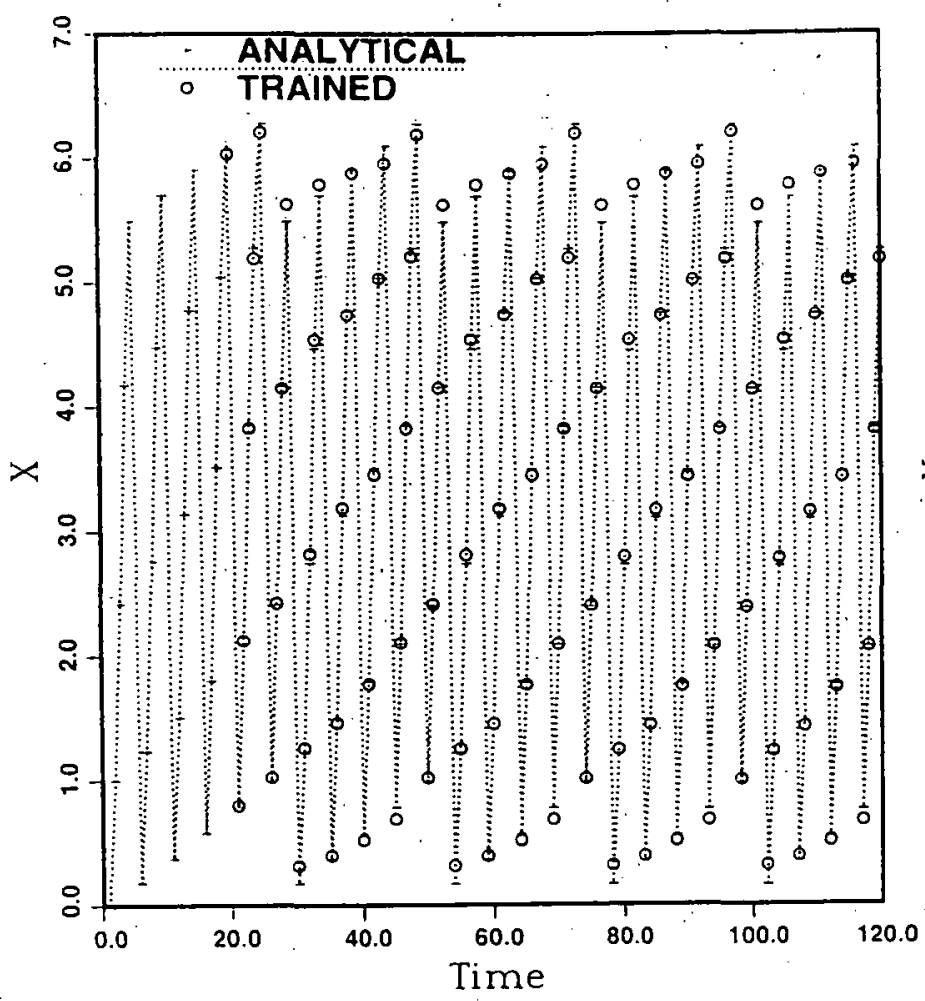

Fig. 1.a Analytical and Training Results

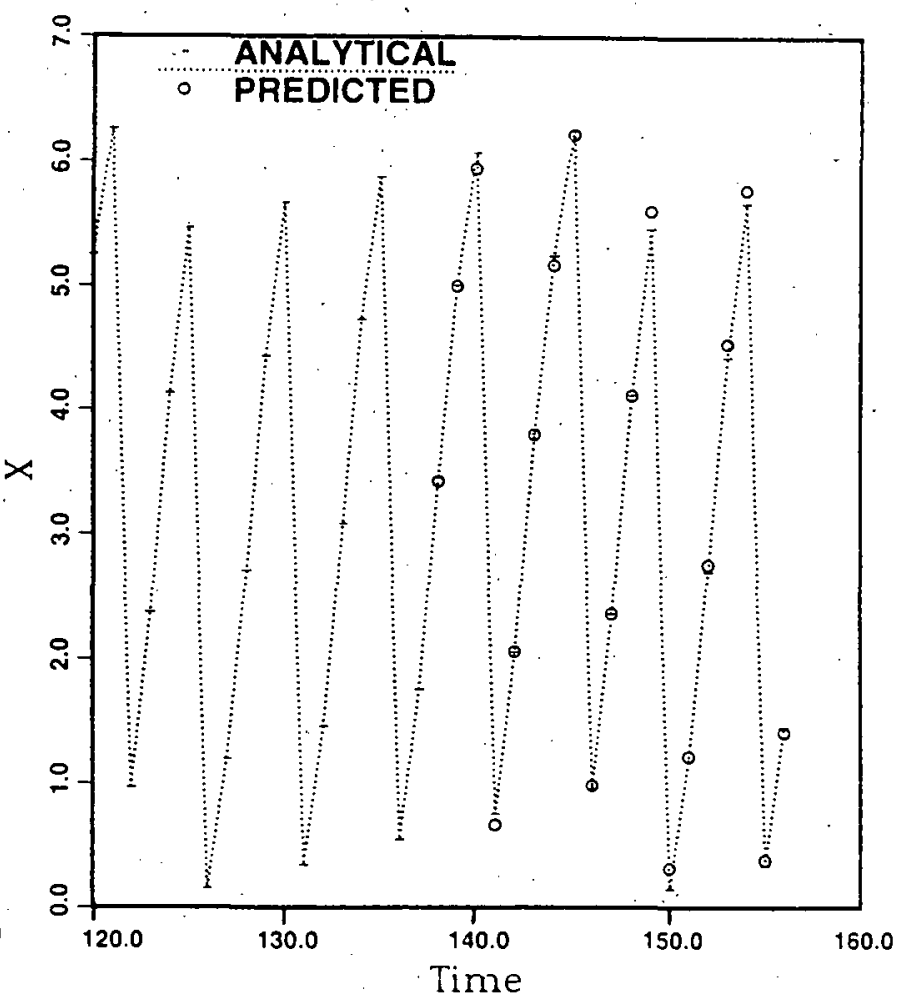

Fig. 1.b Analytical and Predicted Results

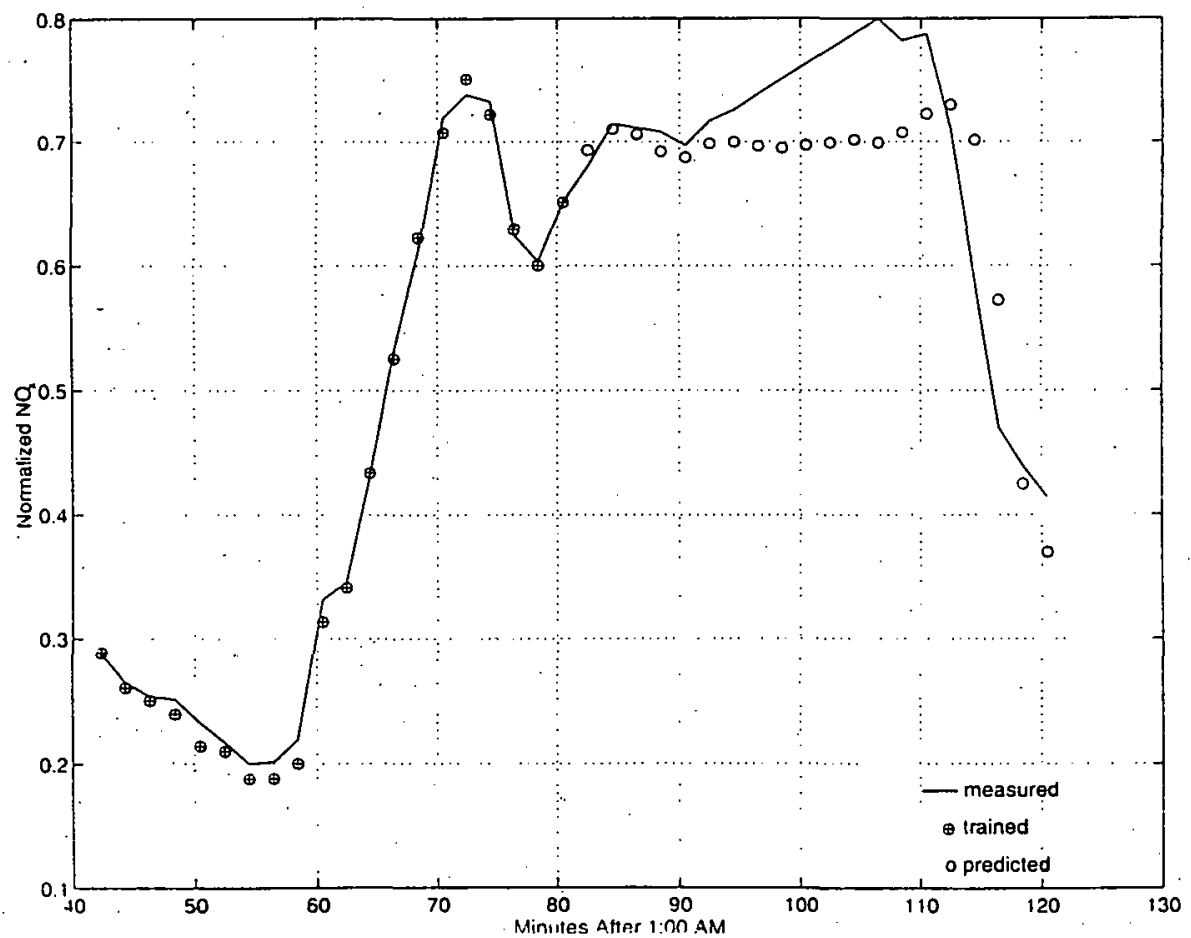

Fig. 2: Measured $\mathrm{NO}_{x}$ and Recurrent Neural Network Trained and Predicted $\mathrm{NO}_{x}$ 


\section{5-247N -- ARTIFICIAL INTELLIGENCE APPLIED TO ADAPTIVE MESH REFINEMENT FOR NONLINEAR FINITE ELEMENT ANALYSIS ${ }^{1}$}

Associate Laboratory Director Area: Engineering Research

Principal Investigator:

Funding Profile:

\author{
E.J. Plaskacz, \\ Reactor Engineering Division
}

FY $1993-0-$

FY $1994-0-$

FY $1995 \$ 74.2 \mathrm{~K}$

FY $1996-0-$

FY $1997-0-$

Purpose: To develop new methods to control the efficient allocation of computational resources using neural networks.

Approach: The effectiveness of mesh adaptivity hinges on the criteria. It is impossible at this time, with the available mathematical tools for error estimation, to estimate the local error in a nonlinear transient solution. Therefore, the decision to divide or regroup elements is often based on response performance criteria, i.e., amount of average plastic work, rotation, etc. Concepts from neural networks were used to incorporate the behavior of adjacent elements as well as the historical record of previous activity in deciding what to do next.

The use of neural networks will be used in conjunction with "fission-fusion" adaptive meshing techniques to allocate spatial resolution to where and when it is needed the most. In this type of adaptive scheme, the mesh is selectively refined in parts of the domain during the evolution of the solution through the "fission" of an element into four elements and coarsening, through the "fusion" of four elements, into one.

Technical Progress and Results: This project was funded in FY95 with the following results:

An assessment of the suitability of various neural network architectures for their potential use in this application was initiated. This assessment was done in tandem with a course offered through IITV entitled Neural Networks. The objective is to assess each neural network introduced in the class and benchmark its performance in fission-fusion mesh adaptivity. A summary of these findings will constitute the final class project and will also be submitted for publication in a technical journal.

The first neural network to be investigated was the ADALINE or Adaptive LINear Element. The ADALINE was invented by Bernard Widrow and has a long history of successful use as an echo suppressant in telephone lines. The goal in our study was to use the ADALINE to suppress the contribution to element strain energy attributable to elastic waves and use this filtered value as the adaptive mesh refinement criteria.

${ }^{1}$ Individual Investigator Project 
Each element in the finite element mesh was associated with its own ADALINE neural network. The weights of this neural network were adjusted in response to the element strain energy history. Four and eight ADALINE neural networks were used. The four neuron ADALINE used the element strain energies from the four previous time steps to guess the current element strain energies while the eight neuron ADALINE used the element strain energies from the eight previous time steps to guess the current element strain energy. After the training period the ADALINE output was used in place of the directly calculated element strain energy as a criterion for mesh adaptivity.

Two important parameters studied were the effect of the learning parameter on the network training rate and the length of training period required before the weights in the ADALINE converged. In general, it was observed that too large a learning parameter and too short a training period were both destabilizing effects. For improving the turn-around time of the numerical experiments, the neural networks were tested on a very coarse mesh with only a single level of mesh adaptivity.

The cylindrical panel problem has been used as a benchmark for many nonlinear transient problems. An initial velocity is applied across the crown of the panel. The panel is simply supported along the sides and clamped at the sides. The result of our studies are summarized in Table 1. All elements in the fixed mesh were fissioned for a total of 288 elements: The adaptive meshes only fissioned a fraction of the elements for an approximate total of 126 elements.

Table 1. Comparison of Computed Results For An Impulsively Loaded Cylindrical Panel

\begin{tabular}{|c|c|c|c|c|}
\hline \multirow[b]{2}{*}{ Point } & \multirow[b]{2}{*}{$\begin{array}{c}\text { Fixed } \\
288 \\
\text { Elements }\end{array}$} & \multicolumn{3}{|c|}{$\begin{array}{l}\text { Adaptive } \\
\text { Approximately } 126 \text { Elements Used }\end{array}$} \\
\hline & & $\begin{array}{l}\text { Total Element } \\
\text { Strain Energy }\end{array}$ & $\begin{array}{l}\text { ADALINE-filtered } \\
\text { Total Element } \\
\text { Strain Energy } \\
\text { 4. Neuron ADALINE }\end{array}$ & $\begin{array}{c}\text { ADALINE-filtered } \\
\text { Total Element } \\
\text { Strain Energy } \\
8 \text { Neuron ADALINE }\end{array}$ \\
\hline A & -1.14 & -1.03 in. & -1.07 in. & -1.09 in. \\
\hline B & -.53 & -0.52 in. & $-0.53 \mathrm{in}$. & -0.53 in. \\
\hline
\end{tabular}

*Point $\mathrm{A}$ is at the crown of the panel 6.28 inches from the fixed end.

*Point $\mathrm{B}$ is at the crown of the panel 9.42 inches from the fixed end.

These results are encouraging for the following reasons:

- In no case did the use of an ADALINE decrease the accuracy of the computed results.

- The coarseness of the mesh hindered the effectiveness of the eight neuron ADALINE. A large percentage of the time steps (160 out of 500 ) were needed just to train the ADALINE. 
Thus, its results were not available for use as criteria for a significant fraction of the simulation.

The ADALINE training time is dependent on the number of neurons in the neural network only. This restriction will be alleviated with finer meshes. Since the maximum time step size will decrease as the elements become smaller, more time steps will be required for the simulation. Hence, the results of the ADALINE will be available sooner which should lead to an even higher improvement in accuracy.

Specific Accomplishments: A paper is in preparation for submission to a technical journal. 
blank page 


\title{
94-047R1 -- 2D-3D/HOLOGRAPHIC IMAGES AND VIRTUAL REALITY PROJECTIONS FOR CONTROL ROOM/SIMULATOR DESIGNS
}

\author{
Associate Laboratory Director Area: Engineering Research \\ Principal Investigators: \\ Engineering Division \\ Funding History: \\ FY $1993-0-$ \\ FY $1994 \$ 179.8 \mathrm{~K}$ \\ FY $1995 \$ 193.1 \mathrm{~K}$ \\ FY $1996-0-$ \\ FY $1997-0-$
}

R.W. King and S.A. Brown-VanHoozer,

Purpose: The project investigates the use of dynamic virtual reality models to visualize, develop, and design complex systems and control consoles to enhance the effectiveness of the engineering process and improve the operator/system interface.

Approach: Virtual Reality (VR) may be used to describe a range of experiences that enable a person to interact with and explore a spatial environment through a computer. The models and images in the virtual environment brings closer the experience of being immersed inside the model or image. Depending upon the desired effects, virtual reality environments can be created by high-end computer systems for total body immersion into the virtual worlds or can be created with low-end workstations for less expensive and more manageable displays.

Various computer systems were investigated by R.C. Singleterry to determine the minimum requirements for either a high-level, totally immersed virtual environment or a low-level windowing environment. Because of the cost effectiveness of the options, a windowing virtual environment was more conducive to engineering applications by using existing hardware and software systems to create the effects of immersion from a desktop system. In addition, outside agencies in industry and government were contacted to determine technical goals and requirements where this technology could be used effectively and to evaluate the potential for follow-on funding for VR prototyping.

The project was divided into four tasks for the development of low-end virtual reality displays. L.H. Hansen developed a $3 \mathrm{D}$ computer model of the remote handling systems in a hot cell environment and used special stereoscopic glasses for viewing the processes and structures. G.G. Peters constructed 3D images for virtual displays of control panels for a fuel handling console. R. Magnuson, a summer student from Western Washington University, developed a 3D model for the engineering design of a future addition to the Sodium Process Facility. D.C. Crites investigated the use of digitized, photographed images for panoramic visualization, training, and other engineering functions.

Technical Progress and Results: In Phase I, a 3D AutoCad model of the EBR-II primary tank was converted and imported into the University of Illinois-Chicago VR-CAVE (Virtual RealityCave Automatic Virtual Environment). The 3D model was developed by L.H. Hansen and C.V. Wiegand. The model was refined for more realistic effects and dynamic movements were 
incorporated. This model was transferred to the new VR-CAVE at ANL-E and further developed by $R$. Hudson and N. Gokhale. Studies were performed to evaluate human interactions with the VR model in a CAVE environment.

For FY 1995 (Phase II), the tasks were refocused to evaluate low-end (PC or workstation scale) VR capability for smaller scale engineering projects where a full VR-CAVE was not required or justifiable. These tasks would be more applicable to training and engineering design of components and consoles.

A 3D model of the remote handling equipment located in the argon cell of the Fuel Conditioning Facility was constructed. The model concentrated on the in-cell cranes, the electromechanical manipulators and the operator control box. As part of this effort, special stereoscopic glasses were used to enhance high-quality graphics and video images by enabling full, untethered, 3D stereoscopic visualization. The eyewear enabled the viewer to experience true-color depth effects by presenting separate perspective images to each eye. The eyewear turned a 3D image normally displayed on a 2D monitor into a virtual window environment. The model and glasses were supported by a Silicon Graphics Crimson Elan workstation. It was demonstrated that this capability could be used to design and verify the operation of future remote handling equipment.

A technique for creating photorealistic 3D images for new control console/control room designs was also developed. The technique was applied to a control panel for a fuel handling console. In addition, a remote monitoring system that allows engineers to visualize approximately 100 fuel-handling signals in real-time on a $2 \mathrm{D} / 3 \mathrm{D}$ virtual reality display was developed.

A 3D model for the future structure of the Sodium Process Facility was constructed from blueprints. The model was a photo-realistic visualization of the planned facility that could be integrated with a human model for work-flow analysis.

Investigation into VR applications with the target of providing data visualization, visual documentation of hot cell equipment, training, and other engineering functions was also performed. A $360^{\circ}$. panoramic photography technique, using images from a standard $35 \mathrm{~mm}$ camera, that enabled VR-like experiences of real-world spaces and objects was used. Realistic spatial interactions could be experienced and viewed since the virtual reality was created from photographs. Scenes were also created based on images generated on a computer. As users change views of a scene (e.g., turning left or right, looking up or down, or zooming in or out) correct.viewing perspective is maintained, giving the illusion of looking around. Similarly, objects can be picked up, manipulated, and viewed from all angles while remaining in perspective. Pixel-accurate hot spots allow the user to click on a designated area of the scene and launch various types of media as designed by the developer.

Finally, a scoping study to determine the various levels of VR capability from PC-based to full immersion was completed and some industry and government agency contacts were made to evaluate outside interest in VR prototyping using ANL VR capability.

Another project identified for future development involves a system to capture video and radiation data from a radiologically contaminated object and create a data visualization technique which incorporates a virtual reality environment. Data from a radiation sensor would be used 
to alter the hue of the images so that areas of high radiation would show as red, low radiation blue, with different colors to show the gradient between them. The process would be automated through the use of scriptable off-the-shelf software. The result would be a manipulatable object with color mapping which signifies areas of various contamination levels.

Specific Accomplishments: Internal report in preparation identifying VR capabilities and potential engineering concept and prototype development applications. Plans for internal seminars on this subject are underway. 
blank page 


\title{
95-269N -- COMPUTER AIDED DRUG DESIGN USING VIRTUAL REALITY
}

\author{
Associate Laboratory Director Area: Energy and Environmental Science and \\ Technology \\ Principal Investigator: \\ P. Bash, Center for Mechanistic Biology and \\ Biotechnology
}

Funding Profile:

FY $1993-0-$

FY $1994-0-$

FY $1995 \$ 99.7 \mathrm{~K}$

FY $1996 \$ 100.0 \mathrm{~K}$

FY 1997 -0-

Purpose: Scientific visualization techniques and advances in computer technology have led to the development of real-time three-dimensional interactive molecular graphics methods. These techniques are routinely used to obtain insights into the properties of biological macromolecules and their interactions with ligands. However, the modeling capabilities of conventional molecular graphics are limited by a reliance on visual information from an outside-looking-in graphics display and the chemical intuition of a user. These shortcomings can be overcome by using methods that provide an immersive sensory experience of a molecular system with quantitative information about atomic interactions that are based on physical and chemical principles. A long-standing goal of molecular science has been to have the capability to visualize, interact with, and obtain quantitative information about the properties of chemical systems. We have accomplished this goal through a combination which includes molecular simulations run on high-performance parallel computers, virtual reality methods, and high speed data communications networks.

The specific accomplishments of the program to date are:

The development of a Virtual Biomolecular Environment (VIBE) consisting of a combination of our parallel computer implemented molecular dynamics simulation methods with advanced visualization techniques from Virtual Reality interconnected via high-speed networks (Information Superhighway) for use in the design of novel pharmaceutical compounds.

The development of VIBE for drug discovery establishes a unique capability which is available only at Argonne National Laboratory. It is now possible to use this new method to gain insights into the fundamental properties associated with the functions of biomolecules, which are beyond the capabilities of traditional experimental methods. The resultant practical application of this new knowledge will have a significant impact on the rational design of novel enzymes and pharmaceutical products.

Approach: Virtual reality tightly coupled to high performance computing and communications ushers in a new era for the study of molecular recognition and the rational design of pharmaceutical compounds. We have created a Virtual Biomolecular Environment (VIBE), which consists of (1) massively parallel computing to simulate the physical and chemical properties of a molecular system, (2) the Cave Automatic Virtual Environment (CAVE) for 
immersive display and interaction with the molecular system, and (3) a high-speed network interface to exchange data between the simulation and the CAVE (see attached manuscript). VIBE enables molecular scientists to have a visual, auditory, and haptic (tactile) experience with a chemical system, while simultaneously manipulating its physical properties by steering, in realtime, a simulation executed on a supercomputer.

The creation of VIBE was facilitated by the unique combination of the IBM SP, Virtual Reality, and computer network facilities that exist at Argonne National Laboratory. We have made maximal utilization of these facilities to develop a capability that exists only at ANL. The current system is a rudimentary one, and work is in progress to generate a fully functional environment that is usable in the pharmaceutical industry.

Technical Progress and Results: At Supercomputing ' 95 in December, we will demonstrate the incorporation of VIBE into an integrated system of supercomputer centers connected via the Internet, which is referred to as the Information Superhighway. Literally thousands of computers of all types will be available to demonstrate the use of Teraflop computing for scientific computations. We will show how drug discovery can be facilitated by such an environment.

In a collaboration between researchers at Iowa State University, Cornell University, IBM Watson Research Center, Biosym Technologies, and Argonne National Laboratory, we are developing the following capability:

1. Run VIBE remotely over a wide area network between ANL (IBM SP) and a CAVE in San Diego (location of Supercomputing '95). We will also incorporate more Virtual Reality features such as a data glove and tactile feedback for a more realistic interaction with a molecular environment.

2. Simultaneously execute a data base search and refinement, i.e. docking, program. This is a typical first step in the drug discovery process utilizing structure-based methods. These methods systematically search a small molecule data base $(\sim 100,000$ compounds $)$, and they are an ideal application for a loosely coupled array of computers. We will demonstrate one of the first actual utilization's of Teraflop computing on a relevant problem.

The current CAVE setup consists of a 10' $\times 10^{\prime} \times 10^{\prime}$ room with projector screens for walls, and high-performance computer graphics processors (one per wall) required to generate images for projection onto the screens. This is a cumbersome and expensive arrangement, which is not suitable for routine molecular modeling $(\sim \$ 1,000,000)$. Our long-range goal is to develop a smaller and more functional version of immersive VR technology, which is optimized for the specific problem area of molecular modeling. The hardware for such a system is available and consists of a desktop single projection and computer graphic system (ImersaDesk), which is in the $\$ 150,000$ range. Significant software development is required for either "CAVE" system to become functional for drug design molecular modeling. Much of our research effort will focus on the necessary software development. This will be done in close collaboration with Carolina Cruz-Neira at Iowa State University. She is the primary developer of the CAVE. 


\section{Specific Accomplishments:}

\section{Publications:}

C. Cruz-Neira and P. A. Bash, "VIBE: A Virtual Biomolecular Environment for Interactive Molecular Modeling," Computers In Chemistry (in press).

Invited Presentations:

Workshop on Methods in Computational Biology, University of Kansas, October 1994 Cambridge Healthtech Institute, "Computational Approaches in Drug Design," March 1994

Supercomputing '95, April 1995

Biosym Technologies, June 1995

IBM Watson Research Center, June 1995

Data Management for Drug Discovery, San Diego, September 1995

Contributed Presentations:

Siggraph '94, August 1994

American Chemical Society National Meeling, August 1994

Biophysical Society Meeting, February 1995 
blank page 


\title{
95-167N -- OPTIMIZATION METHODS FOR MACROMOLECULAR CONFORMATION ON HIGH-PERFORMANCE ARCHITECTURES ${ }^{1}$
}

\author{
Associate Laboratory Director Area: Physical Research \\ Principal Investigators: \\ J. Moré and $\mathrm{Z}$. Wu, \\ Mathematics and Computer Science Division \\ Funding Profile: \\ FY $1993-0-$ \\ FY $1994-0-$ \\ FY $1995 \$ 68.4 \mathrm{~K}$ \\ FY $1996 \$ 70.0 \mathrm{~K}$ \\ FY $1997-0$ -
}

Purpose: The purpose of this project is to develop a parallel version of the continuation-based global optimization technique and to explore its use in solving macromolecular conformation problems. Success in this project will significantly expand the modeling abilities of the macromolecular simulation system XPLOR. Moreover, the project will serve as a mechanism for introducing modern optimization techniques for global optimization into the scientific community, with potential applications in biology, chemistry, and physics.

Approach: A fundamental research issue in modern biophysical chemistry is the determination of the three-dimensional structures of macromolecules as proteins. Researchers have developed various global optimization approaches to the determination of structures, but these approaches have had various limitations, including lack of intuitive appeal, reliance on older optimizatin techniques, and the inability to exploit the power of high-performance computers. The continuation approach that we have developed overcomes these limitations. In this approach, the original function is gradually transformed into a smoother function with fewer local minimizers. An optimization algorithm is then applied to the transformed function, which maintains the overall structure of the function but removes the small and narrow minimizers. Thus, an optimization procedure can skip less interesting local minimizers and concentrate on regions with average low function values, where a global minimizer is most likely to be located.

This project focuses on development of a parallel version of the continuation-based global optimization approach. Only by using massively parallel architectures can we solve the complex global minimization problems involved. Three specific areas. are under investigation: (1) software to transform the original function, focusing in particular on nonbonded potentials; (2) a Monte Carlo procedure to generate the initial starting points; and (3) optimization software, based on the trust region method, to trace solution trajectories.

Technical Progress and Results: Recent advances in the area of optimization methods for macromolecular modeling include the following:

${ }^{1}$ Individual Investigator Project 
- We clarified and strengthened the mathematical theory as a special continuation method for global minimization. In particular, we provided the theoretical support for the transformation method as a robust approach to global minimization. We also provided the mathematical basis for the development of efficient global continuation algorithms for macromolecular conformation.

- We developed and implemented a group of global continuation algorithms on workstations and the IBM SP. One implementation, for the well-studied Lennard-Jones model problem has proved especially effective: the code finds correct solutions where standard methods such as simulated annealing have failed.

- We extended the computational domain of the techniques. Specifically, we applied the global continuation method to the distance geometry problem, and were able to determine the structure of the active fragment of the peptide E. coli STh enterotoxin.

- We investigated optimal configurations of ionic systems (in collaboration with researchers in Argonne's Physics Division).

- We extended our work to the molecular cluster problems.

\section{Specific Accomplishments:}

\section{Refereed Publications:}

J. J. Moré and Z. Wu, "Global continuation for distance geometry problems," SIAM J. Optimization, to be published (MCS preprint MCS-P505-0395).

J. J. Moré and Z. Wu, "Epsilon-optimal solutions to distance geometry problems via global continuation," in Global Minimization of Nonconvex Energy Functions: Molecular Conformation and Protein Folding, P.M. Pardalos, D. Shalloway, and G. Xue, eds., American Mathematical Society, pp. 151-168 (1995) (preprint MCS-P520-0595).

\section{Presentations:}

Z. Wu, "A new continuation approach to global optimization," DIMACS Workshop on Gobal Optimization, DIMACS, Rutgers University at Busch Campus, Piscataway, N.J., March 1995.

J. Moré, "Smoothing techniques for global optimization," Advanced Course on Nonlinear Optimization and Applications, Erice, Italy, June 1995.

J. Moré, "Global continuation for macromolecular modeling on high performance architectures," Conference on High-Performance Software for Nonlinear Optimization: Status and Perspectives, Capri, Itàly, June 1995.

Z. Wu, "Global ootimization methods for macromolecular modeling on high-performance architectures," Workshop on Large Scale Optimization and Applications, Institute for Mathematics and Its Applications, University of Minnesota, Minneapolis, July 1995. 


\title{
95-241N -- IMPROVED FUEL INJECTOR SPRAY MODELING FOR INTERNAL COMBUSTION SIMULATION ${ }^{1}$
}

\author{
Associate Laboratory Director Area: Engineering Research \\ Principal Investigator: \\ C.C. Chu, Reactor Engineering Division \\ Funding Profile: \\ FY $1993-0-$ \\ FY $1994-0-$ \\ FY $1995 \$ 74.2 \mathrm{~K}$ \\ FY $1996-0-$ \\ FY $1997-0-$
}

Purpose: The automotive industry is engaged in intensive research to improve the performance of internal combustion engines to meet increasingly stringent environmental emission standards without unacceptable compromise of performance and efficiency. A major part of this effort involves the computational modeling of internal combustion engine designs. However, significant discrepancies between state-of-the-art three-dimensional computer codes and actual engine behavior continue to exist. Some of these discrepancies are attributed to the overly simplified and inaccurate modeling of the liquid fuel sprays discharged from fuel injectors. The purpose of this project is to achieve an advancement in the state-of-the-art of internal combustion engine modeling and a reduction in emissions into the atmosphere through the development of improved modeling of fuel injector sprays. A computational model has been developed to determine the detailed size and momentum distributions of the liquid droplets created by pressure-driven fuel injectors as well as penetration of the liquid core. The availability of this model will help to better position Argonne to attract future funding from the automotive industry or seek a broader role in future partnerships with the industry.

Approach: Recent years have seen the growth of extensive interest in detailed modeling of the combustion related processes involved in gasoline and diesel internal combustion engines. This has been largely motivated by a desire on the part of the automotive industry to meet increasingly stringent emissions standards without unacceptable compromise of performance and efficiency. The automotive industry uses complex, three-dimensional computational fluid dynamics codes to simulate combustion in cylinder/head geometries. The local combustion behavior depends upon the size and momentum distributions of the liquid droplets injected by the fuel injectors as well as the subsequent motion, evaporation, and burning of the droplets. Experimental characterization of the injected droplet sprays is difficult because of the mist produced that makes it difficult to visualize the liquid breakup and droplet formation. Numerical simulations have therefore had to employ simple approximations to the droplet size and momentum distributions over a specified spray angle. A reliable methodology to calculate the injected spray characteristics did not exit when the present project was initiated.

State-of-the-art modeling for the breakup of liquid streams discharged into another fluid medium and the formation/breakup of liquid droplets had been previously developed by the principal

${ }^{1}$ Individual Investigator Project 
investigator for application to nuclear reactor safety problems. This modeling is incorporated in the ANL THIRMAL-1 melt stream-water interaction code that treats liquid stream/droplet breakup caused by the growth of various fluid instability mechanisms. The existing expertise in this area thus provided a basis for development of an improved model for the atomization of the liquid fuel discharged from a fuel injector.

The project was initiated by conducting a review of the status of technology relevant to atomization of liquid fuel discharged from pressure-driven fuel injectors and subsequent interactions of the atomized liquid with a gaseous atmosphere. This review included discussions with researchers at the University of Wisconsin at Madison and Wayne State University who are also interested in fuel atomization. It was concluded that previously existing fuel injector spray models did not correctly describe the liquid flow pattern near the nozzle exit, did not include all of the physical breakup mechanisms, and did not treat the formation and breakup of liquid ligaments.

A new spray model that addressed these deficiencies was developed at Argonne. The Argonne spray model is both unique and the most comprehensive one available in that it integrates together all of the relevant physical mechanisms for breakup of the liquid stream exiting a circular nozzle. These mechanisms include the growth of small wave (Kelvin-Helmholtz) instabilities on the liquid core surface, the growth of large wave (varicose or sinuous) instabilities of the core, and breakup induced by turbulence in the liquid. Turbulence-induced breakup had heretofore not been incorporated in any spray model. In calculating the liquid core breakup, the effects of variation in the gas velocity with distance from the core (i.e., the gas velocity profile) have been included. The Argonne model also describes the secondary breakup of the droplets resulting from breakup of the liquid core. Secondary breakup mechanisms include bag breakup as well as stripping and penetration; the operative mechanism depends upon the relative velocity between the droplet and gas, the droplet size, as well as the droplet and gas properties. The model equations were implemented in a FORTRAN 77 computer program that with additional work could be implemented in a multidimensional computational fluid dynamics code.

The Argonne spray model was compared with a limited amount of experiment data from the literature selected on the basis of relevance to the new modeling concepts. Good agreement was obtained with data on the length of the liquid core versus relative gas-liquid velocity, the decay of turbulence kinetic energy in the core with distance, and the mean droplet diameter from turbulence induced breakup versus distance from the nozzle exit. These favorable comparisons provide confidence in the application of the model to internal combustion problems.

Technical Progress and Results: Existing research on liquid jet breakup and droplet spray formation relevant to fuel spray injection was reviewed and areas of deficiency identified. A new model for the transient breakup of liquid by fuel injectors having a circular nozzle shape to predict the droplet size and momentum spectra as well as liquid penetration was successfully developed. The Argonne spray model was implemented as a computer code and compared with relevant experiment data. The model is ready for implementation into a larger, computational fluid dynamics internal combustion code such as the DOE-sponsored KIVA-III and CHAD codes or comparable industry codes: 
Specific Accomplishments: A package of viewgraphs describing the status of technology review, the new Argonne spray model, and results of data comparisons was prepared by C.C. Chu and collaborator, J.J. Sienicki. A presentation was made to the automotive industry at the DOE Workshop on Internal Combustion and Fuel Preparation held at Princeton University, March 30-31, 1995.

A detailed paper on the Argonne spray model and data comparisons is being prepared by C.C. Chu, J.J. Sienicki, and B.W. Spencer for submission to the Journal of Atomization and Spray, the leading refereed journal devoted to research on sprays. 
blank page 


\section{5-279N -- EVALUATION OF THE ANL ICRKFLO CODE FOR SIMULATION OF A COMMERCIAL-SCALE FLUID CATALYTIC CRACKER (FCC) SYSTEM}

\author{
Associate Laboratory Director Area:
}

Principal Investigators:

Funding Profile:
Energy and Environmental Science and Technology

\author{
M. Petrick and S.L. Chang \\ Energy Systems Division
}

$\begin{array}{lll}\text { FY } 1993 & -0- \\ \text { FY } 1994 & -0- \\ \text { FY } 1995 & \$ 42.1 \mathrm{~K} \\ \text { FY } 1996 & -0- \\ \text { FY } 1997 & -0-\end{array}$

Purpose: The primary objective of the program is to demonstrate that the ANL computational fluid dynamic computer code ICRKFLO is capable of predicting major flow characteristics within and product yields from a commercial-scale FCC system. Specific objectives of the study are to (a) scope the capabilities and execution efficiency of ICRKFLO, (b) compare predicted results with the observed commercial data, and (c) demonstrate that the ICRKFLO Code can be used to identify critical process parameters (design and operating) that can be modified to adjust the yields of the FCC. The study thus will provide companies such as Exxon Research and Engineering Co. with first-hand knowledge of the capabilities of the ICRKFLO Code and a basis for evaluating the benefits of possible future collaborations with ANL in FCC modeling. ANL, in turn, will gain the opportunity to develop an initial validation of the Code with commercial-scale data and to expand its interactions with and technology transfer to the refining industry. Exxon has agreed to allow access to the relevant data.

Approach: FCC systems play a critical role as the key conversion process in the modern refinery industry. In a modern FCC unit, every effort is made to optimize product yields for each type of feed processed. A review of FCC process improvements and discussions with leading refining companies have underscored the fact that in order to achieve further improvements in FCC performance, an integrated computational fluid dynamics (CFD) kinetics code is needed that can be used as a design/performance/ evaluation tool. This study was therefore structured to explore such a capability and to demonstrate it to those interested in evaluating our capabilities in the area.

The study comprised the following steps: (1) ANL and Exxon identify and agree on an Exxon commercial-scale FCC unit that will be modeled in the study, for which the requisite data is available to test the validity of the ICRKFLO Code; (2) ANL utilizes its ICRKFLO Code to develop a simulation of the riser section of the FCC unit selected; (3) ANL develops an integral reaction submodel for the cracking process utilizing data provided by Exxon, namely, a four-lump kinetic model; (4) Generation of empirical kinetic constants for the integral reaction (cracking) submodel from the baseline test results; (5) Integration of the reaction submodel into ICRKFLO to produce a computer simulation for predicting FCC riser performance characteristics and product yields; (6) Conduct of a limited parametric study and comparison of 
predicted data with measured data; and (7) Evaluate the comparative analyses of data to establish validity of ICRKFLO simulations.

Technical Progress and Results: For FY 1995, the cracking processes including chemical kinetics and hydrodynamics in a commercial-scale FCC system was investigated.

We selected a commercial-scale FCC unit for this study as shown in Fig. 1. The FCC unit is a long tube with an elbow at about $2 / 3$ of the reactor length. The elbow divides the unit into two sections: one slant section (section a, as indicated in Fig. 1) and one vertical section (section b). Catalyst is fed from the bottom and feed oil is injected from both sides of the of the unit. Two feed oil injection locations used in the tests. Exxon provided ANL the operating information of the test unit, the cracking kinetic mechanism, and test results.

The ICRKFLO code was set up for the selected FCC unit. The integral reaction submodel of the ICRKFLO code was modified for the cracking process of the FCC unit. The FCC unit has a neck-down area before oil injection, an elbow, and two sections. New triangular block-cell technique and sectional coupling approach were developed to handle the complex geometry. The FCC unit has a very large capacity. The high flow rates of the unit can cause computer simulation a severe numerical instability problem. Boundary flow condition adjustment and pressure damping technique were implemented to overcome the numerical problem.

A baseline case (feed oil injection, location 1) was selected from Exxon's tests. From the baseline test results, a set of empirical kinetic constants was obtained for the integral reaction submodel. With the new reaction submodel the ICRKFLO code was used to calculate the flow property distributions in both sections of the FCC unit. Figure 2 shows calculated baseline flow properties in section a, including (a) gas velocity, (b) gas temperature, (c) oil droplet number density, and (d) feed oil concentration. The results indicate: (1) the gas is accelerated by the vaporization of the feed oil, (2) the temperature field is highly non-uniform, (3) feed oil droplets penetrate to the center of the reactor and droplets vaporize in a finite distance, (4) most feed oil vapor is converted to products in this section, and (5) gravity force at a slant angle on the flow causes asymmetric property distributions. Figure 3 shows calculated baseline flow properties in section b, including (a) gas velocity, (b) particle droplet number density, (c) light oil vapor concentration, and (d) dry gas concentration. The results indicate: (1) asymmetric flow field is predicted in the elbow section, (2) asymmetric flow causes asymmetric catalyst particle loading, and (3) the light oil portion of the product is over-cracked and converted to dry gas.

With the same set of empirical kinetic constants the ICRKFLO code was used to predict exit product yields for a different test case (feed oil injection, location 2). The predicted product yields agree with test results very well.

A limited parametric study was performed. The parametric results showed a strong effect of oil jet penetration on the cracking products and particle loading distribution. The most desirable product yields can be obtained by adjusting the feed oil injection conditions.

Specific Accomplishments: A continuing dialogue has been established with staff from Exxon Research and Engineering Co. that is exploring options for future collaboration in this area. Also, based on the positive results derived from this study, several papers are being written and 
submitted to refereed journals and for professional society conference presentations. This effort is continuing with non-federal support. 


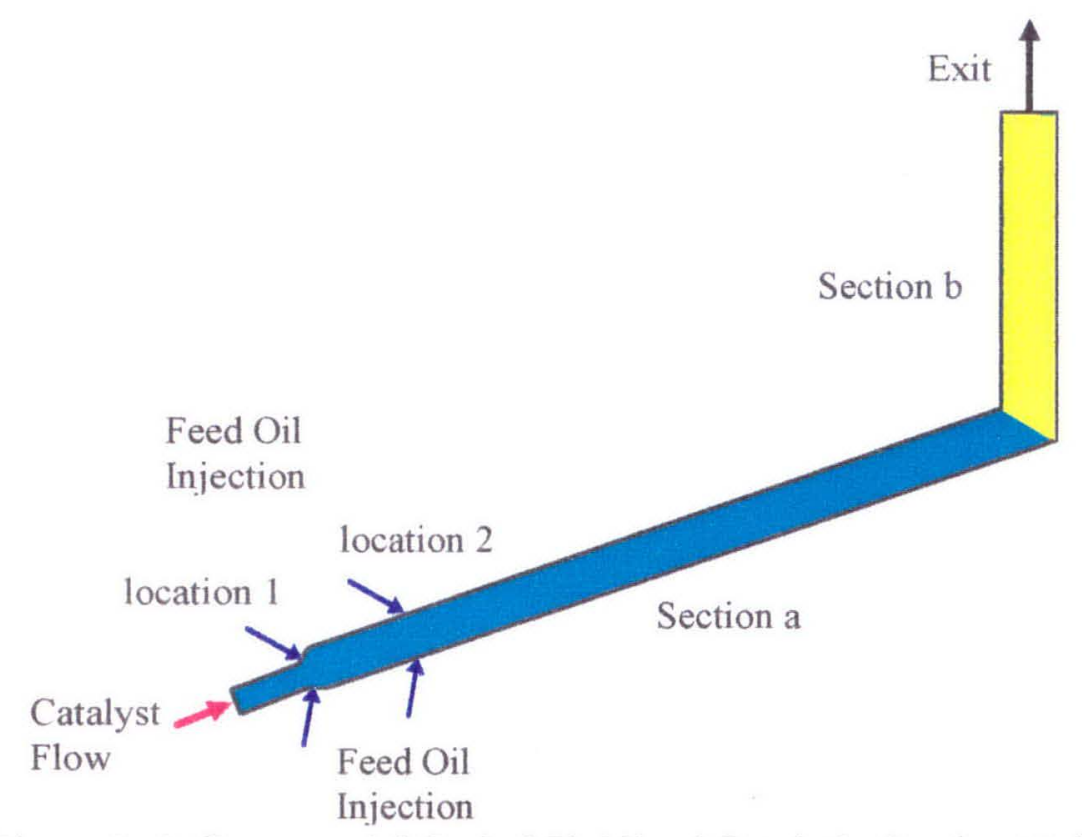

Figure 1 A Commercial-Scaled Fluidized Catalytic Cracker Unit
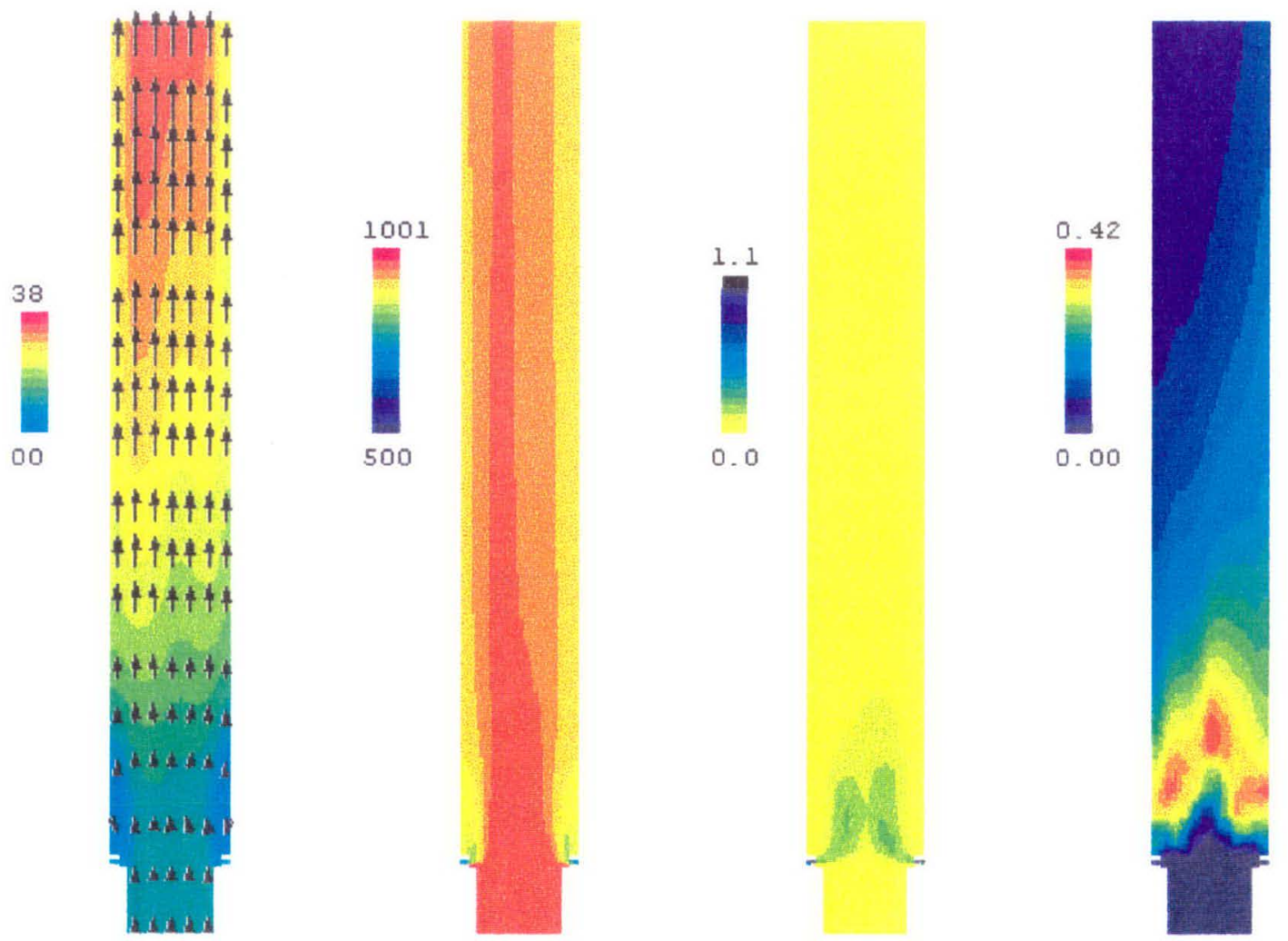
(a) gas velocity
(b) gas temperature
(c) droplet number density
(d) feed oil vapor concentration

Figure 2 Calculated Flow Property Fields in Section a of the FCC Unit 


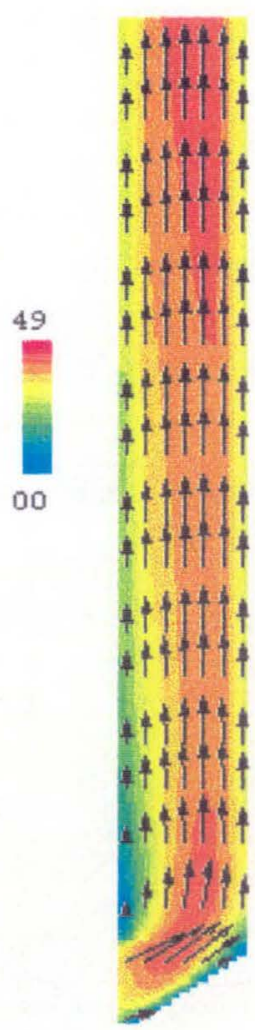

(a) gas velocity

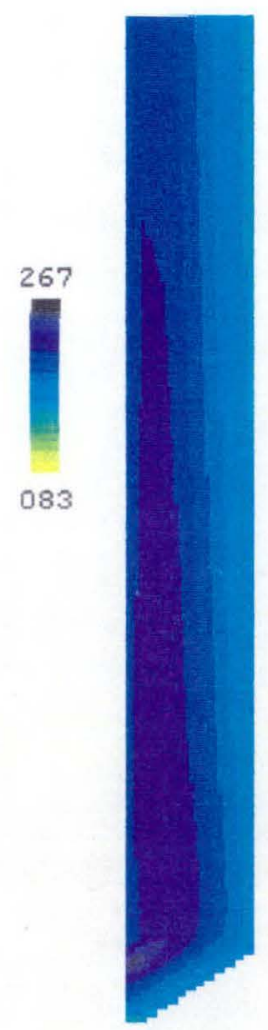

(b) catalyst particle
loading

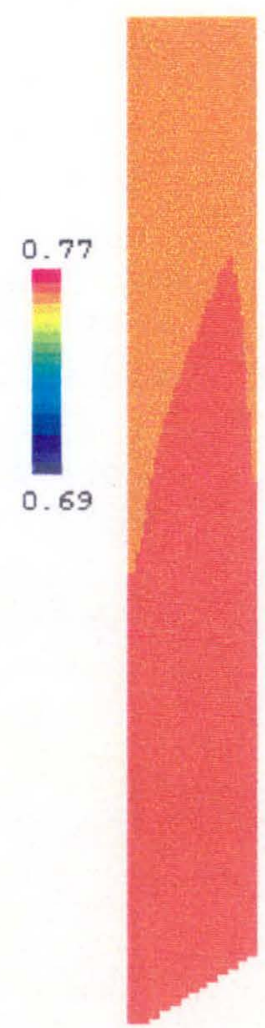

(c) light oil vapor concentration

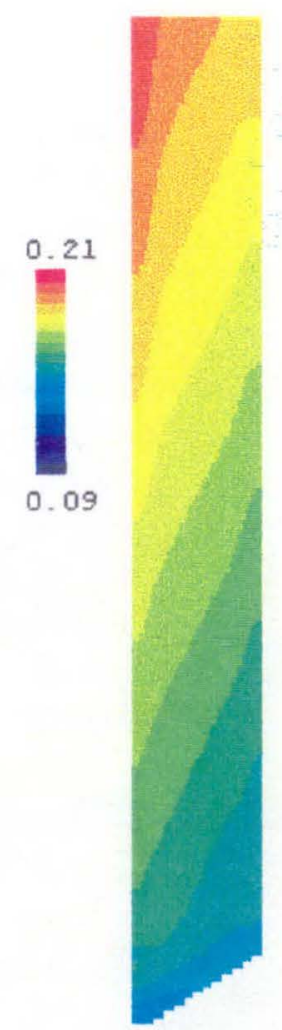

(d) dry gas concentration

Figure 3 Calculated Flow Property Fields in Section b of the FCC Unit 
blank page 


\title{
92-180R3 -- MOLECULAR BIOLOGY AND BIOTECHNOLOGY OF HYPERTHERMOPHILIC ORGANISMS: THE CHAPERONE RELEASE FACTOR ${ }^{1}$
}

\author{
Associate Laboratory Director Area: Energy and Environmental Science and \\ Technology \\ Principal Investigator: \\ Jonathan Trent, Center for Molecular Biology and \\ Biotechnology
}

Funding Profile:
FY $1993 \$ 337.7 \mathrm{~K}^{2}$

FY $1994 \$ 296.0 \mathrm{~K}$

FY $1995 \$ 92.4 \mathrm{~K}^{3}$

FY $1996-0-$

Purpose: We proposed to determine the primary structure of a second heat shock protein discovered in the hyperthermophilic archaeon Sulfolobus shibatae that forms part of the organism's molecular chaperonin complex. This protein was discovered during our previous LDRD research project in which we were searching for functional components of the chaperonin complex, in particular the putative release factor. The new protein, referred to as the a- subunit of combines with a second subunit (b) to form the double ring complex we now call a "rosettasome." Our motivations for wanting to clone and sequence the gene for a were 1 . To compare sequences with other chaperonins to gain additional insights into their phylogenetic relationships, 2. To express the cloned genes in $E$. coli for reconstitution studies and crystallography (collaboration with A. Joachimiak), and 3 . To use this knowledge to expand our understanding of the role of molecular chaperonins in the cell and by extrapolation the potential use of these molecules for biotechnolugy.

Approach: The principal activity in the project was to clone and sequence the gene coding for the $a$ protein and in addition, the gene could be used to study heat-regulated expression, archaeal promoters, and phylogenetic relationships. We planned to identify the gene in $S$. shibatae libraries, using a PCR-screening technique with primers designed around conserved regions of sequences. PCR products will be used as probes for screening genomic libraries and the cloned gene will be sequenced and compared to existing sequences in the data base. In addition, other archaea would be investigated for similar proteins by western blotting.

The cloned gene would be expressed in $E$ coli and these recombinant proteins used in vitro reconstitution experiments for the rosettasome. The structure of the reconstituted complexes will be analyzed by scanning transmission electron microscopy in collaboration. In addition,

\footnotetext{
${ }^{1}$ Project Title in FY92 and FY93: Hyperthermophilic Bacterial Biochemistry and Molecular Biology

${ }^{2}$ Project start date July 1992; FY92 cost $\$ 47.5 \mathrm{~K}$

${ }^{3}$ Project completion date June 1995
} 
over-expressed recombinant proteins will provide material for crystallography, which may lead to high resolution structural analyses (collaboration with Dr. A. Joachimiak at ANL).

Technical Progress and Results: As proposed, we succeeded in cloning, sequencing, and expressing the targeted gene. The protein $(a)$ turned out to be one of the most abundant proteins in Sulfolobus shibatae and stoichiometric with the other chaperonin subunit TF55 (renamed b). The gene and flanking regions of $a$. were cloned and sequenced. The transcription start sites for both genes were mapped and three potential transcription regulatory elements were identified based on sequence homologies in their upstream regions. The inferred amino acid sequences indicated the two proteins have $54.4 \%$ identity and $74.4 \%$ similarity overall. Their expression, indicated by the abundance of their respective mRNAs and the amount of $\mathrm{L}-\left[{ }^{35} \mathrm{~S}\right]$ methionine incorporated into their proteins, was significantly higher at heat shock $\left(85-90^{\circ} \mathrm{C}\right)$ than at normal growth temperatures $\left(70-75^{\circ} \mathrm{C}\right)$. The synthesis and abundance of $a$ was approximately equal to $b$ at all temperatures. They are the most abundant proteins in $S$. shibatae and under extreme heat shock conditions $\left(90^{\circ} \mathrm{C}\right)$, they were the only proteins synthesized.

In vitro, mixtures of purified $a$ and $b$ subunits reconstituted high molecular mass complexes having mobilities on native polyacrylamide gels similar to those of the complexes isolated directly from cells. These results, and symmetry considerations, suggest that the archaeal double ring complex, which we refer to as the "rosettasome," consists of one ring of $a$ and one of $b$.

Polyclonal antibodies against $a$ and $b$ from $S$. shibatae cross-reacted with proteins of similar molecular mass in 16 out of 20 archaeal species tested, indicating the two proteins are highly conserved among the archaea. Phylogenetic analyses revealed the close relationship between the archaeal rosettasomes and the eukaryotic TCP1 chaperonins, and the more distant relationship between these proteins and the bacterial chaperonins.

Specific Accomplishments: The results were published in the Journal of Molecular Biology (Vol. 253: 712-725; appendix 1). More importantly our work with the chaperonin complex led to a series of observations that have clearly indicated an in vivo function that deviates significantly from the established ideas about the role of chaperonins in the cell. Through electron microscopy studies with N. Zaluzec and functional studies with S. Potter at University of British Columbia and P. Londei at University of Rome, Italy, we realized that the ring structure forms polymers and these polymers appear to be the functional analog of the cytoskeleton in eukaryotic cells. We are now pursuing this revolutionary result with a continuing effort supported by DOE-ER. 


\section{2-108R2 -- STRUCTURAL BIOLOGY OF THE HUMAN GENOME: CRYSTAL STRUCTURES OF PROTEIN-NUCLEIC ACID COMPLEXES}

\author{
Associate Laboratory Director Area:
}

Principal Investigator:

Funding Profile:
Energy and Environmental Science and

Technology

A. Joachimiak, Center for Mechanistic Biology and Biotechnology

FY $1993^{1}-0-$

FY $1994 \$ 290.4 \mathrm{~K}$

FY $1995 \$ 188.6 \mathrm{~K}$

FY $1996^{2} \$ 110.0 \mathrm{~K}$

FY $1997-0-$

Purpose: The main goal of this project is to study the structure of biologically important protein-nucleic acid complexes (DNA and RNA) in humans, other eukaryotes, and bactcria, in order to better understand protein/nucleic acid interaction. The program is intended to develop research projects that will take advantage of the $\mathrm{x}$-ray synchrotron radiation beam lines that the Structural Biology Center (SBC) has at the National Synchrotron Light Source (NSLS) and currently constructs at the Advanced Photon Source (APS). This program is also highly relevant to the DOE/OHER Human Genome Initiative.

Approach: The primary experimental method we use is $\mathrm{x}$-ray crystallography using synchrotron sources. We grow x-ray quality crystals of specific protein-nucleic acid complexes for crystallographic analysis. These crystals are used to determine the structures of each proteinnucleic acid complex. Based on the crystal structure, important mutations in proteins and nucleic acids are selected and tested by solution studies. We subsequently verify our observations and hypotheses by cocrystallization of the altered proteins with their targets.

The primary thrust of this proposed project is to study the interactions of proteins with nucleic acids at atomic resolution, thereby elucidating the mechanisms by which these proteins control and modify gene expression, protein biosynthesis, and enzymatic reactions involving DNA or RNA as a substrate. The major methods to carry out these studies are those of molecular biology and protein crystallography. However, the unique capability of the SBC's brilliant synchrotron x-rays are essential for most of the projects, because these complex macromolecules usually do not form large, well-ordered crystals.

\section{Technical Progress and Results:}

DNA repair enzymes - E. coli 8-oxoguanine DNA glycosylase (FPG protein). Crystallization experiments with a free protein and with complexes of FPG with target DNA containing an

\footnotetext{
${ }^{1}$ Project start date January 1992; FY92 cost $\$ 242.1 \mathrm{~K}$

${ }^{2}$ Project completion date February 1996
} 
abasic site or an 8-oxodG analog, 8-oxonebularine, that is recognized by FPG protein but is cleaved very slowly so far did not yield crystals suitable for high resolution $\mathrm{x}$-ray analysis. However, we have discovered that this enzyme (or its folding intermediate) forms very stable complexes with hsp60 chaperonin - GroEL from E. coli and Taq hsp60 from Thermus aquaticus. Our experiments show for the first time direct interaction of DNA repair machinery with protein folding machinery. Both systems respond to stress conditions. Binding of FPG protein to chaperonins is temperature dependent. FPG/GroEL complexes can be separated on native gels. We determined apparent affinity of FPG protein to chaperonin and continue crystallization experiments with FPG/GroEL and FPG/Taq hsp60.

Transcription repressors - Trp repressor of $E$. coli. Crystals of the tandem . Trp repressor/operator complex have been characterized and a complete data set has been collected at the NSLS synchrotron beam line at $100 \mathrm{~K}$. Crystals were obtained with Trp repressor dimer and $27 \mathrm{~N}$ self complementary oligonucleotide. Crystals belong to tetragonal space group $\mathrm{P}_{1} 2_{1} 2$ $\left(\mathrm{P} 4{ }_{3} 22\right), \mathrm{a}=\mathrm{b}=70.5 \AA, \mathrm{c}=100.3 \AA, \alpha=\beta=\gamma=90^{\circ}$ and diffract $\mathrm{x}$-rays to high resolution $(>1.8 \AA$ ). These crystals contain one repressor dimer and 8 bp operators in the asymmetric unit. We have also attempted crystallization of a double mutant DNA operator with repressor that addresses directly the issue of water-mediated interactions observed for this complex. Binding experiments have verified the role of the trp repressor $\mathrm{N}$-terminal arm in DNA binding. We have shown that the $\mathrm{N}$-terminal arms of the trp repressor do not affect specific operator binding. We have found that these arms are dispensable and have no effect on the repressor interaction with $\alpha$ - and $\beta$-centered operator targets.

Restriction enzymes - Eco RII endonuclease from $E$. coli. The restriction endonuclease $E c o$ RII binds with high affinity the duplex DNA $5^{\prime}->\mathrm{CC}(\mathrm{A} / \mathrm{T}) \mathrm{GG} 3^{\prime}$ and specifically cleaves it at the $5^{\prime}$ end of the site. This endonuclease is a $46 \mathrm{kD}$ polypeptide that forms dimers in solution and specifically recognizes DNA sequences. The DNA cleavage reaction is relatively slow $\left(t_{1 / 2}=\right.$ 200 min.) providing the opportunity to study the mechanism of enzyme action using $x$-ray crystallography with synchrotron radiation. It has been shown that EcoRII requires an auxiliary site for activation, and the formation of four-strand "supersite

was suggested. Eco RII shows a sequence homology with integrases (mutation of Tyr 308 to Phe abolishes DNA cleavage but not recognition). Crystallization experiments with this enzyme yielded two crystal forms of the free enzyme. These crystals diffracted to 6 and $5 \AA$ respectively. Cocrystallization experiments with purified synthetic oligonucleotides containing binding site (in the absence of divalent ions) have so far produced microcrystals.

To understand the enzymatic mechanism and the interaction of EcoRII with two recognition sites, we have studied binding and cleavage of DNA duplexes containing canonical or modified EcoRII sites by EcoRII. We found intriguing peculiarity of the recognition and cleavage by restriction nuclease EcoRII its specific DNA target.

The reactions were analyzed by polyacrylamide gel electrophoresis under non-denaturing conditions. We have shown, that ( $\mathrm{T}->5$-fluorodeoxyuridine) or (A-> $\mathrm{N}^{6}$-methyldeoxyadenosine) substitutions in the central base pair did not affect DNA recognition, but they have a dramatic influence on cleavage. At the same time modification or substitution of $C$ or $G$ nucleotides in the recognition sequence affects both recognition and rate of cleavage of DNA. 
These data suggests that specific interaction with central base pair (A/T) contributes to catalytic activity of the enzyme.

DNA binding proteins from Sulfolobus shibatae - DNA chaperones - The archaeon $S$. shibatae thrives at temperatures up to $88^{\circ} \mathrm{C}$ and has a $\mathrm{T}+\mathrm{A}$ rich operon. A DNA duplex would melt at these temperatures. It appears, that $S$. shibatae and other archaebacteria use small basic proteins to protect DNA duplex integrity. We have isolated small proteins (two isoforms $14 \mathrm{kDa}$ and $14.5 \mathrm{kDa}$ ) from this organism. In collaboration with Dr. M. Peak we have characterized the DNA protecting properties of these proteins. From our studies it appears that these small basic proteins would bind to a duplex DNA and protect it from melting at temperatures up to $95^{\circ} \mathrm{C}$. We have attempted crystallization of free protein and obtained crystals.

Human IMP dehygrogenase - a key enzyme in the synthesis of purine bases. Human IMPDH has been purified in large scale from overproducing strains in collaboration with Drs. F. Collart and E. Huberman. Crystals of human IMPDH have been obtained that diffract $\mathrm{x}$-rays to $6 \AA$. Crystals have been characterized using synchrotron $\mathrm{x}$-ray sources and partial data set has been collected to $6 \AA$ resolution.

Protein-RNA recognition - RNA inhibitors of basic fibroblast growth factor and $\alpha$-thrombin. Crystals of RNA fragments with basic fibroblast growth factor (bFGF) were to small to analyze their diffraction using current source (perhaps with SBC beam lines at APS it will be possible). We have also attempted cocrystallization of RNA fragments that bind to $\alpha$-thrombin - a protease. These RNA fragments were designed using molecular evolution and chemically synthesized.

In collaboration with Prof. A. Diamond from the University of Chicago we have attempted cloning of several tRNA molecules from thermophilic fungus Humicola lanuginosa. Several tRNA genes were discovered with specificity to glutamine, glicyne and valine and quite unusual secondary structure.

\section{Specific Accomplishments:}

1. We have obtained specific complex of 8-oxoguanine DNA glycosylase with $E$. coli GroEL and $T$. aquaticus Taq hsp60 and characterized its binding affinities.

2. Crystals of the tandem trp repressor-operator complex that diffracts to high resolution $(>1.8 \AA)$ have been characterized and complete data set has been collected using synchrotron $\mathrm{x}$-ray source.

3. We have characterized two crystal forms the EcoRII endonuclease. Base sequence requirements for DNA target has been determined.

4. Crystals of human IMP dehygrogenase - a key enzyme in the synthesis of purine bases have been obtained that diffract to $6 \AA$. Crystals have been characterized using synchrotron $\mathrm{x}$-ray sources. 
5. We sequenced several tRNA genes from thermophilic fungus Humicola lanuginosa. These tRNAs show quite unusual secondary structures.

6. We have obtained crystals of "DNA chaperones" from S. shibatae.

7. Work will continue under the auspices of DOE-ER.

Publications:

Z. Shakked, G. Guzikevich-Guerstein, F. Frolow, D. Rabinovich, A. Joachimiak, and P.B. Sigler, Sequence-specific gene regulatory interactions: the role payed by the structure and hydration of the DNA target, Structural Biology: The State of Art, Eds. R.H. Sarma and M.H.Sarma, Adenine Press, 199-216 (1994).

Z. Shakked, G. Guzikevich-Guerstein, F. Frolow, D. Rabinovich, A. Joachimiak, and P.B. Sigler, Determinants of repressor/operator recognition from the structure of the trp operator binding site, Nature, 368, 469-473 (1994).

H. Van Kley, E. Quaite-Randall, J. Trent, and A. Joachimiak, Characterization of a low molecular weight proteinase from the hyperthermophile Sulfolobus shibatae, The FASEB Journal, 9, 1259 (1995).

E. Karpova, E. Kubareva, O. Petrauskene, A. Joachimiak, Peculiarity of the recognition and cleavage by restriction endonuclease EcoRII, The FASEB Journal, 9, 834 (1995).

A. Joachimiak, J. Knowlton and E. Quaite-Randall, Formation of stable complexes between proteins and the chaperonin from Thermus aquaticus., The FASEB Journal, 9, 52 (1995).

Publications in preparation 1996:

A. Joachimiak, Armless Trp repressor binds to single and tandem operators with this same affinity.

E.A. Karpova, E.A. Kubareva, Z.A. Shabarova and A. Joachimiak, Binding of the EcoRII endonuclease to synthetic DNA duplexes in the presence or in the absence of magnesium ions.

\section{Patents:}

Invention report: ANL-IN-95-059, A. Joachimiak and E.Quaite-Randall, A binding assay to measure affinities of molecular chaperones to protein substrates. 


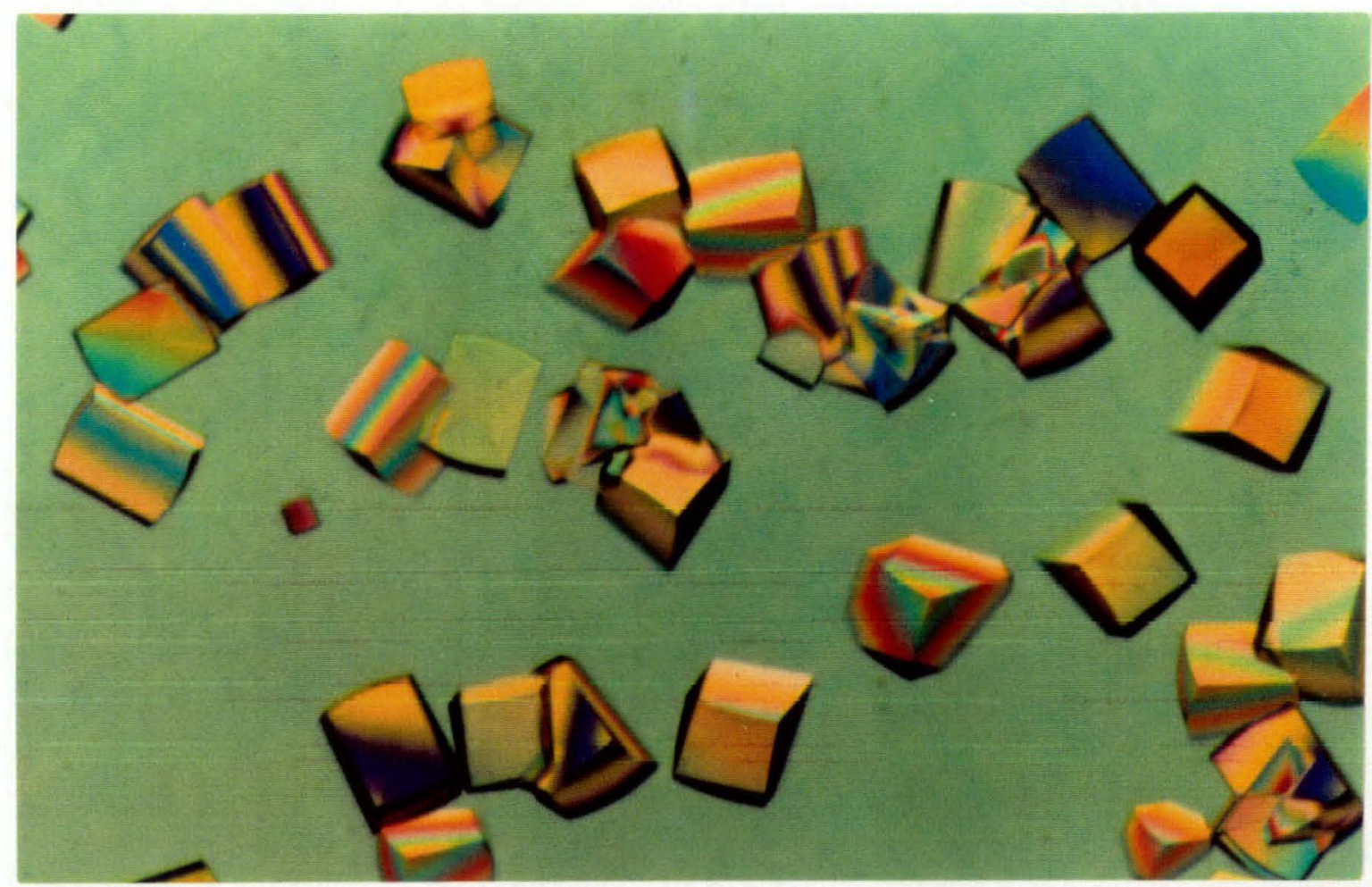

Fig. 1. Crystals of the tandem trp repressor-operator complex.

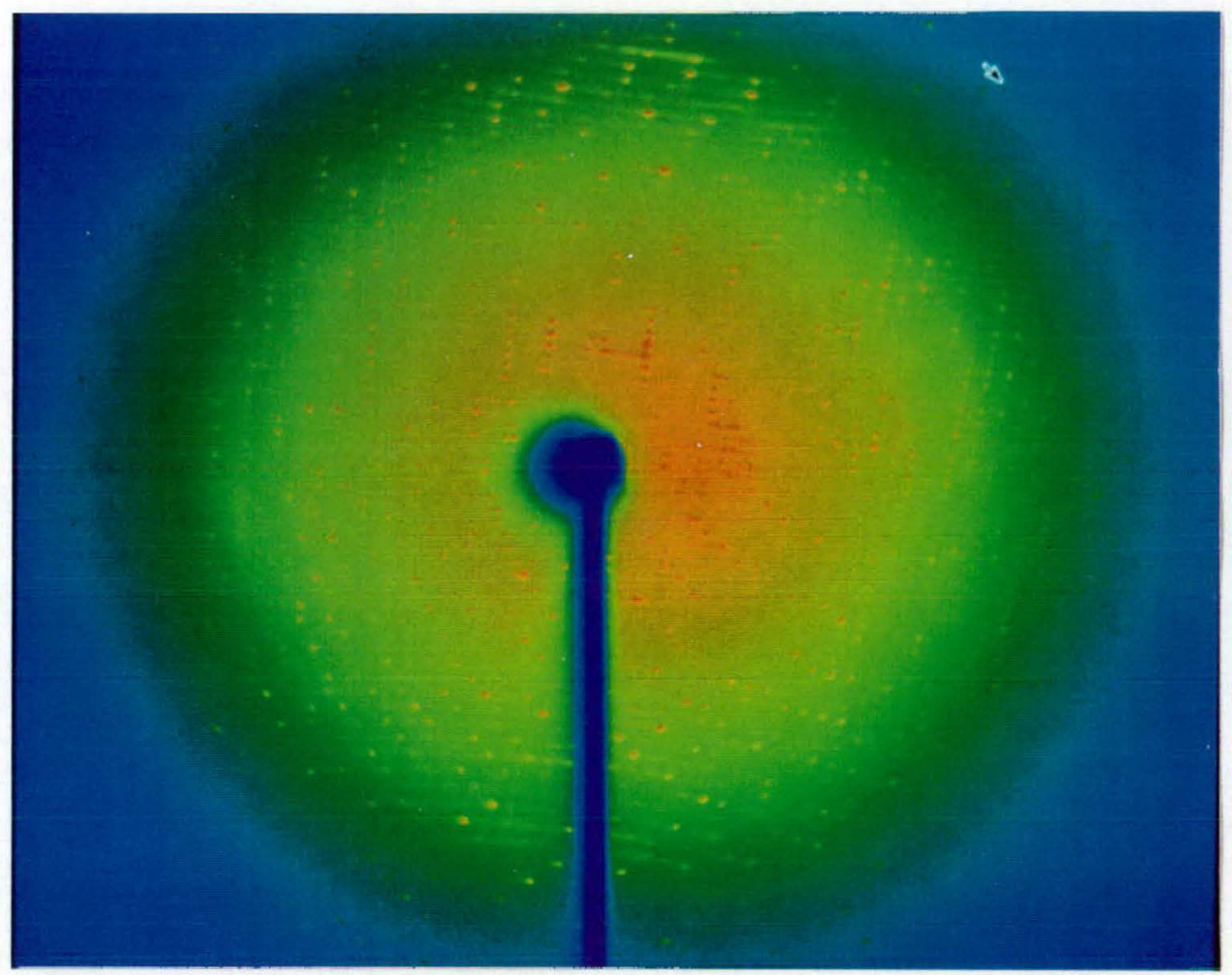

Fig. 2. X-ray diffraction from crystals of tandem repressor (1 degree oscillation photograph. 


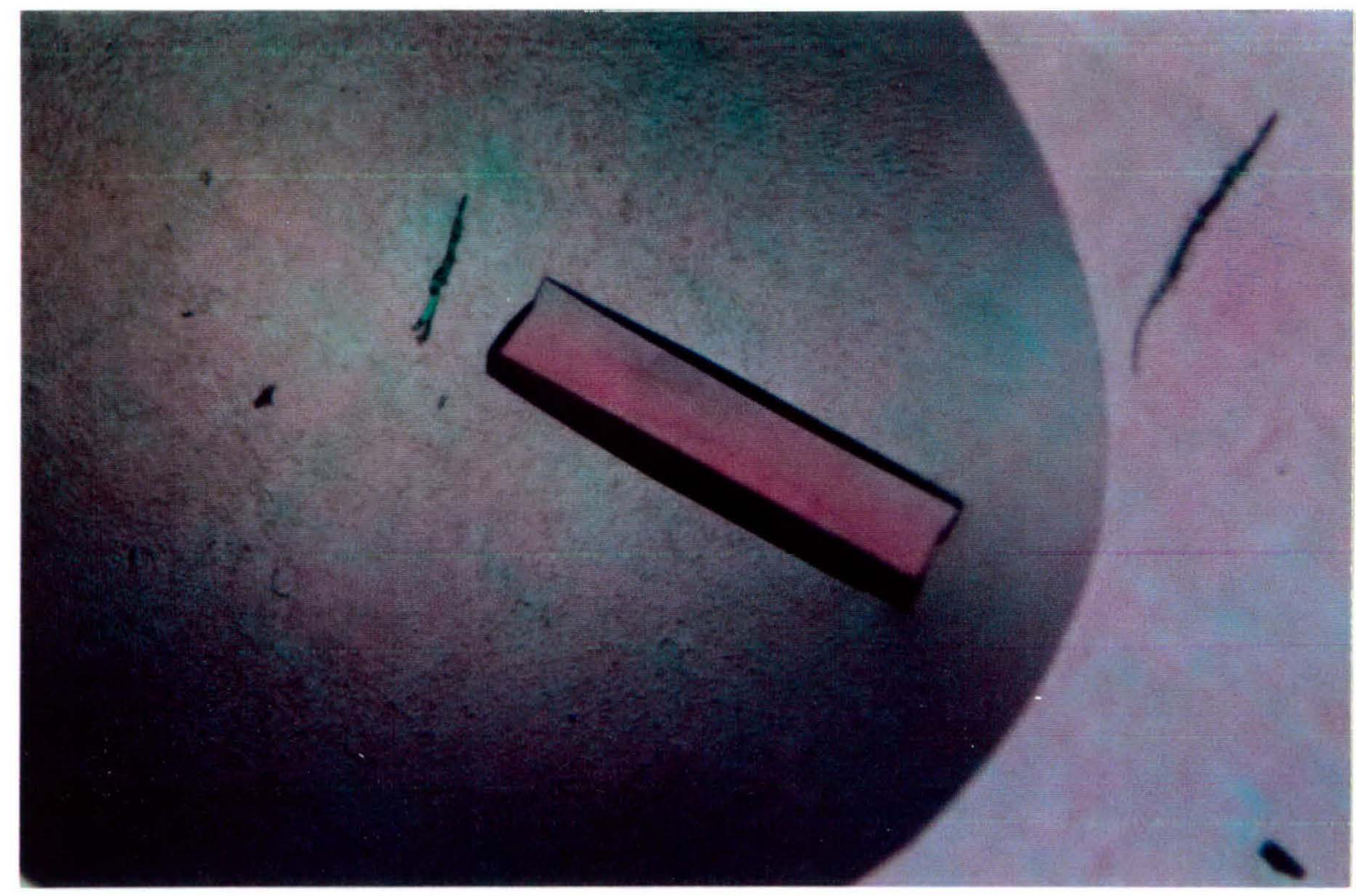

Fig. 3. Crystals of human IMP dehygrogenase

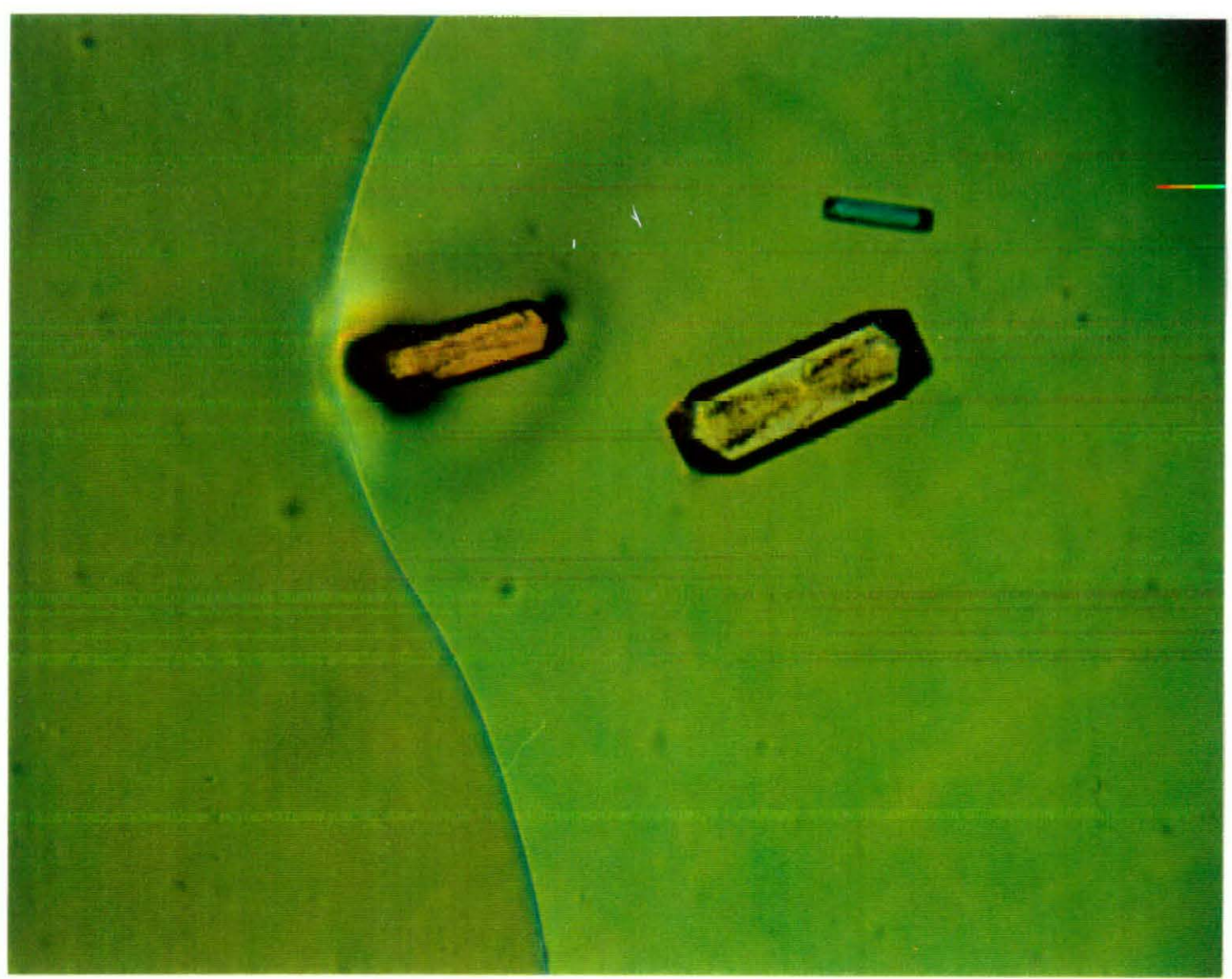

Fig. 4. Crystals of "DNA chaperones" from $S$. shibatae. 


\section{5-176N -- SOLUTION STRUCTURES OF GROEL AND GROES COMPLEXES WITH PROTEINS ${ }^{1}$}

\author{
Associate Laboratory Director Area: \\ Principal Investigators:
}

Physical Research

P. Thiyagarajan, Intense Pulsed

Neutron Source

A. Joachimiak, Center for Mechanistic

Biology and Biotechnology

\section{Funding Profile:}

\author{
FY $1993-0-$ \\ FY $1994-0-$ \\ FY $1995 \$ 75.6 \mathrm{~K}$ \\ FY $1996-0-$ \\ FY $1997-0-$
}

Purpose: The focus of this project was to delineate the solution structures of an important class of proteins, chaperonins and their complexes with other proteins by using the small angle neutron scattering (SANS). Chaperonins are essential proteins that assist in protein folding in the cell and are important in maintaining thermotolerance. These protect proteins and enzymes in the living organisms against thermal aggregation and increase their thermostability. In order to understand the function of these proteins, intensive world-wide investigations have been underway during the past few years on the structure of a representative system, GroEL chaperonin from E.coli and GroEL/protein complexes. What is not known so far is their solution structures. Since the solution state characteristics are the closest to the real biological systems, the solution structures of these systems are more relevant to a better understanding of their function.

Approach: E.coli GroEL chaperonin is a complex consisting 14 subunits, each with a molecular weight of $57.4 \mathrm{kDa}$, while GroES cochaperonin is complex of 7 subunits, each with a molecular weight of $10 \mathrm{kDa}$. GroEL first binds with the denatured protein and the protein folding pathway involves the binding of GroES and ATP hydrolysis. The ATP-dependent protein folding and assembly, and anti-aggregation activity require recognition of the unfolded state of the proteins and the formation of a complex between the chaperonin and the protein in its intermediate folding state.

$\mathrm{X}$-ray crystallography and cryo-electron microscopy were used to obtain the structures of GroEL and GroEL/protein complexes. The $2.8-\AA$ crystal structure of GroEL has shown that the $57.4 \mathrm{kDa}$ subunits are assembled into two 7 -member rings that stack back to back. Each subunit is composed of three domains that are loosely connected by antiparallel strands. In the crystal structure, about $5 \%$ of the amino acid residues comprising $\mathrm{N}$ - and $\mathrm{C}$-terminal sequences have not been resolved. The seven-member ring has an outer diameter of $137 \AA$ and combines with a second ring to form a cylindrical particle with a length of $146 \AA$. Each ring has a cavity (volume $\sim 125 \mathrm{k}^{3}$ ) with a circular mouth $45 \AA$ in diameter. The presence of this cavity was

${ }^{1}$ Individual Investigator Project 
suggested by electron-micrograph reconstructions and was confirmed by $\mathrm{x}$-ray crystallography. The cavity was implicated in protein binding and folding. Calculation of the volumes of the cavity suggests that up to $72 \mathrm{kDa}$ proteins only can be accommodated inside each cavity, but how the unfolded proteins are directed into the cavity is not known.

The relationship between the protein structure in solution and that in crystal has been studied extensively . Due to the difficulties encountered with the crystallization of large multisubunit macromolecular assemblies, solving their structures by $\mathrm{x}$-ray crystallography is highly challenging. Furthermore, the conditions of crystallization and packing in the crystal may alter local three-dimensional structures, and questions are often raised about the functional validity of the crystal structure of macromolecules. Small angle neutron scattering is a powerful technique which can yield structural information on colloidal particles in solution. The energy of the radiation used for SANS applications is in the range of 5 to $80 \mathrm{meV}$ and thus radiation damage is not a concern.

SANS provides information on the size, morphology, and composition of macromolecular complexes and can detect conformational change and substrate binding. When the crystal structure coordinates of the individual components of macromolecular complexes are available, more reliable information on the solution structure of complexes can be delineated by using that information in the model fitting of the SAS data.

The SANS were measured at $15^{\circ} \mathrm{C}$ for GroEL for a number of concentrations of GroEL in $\mathrm{D}_{2} \mathrm{O}$ to determine the concentration range where the interparticle effects are minimal. Since the SANS measurements involve several hours of exposure, it is important to form a GroEL/protein complex which is stable over a period of several hours. Our SANS studies focused on GroEL/rhodanese complex as this has been demonstrated to be stable.

In the present investigation we used the amino acid sequence and the atomic coordinates from the GroEL crystal at $2.8 \AA$ resolution for comparing the GroEL structure in solution and that in the crystal. The modelling of the SANS data was done by using the Monte Carlo method developed by S.J. Henderson, Biology division, Oak Ridge National Laboratory, Oak Ridge, Tennessee. For studying the GroEL/rhodanese complex, we used the solution structure of the GroEL delineated from this study and modelled the SANS data for the complex in order to extract the stoichiometry and the location of the bound protein in GroEL.

The Monte Carlo method used for the modelling of the measured SANS data employs the crystallographic coordinates for the location in space and the scattering power of the elements of GroEL. The solvent-excluded volumes of the amino acid residues are calculated from the van der Waals radii of the atoms and their bond lengths. Random points in space are chosen around the center of the mass of each amino acid, where the number of such points for each amino acid is proportional to the product of the amino acid volume and its scattering length density difference with respect to the solvent. Calculation of all pairwise distances between such points, and binning these as a function of distance generates the pair distribution function $P(r)$ as a function of $r$, and this function is then readily transformed to $I(q)$ vs $q$. The instrumental smearing function for the SANS instrument (due to neutron wavelength spread, beam size and detector pixel size) is then applied to the calculated $\mathrm{I}(\mathrm{q})$ to produce the smeared model intensities and then compared with the measured SANS intensities per molecule. 
Technical Progress and Results: A powerful software has been developed for the modelling of the SANS data by using the crystal structure data and Monte Carlo methods. This program can quantitatively model the SANS data with appropriate scattering cross-sections for the proteins and the solvent. Specific alterations in the structure of the complex can be easily introduced and this helps to study the conformational changes in the GroEL in solution as well as upon binding with a denatured protein.

The present study reveals that the solution structure of GroEL from SANS is similar, but not identical to that in the crystal. The complex resembles a cylinder with two cavities separated by a plug in the middle. The differences between the structure of GroEL in the crystal and that in solution, brought out by the present study, add information to the present understanding of the structure and function of GroEL. For example, the crystal structure data at $2.8 \AA$ resolution could not resolve the location of about $5 \%$ of the total amino acid residues. This study determined the location and packing density of these missing amino acid residues. These seem to be condensed near the equator of the GroEL cylinder. This finding explains why $\mathrm{N}$-terminus is important for the oligomeric state of chaperonin. The C-terminal residues may play only a structural role in blocking the transfer of unfolded proteins between the cavities of GroEL. The present study also shows that the apical domains in the solution structure are projected outward, slightly, more than that in the crystal. The flexibility of the apical domains and the larger opening of the mouth seen in the solution structure may enable GroEL to provide the necessary surface for the binding of the denatured proteins of different sizes. In addition the present study shows the existence of equilibria between the 14-subunit complexes, 7-subunit complexes and monomers. In the case of GroEL/rhodanese complex, this study clearly shows that only one rhodanese is bound to each GroEL complex and the binding site is across the opening of the cavity of the GroEL cylinder, but not in the cavity as proposed in the literature.

The intriguing question, that carries important mechanistic implications, is the location of the binding site in the chaperonin for the unfolded protein. If the unfolded protein is bound inside the chaperonin cavity then the folding process could proceed in an "infinite dilution environment" with chaperonin providing the interactive cavity surface for many topologically different proteins. However, if the protein is bound on the chaperonin surface, then the role of the solvent and other cofactors also have to be considered in determining the mechanism of chaperone-mediated protein folding. Binding of the folding intermediate across the opening of the cavity, as seen in the present study, has important implications on the functional role of chaperonins. It has been shown that GroEL is quite plastic, and therefore, the surface associated with the apical domains can be quite dynamic. This property may be critical for protein folding. With the folding intermediate bound on the chaperonin surface, assembly of oligomeric proteins can also be explained by the interaction of multiple chaperonin/protein complexes.

The present study thus makes an important contribution to the structure-function relationship of GroEL in protein folding and the thermostability. There are still a number of issues which need to be addressed. For example, what happens to the plug in the middle of the GroEL cylinder at elevated temperatures when the actual binding with other proteins occur? What happens to the solution structure of GroEL when it binds with proteins with different molecular weights? What is the location and the stoichiometry of the GroES co-chaperonin in the GroEL/GroES complex which is responsible for the refolding of the denatured protein? Do the GroES and the denatured protein bind on the same side of the GroEL cylinder or they bind on the opposite sides? The 
facilities, software capabilities and expertise presently available at IPNS and CMBB divisions of Argonne National Laboratory will enable us to be visible and well respected research group in this important field. LDRD funding will be sought in the forthcoming years for addressing some of these important issues.

\section{Specific Accomplishments:}

Publication of FY 1995 results - In Press:

P. Thiyagarajan, S.J. Henderson, and A. Joachimiak, "Solution Structures of GroEL and Its Complex with Rhodanese from Small Angle Neutron Scattering," Structure.

Invited Talk:

P. Thiyagarajan, S.J. Henderson, and A. Joachimiak, "SANS Studies of the Solution Structures of E.coli GroEL and its complex with a Protein Substrate," at the American Crystallographic Association meeting in the "Neutron Scattering Session" at Montreal, Canada, July 23-28, 1995. 


\section{5-164N -- RECONSTRUCTING METABOLIC FUNCTION FROM GENOME SEQUENCE DATA ${ }^{1}$}

Associate Laboratory Director Area:

Principal Investigator:

Funding Profile:
Physical Research

T. Gaasterland, Mathematics and Computer Science Division

FY $1993-0-$

FY $1994-0-$

FY $1995 \$ 70.4 \mathrm{~K}$

FY $1996^{2} \$ 75.0 \mathrm{~K}$

FY $1997-0-$

Purpose: The technical objective of this project is to develop an environment that can automatically reconstruct a metabolic structure for an organism from a partial "parts list" of the component proteins that has been provided by genome sequence data. The reconstructed structure is intended to provide essential feedback that both focuses the DNA sequence interpretation process and enables large-scale sequence interpretation to proceed with metabolic accuracy.

Approach: The project is critical because we are on the verge of receiving huge quantities of new genome sequence data for unicellular organisms (i.e., of the order of millions of nucleic acid bases in contiguous sequence fragments). The volume of data is far too great for skilled researchers to analyze by hand (e.g., for one-third of the Mycoplasma capricolum genome, the output of the BLAST sequence database search program exceeds 25 megabytes of human readable data). Methods must be developed to carry the analysis through to the functional reconstruction of metabolism automatically.

The project has the following components:

- Develop reasoning algorithms to feed metabolic data back into the sequence analysis system.

- Define a minimal model of metabolism that contains all the essential modules required for perpetuation of an organism.

- Use the minimal model to drive algorithms that generate a hypothetical metabolic framework for an organism from genome sequence data.

- Encode rules to identify metabolic neighborhood and neighboring proteins for a specific enzyme.

\footnotetext{
${ }^{1}$ Individual Investigator Project

${ }^{2}$ New title in FY 1996: Reconstructing Metabolic Topologies from Whole Genomes
} 
Developing algorithms for reconstruction requires (1) the ability to represent metabolic information in a form that can be used in a deductive reasoning process; (2) a comprehensive volume of metabolic data; and (3) a reasoning framework to drive the reconstruction. To address these requirements, we use EMP, a database on enzymes and metabolic pathways for which we have developed tools that parse EMP's information about connected reaction equations into a logical form that can be used to reason about metabolic pathways. We are also exploring the idea of metabolic neighborhoods, from which we can infer the presence of particular enzymes in the DNA sequence data, and the concept that certain metabolic modules must be present in some form in an organism. The DNA sequence data for Mycoplasma capricolum provides the basis for our genome sequence analysis system.

Collaborators in this work include Natalia Maltsev and E. Selkov, both in the MCS Division at Argonne.

Technical Progress and Results: We created an environment within which partial metabolic topologies from partial genome sequence data can be reconstructed. Particular accomplishments include the following:

- We developed algorithms that automatically reason about metabolic structures at a symbolic level.

- Using these algorithms, we carried out a manual reconstruction of the energy metabolism of Mycoplasma capricolum.

- We set up methods to analyze sets of connected reaction equations and choose potential points of regulation.

- We completed a theoretical reconstruction of the structure of Mycoplasma capricolum metabolism for all metabolic modules represented by the known sequence.

Specific Accomplishments: Research will continue under the auspices of DOE-ER.

\section{Refereed Publications:}

T. Gaasterland, N. Maltsev, R. Overbeek, and E. Selkov, "PUMA: An Integration of Biological Data to Support the Interpretation of Genomes, " Proc. 1995 Albany Conference on Phylogenetic and Structural Relationships among Proteins, September 28-October 1, 1995, extended abstract, p. 25.

T. Gaasterland and E. Selkov, "Automatic Reconstruction of Metabolic Networks Using Incomplete Information," in Proc. of the 3rd International Conference on Intelligent Systems for Molecular Biology, Cambridge, England, July 1995. 
Presentations:

T. Gaasterland, "Combining Expert Knowledge for DNA Sequence Analysis," colloquium talk at the Canadian Institute for Marine Biosciences, Halifax, Canada, April 1995.

T. Gaasterland, "Integrated Databases and Software Tools for Critiquing Protein Molecule Structures," colloquium talk at Brookhaven National Laboratory, January 1995. 


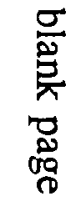




\section{5-183N -- STRUCTURE-FUNCTION CHARACTERIZATIONS OF PROTEIN REGULATORS OF PROGRAMMED CELL DEATH ${ }^{1}$}

\author{
Associate Laboratory Director Area:
}

Principal Investigators:

Funding Profile:

\author{
Energy and Environmental Science and \\ Technology
}

M. Schiffer and P. Wilkins Stevens

Center for Mechanistic Biology and

Biotechnology

FY $1993-0-$
FY $1994-0-$
FY $1995 \$ 75.2 \mathrm{~K}$
FY $1996-0-$
FY $1997-0-$

Purpose: The purpose of this research is to build the framework necessary for developing a programmatic effort in the newly defined field of cell-death regulation. Molecules which regulate cellular pathways of differentiation and cell death have only recently been identified. One family of cell-death regulator proteins, the $B C L-2$ family, contains a number of functionally related molecules which appear to constitute a new structural class of proteins. The long-term goal of our research is to define structure-function relationships for $B C L-2$-family proteins and to provide the detailed molecular structures required for understanding, modifying, and modulating the intracellular activities of these molecules using protein engineering and rational drug design techniques. There is great interest in this protein family from the standpoint of basic science since proper function of these proteins is required for normal cellular development and for maintenance of homeostasis within organisms. These studies are also potentially very important for the fields of medicine and healthcare, since disruption of the regulated process of cell death occurs in many cancers and viral infections. The development of this program at Argonne extends the Laboratory's current focus on structural biology and will allow the Laboratory to become established in the area of programmed cell death, a very important new field of biomedical research.

Approach: The first two proteins in the $B C L-2$ family were isolated in the mid-1980s, but about 10 additional distinct proteins from this family have been identified in the past three years. Virtually all have been shown to play some role in regulating programmed cell death. Most work on these molecules has been biological rather than structural, with its focus to define the roles or effects of these proteins when overexpressed in tissue culture systems. Our objective, however, is to synthesize and purify recombinant $B C L$-2-family proteins and to characterize these isolated proteins using the techniques of biophysical chemistry and structural biology. We will crystallize the purified proteins and begin structural studies using $\mathrm{x}$-ray crystallography. In addition, we will quantitatively analyze the self-dimerization and the hetero-dimerization of various $B C L$-2-family proteins, since dimerization of these molecules appears to be important in cellular regulatory networks which control programmed cell death. Molecular biology and

${ }^{1}$ Individual Investigator Project 
protein chemistry methods are used to synthesize, purify, and crystallize the recombinant proteins. We apply column chromatography and computer simulation methods developed here at Argonne to perform the dimerization analyses. Dr. Xiaoyin Cai, a postdoctoral fellow, has been a vital contributor to this project.

Technical Progress and Results: During FY 1995, we developed expression clones in two different vector systems which encoded full-length or partial $B C L-X$ sequences to produce either individual or fusion proteins. We have developed purification protocols for several of these proteins and have isolated $\sim 5 \mathrm{mg}$ recombinant $B C L-X_{L}$ per liter starting culture of $E$. coli. The single most important technical milestone achieved has been the identification of appropriate buffer conditions to generate small crystals of purified $B C L-X_{L}$ protein. In addition, we have demonstrated the feasibility of using column chromatography and computer simulation methods to analyze the dimerization of isolated $B C L$-2-family proteins. Using Individual Investigator LDRD funding for FY 1996, we will work to develop larger crystals of $B C L-X_{L}$ suitable for crystallographic analyses, and we will measure the dimerization constants for the self-association of $B C L-X_{L}$ and two other members of this protein family, $B C L-2$ and $B A X$.

Specific Accomplishments: In August 1995, we reported on this work to the DOE review team and prepared a poster detailing our results. We have received a second year of LDRD funding for FY 1996 and have also applied for external funding from NIH to continue our work on the biophysical and structural characterization of $B C L$-2-family proteins. Based on amino acid sequences of the dozen or so $B C L$-2-family proteins, we have developed a structural model for a consensus $B C L$-2-type protein structure. A manuscript explaining this model is in preparation for submission to a refereed journal such as Cell or EMBO Journal. In addition, we have recruited Drs. Craig Thompson and Dr. Larry Boise from the University of Chicago as collaborators on this project. 


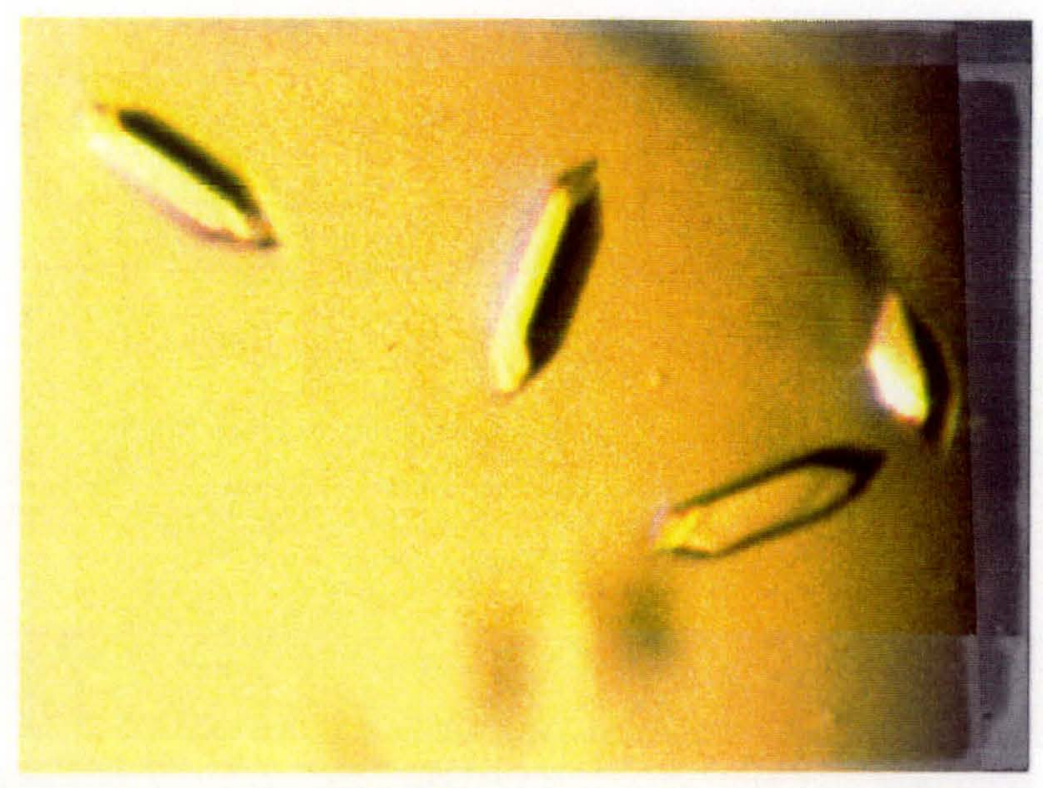

(photo of $B C L-X_{L}$ crystals)

\section{Crystallization of $B C L-X_{L}$ Protein in Ammonium Sulfate}

Purified $B C L-X_{L}$ protein was concentrated to $5 \mathrm{mg} / \mathrm{ml}$, and a $1-\mu \mathrm{l}$ sample was mixed with $1 \mu \mathrm{l}$ of buffer in a hanging drop on a coverslip sealed over a Lindbro plate well containing $0.8 \mathrm{ml}$ of the same buffer. The crystals below formed in the drop containing the buffer $2 \mathrm{M}$ ammonium sulfate, $0.1 \mathrm{M}$ cacodylate, $\mathrm{pH}$ 6.6. Similar crystals were observed in drops formed using three other buffers: $2 \mathrm{M}$ ammonium sulfate, $0.1 \mathrm{M}$ citrate, $\mathrm{pH} 5.7 ; 1.5 \mathrm{M}$ ammonium sulfate, 0.1 $\mathrm{M}$ cacodylate, $\mathrm{pH} 6.5$, and $1.5 \mathrm{M}$ ammonium sulfate, $0.1 \mathrm{M}$ Hepes, $\mathrm{pH}$ 7.7. Crystallization of this protein represents a significant milestone in this project for two reasons: 1) it indicates that our isolated $B C L-X_{L}$ protein is pure and properly folded, and 2) it provides conditions which can be used for preparation of larger crystals suitable for $\mathrm{x}$-ray crystallography. 
blank page 


\section{5-011N -- TIME DOMAIN MOLECULAR STRUCTURE DETERMINATION FOR METASTABLE REACTION INTERMEDIATES IN PHOTOCHEMICAL PROCESSES USING X-RAY ABSORPTION}

\author{
Associate Laboratory Director Area: \\ Principal Investigators:
}

Funding Profile:
Physical Research

\author{
L.X. Chen, J.R. Norris, ${ }^{1}$ D.M. Tiede, \\ and M.C. Thurnauer, Chemistry Division
}

Purpose: The goal of this research is to probe molecular structures of short-lived reaction intermediates created by light in photochemical processes using time-domain X-ray absorption spectroscopy (XAS). The details of such molecular structural information will enhance our understanding of chemical reaction mechanisms, establish structure/function relationships of the molecules, and provide information for developing new materials. The techniques developed in this research will be extended to the time domain studies with sub-nanosecond time resolution at the advanced photon source in the future.

Approach: The development of modern chemistry strongly relies on our knowledge of the molecular structures. For example, much of the progress in understanding natural photosynthesis has been made following the determination of the X-ray structures of the photosynthetic bacterial reaction centers. Currently, structural studies are extended not only into more and more complicated systems, but also into the time evolution of the molecular structures during chemical reactions. Most previous studies on the molecular structures as a function of time are based on indirect observation from changes of the optical and magnetic properties. The direct measurements of the molecular structures with X-ray techniques during a chemical reaction are lacking because of the limitation on X-ray photon flux during the lifetimes of the reaction intermediates. With the prospect of doing experiments at the APS in the near future, we intend to obtain the reaction intermediate (including the triplet state) structures using XAS on a second generation synchrotron source (e.g., NSLS at Brookhaven National Laboratory).

As the initial stage of this study, we measured two photoinduced metastable structures using cryogenic trapping that enables lifetimes of the metastable intermediates to increase significantly, allowing the steady-state X-ray absorption measurements. At this stage of the study, we illuminated samples for a certain period of time using a continuous xenon or mercury lamp. The structural changes were observed by comparing the spectra of the samples at cryogenic temperature before and after light illumination. These experiments on the long-lived metastable intermediates not only helped us to gain insight into the reactions, but also established technical requirements and references for future real time-domain measurements.

\footnotetext{
${ }^{1}$ Left the Laboratory during FY95.
} 
In the past year, we have started real time-resolved XAS experiments. Because the X-ray bunch intervals of the second generation synchrotron are too short to conduct the experiment with a time-resolution of the bunch duration, we intended to conduct the measurements at lower time resolution using the synchrotron radiation as a continuous source. The time resolution is obtained by using energy dispersive X-ray absorption spectroscopy (EDXAS). In contrast to the conventional XAS where the monochromator scans the whole energy range of the spectrum step by step, the EDXAS collects the whole absorption spectrum simultaneously. The "white light" $\mathrm{X}$-ray diffracted from a curved polychromator crystal was arranged in space according to the energy. This X-ray is focused at the sample and then fanned out afterwards. A linear array detector is placed after the sample, collecting the whole spectrum. The time-resolution is limited by the detector readout time (e.g., 5 milliseconds). A pulsed laser, instead of a continuous lamp, is used to illuminate the sample. The structural change of the sample is detected after each laser pulse with 5-millisecond time resolution.

The selection of samples for the EDXAS study is crucial for the success of the experiment for three reasons: (1) only a number of excited states and the reaction intermediates have lifetimes longer than the 5-millisecond limit of the detector readout time; (2) the EDXAS is limited to the transmission detection, where the concentration of the absorbing element must be rather high ( $>$ $50 \mathrm{mM}$ ); and (3) the sample should have the least absorption coefficient discrepancy between the X-ray and the excitation light (the former is always much smaller than the latter), or the sample geometry must compensate such a discrepancy.

We have been collaborating with the synchrotron group at the Material Science Division on developing the time-domain structure determination using EDXAS.

Technical Progress and Results: We have made technical progress from the following three aspects:

(1) Development of the EDXAS with laser excitation. We incorporated a Nd-YAG laser pumped optical parametric oscillator (OPO) system into beamline X6A at NSLS as a photoexcitation source. This laser offers a very broad tunability $(420-2000 \mathrm{~nm})$, high pulse energy (e.g. 50mJ/pulse @ $500 \mathrm{~nm}$ ), 5-6 nanosecond pulse duration (FWHM) at 10Hz repetition rate. The software that synchronizes the laser excitation and detection was written and tested. We conducted an initial experiment at the beamline X6A, NSLS on ZnTPP and ZnOEP (zinc tetraphenylporphine and zinc octaethylporphine). Although we have not obtained what we expected due to limited beamline time and other technical problems, the experiment has proven the technical feasibility and helped us to identify the needs for future experiments. We are scheduled to do another experiment in November, 1995.

(2) Steady-state XAS studies on the systems which are photochemically interesting and are potential candidates for the future time-domain XAS studies. We studied two molecular systems during FY 1995. The steady-state XAS studies was carried out on photoreduction of heavy metal ions (e.g., $\mathrm{Pb}$ and $\mathrm{Hg}$ ) on $\mathrm{TiO}_{2}$ colloid surfaces. A continuous reduction of lead ions $\left(\mathrm{Pb}^{2+}\right)$ by light and by $\mathrm{X}$-ray was observed. We also identified the spectral characteristics in $\mathrm{TiO}_{2}$ colloids as a function of the particle sizes with XAS. A manuscript regarding our results is in preparation. Moreover, we started XAS studies in artificial photosynthetic systems where photoinduced electron transfer reactions occur on metal centered porphyrins and chlorophylls. 
We finished XAS measurements on a covalently linked chlorophyll dimer with $\mathrm{Ni}, \mathrm{Cu}$, and $\mathrm{Zn}$, respectively. This dimer is a model compound for the initial electron transfer in natural photosynthesis. The metal-metal interaction and metal coordination with solvent and ligand were studied. Only $\mathrm{Zn}-\mathrm{Zn}$ interaction was detected for the dimer, and that interaction was interrupted by addition of pyridine ligand to the solution. This implies that the pyridine molecules are inserted between the two chlorophyll rings in the dimer. The coordination of the Ni chlorophyll dimer was observed as a function of the temperature where the ligand only binds to the molecule at lower temperature. A manuscript is in preparation, which will combine the XAS results with photophysical properties of these dimers.

(3) Three manuscripts from steady-state XAS studies on photoinduced metastable states were finished and published. In the study on the photoinduced metastable intermediate of CpNiNO (cyclopentadienyl-nickelnitrosyl), we detected a $0.2 \AA \mathrm{Ni}-\mathrm{N}$ bond elongation and $\mathrm{Ni}-\mathrm{N}-\mathrm{O}$ bending. This structure has given new insight into the photochemical reaction mechanism and prompted further studies with EDXAS. We also published our results on the discovery of a new structure in photoinduced spin state transition in Fe(II) complexes in ethanol solution. The study of chemically modified photosynthetic reaction centers was also published, where strong interactions between the protein and the chlorophylls were identified.

Specific Accomplishments: The following publications resulted from this LDRD funding.

\section{Refereed papers:}

1. L.X. Chen, Z. Wang, J.K. Burdett, P.A. Montano, and J.R. Norris, "X-Ray Absorption Studies on Electronic Spin State Transitions of Fe(ii) Complexes in Different Media," $J$. Phys. Chem. 99:7958-7964 (1995).

2. L.X. Chen, Z. Wang, G. Hartwich, I. Katheder, H. Scheer, A. Scherz, P.A. Montano, and J.R. Norris, "An X-Ray Absorption Study of Chemically Modified Bacterial Photosynthetic Reaction Centers," Chem. Phys. Lett. 234:437-444 (1995).

3. L.X. Chen, M.K. Bowman, Z. Wang, P.A. Montano, and J.R. Norris, "Structural Studies of Photoinduced Intramolecular Electron Transfer in Cyclopentadienylnickelnitrosyl", $J$. Phys. Chem. 98:9467-9474 (1994).

Manuscripts in preparation:

1. L.X. Chen, M.R. Wasielewski, W.A. Svec, and P.A. Montano, "An X-Ray Absorption Study of the Structures and Coordination States in Bis-Metalchlorophyll Cyclophane."

2. L.X. Chen, T. Rajh, Z. Wang, M.C. Thurnauer and D.M. Tiede, "The Structure of $\mathrm{TiO}_{2}$ Colloid Particles and Photoreduction of Heavy Metal Ions." 
Reports:

1. L.X. Chen, Z. Wang, T. Rajh, A. Ostafin, D.M. Tiede, M.C. Thurnauer, P.A. Montano, and J.R. Norris, "Structural Characterization of Quantized $\mathrm{TiO}_{2}$ Particles," NSLS, Brookhaven National Laboratory (1994).

2. L.X. Chen, Z. Wang, T. Rajh, A. Ostafin, D.M. Tiede, M.C. Thurnauer, P.A. Montano, and J.R. Norris, "Monitoring Lead Reduction on the Surface of Titanium Dioxide by X-Ray Abssorption,". NSLS, Brookhaven National Laboratory (1994).

3. L.X. Chen, M:R. Wasielewski, W.A. Svec, K. Huang, and P.A. Montano, "An X-Ray Absorption Study of the Structures and Coordination States in Bis-Metalchlorophyll Cyclophane," NSLS, Brookhaven National Laboratory (1995).

4. L.X. Chen, P.L. Lee, G. Jennings, S. Weber, J.R. Norris, and P.A. Montano, "Capture the Excited State. Structures in ZnTPP Using Laser Excitation and EDXAS,". NSLS, Brookhaven National Laboratory (1995):

Presentation:

L.X. Chen, M.R. Wasielewski, W.A. Svec, K. Huang, P.A. Montano, and J.R. Norris, "X-Ray Absorption Studies of Model Compounds for Photosynthesis --Bischlorophyll Cyclophane," 210th National ACS Meeting, Chicago, Illinois, August 20-25, 1995. 


\section{3-090R2 -- COMPUTATIONAL STRUCTURAL BIOLOGY: COMPUTER SIMULATION OF ENZYME REACTIONS}

\author{
Associate Laboratory Director Area:
}

Principal Investigator:

Funding Profile:
Energy and Environmental Science and

Technology

P. Bash, Center for Mechanistic Biology and Biotechnology

FY $1993 \$ 284.8 \mathrm{~K}$

FY $1994 \$ 270.8 \mathrm{~K}$

FY $1995 \$ 162.6 \mathrm{~K}$

FY $1996-0$

FY $1997-0-$

Purpose: The goal of the Computational Structural Biology Program (CSBP) is to use the advanced computer technologies at Argonne National Laboratory (ANL) to develop unique computational methods that can be used in a concerted fashion with structural biology data obtained from Advanced Photon Source (APS) experiments. This combination of innovative molecular modeling techniques with data obtained from revolutionary APS experiments, such as time-resolved protein crystallography, will lead to breakthroughs in our understanding of the fundamental functions of biological macromolecules at the atomic level of detail. The results from this research will have a significant impact on the solution to problems associated with biomolecular diseases as well as the design of novel enzymes, or biomimetics, that can be used for environmental remediation.

Approach: The objective of the field of Computational Enzymology is to create reliable methods, based on first-principles of physics and chemistry, and use these computational methods to simulate the chemical bond-making and -breaking events in the protein environment of an enzyme. The knowledge gained from such computer "experiments" will provide an understanding of the fundamental properties of enzymes, which are often difficult to determine with traditional experiments, that can be used to facilitate the rational design of new enzyme based catalysts for novel biomedical and industrial applications.

The major problem with the development of a Computational Enzymology is the accurate representation of the chemical transformations that occur in the context of an enzyme's unique protein solvent environment. . The most general approach is to use quantum mechanics (QM) which can, in principle, accurately depict any chemical reaction in an arbitrary environment. In practice, the application of $\mathrm{QM}$ to systems as large as an enzyme, which consists of thousands of atoms, has been problematic. This is due to the fact that QM methods are computationally very intensive when they are applied in their most complete formulations, which is necessary to calculate numerical values of physical and chemical properties of molecules to an accuracy commensurate to data obtained from traditional experiments. Molecular systems containing at most ten atoms can be treated by high-level QM theory to chemical accuracy.

To simulate the properties of enzymes, which contain thousands of atoms, at experimental accuracy required the development of innovative methods. Computational techniques were 
created that provide significant progress toward the solution of the problems associated with the accurate computer simulation of condensed phase chemical reactions. The methods make use of a unique combination of quantum and classical mechanical principles, which are implemented for use on massively parallel computers.

Technical Progress and Results: Most condensed phase reactions involve chemical transformations (i.e., changes in the electronic configuration due to making and braking of chemical bonds) that are localized to a very small region of the system. This fact was used to considerable advantage by proposing that a system can be partitioned into a small region (tens of atoms) treated with computationally expensive QM techniques and another region (thousands of atoms) that uses a "classical" representation, which is computationally efficient. The interaction between the two regions models solvation effects (classical region) on solutes (quantum region), where solvent effects are included in a rigorous fashion within the context of the QM method that is utilized. (Specifically, partial atomic charges on molecular mechanics $(\mathrm{MM})$ atoms interact with electrons and protons on QM atoms, and van der Waals interactions between QM and MM atoms are modeled with a classical potential to emulate electron repulsion and dispersion.) A model consisting of several thousand atoms can provide a realistic representation for most enzyme systems

The specific implementation of these concepts involves the use of a QM method with reasonable, albeit not inherent, chemical accuracy, and a well established classical molecular mechanics method, which has been developed to model biological macromolecules. The semiempirical QM method is the so-called AM1 (Austin Model 1), and the MM method is CHARMM (Chemistry at Harvard for Molecular Modeling). The primary reason for choosing the AM1 QM method was its computational efficiency. Problems related to the accuracy of this QM method are important and they have been solved by new techniques developed in the Computational Structural Biology laboratory, which are described below. The use of these QM and MM methods in their QM/MM scheme has enabled the research group to use the QM/MM concept to calculate statistical thermodynamic quantities which are directly comparable to experimental values for chemical reactions in aqueous solution and protein environments.

In a series of simulations involving proton and hydride reactions in water (J. Phys. Chem., in press), the free energies of transfer were calculated for a proton from methanol to imidazole and a hydride anion from methoxide to nicotinamide, which are model systems for the chemical reactions in the enzyme malate dehydrogenase. The resultant calculated values, 15.1 (proton) and -6.5 (hydride) $\mathrm{kcal} / \mathrm{mol}$, compared remarkably well with the experimental values of 12.8 and $-7.5 \mathrm{kcal} / \mathrm{mol}$. These results demonstrate the potential of the QM/MM method to accurately depict the properties of chemical reactions in complex condensed phase environments.

The simulated data was the result of 100 independent calculations, each of which required about two days of computer time. Such calculations carried out on a single processor supercomputer would have required about 200 days of computing, which would have been impractical. However, the actual computations were completed in about four days through the use of the 128 node IBM parallel computer at ANL. The parallel QM/MM method is therefore providing the means to carry out realistic simulations of complex chemical reaction phenomena. 
This QM/MM method was then applied to the enzyme reaction of malate dehydrogenase (MDH), which involves the same chemical functional groups studied in the above aqueous solution simulations. A first ever calculation of the entire energy surface of an enzyme was carried out. The results (Biochemistry, in press) provide detailed information about the atomic mechanism of the enzyme, which is not accessible through the application of traditional experimental methods. These computer simulations provided new fundamental knowledge about this particular enzyme system, which can now be used to guide rational redesign experiments to produce an enzyme with new functionality. The approach used on MDH is general and will now be used on a series of enzyme systems through collaborations with experimentalists both inside out outside of ANL.

These calculations involved the determination of the energy for 1000 different and independent configurations of the enzyme system, which defines the pathway for the proton and hydride transfers involved with the reaction mechanism. Here again, access to massively parallel computing made it possible to carry out this simulation study. Each configuration required about 12 hours of computer time to determine a value for its energy. Using the 128-processor IBM computer, the energy surface calculations were completed in about ten days versus an estimated 500 days for a more traditional supercomputer.

The basic atomic level data for the structure of MDH, which is an essential input for the enzyme reaction simulations, was determined by the protein crystallography group of Professor Leonard Banazsak at the University of Minnesota. Professor Banazsak is a member of the collaborative action team (CAT) who will be making use of the ANL protein crystallography beam line at the APS. Although the particular protein structural data used as the input for the above enzyme simulations was not determined from APS experiments (APS comes on line the summer of 1996), future collaborative work with the Banazsak and other groups will make use of the structural data determined directly from the APS. The computational methods that have now been developed in the Computational Structural Biology Program will be used to simulate the functions for and analyze the properties of biological macromolecules whose atomic structures will be determined on the APS. The enabling parallel computer technology, which provides the wherewithal to carry out complex computer simulations, exists at ANL in the Mathematics and Computer Science Division, and is routinely used in this program, which is in the Center for Mechanistic Biology and Biotechnology.

The MDH enzyme calculations utilized all of these advanced technology capabilities at ANL. As such, research in this area was singled out by the laboratory director as the prototypical example (see ANL annual report for 1994) of the unique synergy of technologies that are available at ANL for the study of structure/function relationships in biological macromolecules.

A very recent development in the laboratory provides the means to simulate almost any condensed phase system, which includes enzymes, to near experimental accuracy. The process outlined below constitutes a general procedure, which may be a major breakthrough in the field.

The steps of the process include: 
1. Identification of the essential functional groups directly involved in the chemical reaction to be studied. These groups will then be treated with $\mathrm{QM}$ and the remainder of the system with MM.

2. Optimization of adjustable parameters associated with the semiempirical QM method to reproduce physical properties of molecules in the gas phase. The semiempirical QM method used, in general, cannot calculate fundamental properties of molecular systems to chemical accuracy. However, since it is a semiempirical method, there are parameters associated with the atomic elements. (e.g., carbon, nitrogen, hydrogen, and oxygen), which can be adjusted on a case-by-case basis for each molecular system investigated. A procedure was implemented that uses a genetic algorithm to optimize these parameters such that resultant physical properties (e.g., heats of formation, dipole moments, proton affinities, and etc.) are reproduced to chemical accuracy. This procedure has been tested on several molecular systems with spectacular results obtained in all cases. The problem of inaccuracy associated with the computationally efficient semiempirical method is solved.

3. Optimization of the van der Waals parameters associated with specific solute (QM) atoms such that solute-solvent $Q M / M M$ interactions reproduce those determined with high level ab initio $Q M$ calculations. This parameterization process fits the interaction energy of a water molecule (MM) with solute ( $\mathrm{QM})$ to $a b$ initio $\mathrm{QM}$ values. The resultant parameters are then used for bulk phase calculations.

4. Statistical thermodynamic quantities (e.g., free energies of reaction) are calculated using the model developed in steps (1)-(3) on a massively parallel computer to generate the necessary conformations of a system required by well established statistical mechanics principles. This process has been applied to the proton and hydride reactions described above with resultant calculated energy values comparing to within $1 \mathrm{kcal} / \mathrm{mol}$ of experimental values.

The process described above is general and can be applied to any molecular system. A manuscript describing the details of this method has been accepted in the Proceedings of the National Academy of Sciences and transmitted by Kenneth Wiberg from Yale University.

The above methods and procedures are now fully developed and ready for production work on any enzyme system whose 3-D structure is known in atomic detail. The following projects are underway:

1. Malate Dehydrogenase - The work on this enzyme builds from the basic simulations carried out to determine the minimum energy pathway described above. The next step is to calculate the free energy profile and transition states for the enzyme reaction, which can be directly compared to experimental rates of reaction. This will involve a straightforward application of the methods developed, which will be accomplished through the use of the computing resources at ANL and other high-performance computing centers (large grants of computer time have been obtained from the Maui HPCC and the Cornell Theory Center, which have 512 node IBM SP computers). The resultant free energy transition states for the native enzyme and a site-specific mutant will be used as the basic structural input for 
simulations to calculate the relative transition state binding free energies of these molecular systems. These calculations can be compared directly to the enzyme redesign experiments of Mark Donnelly (ANL) and Len Banaszak (U. Minnesota), and they can be used as a quantitative guide for such experiments.

2. Isocitrate dehydrogenase (IDH) - Work on this enzyme system is being done in collaboration with Barry Stoddard at the Fred Hutchinson Cancer Research Center in Seattle. The purpose of the project is to obtain a complete atomic understanding of the mechanisms of this enzyme, which can then be used for enzyme engineering. An initial model of the enzyme-substrate complex has been determined using a combination of Laue $\mathrm{X}$-ray diffraction methods (synchrotron radiation and time-resolved crystallography) and computational molecular dynamics. This is the first time these two methods have been combined in a concerted fashion to determine the structure of a complex biomolecule that is in a dynamic state, and demonstrates the kind of experimental/computational synergism expected to occur when the APS is operational.

3. Alcohol dehydrogenase is being studied in collaboration with Professor Bryce Plapp at the University of Iowa. We will use the available structural and enzymologic data to guide computer simulations to understand the details of this reaction mechanism. The results of this study together with those in (1) and (2) will provide a comparison of the way nature has solved the problems associated with dehydrogenase mechanisms. All three enzymes use a different approach to catalyze proton and hydride transfer reactions.

4. Two projects are in the formative stages of development with professors at the University of Chicago. The first is with Marvin Makinen to study the dynamics of enzyme-substrate complexes as well as the details of the reaction mechanism for beta lactamase. Marvin has developed and uses spectroscopic methods to determine precise structural features of enzyme-substrate interactions. The simulations will complement his experiments. The second project is with Michael Weiss to study the dynamics of proteins in solution. Michael uses nuclear magnetic resonance (NMR) spectroscopy to determine the structures of protein in aqueous solution. Large-scale molecular dynamics calculations will be carried out and compared with his NMR data to understand the dynamical properties of biological macromolecules.

One of the major goals of the Computational Structural Biology group was to establish the wherewithal to interface with experimentalists who collect data at the APS. We now have the program in place to interface with any structural biologist who has atomic resolution data for a macromolecular system. The Computational Structural Biology program is now in production mode and has accomplished the goals set out in the LDRD program.

Specific Accomplishments: Research will continue under the auspices of DOE-ER. Specific accomplishments of the program to date are:

1. The development of a general Computational Enzymology methodology, which is based on a combination of physical principles from quantum and classical mechanics, for the computer simulation of any enzyme reaction mechanism, where the 3-D structure of the enzyme is known in atomic detail. 
2. The implementation of these methods for optimal use on massively parallel computers that exist at ANL and other high-performance computing sites around the world.

3. The first ever computer simulation of the complete reaction mechanism of an enzyme, which made use of our newly developed parallel quantum molecular dynamics methods on Argonne's massively parallel IBM SP computer system. The results from these computations provide essential information about the function of a biomolecular system, which was not possible to determine from traditional biochemical and biophysical experiments. The new insights gained will be used to guide protein redesign experiments.

\section{Publications:}

L. Ho, A. MacKerell, P.A. Bash, "Proton and Hydride Transfers in Solution: Hybrid QM/MM Free Energy Perturbation Study," J. Phys. Chem. (in press).

L. Ho, P.A. Bash, "Computational Enzymology: Computer Simulation of the Enzyme Reaction of Malate Dehydrogenase," Biochemistry (in press).

P.A. Bash, L. Ho, A. MacKerell, D. Levine, "Progress toward Chemical Accuracy in the Computer Simulation of Condensed Phase Chemical Reactions, " PNAS (in press).

P.A. Bash and B. Stoddard, The combination of Laue diffraction and molecular dynamics to determine the structures of enzyme-substrate complexes (manuscript in preparation).

Invited Presentations:

Biochemistry Department, University of Iowa, October 1995.

Workshop on High Performance Computing in Chemistry, August 1995

Enzymes Coenzymes and Metabolic Pathways Gordon Conference, July 1995

Computational Sciences Program, University of Kentucky, November 1994

Workshop on Methods in Computational Biology, University of Kansas, October 1994

Chemistry Department, University of Chicago, December 1994

Physics Department, University of Illinois, Champaign-Urbana, May 1994

Contributed Presentations:

Enzymes Gordon Conference, July 1994

American Chemical Society National Meeting, August 1994

Biophysical Society Meeting, February 1995

Patent Application:

Process for the Computer Simulation of Condensed Phase Chemical Reactions

Patent application - ANL-IN-95-116 


\title{
95-270N -- LIPOGENIN, A POTENTIAL HUMAN "HORMONE" THAT ACTS AS A GENERAL INDUCER OF LIPID FORMATION
}

\author{
Associate Laboratory Director Area: Energy and Environmental Science and \\ Technology \\ Principal Investigator: \\ E. Huberman, Center for Mechanistic \\ Biology and Biotechnology \\ Funding Profile: $\quad$ FY $1993-0-$ \\ FY $1994-0-$ \\ FY $1995 \$ 124 \mathrm{~K}$ \\ FY $1996 \$ 300 \mathrm{~K}$ \\ FY 1997 -0-
}

Purpose: The proposed project will lead to the isolation and characterization of a new protein hormone that may play an important role in obesity, a common yet serious nutritional condition. Several studies have implicated specific hormones and receptors in the regulation of this nutritional condition, but their nature is unknown. We have recently found that a human cell line used in our differentiation studies releases into the culture medium a protein that at low concentrations causes the production of massive lipid droplets in diverse cell types. It is therefore conceivable that this protein, which we termed lipogenin, may be a critical "hormone" involved in the regulation of obesity in humans. Our efforts are therefore directed towards purifying lipogenin in order to clone and sequence its cDNA and produce appropriate antibodies. These DNA and immunological probes will allow us to establish the role that lipogenin and its receptor play in normal development, as well as in the evolution of obesity and its related diseases including hypertension, type II diabetes and certain types of cancer. Eventually, we hope through the use of structural and computational analyses employing the APS and ANL's advanced computers to lead to the development of drugs that will either reduce the production or diminish the action of Lipogenin on its receptor as a means to treat obesity and its associated diseases. Our studies will therefore be important for basic science as well as for medicine and health care.

We anticipate that results from this project will secure future funding from governmental agencies and industrial partners.

Approach: Fat cells (adipocytes) play a pivotal role in lipid homeostasis in humans and other animals. It is believed that the conversion of precursor cells into adipocytes is mediated by specific hormones. Thus, a pathological increase in such hormones may result in obesity.

People are considered obese when their weight is more than $20 \%$ above an ideal weight standard. This condition increases the risk of disorders such as hypertension, hyperlipidemia, hyperglycemia, type II diabetes, and certain types of cancer. Yet, in spite of intense efforts, the molecular basis that underlies obesity in humans is poorly understood.

In the mouse there are at least six recessive gene mutations that lead to an obese phenotype. Studies involving cross-circulation experiments between mutant and wild-type mice indicate that 
some of the controlling factors circulate in the blood. These studies thus implicate potential "hormones" and receptors in the regulation of obesity. A recent interesting development was the position cloning of one of these mouse genes, the $o b$ gene. The speculation is that the product of this gene, a hormone, travels to and affects the hypothalamus (which is considered to be the appetite-controlling organ), which in turn causes proper food intake. In spite of this important observation, there is still a need to identify the critical "molecules" that directly affect the cellular fat reserves in the body.

We have reported earlier the identification of an activity in the culture medium of a human cell line which causes the storage of massive amounts of lipid in many diverse cell types. We subsequently determined that a protein, which we have named lipogenin, is the factor responsible for this activity. Our goals are:

1. To purify lipogenin to homogeneity

2. To obtain a partial protein sequence and an appropriate DNA probe

3. To use this probe for the isolation and sequencing of Lipogenin cDNA

4. To prepare antibodies specific to lipogenin

We have obtained partial purification of lipogenin to date. As this protein is likely present in only small quantities (less than $1 \mu \mathrm{g} / \mathrm{L}$ ) in the cell culture medium, a high degree of purification is required before all traces of contaminating proteins can be removed. In the coming year, we plan to continue to use different methods for Lipogenin purification.

Technical Progress and Results: In the initial part of this project, we have shown that a specific human cell line releases into its growth medium a protein that can induce massive amounts of lipids in a variety of cell types. This inducer, which we termed lipogenin, is present in only small quantities (less than $1 \mu \mathrm{g} / \mathrm{liter}$ ) in the growth medium. To characterize lipogenin, we initiated studies to establish protocols for its purification. After testing a variety of purification approaches, we settled on two initial modes; one involves ethanol precipitation while the other entails the use of a column of agarose gel linked with heparin. These two steps yield partial purification without destroying the biological activity of lipogenin. The final purification will require optimization of these methods and incorporation of additional chromatographic steps.

Among ion-exchange chromatographic methods, cation-exchange chromatography on a Mono-S column gives a degree of purification; optimization of this method will be achieved by using a Pharmacia FPLC system. Ion-exchange chromatography on columns of calcium phosphate also gives promising results. Size-exclusion chromatography, which separates proteins on the basis of molecular mass, may be of use, but practical problems remain which must be overcome before this method can become part of a final purification process.

After we establish these methods for the purification of lipogenin, our future efforts will focus on three areas: final optimization of the procedures described above; investigation of other promising chromatographic techniques which have not yet been evaluated, and finally, the combination of the most effective methods into an efficient process by which highly purified lipogenin may be obtained. 
Specific Accomplishments: We have identified an activity in the culture medium of a human cell line which causes the storage of massive amounts of lipid in many diverse cell types. We subsequently determined that a protein, which we have name Lipogenin, is the factor responsible for this activity. As lipogenin is present in only small quantities (less than $1 \mu \mathrm{g} / \mathrm{l}$ ) in the cell culture medium, a high degree of purification is required for its characterization. At this time, we have achieved partial purification using ethanol precipitation and a column of agarose gel linked with heparin. 


\section{blank page}




\title{
91-034R2 -- INTEGRATED LAND USE MANAGEMENT USING REMOTE SENSING AND ECOLOGICAL MODELS (formerly "Remote Sensing and Global Change")
}

\author{
Associate Laboratory Director Area: Energy and Environmental Science and \\ Technology \\ Principal Investigators: \\ J. Krummel and $\mathrm{H}$. Su, \\ Environmental Assessment Division \\ C. Loehle, Environmental Research Division \\ J. Lee, Energy Systems Division \\ D. Streets, Decision and Information Sciences \\ Division
}

Funding Profile:

FY $1993^{1}-0-$
FY $1994 \$ 105.7 \mathrm{~K}$
FY $1995 \$ 116.3 \mathrm{~K}$
FY $1996-0-$
FY $1997-0-$

Purpose: To develop an integrated analysis tool to address regional sustainable development and land use issues in support of U.S. and international ecosystem management and global change research initiatives. Human activities now alter land cover and associated natural system dynamics (e.g., water runoff, species extinction, soil loss, rainfall patterns) at regional and continental scales. Because of the geographic scale of these changes, spatially-explicit models must be used to project future environmental conditions. Current non-spatial methods (statistical or numerical) can not link local, regional and continental scales. However, spatial models require new techniques to incorporate remote sensing data and tools developed for geographic information systems (GIS). Three divisions are collaborating to produce an integrated set of spatial models and data and GIS tools to address sustainable development and land use issues. This multi-divisional approach will create a new Laboratory capability in computer modeling, GIS technology and remote sensing data analysis that can be used by federal agencies, including DOE, EPA, DOI, and DOD, and international organizations in order to address global change or sustainable development.

Approach: Our approach has been to focus the efforts of three divisions doing spatial analysis, ecosystem modeling and field data collection to develop an advanced, integrated technology that can project future outcomes of human disturbance at several spatial scales. Scientists in each division teamed to address three specific components of the research: image processing of remote sensing data, models of landscape function, including process and spatial dynamics, and field data collection of spatially-explicit ecological parameters. The common technology that was used to integrate the project activities was a set GIS data management tools that allowed us to share and merge a number of distinct data types and also provided the computer code to load parameters and variables when setting initial conditions for dynamic models.

${ }^{1}$ Project initiated in FY91; FY91 cost $\$ 9.9 \mathrm{~K}$ 
Because the use of remote sensing data is fundamental to the simultaneous analysis of local, regional and continental phenomena, the project sought to develop direct links between satellite remote sensing data and digital aerial photography and landscape dynamic models. A key aspect of this research was to determine if remote sensing data could be used to develop a set model parameters for both ecosystem process dynamic models and models vegetation community change. A second data collection activity addressed the use of innovative techniques to develop spatial data of topoedaphic conditions as these change over time and space. The effects of terrain and moisture drive changes in landscape dynamics, but efficiently measuring these changes has been a challenge in the past. The project tested the use of a new instrument, the time domain reflectometer (TDR), to detect soil moisture distribution across an individual field sites.

To assist in guiding large-scale data collection, a theoretical component of the study sought to address the effect of disturbance and habitat fragmentation at regional scales on the risk of loss of species. A specific modeling approach, including parameters and methods for assessing habitat fragmentation effects, had to be developed to determine how species can gradually be lost from human-managed landscapes. A second component of the modeling focused on software development to explicitly link GIS data bases to ecosystem process models. By developing this linkage, a powerful tool would be made available to investigate multi-scale problems of land use management and ecosystem response to climate change.

Technical Progress and Results: The following progress/results were achieved during FY95:

(1) In conjunction with the use of a GIS to construct the data input file, a set of tools was developed that input and edit the spatial data on the computer screen (Figure 1). The package of tools is written in the $C$ language and runs under $X$ Windows, Version 11, Revision 4 or higher. These tools graphically represent the major layers of input data for the distributed parameter model we derived from the ANSWERS code. All data are stored in the ARC/INFO SVF format. Program functions allow the user to make changes to the input data. Once the model simulation has been run, the sediment transport may be viewed and analyzed with the visualization tools and compared with input parameters such as land cover.

(2) Field data of soil moisture variation was collected during the 1995 growing season. The project collected soil moisture data, using the recently developed TDR, from three transects within a 100 acre corn field in northern Illinois that contained a topographical variation of 20 meters in elevation. Three sets of readings were taken at each point along the transect over a period of three months. Each reading included four layers of soil moisture at 15 centimeter intervals. The spatial data collected was placed in the GIS and produced information on finescale spatial dynamics required to adjust the resolution and error checking in the ANSWERS model development.

(3) Spatial and temporal changes in landscape vegetation, including changes caused by agricultural development, were quantified using Landsat thematic mapper (TM) satellite data and digital aerial photography. Land cover classifications derived from this data were then used to develop parameter values for the ANSWERS model and vegetation community models. Two Landsat TM images from Cadiz Township, Wisconsin, collected in 1991 and 1993 provided information on specific cropping practices and remaining natural vegetation patterns, while a 
time series of digitized aerial photographs were used to determine vegetation changes from 1930 to 1986. With this data, the modified ANSWERS model was used to show how ecosystem processes have changed over time.

(4) A model of extinction debt, which is the irreversible, preferential loss of competitive species following prolonged disturbance, was developed that showed that repeated spatially random disturbance can result in potentially severe extinction losses at the landscape and regional scale. For larger fragments of remnant vegetation, such as forest wood lots, some assumptions of the model are violated but extinction debt nevertheless occurs due to gradual stochastic elimination of species that are very rare and isolated in these fragments. For habitat destruction on regional scales (reduction in ecosystem area without disturbance in remnant areas), species-area relations should be based on the distribution of different habitat types (e.g., elevation and rainfall gradients, physiographic and edaphic variability). Such an analysis projects rapid, not delayed, loss of all types of species, not just competitive types. The model forms a community vegetation counterpart to the ANSWERS model so that ecological changes inland cover pattern can be addressed in the integrated framework.

Specific Accomplishments: Based on our model development, the U.S. Army at Aberdeen Proving Ground provided funds to apply the model in the management of sensitive watersheds within the larger Chesapeake Bay Basin. In addition, contacts have been made with EPA and USDA to potentially apply this technology to the DSSAT modeling framework. These models simulate crop production and can be modified to address regional issues if linked to GIS data. Results from this study have produced two publications, a published proceeding and two manuscripts are in preparation. Finally, a three division program development seminar has been prepared to present to potential sponsors.

\section{Publications:}

D. Streets, C. Chung, J. Krummel, and H. Su, "Remote Sensing of Global Change: Growth in China's Jiangsu Province," Internatl. Journal of Sustainable Development and World Ecology, 2:257-266 (1995).

J. Krummel, H. Su, J. Fox, M. Ekasingh, and S. Yarnasan, "Remote Sensing and Land Disturbance: Global Change Indicators," Proc. of the Conf. for Remote Sensing and Environmental Monitoring for the Sustained Development of the Americas, San Juan, Puerto Rico (March 21-22, 1995).

C. Loehle and B.-L. Li, "Habitat Destruction and the Extinction Debt Revisited," Ecological Applications. In press. 


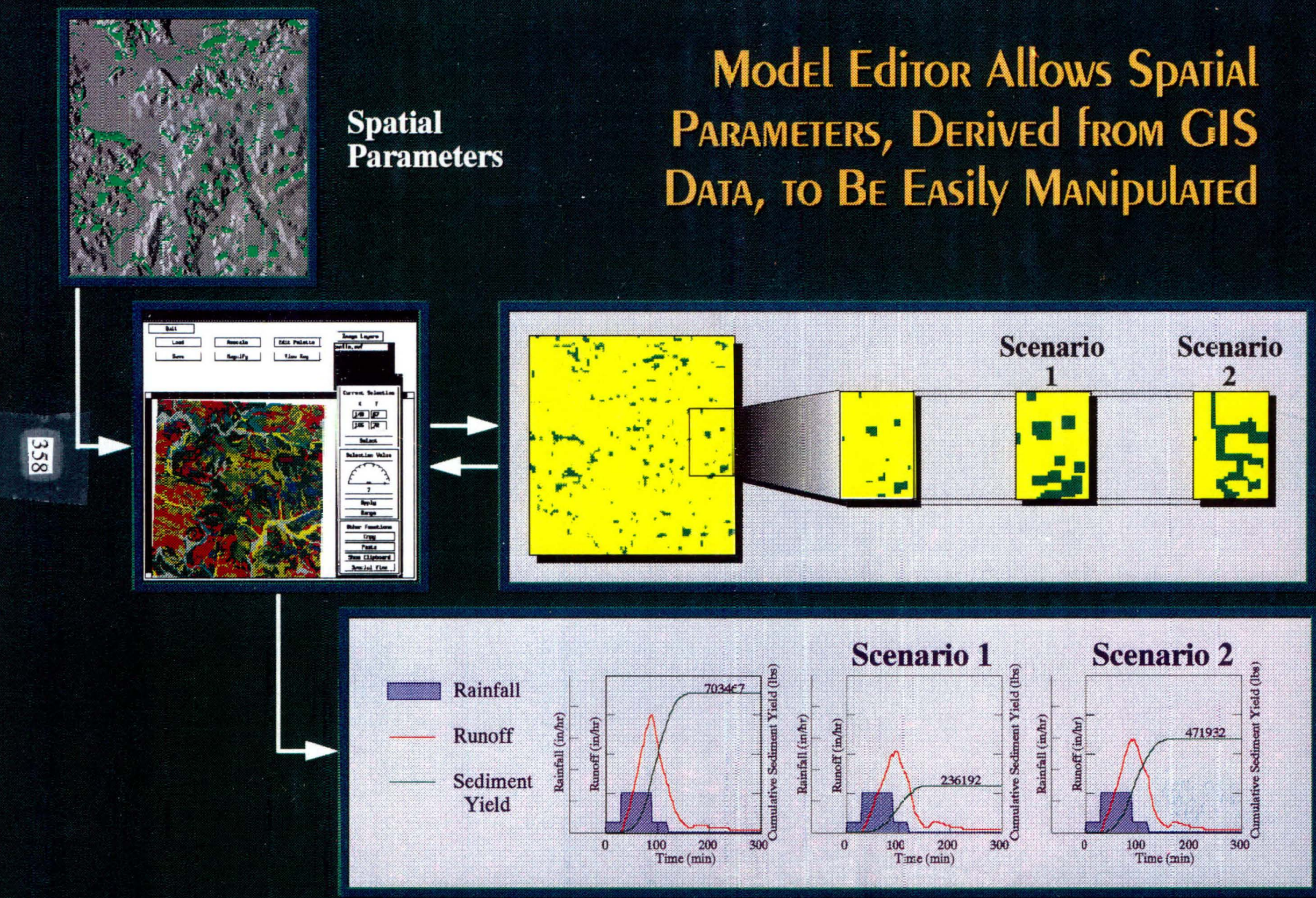




\title{
95-272N -- THREE-DIMENSIONAL REAL-TIME IMAGING OF THE PLANETARY BOUNDARY LAYER ON WATERSHED SCALES
}

\author{
Associate Laboratory Director Area: Energy and Environmental Science and \\ Technology \\ Principal Investigators: \\ R.L. Coulter, B.M. Lesht, J.D. Shannon, \\ D.L. Sisterson, and M.L. Wesely, \\ Environmental Research Division
}

Funding Profile:

FY $1993-0-$

FY $1994-0-$

FY $1995 \$ 39.1 \mathrm{~K}$

FY $1996 \$ 465.0 \mathrm{~K}$

FY $1997 \$ 460.0 \mathrm{~K}$

Purpose: This project will conduct research in the planetary boundary layer on scales most relevant to important questions in atmospheric sciences. The research involves field-based studies using and developing new instrumentation and techniques for real-time three-dimensional atmospheric probing. Instruments will be deployed in a $50 \mathrm{~km} \times 50 \mathrm{~km}$ area in southern Kansas where extremes of mesoscale processes (frontal passages, convective storm development) are known to occur. New techniques of observation and data fusion will be developed and used to develop an understanding of the nocturnal low-level wind maximum and its relationship to synoptic jet features, to develop methods needed for spatial integration of air-surface exchange of heat, gases, and momentum, and to study the horizontal and vertical dispersion in the planetary boundary layer. The data obtained will be provided in real-time to a large user community of atmospheric and ecological scientists in addition to being used by Argonne researchers.

Approach: This research will initially focus on understanding the dynamics of the planetary boundary layer (PBL) by using new combinations of techniques already proven useful in more limited studies. Surface-based observations of the profiles of temperature, humidity, and wind and of the air-surface exchange rates of heat, moisture, and momentum will be made over an area of approximately $50 \mathrm{~km}$ by $50 \mathrm{~km}$, the so-called Argonne Boundary Layer Facility (ABLF). In contrast to other boundary layer studies, these observations will be continuous, will be made in such a way so as to characterize a large volume of atmosphere, and will be continued for several years. By making these observations within the $100000 \mathrm{~km}^{2}$ area now occupied by the Department of Energy's Atmospheric Radiation Measurement (ARM) Program's Southern Great Plains Cloud and Radiation Testbed (SGP/CART) Site, existing data will be leveraged in support of our studies. The data will be provided both to ARM and to other researchers so as to achieve maximum scientific benefit.

The basic suite of measurements will be started in 1996 and will be completed by the end of 1998. Initially, the validity of current models of the planetary boundary layer structure will be tested. The detailed observations will be tied to concurrent numerical calculations to determine the causes of discrepancies between model predictions and actual conditions. The experiments will be adaptive and will be designed to focus on the areas of greatest uncertainty. We will 
devote particular attention to processes involved in the relationships between surface heterogeneity and local circulations and storm development.

Technical Progress and Results: Because this is a field-based research program, work in FY 1995 focused on planning of tasks, developing time schedules, and estimating costs for the deployment of the necessary equipment. The location of the experiment area was determined, and prospective sites for individual instrument systems were identified. Actions were taken to develop documentation to comply with the National Environmental Policy Act, to develop the physical and procedural infrastructure requirements and designs (procurement of instruments, trailers, fencing, concrete pads, safety requirements, etc.), and to arrange for utility services (electricity, telephone, etc.). Instrumentation already available in the Atmospheric Section of the Environmental Research Division was made ready for deployment. Acquisition of a workstation to serve as part of the central data control and acquisition center and acquisition of one $915-\mathrm{MHz}$ wind profiler - Radio Acoustic Sounding System (RASS) were initiated. One existing 915-MHz RWP-RASS existing at Argonne was evaluated and modified for installation at the Walnut River Watershed. These two systems and a $915-\mathrm{MHz}$ system independently supplied by the ARM program will be installed in early 1996 at the vertices of a triangle drawn over the southern half of the CASES site, and the three systems are expected to begin producing data by summer of 1996. A satellite receiver already in use at Argonne has been made available for routine data collection and evaluation of non-dimensional vegetative indices will begin as surface data collection begins. Efforts are also underway to install and begin operation of an eddy correlation system available at Argonne for measuring fluxes of heat, moisture, momentum, and possibly carbon dioxide within the array during 1996.

In June 1995, M.L. Wesely traveled with H. Drucker and C.A. Reilly to DOE Headquarters in Germantown, MD, to brief the Director and staff of the Office of Health and Environmental Research (OHER) on the ABLF initiative. OHER officials subsequently endorsed the ABLF initiative. Although no funding from OHER was committed, statements were made to the effect that OHER funding for the ABLF could probably be expected beyond the lifetime of this LDRD project.

Overall, trips on six separate occasions were made by Argonne staff to visit agency representatives or scientists interested in the ABLF. Some of these efforts were focused on coordination with the Cooperative Atmosphere-Surface Exchange Study (CASES) initiative, a multi-organizational effort to obtain measurements over a somewhat larger domain in the same location. CASES would include hydrological, ecological, and atmospheric chemistry studies in addition to boundary layer research. A very strong dialogue with the CASES community has been established, and it has become apparent that CASES will rely heavily on the ABLF facility for initial investigations.

In FY 1996, work will include adaptation of minisodars existing at Argonne for installation in the boundary layer facility array, acquisition of surface flux stations and site preparation involving leases, equipment installation, data communications, and related tasks. Methods of exchanging data with the ARM program will be developed.

Specific Accomplishments: Partial support for a CASES planning workshop in Wichita, Kansas, on February 22-24, 1995, was provided by this project. This workshop had over 60 
participants from many universities and laboratories to discuss needs for establishing long-term facilities in the Walnut River Watershed and thus effectively established ties to a large user community of users of using the ABLF when it is established. A site visit to the Watershed was included in this meeting. The following presentation was made:

R.L. Coulter, B.M. Lesht, J.D. Shannon, D.L. Sisterson, and M.L. Wesely, "Opportunities for Collaboration with Argonne's Boundary Layer Facility Initiative Planning Workshop for a Cooperative Atmosphere-Surface Study Sites (CASES)," Argonne National Laboratory, Depts. of Agriculture, Commerce, Energy, and Interior, the National Aeronautics and Space Administration, and the National Science Foundation, 22 February 1995, Wichita, Kansas.

On July 17, Marv Wesely participated in a meeting in offices of the National Oceanic and Atmospheric Administration in Silver Spring, Maryland, at which the Cooperative AtmosphericSurface Exchange Study initiative was discussed by scientists and agency representatives. He made the following presentation:

M.L. Wesely, "Instrumentation for the Proposed CASES Facility," Planning Meeting of the Cooperative Atmospheric-Surface Exchange Study, Multi-agency Sponsorship, 17 July 1995, Silver Spring, Maryland. 


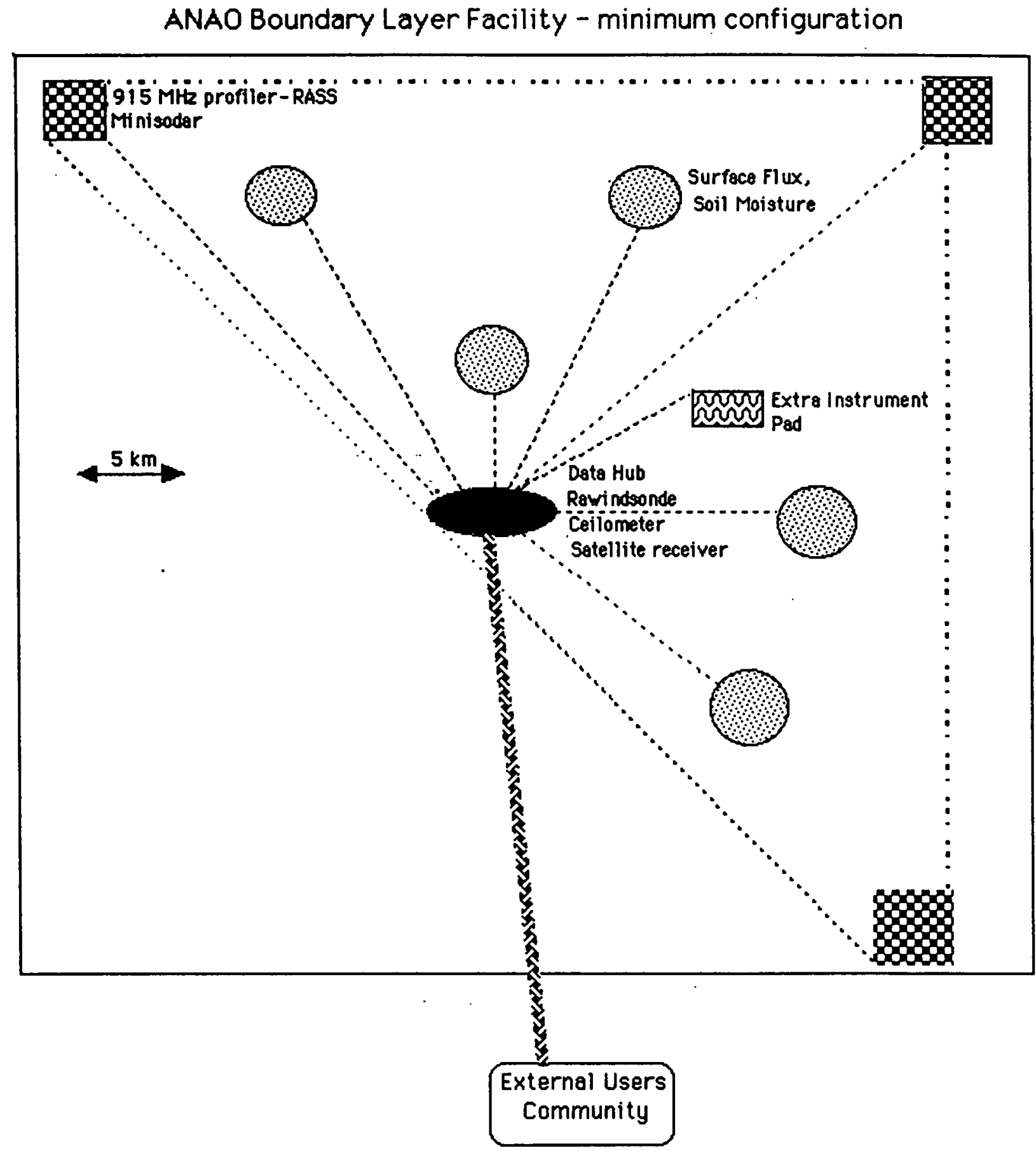

Figure 1. Schematic of the conceptual locations of instrument systems for the minimum configuration deployment. Placement of instruments will take place in FY1996. 


\title{
95-050N -- DEVELOPMENT OF PERFORMANCE ASSESSMENT CAPABILITY FOR WASTE DISPOSAL
}

\author{
Associate Laboratory Director Area: Engineering Research \\ Principal Investigators: \\ S.W. Tam and T. Krause, \\ Chemical Technology Division \\ Funding Profile:

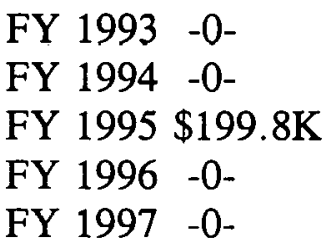

Purpose: The purpose of this LDRD is to develop a performance assessment (PA) capability at Argonne for waste disposal. Performance assessment (PA) involves the use of mathematical or numerical models suitably linked to simulate the long-term degradation of, and contaminant release from, waste disposal systems. PA capability is an important component in identifying technical issues that need to be resolved for the disposal of radioactive and nonradioactive waste. To date, the development and application of PA capability have been focused on radioactive waste disposal; we anticipate that these capabilities will also be useful for future nonradioactive waste disposal actions and decisions. Our purpose is therefore to develop PA capabilities at Argonne to the point where we can use them to complement ongoing experimental programs and to interface with experimental and modeling initiatives in both radioactive and hazardous waste disposal.

Approach: Our approach is to develop and implement an integrated PA package for evaluation of waste management practices and waste disposal options. It includes a number of elements. The first is to identify, evaluate and acquire computer codes that incorporate appropriate theoretical models to simulate the various geochemical and geophysical processes (such as waste form corrosion, radionuclide dissolution, and radionuclide transport) that influence the performance of waste disposal systems. The second is to develop improved models for selected processes and to incorporate these models into the codes identified in the first step. Our primary interest is focused on modeling processes such as degradation of components of the disposal system (e.g., the waste form) and transport of contaminants through the engineered and natural barriers. The third element is to link the selected computer codes into an integrated framework that enables projection of the behavior of the disposal system over the very long time periods of interest (on the order of 10,000 years). The resulting integrated PA methodology will be used for performance assessment analysis which will provide an understanding of the linkage between laboratory investigations, repository design features, and compliance with regulatory requirements and thereby provide a basis for identifying and justifying new experimental and modeling initiatives.

In FY95 the approach has been focused on developing a model for radionuclides transport through the Engineered barrier system (EBS) and on the acquisition and systematic evaluation of the leading PA codes utilized by the waste community. 
Technical progress and results: For FY 1995 we focused on modeling degradation of the engineered part of the disposal system and transport of contaminants through the subsurface from the engineered system to the accessible environment. The strengths and weaknesses of computer codes that can be used to simulate the contaminant release performance of the engineered part of disposal systems and the ensuing groundwater transport of these contaminants were evaluated. Based on this evaluation we have implemented two of the lead codes on the CMT computing environment. They are AREST, a leading code for simulating the performance of the engineered part of disposal systems, and MODFLOW, a hydrological flow code widely used by the ground water flow community. Together the two codes can be applied to describe the nearfield (AREST) as well as the far-field (MODFLOW) transport of radionuclides and other contaminants. AREST is able to examine the effects of many processes that may take place within a repository. Once the radionuclides escape from the waste the major transport medium is the ground water. Figure 1 is a contour plot that demonstrates, using MODFLOW, how local pumping(in the south-west) and the presence of a contaminant source (in the north-east) can interact.

A model has been developed for the transport of contaminants across the low-permeability barriers (e.g., compacted clay layers) that are often used as a key component of the engineered barrier. system intended to inhibit release of contaminants from disposal systems. Part of the motivation for developing this model is that such a model is not in the AREST code because it was designed only to simulate the disposal system concepts under consideration for disposal of high-level radioactive waste at Yucca mountain. However, such a model is needed for the more general applications that we envision. A diffusive transport model has therefore been formulated. Chemical and microstructural effects on transport have been incorporated in terms of an effective diffusion coefficient $D_{\text {eff }}$. The microstructural effect is taken into account by first modeling the electrical conductivity of fluid-bearing rocks using a differential effective medium (DEM) approach. The DEM approach allows one to maintain the long range connectivity of the transport pathways even at porosity that is below the conventional percolation threshold at which the pores would become isolated. This is important since empirically it is well known that for many rocks, minerals and compacted media that conduction/transport pathways are maintained even at low porosity (below 10-20\%) The comparison to experimental data on the conductivity of a fluid-bearing compacted material (as a ratio to the fluid conductivity itself) is shown in Figure 2. Satisfactory agreement (with no adjustable parameter) is found for porosity higher than a few percent. From this result a model for the radionuclide diffusivity in water-bearing porous media is derived using the Nerst-Einstein relation that connects the conductivity to the diffusivity. The resulting diffusivity as a function of porosity for a radionuclide such as $\mathrm{Cs}$ is shown in Figure 3. Depending on the porosity as much as a reduction of an order of magnitude of the diffusivity below its aqueous value is possible.

Another important factor is the effect of chemistry as manifested through the sorption of many of the radionuclide species onto the interior pore surfaces of the diffusion barrier materials. This is taken into account in $\mathrm{D}_{\text {eff }}$ by incorporating a retardation factor $\mathrm{R}$, which is related to the partitioning of the radionuclides population into sorped and 'free' components using a linear sorption isotherm formalism. The effect on $\mathrm{D}_{\text {eff }}$ for $\mathrm{Cs}$ as a function of porosity is shown in Figure 4. The behavior is qualitatively different from the effect of microstructure due to the different dependence on porosity. Note that while the influence of microstructure is largely independent of the particular migrating species the retardation factor is very sensitive to the 
radionuclides involved due to the large variation of the sorption properties among different radionuclides.

Once the chemistry and microstructure effects are accounted for the diffusive transport problem is solved with a Green's function technique. This allows a probabilistic interpretation from which one can extract a characteristic retention time $\tau$ for any one particular radionuclide. $\tau$ is the typical time the nuclide would need to transit the barrier and emerge into the rock formation bordering the outer boundary of the EBS. Our analysis indicates that this retention time is a sensitive function of the porosity which frequently has a range of uncertainty arising from both measurement uncertainty as well as actual variation in the microstructure of the materials. The model allows a Monte Carlo analysis to be performed. The effect of the uncertainty of the barrier microstructure on the retention time has been explored. Figure 5 shows an example of such an analysis for C.s. Note that in the figure only the transport part of the retention time is represented, even though the model includes the radioactive decay effect. The plots represents a probability distribution for $\tau$. Note the unsymmetric long tail of the distribution. This arises from the porosity dependence of $\mathrm{D}_{\text {eff }}$. The results shows that the barrier may be very effective in retaining Cs. Our analysis indicates that there is a sensitive dependence of $\tau$ on the mean porosity which can decrease to only a few hundred years with modest changes in the porosity. This indicates that chemistry and microstructure are crucial design parameters for diffusion barriers. Furthermore the ability of a PA methodology to provide stochastic analysis, in contrast to only a deterministic answer is essential.

In summary we have formulated a model that described radionuclide transport through an impermeable barrier component of the EBS. Chemistry and microstructural effects were found to be important. The retention time of the waste species in the barrier is sensitive to the porosity. Monte Carlo analysis indicates that modest uncertainty in the EBS porosity can lead to a relatively broad distribution in the retention time. Preliminary testing of the AREST and MODFLOW codes have indicated that they should be useful in integrated analysis of the repository/geomedia. The next stage of our work in FY96 will focus on developing models that consider fast transport paths and degree of saturation and the integration of these models into a PA package that include the existing lead PA codes. Among those AREST and MODFLOW are two of the lead candidate codes that we shall consider.

\section{Specific Accomplishments:}

Final report for FY 1995: "Radionuclides transport through engineered barrier systems," submitted for presentation at and publication in the Proceedings of the 1996 International Conference on high level radioactive waste management, Las Vegas, Nevada, April 1996. 
Fig. 1 Head Distribution with 0.5 cu. ft./sec Pump Rate

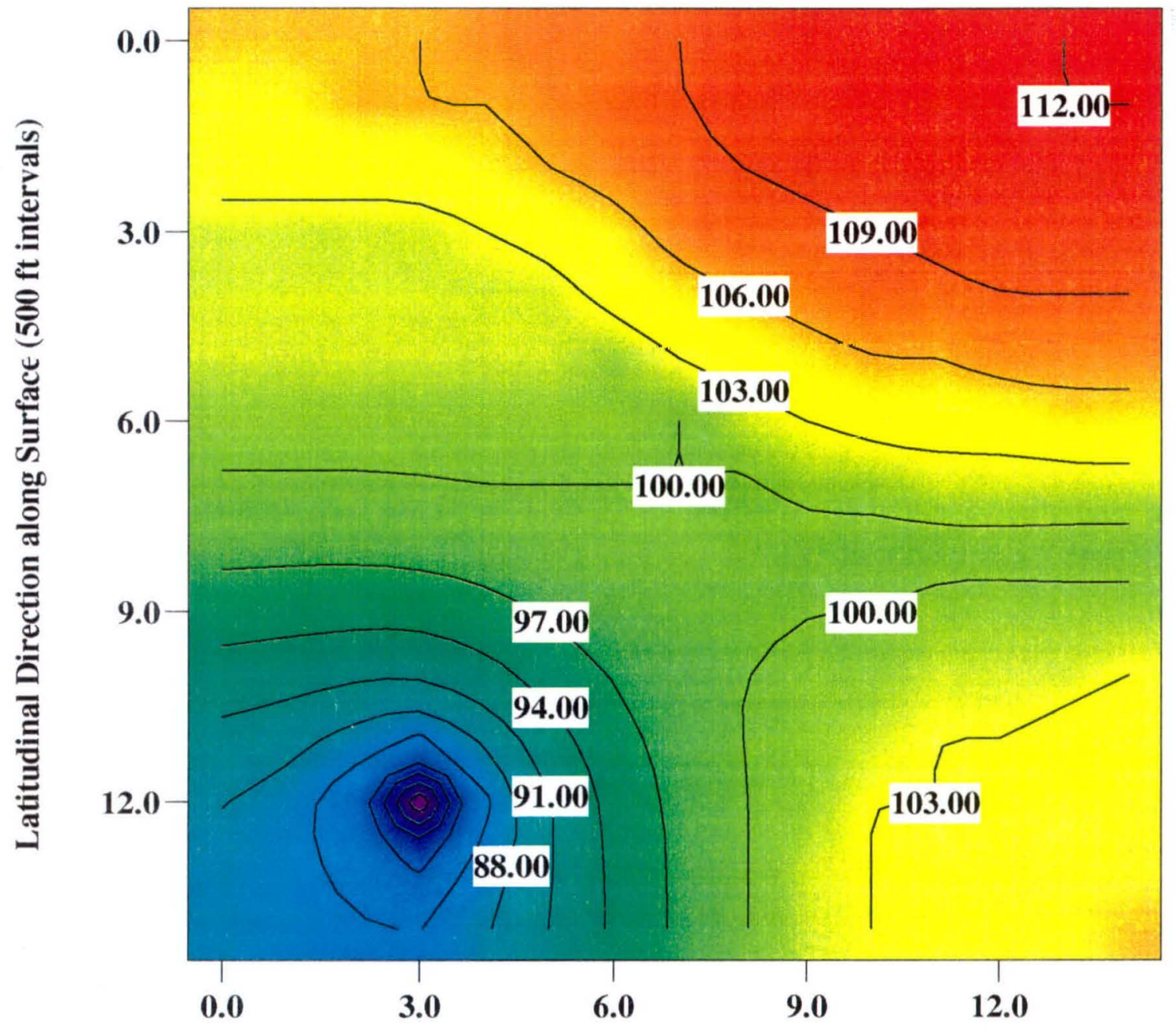

Longitudinal Direction along Surface (500 ft intervals)

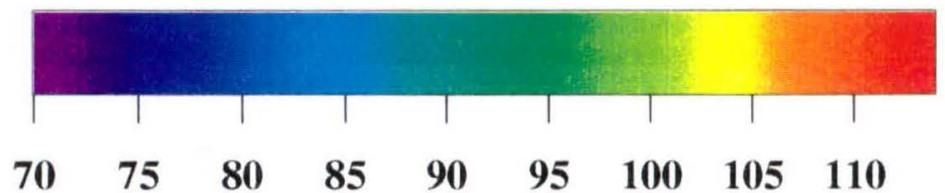

Head (ft) 


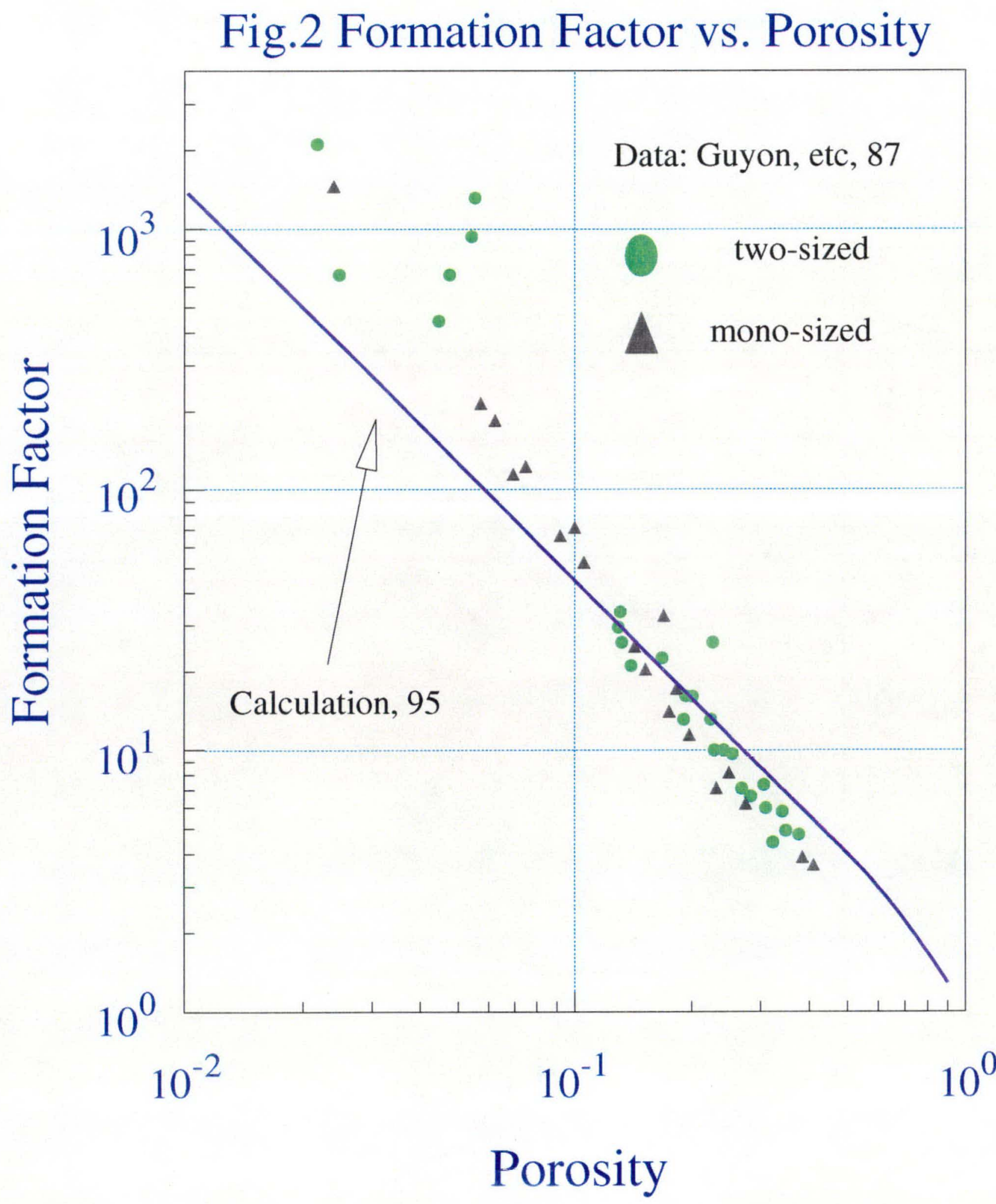




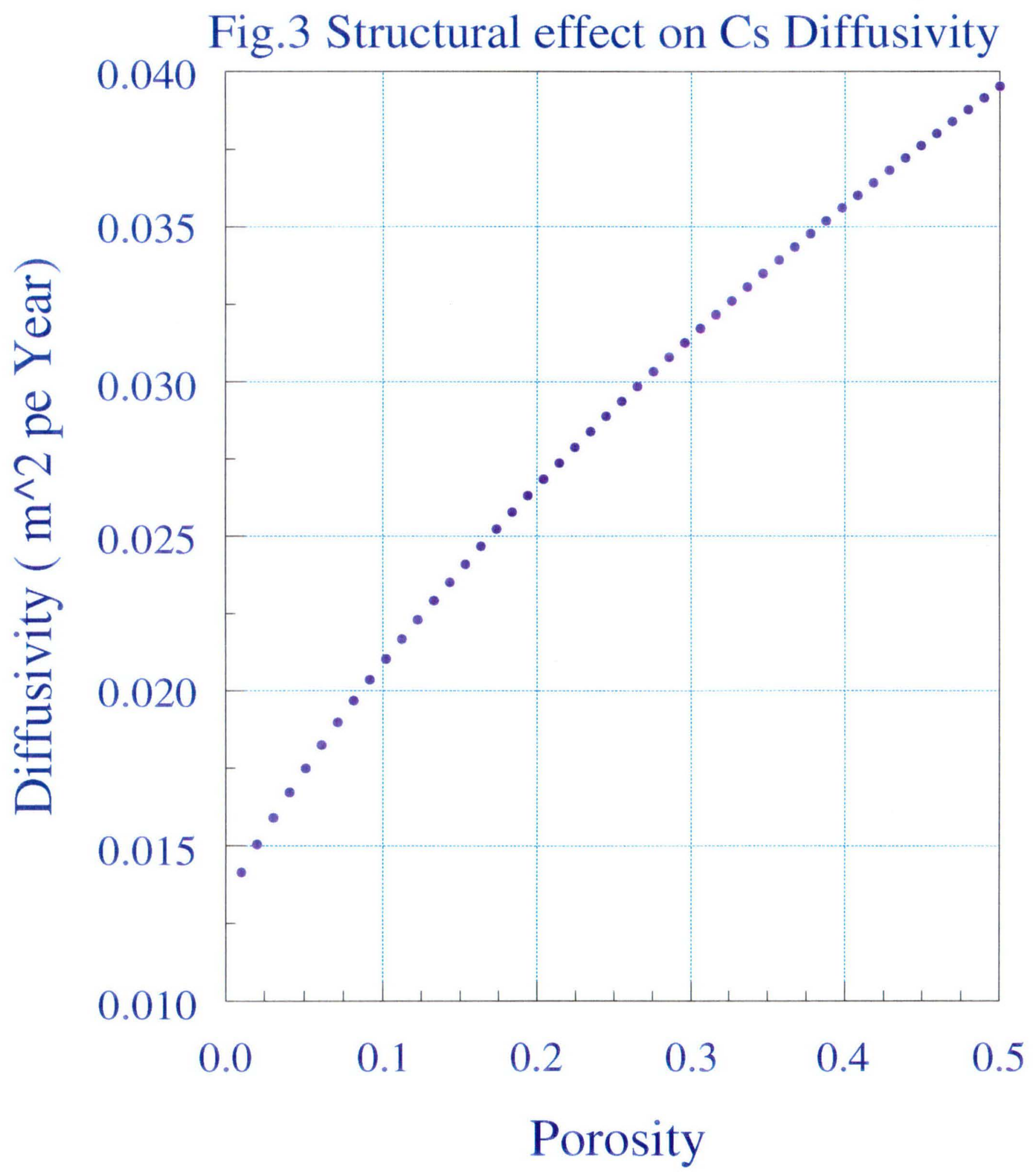




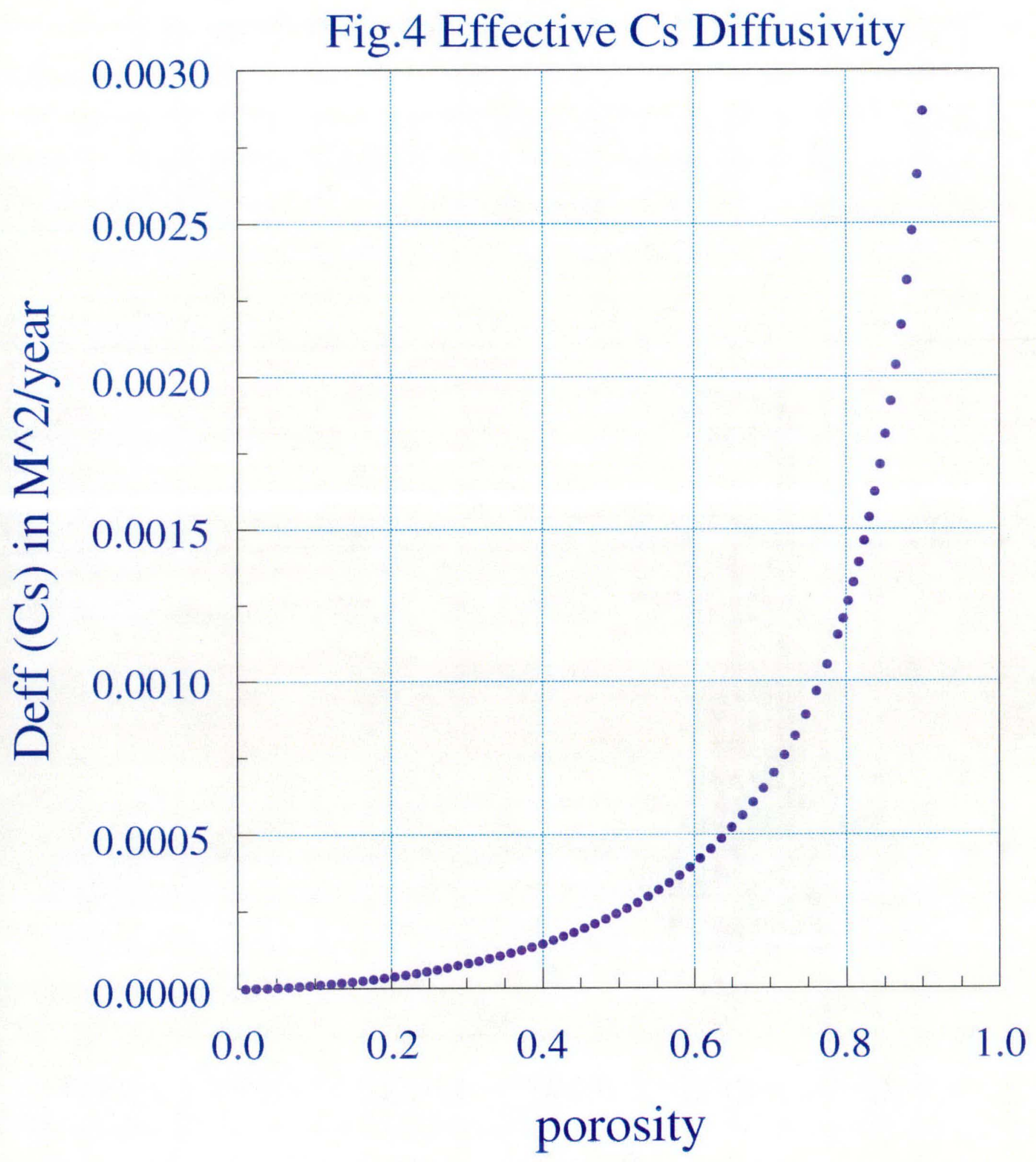




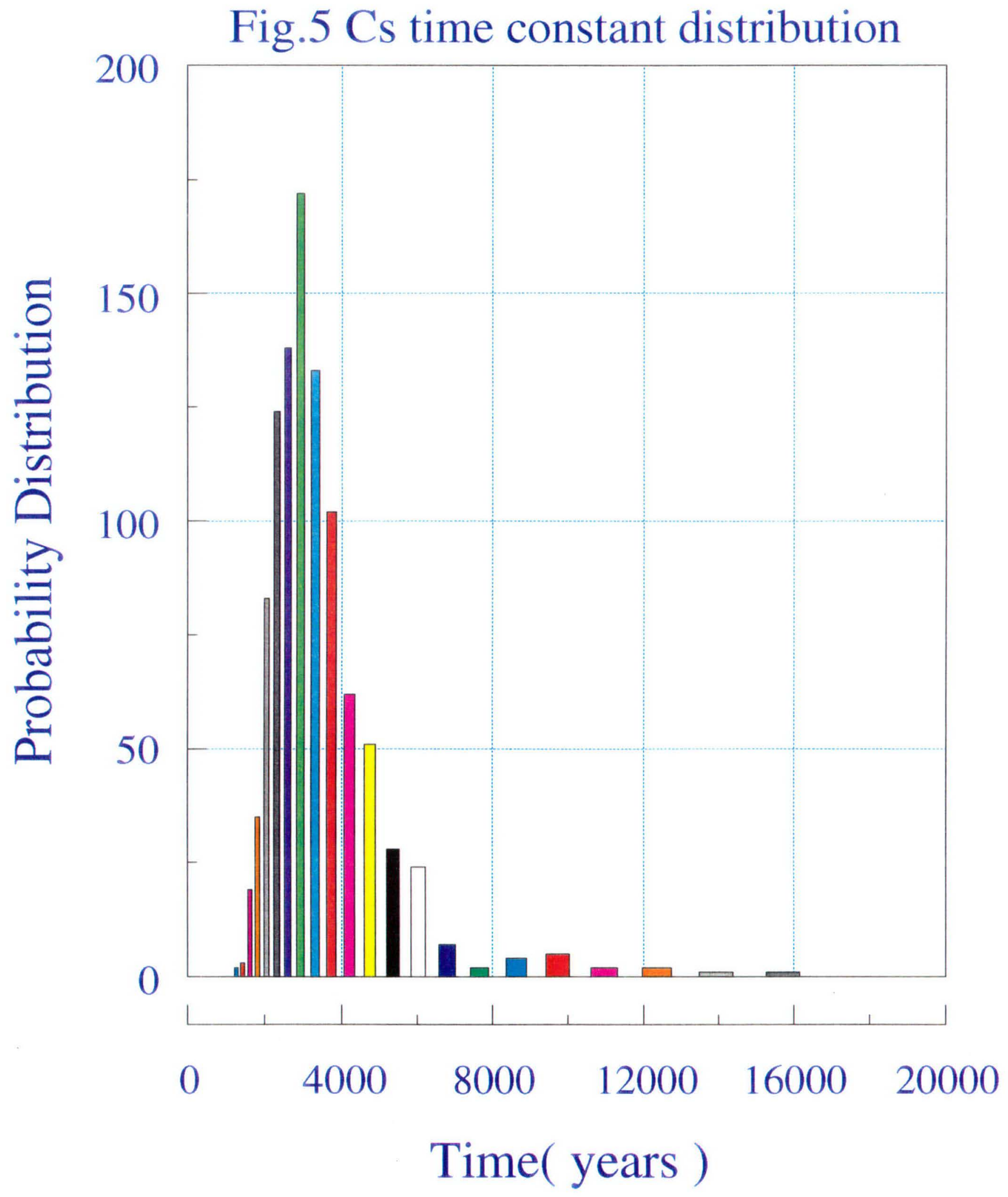




\title{
95-095N -- BENEFICIAL USES OF NUCLEAR WASTE
}

\author{
Associate Laboratory Director Area: Engineering Research
}

Principal Investigators:

D.C. Wade, Reactor Analysis Division

J.J. Laidler, Chemical Technology Division

Funding Profile:

$\begin{array}{lll}\text { FY } 1993 & -0- \\ \text { FY } 1994 & -0- \\ \text { FY } 1995 & \$ 150.1 \mathrm{~K} \\ \text { FY } 1996 & -0- \\ \text { FY } 1997 & -0-\end{array}$

Purpose: Proper disposition of nuclear waste is a current problem in both the private and the public sectors - ranging from private/state disposal of low level wastes which derive from medical, research, and commercial nuclear power plant operations to federal government cleanup of low level, mixed hazardous, and high level wastes from DOE weapons production activities and DOE and Naval reactor operations, to finally, Waste-Policy-Act mandated disposition of spent fuel from civilian nuclear power plants as high level waste.

To address one element of this problem, PYRO-conditioning technologies for DOE and civilian spent nuclear fuel is under development at ANL. Such conditioning will recover the useful uranium and will produce two radioactive waste forms; the first is a metal waste monolith containing noble metal fission products in an iron/zirconium matrix formed from the spent fuel and its cladding. The second is a tile-like ceramic zeolite or sodalite monolith containing rare earth and other fission products. These high level waste forms are expected to be certifiable for disposition in the Geologic Repository.

The purpose of this LDRD project was to address the suitability of the PYRO-waste forms for beneficial issues - as an alternative to burial. The first step was to establish that the PYROgenerated product forms are indeed robust and will not be degraded by the very radiation that is to be employed for beneficial uses.

Approach: The fission products contained in the PYRO-generated waste forms are emitters of both radiation and heat. If it can be established that the waste forms are robust, stable, solidstate products emitting radiation and/or heat, several potential beneficial uses for some fraction of the waste forms produced during disposition of spent fuel can easily be envisioned, e.g., sterilization units for medical equipment or for consumer foodstuffs, radiation emitters for nondestructive assay instruments, radiation emitters for radiation-catalyzed chemical processes, sterilization units for the recycle biological waste used for fertilizer, and sterilization units for water supplies, to name only a few. Most of the ideas enumerated above are not new, but were already envisioned at the dawn of the nuclear age fifty years ago; what is new are the PYROwaste forms and the PYRO waste production process. The novelty of the PYRO process and waste forms may open up possibilities previously unavailable in alternately proposed waste treatment technologies. 
Technical Progress and Results: The first year's work focused on the issues arising from the transmutation of radioactive nuclides sorbed in Zeolite-A as the PYRO-generated salt waste form - and specifically on the chemical changes in the content of the Zeolite, on the possible impact such changes may impart to the stability of the Zeolite, and, in general, the possibility that radiation-induced chemical changes will alter the fission product containment-effectiveness of the Zeolite waste form.

A molecular dynamics model of Zeolite-A was created and the interatomic forces represented as potentials were "tuned" so as to produce agreement between predicted and measured infrared spectra and neutron diffraction data reported in the literature. An understanding of the underlying physics of the Zeolite's ability to bind the salt waste was further refined by confirming the calculated locations of $\mathrm{Na}, \mathrm{K}$, and $\mathrm{Li}$ ions in the Zeolite cage against neutron scattering experiments which had been conducted at the IPNS. The importance of achieving charge balance of the Zeolite/salt compound through the occluded salt ionic bonds with the oxygen in the Zeolite cage was confirmed.

Then the question was addressed of what happens to charge balance when a charged particle radioactive decay of a fission product ion occurs. A search of the fission product libraries showed that while gamma ray decays abound, when it comes to charged particle fission product radioactive decay it is predominantly $\beta^{-}$(beta) decay. Alpha decay is non-existent and only a handful of $\beta^{+}$(positron) fission product emitters exist.

As to the $\beta$ - particle breaking the Zeolite framework bonds directly, the energy required to break an O-Si covalent bond is about $2270 \mathrm{kcal} / \mathrm{mole}$ (and is probably about the same for an O-Al bond). By contrast, ionization energies of first electrons $(115-350 \mathrm{kcal} / \mathrm{mole})$ and even second electrons (320-575 kcal/mole) are, for all atoms, much smaller than the covalent bond strength. The same is true of electron affinities, roughly $90 \mathrm{kcal} / \mathrm{mole}$. Based on scattering kinematics, it is clear that the O-Si covalent bonds which give the waste form its charge balance will not be adversely affected by radioactive $\beta^{-}$decay.

As to the $\beta^{-}$creating a new atom with a different preferred ionization state and undoing the charge balance in the compound, several issues are relevant. First, it was assumed that electrons readily flow through the salt mixture so that (a) the distribution of charges among species is only a function of the instantaneous composition and generally does not depend on past history, and (b) charges on the frame and its contents will balance when averaged over a small number of unit cells.

Second, it was noted that fission products are diluted by a factor of 100 in the salt waste. Thus, for each radioactive atom there are many other atoms to which electrons might be added by means of a ionization state change if required for charge balance.

Third, it was found in the chemical literature that $\beta^{-}$decay tends to lead to new atoms which accommodate an increase in ionization level. So, a $\beta$ - decay leading from a +1 to a +2 ion, will release one electron, in this case the $\beta^{-}$decay particle itself. Similarly, a $\beta^{-}$decay followed by an increase of $n$ in the decay product's ionization level releases $n+1$ electrons to the Zeolite system. As a result, one can expect that, within the whole complex of fission products, many electrons will be available to reduce any electron deficient decay daughter. 
An unresolved issue which might arise if an ionization state changes is the effect of ionic size on the framework. Might the framework be stretched past the breaking point if such a change occurred? Does the reduced size of a +2 ion distort the framework, rather like $\mathrm{LI}^{+}$? A second unresolved issue concerns the creation of neutrals; we can't conclude, a priori, that neutrals cannot be well-contained because they will in fact tend to be polarized by, and therefore attached to charged particles despite their neutrality. Moreover, they may have difficulty squeezing past particles which are large enough to block their transit through the passages in the Zeolite framework. On the other hand, the increased atomic size of a neutral could possibly be large enough to damage the framework.

Specific Accomplishment: Based on the research conducted under this LDRD, it seems unlikely that radionuclide transmutation to $\beta^{-}$decay daughter products will directly cause chemical damage to the Zeolite framework of the PYRO salt waste form. Transmutations exist which, unlike $\beta^{-}$decays, tend to diminish the number of available electrons: i.e., alpha or $\beta^{+}$decay and electron capture. However, if we consider the total number of fission product decays over the life of a waste form product, over $99 \%$ of these are $\beta^{-}$decays. Thus, it seems that in the absence of $\mathrm{Pu}$, or other $\alpha$-emitting actinides in the PYRO salt waste form these decays will be more than compensated by the electrons made available through predominate $\beta$ - decay and will be relatively unimportant.

The effect of radioactive decay on the Zeolite waste form will not lead to its degradation. This is a first step towards its qualification for application to beneficial uses.

A more thorough examination of several aspects of the chemical effects of transmutation should be made in the future. First, quantum chemical and/or molecular dynamics simulations should be used to assess the ionization states of species in the cluster in which free electrons stop. The question is, are individual neutrals or partially reduced ions produced, or does the overall salt cluster take on a free electron? This might be of particular relevance in the case of $\mathrm{Zr}, \mathrm{Pu}$, and other species with more than one possible ionization state. Second, the susceptibility of the framework to damage due to change in ionic size and charge accompanying transmutation and subsequent ionization state changes should be assessed. Molecular dynamics simulations could be applied to deepen understanding of these effects as well.

This effort is continuing under the auspices of DOE-NE. 
blank page 


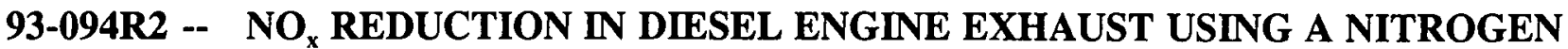 PLASMA}

Associate Laboratory Director Area:

Principal Investigator:

Funding Profile:

\author{
Energy and Environmental Science and \\ Technology
}

\author{
R.R. Sekar, H.K. Ng, and L.O. Hoppie, \\ Energy Systems Division \\ V.J. Novick, Technology Development Division
}

Purpose: This project explores a novel, synergistic technique for the reduction of the $\mathrm{NO}_{\mathrm{x}}$ emissions from engines that preserves all the benefits of the oxygen-enriched combustion concept. The inlet air would be separated in a hollow-fiber membrane into oxygen-rich and nitrogen-rich streams. The oxygen-enriched air would be used for engine combustion and the other stream is generally vented. The purpose of this project is to use the nitrogen in the waste stream as a source of electrically-activated nitrogen which, in turn, can be used in a proposed post-treatment process to reduce $\mathrm{NO}_{x}$ emissions dramatically. Such synergistic use of oxygenenriched air for improved combustion and electrically activated nitrogen for post-treatment may lead to efficient and cleaner diesel and gasoline engines capable of meeting the regulations proposed for the year 2000. The proposed $\mathrm{NO}_{\mathrm{x}}$ standards for engines using gasoline, diesel and other alternative fuels are extremely low and such novel methods will be needed to demonstrate that these levels are technically achievable.

Approach: $\mathrm{NO}_{\mathrm{x}}$ emissions from diesel and gasoline engines are being regulated to very low levels. If a practical means of solving the $\mathrm{NO}_{x}$ problem can be devised, it will benefit all the engine and vehicle manufacturers. This project explores a novel means of $\mathrm{NO}_{\mathrm{x}}$ control. The approach has been entirely experimental research.

The principle involved here is the reduction of NO gases into nitrogen and oxygen by the use of electrically activated atomic nitrogen. While the theory behind this reaction has been known for many years, practical devices to provide electrically activated atomic nitrogen have not been developed. The scope of this project is to evaluate two electrical activation devices and select one of them for subsequent engine tests.

In FY 1993, two electrical activation devices were designed, built and checked out. The first device is a pulsed plasma generator producing a nitrogen corona and the second is an arc discharge device which generates a rotating arc through which nitrogen gas is passed thereby producing atomic nitrogen. No appropriate experimental facility was available before this project was initiated in the Energy Systems Division. Hence a laboratory set-up had to be built almost from scratch. 
Henry $\mathrm{Ng}$ and Vince Novick built up the corona discharge device, with most of the components coming from the Engineering Physics group. The arc discharge device was designed and built by Lyle Hoppie and Raj Sekar. A suitable d.c. power supply was procured. The two devices were installed and Environmental Safety and Health (ES\&H) inspections were completed.

In FY 1994, both the rotating arc and the pulsing devices were used to obtain laboratory data to quantify the $\mathrm{NO}_{x}$ reduction potential of each device. The pulsing system of producing monatomic nitrogen showed $\mathrm{NO}_{\mathrm{x}}$ reduction efficiencies of $55 \%$ to $85 \%$. The arc system employing direct current was better and showed efficiencies of $90 \%$, but the electrodes were burning out after 30 minutes. At this point we concluded that the pulsed arc system offers the best compromise of efficiency and durability.

In FY 1995, the last year of LDRD funding, extensive laboratory data using pulsed arc device were obtained. A Kohler gasoline engine was installed in the laboratory and a limited amount of data were obtained using actual gasoline engine exhaust gases and the monatomic nitrogen device. In addition, a practical spark-plug-like device was designed for use in the engine exhaust manifold. No diesel engine data could be obtained due to lack of funds.

Technical Progress and Results: Most of the objectives set forth for FY 1995 were met. The investigation in this project centers around the following chemical reaction:

$$
2 \mathrm{NO}+2 \mathrm{~N} \rightarrow 2 \mathrm{~N}_{2}+\mathrm{O}_{2}
$$

If this reaction can be made to take place with a practical and economical device attached to an engine exhaust, then the environmental benefits are substantial, since the resultant products are harmless.

In FY 1994 the investigation focussed on collecting data from the devices built in FY 1993. This experimental data will be used in selecting one of the devices and redesign it for engine tests.

A pulsing corona (as compared to a pulsing arc) was obtained but it was too weak to be useful in reducing nitrogen oxides. Therefore all subsequent investigations were done with the arc devices. Both the pulsing arc and rotating arc were shown to be very effective in removing NO and $\mathrm{NO}_{2}$ from a gas stream. For the rotating arc device, over $95 \%$ reduction was observed but this could not be repeated due to premature equipment failure.

During the optimization of the reaction chamber, it was noticed that when the arc hit the side wall instead of the ground electrode, the efficiency was greatly reduced due to the quenching of the reactive nitrogen. Other experiments showed that when nitrogen was replaced with air, no reduction of $\mathrm{NO}$ or $\mathrm{NO}_{2}$ was observed. As far as the electrical power requirement was concerned, it was estimated that $2-5 \mathrm{KW}$ will be needed for a $100 \mathrm{KW}$ vehicle operating with this device. If this device is used in conjunction with oxygen enrichment, then the improved vehicle fuel economy will offset this power requirement.

In FY 1995, a new glass chamber was designed so that the oxygen in the gas stream will not be exposed to the electric field (thus forming undesirable species such as ozone) while the 
reactive nitrogen is delivered to the stream. The test equipment has been moved from building 362 to building 315 . FY 1995 efforts were funded at less than the requested levels. Hence the scope of work was reduced to optimization of the newly designed glass reaction chamber and a limited amount of tests with real exhaust gases from a gasoline engine. Diesel engine tests had to be postponed until additional funding is received. Gasoline engine test data showed conversion efficiencies in the range of $40 \%-45 \%$.

Specific Accomplishments: Several presentations were made to DOE and other potential sponsors and automobile company executives who are interested in $\mathrm{NO}_{\mathrm{x}}$ reduction technologies. The major accomplishment is the collection of experimental data, both in the laboratory test bench and in the engine exhaust. The arc discharge device was entirely designed and built at ANL. With the LDRD support in FY 1995, experimental data were obtained from the test bench with exhaust gases from a gasoline engine. One ANL technical report (ANL-ESD-TM87) and a peer-reviewed conference paper was published in the American Society of Mechanical Engineers conference (ASME-ICE-Vol. 25-1, September 1995). Two patent applications were submitted related to this project. The first patent has been granted and the second one is being evaluated by the patent office. As a result of our deliberate efforts to publish the data one major engine manufacturer has agreed to fund further development of this concept. 
blank page 


\title{
94-167K1 -- WASTE MANAGEMENT OF CHLOROFLUOROCARBONS ${ }^{1}$
}

\author{
Associate Laboratory Director Area: Physical Research \\ Principal Investigators: \\ J.V. Michael and A.F. Wagner, \\ Chemistry Division \\ H.S. Huang, Energy Systems Division
}

Funding Profile:

FY $1994-0-$

FY $1994 \$ 133.6 \mathrm{~K}$

FY $1995 \$ 137.5 \mathrm{~K}$

FY $1996-0-$

FY $1997-0-$

Purpose: The purpose of this work is to further define the chemistry of chlorofluorocarbon (CFC) incineration and to develop practical methods for efficient destruction of these materials or for the conversion to less harmful and/or perhaps more valuable compounds. We measured for the first time the thermal decomposition rate constants for CFC-11, CFC-12, and CFC-22, we applied modern electronic structure and chemical kinetics calculations to understand and generalize these results, and we are conducting incineration experiments on CFC-11 and 12 that are guided by kinetics models which we have developed. During the course of this work, we have discovered a potential mechanism that could lead to the conversion of waste CFCs by pyrolysis and/or incineration into useful chemicals (e.g., Teflon precursors), and this idea will be tested by Wangtech, Inc., a manufacturer of commercial incinerators.

Approach: Incineration is one of the most cost effective methods of disposing of large volumes chemical wastes, but environmental concerns over emissions makes incineration a controversial strategy. It has clearly been shown that the nature and yield of organic emissions from waste incinerators is controlled by chemical kinetics. This is certainly true in the case of two CFC compounds. To optimize complete destruction of these hazardous compounds, chemical models of the incineration process are needed. Such models should be based on experimental data for elementary reactions but at the same time properly describe the performance of practical incinerators. While the chemical mechanisms for destruction of CFCs at the temperatures needed for incineration are not well known, considerable progress at the elementary reaction level in both theory and experiment has occurred in recent years. At the same time, novel incineration devices, such as low-temperature plasma furnaces, are being investigated as alternatives to conventional high temperature combustors.

The three objectives of this work have been: (1) Selected unimolecular dissociation rate constants for some important chlorocarbon molecules were to be measured. (2) These processes would then be theoretically analyzed with the intent being to understand the thermochemical properties of the species involved in the reactions. Also, the experience with theory would then create confidence so that extrapolations to other molecular systems could be made thereby allowing the development of an experimental/theoretical database. (3) With the information so obtained,

${ }^{1}$ CCST Project 
chemical models could be constructed for use in real incinerator applications, and these models were to be tested in experiments performed with a low-temperature plasma furnace incinerator.

Before this work, there were almost no results available for either bimolecular rate constants or unimolecular dissociation processes at the temperatures (800-1500 K) and pressures $(1 \mathrm{~atm})$ used in conventional incinerators. To be sure, work on $\mathrm{OH}$ with several chlorocarbons has appeared, and $\mathrm{O}$-atom studies with the $\mathrm{CH}_{3} \mathrm{Cl}, \mathrm{CH}_{2} \mathrm{Cl}_{2}$, and $\mathrm{CHCl}_{3}$ are complete; however, the rate behavior is strongly non-Arrhenius for all reactions, and this means that extrapolation beyond the T-range of a particular study is not reliable. There are also three studies of note on O-atom and $\mathrm{OH}$-radical with unsaturated $\mathrm{CHC}$ molecules. There are a number of $\mathrm{Cl}$-atom with organic substrate molecule studies. In all cases the mechanism involves simple H-atom abstraction. Most of this work has been carried out in support of atmospheric chemistry and therefore does not extend to the incinerator temperature range. Again, the rate data appear to show strong curvature in Arrhenius plots making new higher temperature studies on this type of reaction to be imperative. Thermal dissociation rate data are also not extensive. There is one report on the thermal decomposition of $\mathrm{CH}_{3} \mathrm{Cl}$, and, due to the work of one of the PIs (Michael), new direct studies have been completed on the decompositions of $\mathrm{CH}_{3} \mathrm{Cl}, \mathrm{CCl}_{4}, \mathrm{CF}_{3} \mathrm{Cl}, \mathrm{COCl}_{2}$, and $\mathrm{CH}_{2} \mathrm{Cl}_{2}$, using the atomic resonance absorption spectrometric method (ARAS) for $\mathrm{Cl}$-atom detection. Before this work, nothing was known about the thermal decompositions of aliphatic chlorocarbon molecules and almost nothing was known about the thermal dissociation of chlorofluorocarbons.

Although primary thermochemical and kinetics rate constants must come from experiment, chemical kinetics and electronic structure theory can play an important role in expanding measurements on a few reactions into a more extensive database for modeling practical devices. In the last decade, the development of both variational versions of conventional statistical theories (e.g., transition state theory and RRKM theory) and accurate approximations to tunneling have provided the framework for a near quantitative understanding of non-Arrhenius curvature in rate constants. At the same time, developments in computer technology and electronic structure theory have made it feasible to calculate features of the potential energy surfaces for reactions that directly translate into thermochemical properties (e.g., heats of formation) or govern reaction rates (e.g., reaction barrier heights) within the variational kinetics theories. Most of this effort has been directed toward hydrocarbon combustion. One of the PIs (Wagner) has been extensively involved in this work. Recently this type of theoretical analysis been applied to halocarbon chemistry with preliminary studies of $\mathrm{CH}_{3} \mathrm{Cl}$ and $\mathrm{CH}_{3} \mathrm{~F}$ dissociation. Extension of this work to CFC thermal dissociations, with a calibration of the theory to critical experiments, has been the second objective of the proposal.

Low temperature (non-equilibrium) plasma destruction is a promising alternative technology for treating CFC compounds. This technology has several advantages over conventional thermal and/or catalytic oxidation processes: operates at ambient temperature; eliminates sensitivity to catalyst poisoning by halogen compounds; requires low maintenance; compact and low cost; can be easily retrofitted to existing equipment.

To further advance this technology an improved understanding of the chemical kinetics steps in the reaction mechanism is required. At Argonne, low-temperature plasma reaction systems have been constructed. Microwave-based systems have been tested for hydrogen sulfide dissociation 
and for evaluating the destruction efficiency of volatile chlorinated organic compounds (e.g., trichloroethylene and trichloroethane). This equipment has been used in the present work.

Technical Progress and Results: The first objective of this work was the measurement of accurate unimolecular rate constants for chlorofluorocarbon molecular dissociations at high temperatures. We used the atomic resonance absorption spectrometric (ARAS) shock tube technique and facility in the Dynamics in the Gas Phase Experimental Group in Chemistry Division at $\mathrm{ANL}$ for measuring $\mathrm{Cl}$-atom formation rates in thermal decomposition studies of $\mathrm{CF}_{2} \mathrm{Cl}_{2}$ (CFC-12), $\mathrm{CFCl}_{3}$ (CFC-11), and $\mathrm{CF}_{2} \mathrm{HCl}$ (HCFC-22). Three papers, one published and two in press, have resulted with the fourth being about $75 \%$ complete. The results for $\mathrm{CF}_{2} \mathrm{Cl}_{2}$ and $\mathrm{CFCl}_{3}$ are shown in Figs. 1 and 2, respectively. Yield measurements in these molecules (2.0 in both cases) indicate that molecular elimination to $\mathrm{CF}_{2}+\mathrm{Cl}_{2}$ or $\mathrm{CFCl}+\mathrm{Cl}_{2}$, respectively, does not occur. Hence, we are able to say with confidence that the primary reactions give $\mathrm{CF}_{2} \mathrm{Cl}+\mathrm{Cl}$ and $\mathrm{CFCl}_{2}+\mathrm{Cl}$, respectively, followed by the subsequent fast decompositions, $\mathrm{CF}_{2} \mathrm{Cl}$ " $\mathrm{CF}_{2}+\mathrm{Cl}$ or $\mathrm{CFCl}_{2}$ " $\mathrm{CFCl}+\mathrm{Cl}$. In the case of $\mathrm{CFCl}_{3}$, we do however note a slow $\mathrm{Cl}$-atom formation rate that drives the yield to values greater than two at longer times. This is attributed to the unimolecular decomposition, $\mathrm{CFCl}$ " $\mathrm{CF}+\mathrm{Cl}$, at very high temperatures with a lower temperature bimolecular contribution being $\mathrm{CFCl}+\mathrm{CFCl}$ " $\mathrm{C}_{2} \mathrm{~F}_{2}+2 \mathrm{Cl}$. With this scheme, rate constants for the bimolecular reaction have been determined. We have finished experimental investigations on $\mathrm{CF}_{2} \mathrm{HCl}$. We developed a multi-pass optical system and have applied it to sensitively detect $\mathrm{CF}_{2}$-radicals. Using the $\mathrm{CF}_{2} \mathrm{Cl}_{2}$ decomposition, a curve-of-growth for $\mathrm{CF}_{2}$ optical absorption was established. Yield measurements for $\mathrm{CF}_{2}$ in $\mathrm{CF}_{2} \mathrm{HCl}$ showed unambiguously that decomposition is entirely by molecular elimination, $\mathrm{CF}_{2} \mathrm{HCl}$ " $\mathrm{CF}_{2}+\mathrm{HCl}$. We also directly observed $\mathrm{Cl}$-atom formation and found it to be negligible. The rate constant results were additionally measured, and these are presented in Fig. 3.

The second objective of this work is theoretical analysis of CFC dissociations. We have collaborated with Dr. David A. Dixon, DuPont Central Research and Development Experimental Station, Wilmington, DE to determine thermodynamic properties for a variety of radical species that are intermediates in the destruction process. He is an expert in electronic structure calculations. Dr. L.B. Harding of our group has also participated in this effort. Transition state structures and potential energy surfaces were theoretically determined, and subsequent dynamics calculations, using RRKM (Rice-Ramsperger-Kassel-Marcus) unimolecular reaction rate theory, were then carried out giving thermal rate constant predictions for all three chlorofluoromethanes. The third objective of this work is the development of a model for the CFC plasma incineration that is verified by measurements. In collaboration with K.P. Lim, a fixed term staff member in $\mathrm{ES}$, and $\mathrm{T}$. Krause of CMT, decomposition of CFC-12 in a low-temperature plasma incinerator has been achieved, and experiments on the decomposition of CFC-11 are in progress. Detailed kinetics modeling of the complex CFC destruction process is being developed. Finally, we have interested Dr. Chi S. Wang, President of Wangtech, Inc., in pursuing the commercial development of this technology with his commercially available incinerators. 


\section{Specific Accomplishments:}

Refereed publications:

Thermal Decomposition of $\mathrm{CF}_{2} \mathrm{Cl}_{2}$, S.S. Kumaran, K.P. Lim, J.V. Michael, and A.F. Wagner, J. Phys. Chem. 99, 8673 (1995).

Thermal Decomposition of $\mathrm{CFCl}_{3}$, S.S. Kumaran, M.-C. Su, K.P. Lim, J.V. Michael, and A.F. Wagner, J. Phys. Chem., in press.

Ab Initio Calculations and Three Different Applications of Unimolecular Rate Theory for the Dissociations of $\mathrm{CCl}_{4}, \mathrm{CFCl}_{3}, \mathrm{CF}_{2} \mathrm{Cl}_{2}$, and $\mathrm{CF}_{3} \mathrm{Cl}$, S.S. Kumaran, M.-C. Su, K.P. Lim, J.V. Michael, A.F. Wagner, and D.A. Dixon, J. Phys. Chem., in press.

Thermal Decomposition of $\mathrm{CF}_{2} \mathrm{HCl}$, M.-C. Su, S.S. Kumaran, K.P. Lim, J.V. Michael, and A.F. Wagner, in preparation.

\section{Talks:}

Thermal Decompositions of Chlorofluorocarbon Molecules, S.S. Kumaran, K.P. Lim, and J.V. Michael, 1994 Annual AICHE Meeting, Nov. 13-18, 1994, San Francisco.

Thermal Decompositions of $\mathrm{CF}_{2} \mathrm{HCl}$ and $\mathrm{CF}_{2} \mathrm{Cl}_{2}$ by Multi-Pass Optical Detection of $\mathrm{CF}$ Radicals, S.S. Kumaran, M.-C. Su, K.P. Lim, J.V. Michael, and A.F. Wagner, 1995 Annual AICHE Meeting, Nov. 12-17, 1995, Miami Beach. 


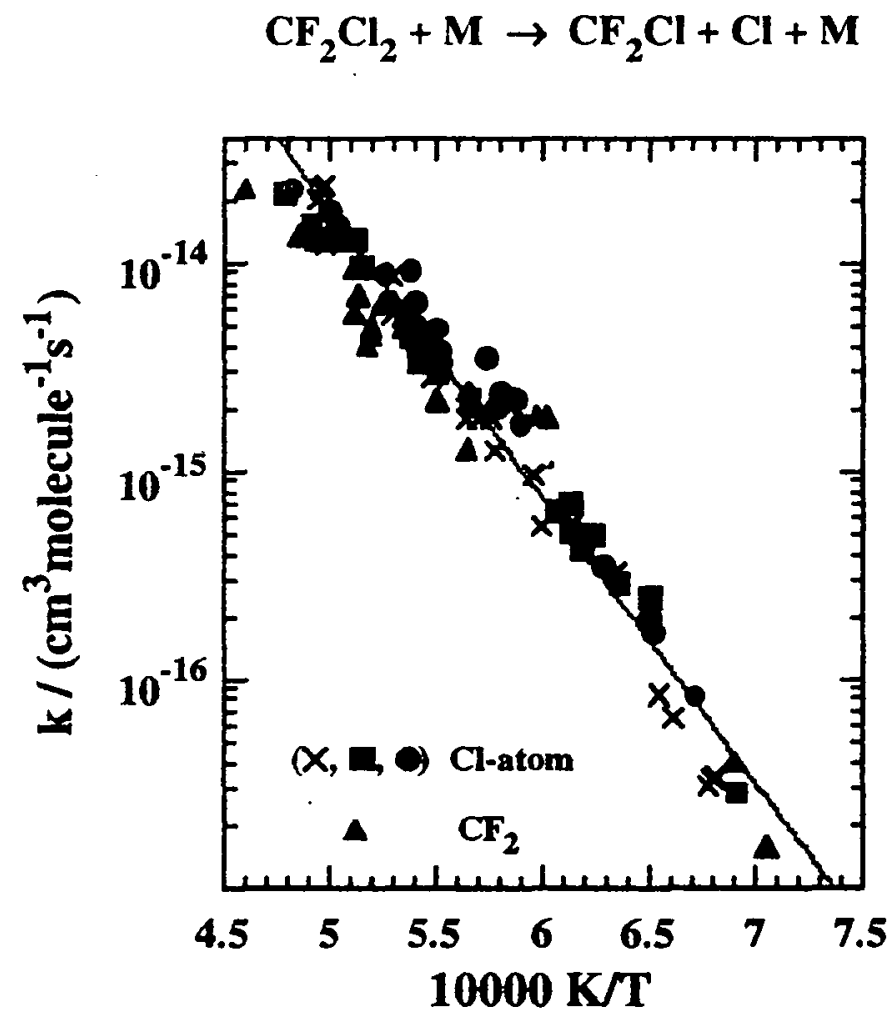

Figure 1

$$
\mathrm{CF}_{2} \mathrm{HCl}+\mathrm{M} \rightarrow \mathrm{CF}_{2}+\mathrm{HCl}+\mathrm{M}
$$

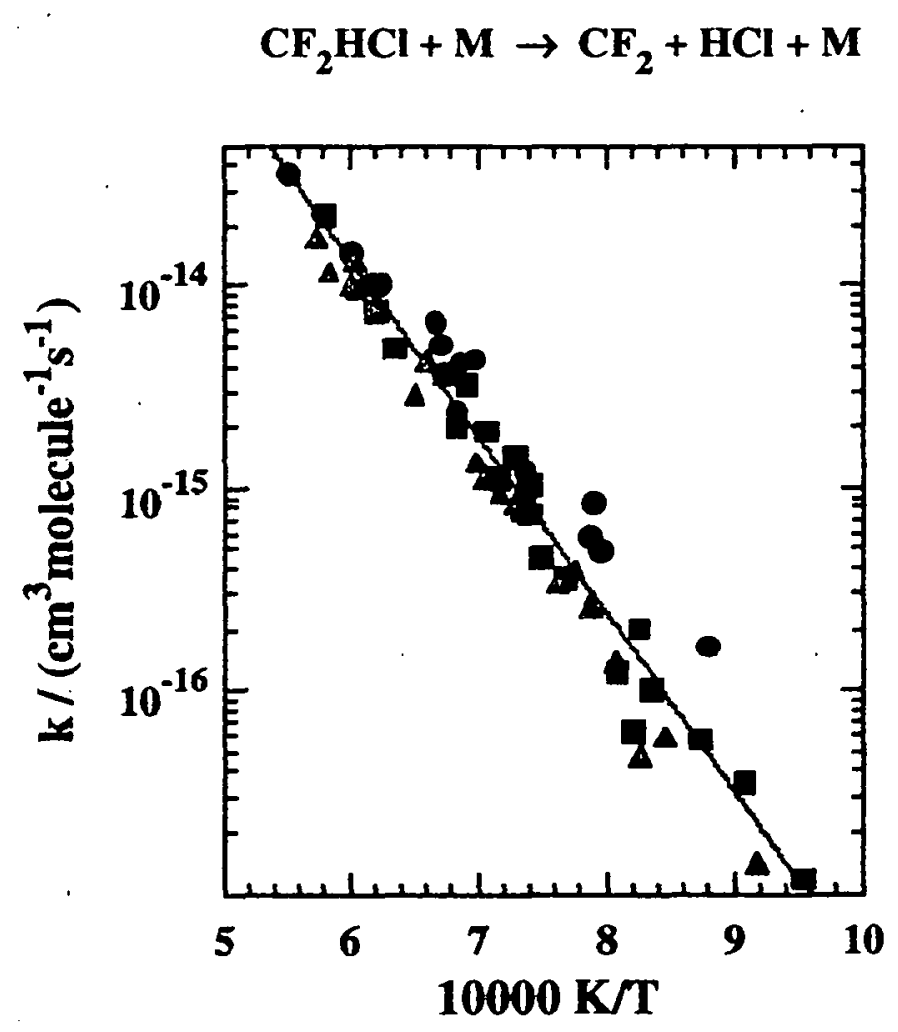

Figure 3

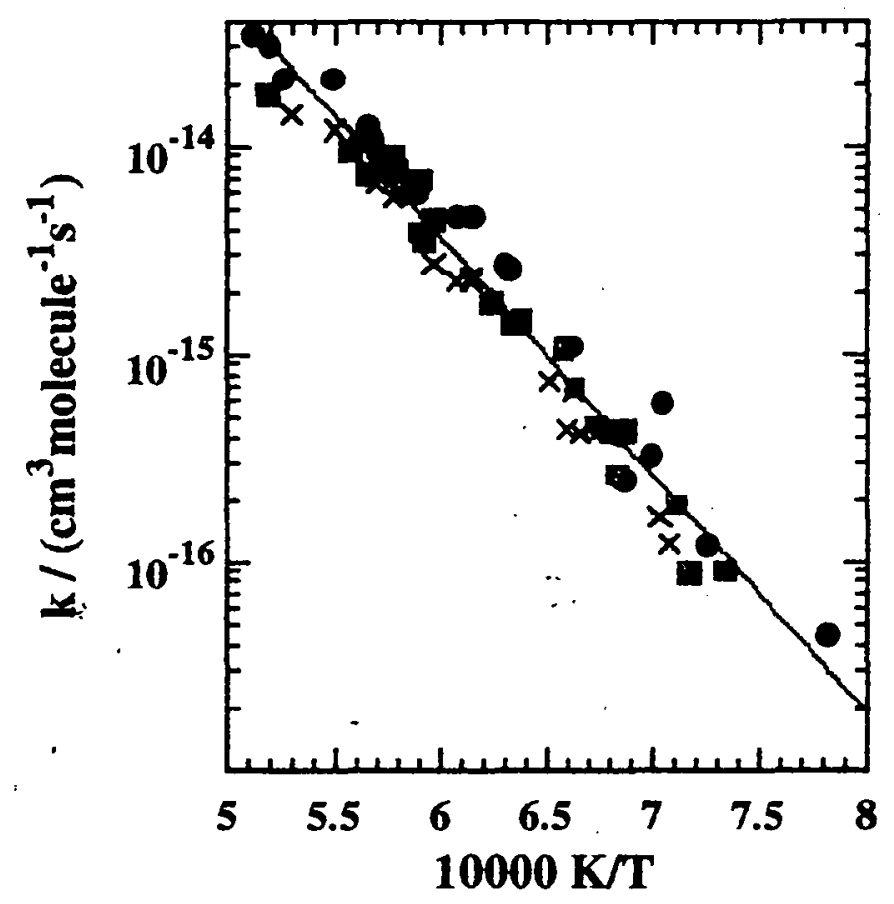

Figure 2 


\section{blank page}




\title{
95-075N -- NEW TECHNOLOGY FOR REMOVAL OF HEAVY NOBLE GASES FROM ARGON AND FROM AIR
}

\author{
Associate Laboratory Director Area: Engineering Research \\ Principal Investigators: \\ Funding Profile:

FY $1993-0-$
FY $1994-0-$
FY $1995 \$ 244.3 \mathrm{~K}$
FY $1996-0-$
FY $1997-0-$

K.C. Gross, J.D. Gabor, K.V. Liu, and

R.E. Nietert, Reactor Analysis Division

F. Markun, Chemical Technology Division

Purpose: To evaluate and select optimal performance parameters for an oil-based apparatus for continuous removal and isolation of radioactive noble gases from the Fuel Cycle Facility of the EBR-II, and radon gas for decontamination and decommissioning (D\&D) of Building 200 hot cells at Argonne East. Performance parameters include breakthrough times, saturation capacities, Henry's Law coefficients, and absorption and degassing rates measured while varying the concentration of noble gases in the inlet gas stream. The apparatus conceived and developed during this LDRD project may replace costly and complex cryogenic charcoal technology for removal of fission gases from the FCF. A second important use of the apparatus is for mitigation of radon gas from DOE hot cell and glove box facilities, and removal of radon from homes, schools, office buildings, and mines.

Approach: The Reactor Analysis division recently identified a phenomenon that offers the possibility of heavy noble gas recovery (Xe, $\mathrm{Kr}$, or $\mathrm{Rn}$ ) from the atmosphere with high efficiency at room temperature, and also permits release at slightly elevated temperatures (50$60^{\circ} \mathrm{C}$ ). This discovery has led to the design of a simple and inexpensive apparatus for continuous separation and concentration of heavy noble gases from air or from a mixture of lighter noble gases (hot cell atmospheres). Such a device offers numerous substantial advantages over costly and complex cryogenic charcoal systems for the removal of radon, xenon, or krypton during D\&D of reactors, hot cells, and other DOE nuclear facilities. Initial proof-of-principle experiments have demonstrated that the process works well with eighteen oils, including four that are biologically benign and cost less than $\$ 1 /$ liter. Experiments have also demonstrated that the process is quite scalable. Small units can be designed for an individual room; intermediate units for homes; and larger, packed column configurations can be designed for schools, offices, mining applications, or facility and site D\&D applications.

The specific objective of this project was to establish feasibility, and to evaluate and select optimal performance parameters for a fluid-based apparatus for removal and isolation of radon from DOE facilities. The approach has been to use controlled sources of Rn in CMT's Radon Research Laboratory to quantitatively assess the performance of a selected class of oils over a range of Rn concentrations prototypic of DOE facility concentrations, and to select and evaluate various system performance parameters. Degassing methods compared included thermal, mechanical agitation, and ultrasound. 
Technical Progress and Results: Experiments conducted to date have demonstrated the ability to concentrate radon at room temperature $\left(20-22^{\circ} \mathrm{C}\right)$. In tests of gas concentration, prototype units have been tested that use a simple bubbling mechanism (by forcing the air through a diffuser at the bottom of the absorption chamber). Or, for surface contact enhancement while minimizing the pressure drop, packed-column units have been designed and tested wherein the air is passed through a vertical plastic tube containing various sizes of glass beads or raschig rings. Laboratory experiments conducted with ambient $\mathrm{Rn}$ have demonstrated the ability to continuously remove and concentrate $\mathrm{Rn}$ gas from the air when it is present at a concentration of only $10^{6}$ atoms per liter.

For degassing of the fluid, a sequence of experiments has been conducted which demonstrate that the working fluid can be purged effectively by several methods. We have demonstrated that warming of the fluid immediately releases $>99 \%$ of the absorbed noble gas. This makes possible construction of a simple apparatus for continuous concentration where the carrier gas passes through an absorption chamber at room temperature, and the working fluid is continuously circulated through a degassing chamber for warming. The desorbed noble gas is drawn into an evacuated steel storage cylinder that is replaced periodically (for radioactive-gas remediation), or into an appropriate counting chamber (for high-sensitivity alpha, beta, or gamma detection), or simply to an outside exhaust line (for home Rn mitigation). The working fluid is passed through a coil in a finned radiator as it is returned to the absorption chamber. Alternatively, for large-scale remediation applications where energy minimization is not a constraint, water chilling or mechanical cooling may be employed. In addition to warming, we have found that continuous degassing may be achieved with mechanical agitation, ultrasound, microwaves, and by passing the working fluid over a surface as a thin film.

Spinoff applications of this system include: Reduction of DOE hot cell emissions and personnel exposures; remediation of DOE site radon problems (e.g. at Fernald, where we have been awarded a contract for $\$ 280 \mathrm{~K}$ to demonstrate feasibility for large-scale $\mathrm{Rn}$ remediation); recovery and isolation of radioactive $\mathrm{Xe} \& \mathrm{Kr}$ fission gases (DOE, DoD facilities, NRC licensees); low cost radon mitigation in subsurface mines; commercial production of noble gases from air (avoiding the costly cryogenic distillation methods currently in use); gas tagging for commercial nuclear fuel rods; improved integrating $\mathrm{Rn}$ detector for earthquake prediction; and an ultrahigh sensitivity $\mathrm{Xe}$ and $\mathrm{Kr}$ radiation detector.

Specific Accomplishments: Effort is continuing under the auspices of DOE-EM, DOE-NE, and U.S. EPA.

"Method of Separating Heavy Noble Gases from Air," K.C. Gross, M.T. Laug, and J.C. Braun, Case No. ANL-IN-93-104B (Patent application pending).

"Device for Removal of Radon and Other Heavy Noble Gases from Air," J.C. Braun, K.C. Gross and D.J. Hill, Case No. $A N L-I N-94-057$. (Invention disclosure filed).

"Ultrahigh Sensitivity Environmental Radiation Detection Apparatus," K.C. Gross, F. Markun, M. Zawadzki, and C. Dickerman (Invention disclosure filed, May 1995). 
"Fluid-Clathrate System for Continuous Removal of Heavy Noble Gases from Mixtures of Lighter Gases," K.C. Gross, F. Markun, and M. Zawadzki, Case No. ANL-IN-95-114 (Patent application filed 10/95).

K.V. Liu, J.D: Gabor, and R.E. Holtz, "Fluid-Based Radon Mitigation Technology Development for Decontamination and Decommissioning," Proc. 4th Int. Conf. on Nuclear Engineering, New Orleans, La. (March 10-14, 1996).

K.C. Gross (Principal Investigator), "Fluid-Based System for Radon Mitigation at Fernald," DOE-EM Technical Task Plan CH2-6-LF-51 (\$280K awarded for FY96). 
blank page 


\title{
95-071N -- RISK EVALUATION OF NUCLEAR WASTE - UNCERTAINTY AND SENSITIVITY ANALYSES
}

\author{
Associate Laboratory Director Area: Engineering Research \\ Principal Investigators: \\ R.N. Hill, G. Palmiotti, \\ and J. Roglans, \\ Reactor Analysis Division \\ Funding Profile: \\ $\begin{array}{lll}\text { FY } & 1993-0- \\ \text { FY } & 1994 & -0- \\ \text { FY } 1995 & \$ 180.2 \mathrm{~K} \\ \text { FY } & 1996 & \$ 200.0 \mathrm{~K} \\ \text { FY } & 1997 \$ 200.0 \mathrm{~K}\end{array}$
}

Purpose: The project objective is to develop a methodology for evaluating the uncertainty of the radiological risk associated with waste forms resulting from the nuclear fuel cycle, and to adapt existing repository performance models to evaluate alternative waste forms. The impact of a wide variety of data uncertainties will be evaluated using time-dependent sensitivity techniques. The sensitivity and uncertainty analyses will be performed for a variety of potential fuel cycle and waste form scenarios.

Approach: The secure and timely disposal of nuclear waste has been the subject of intense debate in recent years; the primary source of waste material is the spent fuel from existing nuclear reactors. Because of various siting difficulties, the schedule for operation of the geological repository has been delayed; and numerous ideas for alternate and/or complementary waste management strategies have been proposed. In general, these proposals involve processing of the spent fuel with two primary goals: first, the most toxic isotopes are separated for destruction ("burning") or monitored storage elsewhere; second, the remaining waste material is processed into a new form specially engineered for geological disposal.

The spent fuel waste consists of radioactive fission product and actinide isotopes. Fission products comprise hundreds of various isotopes with differing decay characteristics. Actinide isotopes include the non-transmuted portion of the fuel material (the vast majority of the initial fuel loading) as well as higher actinides produced by neutron capture. Hazard analyses assess the consequences of exposure to the radioactive isotopes; thus, hazard measures (also called radiotoxicity) evaluate the biological retention/concentration of the various isotopes and the damage caused by decay particles. The impact of data uncertainties on radiotoxicity has been addressed previously by the principal investigators (Palmiotti, NSE, 1994) using time-dependent sensitivity techniques.

A comprehensive risk analysis requires a detailed modeling beyond the consequence (hazard) analysis. The mechanisms for radionuclide release must be identified, and their probability for occurrence specified. This transport is commonly sub-divided into two zones: the 'near-field' where the waste form and package are barriers, and the 'far-field' where migration is dictated by geochemical and ground water hydrology factors. Near-field analysis is complicated by the wide variation of chemical properties (e.g., water solubility) for the hazardous species yielding 
a wide range of release fractions; and far-field analysis is complicated by the potential variability of the disposal environment on geologic time scales. In addition to radiotoxicity release concerns, questions regarding the potential for nuclear criticality in the disposed state have been raised recently. Both the material behavior required to achieve critical configurations and the consequences of criticality in a repository are not well understood.

The project methodology involves the development of tools to assess alternative waste forms. In addition, significant model development and simplification is necessary to achieve a form which is amenable to sensitivity and uncertainty analysis. Time-dependent sensitivity techniques will be applied for the resulting risk model; and the risk uncertainty and sensitivity will be evaluated for a variety of potential fuel cycle and waste form scenarios.

Technical Progress and Results: The state-of-the-art repository performance tools utilize PRA methods (the EPRI code IMARC) or Monte Carlo methods based on probability distribution functions (the Golder code RIP). However, both codes have only been used to model a single waste form (LWR spent fuel) at a specific repository site (Yucca Mountain). These probabilistic total repository system performance assessment models were modified to analyze the release of radionuclides from the mineral and metallic wasteforms that would arise from the pyrometallurgical processing of spent nuclear fuel. The models were developed using the most recent information from the proposed Yucca Mountain repository site and preliminary laboratory leach rate test results for the mineral and metallic wasteforms.

Various fuel cycle scenarios involving pyrometallurgical processing were investigated; in particular, a closed LMR fuel cycle and front-end processing of LWR spent fuel were evaluated. The repository performance results (time-dependent radionuclide transport and release) were compared with those of directly disposed light water reactor spent nuclear fuel. It was found that by removing the actinides from the waste stream (through pyrometallurgical processing), the probability of releasing large amounts of these radionuclides to the environment is reduced significantly as compared to directly disposed light water reactor spent fuel. These studies demonstrated that probabilistic performance assessment models can be developed to analyze the pyrometallurgical process wasteforms using existing tools.

A wide range of parametric studies were performed for the repository performance of the mineral and metallic waste forms. These studies included: variations in the ground water transport time and flow mode, matrix alteration rate, waste container performance parameters, and wasteform design parameters. These analyses demonstrated that the mode of radionuclide transport in flowing ground water (fracture or matrix transport) is a key factor influencing the release characteristics from a geologic repository. An effort is underway to develop a transport model which better accounts for fracture flow. In addition, the transport model is being further modified to account for radio-colloid transport, which has yet to be included in any performance assessment effort.

Concurrently, sensitivity models for the radionuclide migration are being developed; and efforts to specify the data uncertainties have been initiated. The detailed repository performance assessment results will be reflected in these simplified models. The computer code written for uncertainty and sensitivity evaluation of the radionuclide hazard is being modified to perform the risk evaluation. 
The proposed focus for FY1996 continuation of this project is to proceed with the sensitivity and uncertainty analyses of the radiological risk. A variety of fuel cycle and disposal scenarios will be evaluated in a consistent manner. In addition, the repository performance assessment modeling improvements discussed above will be implemented; and the issue of criticality in the repository environment will be investigated.

Specific Accomplishments: A Doctoral dissertation titled "Performance Assessment Modeling of Alternative High Level Nuclear Wasteforms" was completed in partial fulfillment of a $\mathrm{PhD}$ degree (W.M. Nutt, lowa State University) that presents the performance assessment models, the results, and a sensitivity analysis. A technical summary "Performance Assessment Modeling of High-Level Nuclear Wasteforms from the Pyroprocess Fuel Cycle" describing the results obtained was prepared for the 1995 High Level Radioactive Waste Management Conference. The detailed results were presented in Las Vegas, Nevada on May 2, 1995. A technical paper regarding the repository performance of alternate waste forms is being developed for submittal to Waste Management. 
blank page 


\section{5-051N -- EVALUATION OF TRU-BEARING DISPOSAL ALI.OYS}

Associate Laboratory Director Area: Engineering Research

\section{Principal Investigators:}

Funding Profile:

\author{
S.M. McDeavitt, Chemical Technology Division \\ D.D. Keiser, Engineering Division
}

Purpose: The objective of this project is to evaluate the metallurgical behavior of actinide metals, especially plutonium, in stainless steel-zirconium (SS-Zr) alloys. This project was initiated to investigate options for the stabilization and disposition of actinide metals in SS-Zr alloy waste forms. The two source streams of actinide metals that must be stabilized for disposition are (1) uranium and transuranic elements (TRU) refined from spent nuclear fuel by electrometallurgical treatment and (2) surplus plutonium metal extracted from dismantled nuclear weapons. The validation of SS-Zr alloys for use as actinide-bearing waste forms could result in their selection over alternatives for actinide disposition being evaluated by Argonne National Laboratory (ANL) and other national laboratories.

Approach: The SS-Zr alloys are of interest because they have already been selected as metal waste forms for the disposal of metallic wastes from the electrometallurgical treatment of spent nuclear fuel. Two nominal waste form compositions have been selected: (1) stainless steel-15 wt \% zirconium (SS-15Zr) for stainless steel clad fuels and (2) Zircaloy-8 wt \% stainless steel ( $\mathrm{Zr}-8 \mathrm{SS}$ ) for Zircaloy fuels. In addition, alloying and characterization methods for these baseline alloys are currently being developed. Therefore, baseline data already exist. [Interactions with D. P. Abraham (CMT) and J.Y. Park (ET) from with non-TRU alloys waste form development program have been helpful for baseline comparisons.]

The focus of this first year was to generate SS-15Zr and Zr-8SS alloys with different actinide contents to characterize the alloy microstructures. Nine specimens of small-scale simulated waste form alloys $(\sim 30 \mathrm{~g})$ with uranium, plutonium, and neptunium were melted in yttria crucibles in the Plutonium Casting Laboratory (PCL) at ANL-West. Two specimens were made per furnace run, and the melting procedure involved heating the furnace to $1600^{\circ} \mathrm{C}$ for $2 \mathrm{~h}$ with a flowing argon atmosphere, and then cooling slowly. The compositions (in wt \%) include six SS- $15 \mathrm{Zr}$ alloys with $0.5 \mathrm{U}-0.5 \mathrm{Pu}, 2 \mathrm{U}-2 \mathrm{Pu}, 6 \mathrm{Pu}, 10 \mathrm{Pu}, 6 \mathrm{Pu}-2 \mathrm{~Np}$, and $2 \mathrm{~Np}$ and three $\mathrm{Zr}-8 \mathrm{SS}$ alloys with 4,7 , and $10 \mathrm{Pu}$. The samples were sectioned, polished, and examined by scanning electron microscopy and energy dispersive $\mathrm{X}$-ray spectroscopy using equipment in the Alpha-Gamma Hot Cells located in Building 212, ANL.

Technical Progress and Results: A representative microstructure for the SS-15Zr alloys containing transuranics is shown in Fig. 1. The microstructure is similar to the baseline SS-15Zr structure except for the presence of a third phase (bright contrast in Fig. 1) that is rich in 
actinides. The dark phase is an iron solid solution, and the medium contrast phase is an intermetallic phase designated as $\mathrm{Zr}(\mathrm{Fe}, \mathrm{Cr}, \mathrm{Ni})_{2}$. These phase compositions are nearly identical to corresponding phases in the non-TRU samples except that 2 atom $\% \mathrm{Pu}$ is observed in the intermetallic, and negligible actinides are present in the iron solid solution phase. The bright phases in Fig. 1 contain up to 20 atom \% Pu in the SS-15Zr-10Pu specimen. This third phase is apparently an intermetallic phase that is miscible with the $\mathrm{Zr}(\mathrm{Fe}, \mathrm{Cr}, \mathrm{Ni})_{2}$ intermetallic; its composition is 33 atom \% Fe-33 atom \% Ni-20 atom \% actinide (U, Pu, and/or Np) plus small amounts of $\mathrm{Zr}, \mathrm{Cr}$, and minor components. This phase was observed in all of the SS-15Zr alloys with TRU additions, irrespective of the actinide or group of actinides present. Increasing the actinide content resulted in a higher volume fraction of this phase.

A representative microstructure for the $\mathrm{Zr}-8 \mathrm{SS}-\mathrm{xPu}$ alloys is shown in Fig. 2; the microstructure also resembles the corresponding baseline alloy structure. In the baseline $\mathrm{Zr}$-8SS structure, the primary phase is an $\alpha$ - $\mathrm{Zr}$ solid solution phase with $\sim 95$ atom $\% \mathrm{Zr}$ and minor amounts of other alloy constituents. The primary phase is surrounded by a eutectic network of intermetallic compounds ( $\mathrm{Fe}-\mathrm{Zr}$ and $\mathrm{Ni}-\mathrm{Zr}$ ) and secondary $\alpha-\mathrm{Zr}$ solutions. The added $\mathrm{Pu}$ in the present alloys (Fig. 2) was found to be present in the $\alpha$-Zr solid solution phase, with negligible amounts in the intermetallics. In addition, $\mathrm{Pu}$ was observed in higher concentrations at the $\alpha$-phase lath boundaries (bright features in Fig. 2). This Pu segregation can be explained. The $\beta$-Zr phase has a significantly higher solubility for $\mathrm{Pu}$ than $\alpha-\mathrm{Zr}$ since the high-temperature $\beta-\mathrm{Zr}$ and $\epsilon-\mathrm{Pu}$ phases are completely miscible. A likely scenario is that $\beta-\mathrm{Zr}$ metal precipitates with up to $100 \%$ of the $\mathrm{Pu}$ in solution, but as it transforms during cooling to $\alpha-\mathrm{Zr}$ at $\sim 863^{\circ} \mathrm{C}$, Pu becomes concentrated at the newly formed boundaries. For the compositions that were examined, increasing $\mathrm{Pu}$ content of the alloy resulted in higher amounts of $\mathrm{Pu}$ in both the $\alpha-\mathrm{Zr}$ matrix phase and at the lath boundaries.

From the information generated this past year, we have concluded that both the SS-15Zr and the Zr-8SS alloys have promise as actinide-bearing waste forms. In both alloys, actinides are intimately mixed with the other alloying components and do not form pure actinide metal phases (the highest concentration observed for any actinide-rich phase was -20 atom $\%$ ). The elevated level of $\mathrm{Pu}$ at the zirconium lath boundaries does not represent an equilibrium structure, so further work will explore homogenization of the alloy; similar investigations will be pursued for SS-15Zr alloys. In addition, the effectiveness of these alloys as waste forms will be evaluated by leach testing and electrochemical corrosion testing. Experiments show that baseline SS-15Zr and $\mathrm{Zr}$-8SS waste form alloys have superior corrosion resistance. The performance of the actinide-bearing alloys will be evaluated using the test methods developed for the non-TRU metal waste alloys. 
Specific Accomplishments: This work contributed to the following publications:

\section{Conference presentation given:}

S.M. McDeavitt, T.R. Johnson, R.P. Jaqmin, R.N. Hill, and D.D. Keiser, "A Zirconium Metal Alloy for the Immobilization of Surplus Fissile Materials," 19th Annual Actinide Separations Conference, Monterey, California, June 12-15, 1995.

\section{Conference papers accepted/submitted:}

D.D. Keiser and S.M. McDeavitt, "Actinide-Containing Metal Disposition Alloys," DOE Spent Nuclear Fuel \& Fissile Material Management, Embedded Topical Meeting Sponsored by the American Nuclear Society, Reno, Nevada, June 16-20, 1996.

S.M. McDeavitt, D.P. Abraham, D.D. Keiser, and J.Y. Park, "Stainless Steel-Zirconium Alloy Waste Forms," SPECTRUM '96 International Conference on Nuclear and Hazardous Waste Management, Sponsored by the American Nuclear Society, Seattle, Washington, August 18-23, 1996.

S.M. McDeavitt, D.P. Abraham, and D.D. Keiser, "Alloy Waste Forms for Metal Fission Products and Actinides Isolated by Spent Nuclear Fuel Treatment," Second International Symposium on Extraction and Processing for the Treatment and Minimization of Wastes, Sponsored by the Extraction and Processing Division of TMS, Scottsdale, Arizona, October 27-30, 1996.

\section{Patent Application:}

In addition, this work contributed (in a small way) to the advancement of a concept disclosed for patent approval concerning the self-protection of plutonium waste form alloys by neutrons (Invention No. ANL-IN-94-117). 


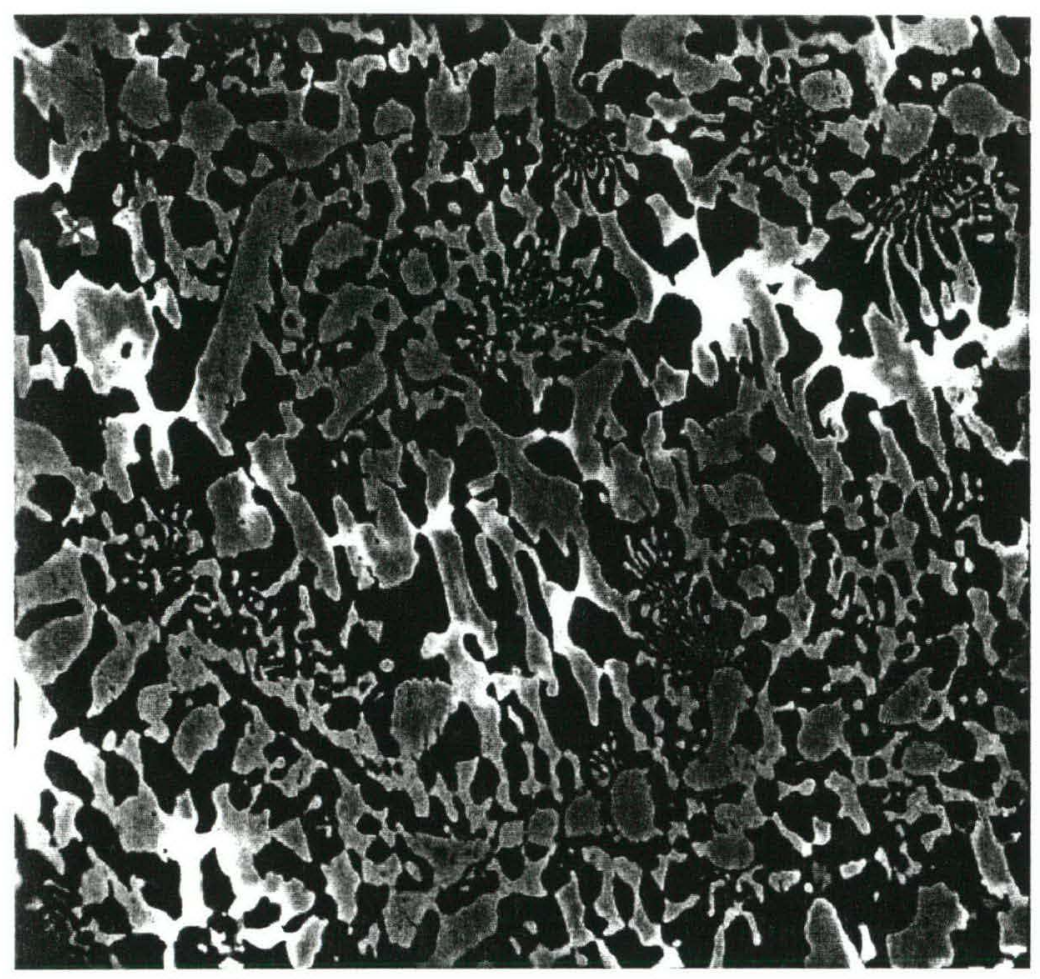

Fig. 1. Backscattered Electron Image of SS-15Zr Alloy Containing 2 wt $\% \mathrm{U}$ and 2 wt $\% \mathrm{Pu}$; the Bright Contrast Phases are Rich in $\mathrm{U}$ and $\mathrm{Pu}$. 


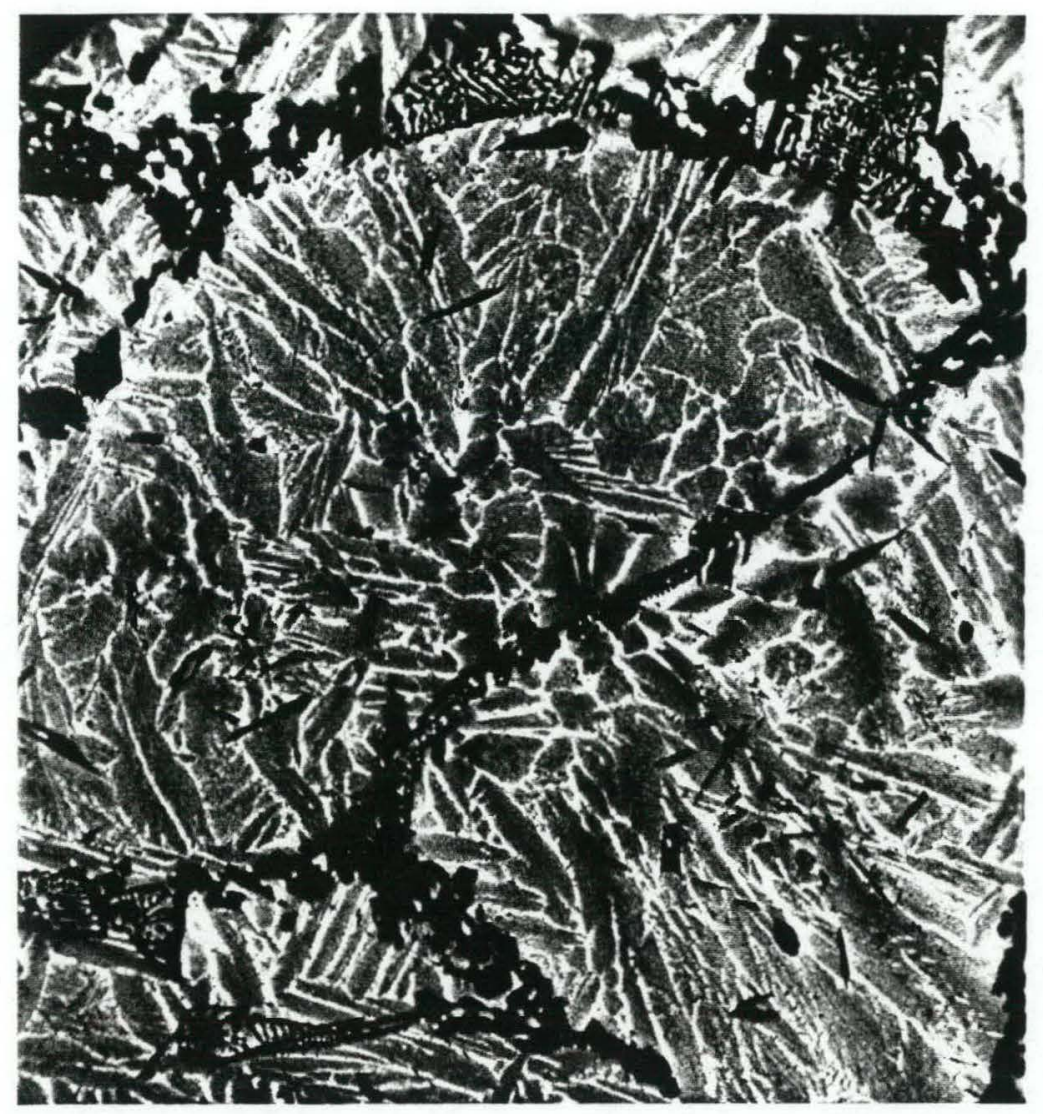

Fig. 2. Backscattered Electron Image of Zr-8SS Alloy Containing $10 \mathrm{wt} \% \mathrm{Pu}$; the $\mathrm{Zr}$ Solution Phase Contains some $\mathrm{Pu}$, but the Bright Features Indicate High $\mathrm{Pu}$ Content at Phase Boundaries. 
blank page 


\section{5-229N -- NEURAL NETWORK $\mathrm{NO}_{x}$ EMISSION CONTROLLER FOR FOSSIL PLANTS $^{1}$}

\begin{tabular}{|c|c|}
\hline Associated Laboratory Director Area: & Engineering Rese \\
\hline Principal lnvestigators: & $\begin{array}{l}\text { J. Reifman, J.E. } \\
\text { Reactor Engineer }\end{array}$ \\
\hline Funding Profile: & $\begin{array}{lll}\text { FY } & 1993 & -0- \\
\text { FY } 1994 & -0- \\
\text { FY } 1995 & \$ 74.6 \mathrm{~K} \\
\text { FY } 1996 & -0- \\
\text { FY } 1997 & -0-\end{array}$ \\
\hline
\end{tabular}

Purpose: The purpose of this project was to perform feasibility studies and develop computational capabilities for controlling dynamic systems with artificial neural networks. The effectiveness of the developed neural network controller (NNC) algorithm was tested for a simple nonlinear control problem. Successful demonstration of the NNC to control discrete-time nonlinear dynamical systems can be readily transferred to the control of complex systems such at the Nitrogen Oxide $\left(\mathrm{NO}_{\mathrm{x}}\right)$ emission levels in coal-fired fossil power plants.

Approach: The introduction of the Clean Air Act Amendments of 1990, delineated environmental constraints requiring many utilities to monitor and reduce the $\mathrm{NO}_{\mathrm{x}}$ emissions from their fossil plants. To monitor $\mathrm{NO}_{\mathrm{x}}$ emission, electric utilities are installing continuous emission monitors that are capable of providing real-time data to plant control rooms and system dispatch centers. To reduce $\mathrm{NO}_{\mathrm{x}}$ emission, utilities are trying to improve boiler control by optimally setting the boiler control variables in order to mcct prespecified $\mathrm{NO}_{x}$ emission levels.

The key basic element for developing a boiler control system is to be able to accurately model the combustion process through real-time simulation programs. The nonlinearity, complexity, and lack of complete understanding of the detailed behavior of the boiler system with respect to $\mathrm{NO}_{\mathrm{x}}$ production, coupled with the real-time performance requirement, add to the difficulty of developing computer models based on physical principles.

An alternative approach for modeling the combustion process in the boiler is to use artificial neural networks. Neural networks are prime candidates for system modeling and identification due to their ability to approximate large classes of nonlinear functions sufficiently accurately in cases where the dynamic system is known only in terms of its input and output. This is indeed the case for modeling $\mathrm{NO}_{\mathrm{x}}$ production since input and output data are now readily available in fossil plants due to the recent installation of continuous emission monitors.

With the ability to model $\mathrm{NO}_{\mathrm{x}}$ production accurately and in real-time through neural networks, a few control systems are currently being developed for steady-state plant conditions. Although these computer systems should improve boiler control, they have some significant limitations.

${ }^{1}$ Individual Investigator Project 
None of these systems (1) model the dynamics of the fossil plant, i.e., the plant is modeled by feedforward neural networks to produce the steady-state $\mathrm{NO}_{\mathrm{x}}$ level for a given set of boiler control actions after all transitory behavior has died out, (2) are sufficiently flexible to allow for slowly degrading boiler conditions and drifting of boiler parameters, and (3) are intended to all types of normal operating conditions including steady-state, load-following, and start-up.

Modeling of dynamic nonlinear systems through recurrent (time-dependent) neural networks was pursued in another FY 1995 funded LDRD project ("Recurrent Artificial Neural Networks for Modeling of Nonlinear Systems" 95-072N). In that project, actual fossil power plant data were used to predict $\mathrm{NO}_{\mathrm{x}}$ emissions levels. Thus, the scope of this investigation was to develop NNC algorithms, implement them into computer programs and gain insight of the issues involved in using NNCs by applying the developed program to control simple dynamical nonlinear systems.

Technical Progress and Results: The research conducted under LDRD auspices during FY 1995 was composed of three tasks: analysis of NNC algorithms, computer program development and program testing. In the first task, various neural network control schemes and learning algorithms were analyzed. From the analyzed NNCs, one was selected and implemented into software in the second task. In the last task, we applied the developed software to control a discrete-time nonlinear dynamical system.

The NNC configuration developed is illustrated in Figure 1, where the NNC was represented by a feedforward neural network and the dynamical system was represented by a set of analytical equations. The dynamical system can also be represented by neural networks through a small program modification. The purpose of the NNC is to provide the appropriate control action $u(k)$, given the system parameters and the present system state $z(k)$, in order to obtain the new state of the system $\mathrm{z}(\mathrm{k}+1)$, such that the state of the system eventually reaches some prespecified objective.

To test the capability of the developed NNC software, we applied it to control a simple nonlinear system. The problem consisted of training the NNC to provide the proper steering angle, i.e. the control $\mathrm{u}(\mathrm{k})$, that guides a boat across a river, subject to a nonlinear stream current, from any initial position $z_{0}$ to a designated final position $z_{\mathrm{f}}$. Figure $2 \mathrm{a}$ shows the results of the trained controller to provide the proper trajectory for twenty-five distinct initial positions in the two-dimensional state-space to one final position. Figure $2 \mathrm{~b}$ shows the results for the case where the trajectories were further constrained by an intermediary "gate" in state-space.

From numerous experiments with the NNC software, we arrived at the following conclusions:

- different control laws $u_{k}=u\left(z_{k}\right)$, with the same objective, can be obtained by using different network topologies, different initial set of weights, as well as by using different training strategies,

- once trained, the resulting NNC provides a unique control law u(z) applicable to all permissible states of the dynamical system, 
- this control law is a continuous function of $z$, provides good acceptable solutions for initial states within certain regions of the state-space, but may also lead to unacceptable solutions outside these regions, and

- state-space constraints may be handled by training the NNC to minimize a sequence of appropriately chosen objective functions.

Specific Accomplishments: An internal report entitled "Neural Network Controller: Prcliminary Results" was distributed; a second one is being prepared. We also plan to summarize the results and publish them as a refereed conference or journal paper. 


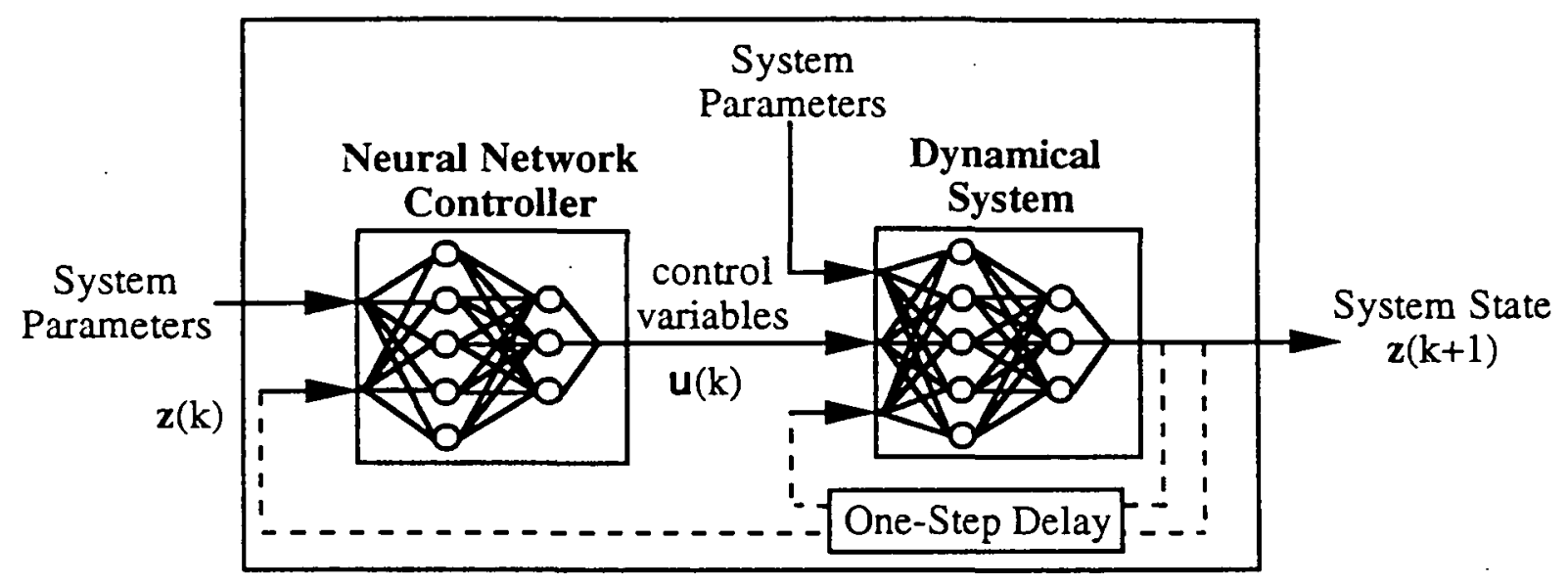

Fig. 1. Neural Network Controller Configuration with One-Step Delay Feedback

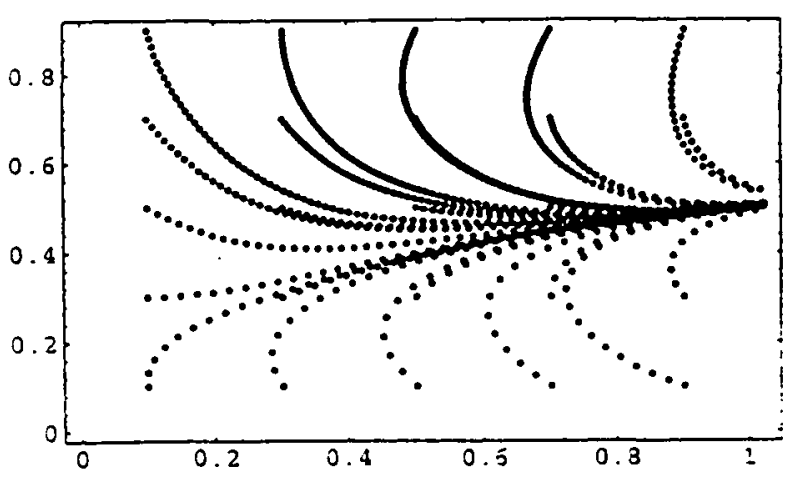

Fig. 2.a Trajectories of Twenty-Five Initial States to the Desired Final State

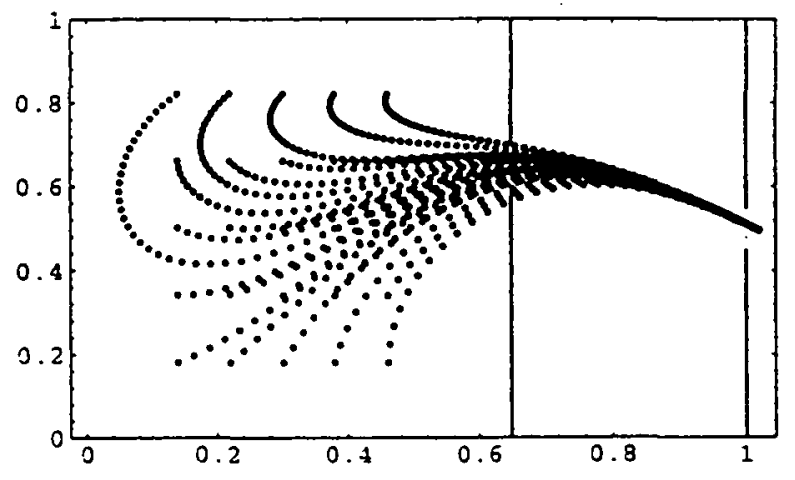

Fig. 2.b Twenty-Five Trajectories for a More Constrained Problem 


\section{5-217N -- FEASIBILITY STUDY FOR THE DEVELOPMENT OF A MINIATURE RAPID-SCAN PASSIVE REMOTE FOURIER TRANSFORM INFRARED (FTIR) SENSOR ${ }^{1}$}

Associate Laboratory Director Area:

Principal Investigator:

Funding Profile:
Engineering Research

S.E. Carpenter, Chemical Technology Division

FY $1993-0-$

FY $1994-0-$

FY 1995 \$74.9K

FY 1996 -0-

FY $1997-0-$

Purpose: The purpose of this project was to determine the feasibility of developing a novel "short-scan" interferometer, which could be used to create a miniature Fourier transform infrared (FTIR) sensor. The proposed sensor would be passive and could be used for remote detection of chemical vapors. The term "passive" indicates that the sensor would not contain an internal infrared (IR) radiation source. Instead, the sensor would be equipped with a special set of input optics to collect ambient IR energy. The term "remote" is used to indicate that target analytes would be located some distance from the instrument. A passive remote FTIR sensor employing a rapid, short-scan interferometer would be smaller and more rugged than existing instruments. This noninvasive sensor would have numerous military and industrial applications and would be useful for environmental waste management.

Approach: This project was originally conceived in order to exploit state-of-the-art developments in signal processing algorithms for passive remote FTIR sensors. These recent advances in data processing have significant implications for improved hardware design. Instead of using conventional spectral processing, these algorithms use digital filtering and pattern recognition methods for automated analysis of short segments of the raw data collected by the sensor. The raw data are collected in the form of an interferogram, which is generated by using an interfcrometer to multiplex incoming IR light. A narrow-band semiconductor IR detector is then used to record variations in modulated intensity versus interferometer scan time. In conventional spectral processing, an interferogram is demultiplexed by applying a mathematical fast Fourier transform (FFT) to obtain a single-beam spectrum (amplitude vs frequency). To obtain a traditional transmittance spectrum, a single-beam spectrum containing analyte information is divided by a single-beam spectrum of the IR background. However, if the IR background changes during the time between the background and analyte scans, undesirable spectral artifacts are produced. Since passive remote FTIR sensors detect ambient IR energy, which is always changing, spectral processing of the resulting data is problematic.

The problems associated with changing IR background can be eliminated by using time-domain (interferogram) processing methods. Time-domain processing eliminates the need for

\footnotetext{
${ }^{1}$ Individual Investigator Project
} 
computationally intensive FFT calculations by using digital filtering to extract characteristic frequency information directly from a short segment of each raw interferogram. This approach also eliminates the need for a stable IR background, as well as the need to compute a ratio. Pattern recognition procedures, namely linear discriminant analysis or piecewise linear discriminant analysis, are then used for qualitative assessment of the data. Since digital filtering and pattern recognition are computationally rapid procedures, real-time automated analysis of the data is possible.

Since time-domain processing requires only a short segment of data, typically 150-200 points, near the centerburst of the interferogram, the rest of the scan is superfluous and need not be collected. Since the scan length is determined by the distance traveled by the moving mirror in the interferometer, movements of only $28 \mu \mathrm{m}$ would be required to collect the short interferogram segments. This type of short-scan interferometer would make it possible to build a rapid-scan passive remote FTIR sensor that provides extraordinary advantages in terms of instrument stability and data integrity. The fragile component in existing FTIR sensors is the interferometer; therefore, special efforts must be made to assure that these sensors are not jarred during data collection. In contrast, an interferometer that provides very short, rapid mirror travel would alleviate common problems associated with maintaining mirror alignment and constant mirror velocity for longer scans. Therefore, the new FTIR sensor would be considerably more rugged than existing instruments. The short scan lengths would also facilitate collection of more scans per unit period of time, thereby enhancing real-time operation for monitoring applications.

To create a short-scan interferometer, it was necessary to identify and evaluate possible mirror drive mechanisms. The primary requirement was the ability to move micrometer and submicrometer distances very rapidly at a velocity of $5000 \mu \mathrm{m} / \mathrm{s}$ with great accuracy and repeatability. Existing mirror drives were not acceptable, since they cannot meet the requirements for precision and accuracy of movement. A high premium was also placed on small size and weight. After careful evaluation of candidate drive mechanisms, only one device with acceptable specifications could be identified. This device is a new, high- precision mechanical positioning stage, which is routinely used for nanometer-scale positioning of fiber optics. The small positioning stage is computer-controlled and is commercially available from Newport (Irvine, California). According to product specifications, this stage can provide up to $2.54 \mathrm{~cm}$ of linear travel in increments of $0.025 \mu \mathrm{m}$ at velocities of $50,000 \mu \mathrm{m} / \mathrm{s}$ with a bidirectional repeatability of $0.025 \mu \mathrm{m}$. Newport is acknowledged for their willingness to lend a positioning stage to Argonne for purposes of testing and evaluation.

To test this stage, a visible-light Michelson interferometer was constructed, and the stage was used as a mirror drive to modulate monochromatic light from a red $\mathrm{He}-\mathrm{Ne}$ laser. The modulated $\mathrm{He}-\mathrm{Ne}$ laser was analyzed by using a silicon photodetector. This was an appropriate test, since existing FTIR sensors employ a He-Ne laser to control the digitization rate of the signal from the IR detector, thereby assuring evenly spaced data points within interferograms. The mirror was driven at a constant velocity of $0.5 \mathrm{~cm} / \mathrm{sec}$, and the modulated signal was sampled at a constant rate of $400 \mathrm{kHz}$, a factor of 25 times faster than the modulated laser frequency of $15.8 \mathrm{kHz}$. A 12-bit analog-to-digital board was used to record the output voltages from the amplified photodetector signal. Extensive data sets were collected for both unidirectional and bidirectional mirror movements. From the data, the zero crossing intervals were computed and 
graphs of stage position versus time were generated. These graphs were used to identify regions of "constant" velocity, and a Fourier transform was applied to the data from these regions to determine the spread in mirror velocities. This information was used to judge the performance of the stage and to evaluate its utility as a mirror drive for a short-scan interferometer. Special acknowledgement is given to Mr. Chris Rush (Science and Engineering Research Semester student) and Mr. Gerald Reedy (Chemical Technology Division/Analytical Chemistry Laboratory) for their vital assistance in performing this work.

Technical Progress and Results: Analysis of the resulting unidirectional and bidirectional data revealed that the velocity of the stage was far from ideal, even for $60-\mu \mathrm{m}$ and $100-\mu \mathrm{m}$ movements. The graphs of stage position versus time revealed that the stage was always accelerating or decelerating and did not maintain a constant velocity. In fact, the unit never reached the specified velocity of $0.5 \mathrm{~cm} / \mathrm{sec}$ for the $60-\mu \mathrm{m}$ movements, and the average velocity was slower for the $60-\mu \mathrm{m}$ movements than for the $100-\mu \mathrm{m}$ movements. According to Newport, the specifications for the stage were obtained by using laser interferometry to determine absolute position accuracy after movement between two points. When positioning fiber optics, this is the primary consideration. However, Newport has never performed tests to characterize the dynamic motion of the stage between two points. The experiments performed in this project indicate that the technical specifications for velocity and acceleration represent maximum obtainable values, but the actual observed values are related to the specified length of travel. This study indicated that the Newport positioning stage is not an acceptable mirror drive for a short-scan interferometer.

The most important technical advancement made during this project was the discovery of a way to design a no-moving-parts interferometer that can collect the same information (short, oversampled interferogram segments) as a rapid, short-scan interferometer. Construction of a passive FTIR sensor with this type of interferometer would utilize a state-of-the-art, uncooled IR focal plane array (FPA) detector. Unlike existing FTIR sensors, which employ a moving mirror design to temporally disperse interferometric information, the proposed sensor would have no moving parts and would spatially disperse interferometric information onto a twodimensional FPA detector. The new FTIR sensor would be even more rugged than the originally proposed short-scan interferometer and would provide both chemical information and IR imaging. This new FTIR sensor would also take advantage of the same types of automated processing algorithms and interferometric data analysis procedures described above. A new FY96 LDRD proposal on this subject has been submitted. An invited proposal has also been sent to the U. S. Department of Energy's Office of Nonproliferation and National Security (NN20). IIT Defense and Electronics (Fort Wayne, IN) has visited Argonne and has signed a nondisclosure agreement in order to discuss the no-moving-parts interferometer concept. ITT has already set aside some funding for collaborative work with Argonne, in the form of a CRADA or a Work-for-Others project.

Specific Accomplishments: An invention report for a no-moving-parts passive remote FTIR sensor has been filed. The Argonne case number for this report is ANL-IN-95-090, and the title of the report is Improved Fourier Transform Infrared (FTIR) Sensor. Final revisions to the invention report are in progress. 


\section{blank page}




\section{5-170N -- NUCLEON AMPLITUDES IN QCD'}

\author{
Associate Laboratory Director Area: Physical Research \\ Principal Investigator: \\ C.D. Roberts, Physics Division
}

Funding Profile:

$\begin{array}{lll}\text { FY } 1993 & -0- \\ \text { FY } 1994 & -0- \\ \text { FY } 1995 & \$ 59.4 \mathrm{~K} \\ \text { FY } 1996 & -0- \\ \text { FY } 1997 & -0-\end{array}$

Purpose: To explain the electromagnetic properties of nucleons solely in terms of the fundamental degrees of freedom in QCD, quarks and gluons, using the manifestly Lorentz invariant Dyson-Schwinger equation framework, which provides an efficacious, less computationally intensive alternative and complement to numerical simulations of discrete lattice QCD actions. Such a description is an important step in understanding nucleonic bound state structure in terms of nonperturbatively dressed quarks and gluons. This structure is to be probed in a series of experiments to be undertaken at the Continuous Electron Beam Accelerator Facility (CEBAF). Our studies form an integral part of the theoretical effort devoted to this facility. They represent a first step in the application of a promising new theoretical tool in nuclear physics, which will greatly assist in the analysis and interpretation of the experimental results.

Approach: Quantum Chromodynamics (QCD) is believed to be the non-Abelian gauge field theory that underlies the strong interaction. In principle, therefore, it should be possible to calculate nucleon masses and electromagnetic properties directly from the Lagrangian of QCD, which is expressed in terms of quark and gluon fields. However, the non-Abelian nature of QCD entails the existence of direct gluon-gluon couplings, which have the effect of generating a quark-quark interaction that is weak at small distances but which grows with separation, becoming large at length-scales greater than $1 \mathrm{fm}$.

Our understanding of QCD indicates that nucleons are composite particles; i.e., bound states of quarks and gluons. The quarks and gluons are confined; i.e., unlike the electron, they do not exist as free particles. Experimentally we know that the nucleon radius is approximately $1 \mathrm{fm}$ and hence any study of the nucleon will confront QCD in the strong coupling regime. Further, as a three-quark bound state, the nucleon is intrinsically a nonperturbative system. As a consequence, a calculation of the nucleon properties in QCD, requires an understanding of nonperturbative dynamics in the strong coupling regime; a very difficult problem.

The identification of the role played by quarks and gluons in the structure of hadrons and nuclei is a primary DOE funding priority. CEBAF has been constructed specifically to address this issue. It is therefore important to develop nonperturbative techniques that are applicable to the study of the quark-gluon substructure of hadrons in order to provide a framework in which the experiments may be interpreted and understood.

${ }^{1}$ Individual Investigator Project 
In the Theory Group of the Physics Division we have been active in developing a nonperturbative approach to QCD based on the Dyson-Schwinger equations (DSEs) of the theory. These equations are the quantum field theory analogue of the Euler-Lagrange equations in classical mechanics (the equations of motion for $\mathrm{QCD}$ ) and have been used successfully in studies of solid state systems. The fundamental quantities in this approach are the nonperturbative quark and gluon propagators and vertex functions, which are obtained as solutions of the DSEs. This is a rapidly developing area of fundamental research in nuclear physics and its application to QCD has met with many successes in the study of quark-antiquark bound states (mesons).

Its application to hadrons (three-quark bound states such as the neutron and proton) is in its infancy. Axel Bender, a postdoctoral fellow employed using these LDRD funds, has received extensive training under this grant and has made significant progress in extending the DSE framework and employing it in a study of the electromagnetic form factors of the proton and neutron.

Explicitly, the nucleon is described as a composite of three quarks. Gluon exchange between quarks is attractive and induces a loose correlation in all quark-quark (diquark) channels: scalar, pseudovector, pseudoscalar and vector. These correlations can have measurable effects on experimental observables. This framework makes it possible to address important questions such as: the structure of the hadronic bound state amplitude; the relative contribution of different quark flavors to the electromagnetic properties of a given hadron; and the importance of quark-quark (diquark) correlations.

Of particular significance is the fact that our DSE approach is manifestly Lorentz invariant, which entails that it is appropriate to the large momentum transfers present in experiments at CEBAF. This overcomes a defect present in all cavity-models of the nucleon, such as the cloudy bag model, which cannot be boosted and hence cannot recoil after absorbing momentum.

Further, in our application of the DSEs, the perturbative aspects of QCD are automatically recovered at large spacelike momenta. This is an important feature and constraint, and makes it possible for us to identify and probe the transition from the perturbative to the nonperturbative regime, which is a primary focus of experiments at CEBAF, and relate observables to the fundamental parameters of QCD.

This research is being carried out in collaboration with Dr. M.R. Frank at the Institute for Nuclear Theory, Seattle.

Technical Progress and Results: A vectorizable computer program was written to calculate the generalized-impulse approximation to the S-matrix element associated with the electric and magnetic form factors of the nucleon. The small spacelike- $q^{2}$ behavior of these form factors yield the charge, magnetic moments, charge and magnetic radii of the nucleon. Based on results obtained in studies of quark-quark systems, it was assumed that the scalar diquark correlation was the most significant component of the nucleon Fadde'ev (bound state) amplitude. The preliminary program only contained the scalar correlation. 
The propagation characteristics of the nonperturbatively-dressed quark-constituents in the nucleon were described by the propagator employed in a phenomenologically successful study of meson systems. The scalar diquark amplitude and nucleon Fadde'ev amplitude were parametrized in accordance with the results of realistic studies of the diquark Bethe-Salpeter equation and nucleon Fadde'ev equation. The nonperturbative quark-photon coupling employed was that obtained in extensive nonperturbative studies of quantum electrodynamics.

The preliminary program, and subsequent versions, are executed using the resources of the National Energy Research Supercomputer Center.

A comparison of calculated with experimental results is presented in Table 1. These preliminary results are encouraging and have the following implications: 1) The dressed-quark constituents of the nucleon must each carry roughly one third of the nucleon momentum; 2) The scalar quark-quark correlation alone is insufficient to describe both the electric and magnetic properties of the nucleon. The latter is a particularly important observation, which will greatly influence studies of the nucleon Fadde'ev equation.

Table 1: Comparison between experiment and preliminary calculated results for a range of static nucleon properties. Charge radii are denoted by $\left\langle r_{E}\right\rangle^{2}$; magnetic radii by $\left\langle r_{\mathrm{M}}\right\rangle^{2}$; and magnetic moments by $\mu$.

\begin{tabular}{||c|c|c|c|c|c|c||}
\hline & $\begin{array}{c}<r_{\mathrm{f}}^{\mathrm{p}}>^{2} \\
\left(\mathrm{fm}^{2}\right)\end{array}$ & $\begin{array}{c}<r_{\mathrm{E}}^{\mathrm{n}}>^{2} \\
\left(\mathrm{fm}^{2}\right)\end{array}$ & $\begin{array}{c}<r_{\mathrm{M}}^{\mathrm{p}}>^{2} \\
(\mathrm{fm} 2)\end{array}$ & $\begin{array}{c}<r_{\mathrm{M}}^{\mathrm{n}}>^{2} \\
\left(\mathrm{fm}^{2}\right)\end{array}$ & $\mu_{\mathrm{p}}\left(\mu_{N}\right)$ & $\mu_{n}\left(\mu_{N}\right)$ \\
\hline Calculated & 0.76 & -0.11 & 0.50 & -0.12 & 0.63 & -0.15 \\
\hline Experiment & 0.74 & 0.12 & 0.74 & -0.77 & 2.79 & -1.91 \\
\hline
\end{tabular}

Extensions of the preliminary program have suggested that pseudovector diquark correlations play an important role in determining the electromagnetic properties of the nucleon. Pseudoscalar and vector correlations do not appear to be important.

The project will continue with the postdoctoral fellow supported by program funds for an additional year. It is anticipated that a thorough study of the electromagnetic properties of the nucleon, including all diquark correlations, will be complete early in 1996.

Specific Accomplishments: This effort is continuing under the auspices of DOE-ER.

Presentations - Axel Bender will give seminars at four German universities in January 1996 describing the results summarized above and more recent developments. 
blank page 


\title{
95-191N -- NEAR-FIELD SCANNING OPTICAL MICROSCOPY ${ }^{1}$
}

\author{
Associate Laboratory Director Area: Energy and Environmental Systems and \\ Technology
}

\author{
Principal Investigators:
}

Funding Profile:
J.L. Dehmer, P.M. Dehmer,

S.T. Pratt, and E.F. McCormack, Environmental Research Division

FY $1993-0-$
FY $1994-0-$
FY $1995 \$ 73.8 \mathrm{~K}$
FY $1996-0-$
FY $1997-0-$

Purpose: The purpose of this work is to establish a new research activity in near-field scanning optical microscopy (NSOM). This new form of optical microscopy achieves a resolution far better than those of traditional far-field microscopies, which are constrained by the diffraction limit. NSOM removes this limitation by imaging a sample through an aperture much smaller than the wavelength $(\lambda)$ of the illuminating radiation, by placing the sample at a distance less than $\lambda$ from the aperture (i.e., in the near field), and by scanning the aperture with respect to the sample. NSOM has been made possible by the technology developed for scanning-tunneling microscopy and by recent developments of nanoscale optical probes that are made by pulling and then coating single-mode optical fibers. This new, cutting-edge technology has enormous potential for nanometer scale optical microscopy in biology, materials research, and a variety of medical and industrial applications. For example, this technique is the first to have the potential for real-time, non-destructive imaging of a living cell. We propose to use our considerable background in pulsed laser technology and gas-phase molecular photophysics to contribute to the development of NSOM by developing new contrast mechanisms using variable wavelength, pulsed excitation and by contributing to the major problem of interpreting the resulting images in terms of the molecular photophysics and tip-sample interactions. We have acquired the basic hardware and software for the NSOM microscope.

Approach: To realize its full potential, NSOM technology must undergo further development in several areas. Perhaps the most important are tip technology (to devise rugged, long-lived tips that will improve on the current $10-20 \mathrm{~nm}$ resolution limit); the efficient coupling of pulsed, high-power, variable-wavelength light sources to the NSOM apparatus (to enable the study of the physics and chemistry of discrete molecular processes); and the theory of evanescent wave interaction with objects in the radiation field (to improve the understanding and interpretation of the detected signal). We propose to concentrate on the second of these - the coupling of pulsed, high-power, variable-wavelength light sources to the NSOM apparatus and the subsequent study of molecular processes on and near surfaces and at interfaces with spatial and temporal resolution.

\footnotetext{
${ }^{1}$ Individual Investigator Project
} 
Variable-wavelength laser sources will greatly extend the potential of NSOM by enabling the selective excitation of specific molecules and chromophores and the study of the time dependence of fluorescence, which can potentially be a new contrast mechanism. Pulsed laser sources would also enable the study of two-color pump-probe processes like those currently used in our gas phase studies. Furthermore, multiphoton excitation would enable the study of the merits of nonlinear contrast mechanisms (i.e., excitation of a two-photon transition followed by observation of fluorescence at a very different wavelength). Because the detection system preserves the light polarization, polarizing optics can be used as a contrast mechanism; of particular interest is the use of circular polarization to enhance contrast in chiral systems. Variable-wavelength and time-dependent detection techniques would further add to the contrast mechanisms available and would enable the study of decay rates and mechanisms. These contrast mechanisms depend only on the absorption and decay characteristics of the molecular species in the sample; hence, they are independent of the characteristics of an externally applied stain. Such a collection of contrast mechanisms might be termed "dynamic staining, " because they are exceptionally versatile and can image a species regardless of whether it is receptive to conventional staining.

We anticipate that our earliest experiments might use pulsed, variable-wavelength lasers to study a submonolayer of dye molecules on an appropriate substrate. We are interested in investigating aspects of both the technology (e.g., the determination of damage thresholds in optical fibers, comparison of single- and multimode laser transmission, efficient coupling and detection of incident and fluorescent radiation over broad wavelength ranges, multiphoton and, possibly, twocolor excitation of the sample) and the physics (determinations of spectral characteristics on surfaces and comparisons with those characteristics in the gas phase and in solution, fluorescence lifetimes, molecule-substrate interactions, molecule-molecule interactions, and the study of the mechanisms of photochemistry/photobleaching of single molecules in real time and as a function of wavelength) of such processes.

Following such early experiments, we will expand our studies to include a number of applications of NSOM. It is not difficult to imagine the array of opportunities afforded by this nondestructive, variable-wavelength optical probe that offers both high spatial and temporal resolution and that can be used on ambient samples. A few of the possible applications might include the study of intracellular molecular structure and dynamics in vivo and in real time; the study of the heterogeneous distribution of microbial cells and the resultant heterogeneous distribution of chemicals within biofilms (which are important in understanding such diverse phenomena as corrosion, product contamination, fouling, and subsurface ecology) under ambient conditions; the study of polymeric materials of all types; clinical pathology; nondestructive nanoinspection and testing of materials using absorption or fluorescence; optical data storage; and control and inspection in optical lithography.

This is a particularly opportune time to begin such a program, since a basic NSOM apparatus has just become commercially available (TopoMetrix); this apparatus provides a $15 \mathrm{~mW} \mathrm{cw}$ argon ion laser, state-of-the-art aluminum-coated optical fiber probes, a piezoelectric scanner, data collection in either the transmission mode or the reflection mode, and software to operate the microscope either as a near-field scanning optical microscope or as an atomic force microscope. Such an apparatus was installed in a newly renovated laboratory in June 1994 . We now anticipate a brief period during which we will learn the details of operating the apparatus 
with the cw laser and interpreting the scanned images of standard samples. Following that, we will begin work to couple pulsed, variable-wavelength lasers to the apparatus. We will start by using our available $(10 \mathrm{~Hz})$ nanosecond $\mathrm{Nd}$ :YAG-pumped dye lasers; however, we anticipate that we will very quickly incorporate high repetition rate, picosecond lasers to improve the duty factor and to enable the study of fast processes.

Technical Progress and Results: Our work in the first year of this LDRD project was aimed at providing the groundwork for our primary effort to improve existing contrast mechanisms and to develop new ones for near-field scanning optical microscopy. The PI and co-PI's received training on the new atomic force microscope/near-field scanning optical microscope and began to learn the details of recording and interpreting scanned images of samples. Much of our effort was directed at investigating the details about how to modify and adapt the apparatus to allow the implementation of alternative illumination and detection techniques. The modifications that were investigated include: adaptations for single-photon counting detection; monitoring dispersed or filtered fluorescence; using area detectors for dispersed fluorescence; determining polarization dependences; synchronizing the NSOM scanning and detection electronics with pulsed laser sources; using variable wavelength $\mathrm{cw}$, chopped-cw, nanosecond, picosecond, and femtosecond lasers; and coupling the output of these lasers into the fiber-optics. For each modification we have both developed the idea of how to incorporate the modification into the apparatus, and determined the most appropriate vendor and parts to make the modification.

We believe that this is a research area that is ready for explosive development in the near future. Unfortunately, during the course of this LDRD project the DOE/OHER base funding for our research group was terminated and, as a result, our group has disbanded. The AFM/NSOM will be "sold" to another division at Argonne, and our AFM/NSOM laboratory will be dismantled.

Specific Accomplishments: None. 
blank page 


\title{
94-003R1 -- INTELLIGENT ROBOTICS GLOVE
}

\author{
Associate Laboratory Director Area: Engineering Research
}

Principal Investigators:

T.Y.C. Wei and D.Y.C. Pan,

Reactor Engineering Division

J. Reifman, Reactor Analysis Division

Funding Profile:

FY $1993-0-$

FY $1994 \$ 128.4 \mathrm{~K}$

FY $1995 \$ 118.4 \mathrm{~K}$

FY $1996-0-$

FY $1997-0-$

Purpose: The project objective is to determine the technical feasibility of an intelligent robotics device using an ANL-derived approach to identify geometrical features of an object through tactile perception. The proposed system is composed of a strain-sensitive glove, a strain tomographer, and a neural network. Establishment of a design would enable ANL to make a technical case to prospective industrial partners for follow-on collaborative work.

Approach: Current research on tactile perception devices is oriented at producing a mechanical duplicate of a human hand with fingers and intelligent sensory feedback. The limitations of this approach appear to be dictated by the need for sensors to measure the displacement of the "fingers" relative to many known reference points. In contrast, the ANL-derived approach is to construct a strain-sensitive glove which can be bent over the contours of the object to be recognized. The use of bending/local curvature information, together with calibration through neural network straining, should substantially reduce the need for known reference points, simplify the design, increase reliability, and produce lower manufacturing costs.

Proof-of-concept feasibility for this ANL approach is addressed through investigating the design issues for a design which combines an intelligent glove composed of layered intelligent composites, an integrated sensor/signal mesh, strain tomography/neural networks, and the plate bending concept. The top-level design issues are:

- Intelligent composite material: flexibility and resilience to adjust to different shapes; sensitivity for electrical signal processing; hysteresis should be minimum for reproducibility of object shape.

- Glove/arm design: conformance of glove to surface of object being recognized; minimal number of electrical connections/wiring for electromechanical interfacing; control over relative position of arm/glove.

- Image reconstruction/tomography: use of curvature for construction of image should not lead to integration of local errors into global image; functional dependence of strain-tocurvature mapping and its inverse should be unique and accurately represented.

The methods used to resolve these top-level design issues were a combination of one-dimensional experiments and two-dimensional simulations. By area, the workscope was: 
- Intelligent composite material: In collaboration with the University of Delaware/Center for Composite Materials, actual samples were fabricated.

- Glove/arm design: One-dimensional experiments were performed to test concepts to obtain surface conformance. The multisensor strip and inflatable bladders were used. A two unit glove was also produced and used.

- Image reconstruction/tomography: Two-dimensional analyses were performed to modify mapping algorithms for image reconstruction. Testing was performed with strain data for a set of objects using simulation calculations for the glove deformation.

Technical Progress and Results: In FY94 an appropriate matrix/preform fibre, RTV silicon/fiberglass yarn had been selected. The choice of sensor was undetermined. A glove design had been produced. A novel doubly connected mesh of sensor and signal strands for the electromechanical interfacing was proposed. The local curvature algorithm with ten sensors/inch produced acceptable image reconstruction results for mild curvature objects using simulation calculations. Difficulties had been encountered with sharp corners.

In summary, the conclusions of the FY95 project work regarding the top-level design issues are:

- Intelligent composite material:

- Matrix/preform fibre: The approach has been completely changed. A Kapton plastic sheet glued to an elastomeric neoprene sheet now forms the glove fabric. This combination allows the required flexibility and resilience for the glove fabric.

- Sensor: Currently available conductive elastomers and inductance coils are not appropriate. Strain gauges (five sensors/inch) were selected. Acceptable techniques to fabricate the one-sensor/one-unit fabric samples and a one-dimensional multisensor/multi-unit strip were developed.

- Glove/arm design:

- Glove mechanical design: Experiments with the inflated glove concept show possible acceptability for surface conformance.

- Glove electromechanical interfacing: The numerical analyses of the doubly connected mesh of sensor and signal strands woven into the preform show good accuracy.

- Image reconstruction/tomography:

- Strain-to-curvature mapping: A correction to account for glove thickness was developed and implemented in the mapping. This, plus refinements in the strain calculation for the set of test objects, has led to improvements for the higher curvature objects. However, additional refinements had to be added in the plot algorithm. 
- Plot algorithm: Addition of the specific capability to plot the one-dimensional ellipses simultaneously from two directions has led to significant improvements for the high curvature ellipses.

Specific Accomplishments: An internal report entitled "System Design Study II: Intelligent Robotics Glove" is in preparation. Detailed results are available in the report. 


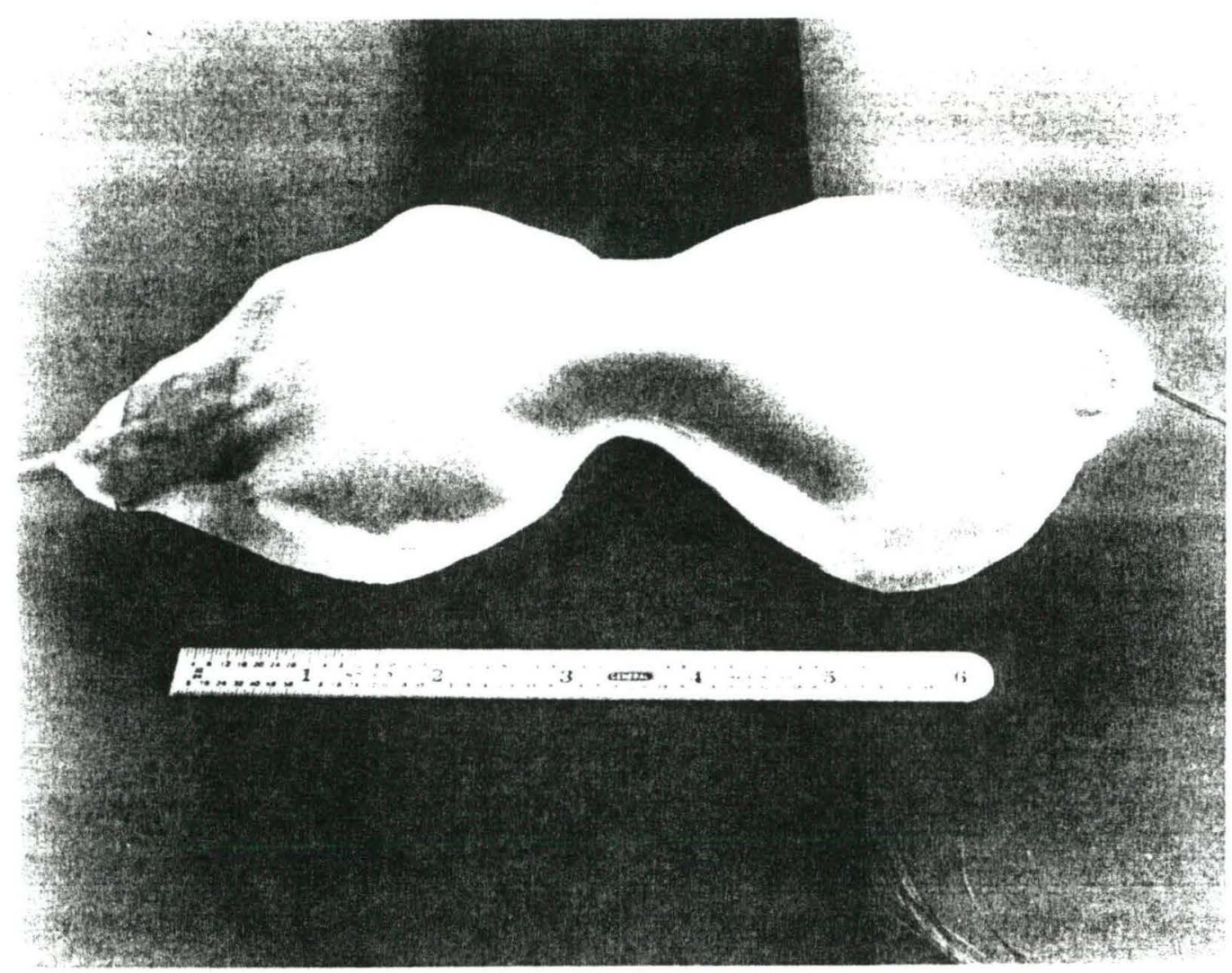

Figure 1. Inflatable glove concept model

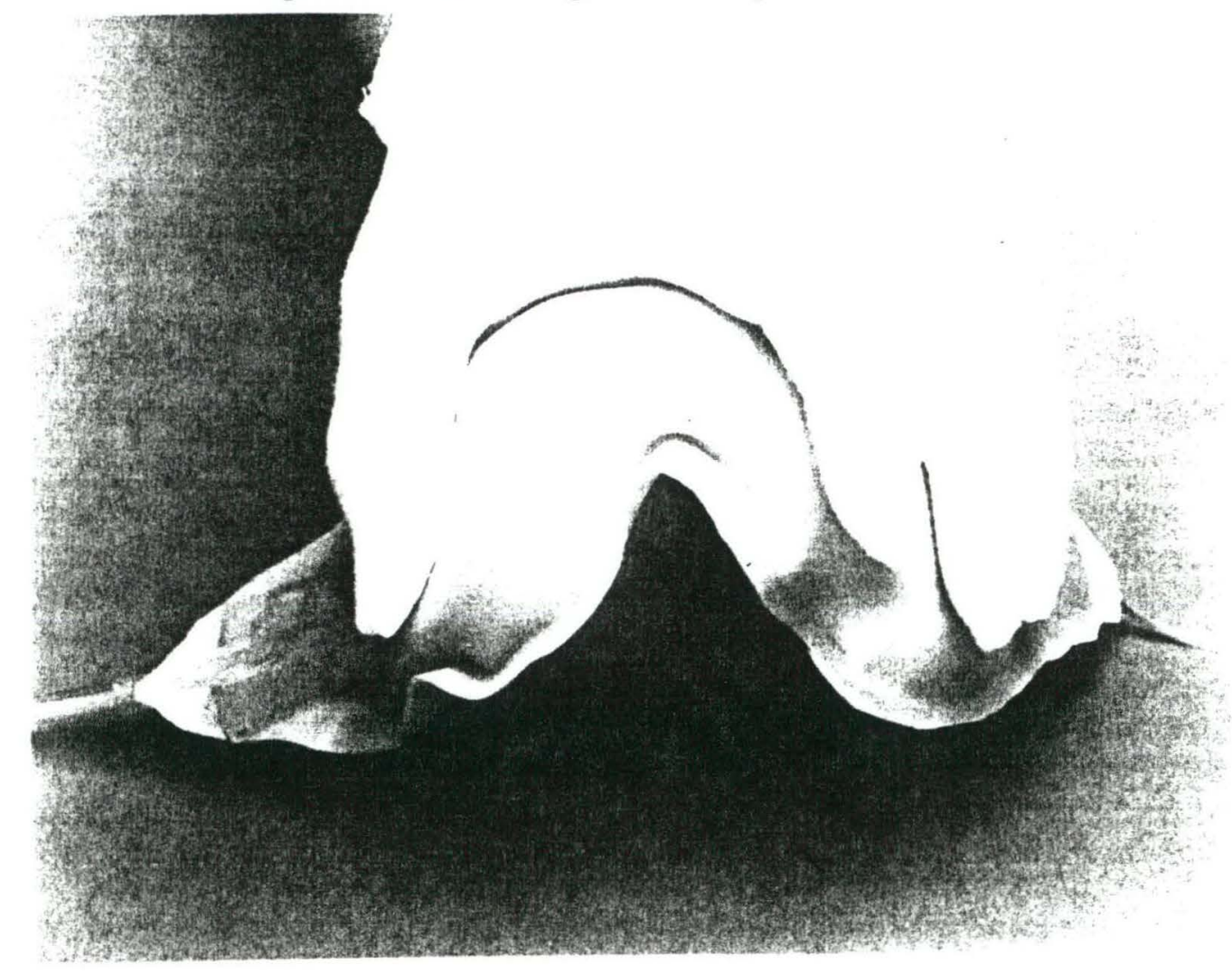

Figure 2. Inflatable glove conforming to object 


\title{
95-159N -- ULTRAPURIFICATION OF TUNGSTEN HEXAFLUORIDE FOR SEMICONDUCTOR USE ${ }^{1}$
}

\author{
Associate Laboratory Director Area: Physical Research \\ Principal Investigator: J.V. Beitz, Chemistry Division \\ Funding Profile: $\quad$ FY $1993-0-$ \\ FY $1994-0-$ \\ FY $1995 \$ 72.7 \mathrm{~K}$ \\ FY $1996-0-$ \\ FY $1997-0-$
}

Purpose: Demonstrate at the proof-of-concept level a novel photochemical process for removal of alpha emitting and other chemically reactive fluoride impurities from tungsten hexafluoride $\left(\mathrm{WF}_{6}\right)$ gas. Ultrapurification of $\mathrm{WF}_{6}$ will enable manufacture of improved semiconductor devices. $\mathrm{WF}_{6}$ gas is the source material for chemical vapor deposition (CVD) of tungsten metal onto silicon surfaces. Chemically reactive impurities in $\mathrm{WF}_{6}$, such as uranium hexafluoride $\left(\mathrm{UF}_{6}\right)$, also are deposited during CVD of tungsten. Alpha emitting impurity elements, such as uranium, are a major concern in integrated circuit (IC) manufacturing and are the major cause of "soft" errors in random access memory (RAM) IC chips that are an essential element of computers.

Approach: Existing methods of removing impurities from $\mathrm{WF}_{6}$ have significant deficiencies. These methods include distillation, reaction with metals, and sorption/desorption from sodium fluoride. Distillation is ineffective in removing species whose volatility is similar to $\mathrm{WF}_{6}$ or are present at trace level. Reaction with metals has the disadvantage that impurities in the metal can contaminate the $\mathrm{WF}_{6}$. Sorption/desorption from sodium fluoride removes hydrogen fluoride but has no influence on many other impurities, including $\mathrm{UF}_{6}$. Our approach, photochemical removal of impurities from $\mathrm{WF}_{6}$, has the advantage that $\mathrm{WF}_{6}$ need come in contact only with inert surfaces and that the expected photochemical reaction product (aside from the removed impurities) is fluorine gas that is recyclable to $\mathrm{WF}_{6}$ production.

No photochemical studies on $\mathrm{WF}_{6}$ gas have been reported. From a literature report, $\mathrm{WF}_{6}$ begins to absorb light at about $191 \mathrm{~nm}$ and increasingly absorbs light as wavelength is decreased. For this reason, a low pressure mercury arc lamp emitting light as short as $184.9 \mathrm{~nm}$ was selected as the photolysis light source. A related challenge is the chemical aggressive nature of $\mathrm{WF}_{6}$ and $\mathrm{UF}_{6}$. These compounds react immediately with moisture (water) in air or on surfaces. Materials of construction, in consequence, are limited to those compatible with strong fluorinating agents and include monel, nickel, sapphire, and polytetrafluoroethlyene. Finally, $\mathrm{UF}_{6}$ poses the challenges associated with handling radioactive materials because all isotopes of uranium are radioactive. Handling $\mathrm{UF}_{6}$ therefore requires special facilities approved for use with radioactive materials.

${ }^{1}$ Individual Investigator Project 
In the process, the expected photochemical reaction sequence in the gas phase is:

$$
\begin{gathered}
2 \mathrm{WF}_{6}+2 \mathrm{~h} \nu \rightarrow 2 \mathrm{WF}_{5}+2 \mathrm{~F} \\
2 \mathrm{WF}_{5}+2 \mathrm{UF}_{6} \rightarrow 2 \mathrm{UF}_{5}+2 \mathrm{WF}_{6} \\
\mathrm{~F}+\mathrm{F}+\mathrm{M} \rightarrow \mathrm{F}_{2}+\mathrm{M}
\end{gathered}
$$

where $\mathrm{h} \nu$ represents an absorbed photon and $\mathrm{M}$ is an inert third body (such as $\mathrm{WF}_{6}$ ). Summing the above steps together results in the overall reaction being

$$
2 \mathrm{WF}_{6}+2 \mathrm{UF}_{6}+\mathrm{M}+2 \mathrm{~h} \nu \rightarrow 2 \mathrm{WF}_{6}+2 \mathrm{UF}_{5}+\mathrm{M}+\mathrm{F}_{2} .
$$

Note that no net loss of $\mathrm{WF}_{6}$ occurs! The ultimate fate of the produced $\mathrm{UF}_{5}$ is expected to be aggregation to form particles that are easily removed from that remaining gases. The advantage of the above sequence is that the major species present, $\mathrm{WF}_{6}$, absorbs the photolysis light but suffers no net loss because reaction with chemically aggressive fluoride impurities, such as $\mathrm{UF}_{6}$, regenerates $\mathrm{WF}_{6}$. In essence, the above reaction sequence represents photocatalytic removal of impurities from $\mathrm{WF}_{6}$. In addition to $\mathrm{UF}_{6}$, removal of many other volatile metal fluorides, such as $\mathrm{CrF}_{5}$, is expected on chemical reactivity grounds.

Technical Progress and Results: A photochemical reactor for $\mathrm{WF}_{6}$ studies that meets the challenges outlined above was designed and constructed. The reactor consisted of a single crystal sapphire tube surrounded by 6 linear low pressure mercury vapor arc lamps. The lamps emit at several ultraviolet wavelengths with the shortest wavelength line of significance occurring at $184.9 \mathrm{~nm}$ (i.e., at a wavelength at which $\mathrm{WF}_{6}$ absorbs light). Oxygen, such that present in air, absorbs $184.9 \mathrm{~nm}$ light to some extent. For this reason, nitrogen gas was used to purge the space between the lamps and the sapphire tube that contained the gases undergoing photolysis. A gas handling vacuum manifold system was constructed by $\mathrm{C}$.W. Williams such that the gases contact only materials that are chemically inert to them. This nickel and monel manifold was constructed in an area approved for use with radioactive materials, specifically including use of $\mathrm{UF}_{6}$, and appropriate safety reviews of the system were carried out prior to commissioning it.

Initial photochemical work consisted of photolysis of WF6 and was successful in that evidence of photoreduction was obtained. Our subsequent work involved use of mixtures of $\mathrm{WF}_{6}$ and $\mathrm{UF}_{6}$. In this work, a gas mixture was photolyzed in the photochemical reactor. The amount of fluorine $\left(\mathrm{F}_{2}\right)$ gas generated was determined and removed from the sapphire reactor tube. The remaining gases were hydrolyzed in nitric acid solution. The solid residue in the cell, if any, was dissolved in nitric acid solution. The uranium content of the hydrolyzed gases and dissolved solids solutions was determined by laser-induced fluorescence and the standard addition method. Photochemical reduction of $\mathrm{UF}_{6}$ concentration in a mixture of $\mathrm{WF}_{6}$ and $\mathrm{UF}_{6}$ gases by as much as a factor of 20 was achieved in a single photolysis run thereby successfully demonstrating the concept. An unexpected consequence of the laser-induced fluorescence analysis effort was discovery of a new means of enhancing uranyl luminescence that may provide the basis for an improved method of chemical analysis for uranium. 
Although substantial photochemical reduction in $\mathrm{UF}_{6}$ content was achieved, even larger reduction would have been expected, given the photolysis conditions, if the only reactions of significance were steps 1 through 3 above. Considering the reported thermal instability of solid $W_{5}$, it seems likely that the following sequence of reactions occurs in the gas phase under the reaction conditions that we used

$$
\begin{gathered}
\mathrm{WF}_{5}+\mathrm{WF}_{5} \rightarrow \mathrm{WF}_{6}+\mathrm{WF}_{4} \\
\mathrm{WF}_{4}+\mathrm{F}_{2} \rightarrow \mathrm{WF}_{6} .
\end{gathered}
$$

If these reactions occur with significant probability, they reduce the amount of lower valent tungsten fluorides $\left(\mathrm{WF}_{5}\right.$ and $\mathrm{WF}_{4}$ ) that are available for reaction with $\mathrm{UF}_{6}$ during photolysis and result in poor utilization of photolysis light.

The milestones for this LDRD effort were: 1) design, approval, and construction of a photochemical reactor system suitable for $\mathrm{WF}_{6}$ studies, 2) design, approval, and construction of a gas handling system for $\mathrm{WF}_{6}$ and mixtures of $\mathrm{WF}_{6}$ and $\mathrm{UF}_{6}$, and 3) proof-of-concept demonstration of photochemical removal of $\mathrm{UF}_{6}$ from a mixture of $\mathrm{WF}_{6}$ and $\mathrm{UF}$. Each of these milestones was meet.

Specific Accomplishments: An invention report (ANL-IN-94-053) was filed concerning this novel photochemical process for removing chemically reactive impurities from tungsten hexafluoride gas.

A publication concerning the photochemical removal of impurities from tungsten hexafluoride gas is in preparation. 
blank page 


\title{
94-168R1 -- A COMPUTER SIMULATION OF AN INTELLIGENT VEHICLE HIGHWAY SYSTEM FOR CHICAGO METROPOLITAN AREA ${ }^{1}$
}

\author{
Associate Laboratory Director Area: Energy and Environmental Science and Technology \\ Principle Investigators: \\ A.M. Tentner, Reactor Analysis Division \\ M.W. Henderson, Mathematics and Computer \\ Science Division \\ T.F. Ewing, Technology Development Division
}

Funding Profile:

FY $1993-0$ -

FY $1994 \$ 123.1 \mathrm{~K}$

FY $1995 \$ 139.7 \mathrm{~K}$

FY $1996 \$ 70.0 \mathrm{~K}$

FY $1997-0$ -

Purpose: The Intelligent Transportation System (ITS) program of the U.S. Department of Transportation (U.S. DOT) is designed to use advanced computing and communications technologies for proactive control and management of traffic flow and transportation facilities in order to improve traveler's safety and mobility, reduce congestion, minimize energy consumption and negative environmental impact, and promote economic competitiveness of the U.S. industry. The ITS Simulation effort at Argonne National Laboratory is directed at advanced modeling and simulation needed to support emerging ITS technologies. The ANL simulator has been developed to provide a large scale, comprehensive simulation of an Intelligent Transportation System running on distributed or parallel computer systems with direct application to the Chicago metropolitan area. The development of the simulator will provide a focal point for the ANL Intelligent Transportation Systems Initiative, an activity which has been identified by the Advanced Transportation Strategic Planning Committee as one of ANL's major initiatives in the transportation area.

Approach: The simulator is designed to model the basic elements of ITS architectures, including conventional and instrumented "smart" vehicles with in-vehicle navigation systems, and traffic management centers (TMC's) capable of two-way communications with smart vehicles. Vehicles are modeled as autonomous computer processes that communicate with other processes by exchanging messages. The simulation architecture provides for comprehensive, scaleable simulations that can run on a single standalone computer, a network of computers, or a massive parallel computer, such as ANL's IBM SP2. Vehicles can perform optimal routing based on minimum distance, minimum time, or other strategies. Traffic Management Centers are capable of broadcasting traffic advisories to smart vehicles, which in turn may automatically reroute to avoid congestion. Functionally and visually realistic graphical user interfaces are used to support human factors work.

Many of the requirements for the simulator were developed in coordination with the Chicagobased ADVANCE project, an ITS pilot program to deploy a fleet of cars with in-vehicle

${ }^{1}$ CCST Project 
Technical Progress and Results: Efforts in FY94 focused on establishing the architecture of the simulator. Emphasis was made for support of distributed and parallel processing to permit scaling to large problem sizes, and developing rudimentary models of a smart vehicle, Traffic Management Center (TMC), and the graphical user interfaces. In addition, a leading traffic simulation code developed for DOT/FHWA called TRAF/NETSIM was acquired for experimentation, and a map database for the Chicago-area was obtained from Navigation Technologies Inc. to support simulator input preparation.

During FY95, our efforts were directed at providing more substantial models for key ITS elements, improving the graphical user interfaces, and establishing efficient algorithms for interprocess communications. A route optimization algorithm was developed which permits onthe-fly computations of optimal routes between any two points. This permits simulated smart vehicles to dynamically adjust their routes due to changing traffic conditions. The memory management of the vehicle process was substantially enhanced to make improved utilization of computer resources. In addition, communications between simulated vehicles and the Traffic Management Center (TMC) were extended to permit 2-way exchanges, so that vehicles can provide tracking data and receive advisories for global optimization of traffic flow. For example, when the TMC broadcasts an advisory about traffic congestion, affected vehicles can reroute automatically. The vehicle code is highly portable, and can be run on a workstation network for modest-size simulations, or on the IBM SP-2 parallel computer for large-scale problems.

In addition, the PC-based TRAF/NETSIM simulator from DOT was ported to the SP-2 parallel computer and extended to permit multiple coupled regions to be processed in parallel. The map database was extended with software tools to permit map regions to be graphically displayed and manipulated to process map files for the ITS simulator.

Specific Accomplishments: A technical paper on the ITS simulator was presented at the 1995 High Performance Computing Symposium, organized by the Society for Computer Simulation (SCS) in Phoenix, Arizona. A second paper on the TRAF/NETSIM parallel computer implementation was given at the ITS World Congress in Yokohama, Japan. Both papers were included in conference proceedings. A World Wide Web (WWW) home page was also established to organize ITS project-related information. The web home page includes a WWW graphical user interface to the simulator.

Contacts were established with other groups involved in ITS simulation activities: Oak Ridge National Laboratory, TASC, Draper Labs, and others. Also, opportunities for using elements of the ANL simulator for various ITS projects, such as the national programs in Automated Highway Systems and Dynamic Traffic Allocation, as well as local Chicago-Gary-Milwaukee corridor activities, are being explored. In addition to ANL's role as evaluation manager in the ADVANCE project, ANL is a founding member of ITS Midwest. 


\title{
95-265N -- HIGH-SENSITIVITY INFRARED CHEMICAL SENSOR DEVELOPMENT ${ }^{1}$
}

\author{
Associate Laboratory Director Area: Physical Research
}

Principal Investigators:

S.A. Johnson, Chemical Technology Division V.A. Maroni, Chemical Technology and Materials Science Divisions

V.J. Novick, Technology Development Division

Funding Profiles:

FY $1993-0-$
FY $1994-0-$
FY $1995 \quad \$ 104.6 \mathrm{~K}$
FY $1996 \quad \$ 140.0 \mathrm{~K}$
FY $1997 \$ 200.0 \mathrm{~K}$

Purpose: We have proposed to develop an ensemble of high-sensitivity chemical sensors by combining several state-of-the-art infrared spectroscopic techniques. The ultimate goal of the effort is economical, portable, research-quality instruments with field and factory floor utility that will detect a broad array of chemical effluents and provide high sensitivity for specific chemical functional groups on a near-real-time basis. Chemical sensors are in high demand in diverse areas, such as atmospheric chemistry, treaty verification, industrial processes, and health effects for both routine monitoring and research studies. Successful development of these sensors would have significant commercial possibilities as well as applications in many federallyand industrially-funded activities.

Approach: The first work (at ANL) that was done on the concepts employed in this research was in the spring of 1995. As initially proposed the research was to be divided into three tasks. In Task 1 we would explore the use of infrared spectroscopy as a sensitive means of detecting species collected on a chemically derivitized inner surface of an infrared transmissive light pipe. In Task 2 we would investigate the utility of fiber evanescent wave spectroscopy (FEWS) as a chemical sensor. For Task 3 we proposed to explore the application of surface enhanced infrared absorption (SEIRA), a powerful new sensitivity boosting technique, and to determine the extent to which it can be coupled with the techniques studied in Tasks 1 and 2 .

Even though infrared spectroscopy (IRS) has been a heavily used analytical tool for decades, it remains a very hot research area, due to the development of some new approaches for interrogating samples and to the discovery of several signal enhancement techniques. The main strength of IRS is its chemical specificity for an extremely broad range of compounds. The main drawbacks for moving IRS out of the lab and into the field (or into manufacturing) have been lack of sensitivity and costly, cumbersome instrumentation. In the case of organic materials, the utility of IRS techniques has heen clearly recognized and, in some instances, exploited, such as in passive remote IRS sensing. However, a comprehensive IR-based detection package remains to be developed in the form that we propose here. The preliminary results from the current work are very encouraging and the proposed future work has the possibility of

${ }^{1}$ CCST Project 
achieving one or more very significant technological advancements in the field of packaged chemical sensors.

One of the major limitations of IRS has been that the chemical species of interest are often present at concentrations well below normal detection limits. Typically, this problem is circumvented by concentrating the species of interest on a surface (e.g., of an IR transparent or reflective substrate) or in a bulk IR-transparent medium. But even when these methods are applied, the concentrated species either remains undetectable or is: detectable but not unambiguously characterizable. Also the process of collecting, concentrating and preparing the sample precludes the possibility of real-time analysis.

Sensitivity enhancement is, therefore, an essential research direction for improving the detection limits of IR-based sensors that operate in real-time or at least in near-real-time (on the order of seconds to a few minutes). Our approach builds on the SEIRA technique to develop an ensemble of high-sensitivity chemical sensors by combining several state-of-the-art infrared spectroscopic techniques.

Technical Progress and Results: Good progress was made in the first months of work. Using a new approach called SEIRA, enhancements of nearly three orders of magnitude have been demonstrated, bringing the limit of detection for the organic analyte, para-nitro benzoic acid (PNBA), into the low picogram range. Also, detection of this low vapor pressure solid (PNBA) placed in a flowing gas stream has been demonstrated. This has been accomplished by using powerful infrared sensitivity enhancement techniques as originally proposed for this project.

Since only half of the requested funding was granted for the first year's work on this LDRD, the FY 1995 effort was directed towards Tasks 1 and 3, with the emphasis on Task 3. The SEIRA technique (Task 3) was perceived to have the greatest potential for major improvement in sensitivity. Indeed, enhancements of nearly three orders of magnitude have been demonstrated (see Figure 1). With the embodiment we have chosen for implementation of the SEIRA technique we can routinely detect quantities of analyte in the low picogram range, using a system which is still not fully optimized. More significantly, this sensitive detection method has been used as a chemical. sensor to detect vapor from a low vapor pressure solid in a flowing air stream. This picogram sensitivity qualifies the concept for near real-time chemical sensor development. Indeed, we have surpassed expectations for progress in this task. One of the first experimental parts of Task 3 was to explore the sputtering process to achieve optimum-size metal islands on the internal reflection elements (IREs) used in attenuated total reflection (ATR) IRS. Appreciable time was expended on this part of Task 3 with good results, and there is potential for even greater improvement. From work done thus far using thermal and plasma sputtered gold, silver, and copper films on substrates of zinc selenite and germanium it has been shown that sputtering conditions are critically important. Thermal sputtering has been shown to be far superior to plasma sputtering due to unwanted chemical and physical interactions when the plasma device is used. For thermal sputtering near optimum enhancement has been achieved routinely. Attempts were made to use colloidal gold, to further simplify the gold film preparation, but this was not successful.

In addition to achieving high sensitivity and demonstrating utility as a vapor phase chemical sensor, some important other features of SEIRA were documented. Several different organic 
compounds were found to have enhancable infrared absorption bands. The compounds used were tri-n-octylphosphine oxide, 2-diethylaminoethanethiol hydrochloride, meta-nitrobenzoic acid, and para-nitrobenzoic acid. In each compound one or more vibrational modes were enhanced, indicating that this type of SIERA chemical sensor system is applicable to a broad range of compounds. Also, the method is very specific, as demonstrated by the differentiation of the two isomers of nitrobenzoic acid. This aspect is important because it highlights the utility of the technique as a tool to understand bonding and surface phenomena as well for chemical sensor applications. Also demonstrated was the detection of para-nitrobenzoic acid from an aqueous solution. Thus, the technique has been shown to be useful for detection of compounds from the vapor phase in air, from liquid organic solutions, and from aqueous solutions.

Progress was also made on Task 1. Existing equipment was modified to accommodate small light pipes or tubes in which the inner surface is the reflective and active surface. Reasonable sensitivities were achieved using simple aluminum foil light pipes with nanogram quantities of the analyte on the inner surface. Informative spectra were recorded with sensitivity at the tenths of nanogram level. In the first trials the small light pipes $(25 \mathrm{~mm}$ long and $5 \mathrm{~mm}$ inside diameter) were prepared from aluminum foil with nanometer gold particles sputtered on the inner surface. This arrangement worked well with nanogram quantities of para-nitro benzoic acid. It was found that this worked with or without the gold island film, which opens up the possibility of inexpensive, disposable sensing elements. As discussed for Task 3 , where internal reflection elements are used, the possibility of enhancement of certain absorption bands via external reflection in a light pipe is possible and needs to be further investigated. Extrapolation of known results suggests that with a conduit of sufficient (but reasonable) surface area and optimized receptors, compound recognition sensitivity could be in the parts-per-billion range and functional group sensitivity in the parts-per-trillion range.

The proposed FY 1996 effort will extend the basic investigations and include work on Task 2 which involves the use of FEWS as a chemical sensor. Emphasis will be on the optimization and integration of the techniques as a combination of chemical sensors to detect both trace and bulk chemical constituents in solid, liquid, or gaseous forms. Toward the end of the second year and into the third year, we expect to demonstrate the potential for miniaturization and packaging of field able sensor instruments with the actual instrument development continued through, e.g., a CRADA or licensing arrangement with a commercial partner.

The specific types of infrared methods that we are developing/demonstrating have great potential for utilization in the Arms Control and Treaty Verification area, in EPA's Environmental Technology Initiative, in the DoD/DOE SERDP activity, and in the DOE Environmental Restoration and Waste Management Program. At the same time, they may be well-suited for applications related to industrial process monitoring and even monitoring in the household and in the workplace.

Specific Accomplishments: Work on this project was not initiated until the spring of 1995. Also, the funding level was only one-half of that requested. Nevertheless, very good progress has been made and about two-thirds of the goals set forth in the original proposal for the first year have been accomplished. The results are very promising in that a very high sensitivity (in the low picogram range) has been achieved. Furthermore, the technique has been demonstrated as a chemical sensor in that it detected a very low vapor pressure compound at room temperature 
by vapor phase transfer. At least one paper is anticipated from this work and a strong basis for an invention disclosure (presumably leading to a patent) should evolve from the proposed continuation of this project. Also, one foreign exchange student was supported by this LDRD project. 


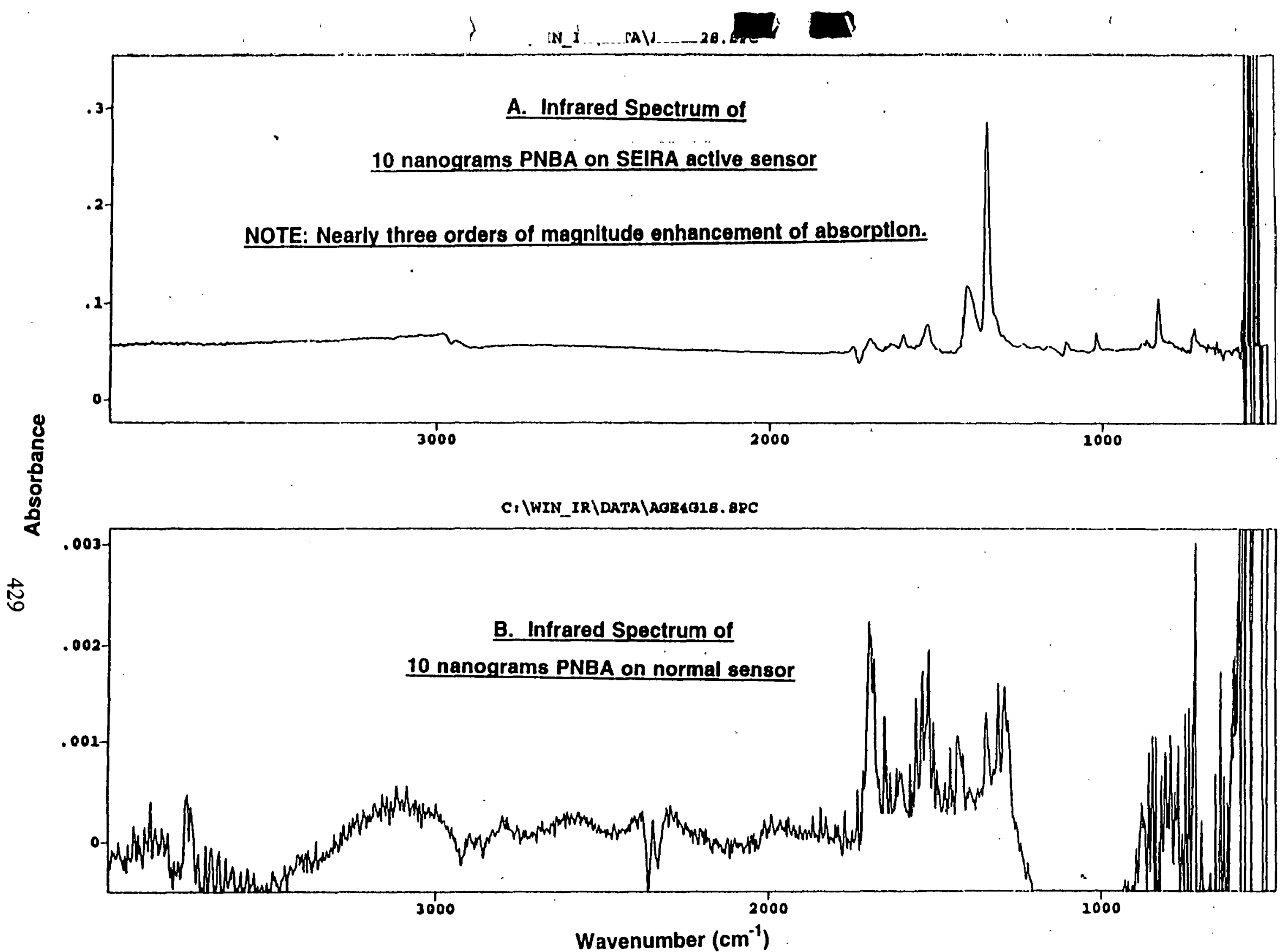

Fig. 1. Comparison of sensitivity on a Surfaced Enhanced Infrared Absorption (SEIRA). sensing element and an unstimulated element. Calculated LOD, based on Spectrum $A$ and other SEIRA spectra, is in the low plcogram range. 
blank page 


\section{FY 1996 LDRD PROJECT SUMMARIES}

*Individual Investigator Projects

${ }^{* \star}$ Authorized funding for FY97 and FY98 is estimated

†There are no new-start CCST projects in FY96. 


\section{FY 1996 LDRD PROJECT SUMMARIES †}

\begin{tabular}{|c|c|c|c|}
\hline $\begin{array}{l}\text { Proposal } \\
\text { Number }\end{array}$ & $\begin{array}{l}\text { Authorized } \\
\text { Funding** }\end{array}$ & Principal Investigators & Title and Summary \\
\hline $96-001 \mathrm{~N}^{*}$ & FY96/\$75K & Jack M. Williams & $\begin{array}{l}\text { Novel Molecular Rulers for Advanced Manufacturing Processes } \\
\text { Unique electrically conducting solid organic substrates (molecular rulers) will be synthesized and } \\
\text { tested [via scanning tunneling microscopy (STM)] in the laboratory for use in advanced ultra-high } \\
\text { precision manufacturing positioning and micromachining operations, based on STM driven } \\
\text { metrology systems and used in the production of next-generation electronic devices. Emphasis will } \\
\text { be on micromachining and cutting operations at } 15-100 \AA \text { repeat distances that will allow much } \\
\text { higher packing densities on integrated chips than are presently achieved. Molecular rulers with } \\
\text { tailored internal repeat separations much larger (10-100 } \dot{A} \text { ) than those in most conducting } \\
\text { compounds and metals (<<10 } \dot{A} \text { ) will be fabricated and characterized crystallographicaly prior to } \\
\text { STM study. The most important advantages of this novel approach to molecular ruler synthesis are } \\
\text { that conducting organic materials uniquely satisfy STM-based equipment requirements, i.e., they are } \\
\text { electrically conductive, can be grown defect free with very large optically flat faces (many mm on a } \\
\text { side), are easily and reproducibly produced, and they can be tailor-made to have exact and } \\
\text { repeatable (necessary for repeat placement and cutting operations) intersolid repeat separations of } \\
10-100 \AA \text {. }\end{array}$ \\
\hline $96-002 N^{*}$ & FY96/\$75K & Neil C. Sturchio & $\begin{array}{l}\text { Stable Isotopic Investigations of the Chemical and Biological Degradation of Chlorinated Organic } \\
\text { Solvents } \\
\text { We will explore and develop innovative methods for investigating the mechanisms of chemical and } \\
\text { biological degradation of chlorinated organic solvents using precise isotopic ratio measurements of } \\
\text { carbon and chlorine in reactant and product species in laboratory experiments and from field } \\
\text { demonstration sites. Specific tasks include (1) optimizing the analytical protocols for determining } \\
\text { compound-specific carbon and chlorine isotope ratios in the appropriate chemical systems; (2) } \\
\text { defining the isotopic fractionation factors during chemical and biological degradation of chlorinated } \\
\text { solvents in laboratory experiments; and (3) using stable isotope ratios to investigate degradation of } \\
\text { chlorinated volatile organic compounds at field demonstration sites where contrasting remedial } \\
\text { techiniques are being employed. }\end{array}$ \\
\hline $96-004 N^{*}$ & FY96/\$75K & Priscilla W. Stevens & $\begin{array}{l}\text { Interactions of Molecules Involved in Cell Death } \\
\text { Molecules which regulate cellular pathways of cell death have only recently been identified and } \\
\text { constitute a new structural and functional class of proteins. Two protein inhibitors of programmed } \\
\text { cell death, BCL-2 and BHRF-1, were sequenced in the mid } 1980 \text { s, but in the past two years, nine } \\
\text { additional distinct proteins in this family have been described. Certain of these molecules } \\
\text { accelerate cell death, while others inhibit it. It is suggested that interactions among various } \\
\text { proteins within this family determine whether a particular cell's fate will be survival or cell death. } \\
\text { This project would add direct biophysical information on molecular-level properties of these } \\
\text { proteins and provide detailed quantitative information about the ways in which these proteins } \\
\text { associate with each other. The long-term goal of this project would therefore be to quantitatively } \\
\text { define the molecular-level interactions of cell-death regulator molecules within the BCL-2 family. }\end{array}$ \\
\hline
\end{tabular}




\begin{tabular}{|c|c|c|c|}
\hline $\begin{array}{l}\text { Proposal } \\
\text { Number }\end{array}$ & $\begin{array}{l}\text { Authorized } \\
\text { Funding** }\end{array}$ & Principal Investigators & Title and Summary \\
\hline $96-007 N^{*}$ & FY96/\$75K & Deborah J. Zurawski & $\begin{array}{l}\text { Zeolite-Based Catalyst Structures for Direct Methanol Fuel Cells } \\
\text { The direct methanol fuel cell is an attractive power source for transportation applications due to the } \\
\text { high energy density of methanol, the portability and ease of distribution of liquid rather than } \\
\text { gaseous fuel, and the elimination of the bulky, power-consuming fuel reformer. The solid polymer } \\
\text { membrane is the electrolyte of choice for mobile applications of fuel cells, since it operates at low } \\
\text { temperatures }(60-100 \mathrm{C}) \text { and is non-corrosive. One of the major limitations on the power output of } \\
\text { the direct methanol fuel cell is the crossover of methanol from the anode to the cathode. } \\
\text { Successful development of a methanol-tolerant cathode catalyst or a methanol-impermeable } \\
\text { membrane will increase the power output of this fuel cell by approximately thirty percent, making } \\
\text { it more viable. }\end{array}$ \\
\hline $96-009 N^{*}$ & FY96/\$75K & Michael M. Thackeray & $\begin{array}{l}\text { Exploratory Research of Novel Oxygen-lon Conducting Structures } \\
\text { We will explore design of novel materials that show anomalously high oxygen ion mobility at room } \\
\text { temperature or moderately high temperature. These materials would be of interest for } \\
\text { electrochemical applications such as sensors and fuel cells and would be particularly suited for } \\
\text { systems that would operate at considerably lower temperature than "state-of-the-art" devices. }\end{array}$ \\
\hline $96-012 N^{*}$ & FY96/\$75K & Deborah K. Hanson & $\begin{array}{l}\text { Engineering Photochemistry for Novel Redox Reactions and Bioremediation } \\
\text { Recent results of the photosynthetic reaction center complex have shown that cavities can be } \\
\text { created at specifically determined sites in the reaction center protein. These cavity mutants provide } \\
\text { a means to test how photochemistry is coupled to the dielectric properties of the protein as it } \\
\text { modulates electron transfer, proton transfer, and charge stabilization. This project will evaluate the } \\
\text { potential for the use of reaction center photochemistry to initiate novel redox reactions. Existing } \\
\text { cavity-containing mutants would allow initial experiments in the binding of organic molecules. } \\
\text { Engineering of metal-binding cavities is a straightforward process. The majority of the effort would } \\
\text { be used to evaluate the redox properties of this system and its potential for application to } \\
\text { environmental contaminants. }\end{array}$ \\
\hline $96-018 N^{*}$ & FY96/\$75K & Ulrich Welp & $\begin{array}{l}\text { Magneto-Optical Imaging of Current Distributions in Josephson Junctions } \\
\text { Josephson junctions (J) in high temperature superconductors have potential to significantly improve } \\
\text { the operating performance of many devices, such as high-speed logic circuits, high sensitivity } \\
\text { SQUID magnetometers and analog-digital converters. This potential has not been realized because } \\
\text { no reliable fabrication process capable of producing junctions with reproducible characteristics has } \\
\text { been found. This is due, in large part, to our poor understanding of the connection between the } \\
\text { atomic structure of junctions and their superconducting behavior. In the first part of this project, } \\
\text { we apply to new Argonne-fabricated JJ a novel high-sensitivity magneto-optical imaging technique } \\
\text { capable of micron resolution showing the interplay of magnetic fields and current within the } \\
\text { junction itself. With the high-resolution information revealed by imaging, we will develop new } \\
\text { models for junction behavior which will focus on the correlation of microstructure and } \\
\text { superconducting operating characteristics. These models will provide background for } \\
\text { understanding junction behavior and for developing new strategies for fabrication processes. In the } \\
\text { second part of this research, we exploit the one dimensional geometry of these junctions as ideal } \\
\text { model systems for the study of vortex dynamics. Periodic arrays of defects have been shown to } \\
\text { cause dramatic enhancements of the critical current. A linear periodic array of defects will be } \\
\text { introduced into the grain boundary junction using electron irradiation and the vortex response will } \\
\text { be characterized by direct magneto-optical vortex imaging. }\end{array}$ \\
\hline
\end{tabular}




\begin{tabular}{|c|c|c|c|}
\hline $96-023 N^{*}$ & FY96/\$75K & Hsien Hau Wang & $\begin{array}{l}\text { Shape and Function Selective Molecular Receptors for Advanced Sensors } \\
\text { Molecular recognition for advanced sensor application bears both scientific and technical } \\
\text { significance. Based on attractive forces such as hydrogen bonding and on molecular shape, } \\
\text { synthetic molecular receptors designed for inclusion and recognition of specific molecules at ultra } \\
\text { low concentrations will be developed. The proposed work for shape and function selection } \\
\text { molecular receptors consists of three stages: (1) organic synthesis of the target "cavitand" } \\
\text { molecules, (2) fabrication of the self assembled monolayer (SAM) with cavitands and gold } \\
\text { substrates; and (3) evaluation of the sensing capability and reversibility of the SAM films with use of } \\
\text { a Raman microscope spectrometer. }\end{array}$ \\
\hline $96-024 N^{*}$ & FY96/\$75K & Gary R. Dyrkacz & $\begin{array}{l}\text { Modification of Catalyst Pores for Low Density Separations } \\
\text { Fluidized catalytic crackers (FCC) are the workhorse of refineries to produce gasoline. The catalyst } \\
\text { used in FCC units loses its activity quite rapidly. A substantial tonnage of catalyst must be replaced } \\
\text { on a daily basis. The removal operation is completely nonselective; active catalyst is removed with } \\
\text { the nonactive material. In addition, disposal of the catalyst, often containing heavy metals, is } \\
\text { looming as an environmental problem. If there were simple methods of separating the inactive } \\
\text { from the active material, then both the economic and the environmental issues would be reduced. } \\
\text { As a new approach to the separation problem, this project will develop reagents that can } \\
\text { reversibly cap off FCC catalyst pores preventing fluid access. Simple, inexpensive low density } \\
\text { fluids can then be used to float away the active from the inactive catalyst. }\end{array}$ \\
\hline $96-031 N^{*}$ & FY96/\$75K & Marion C. Thurnauer & $\begin{array}{l}\text { Investigation of a New Site for Herbicide Action in Green Plants } \\
\text { We will assess the feasibility of utilizing a new herbicide binding site in plants to develop a new } \\
\text { class of herbicides. At the same time, a new tool to rapidly and conveniently screen herbicide } \\
\text { action will be evaluated. Emphasis will be placed on herbicide effectiveness and selectivity } \\
\text { coupled with relative environmental safety. Agriculture relies heavily on the use of herbicides, and } \\
\text { there is a clear need to develop new herbicides which have minimal impact on the environment. } \\
\text { Many broad spectrum herbicides work by interrupting the electron transfer reactions which are } \\
\text { necessary for plants to carry out photosynthesis and grow. Because a site has been found on the } \\
\text { photosynthetic apparatus which differs from the sites which are usually accessed for growth } \\
\text { inhibition, we have the opportunity to search for new classes of herbicides which are potentially } \\
\text { less toxic to the environment. In addition, the concentration in the plant of the native species } \\
\text { which resides at this new site is much lower than the concentration in the plant of the native } \\
\text { species at the traditional herbicide binding site. Therefore, one expects that much lower herbicide } \\
\text { concentrations will be adequate to be effective at the new site. We will attempt to demonstrate. } \\
\text { that this new site can effectively inhibit plant growth. }\end{array}$ \\
\hline
\end{tabular}




\begin{tabular}{|c|c|c|c|}
\hline $\begin{array}{l}\text { Proposal } \\
\text { Number }\end{array}$ & $\begin{array}{l}\text { Authorized } \\
\text { Funding** }\end{array}$ & Principal Investigators & Title and Summary \\
\hline $96-037 N^{*}$ & FY96/\$75K & Jeffrey Tilson & $\begin{array}{l}\text { Ab Initio Characterization of a Model Catalytic HDS Process } \\
\text { We will perform the first all-electron large-scale ab initio calculations of the hydrodesulfurization } \\
\text { (HDS) catalytic process. This is a process routinely used in the oil industry to remove unwanted } \\
\text { heteroatom species from crude oil feedstocks. Calculations of this size are made possible because } \\
\text { of our work in a High Performance Computation and Communications Initiative project that has } \\
\text { produced fully parallelized electronic structure code that runs very efficiently on the } 128 \text {-node SP } \\
\text { computer. The calculations will validate theoretical results by comparison to known experimental } \\
\text { facts about standard HDS catalysts, will clarify remaining contentious experimental issues and will } \\
\text { pave the way for future interactive experimental-theory studies of novel HDS catalysts. }\end{array}$ \\
\hline $96-041 \mathrm{~N}$ & FY96/\$100K & Deming Shu & $\begin{array}{l}\text { Novel Laser Doppler Angular Encoder for High Speed Nanopositioning } \\
\text { We will develop a novel laser Doppler angular encoder with high speed (up to } 3.3 \text { radians } / \mathrm{sec} \text { ) and } \\
\text { sub-nanoradian resolution in the } 5-10 \text { degree measuring range. The new design will have a } \\
\text { compact size (about } 60 \mathrm{~mm}(\mathrm{H}) \times 100 \mathrm{~mm}(\mathrm{~W}) \times 280 \mathrm{~mm}(\mathrm{~L}) \text { ) and potential ultrahigh vacuum } \\
\text { compatibility. Because it has a lower cost and higher performance compared with commercial } \\
\text { laser interferometer systems, there are many potential scientific and industrial applications of this } \\
\text { novel angular encoder. We will also try to determine the feasibility of using a similar optical } \\
\text { method to develop a novel laser doppler linear encoder system that will provide high speed (up to } \\
500 \mathrm{~mm} / \mathrm{sec} \text { ) and near-Angstrom resolution over more than a } 100-\mathrm{mm} \text { range. This new technology } \\
\text { will be valuable for the development of the next generation instrumentation, for example: large } \\
\text { field scanning tunneling microscopes, X-ray microscopes, X-ray lithography and X-ray } \\
\text { micromachining. }\end{array}$ \\
\hline $96-042 N^{*}$ & FY96/\$75K & S. K. Sinha & $\begin{array}{l}\text { Development of X-Ray Waveguide Techniques for Studying Liquids in Confined Geometries } \\
\text { Thin films can act as X-ray waveguide resonators. The increase of coherent photon flux at the APS } \\
\text { by several orders of magnitude over second generation synchrotron sources opens up the possibility } \\
\text { of producing enormous flux concentration of coherent X-ray radiation confined in liquid or } \\
\text { polymeric films of thickness down to as low as } 10 \mathrm{~nm} \text {. The existence of layered and laterally } \\
\text { ordered structures induced in molecular liquids confined to a thickness of < } 10 \text { molecular } \\
\text { diameters between solid surfaces has been inferred from computer simulations and surface force } \\
\text { apparatus measurements, but no direct structural evidence from X-ray diffraction exists. The issue } \\
\text { is important for research in areas such as lubrication (tribology), adhesion, etc. We will carry out } \\
\text { initial experiments using a confined liquid as an X-ray waveguide to exploit the high brilliance of } \\
\text { the APS radiation for directly probing such novel structures. These experiments will lead to the } \\
\text { design and construction of a surface force apparatus fcr carrying out such studies at the APS in a } \\
\text { routine manner. The coherent beam emerging from the microscopic slit will also be used for } \\
\text { demonstrating imaging and holographic capabilities since the device also acts as a microfocusing } \\
\text { device. }\end{array}$ \\
\hline
\end{tabular}




\begin{tabular}{|c|c|c|c|}
\hline $\begin{array}{l}\text { Proposal } \\
\text { Number }\end{array}$ & $\begin{array}{l}\text { Authorized } \\
\text { Funding** }\end{array}$ & Principal Investigators & Title and Summary \\
\hline $96-062 N^{*}$ & FY96/\$75K & David Spence & $\begin{array}{l}\text { A Novel High-Current, High-Brightness } H^{\prime}\left(D^{\prime}\right) \text { lon Source for Advanced Accelerator Applications } \\
\text { A prerequisite for the development of synchrotron-based advanced pulsed neutron sources, and } \\
\text { indeed any circular proton accelerator, is a highly reliable, high-current, high-brightness } H^{\prime} \text { ion } \\
\text { source. For example, the ion source requirements for the proposed IPNS upgrade can barely be } \\
\text { met by the RF driven volume source, which is the most advanced available, and whose durability } \\
\text { and reliability may be questioned. The technical purpose of this project is to develop a novel high } \\
\text { reliability microwave driven } H^{-} \text {source based on an existing high pressure }\left(10^{-3} \text { torr) proton source }\right. \\
\text { but utilizing advanced concepts of } H^{-} \text {production previously developed at ANL. }\end{array}$ \\
\hline $96-076 N^{*}$ & FY96/\$75K & Jeffrey S. Gaffney & $\begin{array}{l}\text { Tracking Atmospheric Aerosols with Natural Radioisotopes - }{ }^{7} \text { Be and }{ }^{210} \mathrm{~Pb} \\
\text { We will determine the extent to which natural radiotracers can be used as direct measures of } \\
\text { tropospheric aerosol sources by applying Argonne's low-level counting facilities to aerosol and } \\
\text { precipitation samples. Increasing evidence indicates that atmospheric aerosols play key roles in the } \\
\text { radiative balance of the atmosphere. Direct effects include shortwave light scattering of incoming } \\
\text { solar radiation and absorption of longwave radiation from the earth's black-body infrared emission. } \\
\text { Aerosols can also act indirectly on the regional radiative balance and can affect wet deposition of } \\
\text { air pollutants by serving as cloud condensation nuclei. To improve our knowledge of the effects of } \\
\text { aerosols on tropospheric air quality and climate, a better understanding of their source, size } \\
\text { distribution, and atmospheric lifetimes is needed. }\end{array}$ \\
\hline $96-081 N^{*}$ & FY96/\$75K & Maury Goodman & $\begin{array}{l}\text { Near Detector for the MINOS Experiment } \\
\text { The near detector is a small but very important part of the MINOS long baseline neutrino } \\
\text { oscillation experiment. Our approach includes the following efforts: Beam Monte Carlo simulation } \\
\text { and design optimization. The design of the near detector must include particular attention to the } \\
\text { understanding of flux differences and similarities between the near and far deteciors. Study of the } \\
\text { timing, spatial resolution, and energy resolution requirements of the detector for neutrino } \\
\text { oscillation using the proposed Fermilab neutrino beam. Study electron neutrino backgrounds in the } \\
\text { beam and determine detector capabilities for electron identification in the near detector. We will } \\
\text { also explore innovative concepts for neutrino detector technology and study magnetic field } \\
\text { configurations in the near detector. }\end{array}$ \\
\hline $96-094 N^{*}$ & FY96/\$75K & Richard L. Coulter & $\begin{array}{l}\text { Development of a Volume-Imaging Sodar } \\
\text { We will develop an atmospheric acoustic remote sensor capable of resolving the three-dimensional } \\
\text { wind and turbulence field throughout a limited region of the lower atmosphere. Present remote } \\
\text { sensors measure the wind field only in a vertical plane oriented along the horizontal wind } \\
\text { direction. Development of this new technology will enable observance of the "instantaneous" } \\
\text { three-dimensional nature of coherent structures within the atmospheric planetary boundary layer, } \\
\text { the effects of surface characteristics on the local wind field, and crosswind energy and momentum } \\
\text { transfer. The data from this instrument will enable calculations of the crosswind spatial } \\
\text { and temporal autocorrelation functions and will aid in the evaluation of atmospheric energy transfer } \\
\text { between the surface and the lower troposphere. }\end{array}$ \\
\hline
\end{tabular}




\begin{tabular}{|c|c|c|c|}
\hline $\begin{array}{l}\text { Proposal } \\
\text { Number }\end{array}$ & $\begin{array}{l}\text { Authorized } \\
\text { Funding** }\end{array}$ & Principal Investigators & Title and Summary \\
\hline $96-101 \mathrm{~N}$ & FY96/\$100K & $\begin{array}{l}\text { David Ayres, } \\
\text { Jonathan Thron }\end{array}$ & $\begin{array}{l}\text { Development of Limited Streamer Tube Technology for MINOS (Main Injector Neutrino } \\
\text { Oscillation Search) } \\
\text { We will develop limited streamer tube (LST) technology for use in the MINOS (Main Injector } \\
\text { Neutrino Oscillation Search) long baseline neutrino oscillation experiment. The MINOS } \\
\text { calorimeter requires the construction of } 32,000 \text { square meters of tracking chambers, which record } \\
\text { the numbers and positions of particle tracks, including electromagetic and hadronic showers. } \\
\text { Unlike previous applications of LSTs, the MINOS chambers must be constructed from material } \\
\text { which does not release toxic gases when burned and must operate with nonflammable gas. Mass } \\
\text { production techniques must be developed for the fabrication of very large areas of chambers at } \\
\text { very low cost. This project supports development of chambers which satisfy these requirements } \\
\text { and the design and construction of a model calorimeter which can test the calorimetry response of } \\
\text { the new chambers in a charged particle test beam. }\end{array}$ \\
\hline $96-103 N$ & FY96/\$100K & Charles D. Jonah & $\begin{array}{l}\text { Feasibility of Microwave Stabilization of a High Energy Pulsed Power Supply That Can Be Used } \\
\text { For A Sub-Picosecond Pulse Radiolysis Facility } \\
\text { We will explore the design and development of a highly stable, pulsed, microwave source that can } \\
\text { be used in the sub-picosecond pulse radiolysis facility. A key advance that is needed for the } \\
\text { realization of sub-picosecond electron accelerators is the development of microwave sources with } \\
\text { commensurate stability. If the inherent time resolution available from a short electron pulse and a } \\
\text { short laser witness pulse is to be maintained, the electron pulse compression must preserve the } \\
\text { synchronization between the electron pulse and the laser witness pulse. This is possible only if the } \\
\text { energy of the electron pulse is very reproducible, which requires a well-regulated microwave } \\
\text { source. These sources supply approximately } 20 \mathrm{MW} \text { of peak power with a pulse width of about } 5 \\
\text { microseconds. The frequency of the microwave power is approximately } 1.4 \mathrm{GHz} \text {. This project } \\
\text { will lead to the development of a pulsed microwave source with one percent energy stability. }\end{array}$ \\
\hline $96-106 \mathrm{~N}$ & $\begin{array}{l}\text { FY96/\$200K } \\
\text { FY97/\$200K }\end{array}$ & $\begin{array}{l}\text { I. Foster, W. Gropp, } \\
\text { E. Lusk, S. Twecke }\end{array}$ & $\begin{array}{l}\text { Enabling Yechnologies for Internetworked Applications } \\
\text { We will develop core technologies required to support applications that exploit computing } \\
\text { resources distributed on national-scale networks. Significant technologv development is required } \\
\text { before the advantages of widespread, low-cost access can be exported by the scientific and } \\
\text { engineering communities. We will investigate issues in runtime systems, communication protocols, } \\
\text { security, and interfaces with standard high-performance and object-based technologies. }\end{array}$ \\
\hline $96-107 \mathrm{~N}$ & FY96/\$100K & J. Proudfoot & $\begin{array}{l}\text { Prototype Calorimeter for ATLAS Detector at CERN LHC } \\
\text { We will build demonstration modules of the hadronic calorimeter for the ATLAS detector at the } \\
\text { CERN Large Hadron Collider (LHC). The LHC is the only planned facility at which physics } \\
\text { investigations formerly planned for the Superconducting Supercollider (SSC) can be pursued. } \\
\text { Argonne and four university collaborators have had a major impact on the work of the group in } \\
\text { ATLAS designing the hadronic calorimeter. The calorimeter has an innovative design, employing a } \\
\text { variation of the scintillating tile/fiber techniques developed by Argonne and our collaborators for } \\
\text { the Solenoidal Detector Collaboration (SDC) detector at SSC. We will test the viability of the } \\
\text { design in } 1996 \text { by fabricating model components and testing them using cosmic rays, radioactive } \\
\text { sources and high-energy particle beams. }\end{array}$ \\
\hline
\end{tabular}




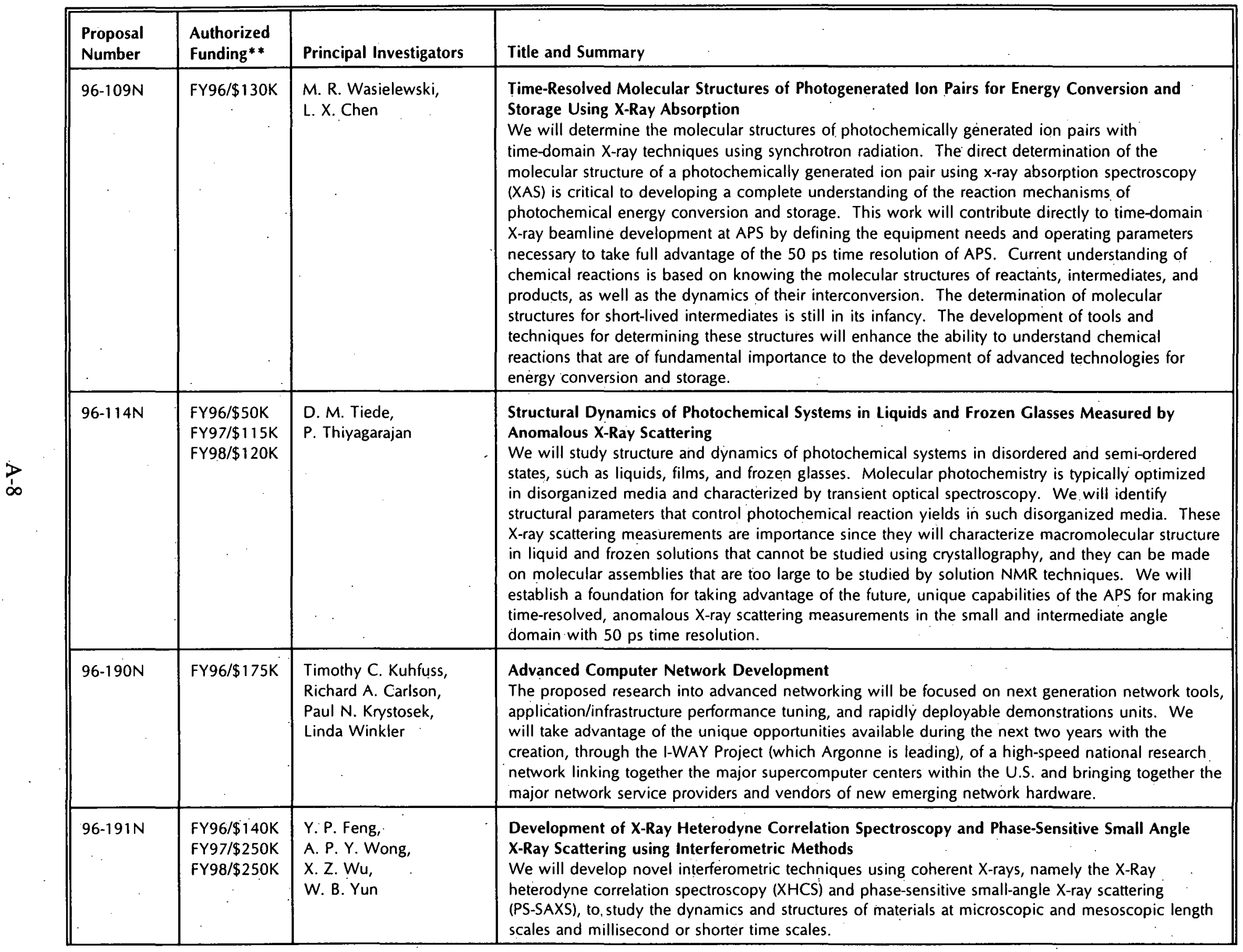




\begin{tabular}{|c|c|c|c|}
\hline $\begin{array}{l}\text { Proposal } \\
\text { Number }\end{array}$ & $\begin{array}{l}\text { Authorized } \\
\text { Funding** }\end{array}$ & Principal Investigators & Title and Summary \\
\hline $96-192 \mathrm{~N}$ & $\begin{array}{l}F Y 96 / \$ 130 K \\
F Y 97 / \$ 230 K \\
F Y 98 / \$ 210\end{array}$ & $\begin{array}{l}\text { Ian McNulty, } \\
\text { Stephan F. Brauer, } \\
\text { G. Brian Stephenson }\end{array}$ & $\begin{array}{l}\text { Development of X-Ray Intensity Fluctuation Spectroscopy for Study of Atomic-Scale Equilibrium } \\
\text { Dynamics with Coherent X-Rays } \\
\text { We will develop X-ray intensity fluctuation spectroscopy (XIFS) as a tool to probe atomic-scale } \\
\text { structural dynamics in several model-disordered systems at equilibrium. IFS is a well developed } \\
\text { scattering technique with visible light that has been highly successful in studies of micron-scale } \\
\text { dynamics in phase separating liquids, liquid crystals, polymers and colloids. Performing IFS } \\
\text { measurements with coherent X-rays would provide the exciting possibility of probing dynamics on } \\
\text { much smaller length scales, extending to atomic-scale structural fluctuations in a wide variety of } \\
\text { systems including materials which are opaque to visible light. Moreover, anomalous absorption } \\
\text { features in the X-ray region are attractive for enhancement of the signal from weakly scattering } \\
\text { samples. Of the various methods used for visible light IFS, the homodyne or self-referencing } \\
\text { method is the most promising for the x-ray region because of its relative optical simplicity and } \\
\text { efficiency. Because the required coherent flux is directly proportional to the source brilliance, this } \\
\text { technique will take full advantage of the unprecedented brilliance of undulator sources at the } \\
\text { Advanced Photon Source. }\end{array}$ \\
\hline $96-193 N$ & FY96/\$75K & $\begin{array}{l}\text { Suk H. Kim, } \\
\text { Charles Doose, } \\
\text { Robert Merl }\end{array}$ & $\begin{array}{l}\text { Temperature Compensation of Permanent Magnets } \\
\text { We will develop permanent magnets which use magnetic flux shunts as a passive scheme to } \\
\text { maintain constant magnetic fields within } 1 \times 10^{-4} \text { under operating temperature variations of }+/-10-20 \\
\text { degrees } C \text {. Permanent magnets have advantages over electromagnets and superconducting magnets } \\
\text { for certain applications such as charged particle beam storage rings and magnetic resonance images } \\
\text { (MRI) which require fixed magnetic fields. In such applications, performance variations due to } \\
\text { changes in the temperature coefficients of the residual induction and coercive forces cannot be } \\
\text { tolerated. }\end{array}$ \\
\hline $96-194 \mathrm{~N}$ & $\begin{array}{l}\text { FY96/\$194K } \\
\text { FY97/\$200K } \\
\text { FY98/\$200K }\end{array}$ & $\begin{array}{l}\text { Marion M. White } \\
\text { Yung Long Qian }\end{array}$ & $\begin{array}{l}\text { Slow-Positron Source } \\
\text { We will design a new type of target for efficient production of slow positrons and determine the } \\
\text { feasibility of using the APS electron linac to create an intense source of very-low-energy positrons } \\
\text { for research. The slow-positron source would operate in parallel with the APS and would provide } \\
\text { exciting, state-of-the-art research opportunities complementary to the synchrotron radiation } \\
\text { program. }\end{array}$ \\
\hline $96-195 N$ & $\begin{array}{l}\text { FY96/\$170K } \\
\text { FY97/\$200K } \\
\text { FY98/\$150K }\end{array}$ & $\begin{array}{l}\text { Brian Rodricks, } \\
\text { Dennis Mills }\end{array}$ & $\begin{array}{l}\text { Investigation of Microstrip Gas Detectors for X-Ray Time-Resolved Studies } \\
\text { We will initiate the investigation of a position-sensitive detector based on the microstrip anode. } \\
\text { This detector is an extension of the multiwire proportional counter (MWPC). A MWPC is a series } \\
\text { of anode wires assembled in a plane and sandwiched between two cathode plates that are } \\
\text { enclosed in a gas environment. X-rays that impinge on the device ionize the gas. The voltage } \\
\text { applied to the anode wires creates an electron avalanche amplification as the electrons approach } \\
\text { the wires. The charge and position are sensed from each wire by means of delay lines or charge } \\
\text { division techniques. In the microstrip gas counter (MSGC), the wires and the support frame are } \\
\text { replaced by an insulator on which anode and cathode strips are photolithographically deposited. }\end{array}$ \\
\hline
\end{tabular}




\begin{tabular}{|c|c|c|c|}
\hline $\begin{array}{l}\text { Proposal } \\
\text { Number }\end{array}$ & $\begin{array}{l}\text { Authorized } \\
\text { Funding** }\end{array}$ & Principal Investigators & Title and Summary \\
\hline $96-196 \mathrm{~N}$ & $\begin{array}{l}\mathrm{FY} 96 / \$ 150 \mathrm{~K} \\
\mathrm{FY} 97 / \$ 160 \mathrm{~K} \\
\mathrm{FY} 98 / \$ 140 \mathrm{~K}\end{array}$ & $\begin{array}{l}\text { Barry Lai, } \\
\text { Wenbing Yun, } \\
\text { Efim Gluskin }\end{array}$ & $\begin{array}{l}\text { Development of Micromachining Technique Based on Deep-Etch X-Ray Lithography } \\
\text { We will study the application, including capabilities and processing issues, of a synchrotron source } \\
\text { such as APS to x-ray-lithography-based micromachining. The capability to fabricate } \\
\text { microelectromechanical systems is considered by many to be a key enabling technology. } \\
\text { Micromachining of miniature sensors, actuators, motors, optics, etc. will improve the performance } \\
\text { of existing devices, allow new devices to be designed and fabricated, and enable a higher level of } \\
\text { integration. The advantages of APS compared to existing sources include high x-ray flux (reduces } \\
\text { exposure time), high collimation and large distance from the source (lead to very high resolution } \\
\text { and as a result fabrication of extremely precise structures), and high energy (makes the fabrication } \\
\text { of ultra-thick structures possible). }\end{array}$ \\
\hline $96-197 N$ & $\begin{array}{l}\text { FY96/\$185K } \\
\text { FY97/\$185K }\end{array}$ & Robert Kustom & $\begin{array}{l}\text { Integrated mm-Wave if Amplifiers and Electron Linac Structures using LIGA Micromachining } \\
\text { Techniques } \\
\text { We will investigate the feasibility of building mm-wave if amplifiers on the same wafers as electron } \\
\text { linac structures using the LIGA (lithographic, galvanoformung, and abformung) micromachining } \\
\text { techniques. Some form of planar klystron or traveling wave structure that is suitable for LIGA } \\
\text { technology will be devised. The amplifiers will be used to directly power the linac cells so that an } \\
\text { external if power source and transmission waveguide system can be eliminated. A } 50 \text {-meV linac } \\
\text { with a 50-microsecond pulse operating at a one Hertz repetition rate will be used as a model. }\end{array}$ \\
\hline $96-199 N$ & FY96/\$120K & Stephen V. Milton & $\begin{array}{l}\text { Vacuum Chambers of Composite Materials } \\
\text { We will explore the use of composite materials and techniques in the construction of vacuum } \\
\text { chambers for particle accelerators. If successful, such a vacuum chamber would provide a more } \\
\text { versatile and cost effective alternative to the use of costly and fragile ceramic vacuum chambers. } \\
\text { Work will combine material compatibilities research for this application and development of } \\
\text { techniques. }\end{array}$ \\
\hline $96-200 N$ & $\begin{array}{l}F Y 96 / \$ 52 K \\
F Y 97 / \$ 225 K \\
F Y 98 / \$ 225 K\end{array}$ & $\begin{array}{l}\text { Robert L. Kustom, } \\
\text { Doug Horan }\end{array}$ & $\begin{array}{l}\text { Cathode Follower Amplifier Test on the IPNS Rapid Cycling Synchrotron } \\
\text { We will build and test a cathode follower amplifier (CFA) on the IPNS rapid cycling synchrotron. } \\
\text { A successful CFA, or some equivalent device, is necessary if a } 1 \mathrm{MW} \text { synchrotron-based pulsed } \\
\text { spallation neutron source is to be built. Success of this project would demonstrate a critical } \\
\text { technology needed for accelerators to be used as sources for } 1 \mathrm{MW} \text { spallation neutron targets. }\end{array}$ \\
\hline $96-201 N$ & FY96/\$125K & $\begin{array}{l}\text { L. Moos, C. Griffin, } \\
\text { N. Meldgin, N. Beskid }\end{array}$ & $\begin{array}{l}\text { Soil Washing/Chemical Extraction to Remediate Lead Contaminated Soils at ANL-E Site } 832 \\
\text { We will develop and utilize innovative soil washing and chemical extraction techniques and verify } \\
\text { the effectiveness of these techniques by removing lead from the soil at a small RCRA (Resource } \\
\text { Conservation and Recovery Act). solid waste management unit at ANL-E, achieving complete } \\
\text { remediation of the unit. Many. facilities within the DOE complex are embarking on the remediation } \\
\text { phase of their environmental projects. Soil washing and chemical extraction of heavy metals from } \\
\text { high clay content soils is an innovative remedial technology which can dramatically reduce waste } \\
\text { volumes and remediation costs. }\end{array}$ \\
\hline
\end{tabular}




\begin{tabular}{|c|c|c|c|}
\hline $\begin{array}{l}\text { Proposal } \\
\text { Number }\end{array}$ & $\begin{array}{l}\text { Authorized } \\
\text { Funding** }\end{array}$ & Principal Investigators & Title and Summary \\
\hline $96-202 \mathrm{~N}$ & $\begin{array}{l}\text { FY96/\$90K } \\
\text { FY97/\$90K }\end{array}$ & $\begin{array}{l}\text { Richard B. Vilim, } \\
\text { Humberto E. Garcia }\end{array}$ & $\begin{array}{l}\text { Predictive Maintenance Research } \\
\text { We will develop tests and evaluate predictive maintenance techniques for the nuclear power } \\
\text { industry. The industry is facing intense competitive pressures to decrease plant operations and } \\
\text { maintenance costs. This effort will provide proof of principle studies that could be used by the } \\
\text { industry to tailor predictive maintenance programs for reducing costs. }\end{array}$ \\
\hline $96-203 N$ & $\begin{array}{l}\text { FY96/\$250K } \\
\text { FY97/\$250K }\end{array}$ & John K. Bates & $\begin{array}{l}\text { Standard Test Method Development for Waste Forms } \\
\text { We will initiate development documentation and standardization of test methods to address the } \\
\text { waste form radionuclide release criteria for repository disposal as required by the Office of Civilian } \\
\text { Radioactive Waste Management (RW). The test methods will be applicable to DOE (EM) spent } \\
\text { fuel, other non-glass waste forms derived from DOE spent fuel and waste forms developed for } \\
\text { disposal of surplus plutonium. These methods would then form the basis for gaining a mechanistic } \\
\text { understanding of processes that control long-term behavior which is necessary in building technical } \\
\text { and regulatory confidence in the performance of these waste forms. The test methods will be } \\
\text { standardized through presentation to the ASTM Committee C26-13 on High Level Waste Disposal. }\end{array}$ \\
\hline $96-204 N$ & $\begin{array}{l}\text { FY96/\$195K } \\
\text { FY97/\$75K }\end{array}$ & $\begin{array}{l}\text { J. L. Willit, } \\
\text { Z. Tomczuk, } \\
\text { W. E. Miller }\end{array}$ & $\begin{array}{l}\text { Precipitation of Metal Nitrides from Chloride Melts } \\
\text { We will investigate the recovery of actinide, lanthanide, and transition metals from molten chloride } \\
\text { melts by formation of nitride precipitates. Thermodynamic equilibrium calculations predict that } \\
\text { metal chloride concentrations can be decreased to sub-ppm levels by this method. Existing } \\
\text { filtration techniques or a submersible centrifuge could be used to separate the nitride precipitate } \\
\text { from the molten salt. One of the unique aspects of nitride formation is the possibility of removing } \\
\text { strontium from molten salts. Thus there is a strong potential for removing one of the major heat } \\
\text { producers as well as rendering these salts as non-transuranic waste. The recovered nitrides can } \\
\text { then be converted to oxides, or the metals can be recovered by electrotransport. If high salt } \\
\text { decontamination levels can indeed be achieved, this approach could be used as a final cleanup } \\
\text { step in treating any waste salt from processing EBR-II spent fuel, molten salt reactor experiment } \\
\text { (MSRE) salt, and molten salt extraction (MSE) salts. }\end{array}$ \\
\hline $96-205 N$ & $\begin{array}{l}\text { FY96/\$300K } \\
\text { FY97/\$600K }\end{array}$ & $\begin{array}{l}\text { Robert W. Benedict, } \\
\text { Brian R. Westphal }\end{array}$ & $\begin{array}{l}\text { Metal Volatility in Waste Processes } \\
\text { Vapor phase metal behavior is an important parameter in the design and operation of high } \\
\text { temperature waste processes such as incineration, vitrification, smelting, and plasma treatment. We } \\
\text { will pursue a fundamental understanding of important process parameters which affect the metal } \\
\text { volatility provides an opportunity to optimize integrated waste treatment processes including the } \\
\text { associated off-gas handling system. }\end{array}$ \\
\hline
\end{tabular}




\begin{tabular}{|c|c|c|c|}
\hline $\begin{array}{l}\text { Proposal } \\
\text { Number }\end{array}$ & $\begin{array}{l}\text { Authorized } \\
\text { Funding*** }\end{array}$ & Principal Investigators & Title and Summary \\
\hline $96-206 \mathrm{~N}$ & FY96/\$200K & $\begin{array}{l}\text { Scott E. Carpenter, } \\
\text { Gerald T. Reedy }\end{array}$ & $\begin{array}{l}\text { Development of a No-Moving-Parts Fourier Transform Infrared (FTIR) Sensor using an Uncooled } \\
\text { Microbolometer Focol Plane Array Detector } \\
\text { We will investigate the possibility of creating a novel Fourier transform infrared (FTIR) sensor with } \\
\text { no moving parts. This passive remote FIIR sensor would utilize a state-of-the-art, uncooled } \\
\text { microbolometer focal plane array (FPA) detector. The sensor would operate in the spectral } \\
\text { wavelength region of } 8-12 \text { microns and provide noninvasive detection of chemical vapors at } \\
\text { distances of a few meters to several kilometers. Unlike existing FTIR sensors, which employ a } \\
\text { moving mirror design to temporally disperse interferometric information, this sensor will have no } \\
\text { moving parts and will spatially disperse interferometric information onto a two-dimensional FPA } \\
\text { detector. The new FTIR sensor would be extremely rugged compared with existing instruments and } \\
\text { could be made into a small hand held unit or incorporated into commercial long-wave infrared (IR) } \\
\text { imaging cameras. }\end{array}$ \\
\hline $96-207 \mathrm{~N}$ & FY96/\$122K & $\begin{array}{l}\text { J. J. Sienicki, } \\
\text { C. C. Chu, } \\
\text { B. W. Spencer }\end{array}$ & $\begin{array}{l}\text { Development and Validation of Atomizer Model for Analysis of Spray Forming Manufacturing } \\
\text { We will create an analytical capability to help in efforts to develop the spray forming } \\
\text { manufacturing process by creating an atomizer model for atomizers of the type used in spray } \\
\text { forming, validating the model with relevant data and using the model to investigate how atomizer } \\
\text { performance can be optimized through selection of design parameters and operating conditions. }\end{array}$ \\
\hline $96-208 \mathrm{~N}$ & FY96/\$176K & $\begin{array}{l}\text { D. Crites, R. Clarksean, } \\
\text { G. Teske }\end{array}$ & $\begin{array}{l}\text { Enhanced End Effector Control of Telerobotic Manipulators for Improved Spent Fuel Handling } \\
\text { An automated means of correcting the end effector position of a robot will be developed using a } \\
\text { vision system in an effort to automate some remote handling tasks associated with conditioning } \\
\text { spent nuclear fuel. The method involves digitizing video from cameras mounted on a remote } \\
\text { robot. Two or three cameras will be used, each containing in the field of view the robot end } \\
\text { effector and an item of known length to be used as a reference. Knowing the reference length and } \\
\text { the pixel count of the image, the location of the end effector will be determined relative to a target } \\
\text { through triangulation. This method is somewhat similar to that used by the Global Positioning } \\
\text { System for identifying locations on earth from satellite information. The target may be some } \\
\text { intermediate location on a trajectory the end effector makes from its beginning to ending location. } \\
\text { Feeding this information back into the control loop will provide an accurate assessment of end } \\
\text { effector location and alleviate accumulated positional error. }\end{array}$ \\
\hline
\end{tabular}




\begin{tabular}{|c|c|c|c|}
\hline $\begin{array}{l}\text { Proposal } \\
\text { Number }\end{array}$ & $\begin{array}{l}\text { Authorized } \\
\text { Funding** }\end{array}$ & Principal Investigators & Title and Summary \\
\hline $96-210 N$ & $\begin{array}{l}\text { FY96/\$100K } \\
\text { FY97/\$175K }\end{array}$ & $\begin{array}{l}\text { M. Lewis, } \\
\text { M. Antonio, } \\
\text { R. Chiarello, } \\
\text { L. Leibowitz }\end{array}$ & $\begin{array}{l}\text { EXAFS Investigation of Transmutation Effects on Waste Form Stability } \\
\text { Identification of potential instabilities in nuclear waste forms using synchrotron radiation and } \\
\text { extended X-ray absorption fine structure (EXAFS). Nuclear waste forms contain cesium-137 and } \\
\text { strontium-90. These radioisotopes decay to barium-137 and zirconium-90 (via yttrium-90), } \\
\text { respectively. The transmutation may affect waste form stability because of the change in ionic } \\
\text { radius and valence. The stable near-neighbor environment for cesium and strontium may be } \\
\text { different from that of their daughters. EXAFS is an element-specific technique which yields } \\
\text { information on the distance, number, and chemical type of near neighbors of a central (excited) } \\
\text { atom. Therefore, it is an ideal technique for determining how fission products are chemically } \\
\text { bonded within the waste form and for identifying potential instabilities. Differences in the } \\
\text { preferred coordination shells for cesium/barium and strontium/zirconium (via yttrium) can be } \\
\text { quantified and instabilities predicted on a rational, thermodynamic basis. This information is } \\
\text { necessary to address concerns regarding waste form stability over long times. Investigators will also } \\
\text { develop and employ three complementary, leading-edge data acquisition techniques to overcome } \\
\text { anticipated experimental difficulties concerning cesium/barium. }\end{array}$ \\
\hline $96-211 N$ & $\begin{array}{l}\mathrm{FY} 96 / \$ 75 \mathrm{~K} \\
\mathrm{FY} 97 / \$ 150 \mathrm{~K} \\
\mathrm{FY} 98 / \$ 150 \mathrm{~K}\end{array}$ & Ezzat Doss & $\begin{array}{l}\text { An Expert System Simulator (ESS) for the Manufacturing Industry } \\
\text { We will develop a real-time computer-based expert system simulator that can simulate discrete } \\
\text { events such as those arising in the manufacturing industry, power plant operation, system } \\
\text { maintenance, etc. This computer capability will improve efficiency in the simulation, design, } \\
\text { integration, and operation processes. Work includes the development of simulation and validation } \\
\text { procedures to support different discrete-event processes such as scheduling, inventory, fabrication } \\
\text { and assembly, material management, and information and control. }\end{array}$ \\
\hline $96-212 N$ & $\begin{array}{l}\mathrm{FY} 96 / \$ 92 \mathrm{~K} \\
\mathrm{FY} 97 / \$ 200 \mathrm{~K}\end{array}$ & $\begin{array}{l}\text { J. E. Cahalan, } \\
\text { E. E. Morris, } \\
\text { B. Shi, } \\
\text { R. N. Hill }\end{array}$ & $\begin{array}{l}\text { Nuclear Criticality of Fissionable Materials in the High-Level Waste Repository } \\
\text { We will assess the potential for migration and criticality of fissile materials initially buried in the } \\
\text { high-level waste repository (Yucca Mountain! and quantify the consequences of such criticality } \\
\text { events should they occur. Specifically, the focus of this work is to apply both limit (equilibrium) } \\
\text { and rate models to the coupled heat transfer and neutron kinetics phenomena that determine the } \\
\text { long-term and short-term power levels in a critical assembly of migrated fissile material. High } \\
\text { short-term power levels have been proposed as a mechanism for overcoming inherent negative } \\
\text { reactivity; feedback effects and for producing energetic, autocatalytic events. The validity of this } \\
\text { hypothesis, and the elements of alternative scenarios will be investigated with appropriate models. }\end{array}$ \\
\hline
\end{tabular}




\begin{tabular}{|c|c|c|c|}
\hline $96-244 \mathrm{~N}$ & $\begin{array}{l}\mathrm{FY} 96 / \$ 200 \mathrm{~K} \\
\mathrm{FY} 97 / \$ 210 \mathrm{~K} \\
\mathrm{FY} 98 / \$ 220 \mathrm{~K}\end{array}$ & $\begin{array}{l}\text { S. T. Pratt, } \\
\text { R. M. Miller, } \\
\text { J. D. Jastrow, } \\
\text { J. A. Manning }\end{array}$ & $\begin{array}{l}\text { Applications of High Brilliance X-Ray Radiation from Synchrotron Sources to Environmental } \\
\text { Science, Technology, and Management } \\
\text { The unique attributes of x-ray synchrotron radiation from sources such as the Advanced Photon } \\
\text { Source are expected to result in exceptional technological advances in each of the major } \\
\text { techniques of } x \text {-ray physics - spectroscopy, scattering, and imaging. As a result, significant } \\
\text { advances in environmental science can be anticipated, particularly in understanding } \\
\text { structures-function relationships and processes occurring in complex, heterogeneous media. Initial } \\
\text { experimental work for this project is directed toward understanding the processes by which } \\
\text { polyvalent cations appear to form bridges between negatively charged clay particles and negatively } \\
\text { charged polysaccharides. This phenomenon is believed to be fundamental to the formation of soil } \\
\text { microaggregates. By using existing available synchrotron sources and by piggy-backing on existing } \\
\text { APS beamlines dedicated to other research areas we hope to establish synergistic links between } \\
\text { field-oriented research environmental research programs and the new generation of analytical tools } \\
\text { represented by the APS. }\end{array}$ \\
\hline $96-254 \mathrm{~N}$ & $\begin{array}{l}\text { FY96/\$175K } \\
\text { FY97/\$200K }\end{array}$ & $\begin{array}{l}\text { D. Singh, A. S. Wagh, } \\
\text { U. Balachandran }\end{array}$ & $\begin{array}{l}\text { Stabilization of Radionuclides in Ceramic Waste Forms by a Novel Electrochemical Approach } \\
\text { We will investigate the use of a novel electrochemical approach for stabilizing and solidifying } \\
\text { radionuclides (Ur, Pu, Sr, Cs) in crystalline ceramics and forming durable final waste forms for their } \\
\text { safe disposal. Because crystalline phosphate ceramics are ionic conductors, the contaminants may } \\
\text { be introduced into the phosphate ceramic crystal structure by electrochemical means.and } \\
\text { subsequently bound, ensuring that the contaminants will not only be chemically fixed by } \\
\text { crystallographic substitutions but also physically encapsulated. This technological approach, for } \\
\text { in-situ radionuclide stabilization, will be particularly attractive in the decontamination of } \\
\text { high-volume wastes such as soils, concrete debris, wastewater, sludges, and liquid wastes. }\end{array}$ \\
\hline $96-255 \mathrm{~N}$ & $\begin{array}{l}\text { FY96/\$130K } \\
\text { FY97/\$90K }\end{array}$ & $\begin{array}{l}\text { Jae K. Lee, } \\
\text { Haiping Su, } \\
\text { John R. Krummel }\end{array}$ & $\begin{array}{l}\text { Hyperspectral Remote Sensing for Environmental Remediation } \\
\text { We will develop a new method of analyzing hyperdimensional remotely sensed data which will be } \\
\text { the mainstream type of future environmental remote sensing data: develop a power analytical } \\
\text { approach of analyzing hyperspectral remotely sensed data to improve current technology } \\
\text { applications; provide a new analysis paradigm of utilizing data from sensors of the next generation; } \\
\text { and open a new application of the state-of-the-art remote sensing technology to environmental } \\
\text { remediation. }\end{array}$ \\
\hline $96-256 \mathrm{~N}$ & FY96/\$190K & $\begin{array}{l}\text { M. M. Thackeray, } \\
\text { D. Dees, } \\
\text { Jai Prakash }\end{array}$ & $\begin{array}{l}\text { Exploratory Development of High Power Density Ultracapacitors } \\
\text { We will develop new materials for use in ultracapacitors which are used for the rapid storage and } \\
\text { release of large quantities of energy. The development of these ultracapacitors would provide the } \\
\text { power needed for the quick acceleration in electric and hybrid vehicles. Load-leveling } \\
\text { characteristics of ultracapacitors have potential to make a significant impact not only on electric } \\
\text { vehicle technologies but also on several military applications, such as electrically pulsed weapons } \\
\text { systems for air defense, artillery, and antiballistic missile weapons. }\end{array}$ \\
\hline
\end{tabular}




\begin{tabular}{|c|c|c|c|}
\hline $\begin{array}{l}\text { Proposal } \\
\text { Number }\end{array}$ & $\begin{array}{l}\text { Authorized } \\
\text { Funding** }\end{array}$ & Principal Investigators & Title and Summary \\
\hline $96-257 \mathrm{~N}$ & $\begin{array}{l}\text { FY96/\$185K } \\
\text { FY97/\$220K }\end{array}$ & S. Ahuja, A. C. Raptis & $\begin{array}{l}\text { Micromachining Technology - Intelligent Microsensor Fabrication } \\
\text { By micromachining for fabricating microsensors and microactuators, we will develop miniaturized } \\
\text { and intelligent microsensor assemblies capable of complete functionality in micromachines and } \\
\text { able to determine physical quantities such as temperature, pressure, flow, strain, etc. The } \\
\text { micromachining technology will utilize hybrid devices that incorporate silicon wafers and optical } \\
\text { fibers. The local measurement of important parameters ior automotive, industrial, biomedical, or } \\
\text { material applications will provide minimal loss of data, time, and effort in sensing while limiting } \\
\text { redundancy in instrumentation. The output signal format of the microassemblies will be } \\
\text { microprocessor compatible to different bus systems and will provide maximum output through } \\
\text { integrated circuits. These microassemblies will bring the utilizable information content to a higher } \\
\text { level than that available in any present device gaining interesting and useful information not } \\
\text { present in a collection of single sources. }\end{array}$ \\
\hline $96-305 \mathrm{~N}$ & $\begin{array}{l}F Y 96 / \$ 150 K \\
F Y 97 / \$ 210 K \\
\text { FY98/\$220K }\end{array}$ & S. T. Pratt & $\begin{array}{l}\text { Coherent Control of Molecular Physics and Chemistry } \\
\text { We will investigate novel techniques for the control of molecular photoionization and } \\
\text { photodissociation processes. Our approach relies on the quantum mechanical interference that } \\
\text { results when two or more pathways exist between indistinguishable initial and final states for a } \\
\text { given process. Unique aspects of this research are (1) control of photoionization and } \\
\text { photodissociation branching ratios rather than total cross sections or angular distributions; (2) a } \\
\text { more complete set of detection methods including photoelectron spectroscopy to determine } \\
\text { photoionization branching ratios; and (3) choice of competing excitation pathways with the } \\
\text { characteristics necessary to maximize control of the product state distributions. This work directly } \\
\text { addresses the ability to control and modify product state distributions of elementary chemical } \\
\text { processes and thus represents an advance toward the goal of laser-controlled chemistry. }\end{array}$ \\
\hline $96-306 \mathrm{~N}$ & $\begin{array}{l}\text { FY96/\$100K } \\
\text { FY97/\$100K }\end{array}$ & $\begin{array}{l}\text { Ross Overbeek, } \\
\text { Terry Gaasterland }\end{array}$ & $\begin{array}{l}\text { An Integrated Environment to Support Bioremediation Studies } \\
\text { We will develop an integrated environment of metabolic, phylogenetic, and sequence structure } \\
\text { information to support bioremediation studies. The environment will be based on Argonne's } \\
\text { PUMA system which provides access to biological data such as metabolic pathways and multiple } \\
\text { sequence alignments. }\end{array}$ \\
\hline $96-308 \mathrm{~N}$ & FY96/\$45K & $\begin{array}{l}\text { Bruce A. Carnes, } \\
\text { S. Jay Olshansky }\end{array}$ & $\begin{array}{l}\text { Biodemography: An Application to Human Life Expectancy } \\
\text { We will estimate the increase in life expectancy for humans that can be attributed to interventions } \\
\text { that delay deaths arising from intrinsic biological processes. This work is an application of theory - } \\
\text { referred to as the biodemographic paradigm - developed by the principal investigators. }\end{array}$ \\
\hline
\end{tabular}




\begin{tabular}{|c|c|c|c|}
\hline $96-310 \mathrm{~N}$ & $\begin{array}{l}\text { FY96/\$50K } \\
\text { FY97/\$150K }\end{array}$ & $\begin{array}{l}\text { David J. Grdina, } \\
\text { Jeffrey S. Murley, } \\
\text { Shie-Chau Liu }\end{array}$ & $\begin{array}{l}\text { Gene Expression Modulated by Amenothiols in Radiation Protection and Chemoprevention } \\
\text { We will develop the necessary database and infrastructure for a new effort in the emerging field of } \\
\text { molecular chemoprevention. Novel chemopreventive agents must be developed that are effective } \\
\text { in inhibiting the carcinogenic process. To facilitate the decision making process in identifying and } \\
\text { implementing effective agents for chemoprevention, an understanding of their underlying } \\
\text { mechanisms of action is required. Work under this project will be to: (1) determine whether the } \\
\text { Tk, PKC-delta, Catalase, CST, Cyclin D, or SOD genes are differentially expressed in cells exposed } \\
\text { to radiation and/or treated with WR-1065 using the identified vectors for Northern analysis; } \\
\text { (2) determine whether the differential expression of these genes is related to the concentration of } \\
\text { WR-1065 that the cells are treated with; (3) confirm differential gene expression by DNA dot blot; } \\
\text { (4) sequence the identified genes and compare these with known sequences in DNA data banks as } \\
\text { additional confirmation. }\end{array}$ \\
\hline $96-311 N$ & FY96/\$250K & $\begin{array}{l}\text { T. Surles, C. Reilly, } \\
\text { S.-W. Tam, K. Kasza }\end{array}$ & $\begin{array}{l}\text { Environimental Management Scientific Research Initiative: Proof of Concept Research } \\
\text { Our effort will focus on four areas: (1) sensor research, (2) separations, extractions, surface } \\
\text { phenomena, (3) waste form research/physical and chemical properties of radionuclides in various } \\
\text { media, and (4) bioremediation and related contaminant fate and transport research. In this } \\
\text { connection, we will study the mechanism of transport of contaminants in fractured rock, both } \\
\text { theoretically and experimentally. We will initiate a study of the chemistry of radionuclides in } \\
\text { alkaline media. And, we will develop sensors applicable to monitoring high-level waste tanks } \\
\text { using novel electrochemical designs. }\end{array}$ \\
\hline $96-312 \mathrm{~N}$ & $\begin{array}{l}\text { FY96/\$260K } \\
\text { FY97/\$450K }\end{array}$ & $\begin{array}{l}\text { D. S. Gemmell, } \\
\text { E. P. Kanter, } \\
\text { T. LeBrum, } \\
\text { S. H. Southworth }\end{array}$ & $\begin{array}{l}\text { Inner-Shell Atomic Structure and Dynamics with High-Energy X-Rays from the APS } \\
\text { We will investigate problems in inner shell atomic structure and dynamics (and related molecular } \\
\text { phenomena) using synchrotron radiation with particular emphasis on problems unique to the } \\
\text { hard-X-ray regime and establish a forefront experimental atomic physics program at the Advanced } \\
\text { Photon Source. In particular, investigators will exploit the high brilliance, polarization and } \\
\text { coherence properties that are expected for X-rays in the } 2.5 \text { to } 100-\mathrm{keV} \text { energy range. }\end{array}$ \\
\hline $96-313 \mathrm{~N}$ & $\begin{array}{l}\text { FY96/\$50K } \\
\text { FY97/\$350K }\end{array}$ & Stephen J. Riley & $\begin{array}{l}\text { The Production of Continuous Beams of Transition Metal Clusters for Studies at the APS } \\
\text { It has been demonstrated that a high-repetition-rate vaporization laser can be used for the } \\
\text { generation of beams of transition metal clusters. These beams are continuous in that they show no } \\
\text { intensity modulation due to the } 5 \mathrm{kHz} \text { vaporization cycle. However, there is some as yet not } \\
\text { understood aspect of the vaporization/cluster growth process under high average power conditions } \\
\text { that leads to cluster beams with substantial shot-to-shot intensity variations. The physics of this } \\
\text { process will be studied under this project in order to understand the source of these instabilities. } \\
\text { By eliminating them, it will become possible to generate truly continuous beams. Such continuous } \\
\text { beams will be essential for future X-ray absorption studies of cluster structure and } \\
\text { structure-reactivity relationships. }\end{array}$ \\
\hline
\end{tabular}

